
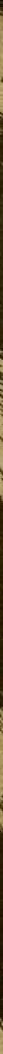

ghymandert

3
4
3
3

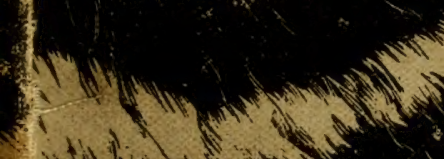

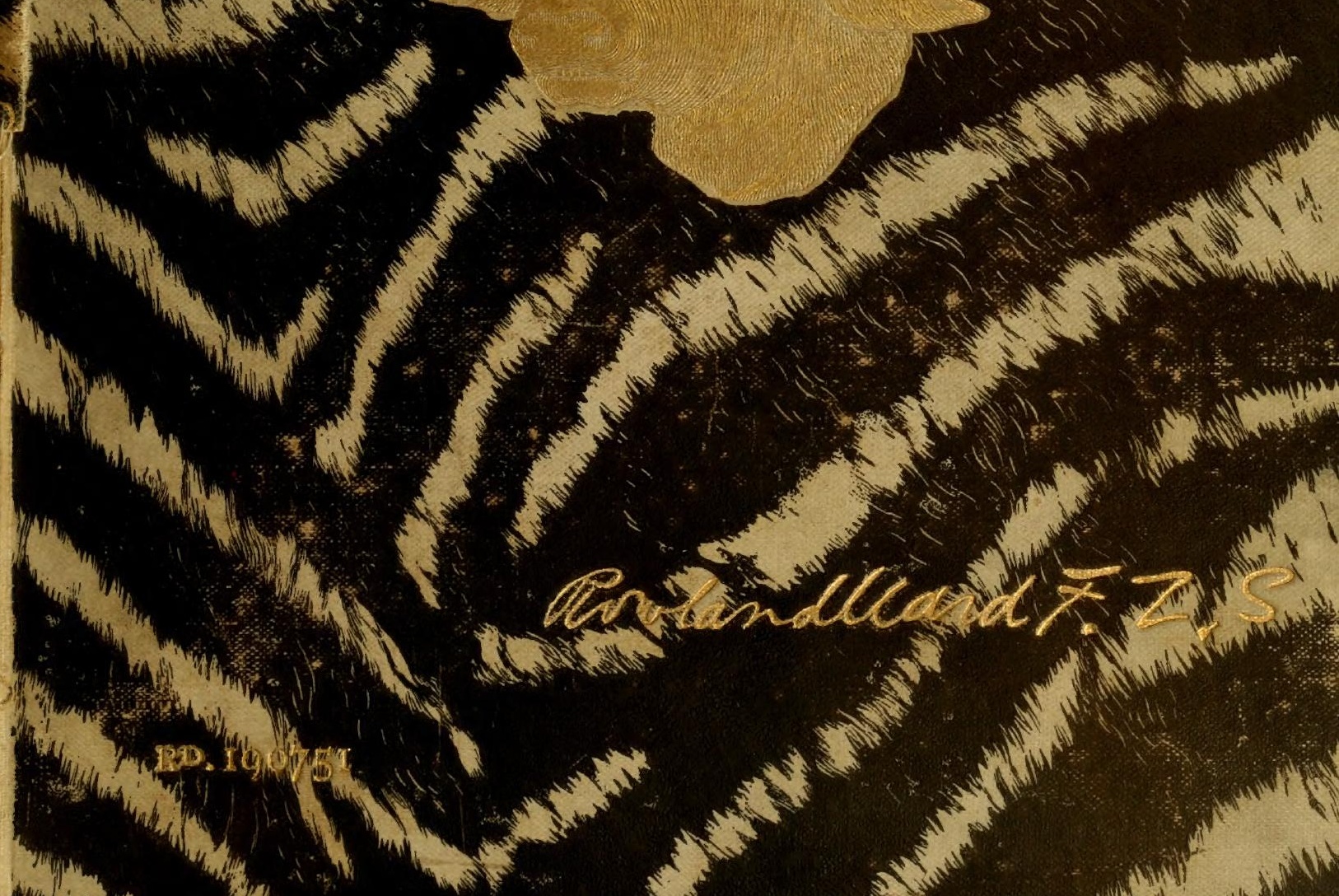


. 
HORN MEASUREMENTS

AND WEIGHTS

OF THE

GREAT GAME OF THE WORLD. 
$+20+4$ 
$44 \omega 26$

A N WEIGHTS,

OF THE

\section{GReat GaMe OF THE WORLD:}

BEING

A RECORD FOR THE USE OF SPORTSMEN AND NATURALISTS.
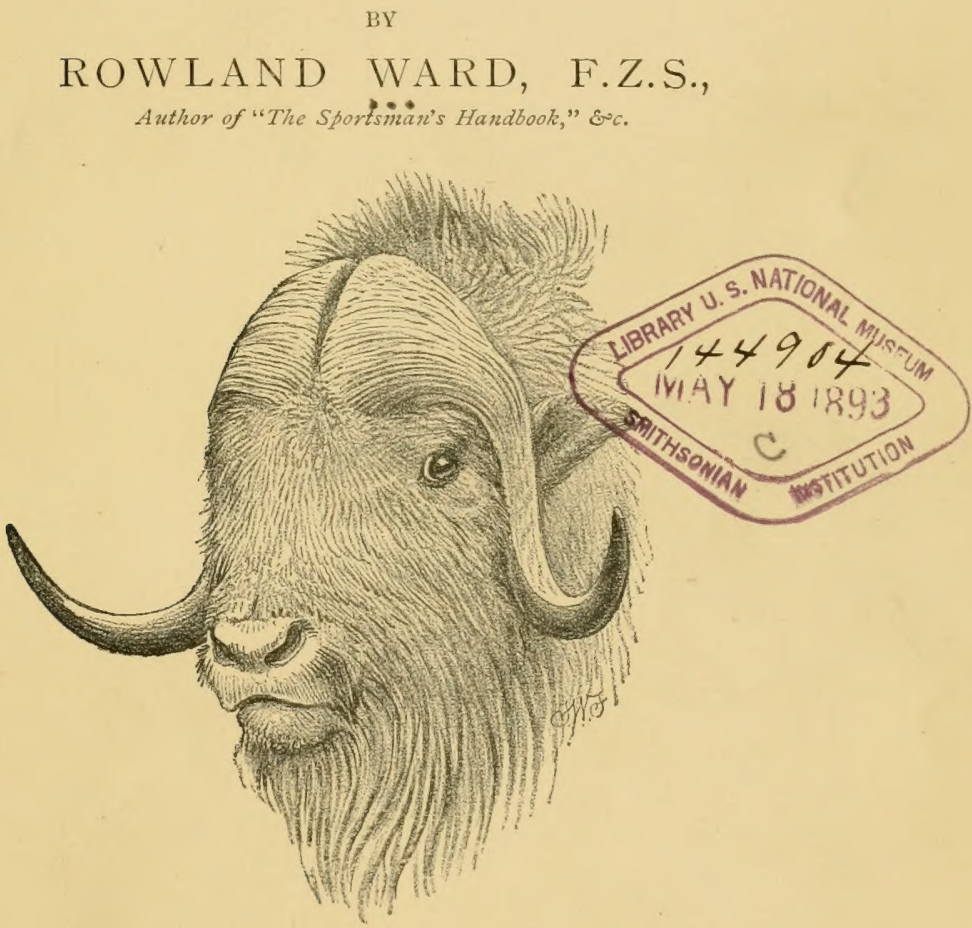

OVIBOS MOSCHATUS.

Londom:

AT “THE JUNGLE," I66, PICCADILLY.

AND OF ALL BOOKSELLERS.

I 892 . 

THIS BOOK IS DEDICATED

TO THE

SPORTSMEN OF THE WORLD,

WITHOUT WHOSE DETERMINED PLUCK AND LOVE OF TRAVEL IT COULD

NOT HAVE BEEN PRODUCED, NOR COULD I HAVE SPENT

A LIFE-TIME IN THE ART WHICH I HAVE

TRIED TO ADVANCE.

ROWLAND WARD. 


\section{PREFACE.}

W $\mathrm{Y}$ object in producing this book is to start a record 1 of Horn Measurements of the Great Game of the World. I only regret that I did not commence it at an earlier date, as it would then have been more complete. But in my earlier life I had but little help, and often worked thirty hours at a stretch; my work necessitated attention to specimens that demanded immediate treatment, and my love for reproducing life-like studics prevented me from keeping records.

The measurements presented here have been taken recently by one hand and, for that reason, I value them the more. The dimensions from acknowledged authorities I am not responsible for. This book is not designed to be in any way a scientific work, but it is prepared for gentlemen sportsmen and scientific men who are interested to see comparable measurements at a glance. I think these have not been produced in like form before. Of course, the record can be added to, and I shall feel indebted to sportsmen who will contribute measurements, for I am not 
producing this work in a completed form-it must grow. It is to be borne in mind that many trophies which have passed through my hands are now scattered all over the world, and the dimensions of them, for the time, are not recorded here. Some that I give now are fine measurements, some are only ordinary statistics, many are of new species altogether; these last being mainly a result of the quite recent opening up of Africa. The advice noted as to the way to measure must be dealt with strictly, for many persons measuring by different methods produce untrustworthy record. We have tried to be fair in producing the statistics, taking them rather under than over the mark; and the tape has not been pressed into corrugations, but carried outside, over all inequalities.

Many gentlemen's trophies I should like to have measured, but those specimens have either changed hands or are now in some remote part of the world, and the book cannot be delayed longer; but distance has not prevented me from obtaining many important statistics.

R. W. 


\section{ABBREVIATIONS AND SIGNS. \\ đ Male. $\quad$ f Female. $\quad \ldots \quad \ldots$... Unrecorded.}

LENGTH is expressed in inches, where not otherwise noted.

Mensurements are on the outside of horn from tip to base. Circumference is at the base; with Deer above first tine.

Memo.-Horns that possess a core have a tendency to shrink.

Weights taken on the field should be accepted as approximate.

P.Z.S., Proceedings of the Zoological Society. 


ERRATA.

\begin{tabular}{|c|c|c|c|c|}
\hline agge & I2 for & "pyaargus" & read & "pygargus." \\
\hline & & "Naivashoe" & & asha." \\
\hline & So & "saltianis" & & “saltianus." \\
\hline & 126 & "hars" & & "ears." \\
\hline & 126 & "sxmmerringii" & , ,, & "søemmerringii." \\
\hline & 150 & "asomaculatus" & , & "'nasomaculatus." \\
\hline
\end{tabular}




\title{
HORN MEASUREMENTS
}

\author{
A ND WEIGHTS
}

OF THE

\section{GREAT GAME OF THE WORLD.}
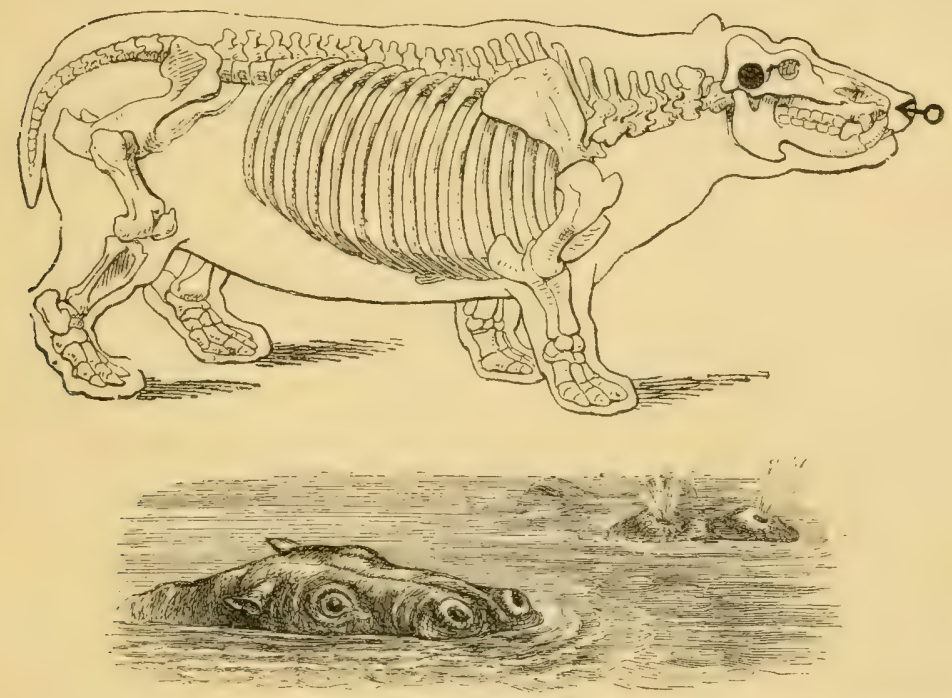

HIPPOPOTAMUS TUSKS (Hippopotamus amphibius).

Habitat-Africa.

\begin{tabular}{|c|c|c|c|c|c|}
\hline $\begin{array}{l}\text { Length round } \\
\text { outside curve. }\end{array}$ & $\begin{array}{l}\text { Circum- } \\
\text { ference. }\end{array}$ & $\begin{array}{l}\text { Across } \\
\text { inside. }\end{array}$ & Habitat. & & Owner. \\
\hline $\begin{array}{l}31 \frac{5}{8} \\
30 \frac{1}{2} \\
30 \\
29 \\
27 \frac{1}{8} \\
27 \frac{1}{8} \\
27 \\
26 \frac{1}{4} \\
25 \frac{1}{8} \\
25 \\
24 \frac{1}{4} \\
23 \frac{1}{4} \\
19 \frac{7}{8}\end{array}$ & $\begin{array}{l}\left.\begin{array}{l}9 \frac{1}{8} \\
9 \frac{1}{8} \\
9\end{array}\right\} * \\
99^{\frac{1}{2}} \\
\cdots \\
8 \\
7 \frac{1}{2} \\
7 \frac{1}{2} \\
7 \frac{1}{4} \\
7 \\
7 \\
\cdots\end{array}$ & $\begin{array}{l}\ldots \\
\ldots \\
14 \\
14 \\
\ldots \\
\ldots \\
12 \frac{1}{2} \\
\ldots \\
12 \frac{1}{3} \\
12 \frac{1}{4} \\
12 \frac{1}{4} \\
\ldots\end{array}$ & $\begin{array}{l}\begin{array}{l}\text { St. Lucia Bay } \\
\text { Do. }\end{array} \\
\text { South Africa } \\
\text { East Africa } \\
\ldots \\
\text { South Africa } \\
\ldots \quad \ldots \\
\ldots \\
\text { E... } \quad \ldots \\
\text { East Africa }\end{array}$ & $\begin{array}{l}\cdots \\
\cdots \\
\cdots \\
\cdots \\
\cdots \\
\cdots \\
\cdots \\
\cdots \\
\cdots \\
\cdots \\
\cdots \\
\cdots\end{array}$ & $\begin{array}{l}\text { Sir Edmund G. Loder, Bart. } \\
\text { Hon. Charles Ellis, I } 870 . \\
\text { Do. } \\
\text { Rowland Ward's Collection. } \\
\text { The late J. S. Jameson. } \\
\text { W. D. James. } \\
\text { E. Gedge. } \\
\text { G. Portal. } \\
\text { The Late J. S. Jameson. } \\
\text { G. Portal. } \\
\text { Do. } \\
\text { Do. } \\
\text { Sir John Kirk, M. D., K.C. B. }\end{array}$ \\
\hline
\end{tabular}

* Weight, $15 \mathrm{lbs}$. the pair. 


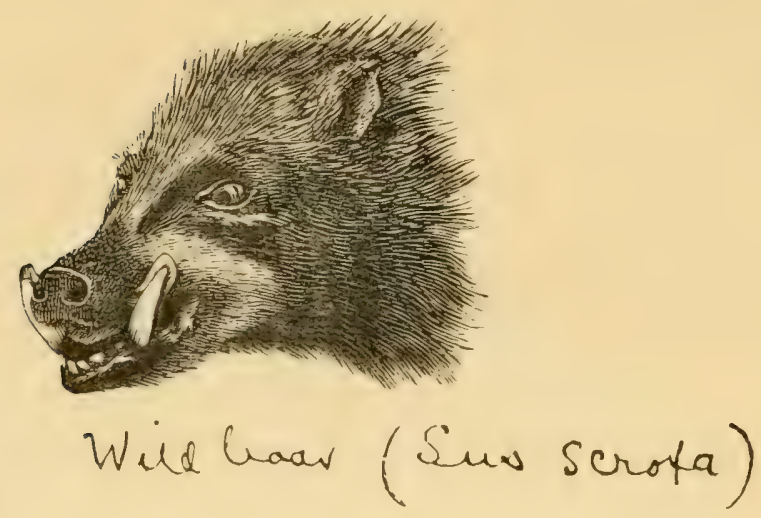

WILD BOAR TUSKS.

Habitat-Europe, Asia Minor, and North Africa.

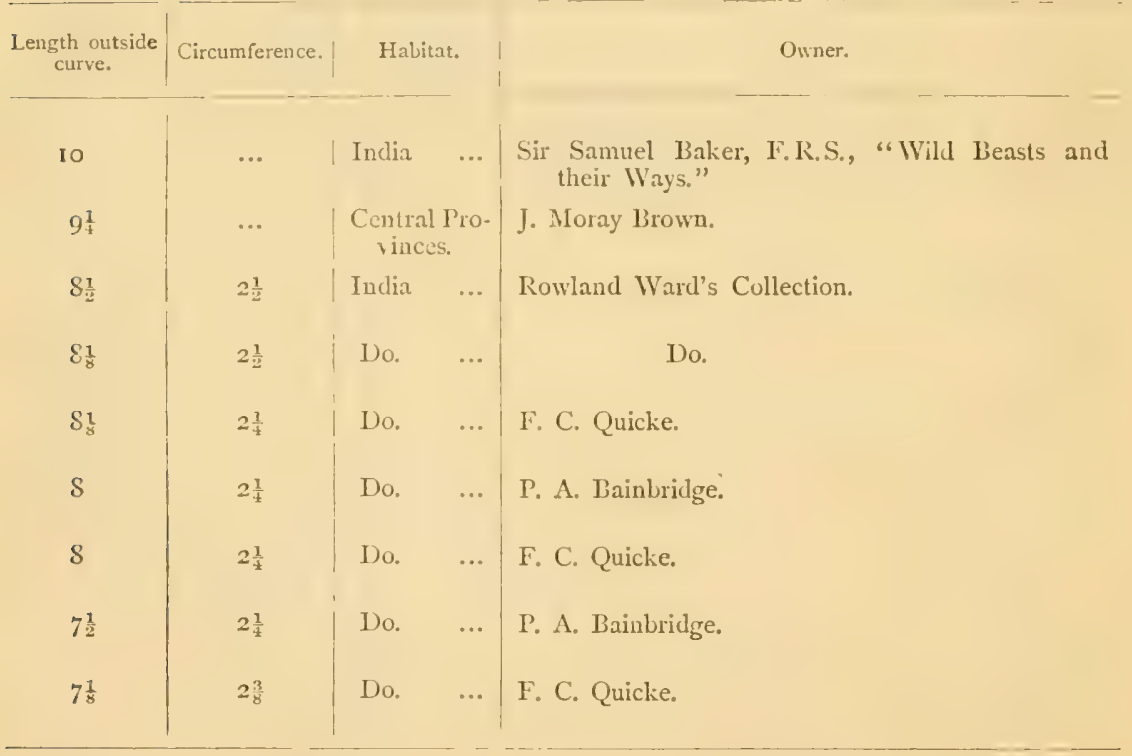



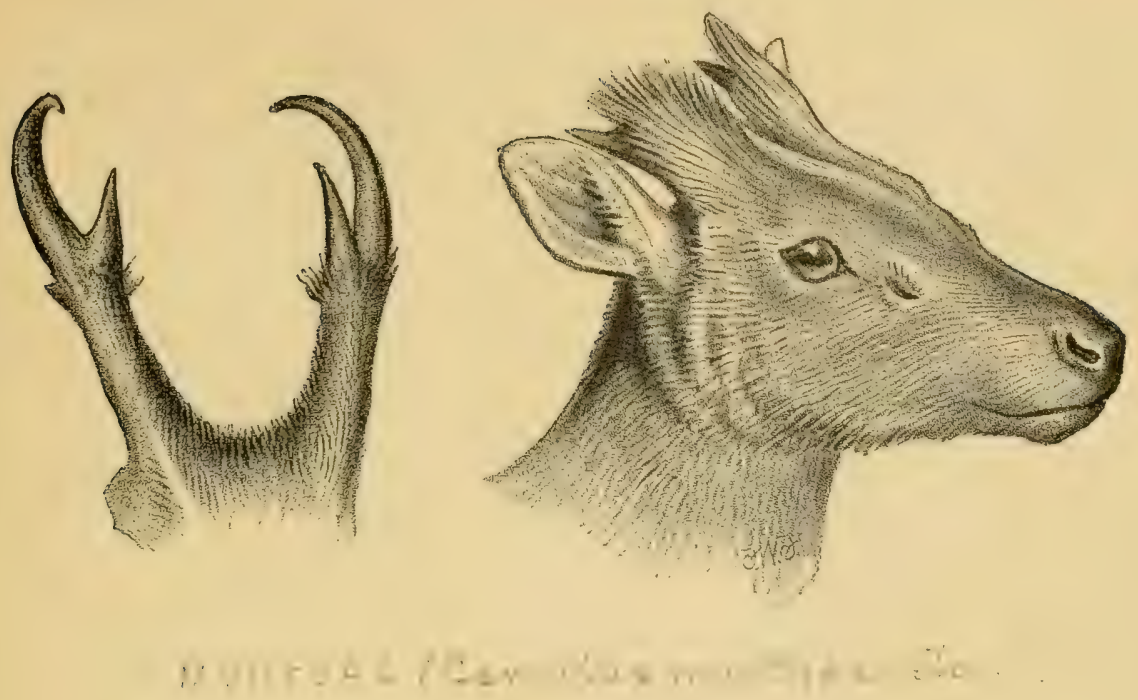

Habitat-British India, Burmah, Malay Peninsula, Java, Sumatra, Borneo, \&c.

\begin{tabular}{|c|c|c|c|c|c|c|}
\hline I ength. & $\begin{array}{l}\text { Circum- } \\
\text { ference. }\end{array}$ & Tip to Tip. & \multicolumn{3}{|c|}{ Habitat. } & Owner. \\
\hline$* 6 \frac{1}{2}$ & $2 \frac{5}{8}$ & $3 \frac{1}{2}$ & $\ldots$ & $\ldots$ & $\ldots$ & B. H. Hodgson, Bıitish Museum. \\
\hline $6 \frac{1}{2}$ & 3 & $3 \frac{3}{8}$ & ... & $\ldots$ & $\ldots$ & A. O. IItume, Private Collection. \\
\hline$* 5 i$ & $2 \frac{3}{4}$ & $3 \frac{1}{t}$ & $\ldots$ & $\ldots$ & $\ldots$ & B. H. Ilorgson, British Museum. \\
\hline $5 \frac{3}{8}$ & $2 \frac{1}{4}$ & $3 \frac{1}{8}$ & $\ldots$ & $\ldots$ & $\ldots$ & IIume Collection, British Museum. \\
\hline $5 \frac{1}{4}$ & 2 & $3 \frac{1}{8}$ & \multicolumn{2}{|c|}{. S. India } & $\ldots$ & $\begin{array}{l}\text { Sir Victor Brooke's Collection., (Shot } \\
\text { by Col. Douglas Hamilton.) }\end{array}$ \\
\hline 5 & $1 \frac{7}{8}$ & 2 & \multicolumn{2}{|l|}{ Do. } & $\ldots$ & Do. \\
\hline $4 \frac{7}{8}$ & $2 \frac{1}{4}$ & $3 \frac{3}{8}$ & \multicolumn{2}{|l|}{ Do. } & $\cdots$ & Do. \\
\hline $4 \frac{3}{4}$ & $I \frac{1}{2}$ & I $\frac{5}{8}$ & $\cdots$ & $\cdots$ & $\cdots$ & Hume Collection, British Museum. \\
\hline $3 \frac{7}{8}$ & $2 \frac{3}{8}$ & $3 \frac{5}{8}$ & $\cdots$ & $\cdots$ & $\ldots$ & Do. \\
\hline$* 3 \frac{1}{t}$ & $I_{0}^{3}$ & $2 \frac{7}{8}$ & ... & $\ldots$ & $\ldots$ & B. II. Hotgson, British Museum. \\
\hline$\nmid 2 \frac{1}{3}$ & $I_{8}^{5}$ & 3 & Ningpo & $0 .$. & $\ldots$ & R. Swinhoe, British Museum. \\
\hline $\pm 1 \frac{1}{2}$ & ... & $4 \frac{1}{2}$ & Do. & $\ldots$ & $\ldots$ & A. Mitchie, British Muscum. \\
\hline
\end{tabular}




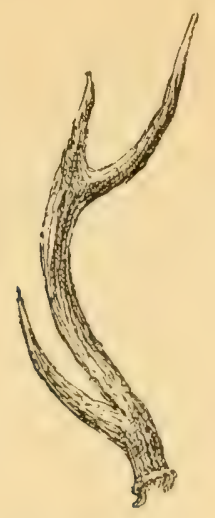

SAIMBUR (Cervus aristotelis).

IV cight -6oo lbs. (about); I $3 \frac{1}{2}$ hands at shoulder.-Sir Samuel Baker, "Wild Beasts and their Ways." Habitat-India, Burmah, and China.

\begin{tabular}{|c|c|c|c|c|c|c|c|}
\hline $\begin{array}{l}\text { Length } \\
\text { round } \\
\text { outside. }\end{array}$ & 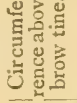 & Tip to Tip. & $\begin{array}{c}\text { WVidest } \\
\text { inside. }\end{array}$ & Points. & Habitat. & & Owner. \\
\hline $4^{6 \frac{1}{2}}$ & $6 \frac{3}{8}$ & $24 \frac{1}{8}$ & $3 \circ \frac{7}{8}$ & $3 \times 3$ & $\cdots$ & $\ldots$ & A. O. Hume, Private Collection. \\
\hline $45 \frac{1}{8}$ & $6 \frac{5}{8}$ & $17 \frac{3}{4}$ & $32 \frac{1}{8}$ & $3 \times 3$ & Indlia ... & ... & Dr. Falconer, British Museum. \\
\hline 45 & $7 \frac{3}{8}$ & $22 \frac{1}{2}$ & $33 \frac{3}{4}$ & $3 \times 3$ & $\begin{array}{l}\text { Mayoghur, C } \\
\text { Provinces. }\end{array}$ & ent. & Sir John Morris, K.C.S.I. \\
\hline 45 & $7 \frac{1}{4}$ & $44 \frac{7}{8}$ & $\cdots$ & $3 \times 3$ & $\cdots$ & ... & Major-Gen. Arthur Ellis, C.S.I. \\
\hline $44 \frac{1}{8}$ & $7 \frac{7}{8}$ & $44 \frac{3}{8}$ & $45^{\frac{7}{8}}$ & $3 \times 3$ & $\begin{array}{r}\text { Rangeer, Ce } \\
\text { Provinces. }\end{array}$ & & \\
\hline 44 & & Dwners' meast & Irement & & $\cdots$ & $\cdots$ & J. D. Invararity. \\
\hline $43 \frac{5}{8}$ & $5 \frac{5}{8}$ & (single horn) & $\cdots$ & 3 & $\cdots$ & $\cdots$ & Hume Collection, British Museum. \\
\hline $43^{\frac{1}{2}}$ & $6 \frac{1}{8}$ & (single horn) & $\cdots$ & 3 & $\cdots$ & $\cdots$ & Do. \\
\hline $43 \frac{3}{8}$ & $6 \frac{1}{4}$ & $20 \frac{3}{5}$ & $29 \frac{1}{8}$ & $3 \times 3$ & $\cdots$ & $\cdots$ & Sir Robert Harvey, Bart. \\
\hline $42 \frac{1}{2}$ & $6 \frac{1}{4}$ & 15 & $27^{\frac{3}{4}}$ & $4 \times 4$ & $\begin{array}{c}\text { Ghâuts of Si } \\
\text { rol. }\end{array}$ & & Col. I. Evans, British Museum. \\
\hline $41 \frac{7}{8}$ & $6 \frac{1}{4}$ & $33 \frac{1}{8}$ & $35 \frac{1}{x}$ & $3 \times 3$ & $\cdots$ & $\cdots$ & Major-Gen. Arthur Ellis, C.S.I. \\
\hline $4 \mathrm{I}^{\frac{3}{4}}$ & 7 & $28 \frac{1}{4}$ & $34 \frac{3}{4}$ & $3 \times 3$ & $\cdots$ & $\cdots$ & Hon. Walter Rothschild. \\
\hline $41 \frac{5}{8}$ & $7 \frac{1}{8}$ & $3 \mathrm{I} \frac{3}{4}$ & $3^{6 \frac{3}{4}}$ & $4 \times 3$ & Nepal... & $\cdots$ & B. H. Hodgson, British Museum. \\
\hline $4 \mathrm{I} \frac{2}{8}$ & $7 \frac{1}{8}$ & (single horn) & $\cdots$ & 5 & $\ldots$ & $\cdots$ & British Museum. \\
\hline
\end{tabular}


SAIMBUR (Cervus aristotelis)-continucd.

\begin{tabular}{|c|c|c|c|c|c|c|}
\hline $\begin{array}{l}\text { Length } \\
\text { round } \\
\text { outside. }\end{array}$ & 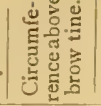 & Tip to Tip. & $\begin{array}{l}\text { Widest } \\
\text { inside. }\end{array}$ & Points. & Habitat. & Owner. \\
\hline $4^{I}\{$. & $\begin{array}{r}8 \frac{1}{2} \mathrm{a} \\
10 \frac{1}{2} \mathrm{r}\end{array}$ & $\left.\begin{array}{l}\text { ove burr } \\
\text { und },,\end{array}\right\}$ paln & mated & $3 \times 3$ & North Gujerat & $\left\{\begin{array}{c}\text { S. C. Lex. } \\
\mathbf{1} 869 .)\end{array} \quad\right.$ (The Field, Oct. 23, \\
\hline $40 \frac{1}{2}$ & $5 \frac{5}{8}$ & 33 & $37 \frac{1}{\ddagger}$ & $3 \times 3$ & $\cdots \quad \cdots$ & Hume Collection, British Museum. \\
\hline $40 \frac{1}{t}$ & $6 \frac{1}{4}$ & $28 \frac{1}{8}$ & 32 & $3 \times 3$ & Neilgherries ... & $\begin{array}{l}\text { Sir Victor Brooke's Collection. } \\
\text { (Shot by Col. Doug. Hamilton.) }\end{array}$ \\
\hline 40 & 7 & $I I \frac{1}{2}$ & $24 \frac{1}{4}$ & $3 \times 3$ & $\cdots \quad \cdots$ & Rowland Ward's Collection. \\
\hline 40 & $5 \frac{7}{8}$ & $32 \frac{1}{4}$ & 36 & $3 \times 3$ & $\begin{array}{l}\text { Ghâuts of Sim- } \\
\text { rol. }\end{array}$ & Col. J. Evans, British Museum. \\
\hline $39 \frac{5}{8}$ & $6 \frac{3}{8}$ & (single horn) & $\cdots$ & 3 & \begin{tabular}{|lll}
$\cdots$ & $\cdots$ & $\cdots$
\end{tabular} & British Museum. \\
\hline $39 \frac{1}{7}$ & $7 \frac{1}{2}$ & $20 \frac{1}{2}$ & $26 \frac{3}{4}$ & $3 \times 4$ & $\cdots \quad \cdots$ & Hume Collection, British Museum. \\
\hline $39 \frac{1}{4}$ & 6 & $16 \frac{1}{2}$ & $20 \frac{1}{2}$ & $2 \times 2$ & $\begin{array}{lll}\cdots & \cdots & \cdots\end{array}$ & H.R.H. Duke of Edinburgh. \\
\hline 39 & $7 \frac{1}{2}$ & 24 & $\cdots$ & $4 \times 4$ & $\begin{array}{lll}\cdots & \cdots & \cdots\end{array}$ & Capt. Henry Streatfield. \\
\hline $3^{8 \frac{7}{8}}$ & $6 \frac{7}{8}$ & (single horn) & $\cdots$ & $\cdots$ & $\ldots \quad \ldots$ & British Museum. \\
\hline $38 \frac{3}{4}$ & $6 \frac{3}{4}$ & 24 & $29 \frac{1}{2}$ & $3 \times 3$ & $\cdots \quad \cdots$ & Major-Gen. Arthur Ellis, C.S.I. \\
\hline $38 \frac{5}{8}$ & 6 & $22 \frac{1}{2}$ & $30 \frac{1}{4}$ & $3 \times 3$ & $\cdots \quad \cdots$ & F. J. Horniman. \\
\hline $38 \frac{1}{2}$ & $6 \frac{33}{4}$ & $23 \frac{x}{t}$ & $28 \frac{7}{8}$ & $3 \times 3$ & $\cdots \quad \cdots$ & Sir Robert Harvey, Bart. \\
\hline $3^{8 \frac{1}{2}}$ & $5 \frac{1}{4}$ & 55 & $\cdots$ & $3 \times 3$ & $\begin{array}{lll}\cdots & \cdots & \cdots\end{array}$ & Major-Gen. Arthur Ellis, C.S.I. \\
\hline $3 S_{\frac{1}{8}}$ & $6 \frac{7}{8}$ & $30 \frac{1}{8}$ & 35 & $3 \times 3$ & Neilgherries... & $\begin{array}{l}\text { Sir Victor Brocke's Collection. } \\
\text { (Shot by Col. Doug. Hamilton.) }\end{array}$ \\
\hline $3^{8}$ & $6 \frac{1}{2}$ & $29 \frac{3}{4}$ & $4 \mathrm{I} \frac{3}{8}$ & $3 \times 3$ & $\begin{array}{l}\text { Moharli Forest, } \\
\text { Chanda District, } \\
\text { Cent. Provinces. }\end{array}$ & Sir John Morris, K.C.S.I. \\
\hline $37^{\frac{7}{8}}$ & 6 & 23 & $3^{I \frac{1}{2}}$ & $3 \times 4$ & $\cdots \quad \cdots \quad \cdots$ & Sir Robert Harvey, Bart. \\
\hline $37 \frac{3}{4}$ & $5 \frac{7}{8}$ & 14 & $21 \frac{1}{4}$ & $3 \times 3$ & Nepal... ... & B. H. Hodgson, British Museum. \\
\hline $37^{\frac{1}{2}}$ & $6 \frac{3}{4}$ & $21 \frac{3}{8}$ & $25 \frac{3}{8}$ & $4 \times 3$ & Cent. Provinces & Major C. S. Cumberland. \\
\hline $37 \frac{1}{2}$ & $5^{\frac{3}{4}}$ & $20 \frac{3}{4}$ & $28 \frac{1}{4}$ & $3 \times 3$ & $\cdots$ & H.R.H. Duke of Edinburgh. \\
\hline $37 \frac{3}{8}$ & $6 \frac{3}{4}$ & $28 \frac{1}{8}$ & $3 x$ & $3 \times 3$ & Neilgherries... & $\begin{array}{l}\text { Sir Victor Brooke's Collection. } \\
\text { (Shot by Col. Doug. Hamilton.) }\end{array}$ \\
\hline $37 \cdot \frac{1}{4}$ & $5 \frac{3}{8}$ & $19 \frac{5}{8}$ & $30 \frac{3}{4}$ & $3 \times 3$ & $\cdots \mid$ & Major Cumberland. \\
\hline 37 & $7 \frac{1}{2} \times 5 \frac{3}{4} \mid$ & 16 & $\cdots$ & $3 \times 3$ & $\cdots$ & Surg.-Major Briggs. \\
\hline 37 & $6 \frac{1}{8}$ & $27 \frac{1}{8}$ & $28 \frac{3}{4}$ & $3 \times 3$ & $\begin{array}{lll}\cdots & \cdots & \cdots\end{array}$ & II. C. V. Hunter. \\
\hline $3^{6 \frac{1}{4}}$ & $5 \frac{7}{8}$ & $19 \frac{7}{8}$ & $24 \frac{1}{8}$ & $3 \times 3$ & Cent. Provinces & Major C. S. Cumberland. \\
\hline $35^{\frac{5}{8}}$ & $6 \frac{1}{4}$ & $19 \frac{3}{4}$ & $24 \frac{1}{2}$ & $3 \times 3$ & $\cdots \quad \ldots$ & H.R.II. Dulse of Edinburgh. \\
\hline
\end{tabular}


SAMBUR (Cervus aristotelis)-continued.

\begin{tabular}{|c|c|c|c|c|c|c|c|}
\hline $\begin{array}{c}\text { Length } \\
\text { round } \\
\text { outside. }\end{array}$ & 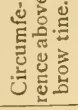 & Tip to Tip. & $\begin{array}{l}\text { Widest } \\
\text { inside. }\end{array}$ & Points. & Habitat. & & Owner. \\
\hline $35 \frac{5}{8}$ & 5 & $22 \frac{1}{4}$ & $28 \frac{1}{2}$ & $3 \times 3$ & Nepal... & $\cdots$ & B. H. Hodgson, British Museum. \\
\hline $35 \frac{1}{2}$ & 6 & $11 \frac{3}{4}$ & ... & $3 \times 3$ & $\ldots \quad \ldots$ & $\ldots$ & Capt. Goulburn, R.A. \\
\hline $35^{\frac{3}{3}}$ & $5 \frac{7}{8}$ & $21 \frac{3}{4}$ & $25 \frac{7}{8}$ & $3 \times 3$ & ... $\quad \cdots$ & $\ldots$ & J. Carr Saunders. \\
\hline $35 \frac{3}{8}$ & $5^{\frac{1}{2}}$ & $23 \frac{1}{8}$ & $28_{3}^{1}$ & $3 \times 3$ & Nepal... & $\cdots$ & B. H. Hodgson, British Museum. \\
\hline $35^{\frac{1}{4}}$ & $7 \frac{3}{8}$ & 20 & 26 & $3 \times 3$ & Bhotan & $\ldots$ & $\begin{array}{l}\text { Sir Victor Brooke's Collection, } \\
\text { I } 869 \text {. }\end{array}$ \\
\hline $35^{\frac{1}{4}}$ & $5 \frac{1}{2}$ & $3^{\circ \frac{3}{4}}$ & $32 \frac{1}{4}$ & $4 \times 3$ & Nepal... & $\cdots$ & B. H. Hodgson, British Museum. \\
\hline $35 \frac{1}{8}$ & $6 \frac{1}{8}$ & $23 \frac{1}{2}$ & 253 & $3 \times 3$ & India ... & $\cdots$ & British Museum. \\
\hline 35 & $5 \frac{5}{8}$ & $22 \frac{1}{2}$ & $26 \frac{7}{8}$ & $3 \times 3$ & Nepal... & $\cdots$ & B. H. Hodgson, British Museum. \\
\hline 35 & $58^{3}$ & $17 \frac{1}{8}$ & $25 \frac{1}{3}$ & $3 \times 3$ & $\ldots \quad \ldots$ & $\cdots$ & Sir Robert IIarvey, Bart. \\
\hline $34 \frac{3}{5}$ & 53 & 33 & $33 \frac{1}{2}$ & $2 \times 2$ & $\cdots$ & $\cdots$ & British Museum. \\
\hline $3 t_{1}^{\frac{1}{1}}$ & $6 \frac{1}{2}$ & $20 \frac{3}{7}$ & $24 \frac{3}{5}$ & $3 \times 3$ & Neilgherries & $\ldots$ & $\begin{array}{l}\text { Sir Victor Brooke's Collection, } \\
\text { (Shot by Col. Doug. Hamiltun.) }\end{array}$ \\
\hline $3 t$ & $4 \frac{7}{8}$ & 30 & $32 \frac{3}{4}$ & $3 \times 3$ & $\ldots \quad \ldots$ & $\cdots$ & J. Carr Saunders. \\
\hline 37 & $4 \frac{7}{8}$ & (single horn) & $\cdots$ & 3 & Nepal... & $\ldots$ & B. H. Hodgson, British Museum. \\
\hline $33 \frac{7}{5}$ & 51 & $27 \frac{1}{8}$ & 301 & $3 \times 3$ & $\cdots \quad \cdots$ & $\cdots$ & H. C. V. Hunter. \\
\hline $33 \stackrel{3}{4}$ & $5 \frac{1}{8}$ & $15 ?$ & $\cdots$ & $3 \times 3$ & Java $\ldots$ & $\cdots$ & Capt. Townley Parker. \\
\hline $33 !$ & $5 i$ & 16 & $1 S_{8}^{5}$ & $3 \times 3$ & Nepal... & $\ldots$ & B. II. Hodgson, British Museum. \\
\hline $33 \frac{1}{2}$ & $5 \%$ & $25:$ & $\cdots$ & $3 \times 6$ & $\ldots \quad \ldots$ & $\cdots$ & II. R.II. Duke of Edinburgh. \\
\hline 33 & $5 \ddot{i}$ & 25 & $\cdots$ & $3 \times 3$ & $\ldots \quad \ldots$ & $\cdots$ & Rowland Ward's Collection. \\
\hline $32 \frac{7}{8}$ & 5 l & $20 ?$ & $24 \times$ & $3 \times 3$ & $\ldots \quad \ldots$ & $\cdots$ & British Museum. \\
\hline $32 \frac{1}{2}$ & $7 !$ & $35^{\frac{1}{2}}$ & $\cdots$ & $7 \times 6$ & $\ldots \quad \ldots$ & $\ldots$ & Hume Collection, British Museum. \\
\hline $32 \frac{1}{3}$ & 4 & $25 \frac{7}{5}$ & $\cdots$ & $3 \times 3$ & $\cdots$ & $\ldots$ & H.R.H. Duke of Edinburgh. \\
\hline 323 & $6 \frac{1}{6}$ & 33 & 318 & $3 \times 3$ & Cent. Provin & aces & Major C. S. Cumberland. \\
\hline $32 \frac{1}{1}$ & $5 \frac{1}{t}$ & $20 \frac{1}{t}$ & $1 S_{3}^{3}$ & $3 \times 3$ & Nepal... & $\cdots$ & B. H. Hodgson, British Museum. \\
\hline $32 \frac{1}{4}$ & $5 \frac{1}{x}$ & $25 \frac{1}{8}$ & $29 \frac{1}{8}$ & $2 \times 2$ & India $\ldots$ & $\cdots$ & Do. \\
\hline 32 & $5^{\frac{1}{2}}$ & 25 & 253 & $3 \times 3$ & $\cdots$ & $\cdots$ & British Museum. \\
\hline 32 & $5 !$ & $20 \frac{3}{1}$ & .. & $3 \times 3$ & $\cdots \quad \cdots$ & $\ldots$ & II. R.H. Duke of Edinburgh. \\
\hline 32 & 5. & 20 & 25 & $3 \times 4$ & Neilgherries & $\ldots$ & St. George Littledale, I 875 . \\
\hline
\end{tabular}


SAIMBUR (Cervus aristotelis)-continued.

\begin{tabular}{|c|c|c|c|c|c|c|c|}
\hline $\begin{array}{c}\text { Length } \\
\text { round } \\
\text { outside. }\end{array}$ & 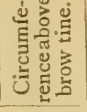 & Tip to Tip. & $\begin{array}{l}\text { Widest } \\
\text { inside. }\end{array}$ & Points. & Habitat. & & Owner. \\
\hline $3^{1 \frac{5}{8}}$ & $5 \frac{3}{8}$ & $21 \frac{3}{4}$ & 25 & $3 \times 3$ & Nepal... & $\ldots$ & B. H. Hodgson, British Museum. \\
\hline $3 \mathrm{I}^{\frac{1}{2}}$ & $5 \frac{3}{8}$ & $17 \frac{1}{t}$ & 24 & $3 \times 3$ & $\ldots \quad \ldots$ & $\cdots$ & Martyn Kennard. \\
\hline $3 \mathrm{I} \frac{1}{2}$ & $5 \frac{3}{8}$ & (one horn bro & oken) & $\mathrm{I} \times 3$ & Nepal... & $\cdots$ & B. H. Hodgson, British Museum. \\
\hline $3^{1 \frac{1}{2}}$ & $5^{\frac{1}{t}}$ & $20 \frac{3}{3}$ & $27 \frac{1}{t}$ & $3 \times 3$ & Deccan & $\cdots$ & Col. J. Sykes, British Museum. \\
\hline $31 \frac{3}{8}$ & $6 \frac{5}{8}$ & $31 \frac{3}{4}$ & $32 \frac{1}{2}$ & $3 \times 3$ & Do. & $\cdots$ & Do. \\
\hline $31 \frac{3}{8}$ & $6 \frac{1}{2}$ & $15^{\frac{1}{2}}$ & 19 & $3 \times 4$ & Nepal... & $\cdots$ & B. H. Hodgson, British Museum. \\
\hline $3^{1 \frac{1}{4}}$ & $4 \frac{1}{2}$ & $22 \frac{3}{4}$ & $\cdots$ & $3 \times 3$ & India ... & $\cdots$ & H. R.H. Duke of Edinburgh. \\
\hline $3 I$ & $5 \frac{3}{t}$ & (single horn) & $\cdots$ & 3 & Do. $\ldots$ & ... & H.R.H. le Duc d'Orleans. \\
\hline $3 \mathrm{I}$ & $5^{\frac{1}{2}}$ & 10 & $17 \frac{1}{4}$ & $4 \times 3$ & Nepal... & $\cdots$ & B. H. Hodgson, British Museum. \\
\hline $30 \frac{3}{4}$ & 5 & I I $\frac{3}{8}$ & $19 \frac{5}{8}$ & $3 \times 3$ & Do. $\ldots$ & $\cdots$ & Do. \\
\hline $3 \circ \frac{1}{8}$ & $4 \frac{5}{8}$ & $2 \mathrm{I} \frac{1}{8}$ & $25 \frac{3}{8}$ & $3 \times 3$ & $\ldots \quad \ldots$ & $\cdots$ & H. C. V. Hunter. \\
\hline $29 \frac{1}{8}$ & $5^{\frac{3}{4}}$ & $15 \frac{1}{3}$ & $20 \frac{1}{2}$ & $3 \times 3$ & $\cdots \quad \cdots$ & $\cdots$ & Do. \\
\hline $29 \frac{1}{8}$ & $4 \frac{5}{8}$ & $21 \frac{1}{8}$ & $22 \frac{1}{3}$ & $3 \times 3$ & India ... & $\cdots$ & British Museum. \\
\hline $28 \frac{1}{8}$ & $5 \frac{7}{8}$ & 23 & $26 \frac{1}{2}$ & $3 \times 3$ & $\ldots \quad \cdots$ & $\cdots$ & H. R.H. Duke of Edinburgh. \\
\hline 28 & 5 & I $8 \frac{3}{t}$ & $22 \frac{7}{8}$ & $3 \times 3$ & India ... & $\cdots$ & British Museum. \\
\hline $27 \frac{3}{4}$ & 5 & $26 \frac{1}{8}$ & $27 \frac{1}{8}$ & $3 \times 3$ & Neilgherries & $\cdots$ & St. George Littledale, I $S_{75}$. \\
\hline $26 \frac{7}{8}$ & $5 \frac{5}{8}$ & $12 \frac{1}{8}$ & 16 & $|3 \times 3|$ & $\cdots \quad \cdots$ & $\cdots$ & A. O. Hume, Private Collection. \\
\hline $26 \frac{7}{8}$ & $5 \frac{1}{t}$ & $23 \frac{1}{2}$ & $23^{\frac{3}{4}}$ & $3 \times 2$ & Neilgherries & $\cdots$ & St. George Littledale, $\mathbf{I} 875$. \\
\hline $23^{\frac{3}{4}}$ & $4 \frac{3}{8}$ & $17 \frac{3}{8}$ & I $8 \frac{1}{8}$ & $4 \times 3$ & $\cdots \quad \cdots$ & $\cdots$ & H.R.H. Duke of Edinburgh. \\
\hline 22 & ... & (single horn) & $\cdots$ & 7 & Nagpore & $\cdots$ & Sir Victor Brooke's Collection. \\
\hline 21 & 5 & III $\frac{1}{8}$ & $\cdots$ & $3 \times 3$ & $\cdots \quad \cdots$ & $\cdots$ & R. Lydekker, Britishı Museum. \\
\hline $18 \frac{1}{4}$ & $4 \frac{1}{2}$ & $7 \frac{5}{8}$ & $8 \frac{3}{8}$ & $3 \times 3$ & India ... & $\cdots$ & B. H. Hodgson, British Muscum. \\
\hline $16 \frac{1}{2}$ & $2 \frac{3}{4}$ & $11 \frac{1}{8}$ & 12 & $\mathbf{I} \times \mathbf{I}$ & Nepal... & $\cdots$ & Do. \\
\hline
\end{tabular}




\section{CENTRAL AND SOUTHERN INDIAN SAMBUR (Rusa hippelaphus).}

Habitat-Central and Southern India.

\begin{tabular}{|c|c|c|c|c|c|c|c|}
\hline I.ength. & $\begin{array}{l}\text { Circum- } \\
\text { ference. }\end{array}$ & $\begin{array}{l}\text { Tip to } \\
\text { Tip. }\end{array}$ & $\begin{array}{l}\text { Widest } \\
\text { inside. }\end{array}$ & Points. & Habitat. & & Owner. \\
\hline $36 \frac{3}{5}$ & $5 \frac{7}{8}$ & $27 \frac{33}{4}$ & ... & $2 \times 3$ & Nepal... & $\cdots$ & B. H. Hodgson, British Muscum. \\
\hline $3 I_{4}^{\frac{1}{4}}$ & $5 \frac{3}{8}$ & I 8 & I $\delta \frac{3}{4}$ & $4 \times 3$ & Do. ... & $\cdots$ & Do. \\
\hline 293 & $4 \frac{1}{8}$ & $20 \frac{1}{2}$ & $\cdots$ & $3 \times 3$ & India ... & $\ldots$ & British Museum. \\
\hline $2 S_{t}^{1}$ & 5 & $18 \frac{7}{8}$ & $\cdots$ & $3 \times 4$ & Nepal... & $\ldots$ & B. H. Hodgson, British Museum. \\
\hline
\end{tabular}

\section{EQUINE DEER (Cervus equinus).}

Habitat-Borneo, Sumatra, and Singapore.

\begin{tabular}{|c|c|c|c|c|c|c|}
\hline I.ength. & $\begin{array}{l}\text { Circum- } \\
\text { ference. }\end{array}$ & $\begin{array}{l}\text { Tip to } \\
\text { Tip. }\end{array}$ & $\begin{array}{l}\text { Widest } \\
\text { inside. }\end{array}$ & Points. & & Iabitat. \\
\hline $30 \frac{7}{5}$ & $6 \frac{1}{2}$ & I $7 \frac{1}{4}$ & $19 \frac{5}{8}$ & $7 \times 7$ & $\cdots$ & $\ldots$ \\
\hline 301 & $4 \frac{3}{8}$ & $20_{ \pm}^{3}$ & $2 I_{4}^{\frac{1}{4}}$ & $6(3 \times 3)$ & ... & $\cdots$ \\
\hline 293 & 6 & $16 \frac{5}{8}$ & $20 \frac{3}{4}$ & $3 \times 3$ & $\ldots$ & $\cdots$ \\
\hline $27 \%$ & $5^{\frac{1}{4}}$ & $19 \frac{1}{2}$ & IS & $3 \times 3$ & $\cdots$ & $\cdots$ \\
\hline $25 !$ & $4 \frac{7}{8}$ & $14 \frac{3}{4}$ & I $S \frac{7}{8}$ & $4 \times 3$ & $\cdots$ & $\ldots$ \\
\hline 23. & 7 & I $5 \frac{3}{8}$ & $\cdots$ & $3 \times 3$ & Borne & \\
\hline $20 \%$ & $4 \frac{7}{8}$ & I $S_{f}$ & ... & $3 \times 3$ & Do. & \\
\hline $2 O_{2}^{\prime}$ & $5 \frac{7}{8}$ & I $5 \frac{1}{2}$ & $\cdots$ & $3 \times 3$ & Do. & \\
\hline $20 \%$ & $4^{\frac{7}{8}}$ & I 3 & $\cdots$ & $3 \times 3$ & Do. & \\
\hline $19 \overline{-}$ & $4 \frac{1}{4}$ & $16 \frac{7}{8}$ & 167 & $4 \times 3$ & $\ldots$ & $\cdots$ \\
\hline IS: & $4^{\frac{1}{2}}$ & I5 & $\cdots$ & $3 \times 3$ & Borne & \\
\hline $17 \%$ & $5 \frac{1}{8}$ & $16 \frac{3}{4}$ & ... & $4 \times 5$ & Do. & \\
\hline $17: 1$ & $4 \frac{5}{8}$ & 13 & ... & $3 \times 3$ & Do. & \\
\hline $16 ?$ & $4 \frac{1}{4}$ & $5^{\frac{5}{8}}$ & $\cdots$ & $2 \times 3$ & Do. & \\
\hline $15 \frac{1}{1}$ & $4 \frac{1}{8}$ & $13 \frac{1}{8}$ & $\cdots$ & $3 \times 3$ & Do. & \\
\hline $11: 3$ & $3 \frac{1}{8}$ & $9 \frac{1}{4}$ & $\ldots$ & $3 \times 2$ & Do. & \\
\hline
\end{tabular}

Owner.

.. Sir Edmund G. Loder, Bart.

... British Museum.

Hume Collection, British Musemm.

Do.

Do.

II. B. Low, British Museum.

Do.

Do.

Do.

Hume Collection, British Museum.

H. B. Low, British Museum.

Do.

Do.

IV. B. Pryer, British Museum.

H. B. Low, British Museum.

Do. 


\section{SWINHOE'S DEER (Cervus swinhoii).}

[Mr. SwinhoE on "Chinese Mammals," P.Z.S. i87o, p. 646.]

["Cervus (Rusa) Swinhoii," Sclater. P.Z.S. 1862, p. I52; Swinhoe, P.Z.S. I862, p. 364.]

IN the central ranges of Formosa, near Mount Morrison, this brown deer is very common; and on a visit I paid to the wild tribes of these parts in February I866, I found them hunting the deer with dogs.

A place is cleared in the forest, where a party of men hide armed with matchlocks; the dogs yelp after the deer and drive them into the open, where the hidden sportsmen get easy shots at them. The son of the chief with whom I was staying had just returned from a successful battue with the robust antlers and flesh of a large buck. I induced him to return for the head, which he had thrown away on the field. I was thus enabled to secure a fine skull for the British Museum.

The young of this species, about half-grown, is reddish brown, with the tail bushy and black, but reddish at its root; sides of the body paler, and the belly blackish brown; legs pale towards the hoofs, the latter black; under surface of tail, abdomen, and inner sides of hind legs down to middle of shank yellowish white, the breast and belly being blackish brown; under surface of head and neck mottled whitey brown; crown of the head, with many of the hairs, tipped with black; from the occiput a dark line runs down to the base of the tail; ears blackish brown, tipped and margined with ochreous white, and whitish on their insides.

The adult, in summer, has its coarse hair deep brown, faintly mottled, rufous on the rump; between the fore legs and the thighs ochreous white; tail bushy and dark. In winter it becomes a deeper brown. 
The Society's Gardens have had two or three examples of this animal alive; and Dr. Sclater is doing it justice in his paper for the "Transactions." Fig. 5 represents the antlers of a mature individual which I procured in South Formosa.

I have nct heard of any species of Rusa occurring on the main of China; but in Hainan the C. hippelaphus appears to abound. From North China we have the Cervus xanthopygus (A. M. Edwards), the Elaphurus davidianus (A. M. Edwards), and the Capreolus pyaargus (Pall).

\begin{tabular}{|c|c|c|c|c|c|c|}
\hline Length. & $\begin{array}{l}\text { Circum } \\
\text { ference. }\end{array}$ & $\begin{array}{l}\text { Tip to } \\
\text { Tip. }\end{array}$ & $\begin{array}{l}\text { Wiclest } \\
\text { inside. }\end{array}$ & Points. & Habitat. & Owner. \\
\hline $19 ?$ & ... & 9 & $12 \frac{7}{8}$ & $3 \times 3$ & Island of Formosa & Zoological Society, British Muscum. \\
\hline $16 \frac{1}{8}$ & $3 \frac{5}{8}$ & 16 & ... & $3 \times 3$ & Do. & British Muscum. \\
\hline $13 \frac{5}{8}$ & $43_{1}^{3}$ & $13 \frac{1}{8}$ & $\ldots$ & $4 \times 4$ & Do. & Zoological Society, British Musetm. \\
\hline $5 \frac{7}{8}$ & $2 \frac{1}{8}$ & $6 \frac{1}{4}$ & $\ldots$ & $2 \times 3$ & Do. & British Muscum. \\
\hline
\end{tabular}

\section{THE PHILIPPINE DEER (Cervus philippinus!}

[Sir Victor BRoOKE, Bart., P.Z.S. I877, p. 51.]

Adult MaLe.-Hair of moderate length and rather harsh. General colour, rich ruddy brown, darkest on the back and lightest on the neck. Forehead and cheeks rufous fawn-colour; dark (almost black) streaks commence over each eye, and, blending together, form a band running down the centre of the face, which is separated from the dark moustache-like mark lordering the muzzle by a narrow track of pale fawn-colour. Breast and kelly uniform brown. Tarsal gland plainly marked by a rufous spot. Ears of moderate size, covered externally by short close-set hair. Horns remarkably rough and massive; a powerful, long brow-antler, and one short tinc directed inwards. Height at shoulder, about 27 inches. 
The skull of this species is an exact miniature of that of Cervus aristotelis.

Female.-In external colouration differs in no way from the male.

YounG.-Of a uniform brown, without spots.

Habitat.-Island of Luzon, Philippines.

The Philippine deer is a very stout-built, short-limbed, powerful animal, in carriage and general appearance closely resembling the common Sambur (Cervus aristotelis).

\begin{tabular}{|c|c|c|c|c|c|c|c|}
\hline Length. & $\begin{array}{l}\text { Circum- } \\
\text { ference. }\end{array}$ & $\begin{array}{l}\text { Tip to } \\
\text { Tip. }\end{array}$ & $\begin{array}{l}\text { Widest } \\
\text { inside. }\end{array}$ & Points. & Habitat. & & Owner. \\
\hline $13 \frac{1}{8}$ & $4 \frac{\pi}{2}$ & II $\frac{5}{8}$ & II $\frac{33}{4}$ & $3 \times 3$ & Basilan & $\cdots$ & Everett, British Museum. \\
\hline I $3 \frac{1}{2}$ & $4 \frac{3}{8}$ & $12 \frac{1}{5}$ & I $2 \frac{1}{2}$ & $3 \times 3$ & Philippine Islanảs & $\ldots$ & Do. \\
\hline
\end{tabular}

\section{MARIANE DEER (Cervus marianus).}

THE late Sir Victor Brooke believed that Cervus philippinus and C. marianus were identical.

Habitat-Philippine Islands.

\begin{tabular}{|c|c|c|c|c|c|c|}
\hline Length. & $\begin{array}{l}\text { Circum- } \\
\text { ference. }\end{array}$ & Tip to Tip. & Points. & \multicolumn{2}{|c|}{ Habitat. } & Owner. \\
\hline $19 \frac{5}{8}$ & $5 \frac{1}{t}$ & $7 \frac{1}{2}$ & $4 \times 4$ & \multicolumn{2}{|c|}{ Philippine Islands... } & Capt. Belcher, British Museum. \\
\hline I $S \frac{7}{8}$ & $5 \frac{1}{4}$ & $9 \frac{3}{4}$ & $3 \times 5$ & Do. & $\ldots$ & Do. \\
\hline $\mathrm{I} S \frac{3}{8}$ & $5 \frac{1}{8}$ & $14 \frac{3}{4}$ & $4 \times 3$ & Do. & $\ldots$ & Do. \\
\hline IS & $4 \frac{5}{8}$ & $13 \frac{7}{8}$ & $4 \times 4$ & Do. & $\ldots$ & Do. \\
\hline I $5 \frac{1}{8}$ & $5 \frac{1}{2}$ & II $\frac{1}{4}$ & $3 \times 3$ & Do. & $\ldots$ & Do. \\
\hline $14 \frac{5}{8}$ & $4 \frac{3}{4}$ & $\delta_{\frac{1}{8}}^{1}$ & $3 \times 3$ & Do. & $\ldots$ & Do. \\
\hline
\end{tabular}




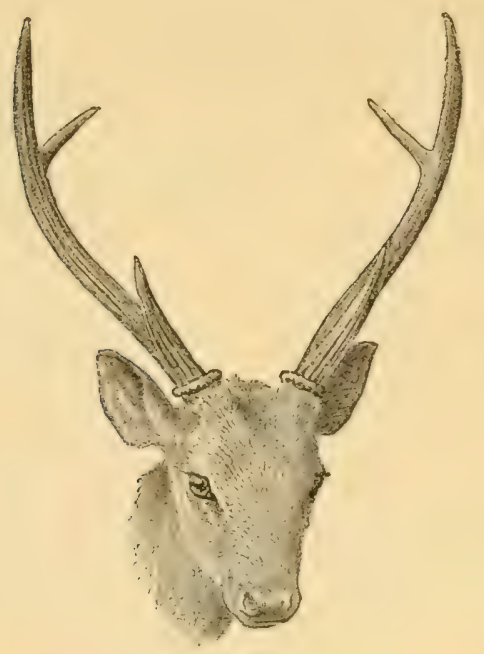

HOG DEER (Cervus porcinus).

Habitat-India.

\begin{tabular}{c|c|c|c|c|c|c} 
Length. & $\begin{array}{c}\text { Circum- } \\
\text { ference above } \\
\text { brow tine. }\end{array}$ & Tip to Tip. & Habitat. & \multicolumn{1}{|c}{ Owner. } \\
\hline $19 \frac{1}{4}$ & $3 \frac{1}{8}$ & $9 \frac{1}{2}$ & India & $\ldots$ & $\ldots$ & A. O. Hume, Private Collection. \\
$19 \frac{1}{8}$ & $3 \frac{1}{4}$ & $16 \frac{1}{2}$ & Burmah & $\ldots$ & $\ldots$ & Sir Victor Brooke's Collection. \\
$19 \frac{1}{8}$ & $3 \frac{3}{8}$ & $18 \frac{3}{4}$ & India & $\ldots$ & $\ldots$ & H. C. V. Hunter. \\
$17 \frac{3}{4}$ & $3 \frac{1}{2}$ & $9 \frac{1}{4}$ & Nepal & $\ldots$ & $\ldots$ & B. H. Hodgson, British Museum. \\
$17 \frac{1}{8}$ & $3 \frac{1}{8}$ & $9 \frac{1}{4}$ & India & $\ldots$ & $\ldots$ & J. Carr-Saunders. \\
17 & $2 \frac{7}{8}$ & $12 \frac{5}{8}$ & Do. & $\ldots$ & $\ldots$ & H. C. V. Hunter. \\
$16 \frac{7}{8}$ & 3 & $10 \frac{3}{8}$ & Do. & $\ldots$ & $\ldots$ & British Museum. \\
$16 \frac{1}{2}$ & $2 \frac{3}{4}$ & 9 & Nepal & $\ldots$ & $\ldots$ & B. H. Hodgson, British Museum. \\
$16 \frac{3}{8}$ & $3 \frac{5}{8}$ & $9 \frac{3}{8}$ & India & $\ldots$ & $\ldots$ & British Museum. \\
$16 \frac{1}{4}$ & $2 \frac{7}{8}$ & 8 & Do. & $\ldots$ & $\ldots$ & H. C. V. Hunter. \\
$16 \frac{1}{8}$ & $2 \frac{7}{8}$ & $8 \frac{5}{8}$ & Do. & $\ldots$ & $\ldots$ & Gen. Hardwicke, British Museum. \\
$15 \frac{5}{8}$ & $3 \frac{1}{8}$ & 8 & Do. & $\ldots$ & $\ldots$ & British Museum. \\
\hline
\end{tabular}


HOG DEER (Cervus porcinus)-continned.

\begin{tabular}{|c|c|c|c|c|c|c|}
\hline Length. & $\begin{array}{c}\text { Circum- } \\
\text { ference above } \\
\text { brow tine. }\end{array}$ & Tip to Tip. & \multicolumn{3}{|c|}{ Habitat. } & Owner. \\
\hline $14 \frac{1}{t}$ & $3 \frac{1}{\frac{1}{4}}$ & $10 \frac{33}{4}$ & Nepal & $\cdots$ & $\cdots$ & B. H. Hodgson, British Museum. \\
\hline $14 \frac{1}{8}$ & $2 \frac{33}{4}$ & (single horn) & India & $\ldots$ & $\cdots$ & Gen. Hardwicke, British Museum. \\
\hline 14 & 3 & $8 \frac{\pi}{8}$ & Do. & $\ldots$ & $\cdots$ & H.R.H. Duke of Edinburgh. \\
\hline $13 \frac{1}{ \pm}$ & $3 \frac{1}{8}$ & $10 \frac{3}{t}$ & Do. & $\ldots$ & ... & Gen, Hardwicke, British Museum. \\
\hline I3 & $3 \frac{1}{8}$ & $12 \frac{3}{4}$ & Do. & $\cdots$ & $\cdots$ & Hon. Walter Rothschild. \\
\hline $12 \frac{3}{t}$ & 3 & $10 \frac{5}{8}$ & Nepal & $\ldots$ & $\cdots$ & B. H. Hodgson, British Museum. \\
\hline I $2 \frac{1}{2}$ & 3 & $12 \frac{7}{8}$ & India & $\ldots$ & $\cdots$ & Gen. Hardwicke, British Museum. \\
\hline II $\frac{1}{4}$ & $3 \frac{1}{\ddagger}$ & $9 \frac{3}{4}$ & Do. & $\cdots$ & $\cdots$ & British Museum. \\
\hline I $1 \frac{1}{4}$ & $2 \frac{5}{8}$ & II & Do. & $\cdots$ & $\cdots$ & Gen. Hardwicke, British Museum. \\
\hline $9 \frac{1}{1}$ & 3 & (single horn) & Do. & $\cdots$ & $\cdots$ & Do. \\
\hline
\end{tabular}

\section{KUHL'S STAG (Cervus kuhli).}

[Sir VICTOR BROOKE, P.Z.S. I878, p. 902.]

[1839-44. Cervus kuhlii, Mull. "Verh. Nat. Gesch. Ned. Bez. Zoo.," p. 223, pls. 44,45 , figs. 12-14.

\section{Habitat-Bavian Islands. Type-Mus. Leyden.}

THIS species was originally described by Muller from specimens collected by Diard in the Bavian Islands, to which locality the species, as far as is at present known, is confined. The auditory bullæ in Cervus kuhli are largely inflated. By this character the skull may be distinguished from that of any of the Rusine deer, with the exception of $\mathrm{C}$. porcinus. The skull of C. kuhli differs from that of $\mathrm{C}$. porcinus in having the facial portion anterior to the orbits much more compressed, and the orbits much more prominent. C. kuhli has proportionally much longer limbs than C. porcinus; and all the hairs of the back and sides are annulated 
in the former and not in the latter species. The young of $\mathrm{C}$. kuhli are unspotted, which is not the case with those of $\mathrm{C}$. porcinus. Both species are devoid of upper canines.

\begin{tabular}{|c|c|c|c|c|c|c|}
\hline Length. & $\begin{array}{l}\text { Circum- } \\
\text { ference. }\end{array}$ & Tip to Tip. & $\begin{array}{l}\text { Widest } \\
\text { inside. }\end{array}$ & Points. & Habitat. & Owner. \\
\hline $9 \frac{\pi}{4}$ & 27 & $10 \frac{7}{8}$ & $10 \frac{7}{8}$ & $3 \cdots 3$ & Java... & British Museum. \\
\hline
\end{tabular}

\section{MOLUCCAN DEER (Cervus moluccensis).}

[Sir Victor BRooke, P.Z.S. 1878, p. 904.]

\section{Dimensions.}

Total length of skull in a straight line

Eng. inches.

From ant. rim of orbit to free extremity of præmaxillæ II

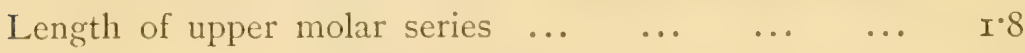

$\begin{array}{lllllll}\text { Greatest length of nasals } & \ldots & \ldots & \ldots & \ldots & 3.5\end{array}$

$\begin{array}{llllllll}\text { Length of præmaxillæe } & \ldots & \ldots & \ldots & \ldots & \ldots & 2.9\end{array}$

Across the lower rims of orbits at the widest place... 4.5

$\begin{array}{lllllll}\text { Length of humerus } & \ldots & \ldots & \ldots & \ldots & \ldots & 7^{\circ} 9\end{array}$

$\begin{array}{lllllllr}" & \text { radius } \ldots & \ldots & \ldots & \ldots & \ldots & \ldots & 7 \cdot 2 \\ , & \text { metacarpal cannon } & \ldots & \ldots & \ldots & \ldots & 6 \cdot 1 \\ , & \text { fernur } \ldots & \ldots & \ldots & \ldots & \ldots & \ldots & 9 \cdot 7 \\ , & \text { tibia } \ldots & \ldots & \ldots & \ldots & \ldots & \ldots & 10{ }^{\circ} 5 \\ " & \text { metatarsal cannon } & \ldots & \ldots & \ldots & \ldots & 7\end{array}$

Habitat- Molucca Islands.

\begin{tabular}{|c|c|c|c|c|c|c|}
\hline Length. & $\begin{array}{l}\text { Circum- } \\
\text { ference. }\end{array}$ & Tip to Tip. & Points. & Habitat. & & Owner. \\
\hline $36 \frac{1}{8}$ & $4 \frac{5}{8}$ & $18 \frac{1}{2}$ & $3 \times 3$ & $\ldots \quad \ldots$ & $\cdots$ & Sir Edmund G. Loder, Bart. \\
\hline$S_{\frac{3}{4}}$ & 4 & $5 \frac{1}{8}$ & $\cdots$ & Batchian & ... & A. R. Wallace, British Museum. \\
\hline $6 \frac{1}{4}$ & $3 \frac{3}{8}$ & $5 \frac{5}{8}$ & $\cdots$ & $\cdots$ & $\ldots$ & British Museum. \\
\hline
\end{tabular}




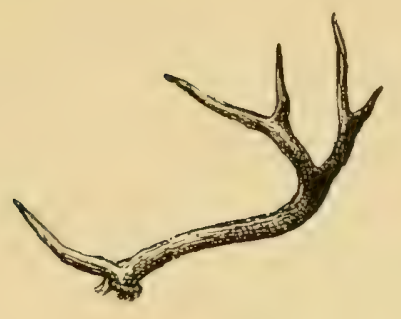

SWAMP DEER (Cervus duvaucelli).

Habitat-India.

Weight-About 40 stone.

\begin{tabular}{|c|c|c|c|c|c|c|c|}
\hline $\begin{array}{c}\text { Length } \\
\text { round } \\
\text { outside. }\end{array}$ & $\begin{array}{l}\text { Circum- } \\
\text { ference. }\end{array}$ & $\begin{array}{l}\text { Tip to } \\
\text { Tip. }\end{array}$ & $\begin{array}{l}\text { Widest } \\
\text { inside. }\end{array}$ & Points. & \multicolumn{2}{|l|}{ Habitat. } & Owner. \\
\hline $4 I$ & $5 \frac{1}{2}$ & \multicolumn{2}{|c|}{$\begin{array}{l}\text { (one horn } \\
\text { broken) }\end{array}$} & $6 \times 6$ & \multicolumn{2}{|c|}{ Central Provinces } & Major C. S. Cumberland. (Shot in I89I.) \\
\hline $39 \frac{1}{4}$ & 5 & $35^{\frac{1}{2}}$ & $37 \frac{1}{2}$ & $5 \times 6$ & India ... & $\cdots$ & Do. \\
\hline $37 \frac{3}{8}$ & $5 \frac{1}{4}$ & $23 \frac{5}{8}$ & $28 \frac{1}{2}$ & $7 \times 6$ & $\cdots$ & $\cdots$ & Sir Edmund G. Loder, Bart. \\
\hline 36 & 5 & $29 \frac{3}{4}$ & $33 \frac{3}{8}$ & $6 \times 5$ & Nepal... & $\cdots$ & B. H. Hodgson, British Museum. \\
\hline $35 \frac{3}{8}$ & $4 \frac{7}{8}$ & 27 & $29 \frac{3}{4}$ & $\cdots$ & $\cdots$ & $\cdots$ & Sir Victor Brooke's Collection. \\
\hline $35 \frac{1}{4}$ & $5 \frac{3}{4}$ & $22 \frac{1}{4}$ & $\cdots$ & $7 \times 6$ & $\cdots$ & $\cdots$ & H. C. V. Hunter. \\
\hline $34 \frac{3}{4}$ & $5 \frac{5}{8}$ & $3^{8 \frac{1}{4}}$ & $\cdots$ & $5 \times 5$ & Central Prov & inces & Major C. S. Cumberland. \\
\hline $33 \frac{1}{2}$ & $4 \frac{3}{4}$ & $26 \frac{1}{2}$ & $29 \frac{1}{4}$ & $5 \times 5$ & Do. & & Do. \\
\hline $33 \frac{1}{8}$ & $5 \frac{1}{2}$ & $3^{8 \frac{3}{8}}$ & $\cdots$ & $5 \times 5$ & Do. & & Do. \\
\hline $32 \frac{7}{8}$ & $5 \frac{1}{4}$ & $22 \frac{3}{8}$ & $26 \frac{3}{8}$ & $5 \times 6$ & $\cdots$ & $\cdots$ & A. O. Hume, Private Collection. \\
\hline $32 \frac{3}{4}$ & 5 & $27 \frac{1}{2}$ & $\cdots$ & $6 \times 7$ & $\cdots$ & $\cdots$ & Capt. Henry Streatfield. \\
\hline $32 \frac{5}{8}$ & 5 & $33 \frac{1}{4}$ & 35 & $6 \times 6$ & $\cdots$ & $\cdots$ & Sir Robert Harvey, Bart. \\
\hline $32 \frac{1}{8}$ & $5 \frac{1}{8}$ & $19 \frac{5}{8}$ & $24 \frac{1}{2}$ & $7 \times 7$ & $\cdots$ & $\cdots$ & H.R.H. Duke of Edinburgh. \\
\hline $31 \frac{7}{8}$ & $4 \frac{5}{8}$ & $26 \frac{1}{2}$ & 30 & $5 \times 6$ & $\cdots$ & $\cdots$ & Major Cumberland. \\
\hline $31 \frac{7}{8}$ & $4 \frac{3}{4}$ & $27 \frac{1}{8}$ & 30 & $7 \times 8$ & Nepal... & $\cdots$ & B. H. Hodgson, British Museum. \\
\hline
\end{tabular}


SWAIMP DEER (Cervus duvaucelli)-continued.

\begin{tabular}{|c|c|c|c|c|c|c|c|c|}
\hline $\begin{array}{l}\text { Length } \\
\text { round } \\
\text { outside. }\end{array}$ & $\begin{array}{l}\text { Circum- } \\
\text { ference. }\end{array}$ & $\begin{array}{l}\text { Tip to } \\
\text { Tip. }\end{array}$ & $\begin{array}{c}\text { Widest } \\
\text { inside. }\end{array}$ & Points. & \multicolumn{3}{|c|}{ Habitat. } & Owner. \\
\hline $3^{1 \frac{5}{8}}$ & $5^{\frac{1}{8}}$ & $30 \frac{1}{4}$ & $32 \frac{5}{8}$ & $6 \times 6$ & \multicolumn{3}{|c|}{ Central Provinces } & Major C. S. Cumberland. \\
\hline $31 \frac{3}{8}$ & $4 \frac{7}{8}$ & $26 \frac{3}{4}$ & $30 \frac{1}{2}$ & $5 \times 5$ & Do & o. & & Do. \\
\hline $31 \frac{1}{8}$ & $4 \frac{5}{8}$ & $22 \frac{1}{2}$ & $26 \frac{1}{4}$ & $6 \times 6$ & Nepal... & & ... & B. H. Hodgson, British Museum, \\
\hline $3 I$ & $5 \frac{3}{8}$ & I6 & $22 \frac{3}{8}$ & $6 \times 5$ & Do. ... & & ... & Do. \\
\hline $30 \frac{3}{8}$ & $5^{\frac{1}{8}}$ & 26 & $27 \frac{1}{2}$ & $7 \times 7$ & ... & .. & ... & A. O. Hume, Private Collection. \\
\hline $29 \frac{3}{8}$ & 5 & I $9 \frac{1}{2}$ & $22 \frac{1}{4}$ & $8 \times 8$ & ... & .. & ... & Hume Collection, British Museum. \\
\hline $29 \frac{1}{4}$ & 5 & 27 & $29 \frac{1}{4}$ & $7 \times 8$ & ... & .. & ... & Do. \\
\hline $29 \frac{1}{8}$ & $6 \frac{1}{4}$ & $20 \frac{1}{4}$ & $28 \frac{1}{4}$ & ... & ... & .. & $\cdots$ & Sir Victor Brooke's Collection, \\
\hline $29 \frac{1}{8}$ & $4 \frac{3}{4}$ & $19 \frac{7}{8}$ & $23 \frac{1}{4}$ & $6 \times 5$ & ... & .. & .. & Earl of Derby, British Museuin. \\
\hline 29 & $5 \frac{1}{8}$ & $24 \frac{5}{8}$ & $27 \frac{3}{8}$ & $6 \times 6$ & $\ldots$ & .. & ... & Capt. Henry Streatfield. \\
\hline 29 & $4^{\frac{1}{x}}$ & $25 \frac{1}{8}$ & $26 \frac{7}{8}$ & $9 \times 8$ & Nepal.. & & $\ldots$ & B. H. Hodgson, British Museum. \\
\hline $28 \frac{1}{2}$ & 5 & 24 & $25 \frac{3}{t}$ & $8 \times 7$ & ... & .. & ... & IIume Collection, British Museum. \\
\hline $27 \frac{3}{8}$ & $5^{\frac{1}{2}}$ & $23 \frac{1}{4}$ & $26 \frac{1}{2}$ & $6 \times 5$ & ... $\quad$. & & .. & British Museum. \\
\hline $27 \frac{1}{8}$ & $4 \frac{3}{8}$ & IS & $23 \frac{1}{4}$ & $5 \times 6$ & Nepal.. & & ... & B. H. Hodgson, British Museum. \\
\hline 27 & $4 \frac{5}{8}$ & $27 \frac{1}{2}$ & ... & $6 \times 5$ & $\cdots$ & & ... & Hon. Walter Rothschild. \\
\hline 26 & 5 & 28 & $\cdots$ & $6 \times 5$ & $\cdots$ & & ... & Do. \\
\hline 25 & $3 \frac{3}{4}$ & $23 \frac{1}{7}$ & $23 \frac{1}{2}$ & $6 \times 6$ & $\ldots$ & & ... & II. C. V. Hunter. \\
\hline 24 & 4 & 20 & $\cdots$ & $6 \times 6$ & $\cdots$ & & ... & Do. \\
\hline $23 \frac{3}{4}$ & 4 & $20 \frac{5}{8}$ & $22 \frac{1}{8}$ & $6 \times 5$ & Nepal.. & & ... & B. H. Hodgson, British Museum. \\
\hline $22 \frac{1}{8}$ & $5 \frac{3}{8}$ & I8 & $19 \frac{3}{4}$ & $7 \times 6$ & Do. .. & & ... & Do. \\
\hline 20 & $4 \frac{1}{2}$ & 17 & ... & $6 \times 6$ & $\cdots$ & .. & $\cdots$ & M. B. Snell. \\
\hline $13 \frac{1}{4}$ & $2 \frac{1}{2}$ & $10 \frac{5}{8}$ & I $1 \frac{1}{2}$ & $4 \times 4$ & ... & .. & $\cdots$ & Hume Collection, British Museum. \\
\hline
\end{tabular}




\section{SCHOIMBURGK'S DEER (Cervus schomburgki).}

[Sir VICTOR BROOKE, Bart., P.Z.S. 1876, p. 306.]

Adult Male.-Antlers with very long powerful brow-antlers, which are frequently forked; beam very short, and more or less laterally compressed; upper part of the antlers strictly dichotomous, each of the main branches about equally developed, and in itself dichotomous, and furnished with long cylindrical tines. External surface of the antlers smooth and polished. Hair in winter rather long and coarse. General colour uniform brown, darkest on the nose and upper surface of the tail, and lightest on the cheeks and lower parts of the sides and haunches. Lower lip, belly, and under surface of tail whitish. Upper lip, occiput, and limbs with a decided tinge of rufous. The hair on the front of the metacarpal cannon bones is lengthened into an everted mane about 2 inches in length.

ImMATURE MALE.-Antlers with the posterior of the two main branches less developed than the anterior branch.

Habitat-Northern parts of Siam.

\begin{tabular}{l|c|c|c|c|c|cc|c}
\hline Length. & $\begin{array}{l}\text { Circum. } \\
\text { ference. }\end{array}$ & $\begin{array}{c}\text { Tip to } \\
\text { Tip. }\end{array}$ & $\begin{array}{l}\text { Widest } \\
\text { inside. }\end{array}$ & Points. & Habitat. & Owner. \\
\hline $30 \frac{1}{8}$ & 5 & $15 \frac{5}{8}$ & 33 & I0 $\times$ IO & Siam & $\ldots$ & $\ldots$ & British Museum. \\
$29 \frac{7}{8}$ & $5 \frac{1}{8}$ & $23 \frac{5}{8}$ & $26 \frac{7}{8}$ & $9 \times 8$ & Do. & $\ldots$ & $\ldots$ & Do. \\
$29 \frac{3}{4}$ & $4 \frac{3}{4}$ & $28 \frac{3}{8}$ & $31 \frac{5}{8}$ & $10 \times$ I I & Do. & $\ldots$ & $\ldots$ & Do. \\
$28 \frac{1}{2}$ & $5 \frac{1}{4}$ & 11 & $29 \frac{3}{8}$ & II $\times 9$ & $\ldots$ & $\ldots$ & $\ldots$ & Sir Victor Brooke's Collection. \\
$27 \frac{7}{8}$ & $5 \frac{3}{8}$ & 14 & $24 \frac{1}{2}$ & $9 \times 8$ & Siam & $\ldots$ & $\ldots$ & British Museum. \\
$27 \frac{3}{4}$ & $4 \frac{3}{8}$ & $18 \frac{1}{2}$ & $24 \frac{7}{8}$ & $7 \times 8$ & Do. & $\ldots$ & $\ldots$ & Do. \\
\hline
\end{tabular}




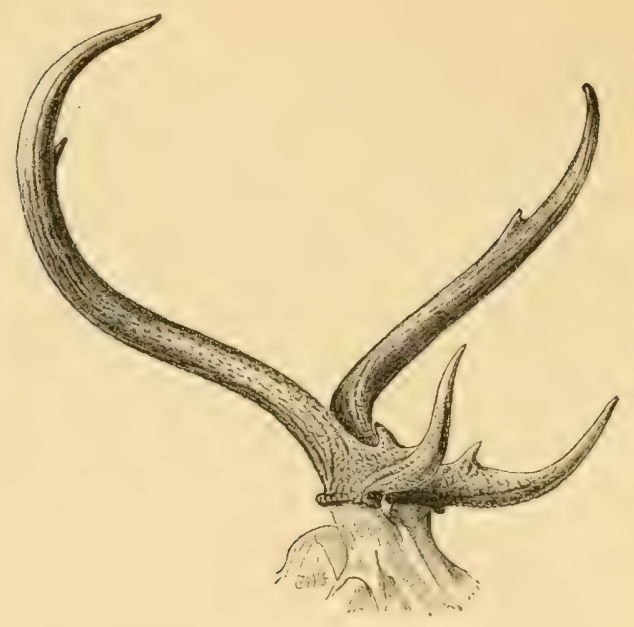

BURMESE STAG or THEMIN (Cervus eldi).

Habitat-Burmah and Hainan.

\begin{tabular}{|c|c|c|c|c|c|c|c|c|c|}
\hline Lingth. & $\begin{array}{l}\text { Circum- } \\
\text { ference. }\end{array}$ & $\begin{array}{l}\text { Tip to } \\
\text { Tip. }\end{array}$ & $\begin{array}{l}\text { Widest } \\
\text { inside. }\end{array}$ & Points. & $\begin{array}{l}\text { Length } \\
\text { of brow } \\
\text { tine. }\end{array}$ & \multicolumn{3}{|c|}{ Habitat. } & Owner. \\
\hline $398^{5}$ & 5 & $31 \frac{3}{8}$ & $36 \frac{5}{8}$ & 20 & $\cdots$ & Siam & $\ldots$ & ... & Sir Fdmund G. Loder, Bart. \\
\hline $38 \frac{7}{8}$ & $6 \frac{1}{4}$ & $25 \frac{1}{2}$ & $30 \frac{3}{8}$ & $16 \times 19$ & ... & India & $\ldots$ & ... & British Museum. \\
\hline $37 \frac{1}{2}$ & $5 \frac{3}{8}$ & $20 \frac{1}{4}$ & $30 \frac{1}{2}$ & $5 \times 5$ & $\cdots$ & Do. & $\ldots$ & ... & Do. \\
\hline 37 & $6 \frac{1}{8}$ & $\cdots$ & $3 I \frac{1}{4}$ & $\cdots$ & $10 \frac{1}{2}$ & $\ldots$ & $\ldots$ & $\ldots$ & Rowland Ward's Collection. \\
\hline $36 \frac{1}{8}$ & $5 \frac{1}{2}$ & $28 \frac{3}{4}$ & $3 \mathrm{I} \frac{3}{4}$ & $4 \times 3$ & $\cdots$ & $\cdots$ & $\cdots$ & $\ldots$ & A. O. Hume, Private Collection. \\
\hline $35^{\frac{3}{4}}$ & $5^{\frac{3}{8}}$ & $30 \frac{7}{8}$ & $34 \frac{1}{2}$ & $4 \times 5$ & $\cdots$ & India & $\cdots$ & $\cdots$ & British Museum. \\
\hline $34 \frac{7}{8}$ & $5 \frac{1}{2}$ & $27 \frac{1}{2}$ & 32 & $6 \times 6$ & $\cdots$ & $\cdots$ & $\ldots$ & ... & Hume Collection, British Museum. \\
\hline $34 \frac{5}{8}$ & $6 \frac{1}{8}$ & 24 & $30 \frac{3}{8}$ & $5 \times 6$ & $\cdots$ & $\cdots$ & $\cdots$ & $\cdots$ & A. O. Hume, Private Collection. \\
\hline $34 \frac{1}{8}$ & 5 & 24 & $32 \frac{1}{4}$ & $6 \times 6$ & $\cdots$ & $\cdots$ & $\cdots$ & $\cdots$ & Hon. Walter Rothschild. \\
\hline 34 & $5 \frac{7}{8}$ & $23^{\frac{1}{2}}$ & $29 \frac{3}{4}$ & $10 \times 10$ & ) $\quad \cdots$ & $\cdots$ & $\cdots$ & $\cdots$ & J. Carr-Saunders. \\
\hline $33 \frac{7}{8}$ & $5 \frac{1}{8}$ & $25 \frac{7}{8}$ & $28 \frac{3}{4}$ & $6 \times 6$ & ... & India & $\ldots$ & ... & British Museum. \\
\hline $33 \frac{1}{2}$ & $5 \frac{1}{2}$ & $\cdots$ & 40 & $\cdots$ & 15 & $\ldots$ & ... & $\cdots$ & Col. Pole Carew. \\
\hline
\end{tabular}


BURMESE STAG or THEMIN (Cervus eldi)-continued.

\begin{tabular}{|c|c|c|c|c|c|c|c|c|}
\hline Length. & $\begin{array}{l}\text { Circum- } \\
\text { ference. }\end{array}$ & $\begin{array}{l}\text { Tip to } \\
\text { Tip. }\end{array}$ & $\begin{array}{l}\text { Widest } \\
\text { inside. }\end{array}$ & Points. & $\begin{array}{l}\text { Length } \\
\text { of brow } \\
\text { tine. }\end{array}$ & \multicolumn{2}{|l|}{ Habitat. } & Owner. \\
\hline $33 \frac{1}{2}$ & $5^{\frac{1}{2}}$ & 25 & $29 \frac{1}{8}$ & $5 \times 4$ & $\cdots$ & $\ldots$ & $\ldots$ & Hume Collection, British Museum. \\
\hline $33 \frac{1}{2}$ & $5 \frac{1}{8}$ & $26 \frac{1}{4}$ & $30 \frac{x}{4}$ & $3 \times 3$ & $\cdots$ & $\ldots \quad \cdots$ & $\cdots$ & Do. \\
\hline $33 \frac{1}{8}$ & $5 \frac{1}{8}$ & $33 \frac{1}{8}$ & $37 \frac{1}{4}$ & $5 \times 5$ & $\cdots$ & British Bur & mah & Sir Edmund G. Loder, Bart. \\
\hline 33 & $4 \frac{5}{8}$ & $28 \frac{7}{8}$ & $32 \frac{1}{8}$ & $3 \times 3$ & $\cdots$ & $\ldots \quad \ldots$ & ... & Sir Victor Brooke's Collection. \\
\hline $3^{2 \frac{1}{8}}$ & $5 \frac{3}{8}$ & $20 \frac{1}{4}$ & $20 \frac{1}{2}$ & $4 \times 3$ & ... & $\ldots \quad \ldots$ & .. & Gen. Sir Henry Collett, K.C.B. \\
\hline $3^{1 \frac{5}{8}}$ & $4 \frac{1}{2}$ & $34 \frac{3}{8}$ & $35 \frac{7}{8}$ & $6 \times 5$ & $\cdots$ & $\cdots \quad \ldots$ & $\cdots$ & Sir Victor Brooke's Collection. \\
\hline $3^{1 \frac{1}{2}}$ & $4 \frac{1}{2}$ & 20 & 27 & $9 \times 9$ & $\cdots$ & $\cdots \quad \ldots$ & $\cdots$ & Do. \\
\hline $3 \mathrm{I}^{\frac{1}{8}}$ & 5 & $25 \frac{7}{8}$ & $29 \frac{1}{8}$ & $6 \times 7$ & $\cdots$ & Nepal... & $\cdots$ & B. H. Hodgson, British Museum. \\
\hline $30 \frac{1}{2}$ & $4 \frac{3}{4}$ & $23 \frac{1}{8}$ & $25 \frac{3}{8}$ & $5 \times 5$ & ... & India ... & ... & British Museum. \\
\hline $30 \frac{1}{2}$ & $4 \frac{3}{4}$ & $22 \frac{7}{8}$ & $25 \frac{7}{8}$ & $6 \times 5$ & $\cdots$ & ... & ... & Do. \\
\hline $30 \frac{1}{2}$ & $4 \frac{3}{4}$ & $19 \frac{1}{4}$ & $23 \frac{1}{4}$ & $5 \times 3$ & ... & Nepal... & ... & Do. \\
\hline 29 & $6 \frac{3}{8}$ & 20 & $24 \frac{3}{4}$ & $6 \times 5$ & ... & ... & ... & Hume Collection, British Museum. \\
\hline $27 \frac{3}{4}$ & $4 \frac{5}{8}$ & $27 \frac{7}{8}$ & $\cdots$ & $5 \times 6$ & ... & ... & .. & Sir Victor Brooke's Collection. \\
\hline $25 \frac{\pi}{8}$ & $4 \frac{1}{2}$ & 22 & $23 \frac{3}{4}$ & $4 \times 4$ & ... & $\ldots$ & .. & British Museum. \\
\hline 25 & $4 \frac{1}{2}$ & I $8 \frac{1}{8}$ & 24 & $6 \times 7$ & $\cdots$ & $\cdots$ & $\cdots$ & Do. \\
\hline $18 \frac{1}{2}$ & $3 \frac{5-5}{8}$ & $12 \frac{1}{2}$ & $13 \frac{1}{2}$ & $5 \times 6$ & $\cdots$ & Formosa & $\cdots$ & R. Swinhoe, British Museum. \\
\hline $17 \frac{1}{2}$ & $3 \frac{1}{8}$ & I $3 \frac{5}{8}$ & $14 \frac{3}{4}$ & $3 \times 5$ & $\cdots$ & Do. & $\cdots$ & Do. \\
\hline $15^{\frac{1}{4}}$ & $2 \frac{7}{8}$ & $9 \frac{3}{4}$ & $10 \frac{5}{8}$ & $3 \times 2$ & $\cdots$ & Do. & ... & Do. \\
\hline $13 \frac{5}{8}$ & $3 \frac{1}{4}$ & $\cdots$ & $12 \frac{3}{8}$ & $5 \times 6$ & $\cdots$ & Siam ... & $\cdots$ & British Museum. \\
\hline $13 \frac{1}{4}$ & $2 \frac{1}{2}$ & $7 \frac{5}{8}$ & $8 \frac{1}{8}$ & $3 \times 2$ & $\cdots$ & Formosa & ... & R. Swinhoe, British Museum. \\
\hline
\end{tabular}




\section{PERE DAVID'S DEER (Cervus davidianus).}

[Sir VICTOR BROOKE, P.Z.S. 1878, p. 906.]

Antrers dichotomous, about twice the length of the head, pedestals short. The anterior branch of the antlers $(b)$ more strongly developed than the posterior branch $(c)$ and in adult specimens bifurcating and carrying many strong tines. Facial portion of the skull much elongated. Ears small and narrow; eye small; tail long; neck maned; stature large; young unspotted.

Distribution-North-eastern Palæarctic region.

\begin{tabular}{|c|c|c|c|c|c|c|c|}
\hline I,ength. & $\begin{array}{l}\text { Circum- } \\
\text { ference. }\end{array}$ & $\begin{array}{l}\text { Tip to } \\
\text { Tip. }\end{array}$ & \begin{tabular}{|l|} 
Widest \\
inside.
\end{tabular} & Points. & Habitat. & & Owner. \\
\hline $32 \frac{7}{8}$ & $6 \frac{3}{8}$ & $13 \frac{5}{8}$ & I $S \frac{1}{2}$ & $8 \times 8$ & Near Pekin & $\ldots$ & Sir Edmund G. Loder, Bart. \\
\hline $28 \frac{1}{4}$ & $5 \frac{3}{8}$ & $26 \frac{1}{2}$ & $\cdots$ & $3 \times 3$ & $\ldots$ & $\ldots$ & Hon. Walter Rothschild. \\
\hline 27 & $5^{\frac{7}{8}}$ & $20 \frac{1}{4}$ & ... & $6 \times 5$ & $\ldots$ & $\ldots$ & British Museum. \\
\hline
\end{tabular}

JAVA DEER (Cervus rusa).

\begin{tabular}{l|c|c|c|c|c|c|c|c|c}
\hline Length. & $\begin{array}{c}\text { Circum- } \\
\text { ference. }\end{array}$ & $\begin{array}{c}\text { Tip to } \\
\text { Tip. }\end{array}$ & $\begin{array}{c}\text { Widest } \\
\text { inside. }\end{array}$ & Points. & Habitat. & Owner. \\
\hline $35 \frac{1}{2}$ & $4 \frac{3}{8}$ & $22 \frac{3}{4}$ & $\ldots$ & $3 \times 3$ & Java & $\ldots$ & $\ldots$ & $\begin{array}{c}\text { Sir Victor Brooke's Collection. } \\
\text { by Col. Douglas Hamilton.) }\end{array}$ & (Shot \\
34 & $4 \frac{3}{4}$ & $16 \frac{3}{8}$ & $17 \frac{1}{2}$ & $3 \times 3$ & $\ldots$ & $\ldots$ & $\ldots$ & \\
\hline
\end{tabular}




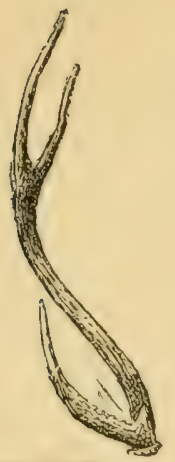

AXIS (Axis maculata).

Native Name-Chital or Chitra.

Habitat-India.

\begin{tabular}{|c|c|c|c|c|c|c|c|}
\hline $\begin{array}{l}\text { Length } \\
\text { outside } \\
\text { curve. }\end{array}$ & $\begin{array}{c}\text { Circum- } \\
\text { ference } \\
\text { above first } \\
\text { point. }\end{array}$ & Tip to Tip. & Points. & & Habitat. & & Owner. \\
\hline $37 \frac{1}{2}$ & $4 \frac{1}{4}$ & $24 \frac{3}{8}$ & $4 \times 4$ & India & $\cdots$ & $\cdots$ & A. O. Hume, Private Collection. \\
\hline $37 \frac{3}{8}$ & 4 & $24 \frac{1}{2}$ & $3 \times 3$ & Do. & $\cdots$ & $\cdots$ & Do. \\
\hline $37 \frac{1}{4}$ & $4 \frac{1}{4}$ & $16 \frac{7}{8}$ & $4 \times 5$ & Do. & $\cdots$ & $\cdots$ & Sir Victor Brooke's Collection. \\
\hline $36 \frac{8}{4}$ & $4 \frac{3}{8}$ & $19 \frac{1}{8}$ & $4 \times 4$ & Do. & $\cdots$ & $\cdots$ & W. C. Oswell. \\
\hline $36 \frac{1}{2}$ & $4 \frac{1}{2}$ & 25 & $3 \times 4$ & Do. & $\cdots$ & $\cdots$ & C. H. Seeley. \\
\hline $3^{6}$ & $4 \frac{1}{4}$ & $25 \frac{1}{4}$ & $3 \times 4$ & Do. & $\cdots$ & $\cdots$ & British Museum. \\
\hline $35^{\frac{1}{2}}$ & $4 \frac{1}{4}$ & $18 \frac{1}{8}$ & $4 \times 4$ & Do. & $\cdots$ & $\cdots$ & Sir Edmund Loder, Bart. \\
\hline $35^{\frac{1}{4}}$ & $4 \frac{1}{8}$ & $22 \frac{7}{8}$ & $3 \times 3$ & Do. & $\cdots$ & $\cdots$ & Major-Gen. Ellis, C.S.I. \\
\hline $34 \frac{3}{4}$ & $4^{\frac{1}{2}}$ & $17 \frac{3}{4}$ & $\ldots$ & Do. & $\ldots$ & $\cdots$ & H.R.H. Duke of Edinburgh. \\
\hline $35^{\frac{3}{8}}$ & $4 \frac{5}{8}$ & 23 & $3 \times 4$ & Do. & $\cdots$ & $\cdots$ & British Museum. \\
\hline $34 \frac{5}{8}$ & $3 \frac{5}{8}$ & $14 \frac{3}{8}$ & $3 \times 3$ & Do. & ... & $\ldots$ & Do. \\
\hline $34 \frac{1}{2}$ & $6 \frac{1}{2}$ & $15^{\frac{1}{2}}$ & $5 \times 4$ & Do. & $\cdots$ & $\cdots$ & Capt. G. Campbell. \\
\hline $34 \frac{1}{2}$ & $4 \frac{1}{4}$ & $20 \frac{1}{8}$ & $3 \times 3$ & Do. & $\cdots$ & $\cdots$ & Sir Victor Brooke's Collection. \\
\hline 34 & $4 \frac{3}{4}$ & $16 \frac{1}{2}$ & $\cdots$ & Do. & ... & $\cdots$ & Col. Pole-Carew. \\
\hline $33 \frac{7}{8}$ & $3 \frac{7}{8}$ & 22 & $4 \times 4$ & Do. & $\ldots$ & $\cdots$ & British Museum. \\
\hline $33 \frac{5}{8}$ & $3 \frac{7}{8}$ & $\begin{array}{c}\text { (one horn } \\
\text { broken) }\end{array}$ & $3 \times 3$ & Do. & ... & $\ldots$ & Do. \\
\hline $33^{\frac{1}{2}}$ & $4 \frac{5}{8}$ & $13 \frac{1}{8}$ & $3 \times 3$ & Do. & $\cdots$ & $\cdots$ & J. Carr-Saunders. \\
\hline $33 \frac{1}{2}$ & $4 \frac{3}{8}$ & $23 \frac{5}{8}$ & $4 \times 5$ & Centra & Provinces & $\cdots$ & Major C. S. Cumberland. \\
\hline
\end{tabular}


AXIS (Axis maculata)-continued.

\begin{tabular}{|c|c|c|c|c|c|c|c|}
\hline $\begin{array}{l}\text { Length } \\
\text { outside } \\
\text { curve. }\end{array}$ & $\mid \begin{array}{c}\text { Circum- } \\
\text { ference } \\
\text { above first } \\
\text { point. }\end{array}$ & Tip to Tip. & Points. & \multicolumn{3}{|c|}{ Habitat. } & Owner. \\
\hline $33 \frac{1}{4}$ & $4 \frac{1}{8}$ & $20 \frac{1}{4}$ & $4 \times 4$ & India & $\ldots$ & $\cdots$ & British Museum. \\
\hline $33^{\frac{1}{4}}$ & $4 \frac{1}{4}$ & $22 \frac{1}{2}$ & $4 \times 5$ & Do. & $\ldots$ & ... & Do. \\
\hline $33 \frac{1}{ \pm}$ & $3 \frac{7}{8}$ & 13 & $2 \times 3$ & $\begin{array}{r}\text { Yeddacu } \\
\text { India }\end{array}$ & & South & $\begin{array}{l}\text { Sir Victor Brooke's Collection. } \\
\text { (Shot by Col. Doug. Hamilton.) }\end{array}$ \\
\hline $33 \frac{1}{8}$ & $3 \frac{7}{8}$ & $14 \frac{33}{8}$ & $3 \times 4$ & India & $\ldots$ & $\cdots$ & Sir James Anderson, Bart. \\
\hline $33 \frac{5}{8}$ & $4 \frac{1}{8}$ & $21 \frac{1}{8}$ & $3 \times 4$ & Do. & ... & ... & British Museum. \\
\hline $32 \frac{7}{8}$ & $4 \frac{3}{8}$ & $21 \frac{1}{2}$ & $3 \times 3$ & Do. & $\ldots$ & ... & Hon. Walter Rothschild. \\
\hline $32 \frac{3^{4}}{4}$ & $3 \frac{7}{8}$ & 14 & $3 \times 3$ & Do. & $\ldots$ & $\cdots$ & Sir James Anderson, Bart. \\
\hline $32 \frac{1}{2}$ & $4 \frac{1}{4}$ & $23 \frac{3}{8}$ & $5 \times 4$ & Karkoor & r, Soutt & h India & Sir Victor Brooke's Collection. \\
\hline $32 \frac{3}{8}$ & $3 \frac{7}{8}$ & $15 \frac{1}{4}$ & $3 \times 3$ & Central & Provin & hces ... & Major C. S. Cumberland. \\
\hline $3 I \frac{1}{2}$ & $3 \frac{5}{8}$ & $19 \frac{1}{2}$ & $5 \times 5$ & India & $\ldots$ & ... & British Museum. \\
\hline $31 \frac{7}{8}$ & $4 \frac{1}{4}$ & 22 & $6 \times 6$ & Do. & ... & ... & Do. \\
\hline $3 I_{4}^{\frac{1}{4}}$ & $3 \frac{3}{4}$ & $10 \frac{7}{8}$ & $3 \times 3$ & Do. & $\cdots$ & ... & H. C. V. Hunter. \\
\hline $30 \frac{1}{2}$ & 4 & $10 \frac{1}{2}$ & $3 \times 3$ & Do. & ... & ... & Do. \\
\hline $30 \frac{3}{8}$ & $4 \frac{1}{8}$ & $21 \frac{3}{1}$ & $4 \times 4$ & Do. & $\cdots$ & ... & Sir Robert Harvey, Bart. \\
\hline $30 \frac{3}{8}$ & $3 \frac{5}{8}$ & $6 \frac{5}{8}$ & $3 \times 3$ & Do. & ... & ... & H.R.H. Duke of Edinburgh. \\
\hline $29 \frac{7}{8}$ & $4 \frac{1}{8}$ & $20 \frac{1}{4}$ & $3 \times 3$ & Do. & $\cdots$ & $\cdots$ & British Museum. \\
\hline $29 \frac{7}{8}$ & $3 \frac{1}{2}$ & $4 \frac{3}{8}$ & $4 \times 4$ & Nepal & $\cdots$ & $\cdots$ & B. H. Hodgson, British Museum. \\
\hline $29 \frac{7}{8}$ & $3 \frac{5}{8}$ & $16 \frac{1}{2}$ & $3 \times 3$ & India & $\cdots$ & ... & Sir Robert Harvey, Bart. \\
\hline $29 \frac{3}{8}$ & $4 \frac{1}{8}$ & $17 \frac{5}{8}$ & $5 \times 4$ & Do. & ... & $\cdots$ & British Museum. \\
\hline 29 & $3 \frac{5}{8}$ & $18 \frac{1}{2}$ & $3 \times 3$ & Nepal & $\cdots$ & .. & B. H. Hodgson, British Museum. \\
\hline $28 \frac{5}{8}$ & $3 \frac{3}{8}$ & $15^{\frac{1}{4}}$ & $3 \times 3$ & India & $\ldots$ & ... & Martyn Kennard. \\
\hline $28 \frac{5}{8}$ & $3 \frac{5}{8}$ & $13 \frac{7}{8}$ & $3 \times 3$ & Do. & $\ldots$ & $\cdots$ & Rowland Ward's Collection. \\
\hline 28 & $3 \frac{1}{2}$ & $12 \frac{5}{8}$ & $3 \times 3$ & Do. & $\cdots$ & $\cdots$ & Sir Robert Harvey, Bart. \\
\hline 27 & $3 \frac{1}{2}$ & $8 \frac{1}{2}$ & $3 \times 3$ & Do. & $\cdots$ & $\cdots$ & Capt. Lewis Jones, R.E. \\
\hline $24 \frac{3}{4}$ & $3 \frac{1}{8}$ & $19 \frac{3}{8}$ & $3 \times 3$ & Do. & $\cdots$ & .. & British Museum. \\
\hline $23 \frac{3}{8}$ & $2 \frac{7}{8}$ & II $\frac{1}{4}$ & $3 \times 3$ & Do. & $\cdots$ & ... & Do. \\
\hline $22 \frac{1}{8}$ & $3 \frac{3}{8}$ & $9 \frac{3}{4}$ & $6 \times 6$ & Nepal & ... & $\cdots$ & B. H. Hodgson, British Museum. \\
\hline
\end{tabular}


JAPANESE DEER (Cervus sika).

Several herds of these Deer have been acclimatized in Great Britain, notably by the late Sir Victor Brooke, Bart., at Colebrooke, Ireland, and Viscount Powercourt, county Wicklow, as well as Sir Robert Harvey, Bart, at Langley, Slough.

\section{Habitat-Japan.}

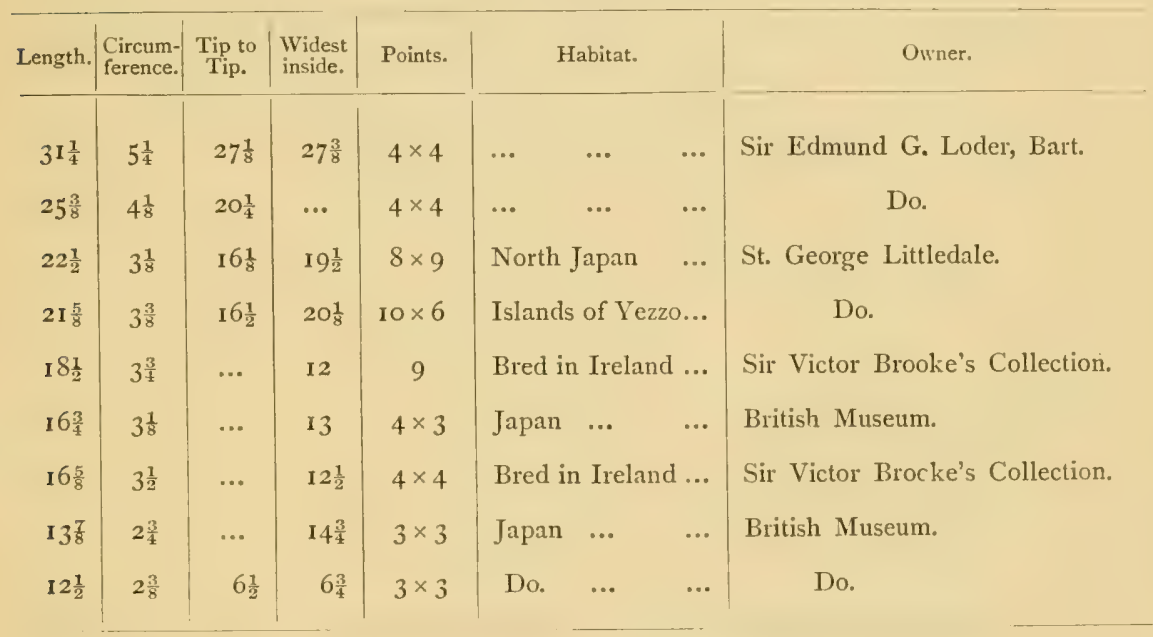

\section{IMANTCHURIAN DEER (Cervus mantchuricus).}

[SWINHOE, P.Z.S. 1865, p. 1.]

FINE buck from Newchwang, still in summer coat, examined 3oth September, I864. General colour reddish brown; under neck and fore thighs covered with rather long dusky brown hair; hair of neck, chiefly on its back part and down part of the back list, long and shaggy; list black between the shoulders, fading towards 
the tail, inner portion of hind thighs, and inside hair of ears; a large spot on the outer portion of ear near its root; rim round the eye and chin; throat white; body ornamented with large spots of white; tail, including apical hair, nearly one foot long; the hair that overhangs the roots of the tail on either side black; basal half of tail white; apical half black with white tips; under the tail and between the hams white; knees and front of tibice brown; head, from crown to tip of nose, about 20 inches long; ear about 9 inches; forehead and before the eyes reddish; rest of face brownish fawn colour; horns, with frontal tine about 5 inches long, bent a little downwards at the tips; about 8 inches of the stem between lowest tine and the next above, which are broken and represented only by a knob; top stem between that and the top knob about 4 inches.

Habitat-China.

\begin{tabular}{|c|c|c|c|c|c|}
\hline Length. & $\begin{array}{l}\text { Circum- } \\
\text { ference. }\end{array}$ & $\begin{array}{l}\text { Tip to } \\
\text { Tip. }\end{array}$ & Points. & Habitat. & Owner. \\
\hline $15^{\frac{3}{3}}$ & 3 & 20 & $4 \times 4$ & South Ussari, Mantchuria ... & British Museum. \\
\hline
\end{tabular}

FORIMOSA DEER (Cervus taëvanus).

\begin{tabular}{c|c|c|c|c|c|c|c}
\hline Length. & $\begin{array}{c}\text { Circum- } \\
\text { ference. }\end{array}$ & $\begin{array}{c}\text { Tip to } \\
\text { Tip. }\end{array}$ & $\begin{array}{c}\text { Widest } \\
\text { inside. }\end{array}$ & Points. & Habitat. & Owner. \\
\hline $19 \frac{3}{4}$ & $3 \frac{3}{8}$ & 13 & $16 \frac{3}{8}$ & $4 \times 4$ & Islands of Formosa & ... & British Museum. \\
\hline
\end{tabular}



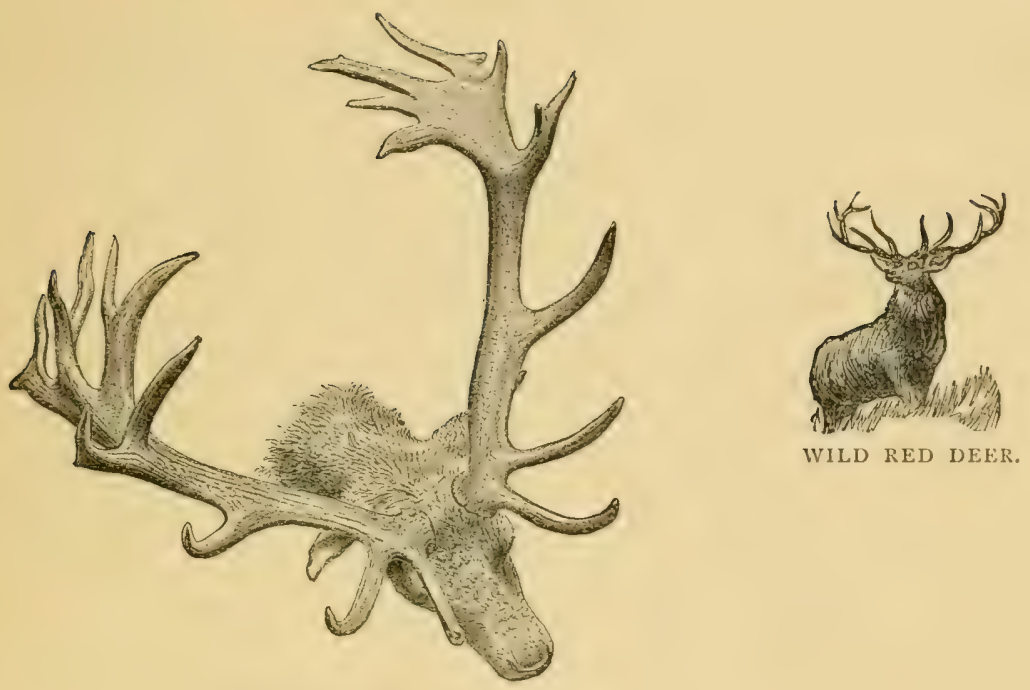

STOKE PARK DEER HEAD.

\section{RED DEER (Cervus elaphus).}

Habitat-Europe, Western Asia, and North Africa.

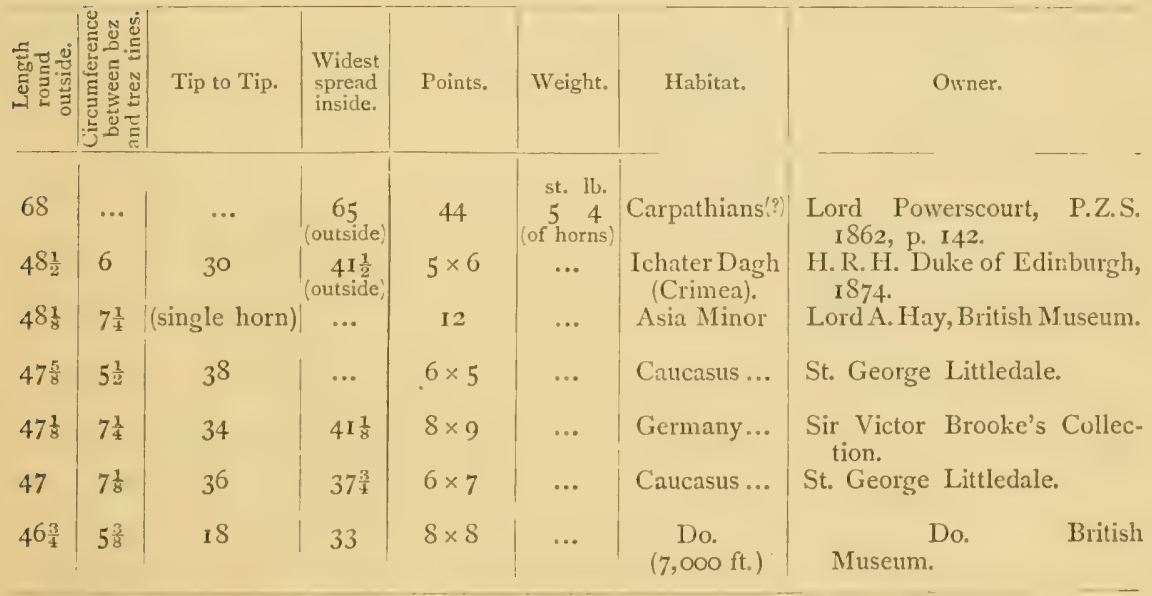


RED DEER (Cervus elaphus)-continued.

\begin{tabular}{|c|c|c|c|c|c|c|c|}
\hline 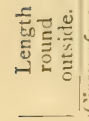 & كّ & Tip to Tip. & $\begin{array}{l}\text { Willest } \\
\text { spreal } \\
\text { inside. }\end{array}$ & Points. & Weight. & Habitat. & Owner. \\
\hline $45{ }^{3}$ & 73 & $25 \frac{1}{5}$ & 35 & $S \times S$ & $\begin{array}{l}\text { st. } 1 \mathrm{his} \text {. } \\
\cdots\end{array}$ & Caucasus... & St. George Littledale. \\
\hline 45 & $6 \frac{1}{5}$ & $263_{3}^{3}$ & $3_{3}^{1}$ & $5 \times 5$ & $\ldots$ & Hungary ... & $\begin{array}{l}\text { Major - Gen. Arthur Ellis, } \\
\text { C.S I., ISS6. }\end{array}$ \\
\hline $45^{\frac{1}{8}}$ & $7 \frac{33}{4}$ & $\cdots$ & $\cdots$ & $9 \times 6$ & $\cdots$ & Asia Minor & Sir Edmund G. Loder, Bart. \\
\hline 44 & $6 !$ & $33:$ & $35 k$ & $7 \times 5$ & $\cdots$ & Caucasus ... & St. George Littledale. \\
\hline 44 & $6 ?$ & 39 & 40 & $7 \times 7$ & $\cdots$ & Hungary ... & $\begin{array}{l}\text { Major-Gen. Arthur Ellis, } \\
\text { C.S.I., I } 8 S 6 \text {. }\end{array}$ \\
\hline $44^{1}$ & 7 & $31 \frac{1}{4}$ & 34 & $6 \times 6$ & $\ldots$ & Caucasus ... & St. George Littledale. \\
\hline $44^{\frac{1}{4}}$ & 67 & (single horn) & $\ldots$ & 9 & $\ldots$ & Asia Minor & $\begin{array}{l}\text { C. G. Danford, IBilish } \\
\text { Museum. }\end{array}$ \\
\hline $43 \frac{1}{2}$ & $6 \%$ & $21 \frac{3}{4}$ & 35 & $7 \times 7$ & $\cdots$ & Caucasus... & St. George Littledale. \\
\hline $43 \frac{1}{\frac{1}{2}}$ & 6 & ... & $3 S \frac{1}{2}$ & $S \times 6$ & $\ldots$ & Asia Minor & E. N. Buxton. \\
\hline 43 & $6 \frac{1}{2}$ & $20 !$ & 25 & $\begin{array}{l}\$ \times 2 \\
\text { (malformed }\end{array}$ & ... & Caucasus... & St. George Littleclale. \\
\hline $42 \frac{1}{2}$ & $6 !$ & $34:$ & 373 & $6 \times 7$ & $\cdots$ & Du. & Do. \\
\hline 428 & $6: 8$ & 371 & 41 & $7 \times 7$ & $\cdots$ & 1). & I 887. \\
\hline $41 \frac{7}{8}$ & $6 \frac{1}{2}$ & $15 \pi$ & 2)! & $6 \times 5$ & $\cdots$ & Do. & Do. \\
\hline $41 \frac{7}{5}$ & $5 i$ & $\cdots$ & 251 & $6 \times 6$ & $\ldots$ & Germany... & British Museum. \\
\hline $41 \frac{1}{8}$ & 54 & $15^{\frac{1}{2}}$ & $2 S !$ & $6 \times 5$ & $\cdots$ & Caucasus... & H. R.H. Duc d'Orleans. \\
\hline 405 & $6 \overline{5}$ & (single horn) & $\cdots$ & s & $\cdots$ & Crimea $\ldots$ & British Museum. \\
\hline $40 \frac{1}{4}$ & 6 & $18 \frac{1}{8}$ & $2 S !$ & $6 \times 8$ & $\cdots$ & Hungary ... & $\begin{array}{l}\text { Major-Gen. Arthur Ellis, } \\
\text { C... I., ISS6. }\end{array}$ \\
\hline $40 \frac{1}{4}$ & 5 & $20 \frac{3}{4}$ & 27 & $7 \times 6$ & $\ldots$ & $\cdots$ & H. R.H. Duke of Edinburgh. \\
\hline 395 & $5 \frac{1}{1}$ & $1+\frac{1}{2}$ & $26 \%$ & $7 \times 7$ & 26) 11 & $\begin{array}{l}\text { Ireland (age } \\
8 \text { years! }\end{array}$ & $\begin{array}{l}\text { Sir Victor Brooke's Collec- } \\
\text { tion, } 1 \$ 86 .\end{array}$ \\
\hline $39 \frac{1}{1}$ & 6 & 30 & $33 \frac{1}{2}$ & $6 \times 5$ & $\cdots$ & Caucasus... & St. George Littledale. \\
\hline $35 \frac{7}{8}$ & 53 & $\cdots$ & $\cdots$ & $5 \times 6$ & $\cdots$ & $\cdots$ & Sir Edmund G. Loder, Bart. \\
\hline $3 S \frac{1}{2}$ & $4 \frac{7}{8}$ & $22 \frac{1}{2}$ & $3 \mathrm{I}$ & $S \times S$ & 2311 & $\begin{array}{l}\text { Ireland (age } \\
\text { Io years). }\end{array}$ & $\begin{array}{l}\text { Sir Victor Brooke's Collec- } \\
\text { tion, I } 88 \mathrm{I} \text {. }\end{array}$ \\
\hline $37 s^{3}$ & $55^{5}$ & $1+\frac{3}{4}$ & $26 \frac{1}{5}$ & $6 \times 7$ & $\cdots$ & $\cdots \quad \cdots$ & H. R.H. Duke of Edinburgh. \\
\hline $3^{\Upsilon \frac{1}{5}}$ & 5 & $26 \frac{1}{1}$ & 30 & $7 \times 7$ & 250 & $\begin{array}{l}\text { Ireland (age } \\
9 \text { years). }\end{array}$ & $\begin{array}{l}\text { Sir Victor Brooke's Collec- } \\
\text { tion, } \mathbf{S} \$ 4 .\end{array}$ \\
\hline $37 \frac{1}{1}$ & 6 & $32 \frac{1}{6}$ & $35 \frac{1}{8}$ & $7 \times 8$ & $\cdots$ & $\begin{array}{l}\text { Ireland (age } \\
12 \text { years). }\end{array}$ & $\begin{array}{l}\text { Sir Victor Brooke's Collec- } \\
\text { tion, I } \delta 7 \mathrm{~S} \text {. }\end{array}$ \\
\hline
\end{tabular}

* Cervus barbarus. 
RED DEER (Cervus elaphus)-continud.

\begin{tabular}{|c|c|c|c|c|c|c|c|}
\hline 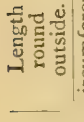 & 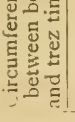 & Tip to Tip. & $\begin{array}{l}\text { Widest } \\
\text { spread } \\
\text { inside. }\end{array}$ & Points. & Weight. & Habitat. & Owner. \\
\hline $37 \frac{1}{4}$ & 5 & 24 & 29 & $7 \times 7$ & $\begin{array}{c}\text { st. Ibs. } \\
\cdots\end{array}$ & Caucasus... & H.R.H. Duc d'Orleans. \\
\hline $37^{\frac{1}{4}}$ & 5 & $14^{\frac{3}{4}}$ & $24 \frac{3}{4}$ & $6 \times 5$ & $\cdots$ & $\cdots$ & H.R.H. Duke of Edinburgh. \\
\hline $3^{6 \frac{1}{2}}$ & $6 \frac{3}{8}$ & $\cdots$ & $32 \frac{1}{2}$ & $9 \times 9$ & $\cdots$ & $\begin{array}{l}\text { Wairarapa, } \\
\text { N.Z. }\end{array}$ & $\begin{array}{l}\text { J. Handyside. (The Field, } \\
\text { Feb. } 6,1891 .)\end{array}$ \\
\hline $3^{6 \frac{1}{2}}$ & $5 \frac{1}{4}$ & $19 \frac{1}{2}$ & $24 \frac{3}{4}$ & $9 \times 7$ & $\cdots$ & $\cdots$ & J. Carr Saunders. \\
\hline$* 3^{6 \frac{1}{2}}$ & $4 \frac{3}{4}$ & $22 \frac{7}{8}$ & $28 \frac{3}{\frac{3}{4}}$ & $4 \times 4$ & $\cdots$ & $\cdots$ & British Museum. \\
\hline$* 36 \frac{3}{8}$ & $4^{\frac{1}{2}}$ & $17 \frac{1}{2}$ & $25 \frac{5}{8}$ & $4 \times 4$ & $\cdots$ & $\cdots$ & Do. \\
\hline $3^{6}$ & $5 \frac{1}{2}$ & 37 & $\cdots$ & I5 & $\cdots$ & South Spain & Lord Lilford. \\
\hline 36 & $6 \frac{1}{2}$ & 24 & 30 & $6 \times 6$ & $\ldots$ & $\begin{array}{l}\text { Vaynol Park, } \\
\text { North Wales. }\end{array}$ & Assheton Smith. \\
\hline $35 \frac{1}{2}$ & $5 \frac{1}{4}$ & $26 \frac{1}{2}$ & $32 \frac{3}{8}$ & $\begin{array}{c}\mathrm{I} 3 \times \mathbf{I} \mathbf{I} \\
\text { (perisherd) }\end{array}$ & $\cdots$ & $\begin{array}{l}\text { Ireland (dug } \\
\text { up). }\end{array}$ & $\begin{array}{l}\text { Sir Victor Brooke's Collec- } \\
\text { tion. }\end{array}$ \\
\hline 35 & 6 & $14 \frac{1}{2}$ & 24 & $6 \times 6$ & $\cdots$ & Vaynol Park, & Assheton Smith. \\
\hline 35 & $\cdots$ & $\cdots$ & 34 & 10 & 161 & North Morar & T. W. Gill. (The Field, \\
\hline 35 & $4 \frac{3}{4}$ & $\cdots$ & 30 & 9 & $\cdots$ & Ireland ... & Hon. A. Charter.s, 1889. \\
\hline $34^{\frac{3}{4}}$ & $5^{\frac{3}{4}}$ & $20 \frac{1}{4}$ & $27 \frac{3}{4}$ & $7 \times 8$ & $\cdots$ & Caucasus ... & H.R.H. Duc d'Orleans. \\
\hline $34 \frac{5}{8}$ & $5 \frac{1}{8}$ & (single horn) & $\cdots$ & 7 & $\cdots$ & Germany... & British Museum. \\
\hline $34 \frac{1}{2}$ & $5 \frac{1}{8}$ & (shed horns) & $\cdots$ & II $\times 9$ & $\cdots$ & $\begin{array}{l}\text { Ireland (still } \\
\text { alive). }\end{array}$ & $\begin{array}{l}\text { Sir Victor Brooke's Collec- } \\
\text { tion. }\end{array}$ \\
\hline $34^{\frac{1}{2}}$ & 5 & $17 \frac{3}{4}$ & $26 \frac{1}{4}$ & $7 \times 7$ & $\cdots$ & $\cdots \quad \cdots$ & J. Carr-Saunders. \\
\hline $34 \frac{3}{8}$ & $5 \frac{1}{8}$ & $25^{\frac{1}{4}}$ & $29 \frac{3}{8}$ & $8 \times 9$ & 290 & $\begin{array}{l}\text { Ireland (age } \\
8 \text { years). }\end{array}$ & $\begin{array}{l}\text { Sir Victor Brooke's Collec- } \\
\text { tion, I } 875 \text {. }\end{array}$ \\
\hline $34 \frac{3}{4}$ & $4 \frac{1}{8}$ & $16 \frac{1}{4}$ & $24 \frac{3}{8}$ & $5 \times 5$ & $\cdots$ & Scotland ... & F. Devas. \\
\hline 34 & $4 \frac{3}{4}$ & $\cdots$ & $3 \mathbf{I}^{\frac{1}{2}}$ & $6 \times 5$ & 268 & Ireland $\ldots$ & Ralph Sneyd. \\
\hline 34 & $5 \frac{1}{4}$ & 34 & $\ldots$ & $5 \times 5$ & $\cdots$ & Caucasus... & H.R.H. Duc d'Orleans. \\
\hline 34 & $\cdots$ & ... & $30 \frac{1}{2}$ & II & 172 & North Morar & $\begin{array}{l}\text { T. W. Gill. (The Fritd, } \\
\text { Dec. 6, I89o. }\end{array}$ \\
\hline 34 & $4 \frac{1}{2}$ & $\cdots$ & 23 & $6 \times 6$ & ... & $\cdots$ & H.R.H. Comtesse de Paris. \\
\hline $33^{\frac{3}{4}}$ & 5 & $\cdots$ & 26 & $5 \times 5$ & ... & $\begin{array}{l}\text { Muckross, } \\
\text { Ireland. }\end{array}$ & Hon. Mrs. Bourke. \\
\hline $33^{\frac{3}{4}}$ & $4 \frac{7}{8}$ & $14 \frac{1}{2}$ & $21 \frac{3}{4}$ & $7 \times 6$ & $\cdots$ & $\cdots \quad \cdots$ & H. R.H. Duke of Edinburgh. \\
\hline $33 \frac{1}{8}$ & $4 \frac{7}{8}$ & $17 \frac{5}{8}$ & $24 \frac{1}{2}$ & $4 \times 4$ & $\cdots$ & Scotland ... & Lord d'Arcy Osborn. \\
\hline
\end{tabular}

* Cervus barbarus. 


\section{RED DEER (Cervus elaphus)-continued.}

\begin{tabular}{|c|c|c|c|c|c|c|c|c|}
\hline 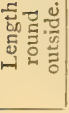 & $\begin{array}{l}0 \\
0\end{array}$ & Tip to Tip. & $\begin{array}{l}\text { Widest } \\
\text { spread } \\
\text { inside. }\end{array}$ & Points. & Weight. & Habitat. & & Owner. \\
\hline 33 & $4 \frac{3}{4}$ & $26 \frac{7}{8}$ & $3 \mathbf{I} \frac{5}{8}$ & $6 \times 6$ & $\begin{array}{c}\text { st. lbs. } \\
\ldots\end{array}$ & Rosshire. & $\cdots$ & E. Lort Phillips. \\
\hline 33 & $6 \frac{1}{2}$ & ... & $3^{\circ}$ & $7 \times 7$ & 29 II & $\begin{array}{l}\text { Muckross, } \\
\text { Ireland. }\end{array}$ & & Hon. A. Bourke. \\
\hline 33 & $4 \frac{3}{4}$ & I6 & $23 \frac{1}{2}$ & $6 \times 6$ & ... & Vaynol Pa & ark, & Assheton Smith. \\
\hline $32 \frac{3}{4}$ & $4 \frac{1}{2}$ & $\ldots$ & $2 S$ & $5 \times 4$ & ... & Scotland. & & Sir Charles Tennant, Bart. \\
\hline $32 \frac{5}{8}$ & $4 \frac{5}{8}$ & $\mid$ (single horn) $\mid$ & ... & 4 & $\cdots$ & $\cdots$ & $\cdots$ & British Museum. \\
\hline $32 \frac{5}{8}$ & $4 \frac{1}{8}$ & $20 \frac{3}{4}$ & $29 \frac{7}{8}$ & $5 \times 5$ & $\cdots$ & $\ldots$ & $\cdots$ & H. R. H. Duke of Edinburgh. \\
\hline $32 \frac{1}{2}$ & 6 & ... & 32 & $6 \times 6$ & ... & Morar & $\ldots$ & $\begin{array}{l}\text { Capt. Gill. (The Field, } \\
\text { Feb. } 6, \text { isgr.) }\end{array}$ \\
\hline $32 \frac{3}{6}$ & $4 \frac{5}{8}$ & $24 \frac{7}{8}$ & $31 \frac{1}{8}$ & $5 \times 5$ & $\cdots$ & $\cdots$ & $\cdots$ & H.K.H. Duke of Edinburgh. \\
\hline 32 & 5 & $\cdots$ & $22 \frac{1}{2}$ & $5 \times 4$ & 190 & Scotland. & $\cdots$ & Sir W. G. Pearce, Bart. \\
\hline $3 \mathbf{I} \frac{3}{4}$ & $3 \frac{3}{4}$ & $\cdots$ & $26 \frac{1}{2}$ & $4 \times 4$ & $\cdots$ & Do. & ... & Capt. F. Cookson. \\
\hline $3 \mathrm{I} \frac{3}{4}$ & $4 \frac{3}{4}$ & $\cdots$ & $25 \frac{1}{2}$ & $6 \times 5$ & $\cdots$ & $\cdots$ & $\cdots$ & A. Brassey. \\
\hline $3 \mathrm{I}^{\frac{1}{2}}$ & 5 & $\cdots$ & $26 \frac{1}{2}$ & 12 & 170 & Scotland. & $\cdots$ & J. F. Laycock. \\
\hline $3 I^{\frac{1}{2}}$ & $4 \frac{3}{4}$ & $\ldots$ & 28 & $4 \times 3$ & $\ldots$ & Norway . & $\cdots$ & E. N. Denny. \\
\hline $3 I^{\frac{1}{2}}$ & $4 \frac{1}{2}$ & $\cdots$ & $26 \frac{1}{2}$ & $6 \times 4$ & $\ldots$ & Scotland. & $\cdots$ & Sir IV. G. Pearce, Bart. \\
\hline $31 \frac{1}{2}$ & $4 \frac{1}{2}$ & $\cdots$ & $24 \frac{1}{2}$ & $5 \times 5$ & $\cdots$ & Norway . & $\cdots$ & I. Seton Karr, M.P. \\
\hline $31 \frac{1}{4}$ & $4 \frac{1}{8}$ & I6 & $24 \frac{1}{8}$ & $7 \times 5$ & $\cdots$ & Scotland . & $\ldots$ & Capt. F. Cookson. \\
\hline $3 I_{4}^{\frac{1}{4}}$ & $4 \frac{5}{8}$ & $24 \frac{7}{8}$ & 32 & $5 \times 3$ & $\cdots$ & Rosshire . & $\cdots$ & E. Lort Phillips. \\
\hline $3 \mathrm{I}$ & 5 & $\cdots$ & $22 \frac{1}{2}$ & I 2 & IS I & Scotland. & $\cdots$ & J. F. Laycock. \\
\hline $3 \mathrm{I}$ & $4 \frac{1}{4}$ & $\cdots$ & $26 \frac{1}{2}$ & $6 \times 4$ & ... & Do. & ... & Sir W. G. Pearce, Bart. \\
\hline $3^{I}$ & $4 \frac{1}{4}$ & $\cdots$ & $20 \frac{1}{2}$ & $6 \times 6$ & ... & Do. & $\cdots$ & W. N. Diggle. \\
\hline $3 \mathrm{I}$ & 4 & $\cdots$ & $28 \frac{1}{2}$ & II & I5 O & Do. & $\cdots$ & J. F. Laycock. \\
\hline $3 \mathrm{I}$ & 4 & $\cdots$ & $24 \frac{1}{2}$ & $4 \times 4$ & $\cdots$ & Do. & $\cdots$ & Sir Chas. Tennant, Bart. \\
\hline 307 & $4 \frac{3}{8}$ & $25 \frac{7}{8}$ & $28 \frac{1}{8}$ & $5 \times 6$ & $\cdots$ & Do. & ... & IV. R. Cookson, \\
\hline $30^{\frac{3}{4}}$ & $4 \frac{5}{8}$ & $15 \frac{7}{8}$ & $2 \mathrm{I} \frac{1}{2}$ & $6 \times 6$ & $\cdots$ & Do. & ... & Capt. F. Cookson. \\
\hline $30 \frac{1}{2}$ & $4 \frac{1}{2}$ & $\cdots$ & 20 & $6 \times 6$ & 147 & Do. & $\cdots$ & J. F. Laycock. \\
\hline $30 \frac{1}{2}$ & $4 \frac{1}{4}$ & 25 & $25 \frac{7}{8}$ & $6 \times 4$ & ... & $\cdots$ & $\cdots$ & Cel. G. A. Percy. \\
\hline
\end{tabular}


RED DEER (Cervus elaphus)-continued.

\begin{tabular}{|c|c|c|c|c|c|c|c|}
\hline 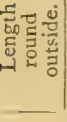 & 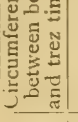 & Tip to Tip. & $\begin{array}{l}\text { Widest } \\
\text { spread } \\
\text { inside. }\end{array}$ & Points. & Weight. & Habitat. & Owner. \\
\hline $30 \frac{1}{2}$ & $4 \frac{1}{8}$ & $23 \frac{1}{8}$ & $26 \frac{5}{8} \mid$ & $5 \times 5$ & $\begin{array}{l}\text { st. lbs. } \\
\ldots\end{array}$ & Scotland ... & Capt. F. Cookson. \\
\hline $30 \frac{3}{8}$ & $4 \frac{1}{2}$ & $14 \frac{1}{8}$ & $24 \frac{1}{8}$ & $5 \times 5$ & ... & Do. $\quad \ldots$ & Miss Laycock. \\
\hline $30 \frac{1}{4}$ & $4 \frac{1}{4}$ & ... & 24 & $6 \times 4$ & $\cdots$ & $\begin{array}{ll}\ldots & \ldots\end{array}$ & E. N. Buxton, ISSo. \\
\hline $30 \frac{1}{8}$ & $4 \frac{1}{x}$ & (single horn) & $\cdots$ & 9 & $\cdots$ & Germany ... & British Museum. \\
\hline 30 & 5 & $\cdots$ & 29 & I 2 & 200 & Norway ... & E. M. Denny. \\
\hline 30 & $4 \frac{1}{8}$ & $25 \frac{1}{8}$ & $28 \frac{7}{8}$ & $4 \times 5$ & $\ldots$ & $\ldots$ & Col. G. A. Percy. \\
\hline $29 \frac{3}{4}$ & $4 \frac{1}{8}$ & $21 \frac{5}{8}$ & $23 \frac{3}{8}$ & $6 \times 6$ & $\ldots$ & Scotland ... & J. F. Laycock. \\
\hline $29 \frac{5}{8}$ & $3 \frac{7}{8}$ & $26 \frac{1}{2}$ & $28 \frac{3}{8}$ & $6 \times 6$ & ... & $\begin{array}{ll}\ldots & \cdots\end{array}$ & Col. G. A. Percy. \\
\hline $29 \frac{1}{2}$ & $4 \frac{1}{2}$ & $\ldots$ & 24 & $4 \times 4$ & I4 I & Scotland ... & J. F. Laycock. \\
\hline $29 \frac{3}{8}$ & $4 \frac{1}{4}$ & $23 \frac{3}{8}$ & $27 \frac{1}{2}$ & $5 \times 5$ & ... & $\cdots \quad \ldots$ & Col. G. A. Percy. \\
\hline $29 \frac{1}{4}$ & $3 \frac{7}{8}$ & $\cdots$ & $2 I \frac{1}{2}$ & $5 \times 6$ & $\cdots$ & Germany... & British Museum. \\
\hline $29 \frac{1}{8}$ & $5^{\frac{1}{4}}$ & $24 \frac{5}{8}$ & $27 \frac{5}{8}$ & $3 \times 4$ & $\cdots$ & Scotland ... & Sir William Eden, Bart. \\
\hline $29 \frac{7}{8}$ & $4 \frac{5}{8}$ & $19 \frac{1}{2}$ & $25^{\frac{1}{4}}$ & $5 \times 5$ & $\cdots$ & $\ldots \quad \ldots$ & Col. G. A. Percy. \\
\hline 29 & $4 \frac{1}{2}$ & $\cdots$ & 24 & $4 \times 4$ & $\cdots$ & Scotland ... & W. N. Diggle. \\
\hline 29 & $4 \frac{1}{4}$ & $\cdots$ & 29 & $6 \times 5$ & $\cdots$ & Do. $\quad \cdots$ & J. F. Laycock. \\
\hline 29 & $3^{\frac{3}{4}}$ & $\cdots$ & $24 \frac{1}{2}$ & $6 \times 4$ & $\cdots$ & Do. $\quad \ldots$ & J. E. Wilmot. \\
\hline $2 S_{\frac{3}{4}}$ & $3_{\frac{3}{4}}^{\frac{3}{4}}$ & $\cdots$ & 23 & $4 \times 3$ & $\cdots$ & Do. $\quad \cdots$ & F. Devas. \\
\hline $28 \frac{1}{2}$ & $5 \frac{1}{2}$ & $\therefore$ & 29 & $6 \times 6$ & $\cdots$ & Norway ... & E. Lee Townshend. \\
\hline $28 \frac{1}{2}$ & $4 \frac{1}{2}$ & $\cdots$ & $24 \frac{1}{2}$ & $6 \times 6$ & $\cdots$ & Scotland ... & Sir Charles Tennant, Bart. \\
\hline $28 \frac{1}{2}$ & $4 \frac{1}{2}$ & $\cdots$ & 22 & $6 \times 5$ & I8 7 & Do. $\quad \cdots$ & Sir W. G. Pearce, Bart. \\
\hline $28 \frac{1}{2}$ & $4 \frac{3}{8}$ & I $8 \frac{5}{8}$ & $24 \frac{5}{8}$ & $4 \times 5$ & $\cdots$ & $\cdots \quad \ldots$ & H. R.H. Duke of Edinburgh. \\
\hline $28 \frac{1}{4}$ & $4 \frac{1}{2}$ & $16 \frac{1}{2}$ & $22 \frac{7}{8}$ & $5 \times 5$ & $\cdots$ & Scotland ... & W. R. Cookson. \\
\hline $28 \frac{1}{8}$ & $4 \frac{1}{2}$ & $29 \frac{1}{4}$ & $33 \frac{3}{8}$ & $6 \times 6$ & $\cdots$ & $\cdots \quad \cdots$ & Col. G. A. Percy. \\
\hline 28 & 5 & $\cdots$ & 23 & $5 \times 3$ & 177 & Scotland ... & J. F. Laycock. \\
\hline $27 \frac{7}{8}$ & $4 \frac{1}{8}$ & $17 \frac{5}{8}$ & $23 \frac{3}{8}$ & $5 \times 5$ & $\cdots$ & Do. $\quad \cdots$ & Lord D'Arcy Osborn. \\
\hline $27 \frac{7}{8}$ & 4 & $20 \frac{3}{8}$ & $27 \frac{1}{4}$ & $4 \times 4$ & $\cdots$ & $\cdots$ & E. N. Buxton. \\
\hline
\end{tabular}


RED DEER (Cervus elaphus)-continued.






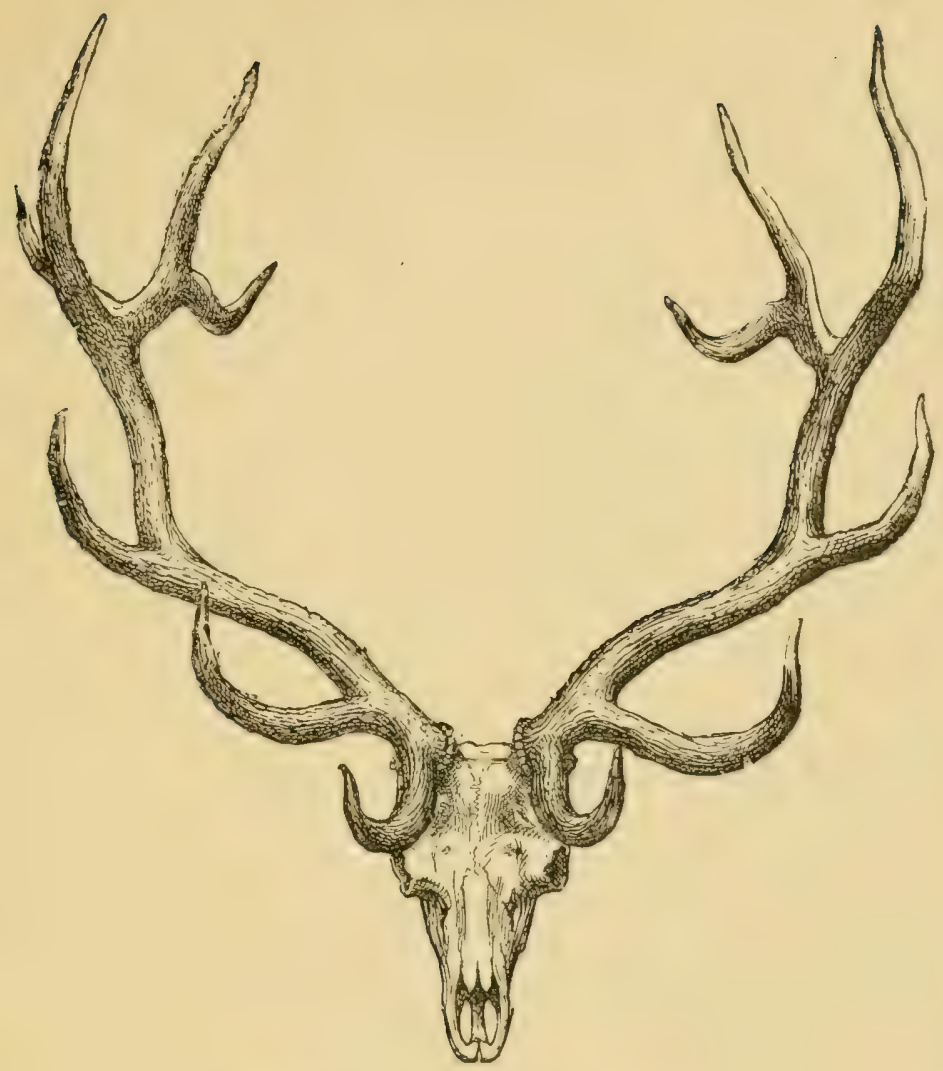

WAPITI (Cervus canadensis).

Weight-About 700 lbs. Height at Shoulder-About I6 hands. Habitat-North America.

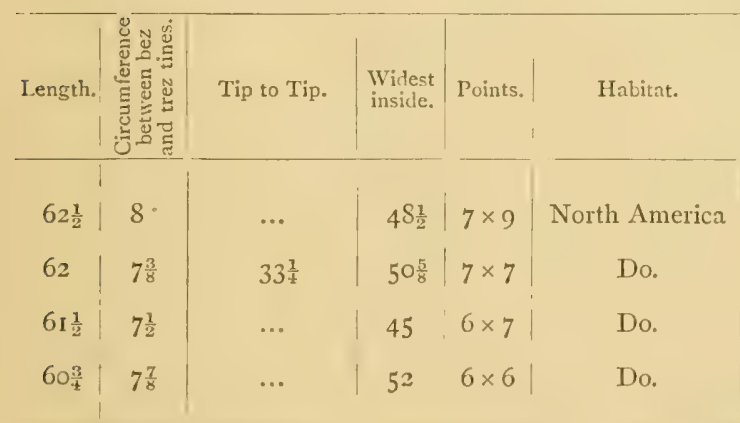

Owner.

Frank Cooper (American Exhibition Catalogue).

Ernest Farquhar.

Do.

W. A. Baillie-Grohman (American Exhibition Catalogue). 
WAPITI (Cervus canajensis)-continued.

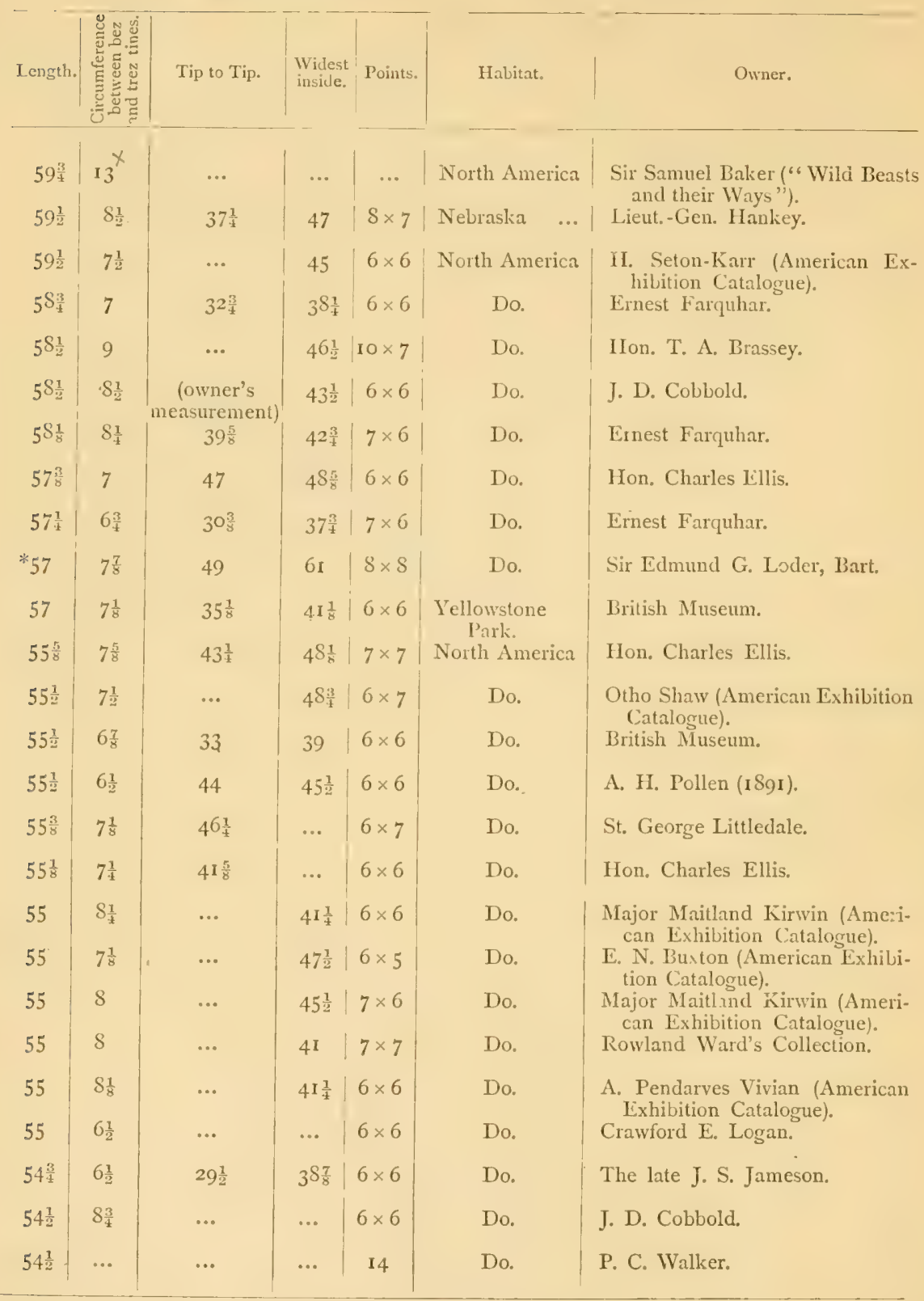

* Weight- $42 \frac{1}{2}$ lbs. With small frontal. 
WAPITI (Cervus canadensis)-continued.

\begin{tabular}{|c|c|c|c|c|c|c|}
\hline Length. & 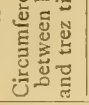 & Tip to Tip. & $\begin{array}{l}\text { Widest } \\
\text { inside. }\end{array}$ & Points. & Habitat. & Owner. \\
\hline $54^{\frac{1}{2}}$ & $7 \frac{1}{8}$ & $\cdots$ & $4 I \frac{1}{8}$ & $6 \times 6$ & North America & $\begin{array}{l}\text { British Museum (American } \\
\text { Exhibition Catalogue). }\end{array}$ \\
\hline $54 \frac{1}{2}$ & 7 & $\cdots$ & $42 \frac{1}{2}$ & $8 \times 7$ & Do. & S. C. Alexander. \\
\hline $54 \frac{3}{8}$ & $6 \frac{1}{3}$ & $38 \frac{3}{8}$ & $44 \frac{1}{4}$ & $7 \times 6$ & Buffalo Fork... & E. N. Buxton. \\
\hline $54 \frac{1}{4}$ & 8 & $\ldots$ & $43^{\frac{1}{2}}$ & $10 \times 10$ & North America & $\begin{array}{l}\text { W, A. Tullock (American } \\
\text { Exhibition Catalogue). }\end{array}$ \\
\hline $54 \frac{1}{4}$ & $7 \frac{5}{8}$ & $49 \frac{7}{8}$ & $\cdots$ & $7 \times 9$ & Do. & Hon. Walter Rothschild. \\
\hline 54 & $7 \frac{7}{8}$ & $\cdots$ & $\cdots$ & $6 \times 6$ & Do. & J. L. Carroll, \\
\hline 54 & $7 \frac{1}{2}$ & $\ldots$ & ... & $6 \times 7$ & Do. & H. Hadden. \\
\hline 54 & $7 \frac{1}{4}$ & $\cdots$ & $4^{8}$ & $7 \times 8$ & Do. & Earl of Rosslyn (American \\
\hline 54 & $7 \frac{1}{4}$ & $\ldots$ & 48 & $7 \times 8$ & Do. & $\begin{array}{c}\text { Moreton Frewen (American } \\
\text { Exhibition Catalogue). }\end{array}$ \\
\hline 54 & $\cdots$ & $\cdots$ & ... & $\cdots$ & Do. & Capt. F. Cookson. \\
\hline 54 & $\cdots$ & $\ldots$ & $\cdots$ & 14 & Do. & W. S. Power. \\
\hline 54 & 7 & $\cdots$ & $40 \frac{1}{4}$ & ... & Do. & British Museum (American \\
\hline $53 \frac{1}{8}$ & $7 \frac{3}{4}$ & $24 \frac{3}{4}$ & $36 \frac{1}{4}$ & $8 \times 8$ & Do. & St. George Littledale. \\
\hline $53 \frac{1}{8}$ & $7 \frac{3}{8}$ & 47 & $49 \frac{3}{4}$ & $8 \times 7$ & Do. & Ford G. Barclay. \\
\hline 53 & $12 \frac{1}{2}^{x}$ & $\ldots$ & $\ldots$ & $\mid \begin{array}{c}\text { (palinated) } \\
\ldots\end{array}$ & Do. & $\begin{array}{l}\text { Sir Samuel Baker ("Wild } \\
\text { Beasts and their Ways"). }\end{array}$ \\
\hline $52 \frac{3}{4}$ & $8 \frac{1}{4}$ & ... & $4 \mathrm{I}$ & $6 \times 6$ & Do. & $\begin{array}{l}\text { Frank Cooper (American EX- } \\
\text { hibition Catalogue). }\end{array}$ \\
\hline $52 \frac{3}{4}$ & $7 \frac{1}{2}$ & $34 \frac{5}{8}$ & $42 \frac{3}{8}$ & $7 \times 7$ & Do. & St. George Littledale. \\
\hline $52 \frac{1}{2}$ & $7 \frac{1}{2}$ & (owner's & $\ldots$ & 12 & Do. & W. H. Hammersley. \\
\hline $52 \frac{1}{2}$ & $7 \frac{3}{8}$ & $\begin{array}{c}\text { measurement) } \\
\ldots\end{array}$ & $37 \frac{1}{t}$ & $6 \times 6$ & Do. & $\begin{array}{l}\text { Sir H. Rae Reid, Bart. (Ameri- } \\
\text { can Exhibition Catalogue) }\end{array}$ \\
\hline $52 \frac{1}{2}$ & $6 \frac{1}{2}$ & $\cdots$ & $34^{\frac{3}{4}}$ & $7 \times 6$ & Do. & Ford G. Barclay. \\
\hline $52 \frac{3}{8}$ & $5^{\frac{3}{4}}$ & $49 \frac{3}{8}$ & $\ldots$ & $6 \times 5$ & Do. & Col. Ralph Vivian. \\
\hline $5^{2}$ & $8 \frac{1}{2}$ & $\cdots$ & $\cdots$ & $6 \times 6$ & Do. & Hon. F. Thellusson. \\
\hline 52 & $6 \frac{1}{2}$ & $\cdots$ & $\cdots$ & 14 & Do. & Do. \\
\hline 52 & 6 & $37 \frac{3}{4}$ & 40 & $6 \times 6$ & Do. & Capt. F. Cookson. \\
\hline $51 \frac{3}{4}$ & $6 \frac{3}{4}$ & 29 & $38 \frac{7}{8}$ & $6 \times 6$ & Do. & A. H. Pollen. \\
\hline $5^{1 \frac{5}{8}}$ & $6 \frac{3}{4}$ & 39 & $41 \frac{1}{2}$ & $6 \times 6$ & Del Nord Creek & E. N. Buxton (I 884 ). \\
\hline
\end{tabular}


WAPITI (Cervus canadensis)-continued.

\begin{tabular}{|c|c|c|c|c|c|c|}
\hline Length. & हूँ & Tip to Tip. & $\begin{array}{l}\text { Widest } \\
\text { inside. }\end{array}$ & Points. & Habitat. & Owner. \\
\hline $5^{1 \frac{1}{2}}$ & $6 \frac{7}{8}$ & $\cdots$ & ... & $6 \times 7$ & North America & J. L. Carroll. \\
\hline $5^{I}$ & 7 & $\cdots$ & $36 \frac{3}{4}$ & $9 \times 8$ & Do. & $\begin{array}{l}\text { J. G. Millais (American Exhibi- } \\
\text { tion Catalogue). }\end{array}$ \\
\hline $5^{I}$ & $\cdots$ & 36 & $\cdots$ & 12 & Do. & W. S. Power. \\
\hline $50 \frac{5}{8}$ & $7 \frac{3}{8}$ & 42 & $4 S_{\frac{1}{4}}$ & $7 \times 7$ & Do. & Col. Ralph Vivian. \\
\hline $50 \frac{1}{2}$ & 8 & 39 & 42 & $6 \times 6$ & Do. & S. Lewis. \\
\hline $50 \frac{1}{2}$ & $7 \frac{1}{2}$ & 35 & $4 I$ & $6 \times 6$ & Do. & H. S. Somerset. \\
\hline $50^{\frac{1}{2}}$ & 6 & $\cdots$ & $\cdots$ & 12 & Do. & E. F. Henenge. \\
\hline $50 \frac{1}{2}$ & $\cdots$ & 28 & $\cdots$ & 13 & Do. & WV. S. Power. \\
\hline $50 \frac{1}{4}$ & $6 \frac{1}{4}$ & $\cdots$ & 43 & $6 \times 6$ & Do. & $\begin{array}{l}\text { Gerald Buxton (American Ex- } \\
\text { hibition Catalogue). }\end{array}$ \\
\hline 50 & II & $\cdots$ & $\cdots$ & 13 & Do. & T. W. H. Clarke. \\
\hline 50 & $1 \mathrm{O}_{\frac{1}{2}}^{\frac{1}{2}}$ & $\cdots$ & 54 & $6 \times 6$ & Do. & Capt. L. Payne. \\
\hline 50 & $7 \frac{1}{8}$ & $\cdots$ & $47^{\frac{1}{2}}$ & $6 \times 6$ & Do. & $\begin{array}{l}\text { J. M. Hanbury (American Lx- } \\
\text { hibition Catalogue). }\end{array}$ \\
\hline $49 \frac{7}{8}$ & $6 \frac{3}{4}$ & $33 \frac{5}{8}$ & 45 & $6 \times 6$ & Do. & H. R.H. Duke of Edinburgh. \\
\hline $49 \frac{3}{4}$ & 7 & $3^{2}$ & $35 \frac{1}{8}$ & $7 \times 8$ & Do. & J. Carr-Saunders. \\
\hline $49^{\frac{3}{4}}$ & $6 \frac{7}{8}$ & 24 & $30 \frac{5}{8}$ & $6 \times 6$ & Do. & Capt. F. Cookson. \\
\hline $49 \frac{5}{8}$ & $6 \frac{5}{8}$ & $34^{\frac{3}{4}}$ & $36 \frac{5}{8}$ & $6 \times 6$ & Do. & IIugh G. Barclay. \\
\hline $49 \frac{1}{2}$ & $7 \frac{3}{8}$ & $45 \frac{1}{4}$ & $45^{\frac{1}{4}}$ & $7 \times 7$ & Do. & H.R.H. Duc d'Orleans. \\
\hline $49 \frac{1}{2}$ & $6 \frac{1}{2}$ & 25 & $30 \frac{1}{2}$ & $6 \times 6$ & Do. & A. H. Pollen. \\
\hline $49 \frac{3}{8}$ & $7 \frac{5}{8}$ & $3 S_{8}^{\tau}$ & $\cdots$ & $10 \times 15$ & Do. & Moreton Frewen. \\
\hline $49 \frac{1}{8}$ & $6 \frac{1}{4}$ & $\cdots$ & $45^{\frac{1}{4}}$ & 13 & Do. & W. A. Fordham. \\
\hline 49 & $\cdots$ & $35^{\frac{1}{2}}$ & $46 \frac{1}{4}$ & 12 & Do. & W. S. Power. \\
\hline $48 \frac{5}{8}$ & 7 & $3 \circ \frac{3}{8}$ & $37^{3}$ & $6 \times 7$ & California $\quad \ldots$ & C. Pentland, British Museum. \\
\hline $48 \frac{1}{2}$ & $8 \frac{3}{8}$ & $31 \frac{1}{2}$ & $34 \frac{1}{2}$ & $6 \times 7$ & North Amierica & Hon, Charles Ellis. \\
\hline $48 \frac{1}{2}$ & 6 & 29 & $33 \frac{1}{4}$ & $6 \times 7$ & Do. & The late J. S. Jameson. \\
\hline 48 & I I $\frac{1}{2}$ & $\cdots$ & $\cdots$ & 17 & Do. & T. W. H. Clarke. \\
\hline 48 & $10 \frac{1}{2}$ & $\cdots$ & $\cdots$ & I3 & Do. & Do. \\
\hline
\end{tabular}




\section{WAPITI (Cervus canadensis)-continued.}

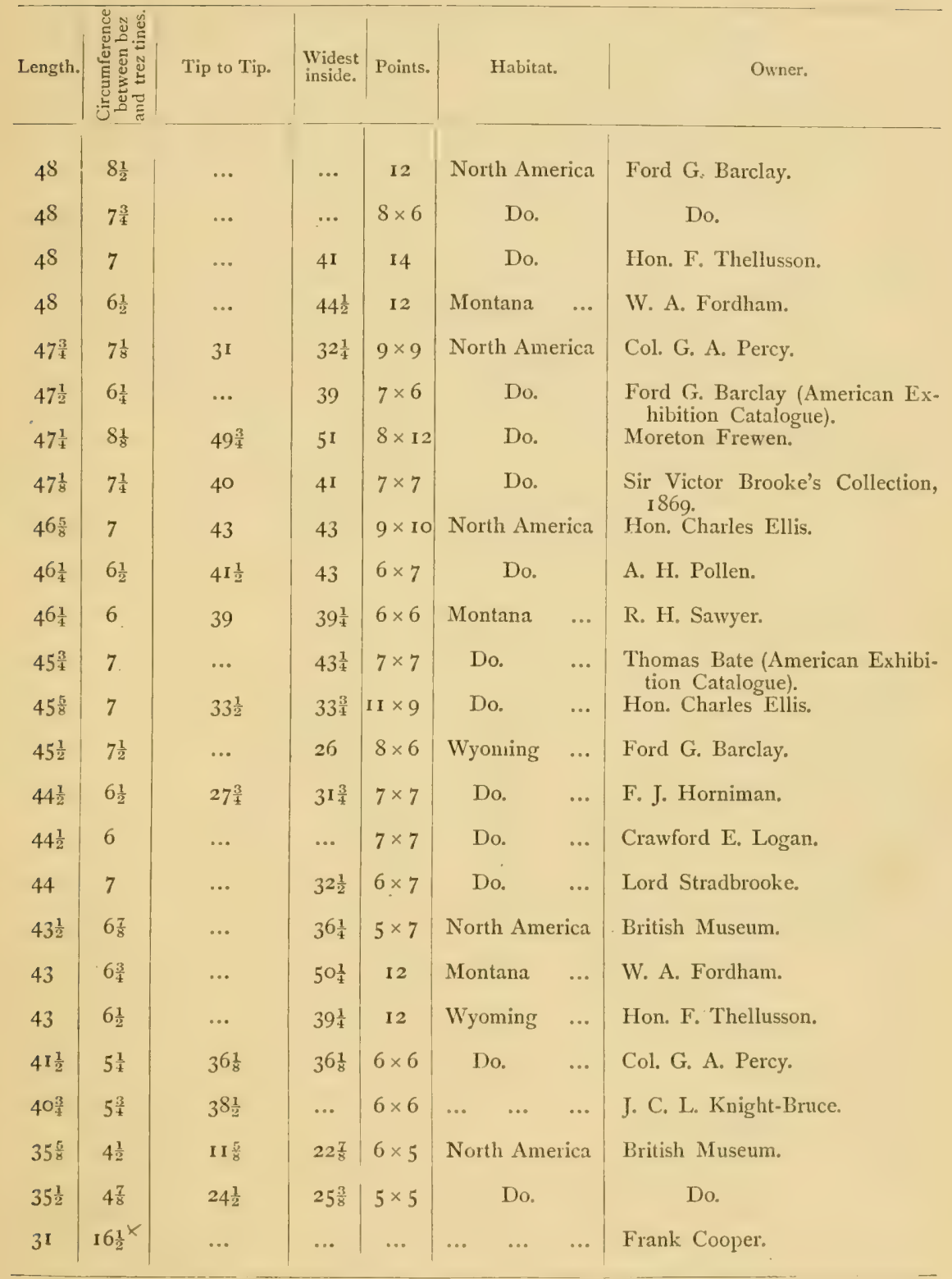




\section{PERSIAN DEER (Cervus maral).}

\begin{tabular}{c|c|c|c|c|c|c|cc|c}
\hline Length. & $\begin{array}{c}\text { Circum- } \\
\text { ference. }\end{array}$ & $\begin{array}{c}\text { Tip to } \\
\text { Tip. }\end{array}$ & $\begin{array}{l}\text { Widest } \\
\text { inside. }\end{array}$ & Points. & & Habitat. & Owner. \\
\hline $48 \frac{5}{8}$ & $6 \frac{1}{8}$ & $38 \frac{1}{4}$ & $40 \frac{7}{8}$ & $6 \times 5$ & Altai & $\ldots$ & $\ldots$ & British Museum. \\
$40 \frac{1}{4}$ & $5 \frac{1}{2}$ & $23 \frac{3}{\frac{3}{4}}$ & $31^{\frac{3}{4}}$ & $5 \times 5$ & $\ldots$ & $\ldots$ & $\ldots$ & Hume Collection, British Museum. \\
$35 \frac{1}{8}$ & $5 \frac{1}{2}$ & $19 \frac{3}{4}$ & 26 & $5 \times 5$ & $\ldots$ & $\ldots$ & $\ldots$ & Do. \\
\hline
\end{tabular}

KASHIMIR STAG, or BARASINGH (Cervus cashmerianus). This stag is closely allied to the Red Deer.

\begin{tabular}{|c|c|c|c|c|c|c|c|}
\hline Length. & $\begin{array}{l}\text { Circum- } \\
\text { ference. }\end{array}$ & $\begin{array}{l}\text { Tip to } \\
\text { Tip. }\end{array}$ & $\begin{array}{c}\text { Widest } \\
\text { inside. }\end{array}$ & Points. & Habitat. & & Owner. \\
\hline 47 & $6 \frac{3}{4}$ & $21 \frac{3}{4}$ & $36 \frac{3}{8}$ & $5 \times 5$ & $\cdots$ & $\ldots$ & Sir Edmund G. Loder, Bart. \\
\hline $45 \frac{5}{8}$ & 6 & $25 \frac{3}{4}$ & 36 & $8 \times 8$ & Kashmir & $\ldots$ & Sir Victor Brooke's Collection. \\
\hline $45 \frac{7}{8}$ & 8 & 35 & $4^{r}$ & $6 \times 6$ & ... & $\ldots \mid$ & Hume Collection, British Museum. \\
\hline $43 \frac{7}{8}$ & $5^{\frac{7}{8}}$ & $15 \frac{7}{8}$ & 32 & $5 \times 5$ & $\cdots$ & $\cdots \mid$ & The Hon. Charles Ellis. \\
\hline $43 \frac{3}{8}$ & $6 \frac{1}{2}$ & $25 \frac{3}{8}$ & $3^{6 \frac{1}{2}}$ & $\cdots$ & $\cdots$ & $\ldots$ & Do. \\
\hline $43 \frac{1}{4}$ & $6 \frac{1}{2}$ & I $S_{8}^{1}$ & $34 \frac{1}{2}$ & $5 \times 5$ & $\cdots$ & $\cdots$ & Hume Collection, British Museum. \\
\hline 43 & $5 \frac{7}{8}$ & $6 \frac{1}{4}$ & $27^{\frac{3}{4}}$ & $5 \times 5$ & Nepal... & $\ldots$ & Dr. Falconer, British Museum. \\
\hline 43 & $5 \frac{7}{8}$ & $26 \frac{1}{8}$ & $37 \frac{7}{8}$ & $6 \times 5$ & $\cdots$ & $\cdots$ & Martyn Kennard. \\
\hline 42 & $5^{\frac{7}{8}}$ & $29 \frac{3}{8}$ & $33 \frac{1}{2}$ & $6 \times 5$ & $\ldots$ & $\ldots$ & British Museum. \\
\hline $41 \frac{3}{4}$ & $6 \frac{3}{8}$ & $27 \frac{1}{4}$ & 35 & $6 \times 6$ & $\cdots$ & $\ldots$ & Martyn Kennard. \\
\hline $41 \frac{3}{8}$ & $5^{\frac{3}{4}}$ & $23 \frac{3}{4}$ & 33 & $5 \times 5$ & $\cdots$ & $\ldots$ & R. Lydekker, British Museum. \\
\hline $41 \frac{1}{4}$ & $5 \frac{1}{4}$ & 34 & 49 & $6 \times 5$ & $\cdots$ & $\cdots$ & C. H. Seeley. \\
\hline $40 \frac{3}{4}$ & $5^{\frac{3}{4}}$ & $15 \frac{3}{8}$ & $31 \frac{1}{4}$ & $5 \times 5$ & $\cdots$ & $\ldots$ & Rowland Ward's Collection. \\
\hline $39 \frac{5}{8}$ & $5 \frac{3}{8}$ & 20 & $26 \frac{1}{8}$ & $5 \times 5$ & $\cdots$ & $\ldots$ & British Museum. \\
\hline 39 & $5 \frac{1}{8}$ & $17 \frac{1}{2}$ & $29 \frac{1}{2}$ & $6 \times 5$ & Kashmir & $\ldots \mid$ & Sir Victcr Brooke's Collection, I $£ 6 g$. \\
\hline
\end{tabular}


KASHIMIR STAG, or BARASINGH (Cervus cashmerianus)continued.

\begin{tabular}{|c|c|c|c|c|c|c|c|c|}
\hline Length. & $\begin{array}{l}\text { Circum- } \\
\text { ference. }\end{array}$ & $\begin{array}{l}\text { Tip to } \\
\text { Tip. }\end{array}$ & $\begin{array}{l}\text { Widest } \\
\text { inside. }\end{array}$ & Points. & & Habitat & & Owner. \\
\hline $38 \frac{3}{4}$ & $6 \frac{1}{4}$ & 26 & $30 \frac{3}{4}$ & $6 \times 6$ & $\ldots$ & $\ldots$ & $\ldots$ & Hume Collection, British Museum. \\
\hline $37 \frac{7}{8}$ & $5 \frac{3}{4}$ & $19 \frac{1}{4}$ & $28 \frac{3}{4}$ & $8 \times 7$ & ... & ... & $\cdots$ & The Hon. Charles Ellis. \\
\hline $3^{6 \frac{1}{2}}$ & $5 \frac{5}{8}$ & $21 \frac{1}{2}$ & $25 \frac{3}{8}$ & $7 \times 5$ & ... & ... & .. & British Museum. \\
\hline $36 \frac{3}{8}$ & $4^{\frac{3}{4}}$ & $17 \frac{3}{7}$ & $22 \frac{1}{4}$ & $6 \times 6$ & ... & $\ldots$ & $\ldots$ & Do. \\
\hline $35 \frac{1}{8}$ & $5 \frac{1}{4}$ & $23 \frac{3}{8}$ & $3 \circ \frac{1}{2}$ & $5 \times 5$ & ... & $\ldots$ & ... & Dr. Falconer, British Museum. \\
\hline 33 & $4 \frac{1}{2}$ & $9 \frac{3}{4}$ & ... & 9 & ... & $\cdots$ & $\cdots$ & Capt. Goulburn. \\
\hline
\end{tabular}

\section{SHOU, or SIKKIII STAG (Cervus affinis).}

THIs is one of the largest deer in Southern Asia, standing sometimes $I_{5}$ hands, and closely approaches the American Wapiti in the measurements of the horns.

Habitat-Eastern Tibet.

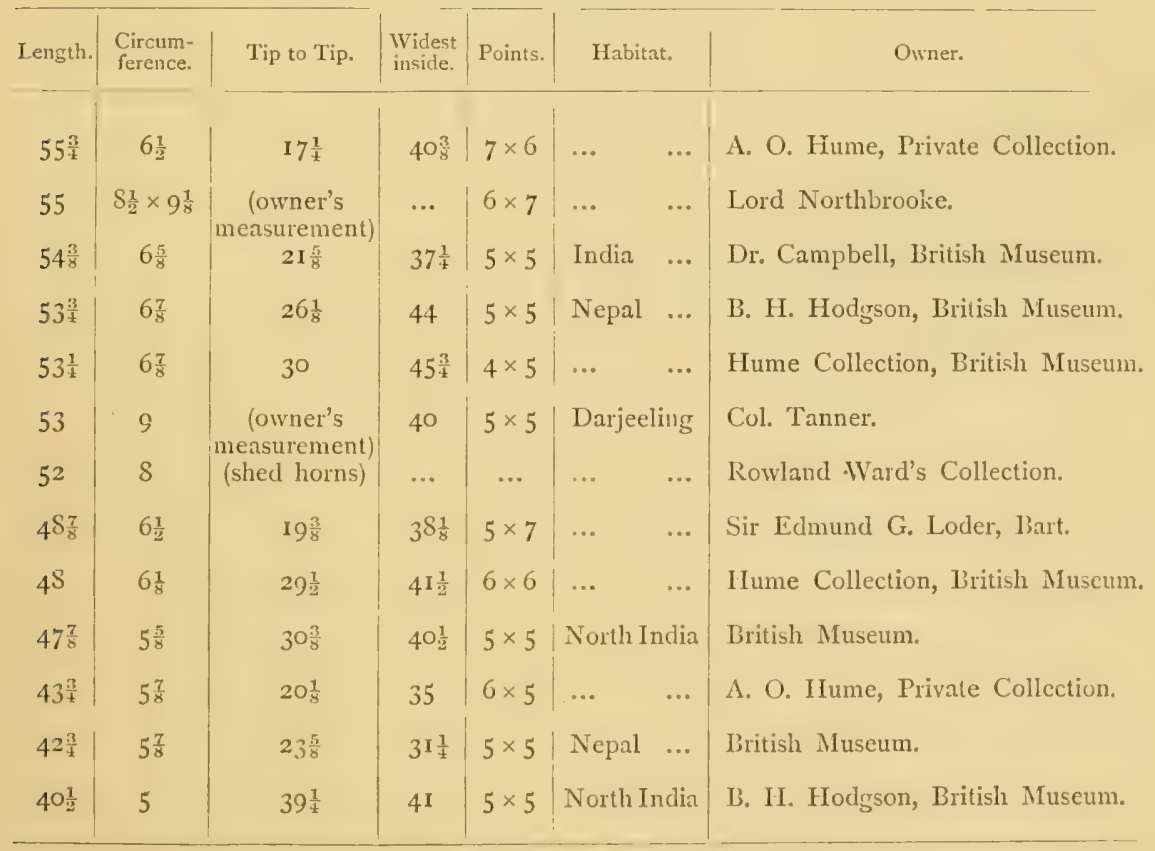




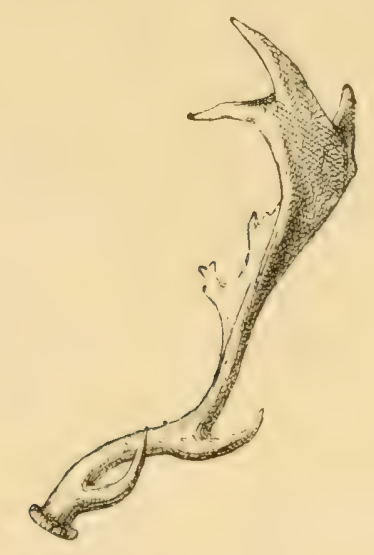

FALLOW DEER (Cervus dama).

Habitat-Europe.

\begin{tabular}{|c|c|c|c|c|c|c|c|c|c|}
\hline Length. & $\begin{array}{l}\text { Circum- } \\
\text { ference. }\end{array}$ & $\begin{array}{l}\text { Tip to } \\
\text { Tip. }\end{array}$ & $\begin{array}{c}\text { Spread } \\
\text { inside. }\end{array}$ & Points. & $\begin{array}{l}\text { Width } \\
\text { of Palm. }\end{array}$ & \multicolumn{3}{|c|}{ Habitat. } & Owner. \\
\hline $21 \frac{3}{8}$ & $4 \frac{3}{8}$ & $\cdots$ & $\cdots$ & $11 \times 10$ & 5 & \multirow{2}{*}{\multicolumn{3}{|c|}{$\begin{array}{l}\text { Colebrooke Woods } \\
\text { (Ireland). } \\
\text { Do. }\end{array}$}} & Sir Victor Brooke's Collec- \\
\hline $28 \frac{3}{8}$ & $4 \frac{1}{8}$ & $\ldots$ & 26 & $10 \times 8$ & 6 & & & & Do. \\
\hline $27 \frac{3}{8}$ & $3 \frac{7}{8}$ & $20 \frac{1}{4}$ & $22 \frac{1}{2}$ & $10 \times 9$ & $5 \frac{1}{8}$ & & Do. & & Do. \\
\hline $27 \frac{1}{ \pm}$ & 4 & 23 & $\cdots$ & $10 \times 8$ & $\cdots$ & ... & .. & $\ldots$ & J. Carr-Saunders. \\
\hline $26 \frac{7}{8}$ & $3 \frac{3}{4}$ & 12 & $17 \frac{1}{2}$ & $7 \times 10$ & ... & ... & $\ldots$ & $\ldots$ & British Museum. \\
\hline $26 \frac{5}{8}$ & 4 & $24 \frac{1}{8}$ & $\ldots$ & $8 \times 12$ & 5 & & and ... & $\ldots$ & Sir Victor Brooke's Collec. \\
\hline $25 \frac{1}{2}$ & $\cdots$ & ... & 26 & ... & $5 \frac{x}{4}$ & & land & $\ldots$ & Rowland Ward's Collection. \\
\hline $25 \frac{3}{8}$ & $4 \frac{1}{4}$ & 26 & $\ldots$ & $8 \times 8$ & $5 \frac{1}{8}$ & & and ... & $\ldots$ & Sir Victor Brooke's Collec- \\
\hline $24 \frac{1}{2}$ & $3 \frac{5}{8}$ & I6 & $19 \frac{7}{8}$ & II $\times$ II & ... & $\ldots$ & .. & $\ldots$ & British Museum. \\
\hline $19 \frac{1}{4}$ & $2 \frac{7}{8}$ & $16 \frac{1}{4}$ & $17 \frac{1}{2}$ & $7 \times 5$ & ... & $\ldots$ & $\ldots$ & $\ldots$ & Do. \\
\hline
\end{tabular}




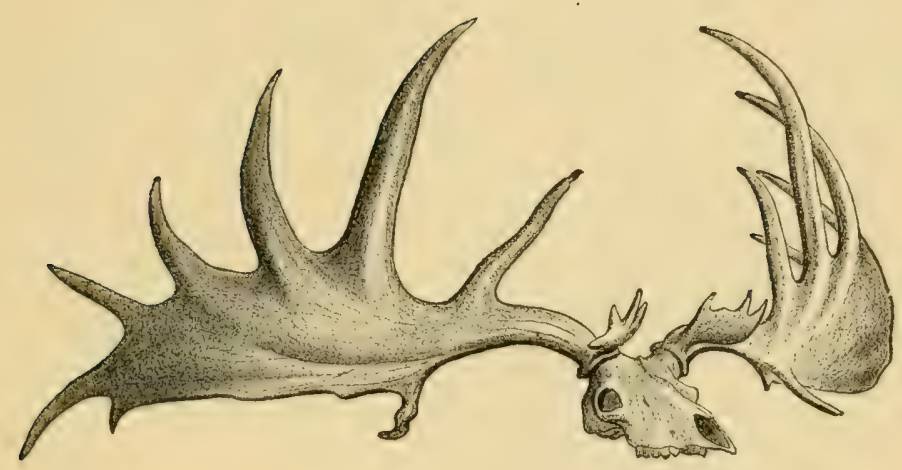

IRISH ELK (Cervus giganteus).

(Extinct.)

\begin{tabular}{|c|c|c|c|c|c|}
\hline $\begin{array}{l}\text { Spread Tip } \\
\text { to Tip. }\end{array}$ & $\begin{array}{l}\text { Length round in- } \\
\text { side of horn. }\end{array}$ & $\begin{array}{c}\text { Circum- } \\
\text { ference } \\
\text { above burr. }\end{array}$ & $\begin{array}{l}\text { Width of } \\
\text { Palm. }\end{array}$ & Points. & Owner. \\
\hline $\begin{array}{l}\text { ft. in. } \\
\text { Io } 2\end{array}$ & $\begin{array}{rl}\text { ft. in. } \\
5 & 8 \frac{1}{2}\end{array}$ & $9 \frac{7}{8}$ & $19 \frac{1}{4}$ & 19 & British Museum. \\
\hline 98 & $\ldots$ & ... & ... & ... & Rowland Ward's Collection. \\
\hline 95 & $\cdots$ & $\cdots$ & 27 & $13 \times 15$ & Sir Edmund G. Loder, Bart. \\
\hline 92 & $\begin{aligned} & \text { I3 } 6 \\
& \text { (of both horns) }\end{aligned}$ & $\ldots$ & $15 \frac{1}{2}$ & $\begin{array}{c}\text { (owner's } \\
\text { measurement) }\end{array}$ & Mrs. Graham Lloyd. \\
\hline $811 \frac{3}{4}$ & $61 \frac{3}{8}$ & $8 \frac{3}{4}$ & $17 \frac{3}{4}$ & $10 \times 13$ & Hon. Charles Ellis. \\
\hline & $\begin{array}{c}12 \text { 10 } \\
\text { (of both horns) }\end{array}$ & $\ldots$ & $13 \frac{1}{2}$ & $\begin{array}{c}\text { (owner's } \\
\text { measurement) }\end{array}$ & Mrs. Graham Lloyd. \\
\hline 76 & $53 \frac{1}{2}$ & $8 \frac{5}{8}$ & $\ldots$ & $10 \times 11$ & Sir Victor Brooke's Collection. \\
\hline
\end{tabular}




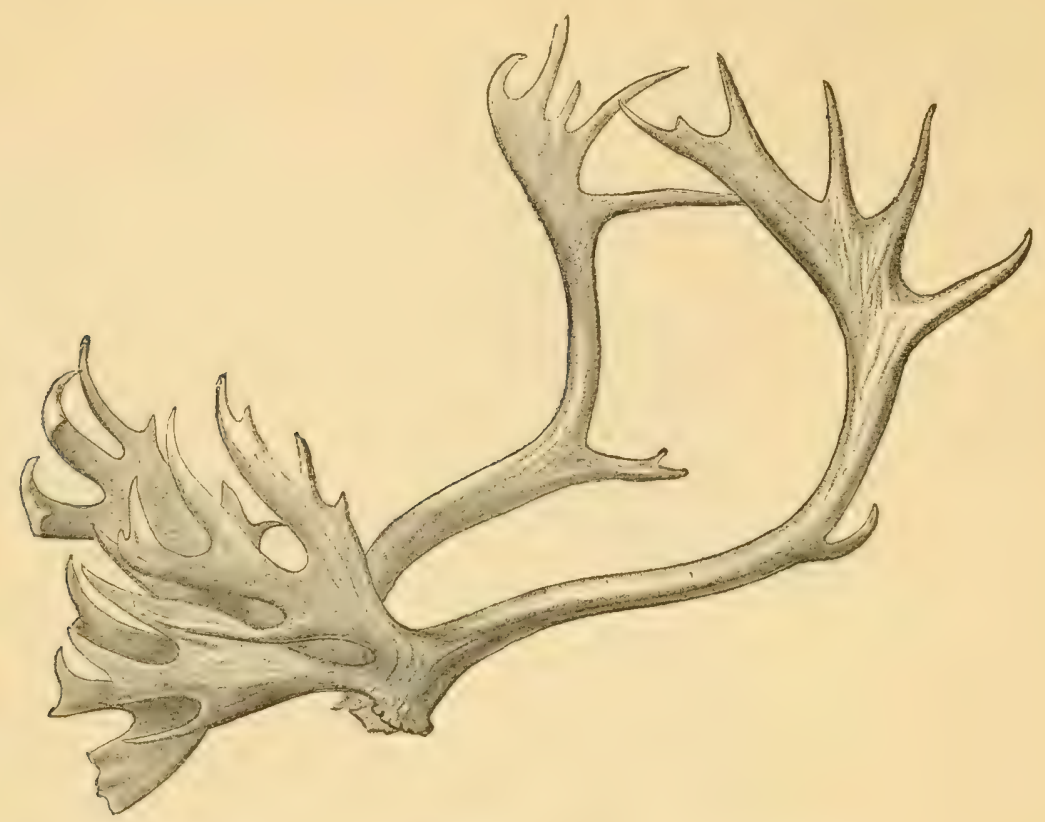

REINDEER, or CARIBOU (Rangifer tarandus).

Weight of a full-grown Reindecr after gralloching-From 250 lbs. to 350 lbs.

Habitat-Europe, Asia, and America.

\begin{tabular}{|c|c|c|c|c|c|c|c|c|}
\hline Length. & $\begin{array}{l}\text { Circum- } \\
\text { ference. }\end{array}$ & $\begin{array}{l}\text { Tip to } \\
\text { Tip. }\end{array}$ & $\begin{array}{l}\text { Widest } \\
\text { inside. }\end{array}$ & Points. & \multicolumn{3}{|c|}{ Habitat. } & Owner. \\
\hline 60 & $5 \frac{5}{8}$ & $38 \frac{5}{8}$ & $4 \mathrm{I} \frac{1}{8}$ & $15 \times 22$ & $\ldots$ & ... & $\cdots$ & Sir Victor Brooke's Collection. \\
\hline $57 \frac{5}{8}$ & $5 \frac{1}{4}$ & $13 \frac{3}{8}$ & $\cdots$ & $13 \times 7$ & Arctic & Regions & $\cdots$ & British Muscum. \\
\hline $57 \frac{1}{8}$ & 5 & $35^{\frac{1}{4}}$ & $\ldots$ & $8 \times 10$ & $\ldots$ & $\cdots$ & $\ldots$ & Do. \\
\hline $54 \frac{3}{8}$ & $4^{\frac{7}{8}}$ & $20 \frac{1}{4}$ & $\cdots$ & I $1 \times 13$ & North & America & $\ldots$ & $\begin{array}{l}\text { Capt. Sir J. Franklin, Iritish } \\
\text { Juseum. }\end{array}$ \\
\hline $51 \frac{3}{8}$ & $4^{\frac{7}{8}}$ & $40 \frac{7}{8}$ & $4 S_{3}^{3}$ & $17 \times 14$ & $\cdots$ & $\cdots$ & $\cdots$ & Sir Edmund G. Loder, Bart. \\
\hline $50 \frac{3}{8}$ & $48^{7}$ & 2.4 & $\begin{array}{c}29 \\
\text { (outside) }\end{array}$ & $13 \times 16$ & ... & $\cdots$ & ... & H. R. H. Duke of Edinburgh. \\
\hline 49 & $4 \frac{1}{8}$ & $24 \frac{5}{8}$ & $32 \frac{3}{8}$ & $7 \times 9$ & $\cdots$ & $\cdots$ & $\cdots$ & Hon. Walter Rothschild. \\
\hline 48 & $6 \frac{1}{2}$ & II $\frac{7}{8}$ & $25 \frac{1}{2}$ & $15 \times 17$ & $\ldots$ & ... & $\cdots$ & Sir Edmund G. Loder, Bart. \\
\hline
\end{tabular}


REINDEER, or CARIBOU (Rangifer tarandus)-contimut.

\begin{tabular}{|c|c|c|c|c|c|c|c|c|}
\hline Length. & $\begin{array}{l}\text { Circum- } \\
\text { ference. }\end{array}$ & $\begin{array}{l}\text { Tip to } \\
\text { Tip. }\end{array}$ & $\begin{array}{l}\text { Widest } \\
\text { inside. }\end{array}$ & Points. & & Iabitat. & & Owner. \\
\hline $47^{3}$ & $5 \frac{1}{8}$ & $32 \frac{1}{8}$ & $\cdots$ & I $2 \times 19$ & North $A$ & America & $\cdots$ & A. Murray, British Museum. \\
\hline 47 & $5 \frac{1}{2}$ & $\cdots$ & $34 \frac{1}{3}$ & $\cdots$ & $\cdots$ & $\cdots$ & $\ldots$ & E. D. Gosling. \\
\hline $46 \frac{1}{4}$ & $5 \frac{3}{8}$ & $29 \frac{3}{8}$ & $\cdots$ & $15 \times 15$ & $\cdots$ & $\cdots$ & $\cdots$ & British Museum. \\
\hline 46 & $4 \frac{1}{2}$ & $\cdots$ & 34 & $\cdots$ & $\cdots$ & $\cdots$ & $\ldots$ & Hon. A. Broderick. \\
\hline $45^{\frac{7}{8}}$ & 6 & 10 & $\cdots$ & $20 \times 27$ & $\cdots$ & $\cdots$ & $\cdots$ & British Museum. \\
\hline $45 \frac{1}{2}$ & $5 \frac{3}{8}$ & $34 \frac{3}{8}$ & $\cdots$ & $23 \times 16$ & $\cdots$ & $\cdots$ & $\cdots$ & Do. \\
\hline $43^{\frac{3}{t}}$ & $5 \frac{3}{8}$ & 40 & $41 \frac{7}{8}$ & $16 \times$ II & Newfou & indland & $\ldots$ & St. George Littledale, I $\$ 76$. \\
\hline $42 \frac{1}{2}$ & $\begin{array}{l}7 \frac{1}{4} \\
\text { (Brow } \\
\text { long }\end{array}$ & $\begin{array}{l}\mid 33^{\frac{1}{4}} \\
\text { v antler } \\
g, 6 \text { in. }\end{array}$ & $\begin{array}{l}38 \frac{1}{2} \\
\text { I } 7 \frac{1}{2} \text { in. } \\
\text { wide) }\end{array}$ & $\begin{array}{c}\text { (Owner's } \\
\text { measure- } \\
\text { ment) }\end{array}$ & $\cdots$ & $\cdots$ & $\ldots$ & R. Gordon Smith. \\
\hline 42 & $6 \frac{1}{2}$ & $\cdots$ & $18 \frac{1}{2}$ & 33 & Alaska & $\cdots$ & $\cdots$ & Earl of Lonsdale. \\
\hline $4^{1} \frac{1}{x}$ & $4 \frac{1}{8}$ & $\cdots$ & $28 \frac{3}{8}$ & $8 \times 6$ & $\cdots$ & $\cdots$ & $\cdots$ & Col. G. A. Percy. \\
\hline $4 \mathrm{I} \frac{1}{8}$ & $4^{\frac{3}{4}}$ & $\cdots$ & $24 \frac{1}{2}$ & $12 \times 9$ & $\cdots$ & $\cdots$ & $\cdots$ & W. D. James. \\
\hline $4^{I}$ & $\cdots$ & $\cdots$ & $\cdots$ & $\cdots$ & Alaska & $\cdots$ & $\cdots$ & Earl of Lonsdale. \\
\hline $40 \%$ & $4 \frac{1}{2}$ & $20 \frac{1}{2}$ & $28 \frac{1}{4}$ & $17 \times 14$ & $\cdots$ & $\cdots$ & $\cdots$ & J. Carr Saunders. \\
\hline 40 & $6 \frac{3}{8}$ & $20 \frac{3}{4}$ & $\begin{array}{c}\text { (In } \\
\text { velvet) }\end{array}$ & $13 \times 14$ & & Bay & $\ldots$ & British Museum. \\
\hline $39 \frac{1}{8}$ & 4 & 16 & ... & $\mathrm{I}_{3} \times \mathrm{I}_{3}$ & North A & America & $\ldots$ & Do. \\
\hline 39 & $5 \frac{5}{8}$ & $\cdots$ & $23 \frac{1}{4}$ & $14 \times 17$ & $\cdots$ & $\cdots$ & $\cdots$ & Sir Victor Brooke's Collection. \\
\hline $38 \frac{3}{4}$ & $4 \frac{7}{8}$ & $15 \frac{3}{8}$ & $19 \frac{3}{4}$ & IO $\times 4$ & $\cdots$ & $\cdots$ & $\cdots$ & J. Carr Saunders. \\
\hline $3 S \frac{3}{4}$ & 4 & $25^{\frac{1}{4}}$ & $\cdots$ & $7 \times 5$ & Norway & $y \ldots$ & $\cdots$ & C. Ingram, British Museum. \\
\hline $3 S^{\frac{3}{8}}$ & $5 \frac{3}{8}$ & $2 \mathbf{I} \frac{3}{4}$ & $30 \frac{1}{8}$ & $16 \times 16$ & $\cdots$ & $\cdots$ & $\cdots$ & Sir James Anderson. \\
\hline $3^{8}$ & $5^{\frac{1}{2}}$ & $2 \mathrm{I} \frac{1}{2}$ & $\cdots$ & $15 \times 13$ & Norway & $y \ldots$ & $\ldots$ & W. H. Ingram, British Museum. \\
\hline $37 \frac{7}{8}$ & $4^{\frac{7}{8}}$ & $3 I^{\frac{3}{4}}$ & $\cdots$ & $14 \times 17$ & $\cdots$ & $\cdots$ & $\cdots$ & British Museum. \\
\hline $37:$ & $4 \frac{7}{8}$ & $2 \mathrm{I} \frac{3}{8}$ & $\cdots$ & $12 \times 10$ & $\cdots$ & $\cdots$ & $\cdots$ & Do. \\
\hline $37 \frac{5}{5}$ & $5^{\frac{1}{4}}$ & $18 \frac{1}{4}$ & $25^{\frac{1}{4}}$ & $18 \times 12$ & $\cdots$ & $\cdots$ & $\cdots$ & F. J. Horniman. \\
\hline $37 \frac{1}{2}$ & $5 \frac{1}{2}$ & $\cdots$ & 29 & $\cdots$ & $\cdots$ & $\cdots$ & $\cdots$ & $\begin{array}{l}\text { Otho Shaw (American Exhili- } \\
\text { tion Catalogue). }\end{array}$ \\
\hline $37 \frac{1}{4}$ & $5 \frac{5}{8}$ & $\cdots$ & 24 & 27 & $\cdots$ & $\cdots$ & $\cdots$ & | Licut. Wm. Tait. \\
\hline $36 \frac{3}{4}$ & $3 \frac{3}{4}$ & $13^{\frac{1}{1}}$ & ... & $7 \times 9$ & North A & America & $\cdots$ & $\begin{array}{l}\text { Capt. Sir J. Franklin, British } \\
\text { Museum. }\end{array}$ \\
\hline $36 \frac{1}{4}$ & $5^{\frac{7}{8}}$ & $24 \frac{3}{8}$ & $\cdots$ & $14 \times 15$ & $\cdots$ & $\cdots$ & $\cdots$ & Bıitish Museum. \\
\hline
\end{tabular}


REINDEER, or CARIBOU (Rangifer tarandus)-continut.

\begin{tabular}{|c|c|c|c|c|c|c|c|}
\hline Length. & $\begin{array}{l}\text { Circum- } \\
\text { ference. }\end{array}$ & $\begin{array}{l}\text { Tip to } \\
\text { Tip. }\end{array}$ & $\begin{array}{l}\text { Widest } \\
\text { inside. }\end{array}$ & Points. & \multicolumn{2}{|l|}{ Habitat. } & Owner. \\
\hline 36 & $6 \frac{3}{8}$ & 17 & ... & $19 \times 13$ & Nova Scotia & $\ldots$ & J. Florence, British Museum. \\
\hline 36 & $4 \frac{1}{2}$ & $\cdots$ & 30 & $\cdots$ & $\cdots$ & $\cdots$ & $\begin{array}{l}\text { Otho Shaw (American Exhibi- } \\
\text { tion Catalogue) }\end{array}$ \\
\hline $34 \frac{7}{8}$ & $5 \frac{5}{8}$ & 23 & ... & $18 \times \mathbf{I} 6$ & Arctic Regions & ... & British Museum. \\
\hline $34 \frac{3}{4}$ & 4 & $12 \frac{3}{4}$ & ... & $17 \times 11$ & $\cdots$ & $\ldots$ & Do. \\
\hline $34^{\frac{1}{2}}$ & 5 & $2 \mathrm{I} \frac{1}{2}$ & $\cdots$ & $18 \times 15$ & North America & $\cdots$ & A. Murray, British Museum. \\
\hline $34 \frac{1}{2}$ & $4 \frac{3}{8}$ & $12 \frac{3}{8}$ & $19 \frac{1}{2}$ & $10 \times 13$ & Do. & $\ldots$ & Capt. F. Cookson. \\
\hline $32 \frac{3}{4}$ & $3 \frac{1}{4}$ & $31 \frac{5}{8}$ & $\cdots$ & $3 \times 14$ & Northern Euro & pe. & British Museum. \\
\hline 32 & $3 \frac{1}{8}$ & $18 \frac{1}{4}$ & $\ldots$ & $7 \times 8$ & North America & ... & Do. \\
\hline $3^{1 \frac{3}{8}}$ & $4 \frac{3}{4}$ & .. & $23 \frac{1}{2}$ & II $\times$ IO & ... & ... & F. J. Horniman, \\
\hline $30 \frac{1}{4}$ & $4 \frac{1}{4}$ & 19 & $23 \frac{1}{2}$ & $7 \times 9$ & Newfoundland & $\cdots$ & St. George Littledale, I 876. \\
\hline $29 \frac{1}{4}$ & $\cdot 3 \frac{3}{8}$ & $20 \frac{3}{8}$ & $\cdots$ & $7 \times 10$ & $\cdots$ & $\cdots$ & E. N. Buxton, 1867. \\
\hline $28 \frac{1}{2}$ & $3 \frac{3}{8}$ & 27 & ... & $6 \times 10$ & North America & $\ldots$ & $\begin{array}{l}\text { Capt. Sir J. Franklin, Brilish } \\
\text { Museun. }\end{array}$ \\
\hline $28 \frac{1}{4}$ & $4 \frac{3}{8}$ & I $3 \frac{1}{8}$ & $21 \frac{3}{4}$ & $12 \times 11$ & Do. & $\cdots$ & Capt. F. Cookson. \\
\hline $24 \frac{1}{4}$ & 3 & $12 \frac{1}{2}$ & $\cdots$ & $4 \times 4$ & Hudson Bay & $\cdots$ & British Museum. \\
\hline $23 \frac{1}{4}$ & $3 \frac{3}{8}$ & $14 \frac{5}{8}$ & $\cdots$ & $I I \times I I$ & $\ldots$ & ... & Do. \\
\hline $2 \mathrm{I} \frac{1}{4}$ & $2 \frac{1}{2}$ & $12 \frac{5}{8}$ & $\cdots$ & I $\times$ I 4 & North America & $\ldots$ & $\begin{array}{l}\text { Capt. Sir J. Franklin, Bitish } \\
\text { Museum. }\end{array}$ \\
\hline I $9 \frac{1}{4}$ & $3 \frac{1}{8}$ & $22 \frac{1}{8}$ & ... & $5 \times 5$ & $\cdots$ & $\cdots$ & British Museum. \\
\hline
\end{tabular}




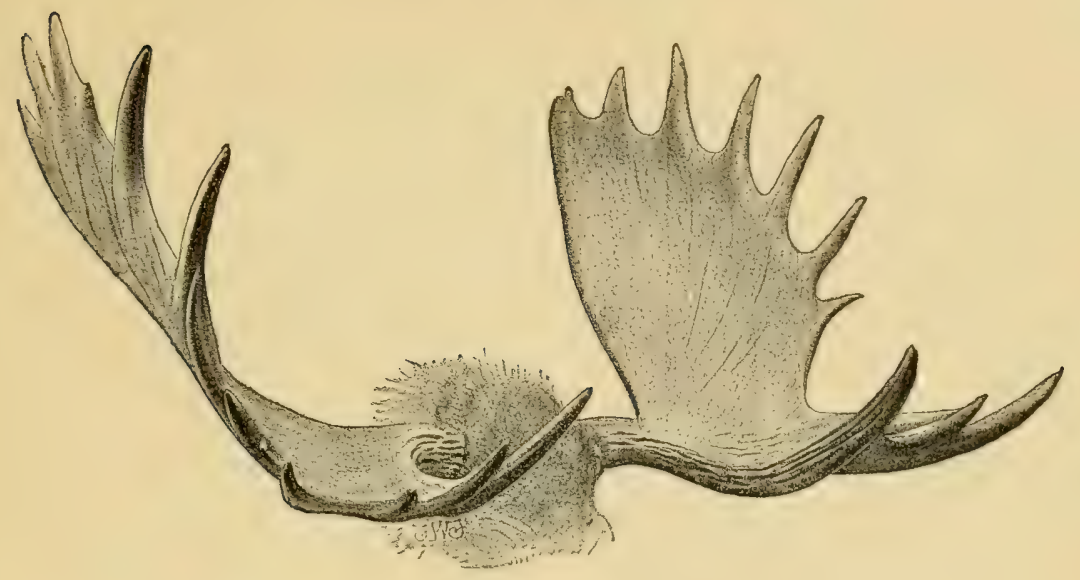

ELK, or, MOOSE (Alces machlis).

Weight-About goo lbs.

Habitat-Northern Europe and North America.

\begin{tabular}{|c|c|c|c|c|c|c|c|}
\hline $\begin{array}{l}\text { Length } \\
\text { t) lung- } \\
\text { eit tine. }\end{array}$ & $\begin{array}{l}\text { Circumference } \\
\text { above burr. }\end{array}$ & $\begin{array}{l}\text { Tip to } \\
\text { Tip. }\end{array}$ & $\begin{array}{l}\text { Greatest } \\
\text { width. }\end{array}$ & $\begin{array}{l}\text { Breadth } \\
\text { of Palm. }\end{array}$ & Points. & Habitat. & Owner. \\
\hline $39 \frac{7}{5}$ & $6 \frac{1}{2}$ & $\cdots$ & $5^{1 \frac{3}{8}}$ & $15 \frac{3}{4}$ & $12 \times 13$ & $\ldots$ & British Museum. \\
\hline $39:$ & $\oint_{\frac{1}{2}}$ & $\cdots$ & $39 \frac{1}{2}$ & I $3 \frac{3}{7}$ & $\cdots$ & N. America & Otho Shaw (American Ex- \\
\hline $39 \frac{1}{4}$ & $7 \frac{5}{8}$ & $33 \frac{1}{\frac{1}{2}}$ & $49 \frac{1}{8}$ & I I $\frac{1}{2}$ & $10 \times 12$ & Do. & J. Carr-Saunders. \\
\hline $39 \frac{1}{3}$ & $7 \frac{5}{8}$ & $3^{6}+\frac{1}{4}$ & $51 \frac{3}{4}$ & $9 \frac{1}{2}$ & $7 \times 8$ & Do. & J. C. L. Knight Bruce. \\
\hline 39 & $S_{\ddagger}^{\frac{1}{t}}$ & $3 S_{\frac{1}{2}}$ & 57 & $12 \frac{1}{2}$ & $15 \times 12$ & Do. & Sir Edmund G. Loder, Bart. \\
\hline $3 S_{:}^{1}$ & 7 & $\cdots$ & $52 \frac{7}{8}$ & 107 & IO $\times$ II & $\cdots$ & British Museum. \\
\hline $3 S_{\frac{1}{4}}$ & $6 \frac{1}{8}$ & 34 & $49 \frac{1}{2}$ & $9 \frac{3}{4}$ & $9 \times 9$ & N. America & $\begin{array}{l}\text { Sir Victor Brooke's Collec. } \\
\text { tion. }\end{array}$ \\
\hline $3^{S}$ & $6 \frac{1}{2}$ & $\cdots$ & $59 \frac{1}{4}$ & $9 \frac{3}{t}$ & I I $\times$ I I & $\cdots$ & H. R. H. Duke of Edinburgh. \\
\hline $37 \frac{3}{1}$ & S & $\cdots$ & $51 \frac{1}{2}$ & II $\frac{1}{4}$ & $14 \times 13$ & N. America & F. Ashby. \\
\hline $37 \frac{1}{2}$ & $\cdots$ & $\cdots$ & $49^{\frac{1}{2}}$ & 20 & $\cdots$ & Do. & $\begin{array}{l}\text { Rowland Ward's Collec- } \\
\text { tion. }\end{array}$ \\
\hline $37 \frac{1}{4}$ & $7 \frac{1}{8}$ & 35 & $50 \frac{5}{8}$ & II $\frac{5}{x}$ & $13 \times 10$ & $\cdots$ & Hon. Charles Ellis. \\
\hline $37 \pm$ & $6 \frac{7}{8}$ & $\cdots$ & $43^{\frac{3}{4}}$ & $8_{4}^{3}$ & II $\times 9$ & $\cdots$ & British Museum. \\
\hline $3^{6 \frac{3}{4}}$ & 67 & $\cdots$ & $47 \frac{7}{8}$ & $10 \frac{1}{2}$ & $8 \times 8$ & $\cdots$ & Do. \\
\hline $3^{65}$ & $6 ?$ & .. & $46 \frac{7}{5}$ & I I & $12 \times 10$ & $\cdots$ & Do. \\
\hline
\end{tabular}


ELK, or IMOOSE (Alces machlis)-continucd.

\begin{tabular}{|c|c|c|c|c|c|c|c|c|}
\hline $\begin{array}{l}\text { Length } \\
\text { to long- } \\
\text { est tine. }\end{array}$ & $\mid \begin{array}{c}\text { Circumference } \\
\text { above burr. }\end{array}$ & $\begin{array}{l}\text { Tip to } \\
\text { Tip. }\end{array}$ & $\begin{array}{c}\text { Greatest } \\
\text { width. }\end{array}$ & $\begin{array}{l}\text { Breadth } \\
\text { of Palm. }\end{array}$ & Points. & Habitat. & & Owner. \\
\hline $34 \frac{1}{2}$ & $6 \frac{5}{8}$ & $\ldots$ & $51 \frac{1}{2}$ & $9 \frac{3}{8}$ & I I $\times I 2$ & $\cdots$ & $\cdots$ & British Museum. \\
\hline $34 \frac{1}{4}$ & $6 \frac{5}{8}$ & 42 & $5^{1 \frac{1}{8}}$ & $9 \frac{1}{2}$ & $10 \times 9$ & $\ldots$ & ... & G. Marchetti. \\
\hline $34^{\frac{1}{4}}$ & $6 \frac{3}{8}$ & $\ldots$ & $47^{\frac{1}{2}}$ & $9 \frac{3}{4}$ & $13 \times$ II & $\ldots$ & ... & British Museum. \\
\hline 32 & $6 \frac{7}{8}$ & $\cdots$ & $43 \frac{3}{5}$ & $9^{\frac{1}{2}}$ & $7 \times 8$ & Russia & $\cdots$ & $\begin{array}{l}\text { Sir Edward Caley, British } \\
\text { Museum. }\end{array}$ \\
\hline $31 \frac{5}{8}$ & $6 \frac{1}{2}$ & ... & $38 \frac{5}{8}$ & $7 \frac{7}{8}$ & $6 \times 5$ & $\ldots$ & ... & British Museum. \\
\hline $3 I_{\frac{1}{2}}$ & $\ldots$ & $\ldots$ & $51 \frac{1}{2}$ & $12 \frac{1}{2}$ & ... & N. Ameri & ica & Earl of Lonsdale. \\
\hline $3 I$ & $6 \frac{1}{2}$ & $\cdots$ & $42 \frac{1}{2}$ & $8 \frac{1}{2}$ & $10 \times 9$ & $\cdots$ & $\cdots$ & F. J. Horniman. \\
\hline $3^{\frac{1}{2}}$ & $\begin{array}{c}\text { (owner's } \\
\text { measurement) }\end{array}$ & 40 & 44 & $8 \frac{3}{8}$ & 17 & Norway & $\ldots$ & Thomas Bate. \\
\hline $30 \frac{1}{8}$ & $7 \frac{1}{8}$ & $29 \frac{1}{2}$ & $44^{\frac{1}{4}}$ & 10 & $8 \times 9$ & $\ldots$ & $\ldots$ & $\begin{array}{l}\text { Sir Victor Brooke's Collec- } \\
\text { tion. }\end{array}$ \\
\hline $29 \frac{3}{4}$ & 6 & $\cdots$ & $40 \frac{1}{8}$ & $8 \frac{1}{2}$ & $8 \times 8$ & $\ldots$ & $\cdots$ & British Museum. \\
\hline $29 \frac{5}{8}$ & $6 \frac{1}{8}$ & $\ldots$ & 45 & $9 \frac{3}{8}$ & $10 \times 9$ & $\ldots$ & $\ldots$ & F. J. Horniman. \\
\hline $29 \frac{x}{4}$ & 7 & $\cdots$ & 40 & $5 \frac{1}{8}$ & $6 \times 5$ & $\ldots$ & $\cdots$ & British Museum. \\
\hline 29 & $7 \frac{1}{2}$ & $\cdots$ & $43^{\frac{1}{2}}$ & $10 \frac{1}{2}$ & 24 & Norway & $\ldots$ & P. C. Walker. \\
\hline $2 S^{3}$ & $7 \frac{3}{8}$ & 35 & $49 \frac{7}{8}$ & $12 \frac{1}{2}$ & II $\times$ II & ... & $\ldots$ & Hon. Charles Ellis. \\
\hline $2 S_{\frac{1}{2}}$ & $\begin{array}{c}6 \frac{3}{4} \text { (owner's } \\
\text { measurement) }\end{array}$ & $3^{1 \frac{1}{2}}$ & $40 \frac{1}{2}$ & $9 \frac{3}{8}$ & II $\times I I$ & Norway & ... & Thomas Bate. \\
\hline 28 & $\mid \begin{array}{c}7 \frac{1}{2} \text { (owner's } \\
\text { measurement) }\end{array}$ & $34 \frac{1}{2}$ & $42 \frac{1}{2}$ & ... & I6 & Do. & ... & Do. \\
\hline $27 \frac{5}{8}$ & $5^{\frac{3}{4}}$ & 30 & $40 \frac{7}{8}$ & $7 \frac{3}{8}$ & $8 \times 7$ & N. Ameri & ica & Capt. F. Cookson. \\
\hline $27 \frac{1}{2}$ & $\cdots$ & $\cdots$ & $49 \frac{1}{2}$ & $10 \frac{1}{2}$ & $\cdots$ & Do. & & Earl of Lonsdale. \\
\hline $27 \frac{3}{8}$ & $6 \frac{1}{8}$ & $24 \frac{3}{4}$ & $37 \frac{3}{8}$ & 7 & $6 \times 6$ & Do. & & J. C. L. Knight Bruce. \\
\hline $26 \frac{7}{8}$ & $\mid \begin{array}{c}6 \frac{5}{8} \\
\text { (single horn) }\end{array}$ & ... & $\cdots$ & $7 \frac{1}{8}$ & $\ldots$ & $\cdots$ & $\ldots$ & British Museum. \\
\hline $26 \frac{5}{8}$ & $6 \frac{1}{2}$ & $\cdots$ & $45 \frac{1}{8}$ & $3^{\frac{3}{4}}$ & $5 \times 5$ & $\cdots$ & $\cdots$ & Do. \\
\hline $26 \frac{1}{8}$ & $6 \frac{1}{2}$ & $\cdots$ & $39 \frac{3}{8}$ & $6 \frac{7}{8}$ & $5 \times 4$ & S. Russia. & a... & Do. \\
\hline 25 & 6 & $\cdots$ & $44 \frac{1}{2}$ & $\cdots$ & 22 & Norway & $\cdots$ & Major Sulivan. \\
\hline $23 \frac{1}{2}$ & $5^{\frac{3}{4}}$ & $\cdots$ & $35^{\frac{3}{4}}$ & $5 \frac{7}{8}$ & $5 \times 3$ & Tydal & ... & E. N. Buxton, I $\$ 86$. \\
\hline 23 & $\cdots$ & $\cdots$ & $28 \frac{1}{2}$ & 8 & $\cdots$ & Norway & $\ldots$ & H. C. Hambro. \\
\hline $19 \frac{3}{4}$ & $5 \frac{1}{2}$ & $\ldots$ & $37^{\frac{3}{4}}$ & 7 & $6 \times 7$ & $\ldots$ & $\ldots$ & E. N. Buxton. \\
\hline I6 & ... & ... & 29 & 5 & ... & Norway & $\cdots$ & H. C. Hambro. \\
\hline
\end{tabular}


ROE DEER (Capreolus caprea).

Habitat-Europe and Western Asia.

\begin{tabular}{|c|c|c|c|c|c|}
\hline Length. & $\begin{array}{l}\text { Circum- } \\
\text { ference. }\end{array}$ & $\begin{array}{l}\text { Tip to } \\
\text { Tip. }\end{array}$ & \multicolumn{2}{|c|}{ Habitat. } & Owner. \\
\hline $9 \frac{7}{8}$ & $3 \frac{3}{4}$ & $5^{\frac{3}{4}}$ & \multicolumn{2}{|c|}{ Kirdels IVood, Ireland } & Sir Victor Brooke's Collection, ISSo. \\
\hline $9 \frac{7}{8}$ & 5 & 5 & Dorsetshire & $\ldots$ & J. E. Harting. \\
\hline $9 \frac{1}{2}$ & $3 \frac{1}{t}$ & $3 \frac{1}{8}$ & Kirdels IVoo & d, Ireland & Sir Victor Brooke's Collection, ISSo. \\
\hline $9 \frac{1}{4}$ & 3 & $7 \frac{3}{8}$ & Easter Elchi & $\ldots$ & Do. $\quad \mathbf{I} 875$. \\
\hline 9 & 4 & $4 \frac{1}{2}$ & $\ldots$ & $\ldots$ & Rowland Ward's Collection. \\
\hline$\delta_{\frac{1}{2}}^{\frac{1}{2}}$ & 3 (malformed) & $5^{\frac{7}{8}}$ & Nairnshire & $\ldots$ & Earl Cawdor, British Museum. \\
\hline $8 \frac{1}{2}$ & 3 & $4 \frac{1}{2}$ & France & $\ldots$ & British Museum. \\
\hline $8 \frac{1}{2}$ & $3 \frac{1}{4}$ & $4 \frac{1}{8}$ & Westphalia & $\ldots$ & Do. \\
\hline$s$ & 4 & $3^{\frac{3}{4}}$ & Inverness & $\ldots$ & Sir Victor Brooke's Collection. \\
\hline $6 \frac{7}{8}$ & 3 & 6 & Dorset & $\ldots$ & J. Fraser. \\
\hline $4 \frac{1}{4}$ & $2 \frac{1}{4}$ (velvet) & $4 \frac{1}{2}$ & Astrabad & $\ldots$ & Col. Beresford Lovett, Brit. Museum. \\
\hline
\end{tabular}

SIBERIAN ROE DEER (Capreolus pygargus).

Habitat-Siberia.

\begin{tabular}{|c|c|c|c|c|c|c|}
\hline Length. & $\begin{array}{l}\text { Circum- } \\
\text { ference. }\end{array}$ & $\begin{array}{l}\text { Tip to } \\
\text { Tip. }\end{array}$ & \multicolumn{3}{|c|}{ Habitat. } & Owner. \\
\hline $13 \frac{3}{4}$ & 3 & 6 & Asia & ... & $\ldots$ & British Museum. \\
\hline $13 \frac{1}{4}$ & 4 & $8 \frac{7}{5}$ & South & Manchuria & $\ldots$ & H. E. M. James, British Museum. \\
\hline II $\frac{3}{8}$ & 4 & $S_{\frac{1}{8}}$ & China & $\ldots$ & $\ldots$ & British Museum. \\
\hline $8 \frac{7}{8}$. & $3 \frac{3}{8}$ & $7 \frac{5}{8}$ & Siberi & $\cdots$ & $\ldots$ & Do. \\
\hline
\end{tabular}

LONG-TAILED DEER (Cariacus leucurus).

\begin{tabular}{r|c|c|c|r|r|r|r}
\hline Length. & $\begin{array}{c}\text { Circum. } \\
\text { ference. }\end{array}$ & $\begin{array}{c}\text { Tip to } \\
\text { Tip. }\end{array}$ & $\begin{array}{c}\text { Widest } \\
\text { inside. }\end{array}$ & Points. & Habitat. & Owner. \\
\hline $25 \frac{1}{\frac{1}{4}}$ & $4 \frac{3}{4}$ & I $2 \frac{7}{8}$ & $17 \frac{7}{8}$ & I I $\times 9$ & $\ldots$ & $\ldots$ & British Museum. \\
$23 \frac{1}{2}$ & $4 \frac{1}{4}$ & $2 \frac{5}{8}$ & 13 & $8 \times 9$ & $\ldots$ & $\ldots$ & Do. \\
$20 \frac{1}{8}$ & $3 \frac{3}{4}$ & IO & $14 \frac{1}{2}$ & I $\times 7$ & $\ldots$ & $\ldots$ & Do. \\
$16 \frac{7}{8}$ & $3 \frac{1}{8}$ & $9 \frac{3}{8}$ & $13 \frac{5}{8}$ & $4 \times 3$ & $\ldots$ & $\ldots$ & Do. \\
\hline
\end{tabular}




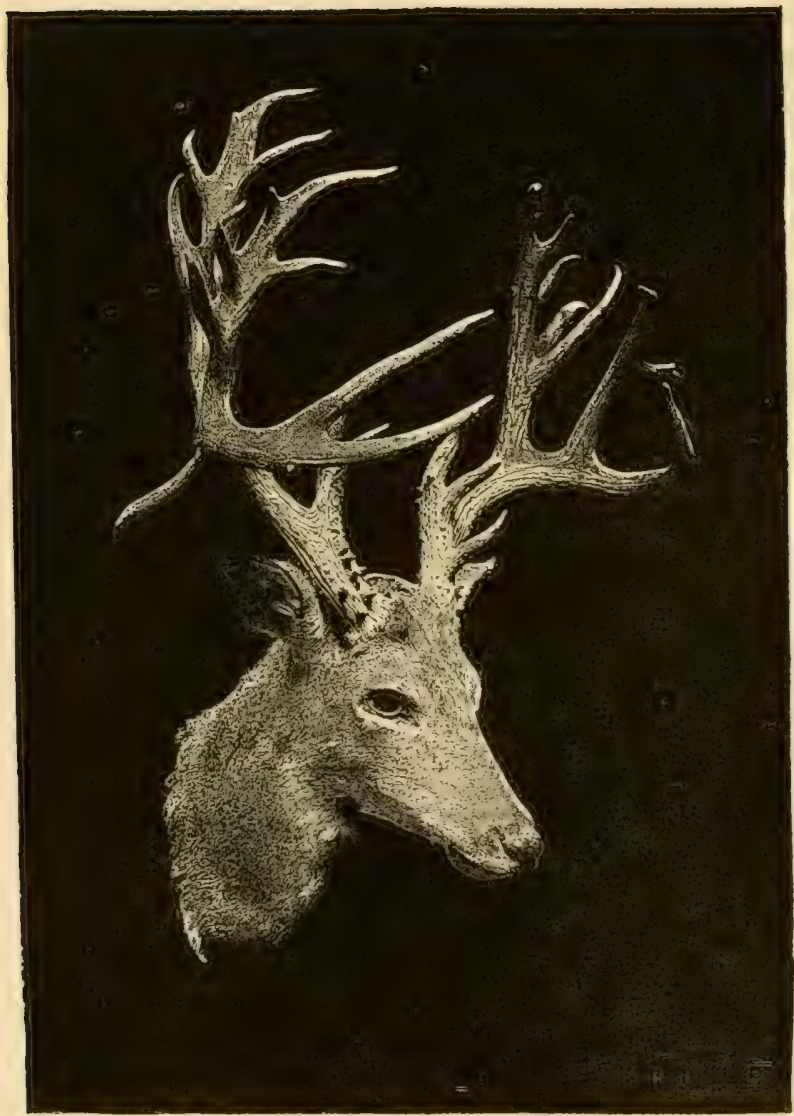

VIRGINIAN Or WHITE-TAILED DEER (Cariacus virginianus). 
VIRGINIAN or WHITE-TAILED DEER (Cariacus virginianus).

Wcight-About I8o lbs.

Habitat-North America from the Atlantic to the Pacific, extending into British Columbia in the North and Mexico in the South.

\begin{tabular}{|c|c|c|c|c|c|c|}
\hline Length. & $\begin{array}{l}\text { Circum- } \\
\text { ference. }\end{array}$ & Tip to Tip. & $\begin{array}{l}\text { Widest } \\
\text { inside. }\end{array}$ & Points. & Habitat. & Owner. \\
\hline $27 \frac{5}{8}$ & $5 \frac{3}{8}$ & (single horn) & $\cdots$ & I6 & North America & British Museum. \\
\hline $27 \frac{1}{8}$ & $4 \frac{3}{8}$ & $14 \frac{3}{4}$ & I 9 & $6 \times 6$ & Do. & Do. \\
\hline $25^{\frac{3}{4}}$ & $4 \frac{1}{2}$ & 9 & I9 & II & Do. & H. S. Wellcome. \\
\hline $25 \frac{3}{8}$ & $4 \frac{5}{8}$ & $10 \frac{3}{8}$ & 19 & $13 \times 15$ & Texas ... ... & Capt. F. Cookson. (See Illus - \\
\hline $24 \frac{7}{8}$ & 5 & (single horn) & ... & I9 & North America & British Museum. \\
\hline $24 \frac{7}{2}$ & $4 \frac{1}{2}$ & $12 \frac{3}{8}$ & I $8 \frac{1}{2}$ & $6 \times 6$ & Do. & J. Carr-Saunders. \\
\hline $24 \frac{1}{2}$ & $4 \frac{1}{8}$ & I I $\frac{1}{8}$ & $19 \frac{1}{2}$ & $6 \times 6$ & Do. & British Museum. \\
\hline 24 & $4 \frac{1}{2}$ & I $4 \frac{1}{2}$ & $19 \frac{7}{8}$ & $10 \times 6$ & $\begin{array}{lll}\cdots & \cdots & \cdots\end{array}$ & Do. \\
\hline $22 \frac{3}{4}$ & $5 \frac{5}{8}$ & $8 \frac{1}{4}$ & $16 \frac{7}{8}$ & $16 \times 19$ & North America & Do. \\
\hline $2 I_{\frac{1}{2}}^{\frac{1}{2}}$ & $3 \frac{3}{1}$ & $7 \frac{1}{4}$ & $16 \frac{3}{8}$ & $7 \times 5$ & Wisconsin $\quad \ldots$ & Do. \\
\hline $2 \mathrm{I}$ & $3 \frac{7}{8}$ & $7 \frac{1}{2}$ & $14 \frac{1}{2}$ & $4 \times 4$ & North America. & Do. \\
\hline $20 \frac{5}{8}$ & $3 \frac{7}{8}$ & $4 \frac{3}{4}$ & $14 \frac{3}{8}$ & $4 \times 5$ & Do. & Do. \\
\hline $20 \frac{1}{2}$ & $4 \frac{1}{8}$ & $9^{\frac{5}{8}}$ & $15 \frac{3}{4}$ & $5 \times 6$ & Do. & Do. \\
\hline $19 \frac{33}{4}$ & $4 \frac{1}{8}$ & $7 \frac{5}{8}$ & I $7 \frac{3}{8}$ & $\cdots$ & Do. & F. J. Horniman. \\
\hline $19 \frac{1}{2}$ & 4 & $\cdots$ & $19 \frac{3}{4}$ & 12 & Do. & H. S. Wellcome. \\
\hline I $S \frac{7}{8}$ & $3 \frac{1}{2}$ & $8 \frac{1}{8}$ & $14 \frac{1}{2}$ & $6 \times 5$ & Do. & Sir Victor Brooke's Collection. \\
\hline $\begin{array}{c}17 \frac{3}{4} \\
\text { (al,out) }\end{array}$ & $4 \frac{3}{4}$ & $\cdots$ & $\cdots$ & $27 \times 25$ & Do. & Moreton Frewen. \\
\hline $16 \frac{3}{4}$ & $4 \frac{1}{4}$ & $3 \frac{3}{4}$ & $12 \frac{5}{8}$ & $5 \times 4$ & Do. & British Museum. \\
\hline $15 \frac{1}{2}$ & $3 \frac{7}{3}$ & 4 & I I $\frac{3}{8}$ & $5 \times 6$ & Do. & Do. \\
\hline $13 \frac{1}{4}$ & 5 & $3 \frac{3}{8}$ & $6 \frac{3}{4}$ & $5 \times 6$ & Do. & Capt. Townley Parker. \\
\hline
\end{tabular}




\section{MEXICAN DEER}

(Cariacus mexicanus; Cervus paludosus; Blastoceros paludosus).

[Sir VictoR BROOKE, P.Z.S. 1878, p. 920.]

\section{Dimensions of Cariacus mexicanus.}

$\begin{array}{lllllll}\text { Height at shoulder } & \ldots & \ldots & \ldots & \ldots & \ldots & 27^{\circ} 5\end{array}$

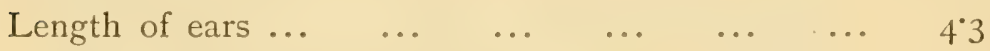

Do. tail exclusive of hair $\quad \begin{array}{lllll} & \ldots & \ldots & \ldots & 5.5\end{array}$

Total length of skull in a straight line $\quad \ldots \quad \ldots \quad$... $9^{\circ}$

Length from ant. rim of orbit to free extremity of $\begin{array}{lllllll}\text { præ } \operatorname{maxill} & \ldots & \ldots & \ldots & \ldots & \ldots & 4{ }^{*}\end{array}$

Length of molar and premolar series (upper) $\quad \ldots \quad 2 * 7$

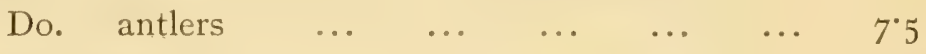

$\begin{array}{llllllll}\text { Greatest } \operatorname{span} & \ldots & \ldots & \ldots & \ldots & \ldots & \ldots & 8 \cdot 2\end{array}$

\begin{tabular}{|c|c|c|c|c|c|c|}
\hline Length. & $\begin{array}{l}\text { Circum- } \\
\text { ference. }\end{array}$ & $\begin{array}{l}\text { Tip to } \\
\text { Tip. }\end{array}$ & $\begin{array}{l}\text { Widest } \\
\text { inside. }\end{array}$ & Points. & Habitat. & Owner. \\
\hline$*_{23} \frac{3}{8}$ & $5 \frac{1}{8}$ & I $5 \frac{3}{1}$ & $16 \frac{1}{t}$ & $4 \times 5$ & South America & British Museum. \\
\hline $23 \frac{1}{\frac{1}{4}}$ & 6 & $\cdots$ & $19 \frac{1}{2}$ & 12 & Do. & G. R. Stuart. \\
\hline$\nmid 22 \frac{5}{8}$ & $6 \frac{1}{4}$ & 20 & $20 \frac{1}{2}$ & $5 \times 5$ & Brazil ... $\quad \ldots$ & Sir Victor Brooke's Collection. \\
\hline$*_{2} \mathrm{I} \frac{1}{4}$ & $7 \frac{1}{8}$ & $9 \frac{5}{8}$ & 16 & $4 \times 3$ & South America & British Museum. \\
\hline$*_{1} S_{\frac{3}{8}}$ & $5^{\frac{1}{2}}$ & $20 \frac{5}{8}$ & $\cdots$ & $4 \times 5$ & Do. & Do. \\
\hline $17 \frac{1}{2}$ & $4 \frac{x}{4}$ & $\cdots$ & I I & Io & Do. & Hon. Walter Rothschild. \\
\hline $13 \frac{5}{8}$ & $3 \frac{3}{8}$ & $6 \frac{7}{8}$ & II $\frac{3}{4}$ & $3 \times 3$ & Mexico $\quad \ldots$ & British Museum. \\
\hline$*_{1} 3^{\frac{1}{2}}$ & $4 \frac{3}{4}$ & 15 & $\cdots$ & $4 \times 4$ & South America & Do. \\
\hline $8 \frac{1}{4}$ & $2 \frac{1}{8}$ & $4 \frac{3}{4}$ & $6 \frac{5}{8}$ & $3 \times 4$ & Mexico & Sir Victor Brooke's Collection. \\
\hline
\end{tabular}




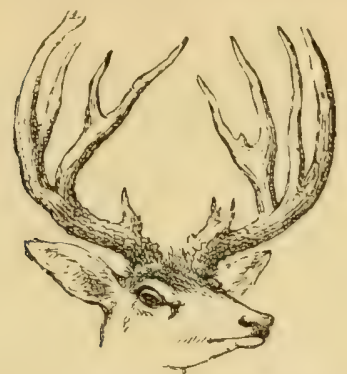

BLACK-TAILED DEER (Cervus columbianus).

Weight-About 200 lbs.

Habitat-California, Oregon, Washington, and along the Pacific Coast.

\begin{tabular}{|c|c|c|c|c|c|c|c|c|}
\hline Length. & $\begin{array}{l}\text { Circum- } \\
\text { ference. }\end{array}$ & $\begin{array}{l}\text { Tip to } \\
\text { Tip. }\end{array}$ & $\begin{array}{l}\text { Widest } \\
\text { inside. }\end{array}$ & Points. & \multicolumn{3}{|c|}{ Habitat. } & Owner. \\
\hline $28 \frac{5}{8}$ & $4 \frac{1}{2}$ & $13 \frac{1}{4}$ & I $7 \frac{3}{4}$ & $5 \times 5$ & ... & $\cdots$ & ... & F. G. Barclay. \\
\hline 27 & $5 \frac{1}{4}$ & $19 \frac{1}{2}$ & $21 \frac{5}{8}$ & $6 \times 5$ & \multicolumn{3}{|c|}{ North America } & Sir Edmund G. Loder, Bart. \\
\hline $26 \frac{1}{8}$ & $4 \frac{1}{8}$ & $15 \frac{7}{8}$ & $22 \frac{7}{8}$ & $6 \times 6$ & \multicolumn{3}{|c|}{ Wyoming } & Capt. F. Cookson. \\
\hline 26 & $5^{\frac{1}{4}}$ & $\cdots$ & 26 & $10 \times 10$ & $\cdots$ & $\cdots$ & $\cdots$ & Wm. Marr. \\
\hline 26 & $\ldots$ & $\cdots$ & I8 & 7 & $\ldots$ & $\cdots$ & $\ldots$ & W. S. Power. \\
\hline $25 \frac{1}{2}$ & $5 \frac{1}{2}$ & $\cdots$ & $2 I \frac{1}{2}$ & 8 & $\cdots$ & $\ldots$ & $\cdots$ & P. C. Walker. \\
\hline $25 \frac{3}{8}$ & $4 \frac{1}{8}$ & 19 & 20 & $5 \times 5$ & $\cdots$ & $\cdots$ & $\cdots$ & C. H. Pollen. \\
\hline $25 \frac{1}{4}$ & $\ldots$ & $\cdots$ & 18 & IO & $\ldots$ & $\cdots$ & $\ldots$ & W. S. Power. \\
\hline 25 & $4 \frac{1}{2}$ & $\cdots$ & 23 & IO & $\cdots$ & $\cdots$ & $\cdots$ & C. P. S. Porter. \\
\hline $24 \frac{1}{2}$ & $5^{\frac{1}{2}}$ & $\cdots$ & 25 & IO & $\cdots$ & $\cdots$ & $\cdots$ & T. W. H. Clarke. \\
\hline $24 \frac{1}{2}$ & 5 & $\cdots$ & I9 & IO & $\cdots$ & $\cdots$ & $\ldots$ & W. G. Rathbone. \\
\hline $24 \frac{1}{2}$ & 5 & $\cdots$ & IS & 12 & $\cdots$ & $\ldots$ & $\ldots$ & Capt. F. Cookson. \\
\hline $24 \frac{1}{2}$ & $5^{\frac{1}{4}}$ & $\cdots$ & $23 \frac{1}{2}$ & IO & $\cdots$ & $\therefore$ & $\cdots$ & H. S. Wellcome. \\
\hline $2 \dot{4} \frac{1}{8}$ & $4 \frac{1}{4}$ & $2 \mathrm{I} \frac{3}{8}$ & $23 \frac{1}{2}$ & $5 \times 5$ & $\ldots$ & $\ldots$ & $\cdots$ & J. Carr-Saunders. \\
\hline 24 & $5 \frac{1}{2}$ & $\cdots$ & $\cdots$ & $8 \times 5$ & $\cdots$ & $\cdots$ & $\cdots$ & F. G. Barclay. \\
\hline $23 \frac{1}{2}$ & $\cdots$ & $\cdots$ & $15 \frac{1}{2}$ & II & $\cdots$ & $\cdots$ & $\cdots$ & W. S. Power. \\
\hline $22 \frac{3}{4}$ & $3 \frac{7}{8}$ & 15 & $19 \frac{3}{4}$ & $5 \times 4$ & $\cdots$ & $\cdots$ & $\cdots$ & J. Carr-Saunders. \\
\hline $22 \frac{1}{4}$ & 4 & $16 \frac{1}{8}$ & $19 \frac{3}{4}$ & $5 \times 5$ & $\cdots$ & $\cdots$ & $\cdots$ & Col. J. A. Percey. \\
\hline 22 & 5 & $\cdots$ & $\cdots$ & $9 \times 7$ & $\cdots$ & $\cdots$ & $\cdots$ & F. G. Barclay. \\
\hline $2 \mathrm{I} \frac{1}{2}$ & $3 \frac{3}{4}$ & $6 \frac{1}{4}$ & $\cdots$ & $7 \times 7$ & & $\cdots$ & $\cdots$ & British Museum. \\
\hline $19 \frac{1}{4}$ & $4 \frac{1}{4}$ & I4 & $16 \frac{1}{2}$ & $3 \times 3$ & \multirow{2}{*}{\multicolumn{3}{|c|}{$\begin{array}{l}\text { Mouterey, Cali- } \\
\text { fornia. } \\
\ldots \\
\ldots\end{array}$}} & Sir Victor Brooke's Collection. \\
\hline $18 \frac{1}{4}$ & $3 \frac{3}{4}$ & $13 \frac{1}{4}$ & $\cdots$ & $3 \times 3$ & & & & British Museum. \\
\hline
\end{tabular}




\section{PAMPAS DEER (Cariacus campestris).}

Habitat-South America.

\begin{tabular}{|c|c|c|c|c|c|c|c|}
\hline Length. & $\begin{array}{l}\text { Circum- } \\
\text { ference. }\end{array}$ & $\begin{array}{l}\text { Tip to } \\
\text { Tip. }\end{array}$ & Points. & \multicolumn{3}{|c|}{ Habitat. } & Owner. \\
\hline $14 \frac{5}{8}$ & $2 \frac{5}{8}$ & $13 \frac{1}{4}$ & $3 \times 3$ & $\mathrm{La}$ & Plata & $\ldots$ & British Museum. \\
\hline $12 \frac{1}{2}$ & $2 \frac{1}{8}$ & $8 \frac{1}{2}$ & $3 \times 3$ & $\ldots$ & $\ldots$ & $\ldots$ & Sir Victor Brooke's Collection. \\
\hline II $\frac{3}{4}$ & $2 \frac{3}{4}$ & $14 \frac{1}{4}$ & $3 \times 3$ & $\cdots$ & $\cdots$ & $\ldots$ & Do. \\
\hline II $\frac{1}{8}$ & $3 \frac{7}{8}$ & $13 \frac{7}{4}$ & $3 \times 3$ & & $\cdots$ & $\ldots$ & Charles Darwin, British Museum. \\
\hline IO $\frac{3}{8}$ & $2 \frac{1}{2}$ & $13 \frac{1}{8}$ & $3 \times 3$ & Nor & th Patag & onia & British Museum. \\
\hline
\end{tabular}

\section{PERUVIAN ROE DEER (Cariacus (furcifer) antisiensis).}

[Dr. J. E. Gray, F.R.S., P.Z.S. I869, p. 498.]

THE horns divide from the base into two branches; the front one is erect, conical, and acute, with a short conical branch on the outer side, and in the middle one or two more or less elongate, basal anterior or interior snags. The hinder part of the base and sheath compressed, diverging horizontally into a strong, angular, tapering branch, which is nearly as long as the erect one, with several irregularly placed, more or less elongate, acute processes; the upper part, near the roots, with one or two cylindrical diverging branches on its upper and lower sides. Like the small branches on the erect part of the horn, they are not exactly similar on the two horns.

The metatarsus is without any glandular tuft on the outside. On the inner side of the hock is a large rounded tuft of such hairs. The fur consists of thick, elastic, tubular quills. The skull has a wellmarked, deep, triangular pit in the front of the orbit. The female is without horns. This animal may be called Xenelaphus huamel.

\begin{tabular}{|c|c|c|c|c|}
\hline Length. & $\begin{array}{l}\text { Circum- } \\
\text { ference. }\end{array}$ & $\begin{array}{l}\text { Tip to } \\
\text { Tip. }\end{array}$ & Habitat. & Owner. \\
\hline $9 \frac{1}{2}$ & $7 \frac{5}{8}$ & $4 \frac{5}{8}$ & Tinta, South Peru ... $\quad \ldots$ & H. Whitely, British Museum. \\
\hline $9 \frac{3}{8}$ & $2 \frac{1}{2}$ & $8 \frac{1}{8}$ & Ceuchepate, Peru ( $\mathrm{I}, 000 \mathrm{ft}$.) & Do. \\
\hline
\end{tabular}




\section{MULE DEER (Cariacus macrotis).}

THIS variety has much larger ears than the White-tailed or Blacktailed Deer, and owing to its having the tip of the tail black, is often called Black-tail.

Habitat-Includes Oregon, Nebraska, Dakota, West of Missouri, Colorado, and Kansas.

\begin{tabular}{|c|c|c|c|c|c|c|}
\hline Length. & $\begin{array}{l}\text { Circum- } \\
\text { ference. }\end{array}$ & $\begin{array}{l}\text { Tip to } \\
\text { Tip. }\end{array}$ & Widest inside. & Points. & Habitat. & Owner. \\
\hline 30 & $\begin{array}{l}53 \\
\text { lowne }\end{array}$ & r's mea & $\underset{\text { asurements) }}{4 \mathrm{I}}$ & 17 & $\begin{array}{l}\text { White River, Colo- } \\
\text { rado, N. America. }\end{array}$ & H. A. James. \\
\hline $28 \frac{1}{2}$ & $\cdots \quad$. & $\cdots \cdots$ & $24 \frac{1}{2}$ & ... & Do. & $\begin{array}{l}\text { Major Maitland Kirwin. (Ameri- } \\
\text { can Exhibition Catalogue.) }\end{array}$ \\
\hline $27 \frac{1}{2}$ & $\cdots$ & $\cdots$ & $26 \frac{1}{2}$ & $\cdots$ & Do. & Do. \\
\hline $26 \frac{3}{4}$ & $\cdots$ & $\cdots$ & ... & 26 & Do. & $\begin{array}{l}\text { W. A. Baillie Grohman, (Ameri- } \\
\text { can Exhibition Catalogue.) }\end{array}$ \\
\hline $26 \frac{3}{4}$ & $4 \frac{3}{4}$ & $19 \frac{5}{8}$ & $20 \frac{1}{8}$ & $5 \times 5$ & Do. & Ernest Farquhar. \\
\hline $26 \frac{1}{2}$ & $5^{\frac{3}{4}}$ & $\cdots$ & I $7 \frac{1}{2}$ & 12 & Do. & Hon. F. Thellusson. \\
\hline $26 \frac{1}{2}$ & $5 \frac{1}{\frac{1}{4}}$ & $\cdots$ & 19 & II & Do. & Do. \\
\hline 25 & $5 \frac{1}{4}$ & $27 \frac{3}{8}$ & $27 \frac{3}{8}$ & $16 \times 13$ & Do. & Moreton Frewen. \\
\hline $24 \frac{1}{2}$ & $5 \frac{1}{2}$ & $\cdots$ & $2 \mathrm{I}$ & 21 & Do. & Ford G. Barclay. \\
\hline $24 \frac{1}{2}$ & 5 & $\ldots$ & (outside points) & 33 & Do. & Hon. F. Thellusson. \\
\hline 24 & $5 \frac{1}{4}$ & $\cdots$ & $20 \frac{1}{4}$ & I0 & Do. & William Fox. \\
\hline 24 & $4 \frac{1}{2}$ & II $\frac{3}{4}$ & $18 \frac{1}{8}$ & $12 \times I I$ & Do. & E. N. Buxton. \\
\hline $23 \frac{1}{4}$ & $\cdots$ & $\cdots$ & $3 \mathrm{I}$ & $5 \times 5$ & Do. & Noble Jenkins. \\
\hline 23 & 6 & $\cdots$ & $12 \frac{1}{2}$ & I3 & Do. & Ford G. Barclay. \\
\hline $22 \frac{3}{8}$ & $4^{\frac{3}{8}}$ & $14 \frac{7}{8}$ & $17 \frac{1}{2}$ & $5 \times 4$ & Yellowstone River & British Museum. \\
\hline $22 \frac{3}{8}$ & 4 & $10 \frac{5}{8}$ & I $8 \frac{1}{4}$ & $6 \times 6$ & Do. & Do. \\
\hline $22 \frac{1}{4}$ & $3 \frac{3}{4}$ & $16 \frac{5}{8}$ & $18 \frac{1}{4}$ & $5 \times 5$ & North America... & Sir Victor Brooke's Collection. \\
\hline $22 \frac{1}{8}$ & 4 & $12 \frac{1}{2}$ & $16 \frac{3}{8}$ & $4 \times 4$ & Yellowstone River & British Museum. \\
\hline $21 \frac{5}{8}$ & $4 \frac{3}{4}$ & $17 \frac{1}{2}$ & $19 \frac{1}{4}$ & $5 \times 4$ & Kansas $\quad \ldots \quad \quad \ldots$ & $\begin{array}{l}\text { Sir Victor Brooke's Collection, } \\
\text { I } 872 \text {. }\end{array}$ \\
\hline 21 & $6 \frac{3}{4}$ & $\cdots$ & 28 & 26 & North America... & J. Turner-Turner. \\
\hline $19 \frac{7}{8}$ & $3 \frac{3}{t}$ & I $1 \frac{1}{2}$ & $15 \frac{1}{2}$ & $5 \times 5$ & Yellowstone River & British Museum. \\
\hline
\end{tabular}




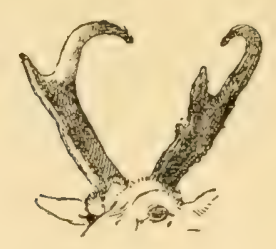

PRONG-HORNED ANTELOPE (Antilocapra americana).

Wcight-About 8 o lbs. clean.

Habitat-North America.

\begin{tabular}{|c|c|c|c|c|c|c|}
\hline Length. & $\begin{array}{l}\text { Circum- } \\
\text { ference. }\end{array}$ & $\begin{array}{l}\text { Tip to } \\
\text { Tip. }\end{array}$ & $\begin{array}{l}\text { Widest } \\
\text { inside. }\end{array}$ & \multicolumn{2}{|l|}{ Habitat. } & Owner. \\
\hline $15^{\frac{3}{x}}$ & $6 \frac{1}{4}$ & $5^{\frac{7}{8}}$ & $\ldots$ & Wyoming & $\ldots$ & St. George Littledale, $\mathbf{I} \$ 79$. \\
\hline $15 \frac{1}{8}$ & $6 \frac{1}{4}$ & $7 \frac{5}{8}$ & ... & North Americ & a... & Col, Ralph Vivian. \\
\hline 15 & $5 \frac{5}{8}$ & $5^{\frac{3}{4}}$ & $\ldots$ & Wyoming & $\cdots$ & St. George Littledale, 1879. \\
\hline $14 \frac{7}{8}$ & 5 & $5 \frac{1}{4}$ & $\therefore$ & New Mexico & ... & Capt. F. Cookson, i 887 . \\
\hline $14 \frac{3}{4}$ & 6 & $5 \frac{7}{8}$ & ... & Wyoming & $\cdots$ & St. George Littledale, 1879. \\
\hline $14 \frac{1}{2}$ & $6 \frac{3}{8}$ & $4 \frac{1}{4}$ & ... & North Americ: & a... & Col. Ralph Vivian. \\
\hline $14 \frac{1}{2}$ & $6 \frac{1}{4}$ & $\cdots$ & 14 & Do. & $\cdots$ & Rev. J. H. Douglas. \\
\hline I $4 \frac{1}{2}$ & 6 & $\cdots$ & $9 \frac{1}{2}$ & Do. & $\cdots$ & Ford G. Barclay. \\
\hline $14 \frac{1}{4}$ & 6 & $2 \frac{5}{8}$ & $8 \frac{1}{8}$ & Do. & $\ldots$ & Sir Victor Brooke's Collection. \\
\hline $14 \frac{1}{4}$ & $5 \frac{1}{4}$ & $2 \frac{7}{8}$ & ... & New Mexico & $\cdots$ & Capt. F. Cookson, 1887 . \\
\hline 14 & $5 \frac{1}{2}$ & $9 \frac{1}{4}$ & $\cdots$ & North Americe & $a \ldots$ & J. Carr-Saunders. \\
\hline $13 \frac{7}{8}$ & $5^{\frac{3}{4}}$ & $5 \frac{5}{8}$ & $\cdots$ & Do. & $\cdots$ & Gerald Buxton, I $S S_{4}$. \\
\hline $13 \frac{1}{2}$ & $5 \frac{?}{8}$ & $5 \frac{3}{8}$ & $\cdots$ & New Mexico & $\cdots$ & Capt. F. Cookson, I887. \\
\hline $13 \frac{x}{4}$ & $5 \frac{1}{2}$ & $\cdots$ & $10_{\frac{3}{4}}^{3}$ & North Americ: & $a \ldots$ & William Fox. \\
\hline 13 & $6 \frac{1}{4}$ & $9 \frac{1}{2}$ & ... & Do. & $\ldots$ & Rowland Ward's Collection. \\
\hline$I_{3}$ & $5 \frac{3}{4}$ & $\cdots$ & ... & Do. & $\ldots$ & P. C. Walker. \\
\hline I3 & $5 \frac{1}{2}$ & $\cdots$ & $5 \frac{1}{2}$ & Do. & $\cdots$ & Crawford E. Logan. \\
\hline $12 \frac{7}{8}$ & $6 \frac{1}{2}$ & $9 \frac{3}{8}$ & ... & Wyoming & $\cdots$ & St. George Littledale, I 879 . \\
\hline $12 \frac{7}{8}$ & $5 \frac{1}{4}$ & $6 \frac{5}{8}$ & ... & North America & & The late J. S. Jameson, \\
\hline
\end{tabular}


PRONG-HORNED ANTELOPE (Antilocapra americana)-

continued.

\begin{tabular}{|c|c|c|c|c|c|}
\hline Length. & $\begin{array}{l}\text { Circum- } \\
\text { ference. }\end{array}$ & $\begin{array}{l}\text { Tip to } \\
\text { Tip. }\end{array}$ & $\begin{array}{l}\text { Widest } \\
\text { inside. }\end{array}$ & Habitat. & Owner. \\
\hline $12 \frac{5}{8}$ & $6 \frac{1}{2}$ & 5 & $\ldots$ & North America... & Hon. Walter Rothschild, \\
\hline $12 \frac{1}{2}$ & $6 \frac{3}{8}$ & $5^{\frac{1}{2}}$ & $10 \frac{1}{2}$ & Do, & J. L. Carroll. \\
\hline $12 \frac{1}{2}$ & $5 \frac{1}{2}$ & ... & $9 \frac{1}{2}$ & Do. & William Fox. \\
\hline $12 \frac{3}{8}$ & $5^{\frac{3}{4}}$ & $4 \frac{7}{8}$ & .. & Do. & E. N. Buxton, I $88_{4}$. \\
\hline 12 & $5 \frac{1}{2}$ & ... & 15 & Do. & M. Tilden. \\
\hline $\mathrm{II} \frac{3}{4}$ & $5^{\frac{3}{4}}$ & ... & $\ldots$ & Do. & $\begin{array}{l}\text { Gerald Buxton. (American Ex- } \\
\text { hibition Catalogue.) }\end{array}$ \\
\hline I I $\frac{7}{8}$ & $5 \frac{1}{8}$ & 4 & $\cdots$ & Do. & F. J. Homiman. \\
\hline $10 \frac{3}{4}$ & $5 \frac{7}{8}$ & $6 \frac{5}{8}$ & $\cdots$ & Mexico ... & British Museum. \\
\hline $10 \frac{1}{2}$ & $5^{\frac{1}{2}}$ & 7 & 8 & North America. & J. L. Carroll. \\
\hline $9 \frac{1}{2}$ & $6 \frac{1}{2}$ & .. & 10 & Do. & H. G. C. Bishop. \\
\hline $6 \frac{3}{8}$ & $4 \frac{1}{2}$ & 10 & .. & Yellowstone Riv & British Museum. \\
\hline $6 \frac{1}{8}$ & $\ldots$ & $8 \frac{3}{4}$ & ... & Do. & Do. \\
\hline $5^{\frac{3}{4}}$ & $5 \frac{1}{8}$ & ... & $\ldots$ & North America. & $\begin{array}{l}\text { Otho Shaw. (American Exhibition } \\
\text { Catalogue.) }\end{array}$ \\
\hline
\end{tabular}

BUBALINE ANTELOPE (Bubalis bubalinus).

Habitat-North Africa.

\begin{tabular}{|c|c|c|c|c|c|}
\hline Length. & $\begin{array}{l}\text { Circum- } \\
\text { ference. }\end{array}$ & $\begin{array}{l}\text { Tip to } \\
\text { Tip. }\end{array}$ & Habitat. & & Owner. \\
\hline $14 \frac{1}{2}$ & $8 \frac{5}{8}$ & $9 \frac{3}{8}$ & North Africa ... & $\ldots$ & British Museum. \\
\hline $14 \frac{1}{8}$ & $6 \frac{3}{4}$ & $9 \frac{3}{4}$ & Soudan & ... & Col. Ralph Vivian. \\
\hline $13 \frac{1}{8}$ & 7 & $5 \frac{1}{4}$ & North Africa ... & $\ldots$ & Zoological Society, British Museum. \\
\hline
\end{tabular}




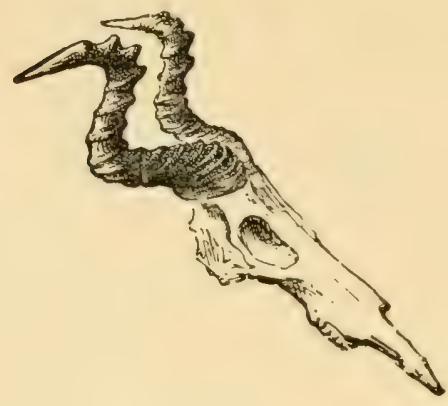

\section{HARTEBEESTE (Bubalis caama).}

Habitat-South Africa: Griqualand West, Eastern border of the Kalahari desert and eastwards to the river Serule on the road from Bamangwato to Tati; also from the Botletlie river to the Zambesi. Further northwards B. caama is replaced by B. lichtensteinii.

\begin{tabular}{l|c|c|cc|l}
\hline Length. & $\begin{array}{c}\text { Circum- } \\
\text { ference. }\end{array}$ & Tip to Tip. & Habitat. & \multicolumn{1}{|l}{ Owner. } \\
\hline $24 \frac{3}{4}$ & $10 \frac{1}{4}$ & $9 \frac{3}{4}$ & South Africa & $\ldots$ & H. Atkinson. \\
24 & 10 & $99 \frac{1}{2}$ & Do. & $\ldots$ & R. C. Dobson. \\
$23 \frac{3}{4}$ & 10 & $8 \frac{3}{8}$ & Do. & $\ldots$ & Sir A. Smith, British Museum. \\
$23 \frac{1}{2}$ & $11 \frac{1}{2}$ & $99 \frac{1}{8}$ & Do. & $\ldots$ & Hon. Walter Rothschild. \\
$23 \frac{1}{2}$ & $11 \frac{1}{2}$ & $10^{\frac{3}{8}}$ & Do. & $\ldots$ & F. C. Selous. \\
$23 \frac{1}{4}$ & $10 \frac{3}{8}$ & $8 \frac{1}{8}$ & Do. & $\ldots$ & The late J. S. Jameson. \\
23 & $11 \frac{1}{4}$ & 6 & Do. & $\ldots$ & F. C. Selous. \\
$22 \frac{1}{2}$ & $10 \frac{3}{4}$ & $10 \frac{1}{8}$ & Do. & $\ldots$ & Sir Edmund G. Loder, Bart. \\
$22 \frac{3}{8}$ & $10 \frac{3}{4}$ & $6 \frac{1}{8}$ & Do. & $\ldots$ & H. Atkinson. \\
$22 \frac{3}{8}$ & $10 \frac{3}{8}$ & $9 \frac{5}{8}$ & Do. & $\ldots$ & A. Beit. \\
22 & $10 \frac{5}{8}$ & $8 \frac{1}{8}$ & Do. & $\ldots$ & Sir Victor Brooke's Collection. \\
22 & 11 & 8 & Do. & $\ldots$ & Major Severas. \\
$211 \frac{3}{4}$ & $11 \frac{1}{\frac{1}{4}}$ & 7 & Do. & $\ldots$ & W. C. Oswell. \\
$21 \frac{3}{4}$ & 10 & $8 \frac{1}{4}$ & Do. & $\ldots$ & A. Beit. \\
\hline
\end{tabular}


HARTEBEESTE (Bubalis caama)-continued.

\begin{tabular}{|c|c|c|c|c|c|}
\hline Length. & $\begin{array}{l}\text { Circum- } \\
\text { ference. }\end{array}$ & Tip to Tip. & Habitat. & & Owner. \\
\hline $21 \frac{1}{2}$ & II & $5 \frac{1}{2}$ & South Africa & $\ldots$ & C. Ansell. \\
\hline $21 \frac{1}{2}$ & $10 \frac{5}{8}$ & $7 \frac{3}{4}$ & Do. & $\ldots$ & A. Beit. \\
\hline $21 \frac{1}{8}$ & $10 \frac{7}{8}$ & $6 \frac{1}{2}$ & Do. & $\ldots$ & F. C. Selous. \\
\hline $20 \frac{1}{2}$ & Io & 8 & Do. & $\ldots$ & Rowland Ward's Collection. \\
\hline $20 \frac{1}{4}$ & II $1 \frac{1}{4}$ & $5 \frac{7}{8}$ & Do. & $\ldots$ & R. C. Dobson, \\
\hline 20 & II & $4 \frac{7}{8}$ & Africa $\quad \ldots$ & $\ldots$ & Sir Edmund G. Loder, Bart. \\
\hline 이 $19 \frac{3}{4}$ & $7 \frac{1}{2}$ & 9 & South Africa & $\ldots$ & The late J. S. Jameson. \\
\hline $19 \frac{1}{2}$ & $8 \frac{1}{4}$ & 8 & Do. & $\ldots$ & F. C. Selous. \\
\hline $19 \frac{1}{3}$ & $7 \frac{1}{2}$ & $8 \frac{1}{2}$ & Africa $\quad \ldots$ & $\ldots$ & J. A. Nicoll. \\
\hline $18 \frac{3}{4}$ & $8 \frac{3}{8}$ & $7 \frac{3}{4}$ & South Africa & $\ldots$ & H.R.H. Duke of Edinburgh. \\
\hline $18 \frac{3}{4}$ & 87 & $9 \frac{1}{2}$ & Do. & $\ldots$ & J. Carr-Saunders. \\
\hline $17 \frac{1}{8}$ & $8 \frac{3}{4}$ & $5 \frac{7}{8}$ & Do. & ... & H.R.H. Duke of Edinburgh. \\
\hline $16 \frac{1}{8}$ & 87 & $\frac{3}{8}$ & Do. & $\ldots$ & British Museum. \\
\hline $10 \frac{3}{8}$ & $7 \frac{1}{4}$ & 3 & Africa $\quad \ldots$ & ... & Earl of Derby, British Museum. \\
\hline
\end{tabular}



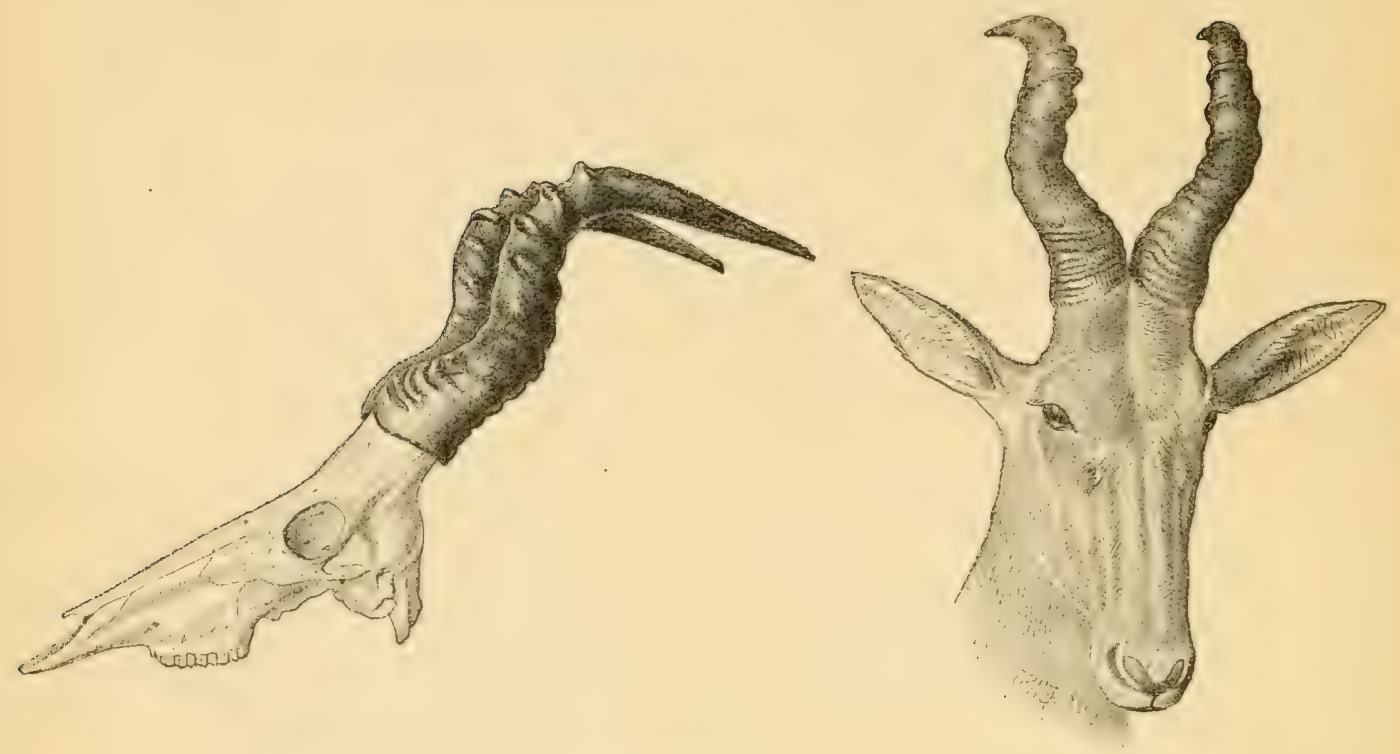

JACKSON'S HARTEBEESTE (Bubalis jacksoni).

Habitat-Central Africa: Country between Lake Victoria Nyanza and Lake Naivashœ.

THIs is a new species of Hartebeeste recently shot by Mr. F. J. Jackson in British East African Territory, who in his letter to me, dated October I89o, says: "I do not think it is likely to extend further south, but of course it may extend to the Cape for what we know ; if it is A. caama it may do so, but I am inclined to doubt its being the same as the S. African animal. I seem to have an idea that the one at the British Museum is very much darker, and the horns different, but this is only from memory, and I cannot be sure. Up north all along the top of the Elgeyo Escarpment (continuation of Mau) into Turquel, to the north and north-east of Mount Elgon, it is common, and takes the place of A. cokei. Round Baringo it is fairly plentiful, but some marches south of Njemis the A. cokei takes its place."

\begin{tabular}{c|c|c|c|c|c|c|c}
\hline Length. & $\begin{array}{c}\text { Circum- } \\
\text { ference. }\end{array}$ & Tip to Tip. & & Habitat. & & \multicolumn{1}{l}{ Owner. } \\
\hline $20 \frac{7}{8}$ & $11 \frac{3}{1}$ & $9 \frac{5}{8}$ & East Africa & $\ldots$ & F. J. Jackson. \\
$23 \frac{1}{2}$ & 12 & 9 & $\ldots$ & $\ldots$ & $\ldots$ & E. Gedge. \\
\hline
\end{tabular}




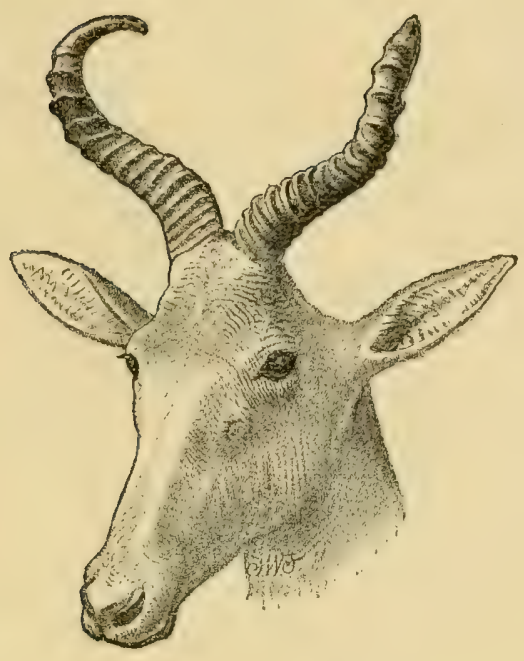

TORA ANTELOPE (Bubalis tora).

Habitat-North Africa. Numerous in Soudan and Abyssinia.

\begin{tabular}{|c|c|c|c|c|}
\hline - Length. & $\begin{array}{l}\text { Circum- } \\
\text { ference. }\end{array}$ & Tip to Tip. & Habitat. & Owner. \\
\hline $19 \frac{3}{4}$ & 9 & $16 \frac{1}{8}$ & Abyssinia... & British Museum. \\
\hline $19 \frac{1}{4}$ & 9 & $13^{\frac{3}{4}}$ & Do. ... & Sir Victor Brooke's Collection. \\
\hline $18 \frac{1}{2}$ & $8 \frac{3}{4}$ & $15 \frac{1}{4}$ & Soudan $\quad \ldots \quad \ldots$ & Col. Ralph Vivian. \\
\hline $18 \frac{1}{8}$ & 10 & $15 \frac{1}{2}$ & Do. $\quad \ldots \quad \ldots$ & Do. \\
\hline $17 \frac{1}{2}$ & $9 \frac{3}{4}$ & 18 & Bogos Iand, Abys- & British Museum. \\
\hline $16 \frac{3}{4}$ & $7 \frac{1}{8}$ & $14 \frac{1}{2}$ & Do. & Do. \\
\hline $15 \frac{5}{8}$ & $7 \frac{7}{8}$ & $17 \frac{3}{4}$ & Do. & Do. \\
\hline $13 \frac{7}{8}$ & $6 \frac{3}{4}$ & 14 & Upper Nubia ... & Sir Edmund G. Loder, Bart. \\
\hline $12 \frac{3}{8}$ & 7 & $6 \frac{7}{8}$ & Abyssinia... & British Museum. \\
\hline II & $6 \frac{1}{8}$ & $15 \frac{1}{8}$ & $\begin{array}{l}\text { Bogos Land, Abys- } \\
\text { sinia. }\end{array}$ & Do. \\
\hline
\end{tabular}




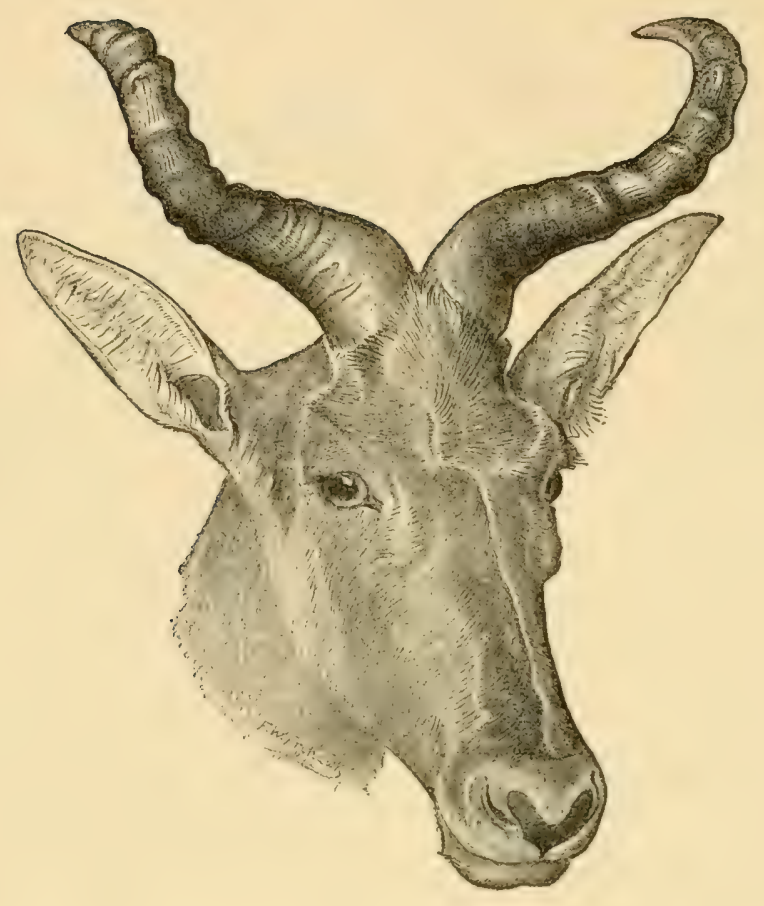

SWAYNE'S HARTEBEESTE (Bubalis swaynei).

Habitat-N. E. Africa: Somali Land.

Closely allied to $B$. cokei and $B$. tora, but can readily be distinguished from either by its dark markings on the face.

\begin{tabular}{|c|c|c|c|c|c|}
\hline Length. & Circumference. & Tip to Tip. & Habitat. & & Owner. \\
\hline $18 \frac{3}{8}$ & $8 \frac{3}{4}$ & I8 & Somali... & $\ldots$ & Capt. H. G. C. Swayne. \\
\hline $17 \frac{1}{2}$ & $8 \frac{1}{8}$ & I $9 \frac{1}{2}$ & Do. ... & ... & T. W. H. Clarke. \\
\hline $16 \frac{1}{2}$ & $9 \frac{3}{4}$ & $12 \frac{3}{4}$ & Do. ... & $\ldots$ & Hon. Walter Rothschild. \\
\hline $16 \frac{3}{8}$ & 9 & $17 \frac{7}{8}$ & Do. ... & $\ldots$ & IV. D. James. \\
\hline I $5 \frac{1}{4}$ & $7 \frac{1}{4}$ & $10 \frac{5}{8}$ & Do. ... & $\ldots$ & E. Lort-Phillips. \\
\hline
\end{tabular}




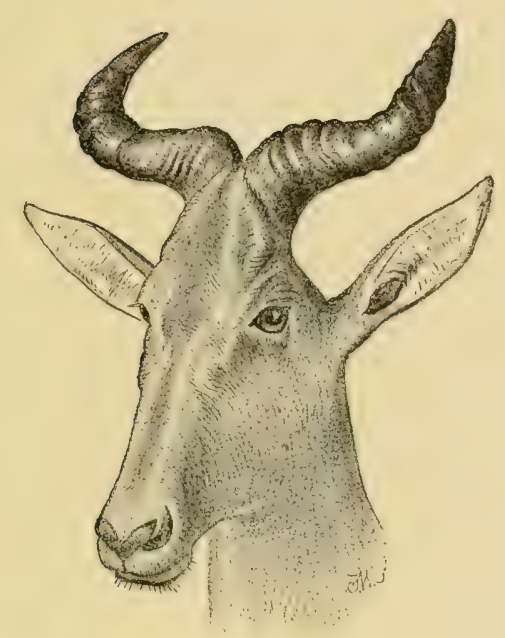

COKE'S HARTEBEESTE (Bubalis cokei).

Habitat-East Africa (Masai Land and adjoining Territory).

\begin{tabular}{|c|c|c|c|c|c|c|}
\hline \multicolumn{2}{|c|}{ Length. } & \multirow{2}{*}{$\begin{array}{l}\text { Circum- } \\
\text { ference. }\end{array}$} & \multirow{2}{*}{$\begin{array}{l}\text { Tip to } \\
\text { Tip. }\end{array}$} & \multirow{2}{*}{ Habitat. } & & \multirow{2}{*}{ Owner. } \\
\hline $\begin{array}{l}\text { Round } \\
\text { bend. }\end{array}$ & Straight. & & & & & \\
\hline 19 & $\cdots$ & $9 \frac{3}{4}$ & 16 & East Africa & $\ldots$ & Sir Robert Harvey, Bart. \\
\hline $18 \frac{5}{8}$ & $\cdots$ & $8 \frac{7}{8}$ & I $6 \frac{1}{4}$ & Do. & ... & F. J. Jackson. \\
\hline $18 \frac{1}{4}$ & $\cdots$ & $9 \frac{1}{2}$ & I $3 \frac{1}{8}$ & Do. & $\cdots$ & H. C. V. Hunter. \\
\hline I8 & $\cdots$ & $9 \frac{1}{4}$ & $16 \frac{3}{4}$ & Do. & $\cdots$ & Sir Robert Harvey, Bart. \\
\hline $17 \frac{5}{8}$ & $\cdots$ & $8_{\frac{3}{4}}$ & I $7 \frac{1}{8}$ & Do. & ... & F. J. Jackson. \\
\hline $17 \frac{3}{8}$ & $\cdots$ & $9 \frac{1}{2}$ & I I & Do. & $\cdots$ & J. Carr-Saunders. \\
\hline $17 \frac{1}{8}$ & $\cdots$ & $9 \frac{1}{4}$ & I $3 \frac{1}{2}$ & Do. & $\cdots$ & H. C. V. Hunter. \\
\hline $16 \frac{3}{8}$ & ... & $9 \frac{1}{8}$ & $13 \frac{5}{8}$ & Do. & $\ldots$ & Do. \\
\hline $16 \frac{3}{8}$ & ... & 8 & $12 \frac{3}{8}$ & Do. & $\ldots$ & British Museum. \\
\hline
\end{tabular}


COKE'S HARTEBEESTE (Bubalis cokei)-continued.

\begin{tabular}{|c|c|c|c|c|c|c|}
\hline \multicolumn{2}{|c|}{ Length. } & \multirow{2}{*}{$\begin{array}{l}\text { Circum- } \\
\text { ference. }\end{array}$} & \multirow{2}{*}{$\begin{array}{l}\text { Tip to } \\
\text { Tip. }\end{array}$} & \multirow{2}{*}{\multicolumn{2}{|c|}{ Habitat. }} & \multirow{2}{*}{ Owner. } \\
\hline $\begin{array}{l}\text { Round } \\
\text { bend. }\end{array}$ & Straight. & & & & & \\
\hline$x 6 \frac{1}{4}$ & $\cdots$ & 9 & I $7 \frac{1}{4}$ & East Africa & ... & Sir Victor Brooke's Collection. \\
\hline $16 \frac{1}{8}$ & ... & $8 \frac{5}{8}$ & $13 \frac{1}{t}$ & Kilima'njaro & ... & Sir John Kirk, M.D., K.C.B. \\
\hline 16 & 12 & $\ldots$ & 12 & East Africa & ... & E. Gedge. \\
\hline $15 \frac{7}{8}$ & ... & $7 \frac{1}{2}$ & $12 \frac{3}{8}$ & Do. & $\ldots$ & Sir Robert Harvey, Bart. \\
\hline $15 \frac{3}{4}$ & $\cdots$ & $8 \frac{3}{4}$ & $10 \frac{1}{8}$ & Taita $\quad \ldots$ & ... & J. Wray, British Museum. \\
\hline $15 \frac{3}{8}$ & $\cdots$ & $7 \frac{3}{8}$ & II $\frac{1}{8}$ & East Africa & ... & Sir Robert Harvey, Bart. \\
\hline$\cdots$ & 9 & $5 \frac{33}{4}$ & II & Do. & ... & W. Astor Chanler. \\
\hline $14 \frac{1}{8}$ & ... & $9 \frac{1}{8}$ & $12 \frac{1}{4}$ & Do. & $\ldots$ & Sir Robert Harvey, Bart. \\
\hline 14 & ... & 10 & II $\frac{7}{8}$ & Do. & ... & Hon, Walter Rothschild. \\
\hline$x_{3} \frac{1}{2}$ & ... & $9 \frac{1}{8}$ & $12 \frac{1}{2}$ & Do. & ... & Roland Ward's Collection. \\
\hline$x I \frac{1}{2}$ & $\cdots$ & ... & $17 \frac{1}{2}$ & Do. & $\cdots$ & $\begin{array}{l}\text { Sir John Willoughby, Bart., "East } \\
\text { Africa and its Big Game." }\end{array}$ \\
\hline
\end{tabular}

WEST AFRICAN HARTEBEESTE (Bubalis major). Habitat-West Africa.

\begin{tabular}{l|c|c|c|cc|c}
\hline Length. & $\begin{array}{c}\text { Circum- } \\
\text { ference. }\end{array}$ & $\begin{array}{c}\text { Tip to } \\
\text { Tip. }\end{array}$ & $\begin{array}{c}\text { Widest } \\
\text { outside. }\end{array}$ & Habitat. & Owner. \\
\hline $20 \frac{3}{8}$ & IO $\frac{1}{4}$ & 7 & $\ldots$ & Africa ... & $\ldots$ & British Museum. \\
\hline
\end{tabular}




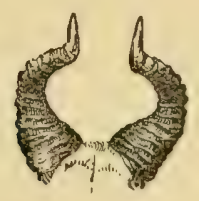

LICHTENSTEIN'S HARTEBEESTE (Bubalis lichtensteinii)

Zambesi name_-"Godoñko."

Habitat-South and East Africa.

\begin{tabular}{|c|c|c|c|c|c|c|}
\hline Length. & $\begin{array}{l}\text { Circum- } \\
\text { ference. }\end{array}$ & Tip to Tip. & $\begin{array}{l}\text { Widest } \\
\text { outside. }\end{array}$ & \multicolumn{2}{|l|}{ Habitat. } & Owner. \\
\hline 20 & $12 \frac{1}{8}$ & 8 & ... & Zanzibar & ... & Sir John Kirk, M. D., K.C.B. \\
\hline $19 \frac{1}{2}$ & II $\frac{7}{2}$ & $8 \frac{1}{8}$ & $\cdots$ & Africa $\ldots$ & $\cdots$ & Hon. Walter Rothschild. \\
\hline I $9 \frac{1}{t}$ & $10 \frac{3}{8}$ & $6 \frac{5}{8}$ & $\ldots$ & Zanzibar & $\cdots$ & Sir John Kirk, M.D., K.C.B. \\
\hline I9 & $\mathrm{II} \frac{1}{2}$ & $4 \frac{7}{8}$ & $\ldots$ & East Africa & ... & British Museum. \\
\hline I $8 \frac{5}{8}$ & I I $\frac{3}{8}$ & $3 \frac{1}{8}$ & $\ldots$ & South Africa & $\cdots$ & F. C. Selous. \\
\hline I $8 \frac{1}{2}$ & $10 \frac{1}{4}$ & 5 & $\cdots$ & Do. & $\cdots$ & F. C. Selous, British Museum. \\
\hline I $8 \underline{\underline{\underline{1}}}$ & 12 & 8 & 12 & East Africa & ... & Gen. Mathews. \\
\hline I $8 \frac{1}{4}$ & II $\frac{1}{4}$ & $5 \frac{1}{8}$ & $\ldots$ & Do. & ... & F. J. Jackson. \\
\hline I $8 \frac{1}{8}$ & $\mathrm{ro}_{4}^{\frac{3}{4}}$ & $4 \frac{5}{8}$ & ... & Do. & $\ldots$ & Do. \\
\hline $16 \frac{5}{8}$ & I I $\frac{1}{8}$ & $6 \frac{1}{8}$ & $\cdots$ & $\begin{array}{l}\text { River Saba (M } \\
\text { shuna Land). }\end{array}$ & Ia. & F. C. Selous, British Museum. \\
\hline $15^{\frac{1}{2}}$ & $1 \mathrm{O}_{\frac{3}{4}}^{3}$ & $5 \frac{3}{8}$ & $\cdots$ & $\cdots \quad \cdots$ & $\cdots$ & W. P. Rylands. \\
\hline q 5 & 87 & $4 \frac{7}{8}$ & $\cdots$ & $\begin{array}{l}\text { River Saba (M } \\
\text { shuna Land). }\end{array}$ & Ia- & F. C. Selous, British Museum. \\
\hline $14 \frac{5}{8}$ & $7 \frac{3}{4}$ & $3 \frac{33}{4}$ & $\cdots$ & East Africa & ... & F. J. Jackson. \\
\hline $14 \frac{1}{2}$ & 8 & $5 \frac{1}{8}$ & ... & Do. & ... & Do. \\
\hline Q $14 \frac{1}{2}$ & $7 \frac{3}{x}$ & $2 \frac{7}{8}$ & $\cdots$ & Do. & ... & Rowland Ward's Collection. \\
\hline $9 \mathrm{I} 4 \frac{1}{8}$ & $8 \frac{1}{2}$ & $5 \frac{5}{8}$ & ... & Transvaal & $\cdots$ & British Museum. \\
\hline $12 \frac{7}{8}$ & 7 & $3 \frac{7}{8}$ & $\cdots$ & Africa $\quad \ldots$ & $\cdots$ & F. L. James. \\
\hline
\end{tabular}




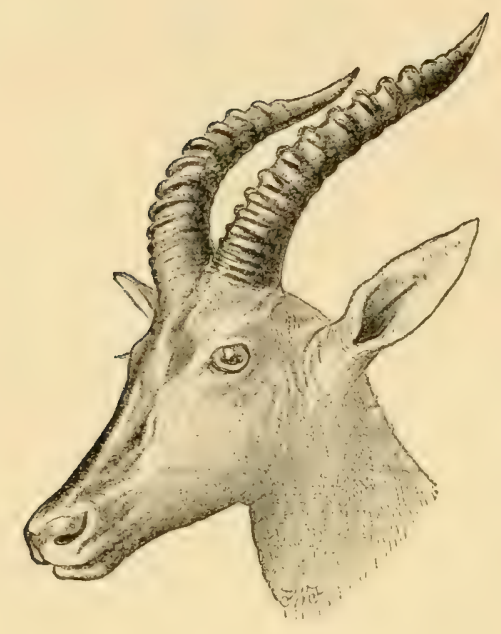

SENEGAL HARTEBEESTE (Bubalis senegalensis).

Habitat-East and West Central Africa.

\begin{tabular}{|c|c|c|c|c|c|}
\hline Length. & $\begin{array}{l}\text { Circum- } \\
\text { ference. }\end{array}$ & Tip to Tip. & Habitat. & & Owner. \\
\hline I $9 \frac{1}{4}$ & $6 \frac{5}{8}$ & $3 \frac{3}{8}$ & East Africa & ... & H. C. V. Hunter. \\
\hline 19 & $6 \frac{3}{4}$ & 10 & Gambia... & ..: & Earl of Derby, British Museum. \\
\hline 19 & $7 \frac{1}{2}$ & 8 & East Africa & $\cdots$ & Rowland Ward's Collection. \\
\hline I $8 \frac{3}{4}$ & $9 \frac{1}{8}$ & II & Gambia... & $\cdots$ & Earl of Derby, British Museum. \\
\hline I $8 \frac{1}{2}$ & $8 \frac{3}{8}$ & $5 \frac{x}{4}$ & East Africa & ... & J. Carr-Saunders. \\
\hline $18 \frac{1}{8}$ & 63 & $5 \frac{7}{8}$ & Do. & $\cdots$ & Sir Robert Harvey, Bart. \\
\hline I 8 & $8 \frac{1}{8}$ & $5 \frac{3}{8}$ & Do. & $\ldots$ & F. J. Jackson. \\
\hline I $7 \frac{3}{4}$ & $8 \frac{1}{2}$ & 8 & Do. & $\ldots$ & Do. \\
\hline
\end{tabular}




\section{SENEGAL HARTEBEESTE (Bubalis senegalensis)-}

cillinu 'ts.

\begin{tabular}{|c|c|c|c|c|c|}
\hline Length. & $\begin{array}{l}\text { Circum- } \\
\text { ference. }\end{array}$ & Tip to Tip. & Habitat. & & Owner. \\
\hline $17 \frac{1}{8}$ & $7 \stackrel{3}{1}$ & 5 & Last $\Delta$ frica & $\ldots$ & II. C. V. IIunter. \\
\hline I 7 & S & 7 & Do. & $\cdots$ & Sir Robert Harvey, Bart. \\
\hline $16 \frac{7}{8}$ & 7 & $5 \frac{\mathrm{I}}{2}$ & Do. & $\cdots$ & F. J. Jackson. \\
\hline $16 \frac{5}{8}$ & $5^{\frac{3}{4}}$ & $5 \frac{3}{8}$ & Do. & $\cdots$ & Do. \\
\hline $16 \frac{3}{8}$ & $7 \frac{\pi}{8}$ & 6 & Do. & ... & H. C. V. Hunter. \\
\hline $16 \frac{3}{8}$ & $6 \frac{1}{8}$ & $5 \frac{1}{2}$ & $\ldots \quad \ldots$ & $\cdots$ & Sir John Kirk, M.D., K.C.B. \\
\hline $16 \frac{1}{t}$ & $7 \frac{5}{8}$ & $8 \frac{1}{4}$ & East Africa & $\cdots$ & Sir Robert Harvey, Bart. \\
\hline $16 \frac{1}{4}$ & $7 \frac{1}{2}$ & $4 \frac{1}{4}$ & Do. & $\cdots$ & Do. \\
\hline $15 \frac{3}{4}$ & $5 \frac{5}{8}$ & $4 \frac{1}{8}$ & Do. & ... & F. J. Jackson. \\
\hline I $5 \frac{5}{8}$ & $7 \frac{5}{8}$ & $5 \frac{3}{4}$ & Do. & $\cdots$ & Hon. Walter Rothschild. \\
\hline $15 \frac{5}{8}$ & $7 \frac{1}{4}$ & $5 \frac{3}{4}$ & Do. & $\cdots$ & F. J. Jackson. \\
\hline $15 \frac{1}{2}$ & $8_{\frac{1}{2}}^{1}$ & 7 & Do. & $\cdots$ & E. Gedge. \\
\hline $14 \frac{3}{4}$ & $7^{\frac{1}{2}}$ & $6 \frac{1}{4}$ & Do. & $\ldots$ & Sir Robert Harvey, Bart. \\
\hline $14 \frac{1}{2}$ & $7 \frac{3}{4}$ & 6 & Do. & $\ldots$ & Do. \\
\hline $13 \frac{3}{8}$ & $8 \frac{1}{8}$ & $5^{\frac{1}{2}}$ & Do. & $\ldots$ & Do. \\
\hline
\end{tabular}

BUBALIS KOBA.

Habitat-East and West Central Africa.

\begin{tabular}{|c|c|c|c|c|}
\hline Length. & $\begin{array}{l}\text { Circum- } \\
\text { ference. }\end{array}$ & Tip to Tip. & Habitat. & Owner. \\
\hline $21 \frac{3}{8}$ & $9 \frac{1}{8}$ & II $1 \frac{3}{4}$ & River Gambia & Dr. P. Rendall, British Museum. \\
\hline 운 $20 \frac{1}{4}$ & $6 \frac{3}{4}$ & $6 \frac{7}{8}$ & Bahr-el-gazal & Consul Petherick, British Museum. \\
\hline 의 $6 \frac{3}{8}$ & $5 \frac{7}{8}$ & $5 \frac{5}{8}$ & River Juba, South & Sir John Kirk, M.D., K.C. B., British \\
\hline $10 \frac{1}{8}$ & $6 \frac{38}{4}$ & $7 \frac{7}{8}$ & Bahr-el-gazal ... & Consul Petherick, British Museum. \\
\hline
\end{tabular}




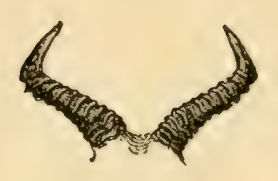

\section{TSESSEBE ANTELOPE or BASTARD HARTEBEESTE (Bubalis lunatus).}

Habitat-South Africa. Open country in territory adjacent to Limpopo River.

\begin{tabular}{|c|c|c|c|c|c|}
\hline Length. & $\begin{array}{l}\text { Circum- } \\
\text { ference. }\end{array}$ & Tip to Tip. & Habitat. & & Owner. \\
\hline $15 \frac{5}{8}$ & $7 \frac{1}{2}$ & II $\frac{3}{4}$ & $\cdots$ & ... & Sir Edmund G. Loder, Bart. \\
\hline I $5 \frac{1}{4}$ & $8 \frac{1}{8}$ & $12 \frac{1}{2}$ & ... & $\cdots$ & F. C. Selous. \\
\hline $15 \frac{1}{4}$ & $8 \frac{1}{8}$ & $10 \frac{1}{2}$ & South Africa & $\ldots$ & W. C. Oswell. \\
\hline $15 \frac{1}{4}$ & 8 & $12 \frac{1}{4}$ & Do. & ... & Sir A. Smith, British Museum. \\
\hline $15 \frac{1}{8}$ & $7 \frac{1}{8}$ & 12 & ... & ... & Sir Victor Brooke's Collection. \\
\hline $14 \frac{x}{2}$ & $7 \frac{7}{8}$ & II $\frac{1}{4}$ & ... & $\cdots$ & F. C. Selous. \\
\hline $14 \frac{1}{2}$ & $7 \frac{5}{8}$ & II & Mashuna Land & $\ldots$ & F. C. Selous, British Museum. \\
\hline I $4 \frac{1}{2}$ & $7 \frac{1}{2}$ & I $3 \frac{5}{8}$ & Central Africa & ... & British Museum. \\
\hline $14 \frac{1}{4}$ & $7 \frac{1}{2}$ & $10 \frac{1}{4}$ & $\cdots$ & $\cdots$ & Hon, Walter Rothschild. \\
\hline $14 \frac{1}{t}$ & $6 \frac{3}{8}$ & II $\frac{1}{4}$ & ... & ... & W. C. Oswell. \\
\hline $14 \frac{1}{8}$ & $6 \frac{1}{8}$ & $9 \frac{7}{8}$ & $\cdots$ & $\cdots$ & F. C. Selous, \\
\hline I4 & 7 & $9 \frac{5}{8}$ & ... & $\cdots$ & Durban Museum. \\
\hline 14 & $6 \frac{3}{4}$ & $12 \frac{7}{8}$ & South Africa & $\cdots$ & A. Beit. \\
\hline $13 \frac{7}{8}$ & $7 \frac{1}{2}$ & $13 \frac{3}{8}$ & Do. & .. & British Museum. \\
\hline 오 $13 \frac{7}{8}$ & $6 \frac{5}{8}$ & I I $\frac{1}{2}$ & Mashuna Land & $\cdots$ & F. C. Selous, British Museum. \\
\hline $13 \frac{7}{8}$ & $6 \frac{1}{4}$ & $12 \frac{3}{8}$ & South Africa & $\cdots$ & H. and C. Beddington. \\
\hline
\end{tabular}


TSESSEBE ANTELOPE OR BASTARD HARTEBEESTE. 67

TSESSEBE ANTELOPE ON BASTARD HARTEBEESTE

(Bubalis Iunatus)-continuci?

\begin{tabular}{|c|c|c|c|c|c|}
\hline Length. & $\begin{array}{l}\text { Circum- } \\
\text { ference. }\end{array}$ & Tip to Tip. & Habitat. & & Owner. \\
\hline $13 \frac{3}{x}$ & $6 \frac{1}{2}$ & $10 \frac{1}{8}$ & South Africa & ... & Hon. Walter Rothschild. \\
\hline $13 \frac{5}{8}$ & $6 \frac{3}{8}$ & $13 \frac{1}{8}$ & Do. & $\ldots$ & The late J. S. Jameson. \\
\hline $13 \frac{1}{2}$ & $7 \frac{3}{8}$ & $10 \frac{7}{8}$ & Do. & $\cdots$ & A. Beit. \\
\hline $13 \frac{1}{2}$ & 6 & $9 \frac{1}{4}$ & $\cdots$ & $\cdots$ & J. A. Nicoll. \\
\hline $13 \frac{\pi}{4}$ & $6 \frac{1}{8}$ & $13^{\frac{1}{4}}$ & South Africa & ... & Sir A. Smith, British Museum. \\
\hline $13 \frac{1}{8}$ & $7 \frac{3}{8}$ & $12 \frac{1}{t}$ & Do. & $\ldots$ & H. and C. Beddington. \\
\hline 13 & $7 \frac{1}{8}$ & $13 \frac{1}{4}$ & Do. & ... & The late J. S. Jameson. \\
\hline $12 \frac{7}{8}$ & $7 \frac{1}{2}$ & $12 \frac{5}{8}$ & Do. & ... & WV. P. Rylands. \\
\hline $12 \frac{3}{4}$ & $5^{\frac{3}{4}}$ & 12 & Do. & $\ldots$ & A. Beit. \\
\hline $12 \frac{1}{2}$ & $7 \frac{1}{2}$ & 12 & Do. & $\cdots$ & Rowland Ward's Collection. \\
\hline 12 & 6 & $12 \frac{7}{8}$ & Do. & $\ldots$ & H. and C. Beddington. \\
\hline
\end{tabular}




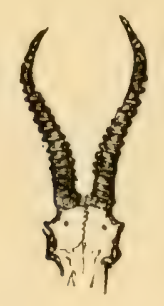

\section{BLESSBOK (Bubalis albifrons).}

\section{Habitat-South Africa.}

\begin{tabular}{|c|c|c|c|c|c|}
\hline Length. & $\begin{array}{l}\text { Circum- } \\
\text { ference. }\end{array}$ & Tip to Tip. & Habitat. & & Owner. \\
\hline $1 S \frac{1}{4}$ & $5 \frac{3}{7}$ & $12 \frac{1}{4}$ & South Africa & $\ldots$ & Sir Edmund G. Loder, Bart. \\
\hline $16 \frac{1}{8}$ & $6 \frac{1}{4}$ & $8 \frac{1}{8}$ & Do. & $\ldots$ & Do. \\
\hline $15 \frac{1}{4}$ & $6 \frac{1}{8}$ & $6 \frac{1}{2}$ & Do. & $\ldots$ & Maj.-Gen. Arthur Ellis, C.S.I. \\
\hline $15 \frac{1}{8}$ & $6 \frac{1}{2}$ & $7^{\frac{3}{4}}$ & Do. & $\ldots$ & Hon, Walter Rothschild. \\
\hline $14 \frac{2}{1}$ & $6 \frac{1}{8}$ & $8 \frac{3}{4}$ & $\ldots$ & $\ldots$ & H.R.H. Duke of Edinburgh. \\
\hline 14 & $6 \frac{3}{8}$ & $7 \frac{1}{2}$ & ... & $\ldots$ & Sir Victor Brooke's Collection. \\
\hline 14 & $5 \frac{1}{8}$ & $7 \frac{1}{8}$ & $\cdots$ & $\ldots \mid$ & Rowland Ward's Collection. \\
\hline $13 \frac{7}{3}$ & $6 \frac{1}{8}$ & $7 \frac{3}{8}$ & South Africa & $\ldots \mid$ & British Museum. \\
\hline $13 \frac{3}{4}$ & $5^{\frac{3}{4}}$ & $6 \frac{1}{8}$ & $\ldots$ & $\ldots$ & W. C. Oswell. \\
\hline $13 \frac{1}{2}$ & 5 & $5^{\frac{3}{4}}$ & $\ldots$ & $\cdots$ & F. C. Selous. \\
\hline $13 \frac{3}{2}$ & 5 & $9 \frac{3}{8}$ & ... & $\ldots \mid$ & H.R.H. Duke of Edinburgh. \\
\hline $12 \frac{3}{4}$ & $5 \frac{3}{8}$ & $4 \frac{\pi}{8}$ & ... & $\ldots \mid$ & Maj.-Gen. Arthur Ellis, C.S.I. \\
\hline $12 \frac{5}{8}$ & $4 \frac{3}{4}$ & 7 & South Africa & $\ldots$ & British Museum. \\
\hline $12 \frac{3}{8}$ & 6 & $9 \frac{3}{4}$ & ... & $\ldots$ & F. J. Horniman. \\
\hline
\end{tabular}


BONTEBOK (Bubalis pygargus).

Habitat-South Africa. The horns closely resemble Blessbok.

\begin{tabular}{|c|c|c|c|c|c|}
\hline Length. & $\begin{array}{l}\text { Circum- } \\
\text { ference. }\end{array}$ & Tip to Tip. & Habitat. & & Owner. \\
\hline $16 \frac{3}{8}$ & $6 \frac{3}{4}$ & $9 \frac{1}{8}$ & ... & $\cdots$ & British Museum. \\
\hline 16 & $6 \frac{1}{4}$ & $6 \frac{3}{4}$ & ... & ... & Rowland Ward's Collecticn. \\
\hline $15^{\frac{3}{4}}$ & 6 & $3 \frac{3}{t}$ & $\cdots$ & ... & Do. \\
\hline $15 \frac{3}{8}$ & $6 \frac{1}{8}$ & $8 \frac{1}{8}$ & Cape Colony & $\ldots$ & Sir Victor Brooke's Collection. \\
\hline $15 \frac{3}{8}$ & $6 \frac{1}{4}$ & $8 \frac{1}{2}$ & ... & $\cdots$ & British Museum. \\
\hline 15 & $5 \frac{1}{4}$ & $4 \frac{1}{2}$ & $\cdots$ & $\ldots$ & Do. \\
\hline 15 & $6 \frac{3}{8}$ & $7 \frac{5}{8}$ & Cape Colony & $\cdots$ & Capt. Freville-Cookson. \\
\hline 15 & $6 \frac{1}{8}$ & $6 \frac{7}{8}$ & South Africa & $\ldots$ & British Museum. \\
\hline $14 \frac{1}{2}$ & $6 \frac{1}{4}$ & $8 \frac{1}{4}$ & De. & $\ldots$ & Hon. Walter Rothschi'd. \\
\hline $14 \frac{1}{2}$ & $6 \frac{5}{8}$ & $7 \frac{1}{4}$ & Do. & $\cdots$ & British Museum. \\
\hline $14 \frac{1}{2}$ & $4 \frac{1}{2}$ & $7 \frac{3}{4}$ & Do. & ... & Do. \\
\hline 14 & $6 \frac{3}{8}$ & $8 \frac{1}{8}$ & $\ldots$ & $\ldots$ & J. Carr-Saunders. \\
\hline 14 & $6 \frac{1}{4}$ & $7 \frac{1}{2}$ & Cape Colony & ... & Sir Victor Brooke's Collection. \\
\hline $13 \frac{3}{4}$ & 5 & $6 \frac{1}{2}$ & South Africa & $\ldots$ & A. W. Davis. \\
\hline $12 \frac{3}{8}$ & $5 \frac{7}{8}$ & $5 \frac{7}{8}$ & Do. & ... & British Museum. \\
\hline $12 \frac{1}{8}$ & $4 \frac{3}{8}$ & $6 \frac{3}{3}$ & Do. & ... & Do. \\
\hline
\end{tabular}




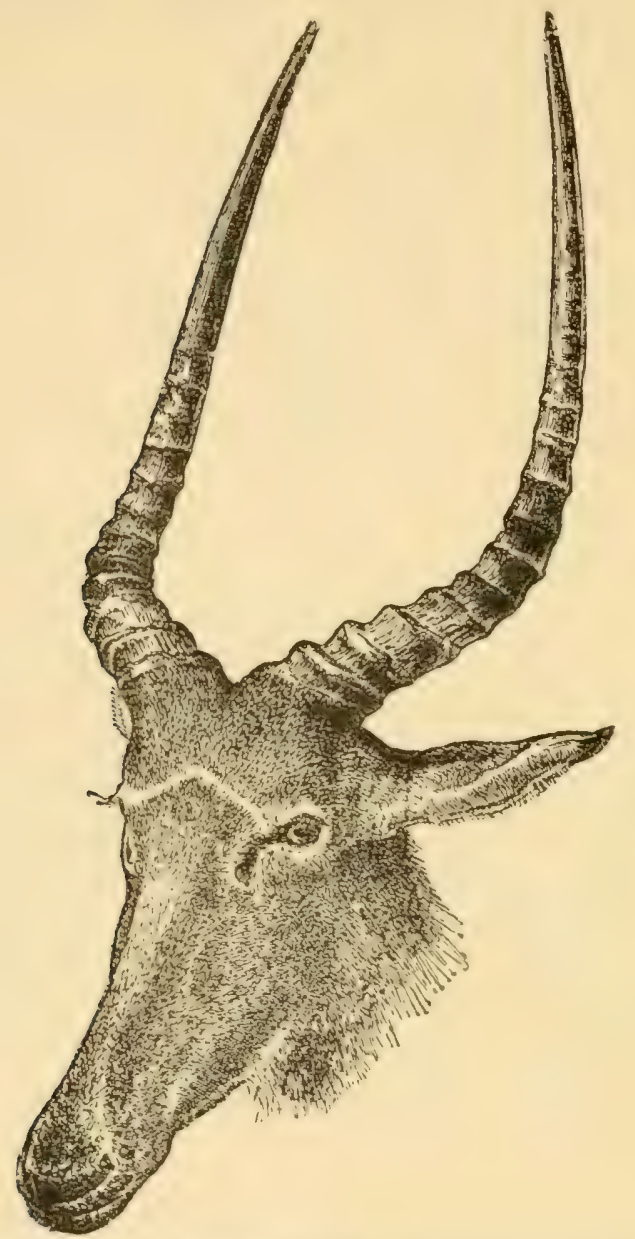

HUNTER'S ANTELOPE (Bubalis hunteri).

Habitat-North banks of the Tana River, East Africa.

\begin{tabular}{|c|c|c|c|c|c|c|}
\hline \multicolumn{2}{|c|}{ Length. } & \multirow{2}{*}{$\begin{array}{l}\text { Circum- } \\
\text { ference. }\end{array}$} & \multirow{2}{*}{$\begin{array}{l}\text { Tip to } \\
\text { I I p. }\end{array}$} & \multirow{2}{*}{ Habitat. } & & \multirow{2}{*}{ Owner. } \\
\hline $\begin{array}{l}\text { Round } \\
\text { bend. }\end{array}$ & Straight. & & & & & \\
\hline $26 \frac{1}{\ddagger}$ & 22 & $S_{\frac{1}{8}}$ & $12 \frac{1}{2}$ & East Africa & $\cdots$ & Sir Robert Harvey, Bart. \\
\hline $24 \frac{7}{8}$ & $\cdots$ & $\delta \frac{1}{8}$ & $15 \frac{1}{2}$ & Do. & $\cdots$ & II. C. V. Hunter. \\
\hline $24 \frac{5}{8}$ & $2 I \frac{1}{4}$ & $8 \%$ & $9 \frac{3}{4}$ & Do. & $\ldots$ & Sir Robert Harvey, Bart. \\
\hline
\end{tabular}


HUNTER'S ANTELOPE (Bubalis hunteri)-continuc.

\begin{tabular}{|c|c|c|c|c|c|c|}
\hline \multicolumn{2}{|c|}{ Length. } & \multirow{2}{*}{$\begin{array}{l}\text { Circum- } \\
\text { ference. }\end{array}$} & \multirow{2}{*}{$\begin{array}{l}\text { Tip to } \\
\text { Tip. }\end{array}$} & \multirow{2}{*}{\multicolumn{2}{|c|}{ Habitat. }} & \multirow{2}{*}{ Owner. } \\
\hline $\begin{array}{l}\text { Round } \\
\text { bend. }\end{array}$ & Straight. & & & & & \\
\hline $24 \frac{3}{8}$ & $\cdots$ & $7 \frac{7}{8}$ & $13 \frac{3}{8}$ & East Africa & $\ldots$ & H. C. V. Hunter. \\
\hline $24 \frac{1}{8}$ & $20 \frac{1}{4}$ & $8 \frac{1}{8}$ & $11 \frac{3}{4}$ & Do. & $\ldots$ & Sir Robert Harvey, Bart. \\
\hline 24 & $20 \frac{1}{2}$ & 8 & $\cdots$ & Do. & $\ldots$ & H. C. V. Hunter. \\
\hline $22 \frac{7}{8}$ & ... & $7 \frac{1}{4}$ & $7 \frac{5}{8}$ & Tana River & $\ldots$ & British Museum. \\
\hline 22 & ... & $\cdots$ & I 6 & East Africa & $\ldots$ & Do. \\
\hline $2 \mathrm{I}$ & $\cdots$ & $5^{\frac{1}{2}}$ & $10 \frac{1}{4}$ & Tana River & $\ldots$ & British Museum. \\
\hline $20 \frac{1}{2}$ & $18 \frac{1}{4}$ & $5 \frac{1}{2}$ & $I I \frac{3}{8}$ & East Africa & $\ldots$ & Sir Robert Harvey, Bart. \\
\hline $20 \frac{1}{8}$ & $\cdots$ & $5 \frac{5}{8}$ & $10 \frac{5}{8}$ & Do. & $\ldots$ & H. C. V. Hunter. \\
\hline $20 \frac{1}{8}$ & $\cdots$ & $5 \frac{5}{8}$ & 87 & Do. & $\cdots$ & Hon. Walter Rothschild. \\
\hline $19 \frac{5}{8}$ & $17 \frac{5}{8}$ & $5 \frac{3}{8}$ & I I $\frac{3}{4}$ & Do. & $\cdots$ & Sir Robert Harvey, Bart. \\
\hline 19 & 17 & $5^{\frac{3}{4}}$ & $10 \frac{1}{4}$ & Do. & $\ldots$ & H. C. V. Hunter. \\
\hline
\end{tabular}




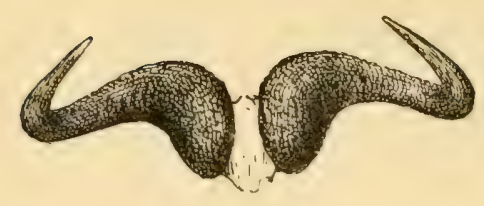

\section{BRINDLED GNU (Connochœtes taurina).}

\section{Habitat-South Central Africa.}

The specimens shot in East Africa may be the sub-species figured on page 75 .

\begin{tabular}{|c|c|c|c|c|c|c|}
\hline Length. & Breadth of Palm. & $\begin{array}{l}\text { Tip to } \\
\text { Tip. }\end{array}$ & $\begin{array}{l}\text { Widest } \\
\text { Inside. }\end{array}$ & Hatitat. & & Owner. \\
\hline $26 \frac{1}{8}$ & 8 & $14 \frac{3}{4}$ & $16 \frac{1}{2}$ & $\cdots$ & $\ldots$ & Sir Edmund G. Loder, Bart. \\
\hline 23 & I I $\frac{1}{2}$ & $19 \frac{1}{2}$ & $3 \mathbf{I}$ & South Africa & $\cdots$ & J. A. Nicoll. \\
\hline 23 & $9 \frac{7}{8}$ & $14 \frac{7}{8}$ & ... & $\ldots \quad \ldots$ & $\ldots$ & H.R.H. Duke of Edinburgh. \\
\hline $22 \frac{7}{8}$ & ... & $16 \frac{1}{2}$ & $23 \frac{3}{8}$ & East Africa & $\cdots$ & F. J. Jackson. \\
\hline $22 \frac{5}{8}$ & $\cdots$ & $18 \frac{3}{4}$ & $26 \frac{1}{4}$ & Do. & ... & H. C. V. Munter. \\
\hline $22 \frac{1}{4}$ & 6 & $18 \frac{1}{8}$ & 26 & South Africa & ... & F. C. Selous. \\
\hline $22 \frac{1}{8}$ & $\cdots$ & $17 \frac{3}{4}$ & $25 \frac{5}{8}$ & East Africa & $\cdots$ & F. J. Jackson. \\
\hline 22 & $5^{\frac{3}{4}}$ & $15 \frac{3}{8}$ & $23 \frac{1}{8}$ & $\ldots \quad \ldots$ & $\cdots$ & The late J. S. Jameson. \\
\hline $2 \mathrm{I} \frac{5}{8}$ & $\begin{array}{c}13 \\
\text { (circun.ference) }\end{array}$ & $23 \frac{3}{4}$ & ... & East Africa & $\ldots$ & F. J. Jackson. \\
\hline $21 \frac{5}{8}$ & $5^{\frac{1}{4}}$ & $17 \frac{3}{8}$ & $28 \frac{1}{2}$ & South Africa & $\cdots$ & G. H. Banks. \\
\hline $2 \mathrm{C} \frac{5}{8}$ & $\cdots$ & $15 \frac{1}{8}$ & 24 & East Africa & $\cdots$ & Sir Robert Harvey, Bart. \\
\hline $20 ?$ & $\begin{array}{c}12 \frac{1}{3} \\
\text { (circuinference) }\end{array}$ & $12 \frac{1}{2}$ & 25 & $\cdots \quad \cdots$ & $\ldots$ & Rowland Ward's Collection. \\
\hline $2 \mathrm{C} \frac{1}{4}$ & $\cdots$ & $12 \frac{2}{4}$ & $2 \mathrm{I} \mathrm{I}^{\frac{2}{4}}$ & Mashunaland & ... & F. C. Selous. \\
\hline 20 & (circumference) & 17 & $\begin{array}{c}27 \\
\text { (outside) }\end{array}$ & East Africa & $\ldots$ & R. P. Carrol. \\
\hline $19 \frac{7}{8}$ & $\cdots$ & $12 \frac{3}{4}$ & $20 \frac{7}{8}$ & Do. & $\cdots$ & Sir Robert Harvey, Bart. \\
\hline $19 \frac{3}{4}$ & $\ldots$ & I 8 & $23 \frac{3}{8}$ & Do. & $\cdots$ & Do. \\
\hline
\end{tabular}


BRINDLED GNU (Connochœtes taurina)-continued.

\begin{tabular}{|c|c|c|c|c|c|c|}
\hline Length. . & $\begin{array}{l}\text { Brendth of } \\
\text { Palm. }\end{array}$ & $\begin{array}{l}\text { Tip to } \\
\text { Iip. }\end{array}$ & $\begin{array}{l}\text { Widest } \\
\text { Inside. }\end{array}$ & Habitat. & & Ownex. \\
\hline $19 \frac{3}{4}$ & 5 & I7 & $23 \frac{3}{4}$ & South Africa & $\cdots$ & R. C. Dobson. \\
\hline $19 \frac{5}{8}$ & $5 \frac{33}{8}$ & $12 \frac{1}{2}$ & $2 I \frac{1}{4}$ & $\cdots$ & $\cdots$ & Hon. Walter Rothschild. \\
\hline I $9 \frac{1}{2}$ & 5 & 15 & $22 \frac{1}{2}$ & $\cdots$ & $\ldots$ & W. C. Oswell. \\
\hline $19 \frac{1}{2}$ & $\cdots$ & 20 & $\cdots$ & East Africa & $\cdots \mid$ & H. C. V. Hunter. \\
\hline I $9 \frac{1}{2}$ & $\begin{array}{c}14 \frac{1}{2} \\
\text { (circumference) }\end{array}$ & $16 \frac{1}{4}$ & 22 & Do. & $\ldots \mid$ & F. J. Jackson. \\
\hline $19 \frac{1}{2}$ & $\cdots$ & I3 $3 \frac{5}{8}$ & $21 \frac{3}{4}$ & Do. & $\cdots$ & Sir Robert Harvey, Bart. \\
\hline $19 \frac{3}{8}$ & $\cdots$ & $15 \frac{1}{8}$ & 22 & Do. & $\cdots$ & H. C. V. Hunter. \\
\hline $19 \frac{1}{4}$ & $\mid \begin{array}{c}\mathrm{II} \frac{1}{8} \\
\text { (circumferenc } 3 \text { ) }\end{array}$ & I $7 \frac{1}{8}$ & $23 \frac{5}{8}$ & South Africa & $\ldots \mid$ & H. Atkinson. \\
\hline $19 \frac{\frac{1}{4}}{4}$ & 5 & $15 \frac{3}{8}$ & $22 \frac{1}{8}$ & Do. & $\cdots$ & H. and C. Beddington. \\
\hline $19 \frac{1}{8}$ & $5 \frac{1}{2}$ & $15 \frac{1}{2}$ & $22 \frac{1}{2}$ & Do. & $\cdots$ & F. C. Selous. \\
\hline I9 & $5 \frac{1}{2}$ & $12 \frac{3}{8}$ & $21 \frac{1}{2}$ & $\cdots \quad \cdots$ & $\cdots$ & J. Carr Saunders. \\
\hline 19 & $5 \frac{1}{2}$ & $11 \frac{1}{2}$ & $13 \frac{5}{8}$ & $\cdots \quad \ldots$ & $\cdots$ & Sir Edmund G. Loder, Bart. \\
\hline I $S \frac{1}{4}$ & $4 \frac{3}{8}$ & $12 \frac{7}{8}$ & $19 \frac{7}{8}$ & $\cdots \quad \cdots$ & $\cdots$ & The late J. S. Jameson. \\
\hline I8 & $\cdots$ & 16 & $\cdots$ & South Afi ica & $\cdots$ & British Museum. \\
\hline $17 \frac{1}{4}$ & $\cdots$ & II $\frac{1}{8}$ & $20 \frac{1}{4}$ & East Africa & $\cdots$ & F. J. Jackson. \\
\hline 17 & $4 \frac{3}{4}$ & $17 \frac{1}{2}$ & $21 \frac{5}{8}$ & $\cdots \quad \ldots$ & $\cdots$ & Sir Victor Brooke's Collection. \\
\hline 17 & ... & 15 & $\begin{array}{c}24 \\
\text { (outside) }\end{array}$ & East Afica & $\ldots$ & W. Astor Chanler. \\
\hline$\cdots$ & $\cdots$ & I6 & $\begin{array}{c}28 \\
\text { (outside) }\end{array}$ & $\cdots \quad \cdots$ & $\cdots$ & Sir J. Willoughby, Bart., "East \\
\hline . & 1 & $12 \overline{\bar{y}}$ & 22 & 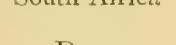 & $\cdots$ & A. W. Daslse \\
\hline $15 \frac{1}{8}$ & $3 \frac{5}{8}$ & $10 \frac{3}{8}$ & I $8 \frac{3}{8}$ & Do. & $\cdots$ & A. Beit. \\
\hline $15 \frac{1}{2}$ & $\cdots$ & $3 \frac{3}{4}$ & $10 \frac{1}{8}$ & Do. & $\cdots$ & British Museum. \\
\hline I 5 & $\cdots$ & I $3 \frac{1}{8}$ & I $8 \frac{1}{2}$ & East Africa & $\ldots$ & Sir Robert Harvey, Bart. \\
\hline 15 & $8 ?$ & $12 \frac{1}{1}$ & $17 \frac{7}{8}$ & South Africa & $\ldots$ & H. and C. Beddington. \\
\hline 147 & 4 & $14 \frac{3}{1}$ & I $8 \frac{7}{8}$ & Do. & $\cdots$ & A. Beit. \\
\hline $14 \frac{1}{2}$ & $\cdots$ & $11 \frac{7}{8}$ & $17 \frac{1}{2}$ & East Africa & $\cdots$ & Sir Robert Harvey, Bart. \\
\hline $13 \frac{3}{4}$ & 83 & $9 \frac{7}{8}$ & $16 \frac{1}{4}$ & Zanzibar & $\cdots$ & Sir John Kirk, M.D., K.C. B. \\
\hline $12 \frac{5}{8}$ & $\cdots$ & $11 \frac{5}{8}$ & $\cdots$ & South Africa & $\ldots$ & British Museum. \\
\hline
\end{tabular}


WHITE-TAILED GNU (Connochœtes gnu).

Habitat-South Africa.

\begin{tabular}{|c|c|c|c|c|c|c|}
\hline Length. & Breadth of Palm. & $\begin{array}{l}\text { Tip to } \\
\text { Tip. }\end{array}$ & $\begin{array}{l}\text { Widest } \\
\text { Inside. }\end{array}$ & Habitat. & & Owner. \\
\hline $22 \frac{3}{8}$ & $8 \frac{1}{2}$ & $15 \frac{1}{4}$ & ... & $\cdots$ & $\ldots$ & F. C. Selous. \\
\hline 22 & 9 & $17 \frac{1}{8}$ & $\cdots$ & $\cdots$ & $\ldots$ & Hon. Walter Rothschild. \\
\hline $21 \frac{3}{4}$ & $10 \frac{5}{8}$ & I $8 \frac{5}{8}$ & $\cdots$ & South Africa & $\ldots \mid$ & British Museum. \\
\hline $21 \frac{3}{8}$ & 8 & $14 \frac{3}{8}$ & $\cdots$ & $\cdots$ & $\ldots$ & F. C. Selous. \\
\hline $20 \frac{3}{8}$ & $\cdots$ & $15 \frac{1}{2}$ & $23 \frac{1}{8}$ & $\ldots$ & $\ldots$ & Bethnal Green Museum. - \\
\hline $16 \frac{3}{4}$ & $10 \frac{1}{2}$ & $12 \frac{5}{8}$ & $17 \frac{3}{4}$ & South Africa & $\ldots$ & British Museum. \\
\hline $15 \frac{7}{8}$ & $3 \frac{3}{4}$ & $13 \frac{1}{8}$ & $19 \frac{3}{8}$ & $\cdots$ & $\cdots$ & Sir Edmund G. Loder, Bart. \\
\hline$*_{14} 4 \frac{5}{8}$ & $\begin{array}{c}\frac{73}{8} \\
\text { (circumference) }\end{array}$ & $\delta_{\frac{1}{2}}$ & $16 \frac{5}{8}$ & Ugarano & $\ldots 1$ & Capt. Speke, British Museum. \\
\hline
\end{tabular}



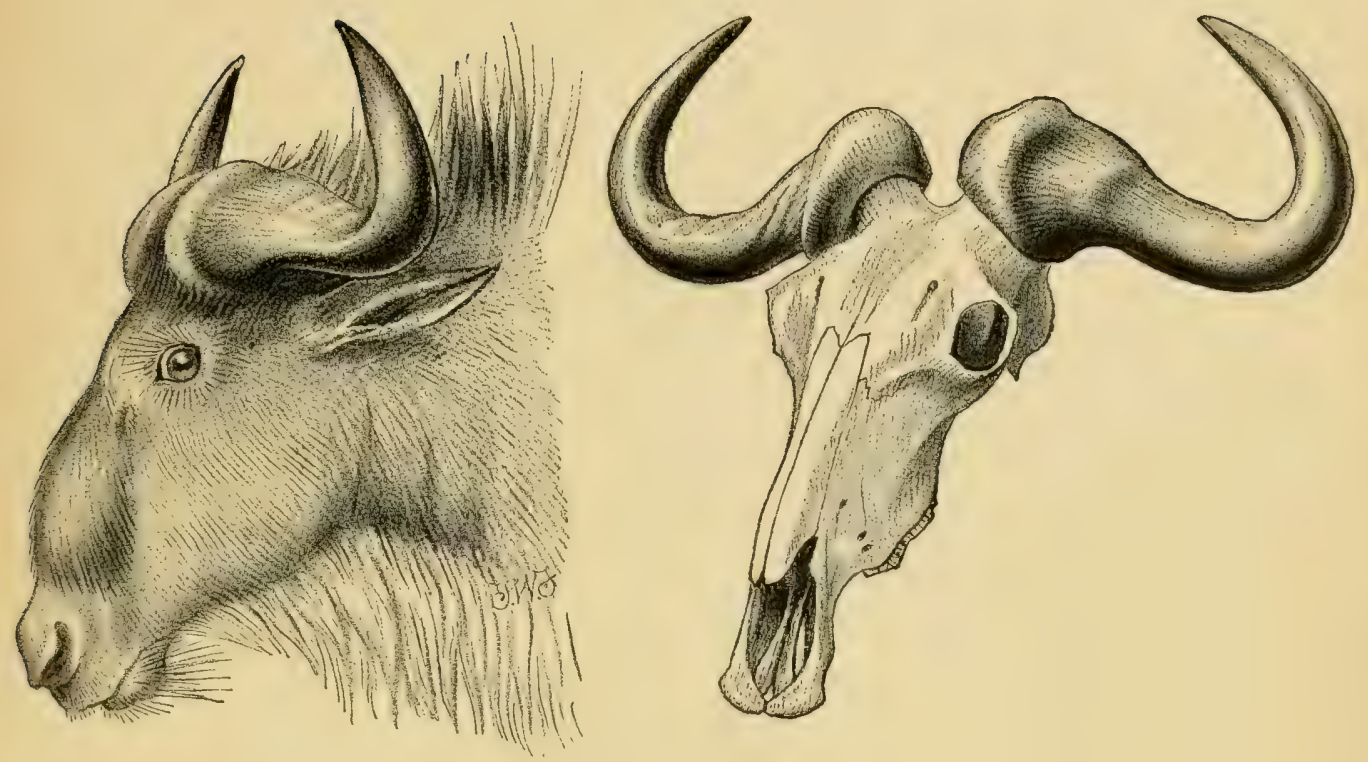

JACKSON'S WILDEBEESTE (Connochœtes taurinus albojubatus).

Habitat-East Africa, Uganda.

Closely allied to the Brindled Wildebeeste, excepting that it has a white mane under the throat instead of black.

\begin{tabular}{r|r|r|r|r|r|r}
\hline Length. & Breadth. & $\begin{array}{c}\text { Tip to } \\
\text { Tip. }\end{array}$ & $\begin{array}{c}\text { Widest } \\
\text { Inside. }\end{array}$ & Habitat. & Owner. \\
\hline $20 \frac{1}{8}$ & $5 \frac{1}{8}$ & $16 \frac{3}{8}$ & $22 \frac{5}{8}$ & East Africa & F. J. Jackson. \\
$19 \frac{1}{8}$ & $5 \frac{3}{4}$ & $16 \frac{1}{4}$ & $21 \frac{7}{8}$ & Do. & $\ldots$ & Do.
\end{tabular}




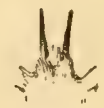

\section{DUIKER ANTELOPE (Cephalophus grimini).}

\section{Habitat-South and East Africa.}

\begin{tabular}{|c|c|c|c|c|c|}
\hline Length. & $\begin{array}{l}\text { Circum- } \\
\text { ference. }\end{array}$ & Tip to Tip. & Habitat. & & Owner. \\
\hline $5^{\frac{3}{x}}$ & $2 \frac{1}{8}$ & $2 \frac{7}{8}$ & South Africa & $\ldots$ & F. C. Selous. \\
\hline 5 & $2 \frac{1}{4}$ & $2 \frac{1}{2}$ & Do. & $\ldots$ & Do. \\
\hline $4 \frac{7}{8}$ & $2 \frac{1}{2}$ & $2 \frac{1}{4}$ & Do. & $\ldots$ & Do. \\
\hline $4 \frac{3}{4}$ & $2 \frac{1}{4}$ & 3 & Do. & $\ldots$ & A. Beit. \\
\hline $4 \frac{1}{2}$ & $\ldots$ & $2 \frac{7}{8}$ & Algoa Bay & $\ldots$ & F. C. Selous, British Museum?, \\
\hline $4 \frac{1}{2}$ & $2 \frac{1}{4}$ & $1 \frac{3}{4}$ & South Africa & $\ldots$ & G. H. Banks. \\
\hline $4 \frac{1}{4}$ & I $\frac{7}{8}$ & $2 \frac{3}{4}$ & $\cdots \quad \cdots$ & $\cdots$ & Sir Robert Harvey, Bart. \\
\hline $4 \frac{1}{8}$ & $2 \frac{1}{8}$ & $I \frac{5}{8}$ & $\ldots \quad \ldots$ & $\ldots$ & J. Carr-Saunders. \\
\hline $4 \frac{1}{8}$ & $1 \frac{7}{8}$ & $2 \frac{5}{8}$ & Natal $\quad \ldots$ & $\ldots$ & J. Wahlberg, British Museum. \\
\hline $4 \frac{1}{8}$ & 2 & 2 & Transvaal & $\cdots$ & F. C. Selous, British Museum. \\
\hline 4 & $1 \frac{7}{8}$ & $I \frac{1}{2}$ & South Africa & $\cdots$ & H. and C. Beddington. \\
\hline 4 & $1 \frac{7}{8}$ & $1 \frac{1}{2}$ & Do. & $\cdots$ & Do. \\
\hline $3 \frac{7}{8}$ & $2 \frac{1}{8}$ & 2 & $\cdots \quad \cdots$ & $\cdots$ & Sir Robert Harvey, Bart. \\
\hline $3 \frac{7}{8}$ & $1 \frac{3}{4}$ & $I \frac{3}{4}$ & East Africa & $\cdots$ & F. J. Jackson, \\
\hline $3^{\frac{3}{4}}$ & 2 & $2 \frac{1}{8}$ & South Africa & $\ldots$ & H. and C. Beddington. \\
\hline $3 \frac{3}{4}$ & $\cdots$ & $\mathbf{I} \frac{3}{1}$ & $\cdots \quad \cdots$ & $\cdots$ & The late J. S. Jameson. \\
\hline $3 \frac{1}{4}$ & $1 \frac{3}{4}$ & $2 \frac{1}{x}$ & East Africa & $\cdots$ & F. J. Jackson. \\
\hline $3 \frac{1}{1}$ & $\cdots$ & $1 \cdot \frac{1}{4}$ & South Africa & $\ldots \mid$ & The late J. S. Jameson. \\
\hline$* 3 \frac{1}{2}$ & $1 \frac{7}{8}$ & $I \frac{3}{4}$ & Abyscinia & $\cdots$ & Sir Victor Brooke's Collection. \\
\hline $2 \frac{3}{4}$ & I $\frac{7}{8}$ & $1 \frac{1}{4}$ & Do. & $\cdots$ & British Museum. \\
\hline
\end{tabular}




\section{NATAL BUSHBUCK (Cephalophus natalensis).}

[Dr. J. E. Gray, P.Z.S., p. 589.]

HAIR rigid, adpressed, of neck and cheeks short, of forehead long, bright red-bay; beneath pale yellowish; forehead red.

There are four specimens in the British Museum, two males and two females.

$$
\text { Habitat-Port Natal. }
$$

\begin{tabular}{c|c|c|cc|c|c}
\hline Length. & $\begin{array}{c}\text { Circum- } \\
\text { ference. }\end{array}$ & Tip to Tip. & Habitat. & Owner. \\
\hline $3 \frac{1}{8}$ & $\ldots$ & $2 \frac{1}{8}$ & Natal & $\ldots$ & $\ldots$ & Dr. A. Kraus, British Museum. \\
$2 \frac{7}{8}$ & 3 & $2 \frac{1}{4}$ & Zanzibar ... & $\ldots$ & Dr. Kirk, British Museum. \\
\hline
\end{tabular}

WHITE-BACKED BUSHBUCK (Cephalophus sylvicultor).

Habitat-Fantee.

\begin{tabular}{|c|c|c|c|c|c|c|}
\hline Length. & $\begin{array}{l}\text { Circum- } \\
\text { ference. }\end{array}$ & Tip to Tip. & & bitat. & & Owner. \\
\hline $4 \frac{5}{8}$ & ... & $2 \frac{1}{4}$ & Fantee & $\ldots$ & $\ldots$ & British Museum. \\
\hline $6 \frac{3}{4}$ & $\cdots$ & $4 \frac{1}{2}$ & Do. & $\ldots$ & $\cdots$ & Do. \\
\hline $6 \frac{5}{8}$ & $3 \frac{5}{8}$ & $4 \frac{3}{8}$ & Do. & $\ldots$ & $\ldots$ & Do. \\
\hline
\end{tabular}

BANDED BUSHBUCK (Cephalophus doriæ).

Habitat-Liberia.

\begin{tabular}{|c|c|c|c|c|c|}
\hline Length. & $\begin{array}{l}\text { Circum- } \\
\text { ference. }\end{array}$ & Tip to Tip. & Habitat. & & Owner. \\
\hline I $\frac{7}{8}$ & ... & I & Liberia ... & $\ldots$ & Dr. J. Buttikofer, British Museum. \\
\hline
\end{tabular}




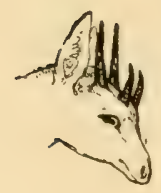

\section{FOUR-HORNED ANTELOPE OP CHOUSINGHA}

(Tetraceros quadricornis).

Horns in male only.

Habitat-India.

\begin{tabular}{|c|c|c|c|c|c|c|c|c|}
\hline \multicolumn{2}{|c|}{ Length. } & \multicolumn{2}{|c|}{ Circumference. } & \multicolumn{2}{|c|}{ Tip to Tip. } & \multicolumn{2}{|c|}{ Habitat. } & \multirow{2}{*}{ Owner. } \\
\hline Rear Horn & Fore Horn. & Rear Horn. & Fore Horn. & | Rear Horn. $\mid$ & Fore Horn. & & & \\
\hline $4 \frac{3}{8}$ & $2 \frac{1}{2}$ & $2 \frac{1}{4}$ & $2 \frac{3}{3}$ & $\mathbf{I} \frac{5}{8}$ & $2 \frac{7}{8}$ & India & $\ldots$ & $\begin{array}{l}\text { Sir Edmund G. Loder, } \\
\text { Bart. }\end{array}$ \\
\hline 4 & $2 \frac{1}{2}$ & $1 \frac{7}{8}$ & I $\frac{7}{8}$ & 3 & $1 \frac{1}{8}$ & Indore & $\ldots$ & $\begin{array}{l}\text { Col. John Evans, Bri- } \\
\text { tish Museum. }\end{array}$ \\
\hline $3 \frac{5}{8}$ & I $\frac{3}{4}$ & $1 \frac{7}{8}$ & 2 & I $\frac{1}{8}$ & $2 \frac{1}{2}$ & India & $\ldots$ & $\begin{array}{l}\text { Sir Robert Harvey, } \\
\text { Bart. }\end{array}$ \\
\hline $3 \frac{1}{2}$ & 2 & I $\frac{6}{8}$ & I $\frac{5}{8}$ & $1 \frac{1}{4}$ & $2 \frac{1}{2}$ & Do. & $\ldots$ & $\begin{array}{l}\text { H. R.H. Duke of Edin- } \\
\text { burgh. }\end{array}$ \\
\hline $3 \frac{1}{8}$ & $\frac{1}{2}$ & $2 \frac{1}{8}$ & $\mathbf{I} \frac{1}{2}$ & $2 \frac{5}{8}$ & I $\frac{1}{8}$ & Nepal & $\ldots$ & British Museum. \\
\hline $2 \frac{3}{4}$ & 1 & $1 \frac{7}{8}$ & $I_{\frac{1}{2}}$ & I $\frac{7}{8}$ & ... & India & $\ldots$ & Do. \\
\hline 2 & $\frac{1}{2}$ & 3 & ... & 3 & I $\frac{1}{8}$ & Do. & $\ldots$ & Do. \\
\hline
\end{tabular}




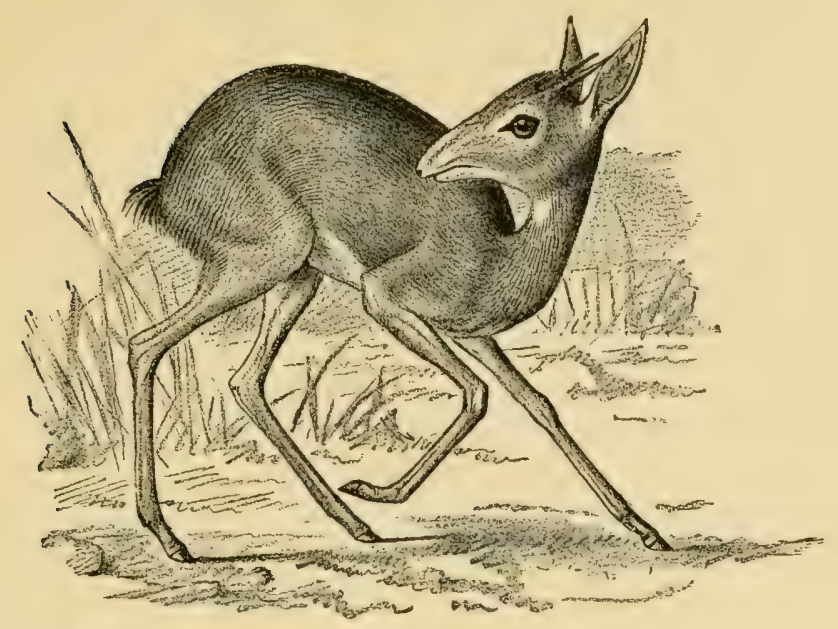

KIRK'S ANTELOPE (Neotragus kirki). Habitat-East Africa.

\begin{tabular}{|c|c|c|c|c|c|}
\hline Length. & $\begin{array}{l}\text { Circum- } \\
\text { ference. }\end{array}$ & Tip to Tip. & \multicolumn{2}{|l|}{ Habitat. } & Owner. \\
\hline 3 & ... & $1 \frac{1}{2}$ & East Africa & $\cdots$ & F. J. Jackson. \\
\hline $2 \frac{3}{4}$ & $\cdots$ & $\mathbf{1} \frac{5}{8}$ & Do. & $\cdots$ & British Museum. \\
\hline $2 \frac{5}{8}$ & ... & $\mathbf{I} \frac{1}{2}$ & Do. & $\ldots$ & Sir Robert Harvey, Bart. \\
\hline $2 \frac{5}{8}$ & I $\frac{1}{2}$ & I $\frac{5}{8}$ & Kilima'njaro & $\cdots$ & H. C. V. Hunter, British Museum. \\
\hline $2 \frac{5}{8}$ & ... & $1 \frac{3}{4}$ & East Africa & $\cdots$ & Sir Robert Harvey, Bart. \\
\hline $2 \frac{1}{2}$ & ... & $1 \frac{7}{8}$ & Do. & ... & F. J. Jackson. \\
\hline $2 \frac{1}{2}$ & $1 \frac{1}{2}$ & $\ldots$ & North-east Afr & & Col. Ralph Vivian. \\
\hline $2 \frac{1}{2}$ & $\cdots$ & I $\frac{5}{8}$ & East Africa & $\ldots$ & Hon. Walter Rothschild, \\
\hline $2 \frac{1}{2}$ & $\cdots$ & $1 \frac{3}{8}$ & Do. & ... & Rowland Ward's Collection. \\
\hline $2 \frac{3}{8}$ & $\cdots$ & $1 \frac{3}{8}$ & Island of Manc & a... & Sir John Kirk, M.D., K.C.B. \\
\hline $2 \frac{3}{8}$ & $\ldots$ & $1 \frac{1}{2}$ & Somali Land & ... & Do. $\quad$ British Museum. \\
\hline $2 \frac{3}{8}$ & $\cdots$ & $1 \frac{1}{2}$ & East Africa & $\cdots$ & Sir Robert Harvey, Bart. \\
\hline $2 \frac{3}{8}$ & $1 \frac{5}{8}$ & I $\frac{3}{8}$ & Do. & $\ldots$ & Sir John Kirk, British Museum. \\
\hline $2 \frac{1}{4}$ & ... & $1 \frac{7}{8}$ & Do. & $\ldots$ & Sir Robert Harvey, Bart. \\
\hline 2 & ... & ... & Do. & $\ldots$ & $\begin{array}{l}\text { Sir John Willoughby, Bart., "East } \\
\text { Africa and its Big Game." }\end{array}$ \\
\hline
\end{tabular}


SALT'S ANTELOPE (Neotragus Saltianis).

Weight-Weight about 6 lbs.

Habita:-Somali Land, North-east Africa.

\begin{tabular}{|c|c|c|c|c|c|}
\hline Length: & $\begin{array}{l}\text { Circum- } \\
\text { ference. }\end{array}$ & Tip to Tip. & Habitat. & & Owner. \\
\hline $2 \frac{3}{4}$ & I. & I & | Somali Land & $\ldots$ & T. IV. H. Clarke. \\
\hline $2 \frac{5}{8}$ & $\ldots$ & $1 \frac{3}{8}$ & Do. & & Sir Victor Brooke's Collection. \\
\hline $2 \frac{1}{2}$ & I & $1 \frac{3}{8}$ & Do. & & Capt. H. G. C. Swayne. \\
\hline $2 \frac{3}{8}$ & $1 \frac{3}{8}$ & $1 \frac{5}{8}$ & Abyssinia... & $\cdots$ & Sir Victor Brooke's Collection. \\
\hline $2 \frac{3}{8}$ & $\cdots$ & $\mathrm{I} \frac{5}{8}$ & North-east Afric & & British Museum. \\
\hline $2 \frac{3}{8}$ & $I_{2}^{\frac{1}{2}}$ & $\ldots$ & Somali Land & $\ldots$ & C. Liddell. \\
\hline
\end{tabular}

ZANZIBAR ANTELOPE (Nanotragus moschatus). Habitat-East Africa.

\begin{tabular}{|c|c|c|c|c|c|}
\hline Length. & $\begin{array}{l}\text { Circum- } \\
\text { ference. }\end{array}$ & Tip to Tip. & Habitat. & & Owner. \\
\hline $3 \frac{1}{\ddagger}$ & $1 \frac{1}{2}$ & 2 & $\begin{array}{l}\text { Coral Islands, } \\
\text { Zanzibar. }\end{array}$ & & Sir John Kirk, M. D., K.C.B. \\
\hline $3 \frac{1}{t}$ & I $\frac{3}{8}$ & $I_{\frac{1}{2}}$ & Zanzibar ... & $\ldots$ & Capt. Speke, British Museum. \\
\hline $3 \frac{1}{4}$ & $I \frac{1}{4}$ & $I \frac{l}{2}$ & Do. $\ldots$ & $\ldots$ & Dr. Kirk, British Museum. \\
\hline $2 \frac{5}{8}$ & $1 \frac{1}{4}$ & I $\frac{3}{4}$ & Do. $\ldots$ & $\ldots$ & Sir Victor Brooke's Collection. \\
\hline
\end{tabular}




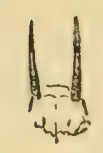

\section{ORIBI ANTELOPE (Nanotragus scoparia).}

Habita:-South Africa.

\begin{tabular}{|c|c|c|c|c|}
\hline Length. & $\begin{array}{l}\text { Circum- } \\
\text { ference. }\end{array}$ & Tip to Tip. & Habitat. & Owner. \\
\hline $4 \frac{5}{8}$ & $1 \frac{3}{4}$ & $3 \frac{1}{2}$ & South Africa $\quad \ldots$ & F. C. Selous. \\
\hline $4^{\frac{3}{4}}$ & 2 & $2 \frac{1}{2}$ & Cape of Good Hope & British Museum. \\
\hline $4 \frac{3}{4}$ & $\mathrm{I}_{4}^{\frac{3}{4}}$ & 2 & South Africa & F. C. Selous. \\
\hline $4 \frac{1}{1}$ & $\mathrm{I}_{4}^{\frac{3}{4}}$ & $1 \frac{7}{8}$ & Do. & Do. \\
\hline $4 \frac{1}{8}$ & ... & $2 \frac{7}{8}$ & Do. & Rowland Ward's Collection. \\
\hline
\end{tabular}

WEST AFRICAN ORIBI (Nanotragus nigricaudatus).

Habitat-River Gambia.

(For description see page 82.)

\begin{tabular}{|c|c|c|c|c|}
\hline Length. & $\begin{array}{l}\text { Circum- } \\
\text { ference. }\end{array}$ & Tip to Tip. & Hatitat. & Owner. \\
\hline 3 & I $\frac{5}{8}$ & $2 \frac{3}{4}$ & River Gambia ... & Charles B. Mosse, British Museum. \\
\hline
\end{tabular}




\section{NANOTRAGUS NIGRICAUDATUS.}

[Sir Victor BROOKE, P.Z.S. I872, p. 875.]

GrizzLy fawn; forehead rufous; tail black, of moderate length; temporal gland distinct, black; knee-brushes large; false hoofs present but small; ears moderate; horns slender, moderate in length, and slightly bent forward.

Height at shoulder, 20 in.; length of ears, $4 \frac{1}{2}$ in.; length of horns, 3 in. A narrow mark about one inch in length on each side of the naked muzzle; a line passing round the eye and suborbital pit, but not meeting in front of the gland, lower lip, chin, spot on throat, belly, inside of limbs nearly as far down as the carpal and tarsal joints, and rump white. Nose and ears externally dusky brown; forehead and occiput rufous; temporal gland large, round, and covered with very short bluish-black hair, that covering the region between the suborbital pit and the eye being of similar character and colour; neck, back, flanks, and sides of a peculiar grizzly fawn colour. This peculiarity is obtained by the base of each hair being almost white, the tip rufous, the intervening part of a deep bluish grey.

\section{ABYSSINIAN ORIBI (Nanotragus montanus).}

Habitat-Abyssinia.

[Sir VICTOR BROOKE, P.Z.S. I872, p. 875.]

SANDY fawn; tail of the same colour as the back, and very short, almost rudimentary; temporal gland and knee-brushes distinct; false hoofs small; ears moderate; horns short and subconical.

\begin{tabular}{|c|c|c|c|c|}
\hline Length. & $\begin{array}{l}\text { Circum- } \\
\text { ference. }\end{array}$ & Tip to Tip. & Habitat. & Owner. \\
\hline $4 \frac{3}{5}$ & $\mathrm{I} \frac{3}{4}$ & $2 \frac{1}{2}$ & $\begin{array}{l}\text { Dembelas, Abys- } \\
\text { sinia. }\end{array}$ & British Museum. \\
\hline
\end{tabular}




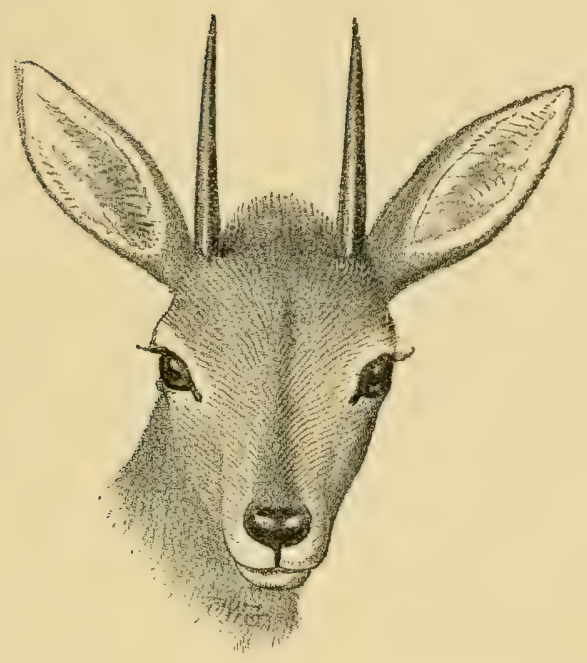

STEINBUCK (Nanotragus tragulus).

Habitat-South and East Africa.

\begin{tabular}{|c|c|c|c|c|c|}
\hline Length. & $\begin{array}{l}\text { Circum- } \\
\text { ference. }\end{array}$ & Tip to Tip. & Habitat. & & Owner. \\
\hline $5 \frac{3}{8}$ & 2 & $1 \frac{3}{4}$ & East Africa & ... & E. Gedge. \\
\hline 5 & $\cdots$ & $2 \frac{1}{2}$ & Do. & ... & Sir John Willoughby, Bart., "East \\
\hline $4 \frac{1}{2}$ & $1 \frac{3}{4}$ & I $\frac{5}{8}$ & South Africa & ... & H. and $\mathrm{C}$. Beddington. \\
\hline $4 \frac{1}{2}$ & I $\frac{5}{8}$ & $2 \frac{5}{8}$ & Do. & ... & F. C. Selous. \\
\hline $4 \frac{3}{8}$ & I $\frac{5}{8}$ & $2 \frac{3}{8}$ & East Africa & ... & Sir Robert Harvey, Bart. \\
\hline $4 \frac{3}{8}$ & 15 & $2 \frac{1}{4}$ & South Africa & ... & F. C. Selous. \\
\hline $4 \frac{1}{4}$ & 2 & $2 \frac{5}{8}$ & Do. & $\ldots$ & The Iate J. S. Jameson. \\
\hline $4 \frac{1}{8}$ & $I \frac{7}{8}$ & $2 \frac{1}{4}$ & Soudan $\ldots$ & $\cdots$ & Col. Ralph Vivian. \\
\hline $4 \frac{1}{8}$ & $1 \frac{5}{8}$ & $2 \frac{3}{8}$ & $\begin{array}{l}\text { Kaokoland, } \\
\text { Africa. }\end{array}$ & West & Capt. F. Cookson, IS9o. \\
\hline $4 \frac{1}{8}$ & $1 \frac{5}{8}$ & I $\frac{3}{4}$ & South Africa & ... & F. C. Selous. \\
\hline
\end{tabular}


STEINBUCK (Nanotragus tragulus)-continued.

\begin{tabular}{|c|c|c|c|c|c|}
\hline Length. & $\begin{array}{l}\text { Circum- } \\
\text { ference. }\end{array}$ & Tip to Tip. & Habitat. & & Owner. \\
\hline 4 & $1 \frac{3}{4}$ & $2 \frac{3}{8}$ & Soudan $\ldots$ & $\ldots \mid$ & Col. Ralph Vivian. \\
\hline 4 & I $\frac{3}{8}$ & $2 \frac{1}{4}$ & East Africa & $\ldots \mid$ & Sir Robert Harvey, Bart. \\
\hline$* 3 \frac{7}{8}$ & I $\frac{5}{8}$ & $2 \frac{3}{8}$ & South Africa & $\ldots$ & British Museum. \\
\hline $3^{\frac{3}{4}}$ & $1 \frac{3}{4}$ & $2 \frac{1}{4}$ & East Africa & $\ldots \mid$ & Sir Robert Harvey, Bart. \\
\hline $3 \frac{3}{t}$ & $1 \frac{5}{8}$ & $1 \frac{7}{8}$ & $\ldots$ & $\ldots$ & J. Carr Saunders. \\
\hline $3 \frac{3}{4}$ & $1 \frac{1}{2}$ & $2 \frac{1}{t}$ & East Africa & $\ldots$ & F. J. Jackson. \\
\hline$* 3 \frac{3}{8}$ & $1 \frac{1}{2}$ & $2 \frac{1}{2}$ & Transvaal & $\ldots$ & British Museum. \\
\hline$* 3 \frac{3}{8}$ & $\ldots$ & $2 \frac{3}{4}$ & $\begin{array}{l}\text { Near Kilima'nja } \\
\text { East Africa. }\end{array}$ & & H. C. V. Hunter, British Museum. \\
\hline
\end{tabular}

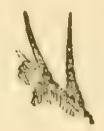

KLIPSPRINGER (Nanotragus oreotragus).

Somali name—"Alakud."

Habitat-Africa.

\begin{tabular}{|c|c|c|c|c|c|}
\hline Length. & $\begin{array}{l}\text { Circum- } \\
\text { ference. }\end{array}$ & Tip to Tip. & Habitat. & & Owner. \\
\hline $4 \frac{3}{8}$ & 2 & $3 \frac{1}{4}$ & South Africa & $\ldots$ & F. C. Selous. \\
\hline $4 \frac{1}{t}$ & $2 \frac{1}{8}$ & $2 \frac{1}{4}$ & Do. & $\cdots$ & Do. \\
\hline $4^{\frac{1}{4}}$ & 2 & $3 \frac{1}{4}$ & East Africa & $\cdots$ & E. Gedge. \\
\hline $4 \frac{1}{8}$ & $2 \frac{1}{8}$ & $2 \frac{3}{5}$ & South Africa & ... & F. C. Selous. \\
\hline 4 & $2 \frac{1}{2}$ & $1 \frac{7}{8}$ & Do. & ... & H. and C. Beddington. \\
\hline
\end{tabular}


REHBOK.

KLIPSPRINGER (Nanotragus oreotragus)-continucd.

\begin{tabular}{|c|c|c|c|c|c|}
\hline Length. & $\begin{array}{l}\text { Circum- } \\
\text { ference. }\end{array}$ & Tip to Tip. & Habitat & & Owner. \\
\hline$*_{4}$ & 2 & 3 & South Africa & $\ldots$ & British Museum. \\
\hline 4 & $1 \frac{7}{8}$ & 2 & Do. & & The late J. S. Jameson. \\
\hline $3 \frac{3}{7}$ & 2 & 2 & $\begin{array}{l}\text { Somali, North- } \\
\text { East Africr. }\end{array}$ & & \\
\hline$* 3 \frac{3}{4}$ & $\cdots$ & I $\frac{7}{8}$ & Abyssinia & $\cdots$ & British Museum. \\
\hline $3 \frac{1}{4}$ & $\mathbf{I} \frac{3}{4}$ & $\mathbf{I} \frac{5}{8}$ & Somali $\quad \ldots$ & ... & E. Lort-Phillips. \\
\hline $3 \frac{1}{8}$ & $\mathrm{I} \frac{3}{4}$ & $2 \frac{1}{2}$ & South Africa & $\ldots$ & A. Beit. \\
\hline$* 3 \frac{1}{8}$ & $\mathbf{I} \frac{3}{4}$ & $2 \frac{3}{8}$ & Do. & $\ldots$ & British Museum. \\
\hline 3 & $1 \frac{7}{8}$ & $3 \frac{3}{4}$ & Do. & $\ldots$ & Hon. Walter Rothschild. \\
\hline
\end{tabular}

REHBOK (Pelea capreola).

Habitat-South Africa.

\begin{tabular}{c|c|c|cc|l}
\hline Length. & $\begin{array}{c}\text { Circum- } \\
\text { ference. }\end{array}$ & Tip to Tip. & Habitat. & \multicolumn{1}{|c}{ Owner. } \\
\cline { 1 - 3 } $8 \frac{5}{8}$ & $2 \frac{1}{2}$ & $2 \frac{5}{8}$ & South Africa & $\ldots$ & Dr. Burchell, British Museum. \\
$7 \frac{7}{8}$ & $2 \frac{3}{8}$ & $3 \frac{1}{2}$ & Do. & $\ldots$ & R. G. Breakes, British Museum. \\
$7 \frac{3}{8}$ & $2 \frac{1}{4}$ & $3 \frac{1}{4}$ & Do. & $\ldots$ & Rowland Ward's Collection. \\
$7 \frac{1}{2}$ & $2 \frac{3}{8}$ & 3 & Do. & $\ldots$ & H. Atkinson. \\
$6 \frac{7}{8}$ & $2 \frac{1}{2}$ & $3 \frac{1}{8}$ & Do. & $\ldots$ & A. Beit. \\
$6 \frac{1}{4}$ & $2 \frac{3}{8}$ & $2 \frac{3}{4}$ & Do. & $\ldots$ & British Museum. \\
$5 \frac{1}{2}$ & $2 \frac{3}{8}$ & $1 \frac{3}{4}$ & Do. & $\ldots$ & Hon. Walter Rothschild. \\
\hline
\end{tabular}




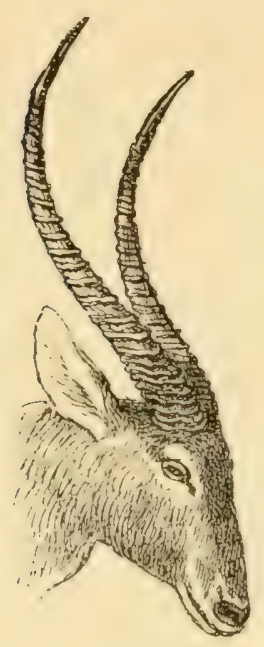

WATERBUCK (Cobus ellipsiprymnus).

Bechuana name-"Tumoha."

Lower Zambesi name_-"Myakobswe."

Habitat-South and East Africa.

\begin{tabular}{|c|c|c|c|c|c|}
\hline Length. & Circumference. & Tip to Tip. & Habitat. & & Owner. \\
\hline $33 \frac{1}{2}$ & $9 \frac{1}{4}$ & $2 I \frac{1}{2}$ & $\cdots$ & .. & Hon. Walter Rothschild. \\
\hline 33 & $9 \frac{3}{8}$ & I $1 \frac{1}{2}$ & Mashuna Land & & F. C. Selous, British Museum. \\
\hline $32 \frac{3}{4}$ & $9 \frac{3}{4}$ & $21 \frac{3}{8}$ & South Africa .. & .. 1 & Sir Victor Brooke's Collection. \\
\hline $31 \frac{3}{8}$ & $8 \frac{1}{2}$ & $2 I \frac{7}{8}$ & Do. &.. & British Museum. \\
\hline $3 \mathrm{I} \frac{1}{4}$ & $9 \frac{5}{8}$ & $19 \frac{1}{4}$ & ... & ... & Sir Edmund G. Loder, Bart. \\
\hline 31 & .. & $\begin{array}{l}\text { (owner's mea- } \\
\text { surements) }\end{array}$ & Mashuna Land & & F. C. Selous. \\
\hline $30 \frac{1}{2}$ & $8 \frac{7}{8}$ & $16 \frac{1}{8}$ & Algoa Bay &. .1 & C. Wemys, British Museum. \\
\hline $30 \frac{1}{8}$ & $8 \frac{1}{2}$ & $13 \frac{7}{8}$ & $\cdots$ & ... & F. C. Selous. \\
\hline
\end{tabular}


WATERBUCK (Cobus ellipsiprymnus)-continued.

\begin{tabular}{|c|c|c|c|c|c|}
\hline Length. & $\begin{array}{l}\text { Circum- } \\
\text { ference. }\end{array}$ & Tip to Tip. & Habitat. & & Owner. \\
\hline 30 & 9 & $22 \frac{1}{2}$ & $\cdots$ & $\ldots$ & W. Ogilby (P.Z.S. I883, pt. I, p. 47). \\
\hline 30 & $8 \frac{7}{8}$ & $16 \frac{1}{2}$ & South Africa & ... & British Museum. \\
\hline $29 \frac{7}{8}$ & $9 \frac{1}{8}$ & $12 \frac{7}{8}$ & $\ldots$ & $\ldots$ & Hon. Charles Ellis。 \\
\hline $299^{3}$ & $9 \frac{1}{2}$ & $2 I \frac{7}{8}$ & $\ldots$ & ... & F. C. Selous. \\
\hline $29 \frac{1}{4}$ & 8 & $24 \frac{1}{2}$ & ... & ... & Hon. Charles Ellis. \\
\hline 29 & $\begin{array}{c}9 \frac{1}{4} \\
\text { (malformed) }\end{array}$ & $5^{\frac{1}{2}}$ & South Africa & ... & A. W. Davis. \\
\hline $28 \frac{7}{8}$ & 9 & 21 & $\cdots$ & $\cdots$ & Rowland Ward's Collection. \\
\hline $2 S_{i}^{3}$ & 8 & $15 \frac{1}{4}$ & South Africa & ... & G. H. Banks. \\
\hline $28 \frac{5}{8}$ & 8 & $16 \frac{3}{4}$ & Do. & $\ldots$ & Do. \\
\hline $28 \frac{3}{4}$ & $9 \frac{1}{8}$ & $21 \frac{3}{8}$ & $\cdots$ & $\ldots$ & H.R.H. Duke of Edinburgh. \\
\hline $28 \frac{3}{8}$ & 8 & $19 \frac{1}{4}$ & $\ldots$ & ... & Hon. Charles Ellis. \\
\hline 28 & $\left(\begin{array}{c}\text { (owner's mea- } \\
\text { surements) }\end{array}\right.$ & 18 & East Africa & $\ldots$ & \\
\hline $2 S$ & $9^{\frac{1}{4}}$ & $16 \frac{3}{4}$ & $\cdots$ & $\cdots$ & Hon. Walter Rothschild. \\
\hline $27 \frac{3}{4}$ & $9 \frac{1}{4}$ & $14^{\frac{3}{4}}$ & $\cdots$ & .. & The late J. S. Jameson. \\
\hline $27 \frac{5}{8}$ & 8 & $9 \frac{7}{8}$ & $\cdots$ & $\ldots$ & Maj.-Gen. Arthur Ellis, C.S.I. \\
\hline $27 \frac{1}{2}$ & $8 \frac{7}{8}$ & $25 \frac{1}{2}$ & $\cdots \quad \cdots$ & $\cdots$ & Hon. Charles Ellis. \\
\hline $27 \frac{1}{2}$ & $8 \frac{3}{4}$ & 14 & South Africa & .. & Durban Museum. \\
\hline $27 \frac{1}{4}$ & $9 \frac{1}{2}$ & $16 \frac{3}{4}$ & $\cdots \quad \ldots$ & $\ldots$ & Maj.-Gen. Arthur Ellis, C.S.I. \\
\hline 27 & 9 & 24 & East Africa & $\cdots$ & E. Gedge. \\
\hline $266_{4}^{3}$ & $9 \frac{1}{4}$ & $2 \mathrm{I}$ & Limpopo & $\cdots$ & W. C. Oswell. \\
\hline $26 \frac{1}{2}$ & $8 \frac{5}{5}$ & $15 \frac{7}{8}$ & South Africa & $\cdots$ & A. M. Sagar Musgrave. \\
\hline $26 \frac{1}{2}$ & $7 \frac{1}{2}$ & I $3 \frac{7}{8}$ & Do. & $\cdots$ & Bethnal Green Museum. \\
\hline $26 \frac{3}{8}$ & $9 \frac{5}{8}$ & II $\frac{1}{2}$ & Do. & $\cdots$ & The late J. S. Jameson. \\
\hline $26 \frac{3}{8}$ & $8 \frac{1}{2}$ & $18 \frac{1}{8}$ & Do. & $\cdots$ & J. Carr-Saunders. \\
\hline 26 & $9 \frac{3}{8}$ & $12 \frac{3}{4}$ & Limpopo & $\cdots$ & W. C. Oswell. \\
\hline $25 \frac{7}{8}$ & $8 \frac{3}{8}$ & $17 \frac{1}{2}$ & Soudan & $\cdots$ & Col. Ralph Vivian. \\
\hline $25 \frac{5}{8}$ & $8 \frac{1}{4}$ & II & $\ldots$ & $\ldots$ & Capt. Speke, British Museum. \\
\hline
\end{tabular}


WATERBUCK (Cobus ellipsiprymnus)-continued.

\begin{tabular}{|c|c|c|c|c|c|}
\hline Length. & $\begin{array}{l}\text { Circum- } \\
\text { ference. }\end{array}$ & Tip to Tip. & Habitat. & & Owner. \\
\hline $25 \frac{3}{8}$ & $9 \frac{3}{8}$ & 16 & $\ldots$ & $\ldots$ & The late J. S. Jameson. \\
\hline $25 \frac{3}{8}$ & $8 \frac{1}{2}$ & 22 & South Africa : & $\therefore$ & W. P. Rylands. \\
\hline $25 \frac{1}{4}$ & $8 \frac{3}{4}$ & $13 \frac{7}{8}$ & East Africa . & $\cdots$ & Sir Robert Harvey, Bart. \\
\hline $25 \frac{1}{8}$ & 8 & $13 \frac{3}{8}$ & Do. & $\cdots$ & II. C. V. Ifunter. \\
\hline 25 & $9^{\frac{1}{4}}$ & $15^{\frac{1}{2}}$ & Soudan & $\cdots$ & Col. Ralph Vivian. \\
\hline 25 & $8 \frac{1}{2}$ & $13 \frac{1}{2}$ & South Africa . & $\cdots$ & J. A. Nicoll. \\
\hline 25 & $7^{\frac{3}{4}}$ & $16 \frac{1}{4}$ & East Africa . & $\cdots$ & Sir Robert Harvey, Bart. \\
\hline $24 \frac{7}{8}$ & $8 \frac{1}{2}$ & $16 \frac{1}{2}$ & South $\Lambda$ frica . & $\cdots$ & W. P. Rylands. \\
\hline $24 \frac{7}{8}$ & $8 \frac{1}{8}$ & 13 & East Africa . & $\cdots$ & Sir Robert IIarvey, Bart. \\
\hline $24 \frac{7}{8}$ & $7^{\frac{3}{4}}$ & $9 \frac{1}{2}$ & Do. & $\cdots$ & Do. \\
\hline $24 \frac{1}{4}$ & $7 \frac{1}{2}$ & $9 \frac{3}{4}$ & $\begin{array}{ll}\cdots & \ldots\end{array}$ & $\cdots$ & IV. D. James. \\
\hline $24 \frac{1}{8}$ & 8 & $177 \frac{7}{8}$ & East $A$ frica & $\cdots$ & Sir Robert Harvey, Bart. \\
\hline $24 \frac{1}{8}$ & $7^{\frac{3}{4}}$ & $16 \frac{1}{8}$ & South Africa . & $\cdots$ & British Museum. \\
\hline $23 \frac{7}{8}$ & $8 \frac{3}{8}$ & $12 \frac{5}{8}$ & East Africa . & $\cdots$ & II. C. V. Hunter. \\
\hline $23 \frac{7}{8}$ & 8 & $15 \frac{3}{8}$ & Do. & $\cdots$ & Sir Robert Harvey, Bart. \\
\hline 23 & 9 & 9 & Do. & $\cdots$ & E. Gedge. \\
\hline $22 \frac{3}{3}$ & $8_{\frac{1}{2}}^{1}$ & $13 \frac{1}{8}$ & Do. & $\cdots$ & Sir Robert Harvey, Bart. \\
\hline $2 \mathrm{I} \frac{3}{4}$ & $7 \frac{3}{8}$ & II $\frac{1}{8}$ & Do. & $\cdots$ & Do. \\
\hline $2 \mathrm{I} \frac{3}{8}$ & $7 \frac{5}{8}$ & $13^{\frac{3}{4}}$ & Cape Delgado & & Sir John Kirk, M.D., K.C.B. \\
\hline $2 I \frac{3}{8}$ & 7 & $I I \frac{1}{t}$ & $\cdots \quad \ldots$ & $\cdots$ & IV. D. James. \\
\hline $2 \mathrm{I}$ & $7^{\frac{3}{4}}$ & 13 & East Africa . & & IV. Astor Chandler. \\
\hline 20 & $7 \frac{1}{8}$ & $12 \frac{1}{8}$ & Cape Delgado & & Sir John Kirk, M.D., K.C.B. \\
\hline
\end{tabular}




\section{SINGSING ANTELOPE (Cobus defassus).}

\section{Habitat-Africa.}

[Dr. SClAter on Capt. Speke's trophies, P.Z.S. I864, p. 102.]

THE general aspect of the head resembles Kobus ellipsiprymnus, but the face is blacker, and the top of the head between the horns dark rufous. "It does not possess the lunate mark on the rump of the Waterbuck, and does not stand so high, but is rather more stoutly built." (Note by Capt. Speke on above.)

[Dr. JaMes Murie, P.Z.S. I 867, p. 5.]

Baron Hernier. British Museum, Capt. Speke's specimen.

Height at shoulder $\quad \ldots \quad 45^{\frac{3}{4}}$

Length of body, rump, to

front of shoulder $\quad \ldots \quad 52$

Length of head from between the horns to tip of muzzle $\quad \ldots \quad \ldots \quad$ I $3 \frac{1}{2}$

Length of tail ... $\quad \ldots \quad$ I6

Length of ears ... ... ... $45^{\frac{1}{2}}$

\section{2}




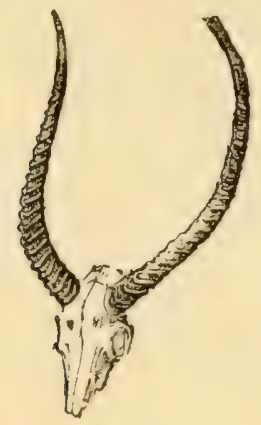

LECHWE ANTELOPE (Cobus leche).

Habitat-South Africa.

\begin{tabular}{|c|c|c|c|c|c|}
\hline Length. & $\begin{array}{l}\text { Circum- } \\
\text { ference. }\end{array}$ & Tip to Tip. & Habitat. & & Owner. \\
\hline $27 \frac{1}{2}$ & $10 \frac{3}{4}$ & I $9 \frac{1}{8}$ & Chobe River & $\ldots$ & F. C. Selous, British Museum. \\
\hline 27 & $\cdots$ & $\cdots$ & Do. & $\cdots$ & $\begin{array}{l}\text { Do. } \\
\text { Wanderings." }\end{array}$ \\
\hline $26 \frac{1}{4}$ & $\delta_{\frac{3}{3}}$ & 21 & South Africa & $\cdots$ & A. Beit. \\
\hline $26 \frac{1}{8}$ & 8 & $\begin{array}{l}\text { I5 } \\
\text { (about) }\end{array}$ & Do. & $\ldots$ & F. C. Selous. \\
\hline 25 & $\cdots$ & ... & Lukanga ... & $\cdots$ & $\begin{array}{c}\text { Do. } \\
\text { Wanderings." }\end{array}$ \\
\hline $24 \frac{3}{5}$ & $7 \frac{5}{8}$ & $13 \frac{5}{8}$ & South Africa & $\cdots$ & The late J. S. Jameson. \\
\hline 24 & $8 \frac{1}{8}$ & $14 \frac{1}{4}$ & Do. & $\cdots$ & F. C. Selous. \\
\hline 24 & S & $15^{\frac{1}{2}}$ & Zambesi ... & $\cdots$ & W. C. Oswell. \\
\hline $23 \frac{3}{4}$ & $7 \frac{1}{2}$ & $14 \frac{1}{8}$ & South Africa & $\cdots$ & F. C. Selous. \\
\hline 23 & 9 & 9 & East Africa & $\cdots$ & E. Gedge. \\
\hline $22 \frac{7}{8}$ & $7 \frac{1}{1}$ & $6 \frac{5}{8}$ & South Africa & $\cdots$ & A. Beit. \\
\hline $22 \frac{5}{8}$ & 73 & I I $\frac{1}{4}$ & Do. & $\cdots$ & The late J. S. Jameson. \\
\hline $22 \frac{5}{5}$ & $6 \frac{5}{5}$ & I I $\frac{3}{4}$ & Chobe River & $\cdots$ & F. C. Selous, British Museum. \\
\hline 22 & $7 \frac{1}{4}$ & $10 \frac{3}{4}$ & South Africa & $\cdots$ & J. Carr-Saunders. \\
\hline $2 \mathrm{I} \frac{1}{2}$ & 7 & 10 & Africa $\quad \ldots$ & $\cdots$ & J. A. Nicoll. \\
\hline $2 I \frac{1}{4}$ & $6 \frac{1}{2}$ & $13 \frac{7}{8}$ & Do. $\quad \ldots$ & $\cdots$ & Capt. F. Vardon, British Museum. \\
\hline
\end{tabular}




\section{WHITE-EARED WATERBUCK (Cobus leucotis).}

Habitat-East Africa.

\begin{tabular}{|c|c|c|c|c|c|}
\hline Length. & $\begin{array}{l}\text { Circum- } \\
\text { ference. }\end{array}$ & Tip to Tip. & Habitat. & & Owner. \\
\hline $19 \frac{7}{8}$ & $6 \frac{3}{8}$ & $7 \frac{1}{4}$ & Bahr el Gazal & $\ldots$ & British Museum. \\
\hline $19 \frac{1}{8}$ & $5 \frac{7}{8}$ & $9 \frac{1}{2}$ & Do. & ... & Consul J. Petherick, British Museum. \\
\hline 19 & 6 & $8 \frac{1}{4}$ & Do. & $\ldots$ & Do. \\
\hline $17 \frac{1}{4}$ & 6 & $7 \frac{1}{4}$ & Africa $\quad \ldots$ & ... & Sir Victor Brooke's Collection. \\
\hline
\end{tabular}

COBUS KOB.

Habitat-Africa.

\begin{tabular}{|c|c|c|c|c|c|}
\hline Length. & $\begin{array}{l}\text { Circum- } \\
\text { ference. }\end{array}$ & Tip to Tip. & Habitat. & & Owner. \\
\hline 18 & $7 \frac{1}{4}$ & II $\frac{1}{2}$ & East Africa & ... & E. Gedge. \\
\hline $17 \frac{3}{4}$ & $7 \frac{3}{4}$ & I I $\frac{1}{2}$ & Uganda & $\ldots$ & Do. \\
\hline I $7 \frac{3}{8}$ & $6 \frac{1}{2}$ & $7 \frac{3}{4}$ & Do. & $\cdots$ & F. J. Jackson. \\
\hline $17 \frac{1}{4}$ & $7 \frac{1}{2}$ & $5 \frac{1}{2}$ & Do. & ... & 1890. \\
\hline I $7 \frac{1}{8}$ & 7 & 5 & Masai Land & $\ldots$ & British Museum. \\
\hline I 7 & $6 \frac{3}{4}$ & $9 \frac{1}{2}$ & East Africa & $\ldots$ & E. Gedge. \\
\hline $9 \frac{1}{2}$ & 5 & 3 & Gambia ... & ... & Earl of Derby, British Museum. \\
\hline 8 & $4 \frac{5}{8}$ & $4 \frac{5}{8}$ & Do. ... & $\ldots$ & Do. \\
\hline
\end{tabular}

MRS. GRAY'S WATERBUCK (Cobus maiæ).

Habitat-East Africa.

\begin{tabular}{c|c|c|c|c}
\hline Length. & $\begin{array}{c}\text { Circum- } \\
\text { ference. }\end{array}$ & Tip to Tip. & Habitat. & Owner. \\
\hline $26 \frac{7}{8}$ & $6 \frac{7}{8}$ & $13 \frac{3}{\frac{3}{4}}$ & $\begin{array}{c}\text { Arwan, Bahr el } \\
\text { Gazal. }\end{array}$ & Consul J. Petherick, British Museum. \\
\hline
\end{tabular}




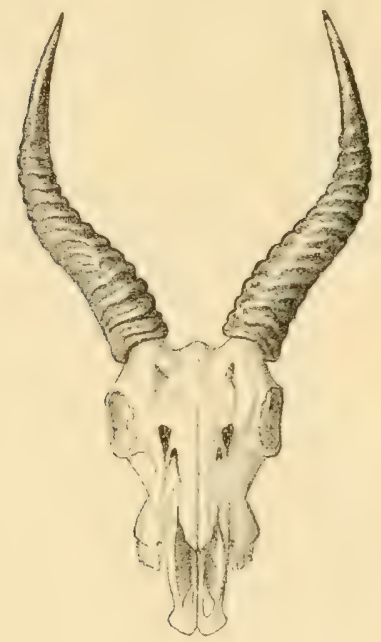

\section{POOKOO ANTELOPE (Cobus vardoni).}

Native name-" Poku."

Mabitat-South Africa.

\begin{tabular}{|c|c|c|c|c|c|}
\hline Length. & $\begin{array}{l}\text { Circum- } \\
\text { ference. }\end{array}$ & Tip to Tip. & Habitat. & & Owner. \\
\hline $19 \frac{1}{8}$ & $6 \frac{3}{4}$ & $8 \frac{1}{2}$ & South Africa & $\ldots$ & J. Carr-Saunders. \\
\hline I $6 \frac{1}{4}$ & $6 \frac{5}{8}$ & $7 \frac{1}{2}$ & Do. & $\cdots$ & British Museum. \\
\hline 16 & $6 \frac{3}{4}$ & $6 \frac{3}{8}$ & Chobe River & .. & F. C. Selous. \\
\hline $15 \frac{1}{8}$ & 6 & IO & South Africa & $\cdots$ & J. Carr-Saunders. \\
\hline $14 \frac{1}{2}$ & $6 \frac{3}{8}$ & $6 \frac{5}{8}$ & Do. & ... & F. C. Selous. \\
\hline $14 \frac{1}{8}$ & $6 \frac{5}{8}$ & $5 \frac{1}{8}$ & Chobe River & $\ldots$ & British Museum. \\
\hline I $3 \frac{7}{8}$ & $5 \frac{7}{8}$ & $7 \frac{1}{4}$ & South Africa & $\ldots$ & Rowland Ward's Collection. \\
\hline
\end{tabular}




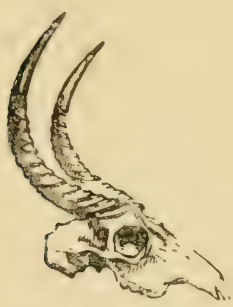

REEDBUCK (Cervicapra arundineum).

(Cervicapra bohor).

Sena and Tete name-" N'sengo."

Habitat-Africa.

\begin{tabular}{|c|c|c|c|c|c|c|}
\hline Length. & $\begin{array}{l}\text { Circum- } \\
\text { ference. }\end{array}$ & Tip to Tip. & & Habitat. & & Owner. \\
\hline $15 \frac{7}{8}$ & $6 \frac{1}{4}$ & $14 \frac{1}{4}$ & $\ldots$ & $\ldots$ & $\ldots$ & British Museum. \\
\hline $15 \frac{3}{4}$ & $6 \frac{1}{8}$ & $\begin{array}{l}\text { (one point } \\
\text { broken) }\end{array}$ & ... & $\ldots$ & ... & The late J. S. Jameson. \\
\hline $15 \frac{1}{8}$ & $6 \frac{5}{8}$ & II $\frac{1}{x}$ & $\cdots$ & $\cdots$ & $\ldots$ & F. C. Selous. \\
\hline I 5 & $\cdots$ & $\cdots$ & $\cdots$ & $\ldots$ & $\cdots$ & $\begin{array}{l}\text { Do. } \\
\text { derings." "A Hunter's Wan- }\end{array}$ \\
\hline 15 & $6 \frac{1}{2}$ & $9 \frac{3}{4}$ & Sot & Africa & ... & Sir John Willoughby, Bart. \\
\hline $14 \frac{5}{8}$ & $7 \frac{3}{8}$ & $9 \frac{1}{8}$ & ... & ... & $\ldots$ & Hon. Walter Rothschild. \\
\hline I $4 \frac{\frac{1}{2}}{2}$ & $6 \frac{3}{8}$ & $7 \frac{5}{8}$ & $\cdots$ & ... & $\ldots$ & Sir Ednuund G. Loder, Bart. \\
\hline $14 \frac{1}{2}$ & $6 \frac{1}{4}$ & 7 & $\cdots$ & ... & $\ldots$ & F. C. Selous. \\
\hline $14 \frac{1}{2}$ & $5 \frac{5}{8}$ & $8 \frac{1}{4}$ & .. & $\cdots$ & ... & Do. \\
\hline $14 \frac{1}{2}$ & $5 \frac{3}{5}$ & $14 \frac{3}{8}$ & $\cdots$ & $\ldots$ & $\ldots$ & W. C. Oswell. \\
\hline I 4 & $\cdots$ & $\ldots$ & \multirow{2}{*}{\multicolumn{3}{|c|}{$\begin{array}{l}\text { River Tati, South } \\
\text { Africa. } \\
\text { South Africa }\end{array}$}} & $\begin{array}{l}\text { F. C. Selous, "A Hunter's Wan- } \\
\text { derings." }\end{array}$ \\
\hline $13 \frac{7}{8}$ & $5^{\frac{3}{4}}$ & $11 \frac{1}{2}$ & & & & A. Beit. \\
\hline$*_{I} 3^{\frac{3}{ \pm}}$ & $5 \frac{7}{8}$ & $8 \frac{1}{8}$ & $\cdots$ & $\cdots$ & $\ldots$ & British Museum. \\
\hline $13 \frac{5}{8}$ & $5 \frac{1}{2}$ & $9 \frac{3}{8}$ & $\cdots$ & $\cdots$ & $\cdots$ & The late J. S. Jameson. \\
\hline $13 \frac{1}{2}$ & 6 & $9 \frac{1}{x}$ & $\cdots$ & $\cdots$ & .. & Hon. Walter Rothschild. \\
\hline $13 \frac{1}{2}$ & $\cdots$ & $12 \frac{1}{4}$ & $\cdots$ & $\cdots$ & ... & $\begin{array}{l}\text { Dr. Knight-Bruce, Bishop of Bloom- } \\
\text { fontein. }\end{array}$ \\
\hline $13 \frac{1}{2}$ & $5 \frac{7}{8}$ & $6 \frac{1}{8}$ & $\cdots$ & $\cdots$ & $\cdots$ & The late J. S. Jameson. \\
\hline $13 \frac{1}{8}$ & $6 \frac{1}{8}$ & $10 \frac{5}{8}$ & $\ldots$ & ... & $\ldots$ & Do. \\
\hline
\end{tabular}

* Cervicapra bohor. 
REEDBUCK-continusd.

\begin{tabular}{|c|c|c|c|c|c|}
\hline Length. & $\begin{array}{l}\text { Circum- } \\
\text { ference. }\end{array}$ & Tip to Tip. & Habitat. & & Owner. \\
\hline I $3 \frac{1}{8}$ & $5 \frac{3}{8}$ & $9 \frac{5}{8}$ & ... & $\ldots$ & G. H. Banks. \\
\hline I3 & $7 \frac{3}{8}$ & II $\frac{3}{8}$ & $\cdots$ & $\cdots$ & F. C. Selous. \\
\hline I3 & $6 \frac{1}{2}$ & I I $\frac{1}{2}$ & ... & $\ldots$ & Col. G. W. Thompson. \\
\hline $12 \frac{7}{8}$ & $6 \frac{3}{4}$ & $12 \frac{3}{8}$ & $\cdots$ & $\ldots$ & F. C. Selous. \\
\hline $12 \frac{7}{8}$ & $5 \frac{3}{8}$ & II & South Africa & $\ldots$ & A. Beit. \\
\hline I $2 \frac{3}{4}$ & $6 \frac{1}{8}$ & $8 \frac{3}{3}$ & $\cdots$ & $\ldots$ & A. M. Sagar-Musgrave. \\
\hline$*_{12 \frac{1}{2}}$ & $5 \frac{5}{8}$ & $5 \frac{3}{8}$ & $\cdots$ & $\ldots$ & $\begin{array}{l}\text { Sir John Kirk, M.D., K.C. B., Brilish } \\
\text { Museum. }\end{array}$ \\
\hline $12 \frac{1}{2}$ & $5 \frac{1}{2}$ & II $\frac{3}{4}$ & South Africa & $\cdots$ & A. Beit. \\
\hline$*_{12}$ & $5^{\frac{1}{2}}$ & II $\frac{7}{8}$ & Do. & $\ldots$ & British Museum. \\
\hline II $\frac{7}{8}$ & $5 \frac{5}{8}$ & $\mathrm{xO}_{\frac{3}{8}}^{3}$ & $\cdots$ & $\ldots$ & J. Carr-Saunders. \\
\hline $11 \frac{3}{4}$ & $5^{\frac{1}{2}}$ & $8 \frac{1}{2}$ & South Africa & $\ldots$ & A. C. Fountaine. \\
\hline II & 6 & 14 & Do. & $\ldots$ & Lieut. C. F. Gisborne. \\
\hline I I & 6 & $9 \frac{1}{4}$ & Do. & $\cdots$ & J. A. Nicoll. \\
\hline$*_{\mathrm{IO}} \mathrm{O} \frac{5}{8}$ & $4 \frac{1}{2}$ & $5^{\frac{1}{2}}$ & East Africa & $\ldots$ & Sir Robert Harvey, Bart. \\
\hline$*_{1} \mathrm{O}_{2}^{\frac{1}{2}}$ & $5 \frac{1}{2}$ & $8 \frac{3}{8}$ & South Africa & $\cdots$ & British Museum. \\
\hline$* 9 \frac{1}{4}$ & $5 \frac{1}{4}$ & $4 \frac{1}{2}$ & Do. & $\cdots$ & Rowland Ward's Collection. \\
\hline 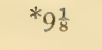 & $5 \frac{3}{8}$ & $8 \frac{1}{2}$ & Do. & $\cdots$ & Dr. Burchell, British Museum. \\
\hline$* 9 \frac{1}{8}$ & $4 \frac{7}{8}$ & $4 \frac{5}{8}$ & East Africa & $\cdots$ & H. C. V. Hunter. \\
\hline *9 & $5^{\frac{1}{4}}$ & $3 \frac{1}{8}$ & Zanzibar ... & $\cdots$ & Sir John Kirk, M.D., K.C.B. \\
\hline$* 8 \frac{3}{4}$ & 5 & $3 \frac{7}{8}$ & East Africa & $\cdots$ & Sir Robert Harvey, Bart. \\
\hline$* 8 \frac{3}{4}$ & $4 \frac{7}{8}$ & $3 \frac{33}{4}$ & Do. & $\cdots$ & H. C. V. Hunter. \\
\hline $8 \frac{1}{2}$ & $5^{\frac{1}{2}}$ & 4 & South Africa & $\ldots$ & The Iate J. S. Jameson. \\
\hline$* 8 \frac{1}{4}$ & $5 \frac{3}{*}$ & 5 & East Africa & $\cdots$ & R. P. Carroll. \\
\hline$* 8 \frac{1}{8}$ & $4^{\frac{3}{4}}$ & $2 \frac{1}{2}$ & Masai Land & $\cdots$ & H. C. V. Hunter, British Museum. \\
\hline$* 7^{\frac{3}{4}}$ & $4 \frac{7}{8}$ & $2 \frac{3}{4}$ & East Africa & $\cdots$ & F. J. Jackson. \\
\hline 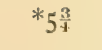 & $3 \frac{7}{8}$ & $3 \frac{1}{2}$ & Do. & $\ldots$ & Do. \\
\hline
\end{tabular}




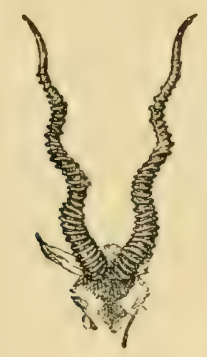

BLACKBUCK or INDIAN ANTELOPE (Antilope cervicapra).

Habitat-India. Average weight $-85 \mathrm{lbs}$.

\begin{tabular}{|c|c|c|c|c|c|c|}
\hline \multicolumn{2}{|c|}{ Length. } & \multirow{2}{*}{$\begin{array}{l}\text { Circum- } \\
\text { ference. }\end{array}$} & \multirow{2}{*}{ Tip to Tip. } & \multirow{2}{*}{\multicolumn{2}{|c|}{ Habitat. }} & \multirow{2}{*}{ Owner. } \\
\hline Straight. & Round Curve. & & & & & \\
\hline $2 S_{\frac{1}{4}}$ & $40 \frac{1}{2}$ & 5 & $17 \frac{3}{4}$ & India & $\ldots$ & $\begin{array}{l}\text { A. O. Hume (Private Col- } \\
\text { lection). }\end{array}$ \\
\hline 27 & $\ldots$ & $\ldots$ & $\begin{array}{l}\text { (owner's mea- } \\
\text { surement) }\end{array}$ & Punjaub & $\ldots$ & $\begin{array}{l}\text { Capt. H. V. Brooke, late } \\
\text { 92nd Highlanders. }\end{array}$ \\
\hline 27 & 34 & 5 & $19 \frac{1}{2}$ & Do. & $\ldots$ & $\begin{array}{l}\text { Sir Victor Brooke's Collec- } \\
\text { tion. }\end{array}$ \\
\hline $26 \frac{3}{8}$ & 33 & 5 & $17 \frac{7}{8}$ & Do. & $\ldots$ & $\begin{array}{l}\text { A. O. Hume (Private Col- } \\
\text { lection). }\end{array}$ \\
\hline $25 \frac{5}{8}$ & $35 \frac{1}{8}$ & $4^{\frac{3}{4}}$ & $20 \frac{3}{4}$ & Do. & $\ldots$ & Do. \\
\hline $25 \frac{1}{2}$ & $\cdots$ & $\cdots$ & 15 & Do. & $\ldots$ & Col. Martin. \\
\hline $25^{\frac{3}{8}}$ & $35^{\frac{1}{x}}$ & $5 \frac{1}{2}$ & $14 \frac{1}{2}$ & Do. & $\ldots$ & $\begin{array}{l}\text { Hume Collection, British } \\
\text { Museum. }\end{array}$ \\
\hline $25 \frac{1}{8}$ & $34^{\frac{3}{4}}$ & $5 \frac{1}{8}$ & $21 \frac{3}{4}$ & $\begin{array}{l}\text { Gadaruar } \\
\text { trict.Cen }\end{array}$ & $\begin{array}{l}\text { Dis- } \\
\text { rov. }\end{array}$ & Sir John H. Morris, \\
\hline 25 & $35 \frac{1}{2}$ & $4 \frac{7}{8}$ & 19 & India & $\ldots$ & $\begin{array}{l}\text { Hume Collection, British } \\
\text { Museum. }\end{array}$ \\
\hline $24 \frac{1}{4}$ & $\cdots$ & 5 & $18 \frac{1}{2}$ & Do. & $\cdots$ & Marquis of Ailsa. \\
\hline 24 & 33 & $5^{\frac{1}{2}}$ & $14 \frac{3}{4}$ & Do. & $\ldots$ & $\begin{array}{l}\text { Rowland Ward's Collec- } \\
\text { tion. }\end{array}$ \\
\hline 24 & $\cdots$ & $\cdots$ & 20 & Do. & $\cdots$ & Lord Herbrand Russell. \\
\hline 24 & 37 & 5 & $18 \frac{7}{8}$ & Aligur & $\cdots$ & St. George Littledale, 1877. \\
\hline $23 \frac{3}{4}$ & $33 \frac{7}{8}$ & $5 \frac{3}{8}$ & 21 & India & $\ldots$ & $\begin{array}{l}\text { H.R.H. Duke of Edin- } \\
\text { burgh. }\end{array}$ \\
\hline
\end{tabular}


BLACKBUCK or INDIAN ANTELOPE (Antilope cervicapra)continued.

\begin{tabular}{|c|c|c|c|c|c|c|}
\hline \multicolumn{2}{|c|}{ Length. } & \multirow{2}{*}{$\begin{array}{l}\text { Circum- } \\
\text { ference. }\end{array}$} & \multirow{2}{*}{ Tip to Tip. } & \multirow{2}{*}{\multicolumn{2}{|c|}{ Habitat, }} & \multirow{2}{*}{ Owner. } \\
\hline Straight. & Round Curve. & & & & & \\
\hline $23 \frac{5}{8}$ & 34 & $5 \frac{1}{8}$ & $20 \frac{7}{8}$ & India & $\cdots$ & $\begin{array}{c}\text { H.R.H. Duke of Edin- } \\
\text { burgh. }\end{array}$ \\
\hline $23 \frac{1}{2}$ & $29 \frac{1}{4}$ & $5 \frac{1}{8}$ & $16 \frac{3}{7}$ & Do. & $\cdots$ & Lieut. F. E. Shafto-Adair. \\
\hline $23 \frac{\mathrm{I}}{2}$ & ... & $\cdots$ & $\cdots$ & Do. & $\cdots$ & $\begin{array}{l}\text { Sir S. Baker, Bart., "Wild } \\
\text { Beasts and their Ways." }\end{array}$ \\
\hline $23 \frac{1}{t}$ & $33^{3}$ & $5 \frac{1}{2}$ & I $8 \frac{1}{2}$ & Do. & $\cdots$ & $\begin{array}{l}\text { Hume Collection, British } \\
\text { Museum. }\end{array}$ \\
\hline $23^{\frac{1}{4}}$ & $34 \frac{1}{1}$ & $4 \frac{7}{8}$ & $19 \frac{3}{8}$ & Do. & $\cdots$ & Do. \\
\hline $23 \frac{1}{4}$ & $29 \frac{7}{3}$ & $4 \frac{5}{8}$ & $15 \frac{1}{2}$ & Do. & $\cdots$ & Do. \\
\hline 23 & $30 \frac{1}{8}$ & $5 \frac{1}{8}$ & $13 \frac{3}{8}$ & North & India & $\begin{array}{l}\text { Sir Victor Brooke's Collec- } \\
\text { tion, } 1869 \text {. }\end{array}$ \\
\hline $22 \frac{3}{4}$ & $32 \frac{5}{8}$ & 5 & $15 \frac{7}{8}$ & India & $\cdots$ & Martyn Kennard. \\
\hline $22 \frac{5}{8}$ & $30 \frac{1}{8}$ & $5^{\frac{3}{1}}$ & II $\frac{1}{8}$ & Do. & ... & $\begin{array}{l}\text { Mrs. Stirling, British Mu- } \\
\text { seum. }\end{array}$ \\
\hline$* 22 \frac{5}{8}$ & $32 \frac{3}{4}$ & $5^{\frac{3}{4}}$ & $\cdots$ & Do. & $\cdots$ & Hon. Walter Rothschild. \\
\hline $22 \frac{1}{2}$ & $\cdots$ & $5^{\frac{1}{t}}$ & $18 \frac{1}{2}$ & Do. & $\cdots$ & Lieut. F. E. S. Adair. \\
\hline $22 \frac{3}{6}$ & $33 \frac{5}{8}$ & 5 & $23 \frac{1}{2}$ & Do. & $\cdots$ & Martyn Kennard. \\
\hline $22 \frac{1}{4}$ & $\cdots$ & $4 \frac{3}{4}$ & 13 & Do. & $\cdots$ & Capt. Henry Streatfield. \\
\hline $22 \frac{1}{8}$ & $3 \mathbf{I}$ & 5 & $18 \frac{7}{8}$ & Do. & $\cdots$ & Lord Ashburton. \\
\hline 22 & 30 & 5 & $14 \frac{3}{4}$ & Do. & $\cdots$ & IV. E. Rowley. \\
\hline 22 & 29 & $4 \frac{3}{3}$ & $16 \frac{1}{2}$ & Do. & $\cdots$ & Martyn Kennard. \\
\hline 22 & $29 \frac{1}{2}$ & $4 \frac{3}{8}$ & I $8 \frac{1}{8}$ & Do. & $\cdots$ & Sir Robt. Harvey, Bart. \\
\hline $2 I \frac{7}{8}$ & $32 \frac{3}{8}$ & $4 \frac{3}{8}$ & $14 \frac{7}{8}$ & Do. & $\cdots$ & F. J. Horniman. \\
\hline $21 \frac{3}{4}$ & $3 I^{\frac{1}{2}}$ & $4^{\frac{1}{2}}$ & I $8 \frac{5}{8}$ & Do. & $\cdots$ & Major Cumberland. \\
\hline $21 \frac{1}{2}$ & $28 \frac{5}{8}$ & $4 \frac{7}{8}$ & $17 \frac{5}{8}$ & Do. & $\cdots$ & Martyn Kennard. \\
\hline $2 \mathrm{I} \frac{3}{8}$ & $27 \frac{3}{8}$ & $4^{\frac{3}{4}}$ & $18 \frac{1}{4}$ & Do. & $\cdots$ & Do. \\
\hline $21 \frac{1}{\frac{1}{4}}$ & $28 \frac{5}{8}$ & $4 \frac{5}{8}$ & I6 & Do. & $\cdots$ & Sir Robert Harvey, Bart. \\
\hline $2 I \frac{1}{4}$ & 30 & $4 \frac{7}{8}$ & $18 \frac{1}{4}$ & Do. & $\ldots$ & $\begin{array}{l}\text { H.R.H. Duke of Edin- } \\
\text { burgh. }\end{array}$ \\
\hline $21 \frac{1}{4}$ & $33 \frac{1}{4}$ & $4 \frac{5}{8}$ & I67 & Do. & $\cdots$ & Martyn Kennard. \\
\hline$\cdots$ & 28 & $4 \frac{1}{2}$ & I6 & Do. & $\ldots$ & Capt. G. Campbell. \\
\hline
\end{tabular}




\section{BLACKBUCK or INDIAN ANTELOPE (Antilope cervicapra)-} continued.

\begin{tabular}{|c|c|c|c|c|c|c|}
\hline & Length. & & & & & \\
\hline Straight. & Round Curve. & & & & & \\
\hline $21 \frac{1}{8}$ & $28 \frac{3}{4}$ & 5 & I $8 \frac{1}{4}$ & India & ... & Lord Ashburion. \\
\hline $21 \frac{1}{8}$ & $31 \frac{7}{8}$ & $4^{\frac{1}{2}}$ & $7^{\frac{3}{4}}$ & Do. & $\cdots$ & The Hon. Charles Ellis. \\
\hline $2 I$ & $25 \frac{3}{4}$ & $4 \frac{7}{8}$ & $15 \frac{1}{4}$ & Do. & $\ldots$ & H. C. V. Hunter. \\
\hline $2 I$ & $\cdots$ & $5^{\frac{1}{4}}$ & I 8 & Do. & $\ldots$ & C. H. Seeley. \\
\hline 21 & 32 & $5^{\frac{1}{t}}$ & $12 \frac{5}{8}$ & Do. & $\ldots$ & $\begin{array}{l}\text { Hume Collection, British } \\
\text { Museum. }\end{array}$ \\
\hline $20 \frac{3}{4}$ & $28 \frac{1}{8}$ & $5 \frac{1}{4}$ & I 4 & Do. & $\cdots$ & $\begin{array}{l}\text { Maj.-Gen, Arthur Ellis, } \\
\text { C.S.I., I } 859 \text {. }\end{array}$ \\
\hline $20 \frac{1}{2}$ & $30 \frac{5}{8}$ & $4^{\frac{3}{4}}$ & 14 & Do. & $\cdots$ & Do. \\
\hline $20 \frac{1}{2}$ & 28 & $5 \frac{1}{8}$ & $2 I \frac{1}{2}$ & Jullunder & $\cdots$ & $\begin{array}{l}\text { Sir Victor Brooke's Collec- } \\
\text { tion, I } 869 .\end{array}$ \\
\hline $20 \frac{1}{2}$ & 28 & $4 \frac{7}{8}$ & 14 & India & $\cdots$ & H. C. V. Hunter. \\
\hline $20 \frac{1}{2}$ & $28 \frac{3}{8}$ & 5 & $19 \frac{3}{4}$ & Do. & $\cdots$ & Martyn Kennard. \\
\hline$\cdots$ & 27 & $\begin{array}{c}\text { (owner's mea- } \\
\text { surement) }\end{array}$ & $\ldots$ & & $\cdots$ & Capt. Townley Parker. \\
\hline $20 \frac{1}{2}$ & $28 \frac{3}{8}$ & $5 \frac{3}{8}$ & $18 \frac{1}{4}$ & Do. & $\cdots$ & $\begin{array}{l}\text { H.R.H. Duke of Edin- } \\
\text { burgh. }\end{array}$ \\
\hline $20 \frac{3}{8}$ & 27 & $4 \frac{5}{8}$ & $8 \frac{3}{4}$ & Do. & $\cdots$ & British Museum. \\
\hline $20 \frac{1}{8}$ & $26 \frac{5}{8}$ & $5^{\frac{1}{4}}$ & I $3 \frac{1}{8}$ & Central In & ndia & Do. \\
\hline $20 \frac{1}{8}$ & $29 \frac{1}{t}$ & 5 & $16 \frac{7}{8}$ & India & $\cdots$ & H. C. V. Hunter. \\
\hline 20 & $27 \frac{3}{4}$ & $5 \frac{1}{4}$ & $16 \frac{3}{8}$ & Do. & $\cdots$ & H. R.H. Le Duc d'Orleans. \\
\hline 20 & 27 & 5 & $12 \frac{1}{2}$ & Do. & $\cdots$ & $\begin{array}{l}\text { Maj.-Gen, Arthur Ellis, } \\
\text { C.S. I., I } 859 \text {. }\end{array}$ \\
\hline $19 \frac{7}{8}$ & $26 \frac{1}{4}$ & $5 \frac{5}{8}$ & I I $\frac{1}{2}$ & Do. & $\cdots$ & H. C. V. Hunter. \\
\hline $19 \frac{7}{8}$ & $26 \frac{1}{2}$ & 5 & $18 \frac{5}{8}$ & Do. & $\ldots$ & Martyn Kennard. \\
\hline $19 \frac{3}{4}$ & 24 & $4 \frac{5}{8}$ & $15 \frac{3}{8}$ & Do. & $\cdots$ & $\begin{array}{l}\text { H.R.H. Duke of Edin- } \\
\text { burgh. }\end{array}$ \\
\hline $19 \frac{5}{8}$ & $29 \frac{3}{t}$ & $5 \frac{1}{8}$ & $16 \frac{1}{8}$ & Nepal & $\ldots$ & British Museum. \\
\hline $19 \frac{1}{2}$ & $\cdots$ & $4 \frac{3}{4}$ & $19 \frac{1}{4}$ & India & $\cdots$ & Noel Fenwick. \\
\hline $19 \frac{1}{2}$ & $\begin{array}{l}\text { (owner's mea- } \\
\text { surements) }\end{array}$ & $5 \frac{1}{2}$ & ... & H. Haran & $\cdots$ & J. H. Mann. \\
\hline $19 \frac{1}{4}$ & $25^{\frac{1}{4}}$ & $4 \frac{3}{4}$ & $14 \frac{1}{8}$ & India & $\cdots$ & F. J. Horniman. \\
\hline $18 \frac{7}{8}$ & $27 \frac{1}{8}$ & $4 \frac{3}{4}$ & $16 \frac{7}{8}$ & Do. & ... & $\begin{array}{l}\text { Sir Victor Brooke's Collec. } \\
\text { tion. }\end{array}$ \\
\hline $18 \frac{7}{8}$ & $25 \frac{3}{4}$ & $4 \frac{5}{8}$ & $15 \frac{1}{3}$ & Do. & $\cdots$ & J. Carr-Saunders. \\
\hline
\end{tabular}


BLACKBUCK or INDIAN ANTELOPE (Antilope cervicapra)continued.

\begin{tabular}{|c|c|c|c|c|c|c|}
\hline & Length. & & & & & \\
\hline Straight. & Round Curve. & & & & & \\
\hline $\begin{array}{l}1 S_{4}^{3} \\
\mathbf{I} S_{4}^{3}\end{array}$ & $\begin{array}{l}25 \\
25 \frac{1}{2}\end{array}$ & $\begin{array}{l}4 \frac{1}{8} \\
5 \frac{\pi}{5}\end{array}$ & $\begin{array}{l}\mathrm{I} 2_{\frac{3}{4}}^{\frac{3}{4}} \\
\mathrm{I} 4^{\frac{3}{4}}\end{array}$ & $\begin{array}{l}\text { Madras } \\
\text { India }\end{array}$ & $\cdots$ & $\begin{array}{l}\text { Sir Walter Elliot, Bart, } \\
\text { British Museum, } \\
\text { H. R. H. Le Duc d'Orleans. }\end{array}$ \\
\hline I $S \frac{1}{3}$ & $2 S \bar{z}$ & $5 i$ & $12 \frac{1}{8}$ & Do. & $\cdots$ & H.R.H. Duke of Edin- \\
\hline$I S_{g}^{1}$ & $20 \%$ & $5+\frac{1}{5}$ & $10 \frac{3}{8}$ & Do. & $\ldots$ & $\begin{array}{l}\text { Hume Collection, British } \\
\text { Museum. }\end{array}$ \\
\hline $\mathbf{I} S \frac{1}{3}$ & 26 & 5 & $13 \frac{1}{1}$ & Do. & $\cdots$ & East India Club. \\
\hline $\mathbf{I} S_{3}^{3}$ & $25 \frac{7}{8}$ & $5 \frac{1}{2}$ & $15 \frac{3}{4}$ & Aligur & $\ldots \mid$ & St. George Littledale, 1877 . \\
\hline IS & $\begin{array}{l}\text { (killed by a } \\
\text { Cheetah) }\end{array}$ & 4 & $\begin{array}{c}\text { (owner's mea- } \\
\text { surement) }\end{array}$ & Seroom nus & |ggar| & J. H. Mann. \\
\hline $17 ?$ & $24 \frac{1}{4}$ & 5 & $7 \frac{1}{4}$ & Meerut & $\ldots$ & $\begin{array}{l}\text { R. Lydekker, British Mu- } \\
\text { seum. }\end{array}$ \\
\hline $177_{1}^{3}$ & 27 & $4 \%$ & $10 \frac{1}{4}$ & India & $\ldots$ & Sir Robert Harvey, Bart. \\
\hline 173 & $2+\frac{1}{2}$ & $4 i$ & $15^{\frac{3}{4}}$ & Aligur & $\ldots$ & St. George Littledale, $\mathbf{1} 877$. \\
\hline $17 \frac{1}{2}$ & $23 \frac{1}{1}$ & $44^{3}$ & I $2 \frac{1}{2}$ & Burmah & ... & British Museum. \\
\hline $178^{3}$ & $22 \frac{1}{4}$ & 5 & $9 \frac{5}{8}$ & India & $\cdots \mid$ & Sir Robert Harvey, Bart. \\
\hline 17 & $21 \frac{3}{4}$ & $5 \frac{1}{2}$ & 17 & Aligur & ... & St. George Littledale, 1877. \\
\hline 16 & $\ldots$ & 5 & $\begin{array}{c}\text { (owner's mea- } \\
\text { surement) }\end{array}$ & H. Haran & $\ldots$ & J. H. Mann. \\
\hline
\end{tabular}





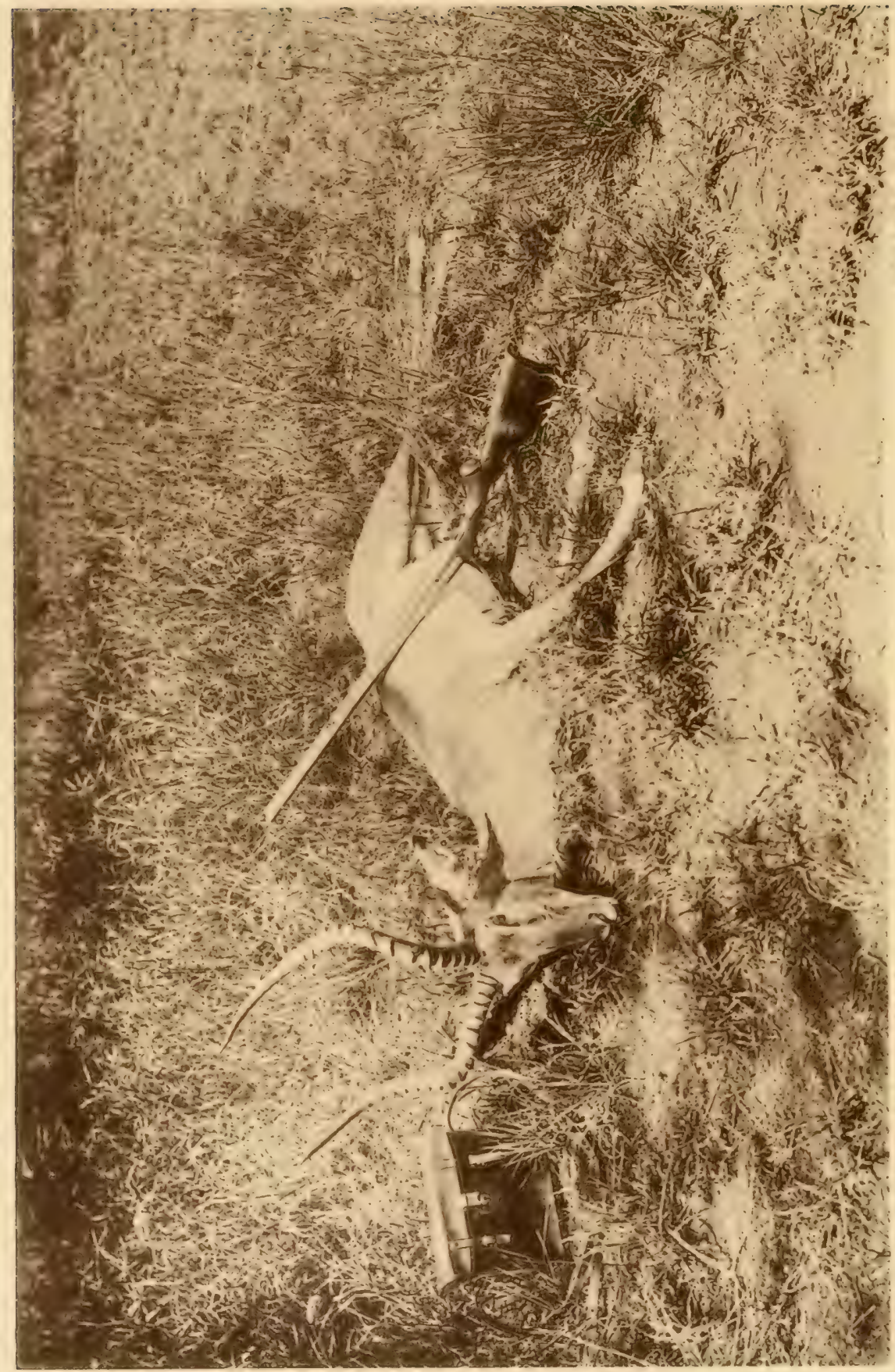




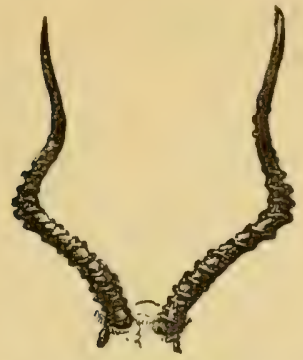

IMPALA ANTELOPE (Æpyceros melampus).

Bechuana name_-"Pallah."

Habitat-South, East, and West Africa.

\begin{tabular}{|c|c|c|c|c|c|c|}
\hline \multicolumn{2}{|c|}{ Length. } & \multirow{2}{*}{$\begin{array}{l}\text { Circum- } \\
\text { ference. }\end{array}$} & \multirow{2}{*}{ Tip to Tip. } & \multirow{2}{*}{$\begin{array}{l}\text { Widest } \\
\text { outside. }\end{array}$} & \multirow{2}{*}{ Habitat. } & \multirow{2}{*}{ Owner. } \\
\hline Straight. & $\begin{array}{l}\text { Round } \\
\text { Bend. }\end{array}$ & & & & & \\
\hline $21 \frac{1}{2}$ & $\ldots$ & 6 & I6 & 17 & East Africa... & Rowland Ward's Collection. \\
\hline $21 \frac{1}{8}$ & 25 & $5^{\frac{7}{8}}$ & I $7 \frac{5}{8}$ & $\cdots$ & Do. $\quad \ldots$ & F. J. Jackson. \\
\hline $2 \mathrm{I}$ & $25^{\frac{1}{4}}$ & $5 \frac{7}{8}$ & I 8 & $\cdots$ & Do. & Do. \\
\hline 21 & $27 \frac{1}{2}$ & $5^{\frac{7}{5}}$ & $14 \frac{1}{4}$ & $\cdots$ & South Africa & W. Burchell, British Museum. \\
\hline $20 \frac{1}{2}$ & .. & $5^{\frac{3}{4}}$ & 15 & IS & East Africa... & W. Astor Chanler. \\
\hline $20 \frac{1}{2}$ & $\ldots$ & $5 \frac{5}{8}$ & II $\frac{1}{2}$ & ... & Do. & H. C. V. Hunter. \\
\hline $20 \frac{1}{2}$ & $25^{\frac{1}{4}}$ & 5 & $12 \frac{5}{8}$ & ... & Do. & F. J. Jackson. \\
\hline $20 \frac{3}{8}$ & $23 \frac{3}{x}$ & 6 & $14 \frac{1}{2}$ & ... & Do. & Sir Robert Harvey, Bart. \\
\hline 20 & $\ldots$ & ... & $\ldots$ & I 6 & Chobe River & $\begin{array}{l}\text { F. C. Selous, I879, " A Hun } \\
\text { ter's Wanderings." }\end{array}$ \\
\hline 20 & ... & ... & $\ldots$ & IS & East Africa ... & $\begin{array}{l}\text { Sir J. Willoughby, "East Africa } \\
\text { and its Big Game." }\end{array}$ \\
\hline 20 & $\cdots$ & $5^{\frac{3}{4}}$ & $14 \frac{3}{4}$ & IS & Africa & R. P. Carrol. \\
\hline $19 \frac{3}{4}$ & $24 \frac{7}{8}$ & $5 \frac{7}{8}$ & II $\frac{5}{8}$ & $\cdots$ & Do. & Sir Edmund G. Loder, Bart. \\
\hline $19 \frac{3}{t}$ & $24 \frac{1}{2}$ & $5 \frac{1}{2}$ & $12 \frac{1}{4}$ & ... & South Africa & F. C. Selous. \\
\hline $19 \frac{5}{8}$ & $23 \frac{3}{8}$ & $5^{\frac{3}{4}}$ & $10 \frac{1}{4}$ & ... & Do. & The late J. S. Jameson. \\
\hline $19 \frac{1}{2}$ & $24 \frac{1}{4}$ & 6 & $15 \frac{3}{8}$ & ... & East Africa ... & Sir Robert Harvey, Bart. \\
\hline $19 \frac{1}{2}$ & $23 \frac{7}{8}$ & $5^{\frac{3}{4}}$ & $13 \frac{3}{4}$ & ... & Do. & H. C. V. Hunter. \\
\hline $19 \frac{1}{4}$ & $24 \frac{3}{8}$ & 6 & $9 \frac{5}{8}$ & ... & Do. & Sir Robert Harvey, Bart. \\
\hline I9! & $25 \frac{7}{8}$ & 6 & 7 & ... & Do. & H. C. V. Hunter. \\
\hline $19 \frac{1}{8}$ & $23 \frac{3}{8}$ & 6 & $10 \frac{1}{4}$ & $\ldots$ & South Africa & A. Beit. \\
\hline
\end{tabular}


IMPALA ANTELOPE (स्सpyceros melampus)-continued.

\begin{tabular}{|c|c|c|c|c|c|c|}
\hline \multicolumn{2}{|c|}{ Length. } & \multirow{2}{*}{$\begin{array}{l}\text { Circum- } \\
\text { ference. }\end{array}$} & \multirow{2}{*}{ Tip to Tip. } & \multirow{2}{*}{$\begin{array}{l}\text { Widest } \\
\text { outside. }\end{array}$} & \multirow[b]{2}{*}{ Habitat. } & \multirow[b]{2}{*}{ Owner. } \\
\hline Straight. & $\begin{array}{l}\text { Round } \\
\text { Bend. }\end{array}$ & & & & & \\
\hline $19 \frac{1}{8}$ & $24 \frac{1}{2}$ & $5 \frac{5}{8}$ & $12 \frac{3}{8}$ & $\ldots$ & South Africa & British Museum. \\
\hline 19 & $24 \frac{1}{2}$ & 6 & II $\frac{5}{8}$ & $\ldots$ & $\mid \begin{array}{lll}\ldots & \ldots & \ldots\end{array}$ & | J. Carr-Saunders. \\
\hline 19 & $24 \frac{1}{8}$ & $5 \frac{7}{8}$ & $8 \frac{1}{8}$ & $\cdots$ & East Africa ... & H. C. V. Hunter. \\
\hline 19 & $\cdots$ & $5^{\frac{1}{2}}$ & $12 \frac{1}{2}$ & is & South Africa & Gen. Mathews. \\
\hline 19 & $23 \frac{1}{2}$ & $5 \frac{7}{8}$ & $14 \frac{1}{4}$ & $\cdots$ & East Africa... & Sir Robert Harvey, Bart. \\
\hline 19 & $\cdots$ & 6 & $2 \mathrm{I}$ & 20 & South Africa & Rowland Ward's Collection. \\
\hline $18 \frac{7}{8}$ & 24 & $5 \frac{1}{2}$ & 14 & $\cdots$ & | Limpopo River & W. C. Oswell. \\
\hline I $8 \frac{3}{4}$ & $23 \frac{1}{4}$ & 6 & $12 \frac{1}{t}$ & $\ldots$ & $\begin{array}{l}\text { Kaoko Land, } \\
\text { WVest Africa. }\end{array}$ & Capt. F. Cookson. \\
\hline $18 \frac{5}{8}$ & 23 & $6 \frac{1}{8}$ & II $\frac{3}{8}$ & $\cdots$ & East Africa ... & H. C. V. Hunter. \\
\hline $18 \frac{5}{8}$ & $24 \frac{3}{8}$ & $5^{\frac{3}{4}}$ & $10 \frac{1}{x}$ & $\cdots$ & Do. $\quad \ldots$ & Sir Robert Harvey, Bart. \\
\hline $18 \frac{5}{8}$ & 23 & $5 \frac{7}{8}$ & $13 \frac{3}{8}$ & $\cdots$ & Do. $\quad \ldots$ & Do. \\
\hline$\cdots$ & $23 \frac{5}{8}$ & $5^{\frac{1}{2}}$ & $9 \frac{3}{8}$ & $\cdots$ & Do. $\quad \ldots$ & Hon. Walter Rothschild. \\
\hline I $8 \frac{1}{2}$ & $23 \frac{1}{2}$ & $5 \frac{7}{8}$ & $I^{1} \frac{1}{4}$ & $\cdots$ & South Africa & The late J. S. Jameson. \\
\hline I 83 & $22 \frac{1}{4}$ & $5 \frac{1}{2}$ & $13 \frac{1}{8}$ & $\cdots$ & East Africa ... & H. C. V. Hunter. \\
\hline I $8 \frac{1}{8}$ & $25 \frac{1}{8}$ & $5 \frac{1}{2}$ & $10 \frac{1}{2}$ & $\cdots$ & South Africa & F. C. Selous. \\
\hline IS & $22 \frac{1}{2}$ & $5^{\frac{3}{4}}$ & $10 \frac{5}{8}$ & $\cdots$ & East Africa ... & Sir Robert Harvey, Bart. \\
\hline I8 & $21 \frac{1}{2}$ & $5 \frac{5}{8}$ & $12 \frac{3}{4}$ & $\cdots$ & South Africa & A. Beit. \\
\hline $17 \frac{3}{1}$ & $22 \frac{7}{8}$ & $5 \frac{5}{8}$ & $6 \frac{1}{4}$ & $\cdots$ & East Africa... & Sir Robert Harvey, Bart. \\
\hline $17 \frac{3}{4}$ & $22 \frac{3}{4}$ & $5 \frac{1}{2}$ & $13 \frac{1}{8}$ & $\cdots$ & South Africa & British Museum. \\
\hline $17 \frac{5}{8}$ & $21 \frac{3}{4}$ & 6 & $10 \frac{1}{8}$ & $\cdots$ & Do. & F. C. Selous. \\
\hline $17 \frac{1}{4}$ & $21 \frac{1}{2}$ & $5^{\frac{1}{2}}$ & $12 \frac{3}{4}$ & $\cdots$ & $\begin{array}{lll}\cdots & \cdots & \cdots\end{array}$ & E. Lort-Phillips. \\
\hline $17 \frac{1}{8}$ & $20 \frac{1}{2}$ & $5 \frac{7}{8}$ & $1 \mathrm{I} \frac{1}{2}$ & $\cdots$ & East Africa ... & Sir Robert Harvey, Bart. \\
\hline $17 \frac{1}{8}$ & $23 \frac{1}{4}$ & $5 \frac{5}{8}$ & $7 \frac{7}{8}$ & $14 \frac{3}{4}$ & Africa & Hon. A. O. Murray. \\
\hline $\mathbf{I} 7$ & $\cdots$ & $5 \frac{3}{4}$ & $8 \frac{3}{4}$ & 14 & South Africa & A. W. Davis. \\
\hline $16 \frac{3}{8}$ & $19 \frac{3}{8}$ & $5 \frac{3}{8}$ & $10 \frac{1}{4}$ & $\cdots$ & Do. & Sir Victor Brooke's Collection \\
\hline $16 \frac{3}{8}$ & $19 \frac{7}{8}$ & $4 \frac{3}{4}$ & $7 \frac{3}{8}$ & $\ldots$ & Do. & G. H. Banks. \\
\hline $16 \frac{1}{4}$ & $19 \frac{3}{4}$ & $5 \frac{1}{8}$ & $12 \frac{7}{8}$ & $\cdots$ & Do. & F. C. Selous. \\
\hline $15 \frac{7}{8}$ & $21 \frac{3}{4}$ & $5 \frac{3}{4}$ & $6 \frac{1}{2}$ & $\cdots$ & Do. & W. Burchell, British Museum \\
\hline
\end{tabular}




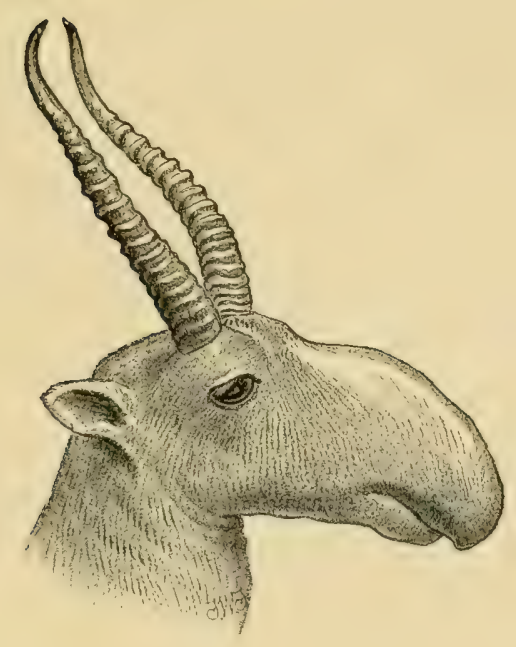

SAIGA (Saiga tartarica).

Habitat-Eastern Europe and Western Asia.

\begin{tabular}{|c|c|c|c|c|c|}
\hline Length. & $\begin{array}{l}\text { Circum- } \\
\text { ference. }\end{array}$ & Tip to Tip. & Habitat. & & Owner. \\
\hline $14 \frac{3}{8}$ & $5 \frac{1}{4}$ & $3 \frac{1}{2}$ & $\cdots$ & $\cdots$ & Hon. Walter Rothschild. \\
\hline $13 \frac{3}{4}$ & 5 & ... & ... & $\cdots$ & Sir Edmund G. Loder, Bart. \\
\hline $13^{\frac{3}{4}}$ & $4 \frac{3}{8}$ & $3 \frac{1}{2}$ & $\cdots$ & ... & Sir Victor Brooke's Collection. \\
\hline $13 \frac{5}{8}$ & 5 & $5 \frac{1}{2}$ & Sarepta, South & & British Museum. \\
\hline $13 \frac{1}{8}$ & $5 \frac{33}{8}$ & $4 \frac{3}{8}$ & $\ldots \quad \ldots$ & ... & Hon. Walter Rothschild. \\
\hline $12 \frac{7}{8}$ & $5 \frac{3}{8}$ & $\begin{array}{l}\text { (single } \\
\text { horn) }\end{array}$ & Russia $\ldots$ & $\ldots$ & British Museum. \\
\hline $12 \frac{7}{8}$ & $4 \frac{5}{8}$ & $5 \frac{5}{8}$ & $\begin{array}{l}\text { Sarepta, South } \\
\text { Russia. }\end{array}$ & & Do. \\
\hline $12 \frac{7}{8}$ & $4 \frac{5}{8}$ & $\begin{array}{l}\text { (single } \\
\text { horn) }\end{array}$ & Ruscia $\ldots$ & $\ldots$ & Do. \\
\hline $12 \frac{3}{4}$ & $4 \frac{7}{8}$ & $4 \frac{1}{2}$ & $\cdots$ & $\cdots$ & Do. \\
\hline$I I \frac{1}{2}$ & $4 \frac{3}{4}$ & $4 \frac{5}{x}$ & $\cdots$ & ... & Rowland Ward's Collection. \\
\hline $11 \frac{1}{4}$ & $4^{\frac{3}{4}}$ & 5 & $\ldots$ & ... & British Museum. \\
\hline II & $4 \frac{7}{8}$ & $4 \frac{1}{2}$ & Siberia ... & $\cdots$ & Do. \\
\hline
\end{tabular}




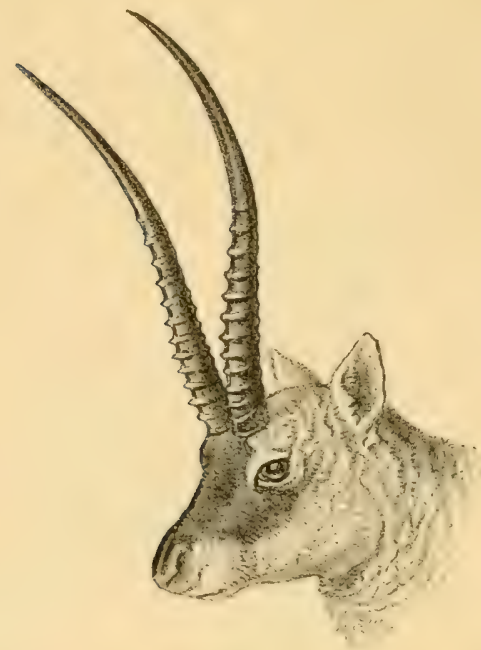

THIBET ANTELOPE (CHIRU) (Pantholops hodgsoni).

Habitat-Western Thibet and Turkestan.

\begin{tabular}{|c|c|c|c|c|c|c|}
\hline Length. & $\begin{array}{l}\text { Circum- } \\
\text { ference. }\end{array}$ & Tip to Tip. & \multicolumn{3}{|c|}{ Habitat. } & Owner. \\
\hline $27 \frac{7}{8}$ & $5 \frac{7}{8}$ & $13 \frac{1}{2}$ & India & $\ldots$ & $\ldots$ & A. O. Hume, Private Collection. \\
\hline $27 \frac{5}{8}$ & $5^{\frac{1}{2}}$ & $I I \frac{1}{4}$ & Do. & $\ldots$ & $\ldots$ & Sir Robert Harvey, Bart. \\
\hline $27 \frac{1}{8}$ & $5 \frac{1}{2}$ & $15 \frac{1}{8}$ & Do. & $\ldots$ & $\ldots$ & A. O. Hume, Private Collection. \\
\hline $26 \frac{3}{8}$ & $5 \frac{7}{8}$ & $13 \frac{5}{8}$ & Do. & $\ldots$ & $\cdots$ & Hon. Charles Ellis. \\
\hline $25 \frac{1}{2}$ & $5^{\frac{1}{2}}$ & $12 \frac{1}{2}$ & Do. & $\ldots$ & $\ldots$ & Capt. G. Campbell. \\
\hline $25 \frac{3}{8}$ & $5 \frac{5}{8}$ & $12 \frac{1}{2}$ & Do. & $\cdots$ & $\ldots$ & Itume Collection, Brilish Museum. \\
\hline $25^{\frac{1}{4}}$ & $5^{3 \frac{3}{4}}$ & $12 \frac{3}{4}$ & Do. & $\cdots$ & $\ldots$ & Do. \\
\hline $24 \frac{7}{8}$ & $5^{\frac{3}{4}}$ & $13 \frac{7}{8}$ & Do. & $\cdots$ & $\cdots$ & Do. \\
\hline $24^{\frac{3}{4}}$ & $5^{\frac{3}{4}}$ & Io & Do. & $\cdots$ & $\ldots$ & Do. \\
\hline $24^{\frac{3}{1}}$ & $5 \frac{5}{8}$ & II $\frac{1}{2}$ & Do. & $\cdots$ & $\cdots$ & Sir Edmund G. Loder, Bart. \\
\hline $24 \frac{3}{4}$ & $5 \frac{5}{8}$ & $14 \frac{1}{2}$ & Do. & $\cdots$ & $\cdots$ & Hume Collection, British Museum. \\
\hline $24 \frac{5}{8}$ & $5 \frac{3}{8}$ & $11 \frac{3}{4}$ & Do. & $\cdots$ & $\ldots$ & Sir Robert Harvey, Bart. \\
\hline $24 \frac{3}{8}$ & $5 a^{\circ}$ & $15 \frac{1}{4}$ & Do. & $\cdots$ & $\ldots$ & Hume Collection, British Museum. \\
\hline
\end{tabular}




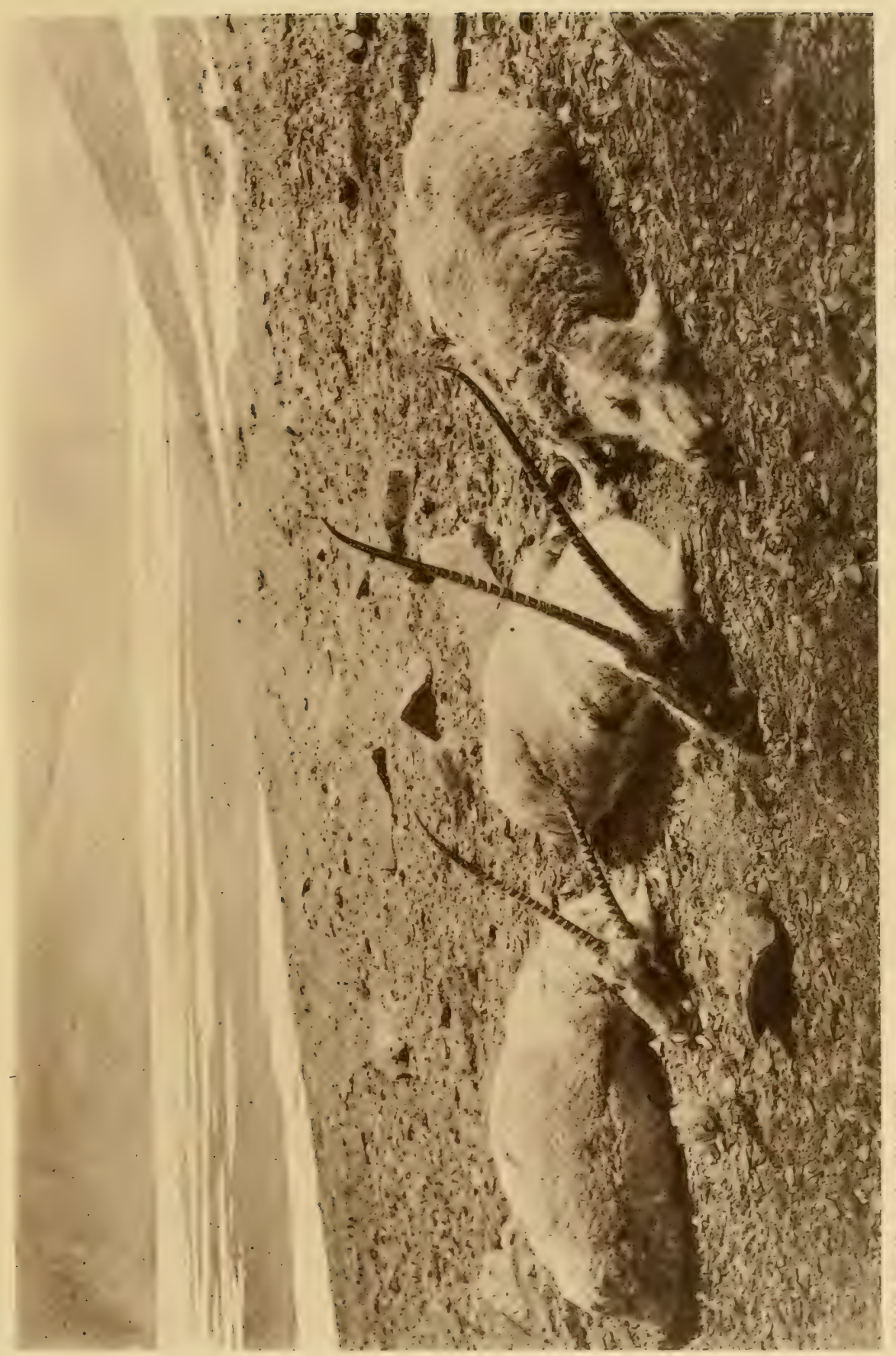



THIBET ANTELOPE (CHIRU) (Pantholops hodgsoni)-continuid.

\begin{tabular}{|c|c|c|c|c|c|c|}
\hline Length. & $\begin{array}{l}\text { Circum- } \\
\text { ference. }\end{array}$ & Tip to Tip. & \multicolumn{3}{|c|}{ Habitat. } & Owner. \\
\hline $24 \frac{3}{8}$ & $5^{\frac{1}{4}}$ & $16 \frac{1}{2}$ & India & $\cdots$ & $\cdots$ & British Museum. \\
\hline $24 \frac{x}{4}$ & $5 \frac{1}{2}$ & II & Do. & $\cdots$ & $\cdots$ & Sir Robert Harvey, Bart. \\
\hline $24 \frac{1}{4}$ & $5 \frac{1}{8}$ & $13 \frac{3}{4}$ & Do. & $\cdots$ & $\cdots$ & British Museum. \\
\hline 24 & $5 \frac{1}{2}$ & $12 \frac{1}{2}$ & Do. & $\cdots$ & $\cdots$ & Hume Collection, British Museum. \\
\hline 24 & $5 \frac{3}{8}$ & $14 \frac{3}{8}$ & Do. & $\cdots$ & $\cdots$ & B. H. Hodgson, British Museum. \\
\hline $23 \frac{3}{4}$ & $5 \frac{1}{4}$ & I $4 \frac{1}{2}$ & Do. & $\cdots$ & $\ldots$ & British Museum. \\
\hline $23 \frac{5}{8}$ & $5^{\frac{1}{4}}$ & II $\frac{5}{8}$ & Do. & $\cdots$ & $\cdots$ & H. C. V. Hunter. \\
\hline $23 \frac{1}{2}$ & 5 & $14^{\frac{3}{4}}$ & Do. & $\ldots$ & $\ldots$ & Reginald Beech. \\
\hline $23 \frac{3}{8}$ & $5 \frac{1}{8}$ & $10 \frac{3}{8}$ & Do. & $\cdots$ & $\cdots$ & H. C. V. Hunter. \\
\hline $23 \frac{3}{8}$ & $5 \frac{1}{8}$ & $9 \frac{7}{8}$ & Do. & $\cdots$ & $\ldots$ & Do. \\
\hline $23^{\frac{1}{ \pm}}$ & $5 \frac{3}{8}$ & $14 \frac{5}{8}$ & Do. & ... & $\ldots$ & Hon. Walter Rothschild. \\
\hline $23 \frac{1}{4}$ & $5^{\frac{1}{4}}$ & $14 \frac{1}{8}$ & Do. & $\ldots$ & $\ldots$ & Rowland Ward's Collection. \\
\hline $23 \frac{1}{t}$ & $5^{\frac{1}{x}}$ & $10 \frac{7}{8}$ & Do. & $\ldots$ & $\ldots$ & J. Carr-Saunders. \\
\hline $23 \frac{1}{8}$ & $5 \frac{1}{8}$ & $9 \frac{1}{2}$ & Do. & $\cdots$ & $\ldots$ & Sir Victor Brooke's Collection. \\
\hline $22 \frac{7}{8}$ & $5 \frac{3}{8}$ & $13 \frac{1}{2}$ & Ladak & $(\mathbf{I} 5,000 \mathrm{f}$ & ft.) & R. Lydekker, British Museum. \\
\hline $22 \frac{3}{4}$ & $5 \frac{1}{2}$ & $11 \frac{3}{8}$ & $\cdots$ & .. & $\ldots$ & H. C. V. Hunter. \\
\hline $22 \frac{5}{8}$ & $5 \frac{1}{8}$ & $15 \frac{1}{8}$ & Nepal & $\cdots$ & $\cdots$ & B. H. Hodgson, British Museum. \\
\hline $22 \frac{1}{2}$ & 5 & 12 & India & ... & $\cdots$ & F. Morrice. \\
\hline $22 \frac{33}{8}$ & $5 \frac{5}{8}$ & $10 \frac{3}{4}$ & Do. & ... & $\ldots$ & H. C. V. Hunter. \\
\hline $22 \frac{3}{8}$ & $5^{\frac{1}{4}}$ & II $\frac{1}{8}$ & Do. & $\cdots$ & $\cdots$ & Hen. Charles Ellis. \\
\hline 22 & $5 \frac{1}{8}$ & II $\frac{3}{5}$ & Do. & $\ldots$ & $\cdots$ & II. C. V. Hunter. \\
\hline $21 \frac{3}{4}$ & $5 \frac{3}{8}$ & $12 \frac{1}{4}$ & Do. & $\cdots$ & $\cdots$ & Hume Collection, British Múseum. \\
\hline $20 \frac{5}{8}$ & 5 & II $\frac{7}{8}$ & Do. & $\cdots$ & $\cdots$ & H. C. V. Hunter. \\
\hline $20 \frac{1}{2}$ & 5 & $9 \frac{1}{2}$ & Do. & $\cdots$ & $\cdots$ & Capt. M. Murphy. \\
\hline $20_{\mathrm{s}}^{3}$ & $5 \frac{1}{4}$ & $10 \frac{1}{4}$ & Do. & $\cdots$ & $\cdots$ & Hume Collection, British Museum. \\
\hline $19 \frac{3}{4}$ & $5 \frac{1}{2}$ & $1 I_{\frac{1}{3}}$ & Do. & $\cdots$ & $\cdots$ & H. C. V. Hunter. \\
\hline I9 & $5 \frac{1}{8}$ & $9 \frac{3}{4}$ & Do. & $\cdots$ & $\cdots$ & Do. \\
\hline
\end{tabular}




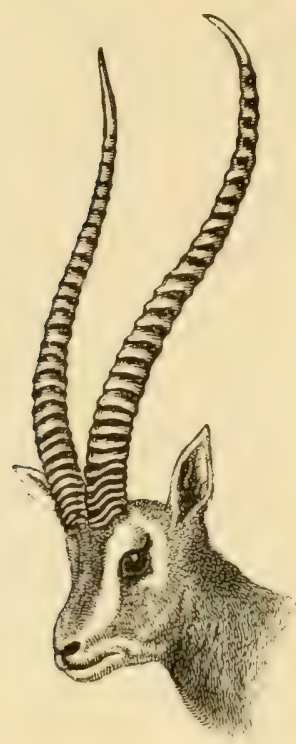

\section{GRANT'S GAZELLE (Gazella granti).}

[Sir Victor BROOkE, Bart., P.Z.S. I878, p. 723.]

GENERAL colour, very rich fawn tinged with purple, the neck and back in certain lights mottled like watered silk; this pattern is gained by indefinitely bounded bands of darker hair. Central facial band darker than the neck and body and strongly tinged with rufous on the forehead; a large black patch above the nostrils; large facial streaks very definite, white; dark facial streak black, extending from the base of the horn to the eye, which it almost encircles, and from the eye to above the corners of the mouth; posterior to the eye is a small white patch; external surface of the ear-conchs streaked with dark brown; dark lateral and pygal bands distinctly marked, the former grizzled, the latter very broad and jet-black; lower two thirds of the tail black, the upper third white; the tip of the tail is tufted; for the remainder of its length, it is furnished with coarse hairs which project at right angles to its axis; chin, lower surface of the head, breast, belly, inside of limbs, and 
rump white; the white of the rump extending forwards in an angle into the darker colour of the body; knee-brushes of moderate length, grizzled; horns of great length in both sexes, much compressed from side to side, marked by strong annulations which completely surround the horn for the proximal half of its length, but become obsolete on the posterior surface of its distal or upper half. The horns diverge at first gradually, then more boldly, their tips converging and giving to the set of the horns a remarkably perfect lyrate form.

\section{Comparison with allied Species.}

Gazella granti belongs, as pointed out in my former description, to the group of long-limbed large Gazelles which are distinguished by the white of the rump projecting in an angle into the fawncolour of the back and sides (vide P.Z.S. I873, p. 537, B.). The allied species are G. sœmmerringi, mohr, and dama. From all these it differs in the very much greater size, and in the form of the horns. It is also peculiar in the presence of the dark lateral band, which is wanting in the three above-named species, and in the remarkably definite black pygal band, which is only shown in very young specimens of $G$. sommerringi, is absent entirely in G. dama, and only indefinitely shown in G. mohr. In the wateredsilk mottling of the neck and back Gazella granti differs from all other known Gazelles.

Habitat-East Africa.

\begin{tabular}{c|c|c|c|c|l}
\hline Length. & $\begin{array}{c}\text { Circum- } \\
\text { ference. }\end{array}$ & Tip to Tip. & Habitat. & \multicolumn{1}{c}{ Owner. } \\
\hline 30 & $\ldots$ & Io & East Africa & $\ldots$ & $\begin{array}{l}\text { Sir John Willoughby, Bart., "East } \\
\text { Africa and its Big Game." }\end{array}$ \\
$28 \frac{1}{4}$ & $6 \frac{5}{8}$ & $15 \frac{1}{8}$ & Do. & $\ldots$ & F. J. Jackson. \\
$27 \frac{7}{8}$ & $6 \frac{5}{8}$ & $18 \frac{3}{8}$ & Do. & $\ldots$ & II. C. V. Hunter. \\
$27 \frac{5}{8}$ & $6 \frac{3}{4}$ & $21 \frac{1}{2}$ & Do. & $\ldots$ & F. J. Jackson. \\
$27 \frac{1}{4}$ & $6 \frac{1}{2}$ & $18 \frac{7}{8}$ & Do. & $\ldots$ & Sir Robert Harvey, Bart.
\end{tabular}


GRANT'S GAZELLE (Gazella granti)-continucd.

\begin{tabular}{|c|c|c|c|c|c|}
\hline Length. & $\begin{array}{l}\text { Circum- } \\
\text { ference. }\end{array}$ & Tip to Tip. & Habitat. & & Owner. \\
\hline 27 & $\cdots$ & 16 & East Africa & $\ldots$ & Sir John Willoughby, Bart. \\
\hline $26 \frac{3}{4}$ & $6 \frac{3}{4}$ & $12 \frac{1}{2}$ & Do. & $\ldots$ & H. C. V. Hunter. \\
\hline $26 \frac{1}{2}$ & $7 \frac{1}{2}$ & I $3 \frac{1}{4}$ & Do. & $\ldots$ & F. J. Jackson. \\
\hline $26 \frac{3}{8}$ & $6 \frac{7}{8}$ & $17 \frac{7}{8}$ & Do. & $\ldots$ & H. C. V. Hunter. \\
\hline $26 \frac{1}{ \pm}$ & $6 \frac{3}{8}$ & $16 \frac{3}{4}$ & Do. & $\cdots$ & Sir Victor Brooke's Collection. \\
\hline 26 & $6 \frac{3}{4}$ & $15 \frac{3}{4}$ & Do. & $\ldots$ & Sir Robert Harvey, Bart. \\
\hline $25 \frac{3}{4}$ & $6 \frac{7}{8}$ & I $5 \frac{5}{8}$ & Do. & $\ldots$ & Do. \\
\hline $25 \frac{1}{2}$ & $6 \frac{5}{8}$ & I I & Do. & $\cdots$ & F. J. Jackson. \\
\hline $25 \frac{1}{3}$ & 7 & $14 \frac{1}{2}$ & $\ldots$ & $\ldots$ & Rowland Ward's Collection. \\
\hline 25 & $6 \frac{1}{2}$ & 14 & East Africa & $\ldots$ & T. W. H. Greenfield. \\
\hline $24 \frac{1}{8}$ & $6 \frac{3}{4}$ & II $\frac{7}{4}$ & Do. & $\cdots$ & H. C. V. Hunter. \\
\hline 24 & 6 & 14 & Do. & $\cdots$ & Sir John Willoughby, Bart. \\
\hline 24 & $6 \frac{1}{3}$ & $9 \frac{1}{4}$ & Do. & $\cdots$ & Rowland Ward's Collection. \\
\hline $23 \frac{5}{8}$ & 7 & $12 \frac{1}{4}$ & Do. & $\cdots$ & Sir Robert Harvey, Bart. \\
\hline $23 \frac{5}{8}$ & $6 \frac{1}{2}$ & $13 \frac{1}{1}$ & Do. & $\ldots$ & H. C. V. Hunter. \\
\hline $23 \frac{\pi}{8}$ & $6 \frac{3}{8}$ & II $\frac{1}{8}$ & Do. & $\cdots$ & Sir Robert Harvey, Bart. \\
\hline $23^{\frac{1}{2}}$ & $7 \frac{1}{8}$ & III $\frac{3}{4}$ & Do. & $\ldots \mid$ & Do. \\
\hline 23 & $7 \frac{1}{4}$ & 9 & Do. & $\cdots \mid$ & E. Gedge. \\
\hline 23 & $6 \frac{5}{8}$ & $10 \frac{5}{8}$ & Do. & $\ldots \mid$ & Sir Robert Harvey, Bart. \\
\hline $22 \frac{33}{4}$ & $6 \frac{3}{5}$ & $12 \frac{1}{8}$ & Do. & $\ldots$ & IIon. Walter Rothschild. \\
\hline $22 \frac{5}{8}$ & $6 \frac{1}{8}$ & $16 \frac{1}{4}$ & Do. & $\ldots$ & Sir Robert Harvey, Bart. \\
\hline $22 \frac{1}{2}$ & $6 \frac{3}{8}$ & II $\frac{1}{2}$ & Do. & $\cdots \mid$ & Do. \\
\hline $22 \frac{1}{2}$ & $6 \frac{1}{3}$ & $9 \frac{3}{t}$ & Do. & $\cdots 1$ & F. J. Jackson. \\
\hline $22 \frac{1}{t}$ & $6 \frac{1}{2}$ & $9 \frac{7}{8}$ & Do. & $\cdots \mid$ & H. C. V. Hunter. \\
\hline $22 \frac{1}{8}$ & $6 \frac{3}{8}$ & $5 \frac{7}{8}$ & Mount Land & $\ldots 1$ & F. J. Jackson, British Museum. \\
\hline 22 & 7 & 9 & East Africa & $\cdots 1$ & E. Gedge. \\
\hline 22 & $6 \frac{1}{2}$ & $14 \frac{1}{2}$ & Do, & $\ldots$ & W. Astor Chanler. \\
\hline
\end{tabular}


GRANT'S GAZELLE (Gazella granti)-continued.

\begin{tabular}{|c|c|c|c|c|}
\hline Length. & $\begin{array}{l}\text { Circum- } \\
\text { ference. }\end{array}$ & Tip to Tip. & Habitat. & Owner. \\
\hline $2 I \frac{3}{4}$ & $6 \frac{1}{2}$ & 15 & East Africa & R. P. Carrol. \\
\hline $2 \mathrm{I}$ & $5 \frac{3}{8}$ & 7 & Do. & F. J. Jackson. \\
\hline $20 \frac{3}{4}$ & $6 \frac{3}{8}$ & $9 \frac{7}{3}$ & Do. & J. Carr-Saunders. \\
\hline $20 \frac{1}{t}$ & $5 \frac{5}{8}$ & $8 \frac{1}{8}$ & Do. & British Museum. \\
\hline $19 \frac{7}{8}$ & $5 \frac{3}{4}$ & $6 \frac{5}{8}$ & Do. & F. J. Jackson. \\
\hline $19 \frac{5}{8}$ & $6 \frac{1}{2}$ & $7 \frac{1}{8}$ & $\begin{array}{l}\text { River Juba, } \\
\text { Somali Land. }\end{array}$ & Sir John Kirk, M.D., K.C.B. \\
\hline $18 \frac{5}{8}$ & $6 \frac{1}{4}$ & $8 \frac{1}{4}$ & Do. & Do. \\
\hline $17 \frac{3}{ \pm}$ & $6 \frac{1}{8}$ & $5^{\frac{3}{4}}$ & Do. & Do. \\
\hline 우 $17 \frac{1}{2}$ & $3 \frac{3}{4}$ & 9 & East Africa & H. C. V. IIunter. \\
\hline q17 & $\ldots$ & 6 & $\cdots \quad \cdots$ & $\begin{array}{l}\text { Sir John Willoughby, Bart., "East } \\
\text { Africa and its Big Game." }\end{array}$ \\
\hline I 7 & $6 \frac{1}{8}$ & I $1 \frac{1}{4}$ & South Somali Land & $\begin{array}{l}\text { Sir John Kirk, M.D., K.C.B., } \\
\text { British Museum. }\end{array}$ \\
\hline q15 & 4 & $6 \frac{3}{4}$ & East Africa & E. Gedge. \\
\hline Q $12 \frac{3}{8}$ & $3 \frac{1}{2}$ & $7 \frac{3}{4}$ & South Somali Land & $\begin{array}{l}\text { Sir John Kirk, M.D., K.C.B., } \\
\text { British Museum. }\end{array}$ \\
\hline 오 $12 \frac{1}{4}$ & $3 \frac{3}{8}$ & $5 \frac{5}{8}$ & $\begin{array}{l}\text { River Juba, } \\
\text { Somali Land. }\end{array}$ & Sir John Kirk, M.D., K.C.B. \\
\hline
\end{tabular}




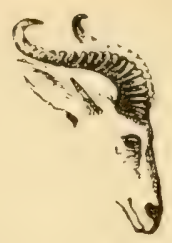

SEIMMERRING'S GAZELLE (Gazella sœmmerringi).

(Somali name-"Awal.")

[Sir Victor BROOKE, Bart., P.Z.S., 1873, p. 549.]

HAIR smooth and short. Facial band blackish, slightly rufous Letween the horns; light facial streak white, well marked; dark facial streak blackish, very definite, running through the eye and leaving its upper end as a black spot isolated above it. A spot below the eye, chin, throat, and a spot in front of the neck white; lateral bands wanting; breast, belly, tail, and rump white, the white of the latter encroaching into the sandy fawn-colour of the rest of the body and the haunches. Knee-brushes well developed. Ears long, bordered externally with black. Horns in the male lyrate, massive, and strongly annulated, well developed but less massive in the female. Height about 30 in.

Habitat-Abyssinian coast of the Red Sea, Danakil and Somali countries, Berber, and East Senaar (Henglin).

\begin{tabular}{|c|c|c|c|c|}
\hline Length. & $\begin{array}{l}\text { Circum- } \\
\text { ference. }\end{array}$ & Tip to Tip. & Habitat. & Ow \\
\hline $19 \frac{1}{2}$ & $5 \frac{1}{2}$ & $5 \frac{5}{8}$ & Somali Land ... & T. W. H. Clarke. \\
\hline $19 \frac{1}{4}$ & $5^{\frac{1}{2}}$ & $6 \frac{1}{2}$ & Do. & Do. \\
\hline 19 & $5^{\frac{3}{4}}$ & 3 & Do. & Gen. Trewell. \\
\hline 19 & 5 & 4 & Do. & Col. A. Paget. \\
\hline ㅇ I $S \frac{1}{t}$ & $5^{\frac{1}{1}}$ & 6 & Do. & T. W. H. Clarke. \\
\hline $1 S_{\frac{1}{8}}$ & $5 \frac{3}{8}$ & $4 \frac{1}{2}$ & Do. & W. D. James. \\
\hline 18 & $5 \frac{1}{\frac{1}{4}}$ & 7 & Do. & Marquis of Ailsa. \\
\hline IS & 5 & $3^{\frac{1}{4}}$ & 1)o. & T. W. H. Clarke. \\
\hline
\end{tabular}


SCEMMERRING'S GAZELLE (Gazella sœmmerringi)-continu'd.

\begin{tabular}{|c|c|c|c|c|c|}
\hline Length. & $\begin{array}{l}\text { Circum- } \\
\text { ference. }\end{array}$ & Tip to Tip. & \multicolumn{2}{|l|}{ Habitat. } & Owne:. \\
\hline I $7 \frac{3}{4}$ & $5 \frac{5}{8}$ & $3 \frac{1}{2}$ & \multicolumn{2}{|c|}{ Somali Land ... } & W. D. James. \\
\hline $17 \frac{3}{8}$ & $5 \frac{1}{2}$ & $5 \frac{3}{4}$ & \multicolumn{2}{|l|}{ Do. } & A. H. Straker. \\
\hline 17 & $5 \frac{1}{2}$ & $4 \frac{3}{4}$ & \multicolumn{2}{|l|}{ Do. } & Capt. Bartelot. \\
\hline $17 \frac{3}{8}$ & $5 \frac{1}{8}$ & $2 \frac{1}{2}$ & \multicolumn{2}{|l|}{ Do. } & C. Liddell. \\
\hline I $7 \frac{1}{t}$ & 5 & $4 \frac{1}{4}$ & \multicolumn{2}{|l|}{ Do. } & C. Liddell. \\
\hline I 7 & $5^{\frac{1}{4}}$ & $3 \frac{1}{4}$ & \multicolumn{2}{|l|}{ Do. } & Hon. Walter Rothschild. \\
\hline $16 \frac{7}{8}$ & $5 \frac{3}{8}$ & $6 \frac{1}{8}$ & Abyssinia & $\ldots$ & British Museum. \\
\hline $16 \frac{1}{2}$ & $5^{\frac{1}{4}}$ & 7 & \multicolumn{2}{|c|}{ Somali Land ... } & Capt. H. G. C. Swayne. \\
\hline $16 \frac{1}{2}$ & $5 \frac{1}{8}$ & $3 \frac{3}{8}$ & \multicolumn{2}{|l|}{ Do. } & T. W. H. Clarke. \\
\hline $16 \frac{3}{8}$ & 5 & $\mathrm{I} \frac{3}{4}$ & \multicolumn{2}{|l|}{ Do. } & E. Lort-Phillips. \\
\hline 16 & $3 \frac{3}{8}$ & $3 \frac{3}{8}$ & \multicolumn{2}{|l|}{ Do. } & E. P. Hare. \\
\hline 16 & 5 & $4 \frac{7}{8}$ & \multicolumn{2}{|l|}{ Do. } & E. Lort-Phillips. \\
\hline +16 & $3 \frac{1}{8}$ & 6 & \multicolumn{2}{|l|}{ Do. } & Rowland Ward's Collection. \\
\hline $15 \frac{1}{2}$ & $5 \frac{1}{2}$ & $\begin{array}{c}4 \text { (owner's } \\
\text { measurement) }\end{array}$ & \multicolumn{2}{|l|}{ Do. } & J. D. Inverarity. \\
\hline $15 \frac{3}{8}$ & $5 \frac{1}{8}$ & $7^{\frac{1}{8}}$ & \multicolumn{2}{|l|}{ Do. } & J. Carr-Saunders. \\
\hline $15 \frac{1}{2}$ & $5 \frac{3}{8}$ & 5 & Soudan & $\cdots$ & Col. Ralph Vivian. \\
\hline $15 \frac{3}{8}$ & $5 \frac{1}{8}$ & $3 \frac{3}{8}$ & \multicolumn{2}{|c|}{ Somali Land ... } & Lord Delamere. \\
\hline 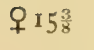 & $3 \frac{3}{8}$ & $5 \frac{3}{8}$ & \multicolumn{2}{|l|}{ Do. } & W. D. James. \\
\hline $14 \frac{3}{1}$ & $5 \frac{1}{2}$ & $4 \frac{1}{4}$ & $\ldots \quad \ldots$ & $\ldots 1$ & B. Cotton. \\
\hline $14 \frac{1}{2}$ & $5 \frac{1}{8}$ & $5 \frac{1}{3}$ & $\ldots \quad \ldots$ & $\ldots$ & Capt. W. H. Besant. \\
\hline I $4 \frac{1}{2}$ & 5 & $6 \frac{1}{8}$ & $\cdots \quad \ldots$ & $\cdots$ & Do. \\
\hline $14 \frac{1}{2}$ & 5 & 4 & Soudan & $\cdots$ & Col. Ralph Vivian. \\
\hline $14 \frac{3}{8}$ & $5 \frac{1}{8}$ & $5 \frac{7}{8}$ & Somali Land & $\cdots$ & Lord Delamere. \\
\hline ㅇ $14 \frac{1}{t}$ & $2 \frac{3}{4}$ & $10 \frac{1}{4}$ & Do. & & C. H. Villiers. \\
\hline I $4 \frac{1}{8}$ & $5 \frac{3}{8}$ & I $\frac{1}{4}$ & $\begin{array}{l}\text { Bogos Country } \\
\text { Abyssinia. }\end{array}$ & & British Museum. \\
\hline I4 & $3 \frac{5}{8}$ & $6 \frac{1}{4}$ & Somali & $\cdots$ & Lord Delamere, \\
\hline I4 & $5 \frac{1}{2}$ & $5^{\frac{1}{2}}$ & Soudan & $\ldots \mid$ & Col. A. Paget. \\
\hline
\end{tabular}


SCMMERRING'S GAZELLE (Gazella sœmmerringi)-continued.

\begin{tabular}{|c|c|c|c|c|c|}
\hline Length. & $\begin{array}{l}\text { Circum- } \\
\text { ference. }\end{array}$ & Tip to Tip. & Habitat. & & Owner. \\
\hline 139 & $5 \frac{1}{2}$ & 6 & $\ldots$ & $\cdots$ & W. D. James. \\
\hline $13 \frac{3}{1}$ & $5 \frac{1}{2}$ & $5 \frac{3}{8}$ & $\ldots$ & $\ldots$ & Capt. W. H. Besant. \\
\hline $13 \frac{1}{2}$ & $5^{\frac{1}{4}}$ & 4 & $\begin{array}{l}\text { North-East } \\
\text { Africa. }\end{array}$ & & \\
\hline $13 \frac{1}{2}$ & $5 \frac{3}{5}$ & $5 \frac{\pi}{8}$ & $\ldots \quad \ldots$ & $\cdots$ & Hon. Walter Rothschild. \\
\hline $13 \frac{1}{t}$ & $5 \frac{1}{8}$ & $6 \frac{1}{2}$ & $\cdots$ & $\cdots$ & Capt. W. H. Besant. \\
\hline$I_{3}$ & $5^{\frac{1}{2}}$ & 5 & $\cdots$ & $\cdots$ & Do. \\
\hline Q $12 \frac{5}{6}$ & $3 \frac{1}{2}$ & - $8 \frac{1}{t}$ & Soudan & $\cdots$ & Col. Ralph Vivian. \\
\hline Q 121 & $3 \frac{5}{8}$ & $7 \frac{1}{8}$ & Do. & $\cdots$ & Do. \\
\hline $123^{3}$ & $5 \frac{1}{8}$ & $4 \frac{3}{4}$ & $\cdots$ & $\cdots$ & Rowland Ward's Collection. \\
\hline q I2 & $3 \frac{1}{t}$ & $5 \frac{1}{8}$ & Abysinia & $\cdots$ & Sir Victor Brooke's Collection. \\
\hline 12 & $4 \frac{7}{8}$ & 3 & $\cdots$ & $\cdots$ & Capt. J. J. Palmer. \\
\hline $11 \frac{1}{8}$ & $4 \frac{3}{4}$ & 4 & $\cdots$ & $\cdots$ & J. Carr-Saunders. \\
\hline ㅇ $8 \frac{1}{2}$ & $3 \frac{3}{4}$ & $5^{\frac{1}{2}}$ & $\cdots$ & .. & Hon. Walter Rothschild. \\
\hline
\end{tabular}




\section{MOHR (Gazella mohr).}

[Sir Victor BROOKE, Bart., P.Z.S. I873, p. 548.]

HAIr close-set and smooth; central facial band grizzled rufous above, fading away below into the dirty white of the lower parts of the face and cheeks; dark facial streak thin, but definite, black; spot on the throat, breast, belly, anterior of hind legs and posterior of fore legs, rump, and tail (with the exception of the black tip) white; the white of the rump projects forwards into the deep rufous fawn-colour of the neck, back, sides, and haunches; this colour, which is darkest on the neck, runs down the front of the fore and outside of the hind extremities; lateral bands wanting; pygal band blackish rufous, very thin and short; ears moderate; horns in both sexes; in the male very massive and rather short, strongly annulated, the points projecting suddenly and boldly forwards; knee-brushes long; height about 34 in.

The Mohr or Mhore, is $4 \mathrm{ft} .2 \mathrm{in}$. long from the nose to the origin of the tail, $2 \mathrm{ft}$. $6 \mathrm{in}$. high at the shoulder, and $2 \mathrm{ft}$. $8 \mathrm{in}$. at the croup; the length of the head from the nose to the root of the horn is $7 \mathrm{in}$.; that of the horn $9 \frac{1}{2} \mathrm{in}$. on the curve, and $7 \frac{1}{2} \mathrm{in}$. in a straight line; and that of the tail 7 in. without the terminal tuft.

Habitat-Senegal.

\begin{tabular}{|c|c|c|c|c|c|}
\hline Length. & $\begin{array}{l}\text { Circum- } \\
\text { ference. }\end{array}$ & Tip to Tip. & Habitat. & & Owner. \\
\hline Q 12 & $3 \frac{1}{1}$ & $5^{\frac{1}{8}}$ & $\Lambda$ byssinia & ... & Sir Victor Brooke's Collection. \\
\hline 7 & $4 \frac{1}{4}$ & $2 \frac{1}{2}$ & Do. & $\cdots$ & Dr. Ruppell, British Museum. \\
\hline
\end{tabular}




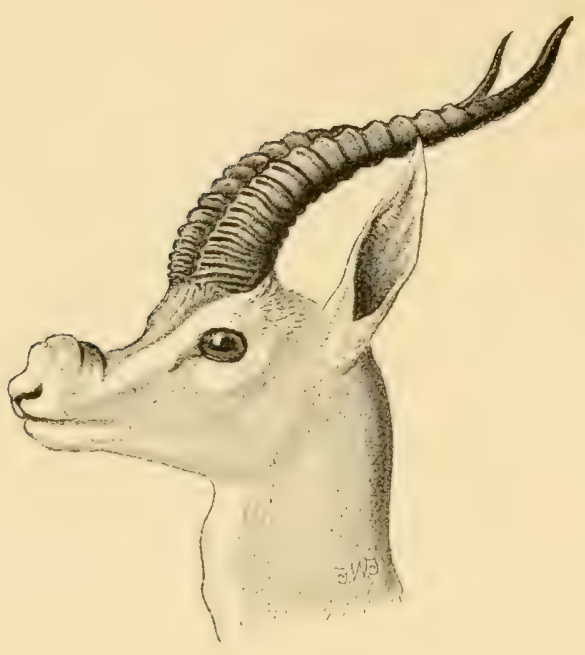

SOMALI LOWLAND GAZELLE (Gazella spekei).

(Somali name-"Dhero.")

Habitat-Somali, North-East Africa.

This gazelle has also been named Gazella nasu.

\begin{tabular}{|c|c|c|c|c|c|}
\hline Length. & $\begin{array}{l}\text { Circum- } \\
\text { ference. }\end{array}$ & Tip to Tip. & Habitat. & & Owner. \\
\hline$I I \frac{1}{2}$ & $4 \frac{3}{8}$ & $2 \frac{3}{4}$ & Somali Land & $\ldots$ & T. W. H. Clarke. \\
\hline$I I \frac{1}{4}$ & $4 \frac{1}{2}$ & $4 \frac{1}{4}$ & Do. & $\ldots 1$ & Do. \\
\hline$I I \frac{1}{8}$ & 4 & $5^{\frac{1}{4}}$ & Do. & ... & C. Liddell. \\
\hline II & $3 \frac{7}{8}$ & $4 \frac{1}{8}$ & Do. & $\ldots$ & Capt. H. G. C. Swayne. \\
\hline $10 \frac{7}{8}$ & $3 \frac{7}{8}$ & $2 \frac{7}{8}$ & Do. & $\ldots$ & T. W. H. Clarke. \\
\hline Io & $4 \frac{1}{2}$ & 4 & Do. & $\ldots$ & Do. \\
\hline 10 $\frac{3}{7}$ & $3^{\frac{3}{4}}$ & $3^{\frac{3}{4}}$ & Do. & $\cdots$ & E. Lort-Phillips. \\
\hline
\end{tabular}


SOMALI LOWLAND GAZELLE (Gazella spekei)-continucd.

\begin{tabular}{|c|c|c|c|c|c|}
\hline Length. & $\begin{array}{l}\text { Circum- } \\
\text { ference. }\end{array}$ & Tip to Tip. & Habitat. & & Owner. \\
\hline $10 \frac{3}{4}$ & $4 \frac{1}{8}$ & $4 \frac{1}{t}$ & Somali Land. & $\ldots$ & Col. A. Paget. \\
\hline $10 \frac{1}{2}$ & $3 \frac{7}{8}$ & $4 \frac{1}{8}$ & Do. & ... & A. H. Straker. \\
\hline $10 \frac{3}{8}$ & $3^{\frac{3}{4}}$ & $3 \frac{3}{4}$ & Do. & ... & E. Lort Phillips. \\
\hline $10_{4}^{\frac{1}{4}}$ & 4 & $3 \frac{7}{8}$ & Do. & $\ldots$ & T. W. H. Clarke. \\
\hline IO $\frac{1}{8}$ & $3 \frac{7}{8}$ & $4 \frac{1}{2}$ & Do. & ... & Hon. Walter Rothschild. \\
\hline IO $\frac{1}{8}$ & $3 \frac{5}{8}$ & $3 \frac{1}{2}$ & Do. & $\cdots$ & IV. D. James. \\
\hline 10 & 4 & $4 \frac{3}{4}$ & Do. & $\ldots$ & Rowland Ward's Collection. \\
\hline Io & 4 & $\begin{array}{c}\text { (owner's } \\
\text { measurement) }\end{array}$ & Do. & $\cdots$ & J. D. Inverarity. \\
\hline 오 $9 \frac{1}{2}$ & $2 \frac{7}{8}$ & $3 \frac{1}{4}$ & Do. & $\ldots$ & T. W. H. Clarke. \\
\hline ㅇ 9 & $2 \frac{1}{2}$ & $\left.\right|_{\text {measurement) }} ^{\text {(owner's }}$ & Do. & $\cdots$ & J. D. Inverarity. \\
\hline
\end{tabular}

SOMALI PLATEAU GAZELLE (Gazella pelzelni).

THIs is common round Berbera, but is replaced by Gazella spekei. when the Somali plateau is reached. It has often been mistaken for Gazella spekei, but can readily be distinguished from that species by its straighter horns and the absence of the loose skin on the nose.

Habitat-North-East Africa, Somali.

\begin{tabular}{|c|c|c|c|c|c|}
\hline Length. & $\begin{array}{l}\text { Circum- } \\
\text { ference. }\end{array}$ & Tip to Tip. & Habitat. & & Owner. \\
\hline $12 \frac{1}{4}$ & $4 \frac{1}{8}$ & $5 \frac{1}{2}$ & Somali Land & $\ldots$ & Lord Delamere. \\
\hline $11 \frac{1}{2}$ & $3 \frac{1}{2}$ & $5 \frac{1}{2}$ & Do. & $\ldots$ & T. W. H. Clarke. \\
\hline 우 $7 \frac{5}{8}$ & $\mathbf{I} \frac{3}{4}$ & $2 \frac{3}{4}$ & Do. & $\ldots$ & Do. \\
\hline $5 \frac{3}{8}$ & $1 \frac{1}{8}$ & $3 \frac{3}{8}$ & Do. & $\ldots$ & C. H. Villiers. \\
\hline
\end{tabular}




\section{ARABIAN GAZELLE (Gazella arabica).}

[Sir VICTOR BROUkE, Bart., P.Z.S. I873, p. 544.]

HAIR very short and smooth; central facial band rich bay above, darker below, forming a nose-spot; light facial streak very strongly marked, running from the base of the horn over the eye, stopping just below the nose-spot; dark facial streak distinct, blackish; breast and belly white; dark lateral and pygal bands grizzly brown; light lateral band grizzly fawn; upper parts of the sides and back rich grizzled bay in adult specimens, the younger animals being greyer; knee-brushes well developed, blackish; tail black; horns massive, the annulations wide apart, diverging gradually from two-thirds their length, the upper third diverging more decidedly, and leaning a little forwards; ears moderate; size about equal to that of Gazella dorcas.

Habitat-South Arabia.

\begin{tabular}{|c|c|c|c|c|c|}
\hline Length. & $\begin{array}{l}\text { Circum- } \\
\text { ference. }\end{array}$ & Tip to Tip. & Habitat. & & Owner. \\
\hline $\begin{array}{l}8 \frac{1}{3} \\
4 \frac{7}{3}\end{array}$ & $\begin{array}{l}4 \\
3 \frac{1}{8}\end{array}$ & $\begin{array}{l}2 \\
3 \frac{5}{8}\end{array}$ & $\begin{array}{l}\text { Mosha, South } \\
\text { Arabia. } \\
\ldots \quad \ldots\end{array}$ & $\ldots$ & $\begin{array}{l}\text { W. T. Blandford, British Museum. } \\
\text { Sir Victor Brooke's Collection. }\end{array}$ \\
\hline
\end{tabular}

\section{DORCAS GAZELLE (Gazella Dorcas).}

[Sir Victor Brooke, Bart., P.Z.S. 1873, p. 538.]

HAIR rather long and coarse; central facial band rufous above, inclining to fawn-colour towards the nostrils; a dark nose-spot sometimes present, not depending on age or sex; light facial streak distinct, encircling the eye; dark facial streak definite, brownish rufous; upper and under lip, breast, and belly white; dark lateral and pygal bands very definite, rufous brown; light lateral band distinguishable from the rich fawn-colour of the upper parts of the back by 
its more silvery hue; knee-brushes well developed, in colour variable; tail black, long, and tapering; horns long and annulated, rather slender, with their points bent inwards and forwards; ears about $5 \frac{1}{2}$ in. ; height at shoulder barely 24 in.

Habitat-Syria, North Arabia, Egypt, Algeria, Morocco, and Senegal.

\begin{tabular}{|c|c|c|c|c|c|c|}
\hline Length. & $\begin{array}{l}\text { Circum- } \\
\text { ference. }\end{array}$ & Tip to Tip. & \multicolumn{3}{|c|}{ Habitat. } & Owner. \\
\hline $13 \frac{1}{\frac{1}{4}}$ & $3 \frac{5}{8}$ & (touch) & \multicolumn{3}{|c|}{ Algeria ... } & Rowland Ward's Collection. \\
\hline $13 \frac{1}{8}$ & $4 \frac{1}{t}$ & $2 \frac{1}{2}$ & Do. & ... & ... & Sir Edmund G. Loder, Bart. \\
\hline $13 \frac{1}{8}$ & $3 \frac{1}{2}$ & 2 & Do. & $\cdots$ & $\cdots$ & Do. \\
\hline $12 \frac{1}{2}$ & $3 \frac{5}{8}$ & $4 \frac{3}{4}$ & Do. & $\ldots$ & $\ldots$ & Rowland Ward's Collection. \\
\hline $12 \frac{3}{8}$ & $3^{\frac{3}{4}}$ & $2 \frac{3}{4}$ & Do. & $\ldots$ & $\ldots$ & Sir Edmund G. Loder, Bart. \\
\hline $12 \frac{3}{8}$ & $3 \frac{3}{8}$ & $\cdots$ & $\cdots$ & $\cdots$ & $\cdots$ & British Museum. \\
\hline $12 \frac{1}{8}$ & 4 & $2 \frac{1}{2}$ & Algeria & $\ldots$ & $\ldots$ & Rowland Ward's Collection. \\
\hline II $\frac{7}{8}$ & $3 \frac{5}{8}$ & $4 \frac{1}{2}$ & ... & $\ldots$ & $\ldots$ & Sir Victor Brooke's Collection. \\
\hline II $\frac{1}{4}$ & $3 \frac{1}{2}$ & $3 \frac{7}{8}$ & $\ldots$ & $\cdots$ & $\cdots$ & E. N. Buxton. \\
\hline II & $3 \frac{7}{8}$ & $3 \frac{1}{8}$ & $\ldots$ & ... & $\ldots$ & British Museum. \\
\hline I0 $\frac{1}{8}$ & 4 & $2 \frac{1}{4}$ & \multicolumn{3}{|c|}{ Shores of Red Sea } & Do. \\
\hline $10 \frac{1}{8}$ & $3 \frac{5}{8}$ & $2 \frac{3}{4}$ & Nubia & ... & $\ldots$ & Sir Victor Brooke's Colleciion. \\
\hline $9 \frac{7}{8}$ & 4 & $4 \frac{1}{8}$ & $\ldots$ & ... & $\ldots$ & E. Lort-Phillips. \\
\hline $9 \frac{1}{2}$ & $3 \frac{1}{2}$ & $3 \frac{7}{8}$ & ... & ... & $\ldots$ & British Museum. \\
\hline $8 \frac{7}{8}$ & 4 & $4 \frac{1}{8}$ & ... & ... & $\ldots$ & E. Lort-Phillips. \\
\hline $8 \frac{7}{8}$ & $3 \frac{3}{8}$ & $3 \frac{1}{4}$ & ... & $\cdots$ & $\ldots$ & British Museum. \\
\hline $8 \frac{1}{8}$ & $3 \frac{5}{8}$ & $2 \frac{3}{4}$ & $\ldots$ & $\ldots$ & $\ldots$ & Capt. J. J. Palmer. \\
\hline 8 & $3 \frac{1}{2}$ & $4 \frac{1}{2}$ & \multicolumn{3}{|c|}{ Shores of Red Sea } & British Museum. \\
\hline$q 6 \frac{5}{8}$ & $2 \frac{1}{4}$ & $2 \frac{1}{4}$ & $\ldots$ & ... & ... & Capt. J. J. Palmer. \\
\hline
\end{tabular}




\section{ISABELLA GAZELLE (Gazella isabella).}

[Sir Victor BROOKE, Bart., P.Z.S. 1873, p. 539.]

HAIR very short and soft; central facial band sandy-rufous; nosespot absent; light facial streak present but indefinite, running into the dark facial streak, which is in some specimens but faintly marked; dark lateral band almost obsolete; light lateral band of a delicate pale fawn-colour, distinctly visible against the isabelline sandy fawn-colour of the back and haunches; tail variable, but generally rufous for a large part of its length, the tip blackish; horns short, massive, and strongly ringed, the tips bent suddenly forwards and inwards; ears very long; height less than that of the last species (barely 24 in. at shoulder).

\section{Habitat-Kordofan, Senaar.}

\begin{tabular}{|c|c|c|c|c|}
\hline Length. & $\begin{array}{l}\text { Circum- } \\
\text { ference. }\end{array}$ & Tip to Tip. & Habitat. & Owner. \\
\hline $10 \frac{1}{4}$ & $3 \frac{3}{4}$ & $2 \frac{3}{4}$ & \multirow{2}{*}{$\begin{array}{l}\text { Anseba River, } \\
\text { Abyssinia. } \\
\text { Komayli, Abyssinia }\end{array}$} & Sir Victor Brooke's Collection. \\
\hline $10 \frac{1}{8}$ & $3 \frac{3}{4}$ & 4 & & WV. T. Blandford, British Museum. \\
\hline $9 \frac{3}{t}$ & $3 \frac{3}{t}$ & $2 \frac{3}{8}$ & Bogos Land & Sir Victor Brooke's Collection. \\
\hline q 7 & 2 & 3 & $\ldots$ & Do. \\
\hline ㅇ 7 & $I \frac{7}{8}$ & $2 \frac{3}{x}$ & Kordofan & British Museum. \\
\hline ㅇ $6 \frac{7}{5}$ & $2 \frac{1}{2}$ & $2 \frac{1}{8}$ & Do. $\ldots$ & Do. \\
\hline
\end{tabular}

\section{GAZELLA DAIMA.}

[Sir Victor BROOKE, Bart., P.Z.S. I873, p. 547.]

HAIR short and smooth; facial and lateral markings wanting; neck, and upper part of the neck, sandy-red; face, spot on the throat, and the rest of the body and limbs white; knee-brushes well developed; ears moderate; horns lyrate, strongly annulated, well developed in both sexes.

Habitat-Desert of Korti (Rupp.), Southern Nubia, and Kordofan (Heugl.). 
GAZELLA DAIMA-continued.

\begin{tabular}{|c|c|c|c|c|}
\hline Length. & $\begin{array}{l}\text { Circum- } \\
\text { ference. }\end{array}$ & Tip to Tip. & Habitat. & Owner. \\
\hline $\begin{array}{l}\text { I } 1 \frac{3}{4} \\
10 \frac{1}{2}\end{array}$ & $\begin{array}{l}6 \\
4 \frac{5}{8}\end{array}$ & $\begin{array}{l}3 \frac{3}{4} \\
6 \frac{3}{8}\end{array}$ & $\begin{array}{l}\text { Wednoon, } \\
\text { dor. } \\
\text { Senegal ... }\end{array}$ & $\begin{array}{l}\text { W. Willshire, British Museum. } \\
\text { Sir Victor Brooke's Collection. }\end{array}$ \\
\hline
\end{tabular}

\section{GAZELLA RUFIFRONS.}

[Sir VICTOR BROOKE, Bart, P.Z.S. 1873, p. 540.]

CEnTRAL facial streak uniform sandy-yellow; light facial streak indefinite; dark facial hardly distinguishable from the colour of the cheeks, which are sandy-yellow; dark lateral band distinctly marked, brown; light lateral band plainly shown between the dark streak and the yellowish fawn-colour of the back and haunches; pygal-band indistinct; no knee-brushes; height, a little over 2 feet.

\section{Habitat-Senegal.}

\begin{tabular}{c|c|c|c|c}
\hline Length. & $\begin{array}{c}\text { Circum- } \\
\text { ference. }\end{array}$ & Tip to Tip. & Habitat. & Owner. \\
\hline $6 \frac{1}{8}$ & $2 \frac{1}{4}$ & $2 \frac{7}{8}$ & $\begin{array}{c}\text { Bogos Country, } \\
\text { Abyssinia. }\end{array}$ & British Museum. \\
\hline
\end{tabular}

\section{ADDRA GAZELLE (Gazella ruficollis).}

IT is about $5 \mathrm{ft}$. 4 in. long, and $3 \mathrm{ft}$. at shoulders; the head is 8 in. long, and tail about 9 in.

\section{Habitat-North Africa.}

\begin{tabular}{|c|c|c|c|c|c|}
\hline Length. & $\begin{array}{l}\text { Circum- } \\
\text { ference. }\end{array}$ & Tip to Tip. & Habitat. & & Owner. \\
\hline I $2 \frac{1}{4}$ & 4 & $5 \frac{5}{8}$ & Senaar ... & $\ldots$ & British Museum, \\
\hline II $\frac{7}{8}$ & $5 \frac{5}{8}$ & $\frac{5}{8}$ & Kordofan & $\ldots$ & Do. \\
\hline
\end{tabular}




\section{PERSIAN and YARKUND GAZELLE (Gazella subgutturosa).}

[Sir Victor Brooke, Bart., P.Z.S. 1873, p. 545.]

HAIR in winter rough and coarse; in summer, much softer and smoother. During both seasons the dirty-white of the face and cheeks is only relieved by the dark facial streak, which is short and narrow, but defined by a sprinkling of rufous hairs; the lateral and pygal bands are very faintly indicated, the dark bands being more rufous, the light band rather paler than the grey fawn-colour of the upper parts of the body; breast and belly white; tail and ears moderate in length, the former blackish-rufous; horns absent in the female; in the male long, annulated, and lyrate, the points projecting inwards; height, about 26 in.

Habitat-High plateau of Persia, Northern Beloochistan, Afghanistan.

\begin{tabular}{|c|c|c|c|c|c|c|}
\hline Length. & $\begin{array}{l}\text { Circum- } \\
\text { ference. }\end{array}$ & Tip to Tip. & \multicolumn{3}{|c|}{ Habitat. } & Owner. \\
\hline I $3 \frac{3}{4}$ & $4 \frac{1}{2}$ & 6 & \multicolumn{3}{|c|}{$\begin{array}{l}\text { Saissan, West } \\
\text { Siberia. }\end{array}$} & Dr. O. Finsch, British Museum. \\
\hline $13 \frac{3}{8}$ & $4^{\frac{3}{4}}$ & $5^{\frac{1}{2}}$ & $\cdots$ & $\cdots$ & $\cdots$ & Hume Collection, British Museum. \\
\hline 13 & $4 \frac{1}{2}$ & 5 & $\cdots$ & $\ldots$ & $\cdots$ & Do. \\
\hline $12 \frac{9}{4}$ & $4 \frac{3}{t}$ & $4 \frac{3}{8}$ & $\cdots$ & $\ldots$ & $\cdots$ & Do. \\
\hline $12 \frac{5}{8}$ & $4 \frac{3}{4}$ & $5 \frac{1}{4}$ & $\cdots$ & $\ldots$ & $\cdots$ & Do. \\
\hline $11 \frac{3}{4}$ & 5 & $5 \frac{1}{8}$ & ... & $\ldots$ & $\ldots$ & Do. \\
\hline $10 \frac{33}{4}$ & $4^{\frac{1}{4}}$ & $6 \frac{7}{8}$ & $\cdots$ & $\ldots$ & $\cdots$ & Hon. Walter Rothschild. \\
\hline $10 \frac{5}{8}$ & 4 & $3 \frac{1}{8}$ & $\cdots$ & $\ldots$ & $\cdots$ & Hume Collection, British Museum. \\
\hline $6 \frac{1}{8}$ & $3 \frac{7}{8}$ & 2 & $\cdots$ & $\cdots$ & $\cdots$ & Do. \\
\hline $5^{\frac{3}{ \pm}}$ & $3 \frac{3}{3}$ & $\frac{5}{8}$ & $\ldots$ & $\ldots$ & $\ldots$ & Do. \\
\hline
\end{tabular}




\section{GUTTUROSA ANTELOPE (Gazella gutturosa).}

[Sir VICTOR BROOKE, Bart., P.Z.S. 1873, p. 546.]

HAIR in winter long and coarse; anterior part of the face slightly darker than the pale grizzled fawn-colour of the sides of the face, neck, back, sides, and haunches; facial and lateral markings wanting; belly and anal disc, which surrounds the very short tail (the root of which is white, the tip fawn-colour), dirty white.

In summer all the parts which are grizzled fawn in winter are of an "intense isabel yellow" (vid. Radde); ears very short; horns short, weakly annulated, the curves as in the last species (G. Subgutturosa), but much less boldly shown; height about 30 in.

Skull : anteorbital fossa very shallow; nasals converge to a point and are rather elongated.

Habitat-Mongolia.

\begin{tabular}{|c|c|c|c|c|c|c|}
\hline Length. & $\begin{array}{l}\text { Circum- } \\
\text { ference. }\end{array}$ & Tip to Tip. & \multicolumn{3}{|c|}{ Habitat. } & Owner. \\
\hline $13 \frac{1}{8}$ & $4 \frac{5}{8}$ & $6 \frac{1}{4}$ & \multicolumn{3}{|c|}{ Mongolia } & Sir Edmund G. Loder, Bart. \\
\hline $12 \frac{3}{4}$ & $4 \frac{3}{8}$ & $3 \frac{3}{8}$ & Altai & ... & ... & St. George Littledale. \\
\hline $12 \frac{1}{4}$ & $4 \frac{3}{8}$ & $4^{\frac{3}{4}}$ & $\ldots$ & ... & $\ldots$ & Do. \\
\hline I $1 \frac{1}{2}$ & $4 \frac{7}{8}$ & 3 & \multicolumn{3}{|c|}{ Chinese Mongolia } & Reginald Beech. \\
\hline I0 $\frac{1}{4}$ & $4 \frac{3}{8}$ & $2 \frac{3}{4}$ & Russia & $\ldots$ & $\ldots$ & British Museum. \\
\hline $9 \frac{3}{4}$ & $4 \frac{1}{8}$ & $4 \frac{3}{8}$ & Pekin & $\ldots$ & ... & R. Swinhoe, British Museum. \\
\hline $7 \frac{3}{8}$ & $3 \frac{3}{4}$ & $2 \frac{1}{2}$ & Do. & $\ldots$ & ... & Do. \\
\hline
\end{tabular}




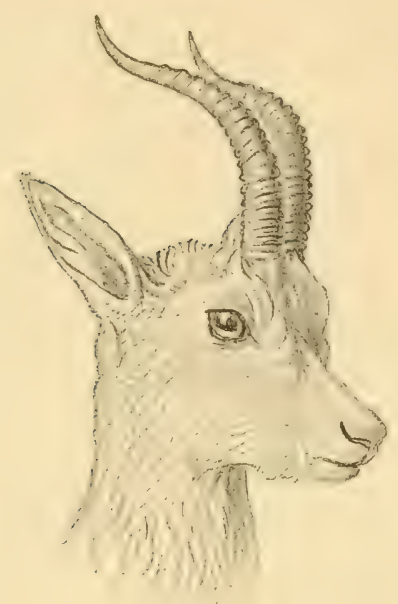

THIBETAN RAVINE DEER (Gazella picticaudata).

Female without Horns.

Habitat-Western Thibet and Mongolia.

\begin{tabular}{|c|c|c|c|c|c|c|}
\hline Length. & $\begin{array}{l}\text { Circum- } \\
\text { ference. }\end{array}$ & Tip to Tip. & & abitat. & & Owner. \\
\hline $13 \frac{1}{2}$ & $3 \frac{5}{8}$ & $5 \frac{1}{4}$ & .. & $\cdots$ & $\ldots$ & Hume Collection, British Museum. \\
\hline $13 \frac{1}{8}$ & 4 & $3 \frac{5}{8}$ & Thibet & $\cdots$ & $\ldots$ & II. C. V. II unter. \\
\hline 13 & $3^{\frac{3}{4}}$ & $3 \frac{1}{2}$ & .. & $\ldots$ & ... & Do. \\
\hline $12 \frac{7}{8}$ & $3 \frac{7}{8}$ & $44^{3}$ & ... & $\cdots$ & .. & A. O. Hume, Private Collection. \\
\hline $12 \frac{7}{8}$ & $3 \frac{7}{8}$ & $4 \frac{3}{8}$ & $\ldots$ & $\cdots$ & .. & B. H. Hodgson, British Museum. \\
\hline $12 \frac{3}{4}$ & $3 \frac{7}{8}$ & $2 \frac{1}{2}$ & ... & $\ldots$ & ... & Hume Collection, British Museum. \\
\hline $12 \frac{5}{8}$ & $3 \frac{1}{2}$ & $2 \frac{1}{2}$ & & ... & ... & Do. \\
\hline $12 \frac{1}{4}$ & $3^{\frac{3}{4}}$ & $4^{\frac{3}{4}}$ & Thibet & $\ldots$ & ... & H. C. V. Hunter. \\
\hline $12 \frac{1}{8}$ & $3 \frac{5}{8}$ & $3 \frac{1}{2}$ & Do. & $\ldots$ & $\ldots$ & Do. \\
\hline
\end{tabular}


THIBETAN RAVINE DEER (Gazella picticaudata)-continucl.

\begin{tabular}{|c|c|c|c|c|c|c|}
\hline Length. & $\begin{array}{l}\text { Circum- } \\
\text { ference. }\end{array}$ & Tip to Tip. & & labitat. & & Owner. \\
\hline 12 & $3 \frac{33}{ \pm}$ & $6 \frac{5}{8}$ & $\ldots$ & $\cdots$ & ... & A. O. Hume, Private Collection. \\
\hline 12 & $3 \frac{5}{8}$ & $2 \frac{1}{2}$ & Thibet & ... & $\cdots$ & H. C. V. Hunter. \\
\hline II $\frac{7}{8}$ & $3 \frac{5}{8}$ & $4 \frac{1}{2}$ & $\ldots$ & $\cdots$ & $\cdots$ & Sir Edmund G. Loder, Bart. \\
\hline II $\frac{5}{8}$ & $3 \frac{3}{8}$ & $5 \frac{7}{8}$ & Thibet & $\ldots$ & $\cdots$ & B. H. Hodgson, British Museum. \\
\hline $11 \frac{1}{2}$ & $3 \frac{5}{8}$ & $4 \frac{1}{8}$ & $\cdots$ & $\cdots$ & $\cdots$ & Hon. Walter Rothschild. \\
\hline$I I \frac{3}{8}$ & $3 \frac{1}{4}$ & $4 \frac{7}{8}$ & $\cdots$ & $\cdots$ & $\cdots$ & Rowland Ward's Collection. \\
\hline I I $\frac{1}{4}$ & $3 \frac{1}{2}$ & $4 \frac{3}{4}$ & Thibet & $\cdots$ & $\cdots$ & B. H. Hodgson, British Museum. \\
\hline II & $3 \frac{5}{8}$ & $3 \frac{3}{8}$ & Ladak & $\cdots$ & $\cdots$ & R. Lydekker, British Museum. \\
\hline $9 \frac{7}{8}$ & $3 \frac{1}{4}$ & $3 \frac{5}{8}$ & Thibet & $\cdots$ & $\cdots$ & H. C. V. Hunter. \\
\hline 8 & $3 \frac{3}{1}$ & $3 \frac{1}{8}$ & $\cdots$ & $\cdots$ & $\cdots$ & Sir Victor Brooke's Collection. \\
\hline
\end{tabular}




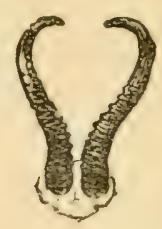

\section{SPRINGBUCK (Gazella euchore).}

[Sir Victor Brooke, Bart., P.Z.S. I873, p. 550.]

HAIR rather long but smooth; the central facial band has the upper part distinctly marked, but ends in a point between the eyes, rufous; the light facial streaks meet below the eyes, white; dark facial streak thin from above, downwards, but very definite, deep rufous; cheeks, line in front of the neck, breast, belly, inside of limbs, dorsal mane, starting from the anterior lumbar region, and tail (with the exception of the long black terminal hairs) white; dark lateral and pygal bands deep rufous, the former very broad and well defined; light lateral band very faintly shown, being of a less rufous tint than the fawn-colour of the shoulders, upper parts of the back, and haunches; ears long; horns in both sexes, but very small in the female; in the male they are truly lyrate, strongly annulated, and massive, of moderate length; knee-brushes absent; height at shoulder about 30 in.

Skull: nasal bones rather elongated and pointed; dentition, i. $\frac{0}{3}$, c. $\frac{0}{1}, \mathrm{p} \cdot \frac{3}{2}, \mathrm{~m} \cdot \frac{3}{3}$; the first premolar in the upper jaw is almost rudimentary, its opposing tooth in the lower jaw is wanting; in the milkdentition, however, the first of the three lower molars is represented by an exceedingly small cylindrical tooth.

Habitat-South and West Africa. This species does not appear to occur in the $Z$ ulu country.

\begin{tabular}{|c|c|c|c|c|c|c|}
\hline $\begin{array}{l}\text { Length on } \\
\text { curve. }\end{array}$ & $\begin{array}{l}\text { Circum- } \\
\text { ference. }\end{array}$ & $\begin{array}{l}\text { Tip to } \\
\text { Tip. }\end{array}$ & $\begin{array}{l}\text { Widest } \\
\text { inside. }\end{array}$ & Habitat. & & Owner. \\
\hline I $5 \frac{1}{ \pm}$ & $6 \frac{1}{4}$ & $3 \frac{7}{8}$ & 9 & $\ldots$ & $\ldots$ & National Liberal Club. \\
\hline $15 \frac{1}{8}$ & $6 \frac{1}{4}$ & $5 \frac{7}{8}$ & ... & West Africa & $\ldots$ & Capt. F. Cookson. \\
\hline $15 \frac{1}{8}$ & $5 \frac{3}{4}$ & $10 \frac{7}{8}$ & ... & $\ldots$ & $\ldots$ & W. C. Oswell. \\
\hline 15 & $5^{\frac{3}{4}}$ & 2 & $7 \frac{1}{2}$ & $\ldots$ & $\ldots$ & J. A. Nicoll. \\
\hline
\end{tabular}


SPRINGBUCK (Gazella euchore)-continucd.

\begin{tabular}{|c|c|c|c|c|c|c|}
\hline $\begin{array}{l}\text { Length on } \\
\text { curve. }\end{array}$ & $\begin{array}{l}\text { Circum- } \\
\text { ference. }\end{array}$ & $\begin{array}{l}\text { Tip to } \\
\text { Tip. }\end{array}$ & $\begin{array}{l}\text { Widest } \\
\text { inside. }\end{array}$ & Habitat. & & Owner. \\
\hline $14 \frac{1}{x}$ & 7 & $2 \frac{1}{2}$ & $\begin{array}{c}8 \\
\text { (outside) }\end{array}$ & South Africa & $\ldots$ & A. W. Davis. \\
\hline $14 \frac{2}{t}$ & $6 \frac{1}{x}$ & 5 & $\left|7 \frac{3}{4}\right|$ & Do. & $\cdots$ & C. Ansell. \\
\hline I. & $6 \frac{1}{2}$ & $2 \frac{1}{4}$ & $\cdots$ & Do. & $\ldots$ & The late J. S. Jameson. \\
\hline 14 & $5^{\frac{3}{4}}$ & $4 \frac{1}{1}$ & 7 & $\cdots$ & $\cdots$ & British Museum. \\
\hline 14 & $5 \frac{1}{2}$ & $5^{\frac{1}{2}}$ & $8 \frac{1}{2}$ & $\cdots$ & $\cdots$ & J. A. Nicoll. \\
\hline $13 \frac{7}{8}$ & 6 & $5 \frac{3}{8}$ & $7 \frac{7}{8}$ & South Africa & $\cdots$ & F. C. Selous. \\
\hline $13 \frac{5}{8}$ & $5 \frac{5}{8}$ & $3 \frac{1}{2}$ & 6 & $\cdots$ & $\cdots$ & British Museum. \\
\hline $13 \frac{5}{8}$ & $5^{\frac{1}{2}}$ & $7 \frac{1}{8}$ & $\cdots$ & $\cdots, \quad \cdots$ & $\cdots$ & Sir Victor Brooke's Collection. \\
\hline $13 \frac{1}{2}$ & $5 \frac{3}{8}$ & $1 \frac{3}{4}$ & $5^{\frac{3}{4}}$ & $\cdots \quad \cdots$ & $\cdots$ & J. Carr-Saunders. \\
\hline I $3 \frac{3}{8}$ & $5 \frac{7}{8}$ & $4 \frac{3}{8}$ & $6 \frac{1}{2}$ & South Africa & $\cdots$ & F. C. Selous. \\
\hline $13 \frac{1}{1}$ & $5 \frac{3}{8}$ & $5 \frac{3}{8}$ & $7 \frac{7}{8}$ & $\cdots$ & $\cdots$ & British Museum. \\
\hline I $3^{\frac{1}{4}}$ & $5^{\frac{1}{2}}$ & 5 & $\cdots$ & $\cdots \quad \cdots$ & $\cdots$ & Sir Edmund G. Loder, Bart. \\
\hline 13 & $6 \frac{1}{2}$ & $4 \frac{5}{8}$ & $\begin{array}{c}9^{\frac{1}{2}} \\
\text { (outside) }\end{array}$ & South Africa & $\ldots$ & C. Ansell. \\
\hline 13 & $5 \frac{5}{8}$ & 5 & $7 \frac{1}{2}$ & Do. & $\ldots$ & A. Beit. \\
\hline $12 \frac{7}{8}$ & $5 \frac{3}{8}$ & $5 \frac{1}{2}$ & ... & $\cdots \quad \cdots$ & $\cdots$ & W. C. Oswell. \\
\hline $12 \frac{3}{4}$ & $5 \frac{1}{7}$ & $3 \frac{1}{4}$ & $6 \frac{1}{2}$ & $\cdots \quad \cdots$ & $\cdots$ & Rowland Ward's Collection. \\
\hline $12 \frac{3}{8}$ & $5 \frac{1}{2}$ & $3 \frac{3}{8}$ & 6 & South Africa & $\ldots$ & F. C. Selous. \\
\hline $12 \frac{1}{8}$ & $5 \frac{1}{8}$ & $5 \frac{1}{4}$ & $7 \frac{1}{8}$ & $\cdots \quad \cdots$ & $\cdots$ & British Museum. \\
\hline II $\frac{7}{8}$ & $5 \frac{3}{\frac{1}{4}}$ & $6 \frac{1}{2}$ & $\cdots$ & $\cdots \quad \cdots$ & $\cdots$ & Hon. Walter Rothschild. \\
\hline II $\frac{5}{8}$ & $5 \frac{7}{8}$ & 4 & $\cdots$ & South Africa & $\cdots$ & British Museum. \\
\hline II $\frac{3}{5}$ & $5^{\frac{1}{4}}$ & 4 & $6 \frac{1}{4}$ & $\cdots \quad \cdots$ & $\cdots$ & Sir Victor Brooke's Collection. \\
\hline$q+\frac{1}{8}$ & 3 & $6 \frac{3}{4}$ & $\cdots$ & South Africa & $\cdots$ & The late J. S. Jameson. \\
\hline 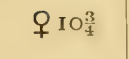 & $3^{\frac{1}{4}}$ & $5^{\frac{3}{4}}$ & $6 \frac{1}{2}$ & Do. & $\cdots$ & F. C. Selous. \\
\hline Q 101 & $3 \frac{3}{8}$ & $2 \frac{1}{2}$ & $\ldots$ & West Africa & $\cdots$ & Capt. F. Cookson. \\
\hline 10 & $4 \frac{5}{8}$ & $4 \frac{1}{8}$ & $\cdots$ & $\cdots$ & $\cdots$ & British Museum. \\
\hline$q S_{5}^{5}$ & $2 \frac{1}{2}$ & 4 & $4 \frac{5}{8}$ & Natal $\ldots$ & $\cdots$ & Do. \\
\hline 오 8 & $3 \frac{1}{8}$ & $2 \frac{1}{2}$ & $3 \frac{7}{8}$ & South Africa & $\ldots$ & A. Beit. \\
\hline
\end{tabular}




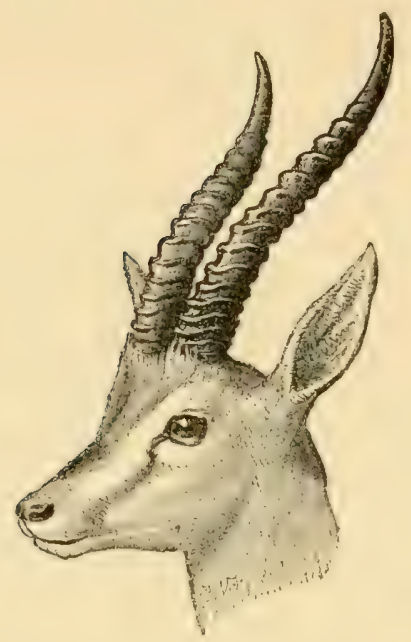

INDIAN GAZELLE or RAVINE DEER (Gazella bennetti).

Habitat-India (Southern Beloochistan) and Southern Persia.

\begin{tabular}{|c|c|c|c|c|c|c|}
\hline Length. & $\begin{array}{l}\text { Circum- } \\
\text { ference. }\end{array}$ & Tip to Tip. & \multicolumn{3}{|c|}{ Habitat. } & Owner. \\
\hline $14 \frac{1}{4}$ & \multicolumn{2}{|c|}{ (owner's measurements) } & Rajah & Waller & $\ldots$ & Capt. H. V. Brooke, late 92nd High- \\
\hline $13^{\frac{3}{4}}$ & $4 \frac{1}{2}$ & 7 & ... & $\cdots$ & $\ldots$ & Sir Victor Brooke's Collection. \\
\hline $12 \frac{3}{4}$ & $4 \frac{3}{8}$ & $7 \frac{1}{2}$ & $\cdots$ & $\cdots$ & ... & H. C. V. Hunter. \\
\hline $12 \frac{1}{2}$ & $4 \frac{1}{2}$ & $6 \frac{1}{4}$ & ... & $\cdots$ & $\cdots$ & Do. \\
\hline $12 \frac{3}{8}$ & 4 & $5 \frac{1}{2}$ & Simrol, & Bengal & $\cdots$ & Col. John Evans, British Museum. \\
\hline II $\frac{5}{8}$ & 4 & $5 \frac{1}{4}$ & $\cdots$ & $\cdots$ & $\cdots$ & British Museum. \\
\hline $11 \frac{5}{8}$ & 4 & $4 \frac{1}{2}$ & $\cdots$ & $\ldots$ & ... & Sir Robert Harvey, Bart. \\
\hline $11 \frac{1}{2}$ & $4 \frac{1}{2}$ & $5 \frac{1}{4}$ & $\ldots$ & $\ldots$ & ... & Lieut. F. E. Shafto-Adair. \\
\hline $11 \frac{1}{4}$ & $4 \frac{3}{8}$ & $6 \frac{7}{8}$ & $\cdots$ & ... & ... & British Museum. \\
\hline II $\frac{1}{8}$ & $4 \frac{5}{8}$ & $5 \frac{3}{8}$ & $\cdots$ & $\ldots$ & ... & Hume Collection, British Museum. \\
\hline II & $4 \frac{1}{8}$ & 6 & $\cdots$ & $\cdots$ & $\cdots$ & Do. \\
\hline
\end{tabular}


INDIAN GAZELLE or RAVINE DEER (Gazella bennetti)continued.

\begin{tabular}{|c|c|c|c|c|c|c|}
\hline Length. & $\begin{array}{l}\text { Circum- } \\
\text { ference. }\end{array}$ & Tip to Tip. & \multicolumn{3}{|c|}{ Habitat. } & Owner. \\
\hline I I & $4 \frac{1}{8}$ & $5 \frac{1}{4}$ & $\ldots$ & ... & $\ldots$ & Sir Robert Harvey, Bart. \\
\hline II & $3 \frac{3}{4}$ & 6 & $\ldots$ & $\cdots$ & $\ldots$ & C. H. Seely. \\
\hline $10 \frac{7}{8}$ & $4 \frac{3}{8}$ & $6 \frac{3}{8}$ & $\ldots$ & $\ldots$ & $\cdots$ & Hume Collection, British Museum. \\
\hline $10 \frac{7}{8}$ & 4 & $5 \frac{1}{8}$ & $\ldots$ & $\cdots$ & $\cdots$ & Maharajah Dhuleep Singh. \\
\hline $10 \frac{7}{8}$ & $3 \frac{3}{4}$ & $7 \frac{7}{8}$ & $\ldots$ & $\cdots$ & $\cdots$ & Sir Victor Brooke's Collection. \\
\hline $10 \frac{3}{4}$ & $3 \frac{5}{8}$ & $4^{\frac{3}{4}}$ & $\cdots$ & $\cdots$ & $\cdots$ & F. J. Horniman. \\
\hline $10 \frac{3}{8}$ & $3^{\frac{3}{4}}$ & $5 \frac{1}{8}$ & $\cdots$ & $\cdots$ & $\cdots$ & Hume Collection, British Museun. \\
\hline Io & $4 \frac{1}{2}$ & $5 \frac{1}{4}$ & Damoor, & , near De & elhi & J. Hugh Mann. \\
\hline IO & 4 & $4 \frac{1}{2}$ & $\ldots$ & $\ldots$ & $\ldots$ & Marquis of Ailsa. \\
\hline $9 \frac{7}{8}$ & 4 & 5 & Simrol, & Bengal & $\cdots$ & Col. John Evans, British Museum. \\
\hline $9^{\frac{3}{4}}$ & $3 \frac{1}{2}$ & $5 \frac{1}{8}$ & Sind & $\ldots$ & ... & R. Lydekker, British Museum. \\
\hline $9 \frac{5}{8}$ & $3 \frac{7}{8}$ & 4 & $\cdots$ & $\ldots$ & $\cdots$ & J. Carr-Saunders. \\
\hline $9^{\frac{1}{2}}$ & $4 \frac{1}{4}$ & $5 \frac{1}{8}$ & $\cdots$ & $\cdots$ & $\ldots$ & Hon. Walter Rothschild. \\
\hline $9 \frac{3}{8}$ & 4 & 4 & $\ldots$ & $\cdots$ & $\cdots$ & Hume Collection, British Musenn. \\
\hline 9 & $3 \frac{5}{8}$ & $3 \frac{7}{8}$ & $\ldots$ & $\cdots$ & $\ldots$ & Do. \\
\hline q $7 \frac{1}{8}$ & I $\frac{5}{8}$ & $2 \frac{1}{4}$ & $\cdots$ & $\cdots$ & $\ldots$ & Sir Victor Brooke's Collection. \\
\hline $94 \frac{7}{8}$ & $\cdots$ & $1 \frac{1}{2}$ & Deccan & ... & $\ldots$ & Col. Sykes, British Museum. \\
\hline
\end{tabular}




\section{ATLAS GAZELLE (Gazella cuvieri).}

[Sir VICTOR I3ROOKE, IJart., P.Z.S. 1873, p. 542.]

Hair long and rather coarse; central facial band grizzled rufous brown above, darker below, sometimes forming a distinct nose-spot; light facial streak present, but indefinite; dark facial streak blackish brown, running into and blending with the grizzled rufous brown of the upper parts of the body; knee-brushes long, blackish; tail black; homs strongly anrulaterd, massive and long, diversing gradually from about two-thirds their length, the upper third leaning more decidedly outwards and a little forward; hars very long.

Adult male: height, $27 \frac{1}{2}$ in.; length of horns, I2 in.; length of ears, $7 \frac{1}{4}$ in.

The above description must be taken as applying to an average specimen, very great variety being exhibited by this large and wellmarked species; in fact, out of a large series, including living specimens, I have never seen two for which the same description would suffice. The species, however, is easily known from all the other small gazelles by its larger size, rough coat, dark colour, and unusually long ears. As I have said above, I think the larger gazelle seen by Tristram in Algeria was probably of this species. A skull in my possession is very nearly as large as that of Gazella sæmmerringii.

\section{Habitat-Morocco and Algeria.}

\begin{tabular}{|c|c|c|}
\hline I ength. & $\begin{array}{l}\text { Circum- } \\
\text { ferenec. }\end{array}$ & Tip to Tiss. \\
\hline
\end{tabular}

\begin{tabular}{|c|c|c|c|c|c|}
\hline $14 \frac{7}{3}$ & 5 & $3 \frac{5}{5}$ & Algeria & $\ldots$ & Sir Edmund (;. Loder, l'art. \\
\hline q $13 \frac{7}{6}$ & $3 \frac{1}{2}$ & $7 \frac{1}{s}$ & $\begin{array}{l}\text { Ben-a-Chouf, } \\
\text { Narth Africa }\end{array}$ & & E. N. I3uxton, 1890. \\
\hline$\delta 12 \frac{3}{3}$ & $4 \frac{\pi}{x}$ & $3 \frac{5}{5}$ & Do. & & Do. \\
\hline $12 \frac{3}{5}$ & $3 \ddot{z}$ & $5 \frac{1}{3}$ & Algeria & ... & Rowland Ward's Collection. \\
\hline
\end{tabular}

\section{BLACK-TAILED GAZELLE (Gazella tilonura).}

[Sir Victor 13ROOKE, I3art., P.Z.S. 1873, p. 542; Antilope melanura (HExGi.o, ANT, und IJÜFF), p. 6 ; 1869, Antilope tilonura (HENGL., REIS, WEISS, NIL.), p. 315.]

CENTRA facial band rufous fawn; light facial streak indistinct, encircling the eye; dark facial streak very indistinct, almost indis- 
tinguishable; neck, back, uppersides, flanks, haunches, shoulders, root of the tail, anterior of fore legs, knee-brushes, and outer sides of the hind legs sandy fawn-colour; dark lateral band distinct, blackish, very narrow from above downwards; light lateral very indefinite; breast and belly white; horns lyrate and strongly annulated.

Height, 29 in.; length of ears, $5 \frac{1}{4}$ in.; length of tail, 8 in.; length of horns, II in.

Habitat-Bushy plains of the Anseba, Bogosland (Hengl.), South Senaar (Casanova).

\begin{tabular}{|c|c|c|c|c|c|}
\hline Length. & $\begin{array}{l}\text { Circum- } \\
\text { ference. }\end{array}$ & Tip to Tip. & Habitat. & & Owner. \\
\hline $10 \frac{3}{4}$ & $3 \frac{3}{4}$ & $4 \frac{1}{2}$ & $\ldots$ & $\ldots$ & E. Lort-Phillips. \\
\hline $10_{4}^{3}$ & 4 & $2 \frac{1}{8}$ & Bogos Country, & & British Museum. \\
\hline $10 \frac{1}{2}$ & $4 \frac{1}{8}$ & $3 \frac{3}{4}$ & Do. & & Do. \\
\hline $10 \frac{1}{4}$ & $4 \frac{1}{8}$ & $2 \frac{7}{8}$ & North Africa & $\ldots$ & Col. Ralph Vivian. \\
\hline $9 \frac{7}{x}$ & $4 \frac{3}{8}$ & $1 \frac{7}{8}$ & $\begin{array}{l}\text { Bogos Country, } \\
\text { Abyssinia. }\end{array}$ & & Sir Victor Brooke's Collection. \\
\hline $9^{\frac{7}{8}}$ & $\cdots$ & $\mathrm{I}_{\frac{1}{2}}^{\frac{1}{2}}$ & $\begin{array}{l}\text { Anseba River, } \\
\text { Abyssinia. }\end{array}$ & & Do. \\
\hline $9 \frac{3}{4}$ & $3 \frac{3}{4}$ & $3 \frac{1}{2}$ & North Africa & $\cdots$ & Col. Ralph Vivian. \\
\hline $9 \frac{1}{2}$ & $4 \frac{1}{4}$ & $1 \frac{3}{4}$ & Do. & $\cdots$ & Do. \\
\hline $8 \frac{1}{4}$ & $3 \frac{3}{8}$ & $2 \frac{1}{2}$ & $\ldots$ & $\cdots$ & Lieut. E. Lacy. \\
\hline $7 \frac{3}{4}$ & $3 ?^{7}$ & I $\frac{5}{8}$ & $\begin{array}{l}\text { Bogos Country, } \\
\text { Abyssinia. }\end{array}$ & & British Museum. \\
\hline ㅇ $5 \frac{1}{8}$ & $1 \frac{3}{4}$ & $1 \frac{3}{4}$ & Abyssinia & $\cdots$ & Sir Victor Brooke's Collection. \\
\hline
\end{tabular}

CORINNE (Gazella corinna).

Habitat-West Africa.

\begin{tabular}{|c|c|c|c|c|c|}
\hline Length. & $\begin{array}{l}\text { Circum- } \\
\text { ference. }\end{array}$ & 'Tip to Tip. & Habitat. & & Owner. \\
\hline q $6 \frac{1}{1}$ & $2 !$ & $2 \frac{3}{8}$ & West $\Lambda$ frica & $\ldots$ & Brilish Museum. \\
\hline
\end{tabular}




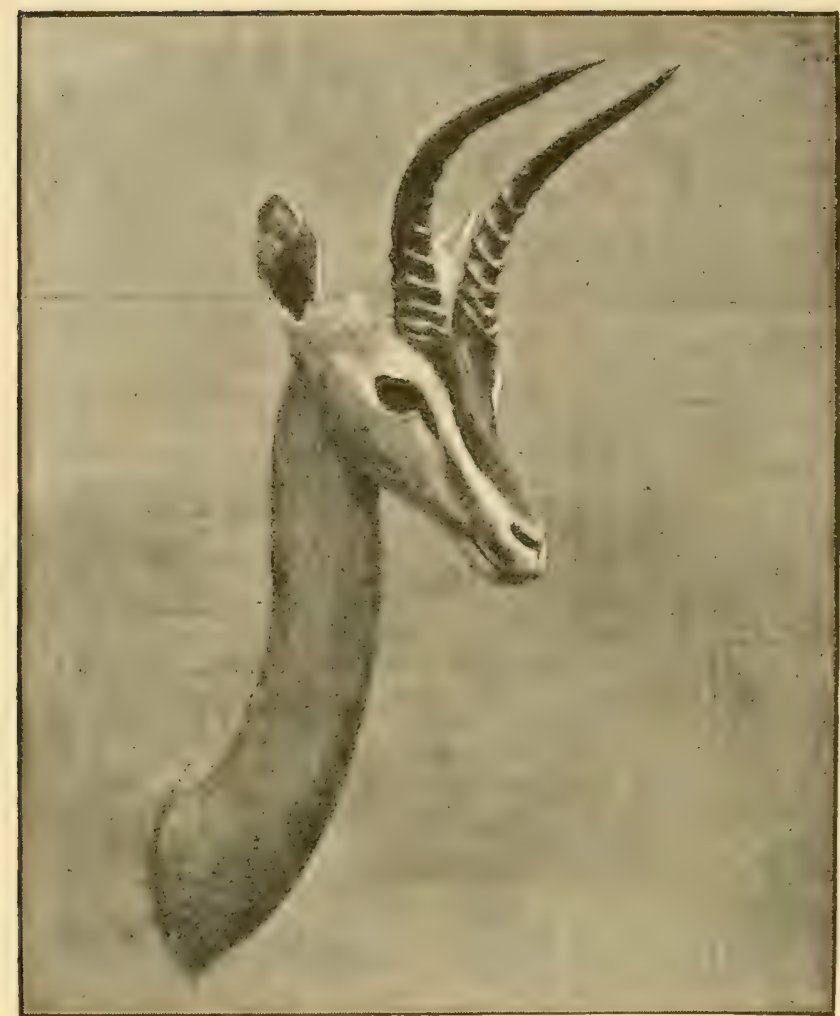

Shot in Somali Land by T. W. H. Clarke, I8gI.

\section{CLARKE'S GAZELLE (Ammodorcas clarkei).}

[OLDFIELD THOMAS, P.Z.S. I891, p. 206.]

This most remarkable animal combines the horns of a Reed-buck (Cervicapra), with the essential characters of a Gazelle, showing a

When I received Mr. Clarke's specimens and examined this particular Gazelle, it was at once apparent to me that it was a new variety-a fact that I had the pleasure to convey to Mr. Clarke, - R. W. 
special relationship to that most aberrant Gazelle the Gerenook (Lithocranius walleri), and appears certainly to be worthy of generic distinction. On the first arrival of the specimens, before the skulls were cleaned, and before Mr. Sclater had received from Mr. Swayne the face-skins mentioned below, the animal was naturally supposed to be a Reed-buck from the character of its horns, little likely as it appeared that a Reed-buck would occur in the dry sandy plateau of Somali Land. Now, however, that better material is available, I am able to draw up a fairly complete account of the more essential characters of this most beautiful Gazelle, of the discovery of which Mr. Clarke, both as a sportsman and naturalist, has so much reason to be proud.

Muzzle, character of face-marking, and presence of anteorbital glands as in Gazella; general form (as described by Mr. Clarke, see below) and skull more as in Lithocranius; horns in curve and general appearance as in Cervicapra.

Coloration of face, using Sir Victor Brooke's phraseology, as follows:-Central facial band deep rich chestnut-rufous, becoming duller towards the bases of the horns; light facial streaks pure white, very prominent and sharply defined, extending the whole length of the head from the bases of the ears to the corners of the nostrils, somewhat duller and more indistinct at their extremities, but broadening in the middle to encircle the eyes; dark facial streak present, but not strongly marked; cheeks and sides of neck pale fawn; throat white; crown between and behind horns brown or grizzled fawn, the latter condition occurring in the older of Mr. Swayne's two head-skins; a marked whorl of hairs situated between the ears; back of ears shorthaired, dark fawn basally, gradually darkening to black terminally; insides white-haired, but with black tips.

Horns evenly curved upwards and forwards, the basal halves running backwards and the terminal halves nearly vertically upwards, the main curvature being therefore exactly in the opposite direction to that found in Gazella and Lithocranius. The horns themselves slender, their smooth unridged portion very long, the first indistinct ridge occurring about five inches from the tip. Ridges widely separate, strongly defined anteriorly, but becoming abruptly obsolete about halfway round, the sides and back showing scarcely a trace of them; 
their number amounting to Io in the oldest specimen available- $\mathrm{a}$ number probably but seldom much exceeded.

Skull diverging from that of Gazella and approaching that of Lithocranius by being unusually low, long in the occipital region with a comparatively straight cranio-facial angle with very small bullæ with the postero-internal wall of the suborbital fossæ imperfect, and with a remarkably small and slender lower jaw. On the other hand, it differs from the same skull by being peculiar to a less extent in all these points by the greater development of the premaxillæ posteriorly, where they have a broad articulation with the nasals, by the larger nasals and smaller interparietal, and by the larger and more powerful teeth, which are absolutely larger in the smaller animal.

In its skull characters, therefore, Ammodorcas stands just intermediate between Gazella and Lithocranius; in its muzzle and in the characters of its markings it agrees fairly with both, but in general form with the latter only, that animal, like it, being peculiar for its extraordinarily long neck, and finally, in the curvature of its horns it differs absolutely from both, as also from any of the other Gazelline genera, while it agrees with a group so different from it in all essential respects that the resemblance must evidently be an accidental one and not indicative of any relationship. Presumably the whole of the horn of this species is homologous with the terminal three or four inches of the Gerenook's horns, that is to say, with the upwardly curved part-the greater part of the horn having become obsolete while the upwardly curved tips have attained to a remarkable proportionate development.

The following are the dimensions of the skull of specimen :-

Length, occiput to gnathion, 2ro millim., tip of nasals to occiput I76, greatest breadth 95; height, crown to angle of lower jaw, 94; nasals, length 67 , breadth 26 ; interparietal, length 24 , breadth 39 ; height of orbital opening 35 , gnathion to front of anterior premolar 63; length of molar series 53; lower jaw, condyle to front of symphysis 153 ; height from coronoid process to angle 70 ; height of ramus at centre of $\mathrm{m}$. $\mathrm{I}^{\circ} 4$, at posterior end of symphysis 9 .

In a letter addressed by Mr. Clarke to Mr. Rowland Ward, the following notes on the appearance and habits of this species are 
given :- "The Somali name for these antelopes is 'Debo Tag.' I shot them in the new country I went into, and did not see them in any other part. They have a very thin long tail, and when they run, throw it up and over towards the neck. The neck is very long and thrown back towards the tail, so that the two look as if they would touch each other."

Shortly after the arrival of Mr. Clarke's specimens, Mr. Sclater received from Mr. Swayne two scalps of this species which he had bought in Berbera, and these scalps Mr. Sclater has most kindly handed over to me for the purposes of the present paper. Fortunately, the muzzle of one of them has been preserved, so that I have been able to make out its strictly Gazelline character, and the description of the colours above given has also been taken from these specimens.

It is much to be hoped that more specimens of this beautiful Gazelle will soon be obtained, and that we may thereby gain a full knowledge of its range, habits, and natural affinities.

P.S. (May I 4 th).-Mr. Clarke has favoured me with the following notes on this new Gazelle:-

"I saw this Gazelle for the first time on December I7th, I8go, about three hours from Bairwell, or about one day from Buroa Well, Habergerhagi's country, and afterwards on the road all the way into the Marchan district, $8^{\circ} \mathrm{N} .47^{\circ} \mathrm{E}$. I killed a male, and found one of the horns broken off close to the skull, which had apparently been done a year or so ago.

"They are very graceful animals, with a long neck and well-proportioned head and horns; the body is rather slender, but considerably larger than in Gazella spekii, about the size of a female G. walleri. The legs are long and slender; the hoofs are not so triangular as those of G. spekii, and small for the size of the animal."

Mr. Clarke informs me that the female of Ammodorcas is without horns, a character which allies it still more closely with Lithocranius, and removes it from the typical Gazelles. The exact locality at which he obtained the specimens is about a day and a half from the Buroa Wells, Central Somali, about Ioo miles south of Berbera. 
CLARKE'S GAZELLE (Ammodorcas clarkei)-continued.

Habitat-Somali Land.

\begin{tabular}{|c|c|c|c|c|c|}
\hline Length. & $\begin{array}{l}\text { Circum- } \\
\text { ference. }\end{array}$ & Tip to Tip. & Habitat. & & Owner. \\
\hline II $\frac{3}{4}$ & $4 \frac{7}{8}$ & $3 \frac{5}{8}$ & Somali Land & $\ldots$ & Col. A. Paget. \\
\hline $11 \frac{3}{4}$ & $4^{\frac{3}{4}}$ & $3 \frac{5}{8}$ & Do. & $\ldots$ & T. W. H. Clarke. \\
\hline$I_{1}^{1}$ & 5 & 5 & Do. & $\ldots$ & Col. A. Paget. \\
\hline II $\frac{1}{4}$ & $4 \frac{1}{2}$ & $4^{\frac{3}{4}}$ & Do. & $\cdots$ & T. W. H. Clarke. \\
\hline $10 \frac{1}{4}$ & $4 \frac{1}{2}$ & $4 \frac{1}{2}$ & Do. & $\ldots$ & Capt. Townley Parker. \\
\hline $10 \frac{1}{8}$ & $4 \frac{7}{8}$ & $3 \frac{3}{3}$ & Do. & $\ldots$ & Hon. Walter Rothschild. \\
\hline $10 \frac{1}{8}$ & $4 \frac{1}{4}$ & $4 \frac{7}{8}$ & Do. & $\ldots$ & Rowland Ward's Collection. \\
\hline $9 \frac{3}{4}$ & $4 \frac{7}{2}$ & $5 \frac{1}{8}$ & Do. & $\ldots$ & T. W. H. Clarke, British Museum. \\
\hline $9^{\frac{3}{4}}$ & $4 \frac{1}{2}$ & $4^{\frac{3}{4}}$ & Do. & $\ldots$ & Capt. H. G. C. Swayne. \\
\hline $9 \frac{1}{2}$ & $4 \frac{5}{8}$ & $3^{\frac{3}{4}}$ & Do. & $\ldots$ & T. W. H. Clarke. \\
\hline 8 & $3 \frac{3}{t}$ & $2 \frac{7}{8}$ & Do. & $\ldots$ & Marquis of Ailsa. \\
\hline $6 \frac{1}{8}$ & $3^{\frac{1}{4}}$ & $2 \frac{1}{8}$ & Do. & $\ldots$ & E. Lort-Phillips. \\
\hline
\end{tabular}




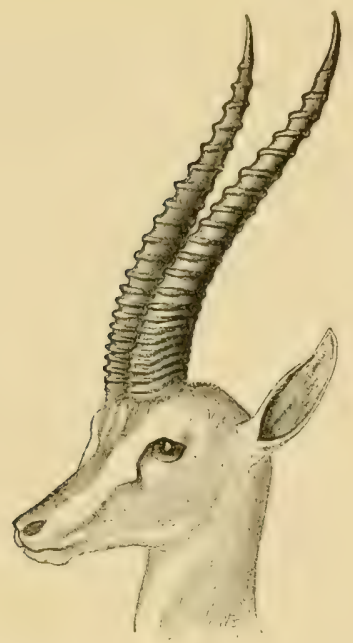

THOMSON'S GAZELLE (Gazella thomsoni).

This Gazelle was discovered in East Africa by Mr. Joseph Thomson, the celebrated explorer, and is rather rare.

\section{Habitat-East Africa.}

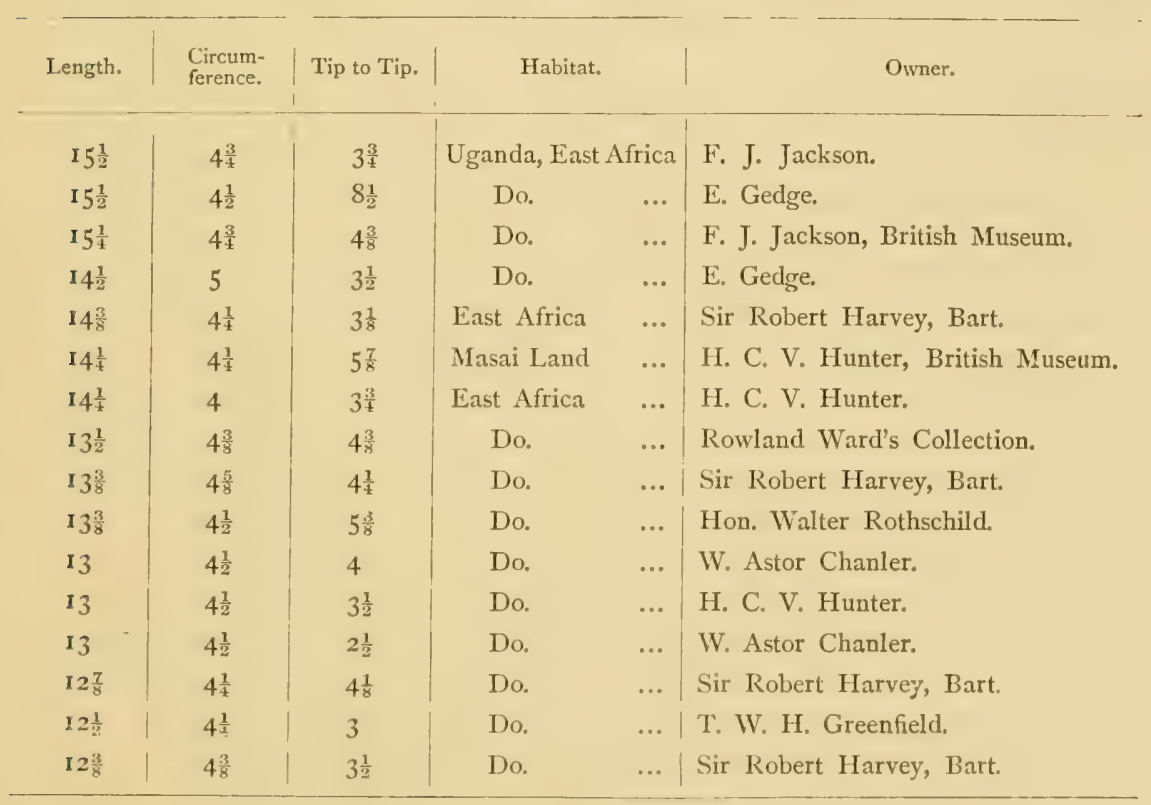




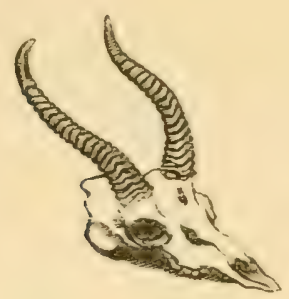

WALLER'S GAZELLE (Lithocranius walleri).

THis Gazelle was described by the late Sir Victor Brooke from a specimen collected by the Revd. Mr. Waller in British East Africa.

Somali name-"Gerenook."

Habitat-Somali Land and East Africa.

\begin{tabular}{|c|c|c|c|c|c|}
\hline Length. & $\begin{array}{l}\text { Circum- } \\
\text { ference. }\end{array}$ & Tip to Tip. & Habitat. & & Owner. \\
\hline $15 \frac{3}{8}$ & $5 \frac{5}{8}$ & 4 & Somali Land & $\ldots$ & Capt. H. G. C. Swayne. \\
\hline $15 \frac{3}{8}$ & $5 \frac{3}{s}$ & $4 \frac{1}{8}$ & Do. & $\ldots$ & C. Liddell. \\
\hline $14^{\frac{3}{4}}$ & $5 \frac{1}{8}$ & $4 \frac{3}{4}$ & Do. & $\ldots$ & Lord Delamere. \\
\hline $14 \frac{1}{2}$ & $5 \frac{1}{2}$ & 3 & Do. & $\ldots$ & Capt. H. G. C. Swayne. \\
\hline $14 \frac{1}{2}$ & 5 & $4 \frac{1}{2}$ & Do. & $\cdots$ & Lord Delamere. \\
\hline $14 \frac{1}{8}$ & $5 \frac{3}{8}$ & $4 \frac{1}{4}$ & Do. & $\ldots$ & Do. \\
\hline 14 & $5 \frac{1}{2}$ & $\mid \begin{array}{r}3 \frac{3}{1} \text { (owner's } \\
\text { measurement) }\end{array}$ & $\ldots \quad \ldots$ & $\ldots$ & J. D. Inverarity. \\
\hline I4 & $\ldots$ & 3 & East Africa & $\ldots$ & $\begin{array}{l}\text { Sir John Willoughby, Bart., I } \delta \delta_{7} \text {, } \\
\text { "East Africa and its Big Game." }\end{array}$ \\
\hline 14 & $5^{\frac{1}{2}}$ & 4 & Somali Land & $\cdots$ & Lord Delamere. \\
\hline $13 \frac{7}{8}$ & $4 \frac{5}{8}$ & $5 \frac{1}{8}$ & East Africa & $\cdots$ & H. C. V. Hunter. \\
\hline $13 \frac{7}{8}$ & $4 \frac{7}{8}$ & $2 \frac{7}{8}$ & Somali Land & $\cdots$ & F. L. James, I 884 . \\
\hline $13 \frac{3}{4}$ & $4 \frac{7}{8}$ & $4 \frac{1}{8}$ & Do. & $\ldots$ & E. Lort-Phillips. \\
\hline $13 \frac{3}{4}$ & $5 \frac{5}{3}$ & $2 \frac{7}{3}$ & North Somali L & and & J. Menges, British Museum. \\
\hline $13 \frac{3}{t}$ & $5 \frac{3}{8}$ & $3 \frac{7}{8}$ & Somali Land & $\cdots$ & Hon. Walter Rothschild. \\
\hline $13 \frac{3}{4}$ & $5 \frac{1}{4}$ & $4 \frac{3}{5}$ & Do. & $\cdots$ & Lord Delanere. \\
\hline
\end{tabular}


WALLER'S GAZELLE (Lithocranius walleri)-continucd.

\begin{tabular}{|c|c|c|c|c|c|}
\hline Length. & $\begin{array}{l}\text { Circum- } \\
\text { ference. }\end{array}$ & Tip to Tip. & Habitat. & & Owner. \\
\hline $13 \frac{5}{8}$ & $5 \frac{5}{8}$ & $3 \frac{1}{t}$ & Somali Land & $\cdots$ & A. II. Straker. \\
\hline $13 \frac{5}{8}$ & $5 \frac{1}{8}$ & $4 \frac{7}{8}$ & Do. & ... & T. W. H. Clarke. \\
\hline $13 \frac{5}{8}$ & $4 \frac{7}{8}$ & $4 \frac{5}{8}$ & De. & $\cdots$ & C. H. Villiers. \\
\hline $13 \frac{1}{2}$ & $5 \frac{7}{3}$ & 6 & $\ldots \quad \ldots$ & $\cdots$ & C. Liddell. \\
\hline $13 \frac{3}{8}$ & $5 \frac{5}{8}$ & $3 \frac{1}{\frac{1}{2}}$ & Somali Land & $\cdots$ & Hon. Walter Rothschild. \\
\hline I $3 \frac{1}{t}$ & $5^{\frac{1}{2}}$ & $3 \frac{1}{2}$ & Do. & $\cdots$ & T: W. H. Clarke. \\
\hline $13 \frac{1}{4}$ & $5 \frac{3}{8}$ & $5 \frac{5}{8}$ & Do. & $\cdots$ & A. H. Straker. \\
\hline $13 \frac{1}{4}$ & $4 \frac{5}{8}$ & $2 \frac{5}{8}$ & $\begin{array}{l}\text { Juba Valley, } \\
\text { mali Land. }\end{array}$ & So- & Sir John Kirk, M.D., K.C. B. \\
\hline 13 & $58^{3}$ & 4 & $\cdots \quad \cdots$ & $\cdots$ & E. Lort-Phillips. \\
\hline 13 & $5^{\frac{1}{2}}$ & $4 \frac{3}{4}$ & Somali Land & $\cdots$ & Marquis of Ailsa. \\
\hline $\mathbf{I}_{3}$ & $5 \frac{1}{2}$ & $3 \frac{1}{2}$ & I)o. & $\cdots$ & J. Carr-Saunders. \\
\hline 13 & $5^{\frac{1}{t}}$ & $\mid \begin{array}{c}5 \text { (owner's } \\
\text { measurement) }\end{array}$ & I). & $\ldots$ & J. D. Inverarify. \\
\hline 13 & $4 \frac{1}{\frac{1}{4}}$ & $4 \frac{1}{t}$ & 1\%. & $\cdots$ & J. Carr-Saunders. \\
\hline $12 \frac{5}{8}$ & $4^{\frac{8}{1}}$ & $4 \frac{31}{1}$ & $1 \%$. & $\cdots$ & Lord Delamere. \\
\hline I $2 \frac{1}{2}$ & $5 \frac{1}{2}$ & $3 \frac{1}{8}$ & $1 \%$ & $\cdots$ & Col. A. Paget. \\
\hline $12 \frac{1}{2}$ & $5 \frac{3}{8}$ & $3 \frac{7}{8}$ & l). & $\cdots$ & Lord Delamere. \\
\hline $12 \frac{1}{2}$ & $5^{\frac{1}{4}}$ & $2 \frac{38}{4}$ & I)o. & $\cdots$ & T. W. H. Clarke. \\
\hline $12 \frac{1}{3}$ & $5^{\frac{1}{2}}$ & $4 \frac{1}{2}$ & Do. & $\cdots$ & Do. \\
\hline $12 \frac{1}{2}$ & $4 \frac{2}{4}$ & $\begin{array}{c}2 \frac{1}{2} \text { (owner's } \\
\text { measurement) }\end{array}$ & $\cdots \quad \ldots$ & $\cdots$ & J. D. Inverarity. \\
\hline $12 \frac{1}{2}$ & $4 \frac{3}{8}$ & $4 \frac{3}{4}$ & $\begin{array}{l}\text { Kilima'njaro, I } \\
\text { Africa. }\end{array}$ & East & F. J. Jackson. \\
\hline $12 \frac{3}{8}$ & $5 \frac{3}{5}$ & $4 \frac{1}{4}$ & Zanzibar ... & $\cdots$ & Sir Edmund G. Loder, Bart. \\
\hline $12 \frac{3}{8}$ & $5 \frac{1}{4}$ & $5^{\frac{1}{2}}$ & Somali Land & $\cdots$ & T. W. H. Clarke. \\
\hline I $2 \frac{3}{8}$ & $4 \frac{5}{8}$ & $3^{\frac{1}{4}}$ & $\begin{array}{l}\text { Juba Valley, } \\
\text { mali Land. }\end{array}$ & So- & Sir John Kirk, M.D., K.C.B. \\
\hline $12 \frac{1}{4}$ & $4 \frac{3}{4}$ & 5 & Do. & & Do. \\
\hline 12 & $5^{\frac{1}{2}}$ & $5^{\frac{1}{2}}$ & Somali Land & $\cdots$ & Gen. Trewell. \\
\hline $11 \%$ & $5: \frac{3}{4}$ & $5^{\frac{3}{4}}$ & Do. & $\cdots$ & C. H. Villiers. \\
\hline II $\frac{5}{8}$ & $4 \frac{3}{4}$ & $4 \frac{3}{8}$ & Enst Africn & $\cdots$ & Sir Robert Harvey, Bart. \\
\hline
\end{tabular}


WALLER'S GAZELLE (Lithocranius walleri)-continued.

\begin{tabular}{|c|c|c|c|c|}
\hline Length. & $\begin{array}{l}\text { Circum- } \\
\text { ference. }\end{array}$ & Tip to Tip. & Habitat. & Owner. \\
\hline III $\frac{5}{8}$ & $4 \frac{5}{8}$ & $4 \frac{1}{4}$ & South Somali Land & Dr. Kirk, British Museum. \\
\hline II $\frac{5}{8}$ & $4 \frac{3}{s}$ & $2 \frac{1}{2}$ & East Africa & Sir Robert Harvey, Bart. \\
\hline II I $\frac{3}{8}$ & $4^{\frac{3}{4}}$ & $3 \frac{7}{8}$ & $\begin{array}{l}\text { Juba Valley, So- } \\
\text { mali Land. }\end{array}$ & Sir John Kirk, M.D., K.C.B. \\
\hline II $\frac{1}{4}$ & $5 \frac{1}{4}$ & $4 \frac{3}{4}$ & Somali Land $\quad \ldots$ & Sir Victor Brooke's Collection. \\
\hline II $\frac{1}{4}$ & $44^{\frac{8}{t}}$ & $4 \frac{1}{t}$ & East Africa & Sir Robert Harvey, Bart. \\
\hline $10_{\frac{3}{4}}^{\frac{3}{4}}$ & 5 & $3 \frac{3}{4}$ & Somali Land $\quad \ldots$ & F. L. James, I $88_{4}$. \\
\hline $10_{\frac{3}{4}}^{3}$ & $4^{\frac{3}{4}}$ & 3 & South Somali Land & $\begin{array}{l}\text { Sir John Kirk, M.D., K. C. B., British } \\
\text { Museun. }\end{array}$ \\
\hline $10 \frac{1}{4}$ & $4 \frac{3}{4}$ & $4^{\frac{1}{3}}$ & East Africa & Sir Robert Harvey, Bart. \\
\hline $10 \frac{1}{4}$ & $4 \frac{3}{t}$ & $4 \frac{1}{x}$ & Do. & Do. \\
\hline $9 \frac{3}{5}$ & $4 \frac{1}{x}$ & $3 \frac{3}{4}$ & South Somali Land & $\begin{array}{l}\text { Sir John Kirk, M. D., K. C. B., British } \\
\text { MInseum. }\end{array}$ \\
\hline
\end{tabular}

The Horns of the specimens from Somali Land are very much longer than those which I have received from British East Africa, as will be seen by reference to the above measurements. 


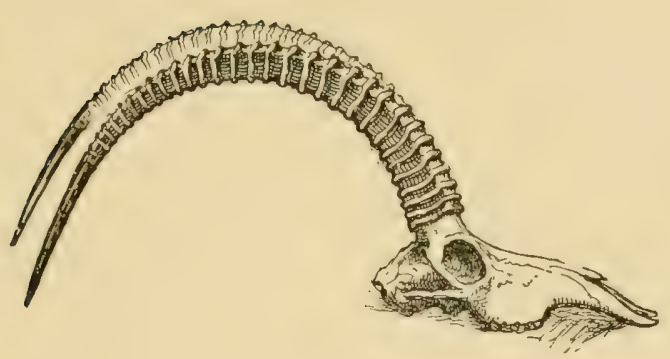

SABLE ANTELOPE (Hippotragus niger)

HeIght at shoulder $4 \mathrm{ft} .6$ in., tail 25 in., ears Io in., extreme length nearly $9 \mathrm{ft}$.

$$
\begin{gathered}
\text { Sena name-"Para-Para." Bechuana name-"Tahetse." } \\
\text { Makalolo name-" Qualata e Enchu." } \\
\text { Habitat-South and East Africa. }
\end{gathered}
$$

\begin{tabular}{|c|c|c|c|c|c|}
\hline Length. & $\begin{array}{l}\text { Circum- } \\
\text { ference. }\end{array}$ & Tip to Tip. & Habitat. & & Owner. \\
\hline 46 & $\ldots$ & $\ldots$ & South Africa & $\ldots$ & F. and H. Barber, "Land and \\
\hline $44 \frac{1}{2}$ & $10 \frac{1}{2}$ & ... & $\cdots$ & $\cdots$ & F. V. Kirby, "Land and Water," \\
\hline 44 & ... & $\cdots$ & Chobe River & ... & F. C. Selous, "A Hunter's Wander- \\
\hline $43^{\frac{1}{2}}$ & $9 \frac{3}{4}$ & 15 & South Africa & $\ldots$ & The late J. S. Jameson. \\
\hline $43^{\frac{1}{4}}$ & $9 \frac{5}{8}$ & $8 \frac{1}{4}$ & Do. & .. & Do. \\
\hline 43 & $9 \frac{2}{4}$ & $6 \frac{1}{2}$ & Mashuna Land & $\ldots$ & F. C. Selous, British Museum. \\
\hline 43 & $10 \frac{1}{4}$ & 13 & $\ldots$ & ... & Major Gould Adams. \\
\hline 43 & ... & $\cdots$ & Chobe River & $\ldots$ & $\begin{array}{l}\text { F. C. Selous, "A Hunter's Wander- } \\
\text { ings." }\end{array}$ \\
\hline $42 \frac{7}{8}$ & $10 \frac{3}{8}$ & $16 \frac{1}{8}$ & ... & ... & F. C. Selous. \\
\hline $42 \frac{7}{8}$ & $9 \frac{3}{4}$ & $10_{4}^{3}$ & ... & ... & J. Carr-Saunders. \\
\hline $42 \frac{3}{4}$ & $9 \frac{5}{8}$ & I $7 \frac{3}{8}$ & ... & $\cdots$ & F. C. Selous. \\
\hline $42 \frac{5}{8}$ & $9 \frac{7}{8}$ & $9 \frac{1}{2}$ & South Africa & $\ldots$ & Bethnal Green Muscum. \\
\hline
\end{tabular}


SABLE ANTELOPE (Hippotragus niger)-continued.

\begin{tabular}{|c|c|c|c|c|c|c|}
\hline Length. & $\begin{array}{l}\text { Circum- } \\
\text { ference. }\end{array}$ & Tip to Tip. & & Habitat. & & Owner. \\
\hline $42 \frac{5}{8}$ & $9 \frac{1}{2}$ & $I I \frac{1}{2}$ & $\ldots$ & $\ldots$ & $\ldots$ & J. A. Jameson. \\
\hline $42 \frac{1}{2}$ & $9 \frac{5}{6}$ & $10 \frac{3}{8}$ & $\ldots$ & $\ldots$ & $\ldots$ & F. C. Selous. \\
\hline $42 \frac{3}{8}$ & 9 & $19 \frac{1}{2}$ & $\ldots$ & $\ldots$ & $\ldots$ & Do. \\
\hline 42 & $9 \frac{1}{4}$ & $12 \frac{3}{4}$ & $\cdots$ & ... & $\ldots$ & J. Carr-Saunders. \\
\hline $41 \frac{7}{8}$ & $9 \frac{1}{2}$ & I4 & $\ldots$ & $\ldots$ & $\ldots$ & J. A. Jameson. \\
\hline $41 \frac{3}{4}$ & $9 \frac{5}{8}$ & 12 & South & Africa & $\ldots$ & A. Beit. \\
\hline $41^{\frac{3}{4}}$ & $\cdots$ & $9 \frac{1}{2}$ & $\cdots$ & $\ldots$ & $\ldots$ & A. C. Fountaine. \\
\hline $41 \frac{5}{8}$ & $9^{\frac{3}{4}}$ & II & $\ldots$ & ... & $\ldots$ & F. C. Selous. \\
\hline $41 \frac{1}{4}$ & 10 & $12 \frac{7}{8}$ & South & Africa & $\ldots$ & Hon. Walter Rothschild. \\
\hline $4 I$ & $10 \frac{1}{4}$ & $7^{\frac{3}{4}}$ & $\cdots$ & $\cdots$ & $\ldots$ & A. Haggard. \\
\hline $4 \mathrm{I}$ & 10 & $7 \frac{5}{8}$ & South & Africa & $\cdots$ & The late J. S. Jameson. \\
\hline $4 \mathrm{I}$ & $\cdots$ & $13 \frac{1}{4}$ & $\cdots$ & $\cdots$ & $\cdots$ & $\begin{array}{l}\text { Dr. Knight Bruce, Bishop of Bloom- } \\
\text { fontein. }\end{array}$ \\
\hline $4 \mathrm{I}$ & 9 & $14 \frac{3}{4}$ & $\cdots$ & $\cdots$ & $\cdots$ & J. A. Jamieson. \\
\hline $40 \frac{7}{8}$ & $8 \frac{7}{8}$ & $12 \frac{1}{x}$ & South & Africa & $\ldots$ & The late J. S. Jameson. \\
\hline $40 \frac{5}{8}$ & $9 \frac{3}{4}$ & $12 \frac{3}{1}$ & $\cdots$ & $\cdots$ & $\cdots$ & F. C. Selous. \\
\hline $40 \frac{5}{8}$ & $9 \frac{1}{2}$ & $16 \frac{1}{t}$ & $\cdots$ & $\cdots$ & $\cdots$ & Do. \\
\hline $40 \frac{1}{8}$ & $9 \frac{3}{8}$ & I $1 \frac{3}{8}$ & $\cdots$ & $\cdots$ & $\cdots$ & Do. \\
\hline 40 & $9 \frac{1}{\frac{1}{2}}$ & $\cdots$ & $\cdots$ & $\cdots$ & $\cdots$ & Rowland Ward's Collection. \\
\hline $39 \frac{1}{8}$ & $9 \frac{1}{8}$ & $9 \frac{1}{2}$ & South & Africa & $\cdots$ & The late J. S. Jameson. \\
\hline 우 $39 \frac{1}{8}$ & $6 \frac{x}{t}$ & $6 \frac{5}{8}$ & $\cdots$ & $\cdots$ & $\ldots$ & F. C. Selous. \\
\hline 39 & Io & II & $\cdots$ & $\cdots$ & $\ldots$ & Rowland Ward's Collection. \\
\hline 39 & $9 \frac{3}{4}$ & $9 \frac{3}{5}$ & $\cdots$ & ... & $\cdots$ & $\begin{array}{l}\text { Dr. Knight Bruce, Bishop of Mashuna } \\
\text { Land. }\end{array}$ \\
\hline 39 & $9 \frac{1}{2}$ & $\cdots$ & $\cdots$ & $\cdots$ & $\cdots \mid$ & H. S. Runcheman. \\
\hline 39 & 9 & $10 \frac{3}{8}$ & $\cdots$ & $\cdots$ & $\cdots$ & Sir Victor Brooke's Collection. \\
\hline $38 \frac{1}{2}$ & $9 \frac{5}{8}$ & $13 \frac{1}{2}$ & South & Africa & $\cdots \mid$ & The late J. S. Jameson. \\
\hline $38 \frac{1}{8}$ & $9 \frac{3}{s}$. & II & $\begin{array}{r}\text { River } \\
\text { Sou }\end{array}$ & $\begin{array}{l}\text { Umferli, } \\
\text { th Africa. }\end{array}$ & & F. C. Selous, British Museum. \\
\hline $3^{8}$ & $9 \frac{1}{2}$ & $12 \frac{1}{2}$ & $\therefore$ & $\cdots$ & $\cdots$ & B. J. Angle. \\
\hline
\end{tabular}


SABLE ANTELOPE (Hippotragus niger)-continued.

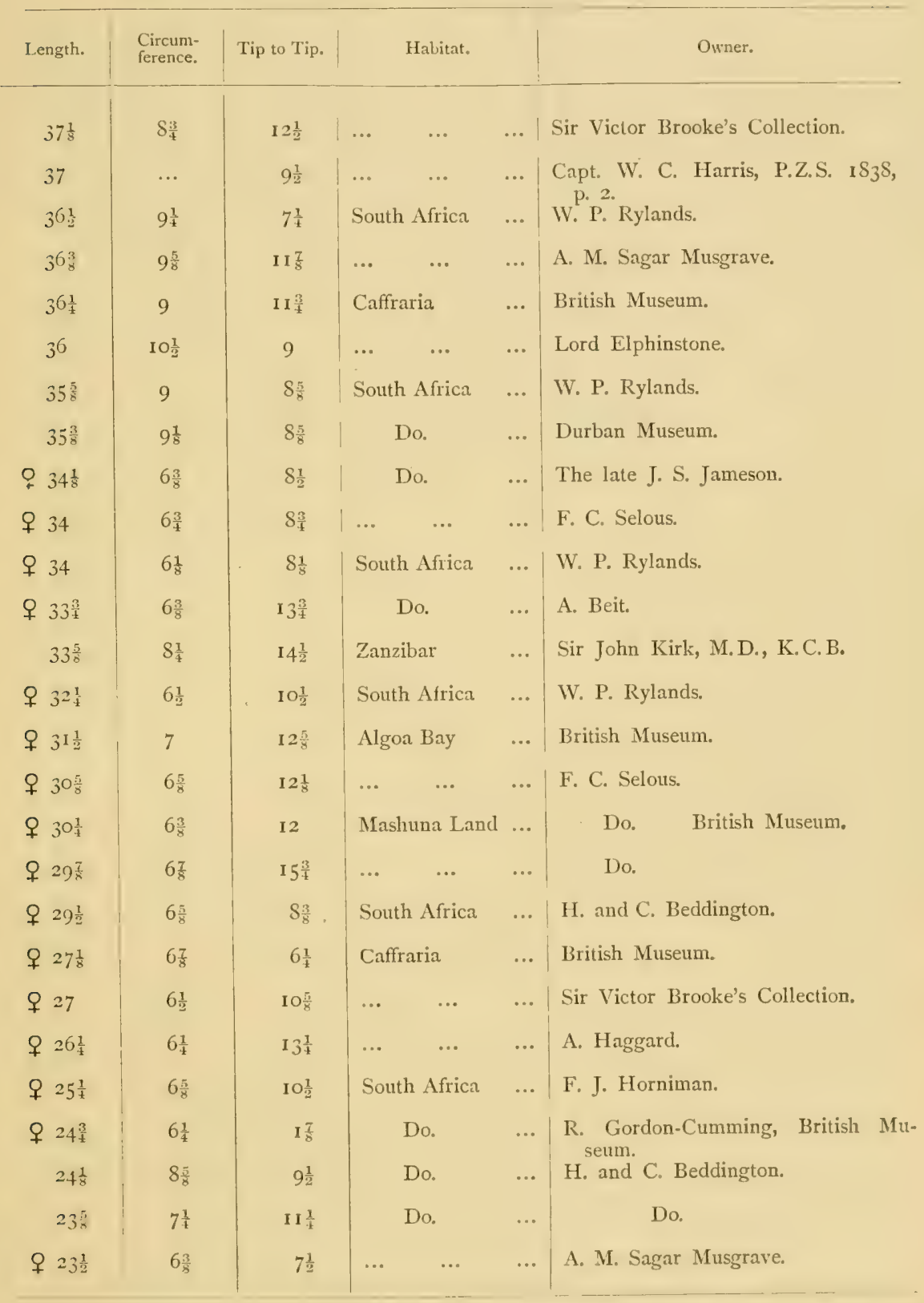




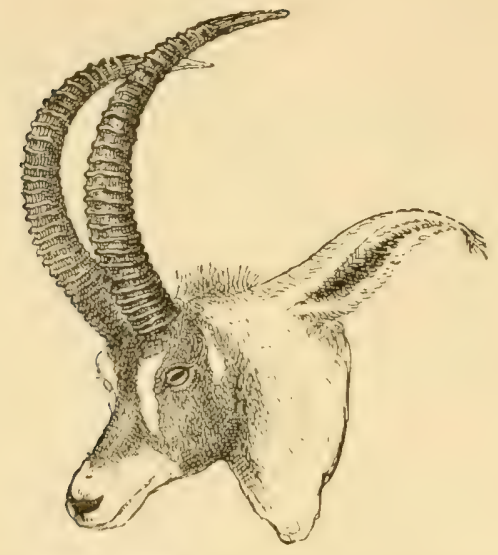

ROAN ANTELOPE (Hippotragus leucophœus).

Habitat-South Africa.

\begin{tabular}{|c|c|c|c|c|c|}
\hline Length. & $\begin{array}{l}\text { Circum- } \\
\text { ference. }\end{array}$ & Tip to Tip. & Habitat. & & Owner. \\
\hline 33 & $\cdots$ & $\cdots$ & South Africa & $\ldots$ & A. C. Fountaine. \\
\hline $31^{\frac{3}{4}}$ & $9 \frac{1}{2}$ & $5 \frac{1}{2}$ & Do. & $\cdots$ & Sir Edmund G. Loder, Bart. \\
\hline $31 \frac{1}{2}$ & $9 \frac{1}{2}$ & $11 \frac{7}{8}$ & Do. & $\ldots$ & F. C. Selous. \\
\hline $30 \frac{7}{8}$ & $9 \frac{1}{2}$ & $10 \frac{5}{8}$ & Do. & $\cdots$ & Do. \\
\hline 우 $30 \frac{1}{2}$ & 7 & $7 \stackrel{3}{s}$ & Do. & $\cdots$ & Do. \\
\hline $30 \frac{1}{4}$ & 10 & $9 \frac{1}{2}$ & Do. & $\cdots$ & Do. \\
\hline $29 \frac{7}{8}$ & Io & 14 & Do. & $\cdots$ & The late J. S. Jameson. \\
\hline $29 \frac{7}{8}$ & $9 \frac{5}{8}$ & $11 \frac{1}{4}$ & Do. & $\cdots$ & Hon. Walter Rothschild. \\
\hline $29 \frac{3}{4}$ & $8 \frac{1}{8}$ & $12 \frac{7}{8}$ & Limpopo & $\ldots$ & W. C. Oswell. \\
\hline $29 \frac{5}{8}$ & $9 \frac{1}{2}$ & 10 & South Africa & $\cdots$ & Hon. Walter Rothschild. \\
\hline $29 \frac{3}{8}$ & $9 \frac{5}{8}$ & $I I \frac{3}{8}$ & Do. & $\cdots$ & F. C. Selous. \\
\hline $29 \frac{1}{8}$ & $9 \frac{1}{8}$ & $7 \frac{3}{4}$ & Do. & $\cdots$ & H. and C. Beddington. \\
\hline 29 & $\cdots$ & $\cdots$ & Mashona Land & $\cdots$ & F. C. Selous, I880, "A Hunter's \\
\hline 29 & $\cdots$ & $\cdots$ & Impaqui River & $\cdots$ & $\begin{array}{l}\text { F. C. Selous, I876, "A Hunter's } \\
\text { Wanderings." }\end{array}$ \\
\hline $28 \div$ & 0 & $7 \frac{1}{4}$ & South Atrica & $\cdots$ & F. C. Selous. \\
\hline $28_{4}^{3}$ & 87 & $12 \frac{7}{8}$ & Do. & $\cdots$ & J. A. Jameson. \\
\hline
\end{tabular}


ROAN ANTELOPE (Hippotragus leucophœus)-coninued.

\begin{tabular}{|c|c|c|c|c|c|}
\hline Length. & $\begin{array}{l}\text { Circum- } \\
\text { ference. }\end{array}$ & Tip to Tip. & Habitat. & & Owner. \\
\hline $28 \frac{5}{8}$ & $9 \frac{3}{4}$ & $9 \frac{5}{8}$ & Mashona Land . & $\ldots \mid$ & F. C. Selous, British Museum. \\
\hline $28 \frac{1}{4}$ & $9 \frac{5}{8}$ & 8 & River Manyane . & $\ldots \mid$ & Do. \\
\hline 28 & $9 \frac{1}{2}$ & 13\% & South Africa & $\ldots$ & F. C. Selous. \\
\hline $27 \frac{7}{8}$ & $8 \frac{3}{8}$ & $8 \frac{1}{t}$ & Do. & $\cdots$ & Sir Edmund G. Loder, Bart. \\
\hline $27 \frac{5}{8}$ & $9 \frac{1}{8}$ & 9 & Do. & $\cdots$ & J. Carr-Saunders. \\
\hline $27 \frac{1}{2}$ & $10 \frac{1}{2}$ & 3 & Do. & $\cdots$ & A. C. Fountaine. \\
\hline $27 \frac{1}{2}$ & 9 & II $\frac{1}{2}$ & Do. & $\cdots$ & G. H. Banks. \\
\hline $27 \frac{3}{8}$ & $9 \frac{3}{4}$ & $8 \frac{1}{2}$ & Do. & $\cdots$ & A. Beit. \\
\hline $27 \frac{1}{8}$ & $9^{\frac{3}{4}}$ & $9 \frac{1}{4}$ & Do. & $\cdots$ & Sir Victor Brooke's Collection. \\
\hline $26 \frac{7}{8}$ & $8 \frac{5}{8}$ & $10 \frac{1}{2}$ & River Gambia . & $\cdots$ & Dr. P. Rendell, British Museum. \\
\hline $26 \frac{1}{2}$ & $10 \frac{1}{8}$ & $10 \frac{3}{4}$ & South Africa & $\cdots$ & The late J. S. Jameson. \\
\hline $26 \frac{1}{4}$ & $9 \frac{3}{4}$ & $7 \frac{5}{8}$ & Do. & ... & F. C. Selous. \\
\hline 26 & 9 & S & South Africa & $\cdots$ & British Museum. \\
\hline 26 & $8 \frac{7}{8}$ & $8 \frac{1}{8}$ & Do. & $\cdots$ & Do. \\
\hline $925 \frac{7}{8}$ & $6 \frac{3}{4}$ & 7 & Do. & $\cdots$ & F. C. Selous. \\
\hline $25 \frac{5}{8}$ & $9 \frac{1}{2}$ & $7 \frac{3}{8}$ & Do. & $\cdots$ & Sir Victor Brooke's Collection. \\
\hline $24 \frac{1}{2}$ & $9^{\frac{1}{2}}$ & $5 \frac{5}{8}$ & Limpopo & $\cdots$ & W. C. Oswell. \\
\hline $24 \frac{1}{t}$ & $8 \frac{7}{8}$ & $8 \frac{1}{2}$ & South Africa & $\cdots$ & A. Beit. \\
\hline $923 \frac{1}{8}$ & 7 & $8 \frac{1}{4}$ & Limpopo & $\cdots$ & W. C. Oswell. \\
\hline 23 & $9 \frac{\bar{\pi}}{t}$ & $9 \frac{1}{8}$ & South Africa & $\cdots$ & Rowland Ward's Collection. \\
\hline 23 & $6 \frac{3}{8}$ & 9 & Do. & $\cdots$ & British Museum. \\
\hline $922 \frac{5}{8}$ & $5^{\frac{3}{4}}$ & 9 & Limpopo & $\cdots$ & W. C. Oswell. \\
\hline $22 \frac{1}{4}$ & $6 \frac{5}{8}$ & $13 \frac{1}{8}$ & \multicolumn{2}{|c|}{ Cape of Good Hope } & Earl of Derby, British Museum. \\
\hline $22 \frac{1}{8}$ & $7 \frac{1}{8}$ & 8 & $\cdots \quad \cdots$ & $\cdots$ & W. D. James. \\
\hline $21 \frac{7}{8}$ & 7 & $9 \frac{1}{8}$ & Abyssinia & $\cdots$ & E. Lort-Phillips. \\
\hline $17 \frac{3}{\frac{3}{4}}$ & $6 \frac{1}{8}$ & $9 \frac{\frac{1}{t}}{x}$ & Do. & $\cdots$ & British Museum. \\
\hline 14 & $9 \frac{1}{2}$ & $7 \frac{1}{2}$ & South Africa & $\cdots$ & A. W. Davis. \\
\hline
\end{tabular}




\section{HIPPOTRAGUS KOBA.}

Habitat-Gambia.

\begin{tabular}{c|c|c|c|c|c|c}
\hline Length. & $\begin{array}{c}\text { Circum. } \\
\text { ference. }\end{array}$ & Tip to Tip. & Habitat. & Owner. \\
26 & $8 \frac{7}{8}$ & $7 \frac{1}{8}$ & Gambia & $\ldots$ & Earl of Derby, British Museum. \\
$20 \frac{5}{8}$ & $6 \frac{7}{8}$ & 7 & $7 \frac{3}{4}$ & Do. & $\ldots$ & Do. \\
\hline
\end{tabular}

\section{BAKER'S ANTELOPE, or MAARIFFE (Hippotragus bakeri).}

This Antelope much resembles the South African Roan Antelope, and was discovered in I860 by Sir Samuel Baker, Bart.

Habitat-Africa-Soudan.

\begin{tabular}{|c|c|c|c|c|c|c|}
\hline Length. & $\begin{array}{l}\text { Circum- } \\
\text { ference. }\end{array}$ & Tip to Tip. & & abitat. & & Owner. \\
\hline $28 \frac{1}{2}$ & $8 \frac{1}{2}$ & $\begin{array}{l}\text { (mal- } \\
\text { formed) }\end{array}$ & Soudan & $\cdots$ & $\ldots$ & Col. Ralph Vivian. \\
\hline $28 \frac{3}{8}$ & 9 & I I $\frac{3}{4}$ & Do. & $\cdots$ & $\cdots$ & Do. \\
\hline 27 & $\cdots$ & $\cdots$ & $\cdots$ & $\cdots$ & $\cdots$ & Sir Saml. Baker, Bart. (P.Z.S. I86I). \\
\hline $25 \frac{1}{8}$ & $9 \frac{3}{4}$ & $5 \frac{3}{8}$ & $\cdots$ & $\cdots$ & $\cdots$ & Sir Edmund G. Loder, Bart. \\
\hline
\end{tabular}




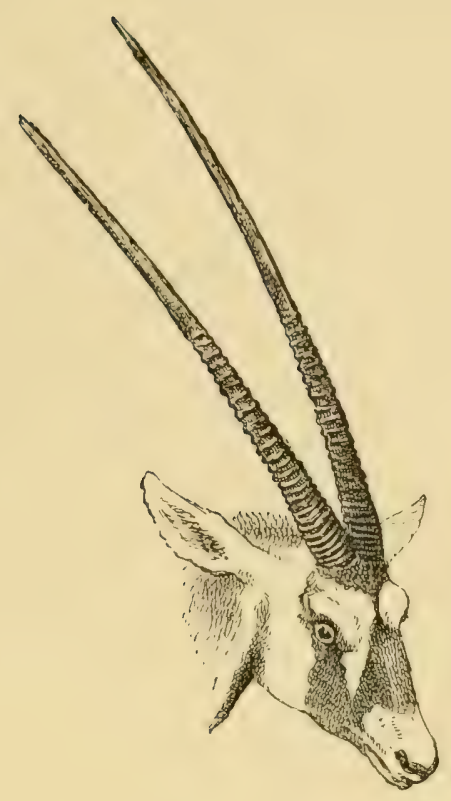

\section{GEMSBUCK (Oryx gazella).}

Habitat-South Africa.

\begin{tabular}{c|c|c|c|c|c}
\hline Length. & $\begin{array}{c}\text { Circum- } \\
\text { ference. }\end{array}$ & Tip to Tip. & Habitat. & \multicolumn{1}{|c}{ Owner. } \\
\hline $47^{\frac{1}{2}}$ & $6 \frac{3}{4}$ & $17 \frac{1}{2}$ & South Africa & $\ldots$ & The late J. S. Jameson. \\
$44 \frac{5}{8}$ & $6 \frac{5}{8}$ & $21 \frac{7}{8}$ & Bakalahari & $\ldots$ & IV. C. Oswell. \\
$43 \frac{1}{2}$ & $7 \frac{5}{8}$ & 17 & Do. & $\ldots$ & Do. \\
$43 \frac{3}{8}$ & $6 \frac{3}{8}$ & $\mathbf{I} 8 \frac{1}{2}$ & South Africa & $\ldots$ & F. C. Selous, British Museum. \\
$43 \frac{1}{\frac{1}{4}}$ & $6 \frac{5}{8}$ & $20 \frac{7}{8}$ & Do. & $\ldots$ & Sir Victor Brooke's Collection. \\
$43 \frac{1}{8}$ & $6 \frac{7}{8}$ & $\ldots$ & Do. & $\ldots$ & F. C. Selous. \\
43 & 7 & $\mathbf{1} 8 \frac{3}{4}$ & Do. & $\ldots$ & Rowland Ward's Collection. \\
\hline
\end{tabular}


GEIMSBUCK (Oryx gazella)-continued.

\begin{tabular}{|c|c|c|c|c|c|}
\hline Length. & $\begin{array}{l}\text { Circum- } \\
\text { ference. }\end{array}$ & Tip to Tip. & Habitat. & & Owner. \\
\hline $42 \frac{1}{2}$ & $6 \frac{1}{2}$ & I9 & Botletlie River & $\ldots 1$ & F. C. Selous. \\
\hline $42 \frac{1}{2}$ & 7 & ... & $\ldots$ & $\ldots 1$ & Major Gould Adams. \\
\hline 42 & $\cdots$ & $\cdots$ & $\cdots$ & $\ldots \mid$ & Mrs. Murray. \\
\hline $41 \frac{3}{4}$ & $7 \frac{1}{8}$ & I $S_{\frac{1}{2}}$ & ... & $\ldots 1$ & F. C. Selous. \\
\hline $4 \mathrm{I}$ & $7 \frac{1}{2}$ & 22 & $\ldots$ & $\cdots$ & T. Gibb. \\
\hline $4 \mathrm{I}$ & $6 \frac{3}{4}$ & I $8 \frac{1}{2}$ & $\ldots$ & $\ldots$ & M. E. Stephens. \\
\hline 우 $40 \frac{3}{4}$ & $6 \frac{5}{8}$ & $17 \frac{1}{8}$ & West Africa & $\cdots$ & Capt. F. Cookson. \\
\hline $40 \frac{3}{4}$ & $6 \frac{7}{8}$ & $16 \frac{1}{2}$ & South Africa & $\ldots$ & R. C. Dobson. \\
\hline $40 \frac{5}{8}$ & $6 \frac{3}{4}$ & $16 \frac{1}{2}$ & Transvaal & ... & H. Atkinson. \\
\hline $40 \frac{1}{2}$ & $6 \frac{3}{4}$ & $21 \frac{5}{8}$ & South Africa & $\ldots \mid$ & F. C. Selous. \\
\hline $40 \frac{1}{x}$ & 7 & 17 & Do. & $\ldots$ & Hon. Walter Rothschild. \\
\hline $40 \frac{1}{8}$ & $6 \frac{3}{ \pm}$ & $18 \frac{1}{3}$ & Do. & $\ldots$ & The late J. S. Jameson. \\
\hline 40 & 7 & $21 \frac{3}{4}$ & Do. & $\ldots \mid$ & F. C. Selous. \\
\hline 40 & $6 \frac{7}{8}$ & I $S_{ \pm}^{1}$ & Do. & $\cdots$ & Sir Edmund G. Loder, Bart. \\
\hline $39 \frac{3}{4}$ & $6 \frac{3}{4}$ & 20 & Do. & $\cdots$ & St. George Littledale. \\
\hline $39 \frac{5}{8}$ & $7 \frac{3}{4}$ & 14 & Do. & $\cdots$ & The late J. S. Jameson. \\
\hline $39 \frac{1}{4}$ & $6 \frac{3}{4}$ & I $7 \frac{5}{8}$ & Do. & $\cdots$ & Do. \\
\hline $38 \frac{7}{3}$ & $6 \frac{3}{1}$ & I $5 \frac{1}{2}$ & Do. & $\cdots$ & A. Beit. \\
\hline $38 \frac{7}{8}$ & $6 \frac{5}{8}$ & I $8 \frac{5}{8}$ & Do. & $\cdots$ & Do. \\
\hline $38 \frac{1}{2}$ & $6 \frac{3}{8}$ & I $\sum_{\frac{1}{4}}$ & Do. & $\cdots$ & Do. \\
\hline $38 \frac{1}{2}$ & $7 \frac{3}{8}$ & $21 \frac{7}{8}$ & West Africa & $\cdots \mid$ & Capt. F. Cookson. \\
\hline $38 \frac{1}{8}$ & $6 \frac{3}{8}$ & I $8 \frac{1}{2}$ & South Africa & $\cdots$ & F. C. Selous. \\
\hline 38 & $\cdots$ & I $8 \frac{3}{4}$ & Do. & $\cdots$ & A. C. Fountaine. \\
\hline 38 & $6 \frac{3}{8}$ & $17 \frac{7}{8}$ & Do. & $\cdots$ & National Liberal Club. \\
\hline 38 & $7 \frac{3}{4}$ & $15 \frac{3}{4}$ & Do. & $\cdots$ & A. W. Davis. \\
\hline $37 \frac{7}{8}$ & $6 \frac{7}{8}$ & I $9 \frac{3}{4}$ & Do. & $\cdots$ & The late J. S. Jameson. \\
\hline $37 \frac{3}{t}$ & $6 \frac{1}{2}$ & I $\delta \frac{1}{2}$ & Do. & $\cdots$ & C. Ansell. \\
\hline
\end{tabular}


GEMSBUCK (Oryx gazella)-continued.

\begin{tabular}{|c|c|c|c|c|c|}
\hline Length. & $\begin{array}{l}\text { Circum- } \\
\text { ference. }\end{array}$ & Tip to Tip. & Habitat. & & Owner. \\
\hline $37 \frac{1}{8}$ & $6 \frac{7}{8}$ & $16 \frac{7}{8}$ & North Bechuana & & F. C. Selous, British Museum. \\
\hline 오 $36 \frac{3}{4}$ & $6 \frac{5}{8}$ & $\cdots$ & Do. & & Do. \\
\hline $36 \frac{1}{4}$ & $7 \frac{3}{8}$ & 16 & South Africa & ... & Hon. Charles Ellis. \\
\hline $35 \frac{7}{8}$ & $7 \frac{3}{4}$ & $15 \frac{1}{4}$ & Algoa Bay & $\ldots$ & J. Wemyss, British Museum. \\
\hline $35 \frac{5}{8}$ & $7 \frac{1}{8}$ & $16 \frac{1}{2}$ & South Africa & $\cdots$ & H. R. H. Duke of Edinburgh. \\
\hline $35 \frac{1}{2}$ & $7 \frac{1}{2}$ & I4 & Do. & $\ldots$ & J. A. Nicoll. \\
\hline 35 & $7 \frac{5}{8}$ & I $3 \frac{3}{4}$ & West Africa & $\cdots$ & Capt. F. Cookson. \\
\hline $34^{\frac{3}{4}}$ & $7 \frac{1}{4}$ & 14 & Transvaal & $\cdots$ & H. Atkinson. \\
\hline $34 \frac{5}{8}$ & $\$ \frac{3}{8}$ & $12 \frac{7}{8}$ & South Africa & $\cdots$ & J. Carr-Saunders. \\
\hline 34 & 6 & $17^{\frac{3}{4}}$ & Do. & $\cdots$ & Hon. Charles Ellis. \\
\hline $33 \frac{7}{8}$ & $7 \frac{1}{4}$ & $16 \frac{1}{4}$ & Do. & $\cdots$ & F. C. Selous, British Museunı. \\
\hline $32 \frac{1}{2}$ & $7 \frac{1}{2}$ & I $7 \frac{3}{8}$ & Do. & $\cdots$ & Bethnal Green Museum. \\
\hline
\end{tabular}

It is interesting to note that the finest Gemsbuck specified above was shot by the late J. S. Jameson when he was pursuing his last hunting journey, accompanied by F. C. Selous, before he started on that expedition which cost him his life. 


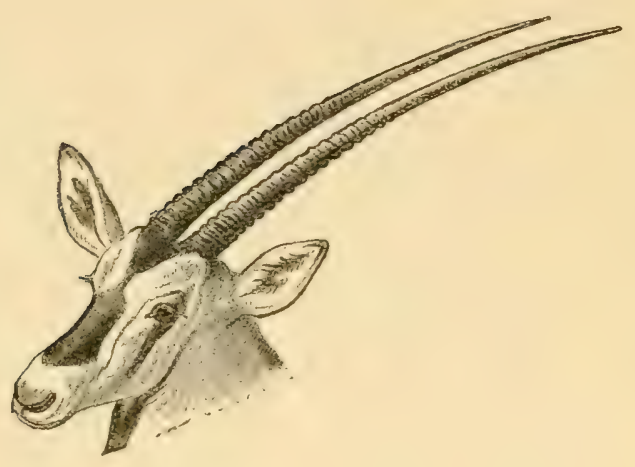

BEISA ANTELOPE (Oryx beisa).

Somali name-"Berid." Habitat-North-East Africa.

\begin{tabular}{|c|c|c|c|c|c|c|}
\hline & Length. & $\begin{array}{l}\text { Circum- } \\
\text { ference. }\end{array}$ & Tip to Tip. & \multicolumn{2}{|l|}{ Habitat. } & Owner. \\
\hline \multirow[t]{5}{*}{ 우 } & 39 & $5^{\frac{1}{2}}$ & 8 & Somali Land & $\ldots$ & E. P. Hare. \\
\hline & $3^{6}$ & $6 \frac{1}{2}$ & ... & Do. & ... & Capt. Brinkley. \\
\hline & 35 & 6 & 9 & Do. & $\cdots$ & Col. A. Paget. \\
\hline & $35^{\frac{1}{2}}$ & $6 \frac{1}{4}$ & $8 \frac{1}{4}$ & Do. & .. & Capt. Townley Parker. \\
\hline & $34 \frac{1}{2}$ & $\cdots$ & $9 \frac{1}{2}$ & Do. & $\cdots$ & Ernest Horn. \\
\hline \multirow[t]{8}{*}{ 우 } & $34^{\frac{3}{4}}$ & $5^{\frac{1}{4}}$ & $S_{\frac{3}{4}}^{3}$ & Do. & ... & Capt. Townley Parker. \\
\hline & $34 \frac{1}{2}$ & ... & $\begin{array}{c}\text { (owner's mea- } \\
\text { surement) }\end{array}$ & Do. & $\ldots$ & T. W. H. Clarke. \\
\hline & $34 \frac{3}{8}$ & $8 \frac{7}{8}$ & II $\frac{5}{8}$ & Do. & $\cdots$ & Do. \\
\hline & $33 \frac{5}{8}$ & $6 \frac{3}{4}$ & $9 \frac{3}{8}$ & Do. & ... & A. H. Straker. \\
\hline & $33 \frac{1}{2}$ & $5 \frac{1}{2}$ & $7 \frac{5}{8}$ & Do. & $\ldots$ & Col. A. Paget. \\
\hline & $33 \frac{3}{8}$ & $4 \frac{7}{8}$ & $7 \frac{5}{5}$ & Do. & $\ldots$ & W. D. James. \\
\hline & $33^{\frac{1}{4}}$ & $5 \frac{5}{8}$ & $2 \frac{3}{4}$ & Do. & $\ldots$ & A. H. Straker. \\
\hline & $33^{\frac{1}{x}}$ & $5 \frac{1}{2}$ & 9 & Do. & $\cdots$ & Rowland Ward's Collection. \\
\hline ㅇ & 33 & $5 \frac{1}{4}$ & $9 \frac{1}{2}$ & Do. & $\ldots$ & Capt. Bartelot. \\
\hline
\end{tabular}


BEISA ANTELOPE (Oryx beisa)-continued.

\begin{tabular}{|c|c|c|c|c|c|}
\hline Length. & $\begin{array}{l}\text { Circum- } \\
\text { ference. }\end{array}$ & Tip to Tip. & Habitat. & & Owner. \\
\hline 오 $32 \frac{5}{8}$ & 5 & S & Somali Land & $\ldots$ & C. H. Villiers. \\
\hline $32 \frac{3}{8}$ & $6 \frac{1}{4}$ & $6 \frac{1}{4}$ & Do. & $\cdots$ & Lord Delamere. \\
\hline 32 & $6 \frac{1}{2}$ & 93 & Do. & $\cdots$ & Capt. Bartelot. \\
\hline 32 & $5 \frac{1}{t}$ & $9 \frac{3}{8}$ & Do. & $\cdots$ & Lord Delamere. \\
\hline 32 & $5 \frac{1}{1 \cdot i}$ & $8 \frac{1}{8}$ & Do. & $\cdots$ & Col. A. Paget. \\
\hline 32 & $5 \frac{1}{8}$ & $9 \frac{7}{8}$ & De. & $\cdots$ & E. Lort-Phillips. \\
\hline $3 I_{\frac{1}{4}}$ & $6 \frac{3}{4}$ & S & Dc. & $\ldots$ & E. P. Hare. \\
\hline $3 \mathrm{I} \frac{1}{8}$ & 6 & S & Do & $\cdots$ & Lord Delamere. \\
\hline $3 \mathrm{I} \frac{1}{8}$ & 5 & $7 \frac{1}{8}$ & Do. & $\cdots$ & Sir John Kirk, M.D., K.C.B. \\
\hline $30 \frac{7}{8}$ & $5 \frac{1}{4}$ & $10 \frac{1}{2}$ & $\begin{array}{l}\text { River Juba, Som } \\
\text { Land. }\end{array}$ & mali & Dr. Kirk, British Museum. \\
\hline 303 & 6 & $7 \frac{1}{8}$ & Somali Land & $\cdots$ & E. Lort-Phillips. \\
\hline $30 \frac{3}{4}$ & $6 \frac{1}{4}$ & S & Do. & $\ldots$ & T. W. H. Clarke. \\
\hline $30 \frac{1}{2}$ & $6 \frac{1}{2}$ & $7 \frac{1}{2}$ & $\cdots$ & $\cdots$ & Marquis of Ailsa. \\
\hline $30 \frac{1}{2}$ & 6 & $7 \frac{3}{4}$ & Somali Land & $\cdots$ & Capt. H. G. C. Swayne. \\
\hline $30 \frac{1}{2}$ & $5^{\frac{1}{2}}$ & 7 & Do. & $\cdots$ & H.R.H. Duke of Edinburgh. \\
\hline $30 \frac{3}{8}$ & $6 \frac{1}{4}$ & 9 & Do. & $\cdots$ & Sir John Kirk, M.D., K.C.B. \\
\hline $30 \frac{1}{5}$ & 5 & $8 \frac{1}{4}$ & Do. & $\cdots$ & C. Liddell. \\
\hline 30 & $4 \frac{7}{8}$ & 9 & $\begin{array}{l}\text { River Juba, Som } \\
\text { Land. }\end{array}$ & & Sir John Kirk, M.D., K.C.B. \\
\hline 30 & $5 \frac{3}{8}$ & $5^{\frac{1}{2}}$ & Do. & & C. Liddell. \\
\hline $29 \frac{1}{4}$ & $5 \frac{5}{8}$ & $9 \frac{7}{8}$ & Somali Land & $\cdots$ & Hon. Walter Rothschild. \\
\hline $28 \frac{5}{8}$ & 6 & $7 \frac{7}{8}$ & Do. & $\cdots$ & Sir Edmund G. Loder, Bart. \\
\hline $2 S_{\frac{1}{2}}$ & $5 \frac{1}{2}$ & IO & Do. & $\cdots$ & Rowland Ward's Collection. \\
\hline $27^{\frac{3}{4}}$ & $5^{\frac{1}{4}}$ & $\begin{array}{l}\text { (one horn } \\
\text { malformed) }\end{array}$ & Do. & $\ldots$ & Lord Delamere. \\
\hline $27_{4}^{3}$ & $44^{3}$ & $6 \frac{3}{4}$ & Do. & $\cdots$ & Sir John Kirk, M.D., K.C.B. \\
\hline $27 \frac{1}{2}$ & $6 \frac{1}{2}$ & $8 \frac{1}{8}$ & Do. & $\cdots$ & H.R.H. Duke of Edinburgh. \\
\hline $26 \frac{1}{2}$ & $6 \frac{3}{4}$ & $\begin{array}{l}8 \frac{3}{4} \text { (owner's } \\
\text { measurement) }\end{array}$ & Do. & $\cdots$ & J. D. Inverarity. \\
\hline $25 ?$ & $4 \frac{5}{8}$ & $8 \frac{3}{8}$ & Abyssinia & $\ldots$ & British Museum. \\
\hline
\end{tabular}




\section{BEATRIX ANTELOPE (Oryx beatrix).}

[DR. J. E. GRAY, P.Z.S. 1857, p. I 57.]

THE horns slender, straight, or only very slightly curved near the tip; White, a spot on the middle of the face, a smaller spot between the base of the horns, a large patch on each cheek, extending above up to the eyes, and united together beneath under the throat; the knees and front of the fore and hind legs, and a large spot on the chest, dark blackish brown; the legs to the posterior grey-brown; end of the tail black.

This specimen is not half the size of the Gemsbok from the Cape, and is immediately known from it by the distribution of its colours.

In form and size it resembles the true Oryx (O. leucoryx), but it differs in the straightness of the horn, the size and form of the cheekspots, and especially in the dark colour of the legs, and the wellmarked white ring around the fetlock joint, just above the hoof.

The hair is whorled on the middle of the haunches like the rest of the genus, and the hairs of the back in front of the withers are directed forwards.

Habitat-Head of Persian Gulf.

\begin{tabular}{c|c|c|c|c}
\hline Length. & $\begin{array}{c}\text { Circum- } \\
\text { ference. }\end{array}$ & Tip to Tip. & Habitat. & Owner. \\
\hline 오 I5 & $3 \frac{3}{8}$ & $4 \frac{1}{2}$ & $\begin{array}{c}\text { Head of Persian } \\
\text { Gulf. }\end{array}$ & B. T. Finch, British Museum. \\
\hline
\end{tabular}

LEUCORYX (Oryx leucoryx).

Habitat-North Africa.

\begin{tabular}{|c|c|c|c|c|c|c|}
\hline Length. & $\begin{array}{l}\text { Circum- } \\
\text { ference. }\end{array}$ & Tip to Tip. & & Habitat. & & Owner. \\
\hline $39 \frac{5}{8}$ & $4 \frac{7}{8}$ & $14 \frac{1}{8}$ & ... & $\ldots$ & $\ldots$ & British Museum. \\
\hline $37 \frac{5}{8}$ & $5^{\frac{1}{4}}$ & $7 \frac{1}{4}$ & ... & ... & ... & Do. \\
\hline $35 \frac{5}{5}$ & $5^{\frac{1}{2}}$ & II $\frac{7}{n}$ & ... & $\ldots$ & $\ldots$ & J. Carr-Saunders. \\
\hline $33 \frac{7}{8}$ & $5 \frac{1}{2}$ & S & $\cdots$ & $\cdots$ & $\ldots$ & Hon. Walter Rothschild. \\
\hline
\end{tabular}




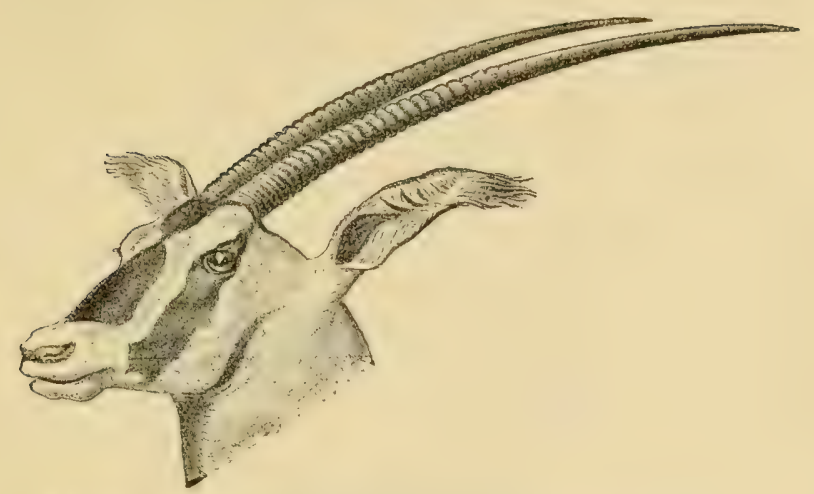

EAST AFRICAN ORYX (Oryx callotis).

Habitat-Africa, Masai Land and adjoining territory.

\begin{tabular}{|c|c|c|c|c|c|}
\hline Length. & $\begin{array}{l}\text { Circum- } \\
\text { ference. }\end{array}$ & Tip to Tip. & Habitat. & & Owner. \\
\hline $30 \frac{1}{2}$ & $5 \frac{3}{4}$ & $\mathrm{IO} \frac{1}{2}$ & East Africa & $\cdots$ & F. J. Jackson. \\
\hline $30 \frac{1}{4}$ & $\cdots$ & 6 & Do. & $\cdots$ & T. W. H. Greenfield. \\
\hline 30 & $\cdots$ & 10 & Do. & $\cdots$ & $\begin{array}{l}\text { Sir John Willoughby, Bart., "East } \\
\text { Africa and its Big Game." }\end{array}$ \\
\hline $29 \frac{1}{2}$ & $5 \frac{5}{8}$ & $7 \frac{3}{8}$ & Do. & $\cdots$ & Sir Robert Harvey, Bart. \\
\hline $28 \frac{7}{8}$ & $6 \frac{1}{2}$ & $10 \frac{1}{8}$ & Do. & $\cdots$ & Do. \\
\hline $28 \frac{3}{4}$ & $5 \frac{5}{8}$ & $6 \frac{1}{2}$ & Do. & $\cdots$ & H. C. V. Hunter. \\
\hline $27 \frac{3}{4}$ & $6 \frac{7}{8}$ & $10 \frac{1}{4}$ & Do. & $\cdots$ & F. J. Jackson. \\
\hline $27 \frac{1}{8}$ & $6 \frac{7}{8}$ & $8 \frac{1}{2}$ & Do. & $\cdots$ & Sir Robert Harvey, Bart. \\
\hline 27 & $7 \frac{1}{8}$ & $7 \frac{3}{8}$ & Do. & $\cdots$ & H. C. V. Hunter. \\
\hline $26 \frac{5}{8}$ & 7 & 13 & Do. & $\cdots$ & Sir Robert Harvey, Bart. \\
\hline $26 \frac{3}{3}$ & $6 \frac{7}{8}$ & $8 \frac{3}{8}$ & Do. & $\cdots$ & Do. \\
\hline $26 \frac{1}{4}$ & $5 \frac{7}{8}$ & $9 \frac{3}{4}$ & Do. & $\cdots$ & F. J. Jackson. \\
\hline 26 & 6 & 9 & Do. & $\cdots$ & E. Gedge. \\
\hline 23 & $5 \frac{1}{2}$ & 8 & Do. & $\cdots$ & W. Astor Chanler. \\
\hline $22 \frac{7}{8}$ & $6 \frac{1}{2}$ & $8 \frac{1}{2}$ & Do. & $\cdots$ & Sir Robert. Havvey, Fart. \\
\hline $2 I \frac{5}{8}$ & 7 & IO & Do. & $\cdots$ & Do. \\
\hline$*_{2 I}$ & $6 \frac{3}{4}$ & IO & Do. & $\cdots$ & Rowland Ward's Collection. \\
\hline
\end{tabular}

* The type specimen from which this Antelope was described I have presented to the British Museum. Until the date of my noticing its points of difference, this variety had been referred to as Oryx beisa, $-\mathrm{R}, \mathrm{IW}$. 


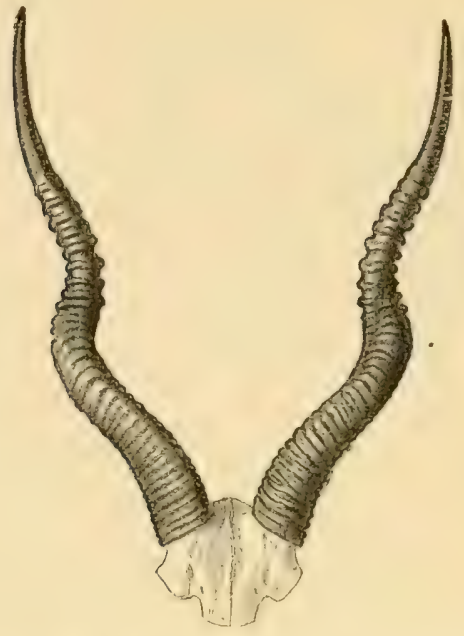

\section{ADDAX (Addax asomaculatus).}

THis Antelope has rarely been shot by Europeans. It closely approaches the South African Gemsbok in size:

\section{Habitat-North Africa.}

\begin{tabular}{|c|c|c|c|c|c|c|}
\hline \multicolumn{2}{|c|}{ Length. } & \multirow{2}{*}{$\begin{array}{l}\text { Circum- } \\
\text { ference } \\
\text { at base. }\end{array}$} & \multirow{2}{*}{$\begin{array}{l}\text { Tip to } \\
\text { Tip. }\end{array}$} & \multirow{2}{*}{\multicolumn{2}{|c|}{ Habitat. }} & \multirow{2}{*}{ Owner. } \\
\hline $\begin{array}{l}\text { Along } \\
\text { curve. }\end{array}$ & $\begin{array}{l}\text { Straight } \\
\text { line. }\end{array}$ & & & & & \\
\hline $35 \frac{3}{7}$ & 28 & $6 \frac{3}{8}$ & $13 \frac{5}{8}$ & North Africa & $\ldots$ & Hon. Walter Rothschild. \\
\hline $35^{\frac{1}{2}}$ & 28 & $6 \frac{1}{2}$ & I $3 \frac{1}{2}$ & Do. & $\ldots$ & Rowland Ward's Collection. \\
\hline $33 \frac{7}{8}$ & $28 \frac{3}{8}$ & $5 \frac{7}{8}$ & 22 & Do. & $\ldots$ & Sir Edmund G. Loder, Bart. \\
\hline $32 \frac{1}{4}$ & $26 \frac{1}{8}$ & $6 \frac{1}{8}$ & $13 \frac{1}{2}$ & Do. & ... & Rowland Ward's Collection. \\
\hline $31 \frac{5}{8}$ & $26 \frac{1}{4}$ & $6 \frac{3}{8}$ & $17 \frac{3}{8}$ & Do. & ... & Do. \\
\hline $3 \mathrm{r}$ & $26 \frac{7}{8}$ & 6 & $13 \frac{1}{2}$ & Do. & ... & Do. \\
\hline $29 \frac{7}{8}$ & 24 & 6 & I $4 \frac{3}{4}$ & Do. & $\cdots$ & Do. \\
\hline $28 \frac{3}{4}$ & $24 \frac{7}{8}$ & $5 \frac{1}{2}$ & $12 \frac{5}{8}$ & Do. & $\cdots$ & British Museum. \\
\hline 28 & $\cdots$ & 5 & $16 \frac{1}{2}$ & Do. & ... & Rowland Ward's Collection. \\
\hline 26 & $22 \frac{1}{2}$ & 6 & $17 \frac{1}{2}$ & Do. & ... & Do. \\
\hline
\end{tabular}




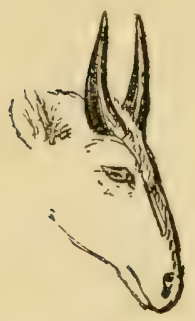

\section{NILGHAI (Boselaphus tragocamelus).}

[From "The Mammals of India," by T. C. Jerdon.]

MALE of an iron-grey colour; lips, chin, lower surface of the tail, stripes inside the ears, rings on the fetlocks and abdomen white; head and limbs tinged with sepia-brown; mane, throat-tuft, and tip of tail, black.

The female is a good deal smaller than the male and tawny or light brown.

Length of male about $6 \frac{1}{2}$ to 7 feet; height at shoulder 4 feet 4 inches to $4 \frac{1}{2}$ feet; horns 8 to 9 inches, rarely Io; ear 7 inches, very broad; tail I8 to $2 \mathrm{I}$ inches.

Habitat-India.

\begin{tabular}{|c|c|c|c|c|c|c|}
\hline Length. & $\begin{array}{l}\text { Circum- } \\
\text { ference. }\end{array}$ & Tip to Tip. & \multicolumn{3}{|c|}{ Habitat. } & Owner. \\
\hline $9 \frac{1}{4}$ & $6 \frac{3}{4}$ & $4 \frac{7}{3}$ & India & $\cdots$ & $\ldots$ & British Museum. \\
\hline $8 \frac{7}{8}$ & $6 \frac{5}{8}$ & $3 \frac{1}{2}$ & Do. & $\ldots$ & $\ldots$ & A. O. Hume, Private Collection. \\
\hline $8 \frac{3}{8}$ & $5^{\frac{3}{4}}$ & $4 \frac{3}{8}$ & Do. & ... & $\cdots$ & Do. \\
\hline $8 \frac{1}{4}$ & $7 \frac{1}{8}$ & $7 \frac{3}{4}$ & N. Ind & $\ldots$ & $\ldots$ & Sir Victor Brooke's Collection. \\
\hline $8 \frac{1}{8}$ & $6 \frac{1}{8}$ & 5 & India & $\ldots$ & $\ldots$ & Hume Collection, British Museum. \\
\hline $7 \frac{3}{4}$ & $5 \frac{1}{2}$ & $5 \frac{1}{4}$ & Do. & ... & ... & Rowland Ward's Collection. \\
\hline $7 \frac{5}{8}$ & $6 \frac{1}{8}$ & $5 \frac{1}{4}$ & Do. & $\ldots$ & $\ldots$ & Hume Collection, British Museum. \\
\hline $7 \frac{1}{2}$ & $5 \frac{3}{8}$ & $7 \frac{3}{8}$ & Cent. I & covinc & $s \ldots$ & G. A. Carmichael, British Museum. \\
\hline $7 \frac{3}{8}$ & $5^{\frac{3}{4}}$ & $5 \frac{7}{8}$ & India & ... & $\ldots$ & Hume Collection, British Museum. \\
\hline $7 \frac{1}{4}$ & $5^{\frac{1}{4}}$ & $6 \frac{1}{2}$ & Do. & $\ldots$ & ... & A. Wilson. \\
\hline $7 \frac{1}{8}$ & $6 \frac{3}{8}$ & $4 \frac{7}{8}$ & Do. & $\cdots$ & ... & Hon. Walter Rothschild. \\
\hline $6 \frac{3}{4}$ & $5 \frac{1}{4}$ & $4 \frac{3}{4}$ & Do. & $\ldots$ & $\cdots$ & Marquis of Ailsa. \\
\hline $6 \frac{5}{8}$ & $5 \frac{1}{4}$ & $5^{\frac{3}{4}}$ & Do. & ... & ... & H. R.H. Duke of Edinburgh. \\
\hline $6 \frac{1}{2}$ & $5 \frac{3}{8}$ & $5 \frac{3}{8}$ & Do. & $\ldots$ & ... & J. Carr-Saunders. \\
\hline $6 \frac{1}{8}$ & $4 \frac{5}{8}$ & $6 \frac{3}{8}$ & Do. & $\ldots$ & $\ldots$ & British Museum. \\
\hline 6 & $6 \frac{1}{2}$ & $5 \frac{\pi}{8}$ & Do. & $\ldots$ & $\ldots$ & Sir Robert Harvey, Bart. \\
\hline
\end{tabular}




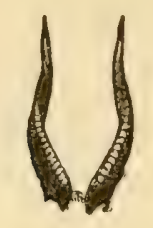

BUSHBUCK (Tragelaphus sylvaticus).

Bechuana name_"M'Pabala."

Lower Zambesi and Shivé name-"M'Bawala."

Habitat-South and East Africa.

\begin{tabular}{|c|c|c|c|c|}
\hline Length. & $\begin{array}{l}\text { Circum- } \\
\text { ference. }\end{array}$ & Tip to Tip. & Habitat. & Owner. \\
\hline $15 \frac{1}{2}$ & $5 \frac{3}{4}$ & $9 \frac{1}{t}$ & South Africa ... & Rowland Ward's Collection. \\
\hline 15 & $5 \frac{3}{8}$ & $4 \frac{1}{2}$ & Do. & F. C. Selous. \\
\hline $14 \frac{5}{8}$ & $5 \frac{3}{t}$ & $2 \frac{3}{4}$ & Do. & G. H. Banks. \\
\hline $14 \frac{1}{4}$ & $\cdots$ & $4 \frac{1}{8}$ & $\cdots$ & Rowland Ward's Collection. \\
\hline 14 & $5^{\frac{7}{8}}$ & $5^{\frac{3}{4}}$ & $\begin{array}{l}\text { Matabele Land, } \\
\text { South Africa. }\end{array}$ & II. and C. Beddington. \\
\hline $13 \frac{7}{8}$ & $5^{\frac{3}{4}}$ & $5^{\frac{1}{2}}$ & $\cdots \quad \ldots$ & II.R.H. Duke of Edinburgh. \\
\hline I $3 \frac{3}{4}$ & $5 \frac{3}{4}$ & $6 \frac{1}{2}$ & $\begin{array}{lll}\cdots & \ldots & \ldots\end{array}$ & Hon. Walter Rothschild, \\
\hline $13 \frac{3}{4}$ & $5 \frac{1}{2}$ & $5 \frac{3}{8}$ & $\begin{array}{lll}\cdots & \ldots & \cdots\end{array}$ & Sir Edmund G. Loder, Bart. \\
\hline I $3 \frac{5}{8}$ & $5 \frac{7}{8}$ & $5 \frac{7}{8}$ & $\begin{array}{l}\text { Matabele Land, } \\
\text { South Africa. }\end{array}$ & H. and C. Beddington. \\
\hline $13 \frac{5}{8}$ & $5 \frac{1}{2}$ & $5 \frac{1}{2}$ & East Africa $\quad \ldots$ & H. C. V. Hunter. \\
\hline $13 \frac{5}{8}$ & $5 \frac{3}{8}$ & $4 \frac{1}{8}$ & $\begin{array}{lll}\cdots & \cdots & \cdots\end{array}$ & Sir Victor Brooke's Collection. \\
\hline I3 $3 \frac{1}{2}$ & $5 \frac{1}{2}$ & 7 & South Africa ... & F. C. Selous. \\
\hline $13 \frac{1}{2}$ & $5 \frac{1}{4}$ & $5 \frac{1}{2}$ & $\begin{array}{l}\text { Matabele Land, } \\
\text { South Africa. }\end{array}$ & H. and C. Beddington. \\
\hline I $3 \frac{3}{8}$ & $5 \frac{3}{8}$ & 5 & South Africa ... & F. C: Selous. \\
\hline $13 \frac{1}{8}$ & $5 \frac{1}{4}$ & 6 & Do. & Do. \\
\hline 13 & $6 \frac{1}{4}$ & $5 \frac{1}{8}$ & East Africa $\quad \ldots$ & F. J. Jackson. \\
\hline 13 & $5 \frac{1}{2}$ & $5 \frac{1}{2}$ & $\begin{array}{l}\text { Matabele Land, } \\
\text { South Africa. }\end{array}$ & H. and C. Beddington. \\
\hline $12 \frac{7}{8}$ & 5 & $6 \frac{5}{8}$ & $\begin{array}{lll}\cdots & \ldots & \ldots\end{array}$ & W. C. Oswell. \\
\hline
\end{tabular}


BUSHBUCK (Tragelaphus sylvaticus)-continued.

\begin{tabular}{|c|c|c|c|c|c|}
\hline Length. & $\begin{array}{l}\text { Circum- } \\
\text { ference. }\end{array}$ & Tip to Tip. & Habitat. & & Owner. \\
\hline I $2 \frac{3}{4}$ & .. & $6 \frac{1}{2}$ & ... & $\ldots$ & Rowland Ward's Collection. \\
\hline $12 \frac{5}{8}$ & 5 & $5 \frac{1}{4}$ & $\ldots$ & $\ldots$ & W. D. James. \\
\hline $12 \frac{1}{2}$ & $5 \frac{1}{2}$ & $4 \frac{1}{2}$ & South Africa & $\ldots$ & J. A. Nicoll. \\
\hline 12 & $4 \frac{3}{8}$ & $4 \frac{5}{8}$ & Do. & $\ldots$ & F. C. Selous. \\
\hline $11 \frac{3}{4}$ & 5 & $6 \frac{3}{8}$ & East Africa & $\ldots$ & Sir Robert Harvey, Bart. \\
\hline$I I \frac{1}{2}$ & (owner's mea- & 4 & Do. & $\ldots$ & Sir John Willoughby, Bart. \\
\hline II $\frac{7}{2}$ & $5^{\frac{1}{4}}$ & $3 \frac{5}{8}$ & $\ldots$ & .. & H. R. H. Duke of Edinburgh. \\
\hline II $\frac{1}{2}$ & $4 \frac{7}{8}$ & $4 \frac{1}{2}$ & South Africa & $\ldots$ & A. Beit. \\
\hline $11 \frac{1}{4}$ & $4 \frac{3}{8}$ & $4 \frac{1}{2}$ & East Africa & ... & F. J. Jackson. \\
\hline I I & $5 \frac{1}{8}$ & 5 & $\ldots$ & $\ldots$ & Col. Ralph Vivian. \\
\hline $10 \frac{7}{8}$ & 5 & $3 \frac{1}{2}$ & $\ldots$ & $\ldots$ & J. Carr-Saunders. \\
\hline
\end{tabular}


HARNESSED ANTELOPE (Tragelaphus scriptus).

Habitat-South and East Africa.

\begin{tabular}{|c|c|c|c|c|c|}
\hline $\begin{array}{l}\text { Length along } \\
\text { the bend. }\end{array}$ & $\begin{array}{l}\text { Circum- } \\
\text { ference. }\end{array}$ & Tip to Tip. & Habitat. & & Owner. \\
\hline $17 \frac{7}{8}$ & $6 \frac{1}{4}$ & $5 \frac{7}{8}$ & Zanzibar ... & $\cdots$ & Sir John Kirk, M.D., K.C.B. \\
\hline $16 \frac{5}{8}$ & $5 \frac{7}{8}$ & $6 \frac{1}{4}$ & Mandar Island, & & Do. \\
\hline $15 \frac{3}{8}$ & $6 \frac{3}{8}$ & $7 \frac{1}{2}$ & South Africa & ... & Maj.-Gen. Arthur Ellis, C.S.I. \\
\hline $15 \frac{3}{8}$ & $5 \frac{1}{2}$ & $7 \frac{1}{8}$ & Do. & $\ldots$ & Hon. Charles Ellis. \\
\hline$*_{1} 3_{\frac{1}{2}}$ & $5 \frac{1}{4}$ & $5 \frac{3}{8}$ & Do. & ... & British Museun. \\
\hline I4 & $5 \frac{1}{2}$ & $4 \frac{1}{8}$ & Do. & $\cdots$ & Hon. Charles Ellis. \\
\hline $13 \frac{1}{2}$ & $5 \frac{1}{8}$ & $4 \frac{3}{3}$ & Do. & $\ldots$ & Sir John Willoughby, Bart. \\
\hline $13 \frac{3}{4}$ & $4 \frac{3}{4}$ & $3 \frac{1}{2}$ & Mombasa & $\cdots$ & Sir John Kirk, British Museum. \\
\hline $13 \frac{1}{8}$ & $5 \frac{1}{8}$ & $3 \frac{1}{2}$ & Lower Zambesi & $\cdots$ & F. C. Selous, British Museum. \\
\hline $12 \frac{3}{4}$ & 5 & $4 \frac{1}{2}$ & South Africa & $\cdots$ & A. E. Capell. \\
\hline $12 \frac{5}{8}$ & $5 \frac{1}{t}$ & $4 \frac{1}{8}$ & Orange River & ... & British Museum. \\
\hline $12 \frac{5}{8}$ & $4 \frac{5}{8}$ & $4 \frac{3}{8}$ & $\ldots \quad \ldots$ & ... & Do. \\
\hline$*_{12 \frac{3}{8}}$ & $5 \frac{1}{8}$ & $4 \frac{5}{8}$ & Chobe River & $\ldots$ & F. C. Selous, British Museum. \\
\hline II $\frac{3}{4}$ & $5 \frac{1}{2}$ & $6 \frac{5}{8}$ & $\begin{array}{ll}\cdots & \cdots\end{array}$ & $\cdots$ & Sir Victor Brooke's Collection. \\
\hline II $\frac{3}{4}$ & $5 \frac{1}{8}$ & $4 \frac{1}{2}$ & Uganda & $\ldots$ & Capt. Speke, British Museum. \\
\hline II & $4 \frac{5}{8}$ & $5 \frac{7}{8}$ & Limpopo River & ... & R. Gordon Cumming, British Museum. \\
\hline $10 \frac{1}{2}$ & $4 \frac{1}{2}$ & $2 \frac{1}{4}$ & Gambia ... & $\ldots$ & Earl Derby, British Museum. \\
\hline
\end{tabular}

* The above are Banded Bushbuck. 


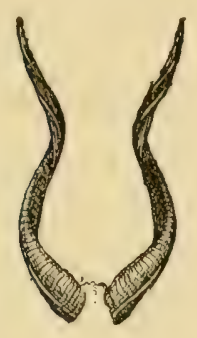

\section{SITUTUNGA ANTELOPE (Tragelaphus spekei).}

[Sir VICTOR BROOKE, Bart., P.Z.S. I871, p. 485.]

HAIR of sides coarse and long, without stripes; hoofs long; general colour rusty brown; neck maned; horns smooth, slender, strongly keeled; hair of sides and body of uniform length.

Native name-" Nakong."

Habitat-Central and Southern Central Africa.

\begin{tabular}{|c|c|c|c|c|c|c|c|}
\hline \multicolumn{2}{|c|}{ Length. } & \multirow{2}{*}{$\begin{array}{l}\text { Circum. } \\
\text { ference. }\end{array}$} & \multirow{2}{*}{ Tip to Tip. } & \multirow{2}{*}{\multicolumn{3}{|c|}{ Habitat. }} & \multirow{2}{*}{ Owner. } \\
\hline $\begin{array}{l}\text { Round } \\
\text { curve. }\end{array}$ & $\begin{array}{l}\text { Straight } \\
\text { lines. }\end{array}$ & & & & & & \\
\hline $32 \frac{5}{8}$ & 27 & $7 \frac{1}{2}$ & $16 \frac{3}{8}$ & $\cdots$ & $\ldots$ & $\ldots$ & F. C. Selous. \\
\hline $3^{1 \frac{7}{8}}$ & $25^{\frac{3}{4}}$ & $8 \frac{1}{8}$ & I $7 \frac{1}{4}$ & $\ldots$ & $\ldots$ & $\ldots$ & Do. \\
\hline $3^{1 \frac{1}{2}}$ & $24 \frac{3}{8}$ & 7 & $16 \frac{1}{8}$ & $\begin{array}{r}\mathrm{Ch} \\
\mathrm{M}\end{array}$ & $\begin{array}{l}\text { e Ri } \\
\text { huna }\end{array}$ & and. & British Museum. \\
\hline$\cdots$ & 25 & $\cdots$ & $\cdots$ & $\cdots$ & $\ldots$ & $\cdots$ & $\begin{array}{l}\text { F. C. Selous, "A Hunter's Wan- } \\
\text { derings." }\end{array}$ \\
\hline $29 \frac{5}{8}$ & $24 \frac{1}{2}$ & $8 \frac{1}{8}$ & $16 \frac{3}{8}$ & $\cdots$ & $\cdots$ & $\cdots$ & F. C. Selous. \\
\hline 28 & $23 \frac{3}{4}$ & $7 \frac{3}{4}$ & $19 \frac{7}{8}$ & $\ldots$ & $\ldots$ & $\ldots$ & W. C. Oswell. \\
\hline 27 & $21 \frac{3}{4}$ & $6 \frac{7}{8}$ & 12 & $\ldots$ & ... & $\ldots$ & The late J. S. Jameson. \\
\hline 26 & $\ldots$ & $7 \frac{3}{8}$ & 87 & $\ldots$ & $\ldots$ & $\ldots$ & Hon. Charles Ellis. \\
\hline 26 & $22 \frac{5}{8}$ & 7 & $17 \frac{1}{2}$ & $\ldots$ & $\ldots$ & $\ldots$ & Sir Victor Brooke's Collection. \\
\hline 26 & $20 \frac{7}{8}$ & $6 \frac{7}{8}$ & 16 & $\ldots$ & $\ldots$ & $\ldots$ & W. C. Oswell. \\
\hline
\end{tabular}


SITUTUNGA ANTELOPE (Tragelaphus spekei)-continut.

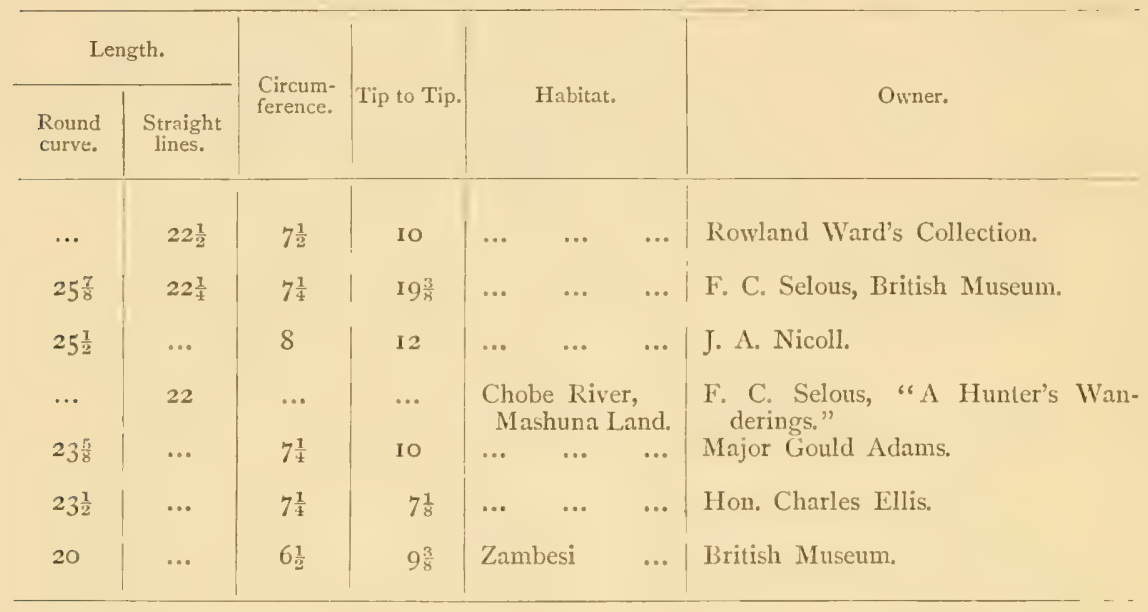

(Tragelaphus gratus).

Habitat-IVest Africa.

\begin{tabular}{|c|c|c|c|c|c|c|}
\hline \multicolumn{2}{|c|}{ Length. } & \multirow{2}{*}{$\begin{array}{l}\text { Circum- } \\
\text { ference. }\end{array}$} & \multirow{2}{*}{$\begin{array}{l}\text { 'ip to } \\
\text { Tip. }\end{array}$} & \multirow{2}{*}{\multicolumn{2}{|c|}{ Habitat. }} & \multirow{2}{*}{ Owner. } \\
\hline $\begin{array}{l}\text { Straight } \\
\text { line. }\end{array}$ & $\begin{array}{l}\text { Round } \\
\text { curve. }\end{array}$ & & & & & \\
\hline I9 & $\cdots$ & $\cdots$ & $\cdots$ & Gaboon ... & .. & British Museum. \\
\hline 18 & $23 \frac{1}{2}$ & $7 \frac{7}{8}$ & I I $\frac{3}{4}$ & Do. & $\cdots$ & Sir Victor Brooke's Collection. \\
\hline
\end{tabular}




\section{INYALA (Tragelaphus angasi).}

[G. F. ANGAS, P.Z.S. I848, p. 89.]

This brilliant Antelope, the Inyala of the Amazulu, appears to be a link between the Koodos and Boshbok, uniting in itself the markings and characteristic features of both these animals. The adult male is about $7 \mathrm{ft}$. 6 in. in total length, and $3 \mathrm{ft} .4$ in. high at the shoulder. Though elegant in form, and much of the grace of the solitary Koodos, the robust and shaggy aspect of the male bears considerable resemblance to that of the Goat. Legs clean; hoofs pointed and black, with two oval cream-coloured spots in front of each fetlock, immediately above the hoof; horns $\mathrm{I} f \mathrm{ft}$. Io in. long, twisted and sublyrate, very similar to those of the Boshbok, but rather more spiral; have polished extremities of a pale straw colour; rest of horns brownish-black, deeply ridged from the forehead to about half the length of the horn; prevailing colour greyishblack, tinged with purplish-brown and ochre; on the neck, flanks, and cheeks, marked with several white stripes like the Koodos; forehead brilliant sienna-brown, almost approaching to orange; mane black down the back and white from the withers to the insertion of the tail; ears 8 in. long, oval, rufous, tipped with black and fringed inside with white hairs; a pale ochreous circle round the eyes, which are connected by two white spots forming an arrowshaped mark on a black ground; nose black; a white spot on each side of the upper lip; chin and gullet white; and three white marks under each eye; neck covered with long shaggy hair, extending also under the belly and fringing the haunches to the knees; two white spots on the flanks, and a patch of long white hair on the anterior portion of the thigh; a white tuft under the belly and another on the dewlap; on the outer side of the fore-legs is a black patch above the knee surrounded by three white spots; legs below the knee bright rufous colour; tail I ft. 8 in. long, black above, with tip and inside white.

Female smaller and without horns.

The young resembles the female, but is rather paler in colour, and has more white spots on the flanks and sides.

Inhabits the lower undulating hills, scattered with Mimosa bushes, that border upon the northern shores of St. Lucia Bay, in the Zulu country, lat. $28^{\circ}$ south. Found in small troops of eight or ten together, feeding amongst the thickets. 
INYALA (Tragelaphus angasi)-continued.

Habitat-South-East Africa and Zulu Country.

\begin{tabular}{|c|c|c|c|c|c|c|}
\hline \multicolumn{2}{|c|}{ Length. } & \multirow{2}{*}{$\begin{array}{l}\text { Circum- } \\
\text { ference.- }\end{array}$} & \multirow{2}{*}{$\begin{array}{l}\text { Tip to } \\
\text { Tip. }\end{array}$} & \multirow{2}{*}{\multicolumn{2}{|c|}{ Habitat. }} & \multirow{2}{*}{ Owner. } \\
\hline $\begin{array}{l}\text { Round } \\
\text { curve. }\end{array}$ & Straight. & & & & & \\
\hline 28 & $\ldots$ & $8 \frac{1}{8}$ & $10 \frac{1}{8}$ & South Africa & ... & British Museum. \\
\hline $27 \frac{5}{8}$ & $22 \frac{1}{2}$ & $7 \frac{7}{8}$ & $9 \frac{1}{2}$ & Do. & $\cdots$ & Sir Victor Brooke's Collection. \\
\hline $27 \frac{3}{8}$ & $\cdots$ & $8 \frac{1}{8}$ & $10 \frac{1}{8}$ & Do. & $\cdots$ & Sir Edmund G. Loder, Bart. \\
\hline $26 \frac{3}{4}$ & 22 & $7 \frac{3}{4}$ & $12 \frac{1}{4}$ & Do. & $\cdots$ & Sir Victor Brooke's Collection. \\
\hline $26 \frac{7}{4}$ & 22 & $6 \frac{7}{8}$ & II $\frac{5}{8}$ & Do. & $\ldots$ & F. C. Selous, \\
\hline 23 & $\cdots$ & 7 & $5 \frac{5}{8}$ & Zulu Country & $\ldots$ & R. S. Fellowes, British Museum. \\
\hline 22 & $\cdots$ & ... & $\ldots$ & $\cdots$ & $\cdots$ & Angas, P.Z.S. 1 848, p. 89 . \\
\hline
\end{tabular}

\section{TRAGELAPHUS EURYCEROS.}

[Sir Victor BROOKE, Bart., P.Z.S. I871, p. 485.]

HAIR of sides smooth and short, striped with white bands, descending from a white dorsal streak; hoofs short; general colour deep chestnut; stripes strongly marked, numerous; horns smooth, massive; neck, back, and belly maneless; tail bovine; fore limbs with dark markings anteriorly.

\section{Habitat-West Africa.}

\begin{tabular}{|c|c|c|c|c|c|c|}
\hline \multicolumn{2}{|c|}{ Length. } & \multirow{2}{*}{$\begin{array}{l}\text { Circum- } \\
\text { ference. }\end{array}$} & \multirow{2}{*}{$\begin{array}{l}\text { Tip to } \\
\text { Tip. }\end{array}$} & \multirow{2}{*}{ Habitat. } & & \multirow{2}{*}{ Owner. } \\
\hline On curve. & Straight. & & & & & \\
\hline $3 I_{\frac{3}{4}}$ & $26 \frac{1}{2}$ & $9 \frac{3}{4}$ & $12 \frac{3}{8}$ & $\ldots$ & $\ldots$ & Sir Victor Brooke's Collection. \\
\hline $30 \frac{3}{4}$ & $24 \frac{7}{8}$ & $10 \frac{7}{8}$ & $12 \frac{3}{4}$ & West Africa & $\ldots$ & Do. \\
\hline 30 & $24 \frac{1}{8}$ & $9^{\frac{5}{8}}$ & II $\frac{1}{4}$ & Ashkanholoo & & British Museum. \\
\hline $29 \frac{1}{2}$ & $25 \frac{7}{8}$ & $9 \frac{3}{4}$ & $10 \frac{1}{8}$ & $\cdots \quad \ldots$ & $\ldots$ & P. Du Chaillu, British Museum. \\
\hline $27 \frac{5}{8}$ & $23 \frac{3}{4}$ & IO $\frac{1}{8}$ & $10 \frac{5}{8}$ & Fantee ... & $\ldots$ & Do. \\
\hline$\ldots$ & $19 \frac{7}{8}$ & 8 & $6 \frac{5}{8}$ & Gaboon ... & $\ldots$ & Do. \\
\hline
\end{tabular}




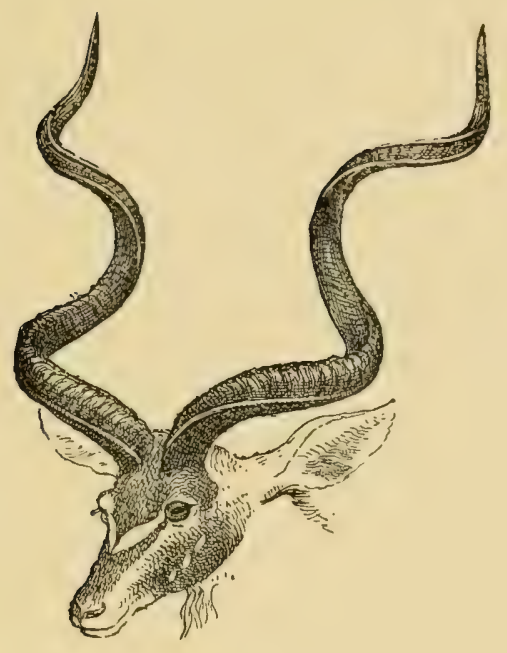

GREATER KOODOO (Strepsiceros kudu).

Somali name-"Godir."

Schuana name-" Tolo."

Lower Zambesi name-"Goma."

Habitat-North, East, and South Africa.

\begin{tabular}{|c|c|c|c|c|c|c|}
\hline \multicolumn{2}{|c|}{ Length. } & \multirow{2}{*}{$\begin{array}{l}\text { Circum- } \\
\text { ference. }\end{array}$} & \multirow{2}{*}{ Tip to Tip. } & \multirow{2}{*}{\multicolumn{2}{|c|}{ Habitat. }} & \multirow{2}{*}{ Owne:. } \\
\hline $\begin{array}{l}\text { Straight } \\
\text { line. }\end{array}$ & $\begin{array}{l}\text { Round } \\
\text { curve. }\end{array}$ & & & & & \\
\hline $45^{\frac{3}{8}}$ & $60 \frac{5}{8}$ & II $\frac{1}{2}$ & 33 & South Africa & ... & F. C. Selous. \\
\hline $45 \frac{1}{8}$ & $55 \frac{7}{8}$ & $11 \frac{1}{4}$ & $37 \frac{1}{8}$ & Do. & $\ldots$ & W. C. Oswell. \\
\hline $44 \frac{7}{8}$ & $57^{\frac{1}{4}}$ & $11 \frac{3}{4}$ & $34 \frac{3}{8}$ & Africa $\ldots$ & $\cdots$ & Sir Edmund G. Loder, Bart. \\
\hline $44 \frac{1}{4}$ & 59 & $10_{4}^{\frac{1}{4}}$ & 26 & South Africa & $\cdots$ & The late J. S. Jameson. \\
\hline $44 \frac{1}{4}$ & $56 \frac{1}{4}$ & II $\frac{3}{8}$ & $33^{\frac{1}{4}}$ & Do. & $\ldots$ & Hon. Walter Rothschild. \\
\hline
\end{tabular}




\section{GREATER KOODOO (Strepsiceros kudu)-continued.}

\begin{tabular}{|c|c|c|c|c|c|c|}
\hline \multicolumn{2}{|c|}{ Length. } & \multirow{2}{*}{$\begin{array}{l}\text { Circum- } \\
\text { ference. }\end{array}$} & \multirow{2}{*}{ Tip to Tip. } & \multirow{2}{*}{\multicolumn{2}{|c|}{ Habitat. }} & \multirow{2}{*}{ Owner. } \\
\hline $\begin{array}{l}\text { Straight } \\
\text { line. }\end{array}$ & $\begin{array}{l}\text { Round } \\
\text { curve. }\end{array}$ & & & & & \\
\hline $43 \frac{7}{8}$ & $61 \frac{1}{4}$ & $10 \frac{5}{8}$ & 29 & South Africa & $\ldots$ & Rowland Ward's Collection. \\
\hline $43 \frac{5}{8}$ & $55^{\frac{1}{2}}$ & 10 & $44 \frac{7}{8}$ & Do. & $\cdots$ & W. C. Oswell. \\
\hline $43 \frac{1}{2}$ & $54 \frac{1}{2}$ & I I $\frac{1}{2}$ & $33 \frac{3}{7}$ & Do. & $\cdots$ & Hon. Walter Rothschild. \\
\hline 43 & $57 \frac{1}{8}$ & II $\frac{5}{8}$ & $34^{\frac{3}{4}}$ & Do. & $\cdots$ & H. and C. Beddington. \\
\hline 43 & $56 \frac{1}{2}$ & $10 \frac{1}{1}$ & 29 & Do. & $\cdots$ & C. Ansell. \\
\hline 43 & 56 & $1 \mathrm{O}_{4}^{\frac{3}{4}}$ & 43 & Do. & $\cdots$ & F. C. Selous. \\
\hline 43 & 55 & II $\frac{1}{2}$ & 25 & Do. & $\cdots$ & Hon. Walter Rothschild. \\
\hline $42 \frac{3}{4}$ & $54 \frac{1}{8}$ & I I $\frac{5}{8}$ & $33 \frac{3}{8}$ & Do. & $\cdots$ & A. Beit. \\
\hline $42 \frac{5}{8}$ & $5^{6 \frac{1}{2}}$ & $10 \frac{3}{8}$ & $38 \frac{3}{4}$ & Do. & $\cdots$ & Sir Victor Brooke's Collection. \\
\hline $42 \frac{1}{2}$ & $58 \frac{1}{2}$ & II & 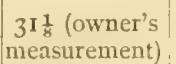 & Africa & $\cdots$ & Edmund Smith. \\
\hline $42 \frac{1}{2}$ & 53 & $\mathrm{II} \frac{1}{2}$ & 30 & South Africa & $\cdots$ & A. W. Davies. \\
\hline $42 \frac{1}{2}$ & 50 & $11 \frac{1}{2}$ & 32 & Africa & $\cdots$ & Major Gould Adams. \\
\hline 42 & 54 & II & 45 & Do. & $\cdots$ & E. R. Thompson. \\
\hline $41 \frac{7}{8}$ & $52 \frac{1}{8}$ & $10 \frac{3}{4}$ & $39 \frac{5}{8}$ & Do. & $\cdots$ & Hon. Charles Ellis. \\
\hline $41 \frac{1}{2}$ & 57 & $9^{\frac{3}{4}}$ & $3 I^{\frac{1}{2}}$ & South Africa & $\cdots$ & James Greenlees. \\
\hline $41 \frac{1}{8}$ & $55^{\frac{3}{4}}$ & $10 \frac{1}{8}$ & $24 \frac{3}{8}$ & Do. & $\cdots$ & F. C. Selous. \\
\hline $\begin{array}{l}4 \mathrm{I} \\
4 \mathrm{I}\end{array}$ & 64 & $\cdots$ & $\begin{array}{l}\cdots \\
24^{\frac{3}{1}}\end{array}$ & $\begin{array}{l}\text { Mashuna Lanc } \\
\text { South Africa }\end{array}$ & & $\begin{array}{l}\text { F. C. Selous, "A Hunter's Wan- } \\
\text { derings," } \\
\text { G. H. Banks. }\end{array}$ \\
\hline 41 & $57 \frac{2}{4}$ & $9 \frac{2}{4}$ & $24 \frac{1}{x}$ & South Arrica & $\cdots$ & G. H. Danks. \\
\hline $4 \mathrm{I}$ & $5^{6 \frac{1}{2}}$ & $10 \frac{1}{4}$ & $28 \frac{1}{2}$ & Do. & $\cdots$ & C. Ansell. \\
\hline $4 \mathrm{I}$ & $53 \frac{5}{8}$ & IO & 25 & Do. & $\cdots$ & J. A. Jameson. \\
\hline $40 \frac{7}{8}$ & $53 \frac{1}{2}$ & IO & $33 \frac{1}{8}$ & Do. & $\cdots$ & The late J. S. Jameson. \\
\hline $40 \frac{3}{4}$ & 62 & $10 \frac{3}{8}$ & $23 \frac{1}{2}$ & Do. & $\cdots$ & F. C. Selous. \\
\hline $40 \frac{5}{8}$ & $49 \frac{7}{8}$ & II $\frac{1}{2}$ & $35^{\frac{3}{8}}$ & Do. & $\cdots$ & H. and $\mathrm{C}$. Beddington. \\
\hline $40 \frac{1}{2}$. & $5^{1}$ & $10 \frac{1}{4}$ & $28 \frac{5}{8}$ & Do. & $\cdots$ & A. Beit. \\
\hline $40 \frac{1}{2}$ & 50 & II $\frac{3}{4}$ & $29 \frac{1}{2}$ & Do. & $\cdots$ & Rowland Ward's Collection. \\
\hline $40 \frac{1}{4}$ & $52 \frac{3}{8}$ & Io & $3^{6 \frac{1}{2}}$ & Do. & $\cdots$ & Maj-Gen, Arthur Ellis, C.S.I. \\
\hline
\end{tabular}


GREATER KOODOO (Strepsiceros kudu)-continued.

\begin{tabular}{|c|c|c|c|c|c|c|}
\hline \multicolumn{2}{|c|}{ Length. } & \multirow{2}{*}{$\begin{array}{l}\text { Circum- } \\
\text { ference }\end{array}$} & \multirow{2}{*}{ Tip to Tip. } & \multirow{2}{*}{\multicolumn{2}{|c|}{ Habitat. }} & \multirow{2}{*}{ Owner. } \\
\hline $\begin{array}{l}\text { Straight } \\
\text { line. }\end{array}$ & $\begin{array}{l}\text { Round } \\
\text { curve. }\end{array}$ & & & & & \\
\hline $40 \frac{1}{4}$ & $50 \frac{1}{2}$ & $10 \frac{1}{8}$ & $39 \frac{1}{8}$ & South Africa & $\ldots$ & H. Atkinson. \\
\hline 40 & $52 \frac{7}{8}$ & $10 \frac{1}{8}$ & 37 & Delagoa Bay & $\cdots$ & Sir John Kirk, M.D., K.C.B. \\
\hline 40 & $52 \frac{3}{8}$ & $9 \frac{7}{8}$ & $27 \frac{1}{4}$ & South Africa & $\ldots$ & Bethnal Green Museum. \\
\hline 40 & $52 \frac{3}{8}$ & $9 \frac{3}{4}$ & $28 \frac{3}{8}$ & Do. & $\cdots$ & F. J. Horniman. \\
\hline $39 \frac{7}{8}$ & $49 \frac{7}{8}$ & $10 \frac{7}{8}$ & $40 \frac{1}{2}$ & Do. & $\cdots$ & R. M. Sagar Musgrave. \\
\hline $39 \frac{3}{4}$ & $54 \frac{1}{8}$ & II $\frac{1}{2}$ & $23 \frac{3}{8}$ & Do. & $\cdots$ & The late J. S. Jameson. \\
\hline $39 \frac{3}{4}$ & $5 I^{\frac{1}{2}}$ & $10 \frac{1}{4}$ & $28 \frac{5}{8}$ & Do. & $\cdots$ & W. P. Rylands. \\
\hline $39 \frac{3}{t}$ & $50 \frac{1}{2}$ & $10 \frac{1}{2}$ & 35 & Do. & $\cdots$ & Hon. Charles Ellis. \\
\hline $39 \frac{5}{8}$ & $5 \mathrm{I} \frac{1}{8}$ & III $\frac{1}{4}$ & $3 \frac{1}{2}$ & Do. & $\cdots$ & W. P. Rylands. \\
\hline $39 \frac{1}{2}$ & $52 \frac{3}{4}$ & $10 \frac{3}{4}$ & $1 S_{\frac{1}{4}}^{1}$ & Do. & $\cdots$ & Do. \\
\hline $39 \frac{1}{4}$ & $53 \frac{1}{2}$ & IO & $3^{1 \frac{1}{8}}$ & Africa $\ldots$ & $\cdots$ & Sir Victor Brooke's Collection. \\
\hline $39 \frac{1}{4}$ & $48 \frac{1}{2}$ & $10 \frac{1}{2}$ & $39 \frac{1}{2}$ & South Africa & $\ldots$ & R. C. Dobson. \\
\hline 39 & $53^{\frac{1}{2}}$ & I I & $27 \frac{1}{4}$ & Do. & $\cdots$ & Rowland Ward's Collection. \\
\hline 39 & 52 & $9 \frac{1}{2}$ & 33 & Do. & $\cdots$ & E. R. Thompson. \\
\hline 39 & $50 \frac{3}{4}$ & $10 \frac{3}{4}$ & $27 \frac{5}{8}$ & Do. & $\cdots$ & Maj.-Gen. Arthur Ellis, C.S.I. \\
\hline $38^{\frac{3}{4}}$ & $51 \frac{1}{8}$ & $10 \frac{1}{1}$ & $28 \frac{5}{8}$ & Do. & $\cdots$ & A. Beit. \\
\hline $38 \frac{1}{2}$ & $50 \frac{1}{4}$ & $8 \frac{1}{2}$ & $29 \frac{1}{2}$ & Africa $\ldots$ & $\ldots$ & F. L. James, I 880. \\
\hline $38 \frac{1}{4}$ & $5^{1 \frac{1}{4}}$ & 10 & $20 \frac{1}{2}$ & South Africa & $\cdots$ & J. Carr-Saunders. \\
\hline $3^{8}$ & $54 \frac{7}{8}$ & IO & I $\delta_{\frac{1}{8}}$ & Do. & $\ldots$ & F. C. Selous. \\
\hline 38 & 49 & $8 \frac{7}{8}$ & $29 \frac{3}{8}$ & Somali Land & $\ldots$ & C. Liddell. \\
\hline 38 & $48 \frac{7}{8}$ & $9 \frac{3}{8}$ & $36 \frac{3}{4}$ & South Africa & $\cdots$ & G. H. Banks. \\
\hline 38 & $\cdots$ & II & $33 \frac{1}{2}$ & Do. & $\cdots$ & Lieut. C. F. Gisborne. \\
\hline 38 & 48 & IO & 36 & Do. & $\cdots$ & J. A. Nicoll. \\
\hline $37 \frac{1}{2}$ & $53 \frac{5}{8}$ & $9^{\frac{3}{4}}$ & $25 \frac{3}{8}$ & Do. & $\cdots$ & Maj.-Gen. Arthur Ellis, C.S.I. \\
\hline $37 \frac{1}{2}$ & $52 \frac{1}{8}$ & $9 \frac{1}{4}$ & $23 \frac{1}{2}$ & Somali Land & $\cdots$ & T. W. H. Clarke. \\
\hline $37 \frac{1}{8}$ & $47^{\frac{1}{4}}$ & $10 \frac{1}{4}$ & $35^{\frac{1}{2}}$ & South Africa & $\cdots$ & H.R.H. Duke of Edinbuigh. \\
\hline
\end{tabular}


GREATER KOODOO (Strepsiceros kudu)-continued.

\begin{tabular}{|c|c|c|c|c|c|c|}
\hline \multicolumn{2}{|c|}{ Length. } & \multirow{2}{*}{$\begin{array}{l}\text { Circum- } \\
\text { ference. }\end{array}$} & \multirow{2}{*}{ Tip to Tip. } & \multirow{2}{*}{\multicolumn{2}{|c|}{ Habitat. }} & \multirow{2}{*}{ Owner. } \\
\hline $\begin{array}{l}\text { Straight } \\
\text { line. }\end{array}$ & $\begin{array}{l}\text { Round } \\
\text { curve. }\end{array}$ & & & & & \\
\hline 37 & $53 \frac{1}{2}$ & $9 \frac{5}{8}$ & 23 & Somali Land & $\ldots$ & E Lort-Phillips. \\
\hline 37 & $49 \frac{1}{2}$ & I I & 20 & Do. & $\ldots$ & A. Haggard. \\
\hline 37 & $49 \frac{1}{2}$ & $9 \frac{1}{4}$ & $22 \frac{1}{4}$ & South Africa & $\cdots$ & F. C. Selous. \\
\hline $36 \frac{5}{8}$ & $50 \frac{1}{2}$ & $9 \frac{1}{8}$ & 34 & Somali & $\cdots$ & E. Lort-Phillips. \\
\hline $36 \frac{1}{2}$ & $47^{\frac{1}{2}}$ & $10 \frac{1}{4}$ & $27 \frac{5}{8}$ & South Africa & $\cdots$ & British Museum. \\
\hline 36 & $56 \frac{1}{2}$ & II & $33 \frac{1}{2}$ & Do. & $\cdots$ & M. S. Runcheman. \\
\hline 36 & $48 \frac{5}{8}$ & $1 \mathrm{O}_{\frac{3}{8}}^{\frac{3}{8}}$ & $3 \mathrm{I} \frac{1}{2}$ & Do. & $\cdots$ & British Museum. \\
\hline 36 & $46 \frac{1}{4}$ & $9 \frac{3}{4}$ & 25 & Africa & $\cdots$ & R. Farrow. \\
\hline $35 \frac{3}{4}$ & $47 \frac{7}{8}$ & $9 \frac{7}{8}$ & $24 \frac{5}{8}$ & Somali & $\cdots$ & A. H. Straker. \\
\hline $35^{\frac{1}{2}}$ & 47 & $9 \frac{1}{4}$ & $33 \frac{5}{8}$ & $\begin{array}{l}\text { Africa ... } \\
\text { (killed by a lio }\end{array}$ & $\ldots$ & F. L. James. \\
\hline $35^{\frac{1}{8}}$ & $47 \frac{1}{8}$ & 10 & $30 \frac{1}{8}$ & South Africa & $\cdots$ & British Museum. \\
\hline 35 & $48 \frac{1}{4}$ & $9^{\frac{3}{4}}$ & 27 & Somali & $\cdots$ & Lieut. Merewether. \\
\hline 35 & $47 \frac{1}{2}$ & $8 \frac{3}{4}$ & $38 \frac{3}{8}$ & Soudan & $\cdots$ & Col. Ralph Vivian. \\
\hline 35 & 45 & $9 \frac{3}{8}$ & $32 \frac{1}{8}$ & West Africa & $\cdots$ & Capt. F. Cookson. \\
\hline $34 \frac{7}{8}$ & $46 \frac{3}{4}$ & $10 \frac{1}{4}$ & $2 \mathbf{I} \frac{1}{4}$ & South Africa & $\cdots$ & British Museum. \\
\hline $34 \frac{1}{2}$ & $46 \frac{1}{2}$ & $9 \frac{1}{2}$ & $\cdots$ & Abyssinia & $\cdots$ & Do. \\
\hline $34 \frac{1}{2}$ & 44 & $9 \frac{5}{8}$ & $21 \frac{3}{8}$ & South Africa & $\cdots$ & G. H. Banks. \\
\hline$\cdots$ & 44 & IO & $3^{1}$ & $\begin{array}{l}\text { Kaoko Land, I } \\
\text { Africa. }\end{array}$ & iVest & Capt. F. Cookson. \\
\hline$\cdots$ & 44 & 9 & $\cdots$ & North Africa & $\cdots$ & Capt. Brinkley. \\
\hline$\cdots$ & $43 \frac{1}{2}$ & $10 \frac{1}{2}$ & 29 & West Africa & $\cdots$ & Capt. F. Cookson. \\
\hline $34 \frac{3}{8}$ & $45^{\frac{3}{8}}$ & $10 \frac{1}{8}$ & $25 \frac{5}{8}$ & South Africa & $\cdots$ & British Museum. \\
\hline $33^{\frac{3}{4}}$ & $46 \frac{1}{2}$ & $9 \frac{1}{2}$ & $24 \frac{1}{2}$ & Somali Land & $\cdots$ & Capt. H. G. C. Swayne. \\
\hline $33^{\frac{1}{2}}$ & $42 \frac{1}{4}$ & $9 \frac{3}{4}$ & $28 \frac{1}{2}$ & West Africa & $\cdots$ & Capt. F. Cookson. \\
\hline$\cdots$ & 42 & $\cdots$ & $\cdots$ & North Africa & $\cdots$ & Capt. Brinkley. \\
\hline $3 \mathrm{I} \frac{1}{2}$ & $4 \mathrm{I} \frac{1}{8}$ & $8 \frac{3}{4}$ & $28 \frac{1}{4}$ & Abyssinia & $\cdots$ & British Museum. \\
\hline $28 \frac{1}{4}$ & $37 \frac{5}{9}$ & $8 \frac{1}{4}$ & $3 I$ & Do. & $\cdots$ & Do. \\
\hline
\end{tabular}




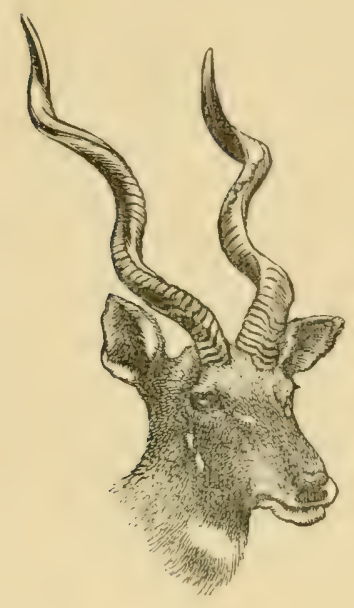

LESSER KOODOO (Strepsiceros imberbis).

Somali name-" Anderio."

Habitat-Africa: Somali Land to Masai Land.

\begin{tabular}{|c|c|c|c|c|c|}
\hline \multicolumn{2}{|c|}{ Length. } & \multirow{2}{*}{$\begin{array}{l}\text { Circum- } \\
\text { ference. }\end{array}$} & \multirow{2}{*}{$\begin{array}{l}\text { Tip to } \\
\text { Tip. }\end{array}$} & \multirow{2}{*}{ Habitat. } & \multirow{2}{*}{ Owner. } \\
\hline $\begin{array}{l}\text { Straight } \\
\text { line. }\end{array}$ & Round Curve. & & & & \\
\hline $25^{\frac{1}{2}}$ & $30 \frac{5}{8}$ & $6 \frac{3}{8}$ & I I $\frac{1}{8}$ & Somali Land & A. H. Straker. \\
\hline $25 \frac{1}{4}$ & $3 I \frac{1}{2}$ & $6 \frac{3}{8}$ & II $\frac{5}{8}$ & Do. & Sir John Kirk, M. D., K. C, B. \\
\hline $24 \frac{1}{2}$ & $31 \frac{1}{4}$ & $6 \frac{3}{\frac{3}{x}}$ & $14 \frac{1}{2}$ & Do. & Capt, H. G. C. Swayne. \\
\hline $24 \frac{1}{2}$ & $\cdots$ & $6 \frac{1}{8}$ & $14 \frac{1}{4}$ & Do. & Rowland Ward's Collection. \\
\hline $24 \frac{3}{8}$ & $30 \frac{3}{4}$ & $6 \frac{5}{8}$ & I I $\frac{7}{8}$ & Do. & W. D. James. \\
\hline 24 & $30 \frac{3}{4}$ & $6 \frac{7}{8}$ & 9 & Do. & E. P. Hare. \\
\hline 24 & $30 \frac{1}{2}$ & $6 \frac{1}{2}$ & 12 & Do. & T. W. H. Clarke. \\
\hline 24 & ... & $\begin{array}{c}\text { (owner's } \\
\text { measurement) }\end{array}$ & $\ldots$ & $\cdots$ & $\begin{array}{l}\text { Bombay Nistural History So- } \\
\text { ciety. }\end{array}$ \\
\hline $23 \frac{1}{2}$ & 29. & $6 \quad 6 \frac{1}{2}$ & II $\frac{1}{8}$ & East Africa... & Sir Robert Harvey, Bart. \\
\hline$\cdots$ & 29 & $\ldots$ & 12 & Somali Land & Ernest Horn. \\
\hline
\end{tabular}


LESSER KOODOO (Strepsiceros imberbis)-continut.

\begin{tabular}{|c|c|c|c|c|c|}
\hline \multicolumn{2}{|r|}{ Length. } & \multirow{2}{*}{$\begin{array}{l}\text { Circum- } \\
\text { ference. }\end{array}$} & \multirow{2}{*}{$\begin{array}{l}\text { Tip to } \\
\text { Tip. }\end{array}$} & \multirow{2}{*}{ Habitat. } & \multirow{2}{*}{ Owner. } \\
\hline $\begin{array}{l}\text { Straight } \\
\text { Line. }\end{array}$ & Round Curve. & & & & \\
\hline 23 & $\ldots$ & 63 & $10 \frac{3}{4}$ & Somali Land & Col. Arthur Paget. \\
\hline 23 & $28 \frac{1}{8}$ & $5 \frac{1}{2}$ & II $\frac{5}{8}$ & Do. & $\begin{array}{l}\text { Sir John Kirk, British Mu- } \\
\text { seum. }\end{array}$ \\
\hline $22 \frac{7}{8}$ & $29 \frac{3}{8}$ & $6 \frac{1}{8}$ & $14 \frac{1}{8}$ & Do. & Lord Delamere. \\
\hline $22 \frac{1}{4}$ & $28 \frac{7}{8}$ & $6 \frac{1}{4}$ & $15^{\frac{1}{4}}$ & Do. & Do. \\
\hline $22 \frac{1}{4}$ & $29 \frac{3}{8}$ & $6 \frac{3}{8}$ & $13 \frac{5}{8}$ & Do. & E. Lort-Phillips. \\
\hline $22 \frac{1}{8}$ & $2 S_{\frac{1}{4}}^{1}$ & $5^{\frac{3}{4}}$ & I $3 \frac{1}{8}$ & Do. & J. Carr-Saunders. \\
\hline $22 \frac{1}{8}$ & $26 \frac{7}{8}$ & $5 \frac{1}{2}$ & $12 \frac{3}{4}$ & $\begin{array}{l}\text { South Somali } \\
\text { Land. }\end{array}$ & Sir John Kirk, M.D., K. C.B. \\
\hline 22 & 28 & $6 \frac{3}{4}$ & $9 \frac{1}{2}$ & Somali Land & C. H. Villiers. \\
\hline $21 \frac{7}{8}$ & $28 \frac{7}{8}$ & $6 \frac{1}{2}$ & 9 & East Africa ... & H. C. V. Hunter. \\
\hline $2 \mathrm{I} \frac{1}{2}$ & $28 \frac{3}{8}$ & $6 \frac{5}{8}$ & $10 \frac{1}{8}$ & Do. & Sir Robert Harvey, Bart. \\
\hline $21 \frac{5}{8}$ & $27 \frac{1}{x}$ & $6 \frac{1}{2}$ & $12 \frac{3}{t}$ & $\begin{array}{l}\text { South Somali } \\
\text { Land. }\end{array}$ & Sir John Kirk, M.D., K.C. B. \\
\hline $21 \frac{3}{8}$ & $27 \frac{1}{4}$ & $6 \frac{5}{8}$ & 7 & Somali Land & Hon. Walter Rothschild. \\
\hline $2 \mathrm{I}$ & $\begin{array}{c}\text { (owner's } \\
\text { measurement) }\end{array}$ & $\cdots$ & $\ldots$ & Do. & J. D. Inverarity. \\
\hline 21 & $27 \frac{1}{4}$ & $6 \frac{1}{2}$ & 7 & Do. & Rowland Ward's Collection. \\
\hline $20 \frac{3}{4}$ & 26 & $6 \frac{3}{8}$ & $12 \frac{1}{8}$ & Do. & E. Lort-Phillips. \\
\hline $20 \frac{1}{2}$ & 25 & $5 \frac{5}{8}$ & $12 \frac{1}{8}$ & East Africa... & II. C. V. II unter. \\
\hline $20 \frac{1}{4}$ & 26 & 6 & $11 \frac{3}{4}$ & Do. & Sir Robert Harvey, Bart. \\
\hline 20 & 24 & 6 & 14 & Do. & W. Astor Chanler. \\
\hline 19 & $23 \frac{1}{2}$ & $5^{\frac{3}{4}}$ & $7 \frac{1}{2}$ & $\begin{array}{l}\text { River Juba, } \\
\text { Somali. }\end{array}$ & $\begin{array}{l}\text { Sir John Kirk, British Mu- } \\
\text { seum. }\end{array}$ \\
\hline $18 \frac{7}{8}$ & $24 \frac{5}{8}$ & $5 \frac{1}{\frac{1}{4}}$ & $4^{\frac{3}{4}}$ & East Africa ... & Sir Robert Harvey, Bart. \\
\hline$\cdots$ & $24 \frac{1}{8}$ & $5 \frac{5}{8}$ & $12 \frac{5}{8}$ & Do. & II. C. V. Hunter. \\
\hline $18 \frac{7}{8}$ & $22 \frac{7}{8}$ & $5^{\frac{1}{4}}$ & 12 & Somali Land & $\begin{array}{l}\text { Sir John Kirk, British Mu- } \\
\text { seum. }\end{array}$ \\
\hline $17 \frac{5}{x}$ & $21 \frac{3}{8}$ & $5 \frac{3}{8}$ & $5 \frac{1}{8}$ & Do. & Sir John Kirk, M.D., K.C.B. \\
\hline 17 & $\cdots$ & ... & I4 & Near Lanjora & $\begin{array}{l}\text { Sir J. Willoughby, Bt., "East } \\
\text { Africa and its Big Game." }\end{array}$ \\
\hline $15 \frac{1}{8}$ & $17 \frac{7}{8}$ & $5 \frac{1}{8}$ & I I & East Africa ... & F. J. Jackson. \\
\hline
\end{tabular}




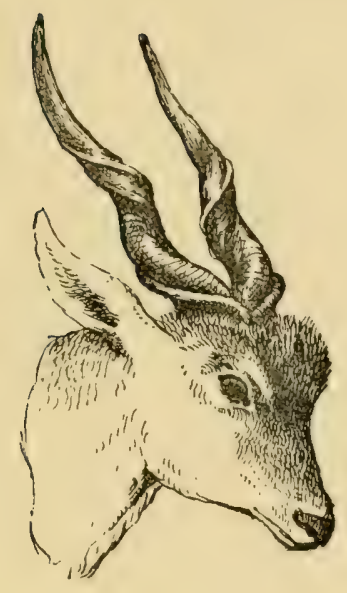

ELAND (Oreas canna),

Do. (Do. derbianus).

\section{Bechuana Name-" Impofu."}

Habitat-South, East, and Central Africa.

\begin{tabular}{|c|c|c|c|c|c|}
\hline Length. & $\begin{array}{l}\text { Circum- } \\
\text { ference. }\end{array}$ & Tip to Tip. & Habitat. & & Owner. \\
\hline$* 34 \frac{1}{t}$ & $12 \frac{5}{8}$ & $22 \frac{3}{4}$ & $\ldots$ & $\therefore$ & Sir Edmund G. Loder, Bart. \\
\hline 34 & $13 \frac{1}{2}$ & $\begin{array}{c}\text { (owner's mea- } \\
\text { surements) }\end{array}$ & $\ldots$ & $\therefore$ & F. Coburn. \\
\hline *34 & $12 \frac{3}{4}$ & $\ldots$ & $\cdots$ & $\therefore$ & J. Carr-Saunders. \\
\hline$* 32 \frac{1}{2}$ & $12 \frac{1}{2}$ & $29 \frac{7}{8}$ & Gambia... & $\ldots$ & Earl of Derby, British Museum. \\
\hline * $+32 \frac{1}{2}$ & $9 \frac{1}{4}$ & $12 \frac{3}{4}$ & $\cdots$ & $\ldots$ & Sir Edmund G. Loder, Bart. \\
\hline$*_{32}$ & $\mathbf{I}_{3}$ & $24 \frac{1}{2}$ & $\ldots$ & $\ldots$ & Hon. Walter Rothschild. \\
\hline$*_{3} \mathrm{I}_{8}^{\frac{7}{8}}$ & $11 \frac{5}{8}$ & $28 \frac{5}{8}$ & Senegambia & $\ldots$ & Earl of Derby, British Museum. \\
\hline $31 \frac{3}{4}$ & $12 \frac{1}{4}$ & $22 \frac{3}{4}$ & ... & $\ldots$ & Hon. Walter Rothschild. \\
\hline
\end{tabular}


ELAND (Oreas canna and Oreas derbianus)-continued.

\begin{tabular}{|c|c|c|c|c|c|}
\hline Length. & $\begin{array}{l}\text { Circum- } \\
\text { ference. }\end{array}$ & Tip to Tip. & Habitat. & & Owner. \\
\hline$\delta 31 \frac{5}{8}$ & $10 \frac{1}{4}$ & $25 \frac{1}{2}$ & East Africa & $\cdots$ & F. J. Jackson. \\
\hline$q 3 r^{\frac{1}{2}}$ & $8 \frac{1}{4}$ & $20 \frac{3}{8}$ & South Africa & $\cdots$ & F. C. Selous. \\
\hline$\delta 3 \mathrm{I} \frac{1}{2}$ & $12 \frac{3}{4}$ & $12 \frac{1}{2}$ & Mashonaland & $\cdots$ & Do. British Museum. \\
\hline$*_{3} \mathrm{I} \frac{1}{8}$ & 12 & $18 \frac{5}{8}$ & $\ldots \quad \ldots$ & $\ldots$ & Sir Victor Brooke's Collection. \\
\hline$\delta 3 \mathrm{I}$ & 13 & 12 & South Africa & $\cdots$ & F. C. Selous. \\
\hline$*_{3} \circ \frac{7}{8}$ & $10 \frac{3}{t}$ & $26 \frac{3}{4}$ & $\cdots \quad \cdots$ & $\ldots$ & F. W. Reade, British Museum. \\
\hline$q 30 \frac{7}{5}$ & $7 \frac{7}{3}$ & $15^{\frac{3}{4}}$ & South Africa & $\cdots$ & F. C. Selous. \\
\hline$\delta 30_{4}^{3}$ & $12 \frac{3}{8}$ & $16 \frac{1}{8}$ & Do. & $\cdots \mid$ & Do. \\
\hline ठ29 & II $\frac{1}{x}$ & $19 \frac{1}{8}$ & Mashonaland & $\ldots \mid$ & Do. British Museum. \\
\hline 인 $29 \frac{5}{8}$ & $7 \frac{1}{2}$ & $21 \frac{1}{8}$ & South Africa & $\cdots 1$ & Do. \\
\hline $29 \frac{3}{8}$ & $7 \stackrel{3}{ \pm}$ & $9 \frac{3}{8}=$ & Algoa Bay & $\cdots \mid$ & British Museum. \\
\hline 을 & $6 \frac{5}{8}$ & $17 \frac{5}{8}$ & $\cdots \quad \ldots$ & $\cdots \mid$ & W. C. Oswell. \\
\hline $29 \frac{1}{8}$ & $12 \frac{1}{2}$ & $14 \frac{7}{x}$ & South Africa & $\ldots 1$ & F. C. Selous. \\
\hline $\begin{array}{r}\$ 29 \\
29\end{array}$ & $\begin{array}{l}\cdots \\
10 \frac{1}{4}\end{array}$ & $\begin{array}{l}\cdots \\
21\end{array}$ & $\begin{array}{l}\text { Mashonaland } \\
\text { South Africa }\end{array}$ & \begin{tabular}{l|l}
$\cdots$ \\
$\cdots$
\end{tabular} & $\begin{array}{l}\text { Do. "A Hunter's Wander- } \\
\text { ings." } \\
\text { Major Gould Adams. }\end{array}$ \\
\hline $28 \frac{1}{4}$ & $10 \frac{1}{2}$ & $19 \frac{1}{2}$ & $\ldots \quad \ldots$ & $\ldots 1$ & Do. \\
\hline$q 28 \frac{1}{4}$ & $7 \frac{7}{8}$ & $13 \frac{3}{4}$ & South Africa & $\ldots$ & F. C. Selous. \\
\hline$\delta 27 \frac{7}{8}$ & $12 \frac{1}{2}$ & $20 \frac{1}{2}$ & Do. & $\ldots 1$ & Do. \\
\hline$q 27 \frac{3}{4}$ & $6 \frac{5}{8}$ & $12 \frac{1}{4}$ & Do. & $\cdots$ & II. Atkinson. \\
\hline$\delta 27 \frac{1}{2}$ & $10 \frac{1}{8}$ & $18 \frac{3}{8}$ & Do. & $\cdots$ & Do. \\
\hline $27 \frac{1}{2}$ & $\cdots$ & $20 \frac{1}{2}$ & Do. & $\cdots \mid$ & $\begin{array}{l}\text { Dr. Knight Bruce (Bishop of Bloom- } \\
\text { fontein). }\end{array}$ \\
\hline$q 27$ & $\cdots$ & ro & $\cdots, \cdots$ & $\cdots \mid$ & $\begin{array}{l}\text { Sir John Willoughby, Bart., "East } \\
\text { Africa and its Big Game." }\end{array}$ \\
\hline 27 & $10 \frac{1}{2}$ & I $S_{\frac{3}{4}}$ & $\begin{array}{ll}\cdots & \cdots\end{array}$ & $\cdots$ & R. C. Dobson. \\
\hline 27 & $8 \frac{1}{8}$ & $12 \frac{5}{8}$ & South Africa & $\ldots 1$ & The late J. S. Jameson. \\
\hline 27 & II $\frac{1}{2}$ & $x 8 \frac{1}{4}$ & $\ldots \cdot \ldots$ & $\cdots 1$ & Rowland Ward's Collection. \\
\hline 27 & $\cdot \ldots$ & $\ldots$ & Mashonaland & $\cdots \mid$ & $\begin{array}{l}\text { F. C. Selous, "A Hunter's Wander- } \\
\text { ings." }\end{array}$ \\
\hline
\end{tabular}


ELAND (Oreas canna and Oreas derbianus)-continucd.

\begin{tabular}{|c|c|c|c|c|c|}
\hline Length. & $\begin{array}{l}\text { Circum- } \\
\text { ference. }\end{array}$ & Tip to Tip. & Habitat. & & Owner. \\
\hline $26 \frac{7}{8}$ & $10 \frac{1}{2}$ & $I 7^{\frac{3}{4}}$ & South Africa & $\ldots$ & The late J. S. Jameson. \\
\hline $26 \frac{3}{4}$ & IO & $19 \frac{1}{2}$ & $\cdots$ & $\cdots$ & H.R.H. Duke of Edinburgh. \\
\hline $26 \frac{5}{8}$ & $7 \frac{5}{8}$ & I 8 & South Africa & $\cdots$ & The late J. S. Jameson. \\
\hline $26 \frac{1}{2}$ & $12 \frac{1}{4}$ & $12 \frac{3}{8}$ & Do. & $\cdots$ & F. C. Selous. \\
\hline $26 \frac{3}{8}$ & $8 \frac{3}{8}$ & $15 \frac{3}{4}$ & Mashonaland & $\cdots$ & Do. British Museum. \\
\hline$+26 \frac{1}{4}$ & $7 \frac{3}{8}$ & $19 \frac{3}{4}$ & $\ldots$ & $\ldots$ & Earl of Derby, British Museum. \\
\hline 인 & $8 \frac{1}{4}$ & IO $\frac{1}{8}$ & West Africa & $\cdots$ & Capt. F. Cookson. \\
\hline $25 \frac{7}{8}$ & I I $\frac{1}{2}$ & $12 \frac{7}{8}$ & Mashonaland & $\ldots$ & F. C. Selous, British Museum. \\
\hline$\delta^{2} 5^{\frac{3}{4}}$ & II $\frac{5}{8}$ & $14 \frac{1}{8}$ & Do. & $\ldots$ & Do. \\
\hline $25 \frac{3}{t}$ & $10 \frac{1}{8}$ & 12 & East Africa & $\cdots$ & H. C. V. Hunter. \\
\hline $25 \frac{5}{8}$ & II $\frac{1}{2}$ & I $3 \frac{1}{2}$ & South Africa & $\cdots$ & J. A. Jameson. \\
\hline Q $25 \frac{5}{8}$ & $8 \frac{1}{8}$ & I $4 \frac{1}{2}$ & Mashonaland & $\cdots$ & F. C. Selous, British Museum. \\
\hline $25 \frac{5}{8}$ & 13 & $8 \frac{1}{8}$ & South Africa & $\ldots$ & J. A. Jameson. \\
\hline $25^{\frac{1}{2}}$ & 12 & 7 & Do. & $\ldots$ & A. Beit. \\
\hline$\Upsilon_{25 \frac{1}{2}}$ & $7 \frac{5}{8}$ & $10 \frac{1}{4}$ & Do. & $\ldots$ & Do. \\
\hline $25 \frac{1}{8}$ & $9 \frac{7}{8}$ & $16 \frac{1}{8}$ & East $A$ frica & $\cdots$ & H. C. V. Hunter. \\
\hline$q 25 \frac{1}{8}$ & $6 \frac{1}{4}$ & $9 \frac{1}{8}$ & Do. & ... & Sir Robert Harvey, Bart. \\
\hline$\$ 25$ & 7 & $14 \frac{1}{2}$ & South Africa & ... & A. W. Davis. \\
\hline $24 \frac{5}{8}$ & $10 \frac{1}{8}$ & 13 & Do. & $\cdots$ & The Hon. Charles Ellis. \\
\hline $24 \frac{1}{2}$ & II & 15 & $\cdots$ & $\ldots$ & J. A. Nicoll. \\
\hline $24 \frac{1}{2}$ & $6 \frac{3}{3}$ & $10 \frac{1}{2}$ & East Africa & $\cdots$ & H. C. V. Hunter. \\
\hline $24 \frac{3}{8}$ & $6 \frac{1}{4}$ & $15 \frac{1}{8}$ & Do. & $\cdots$ & F. J. Jackson. \\
\hline $24 \frac{1}{4}$ & I $2 \frac{3}{4}$ & 13 & Mashonaland & $\ldots$ & F. C. Selous, British Museum. \\
\hline$*_{24 \frac{1}{4}}$ & 9 & $6 \frac{1}{4}$ & Senegambia & ... & F. W. Reade, British Museum. \\
\hline 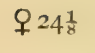 & $6 \frac{1}{4}$ & 2 & $\cdots$ & $\ldots$ & Sir Victor Brooke's Collection. \\
\hline 24 & $7 \frac{1}{2}$ & 12 & East Africa & ... & Gen. Matthews. \\
\hline
\end{tabular}

* Oreas derbianus. 
ELAND (Oreas canna and Oras derbianus)-continucd.

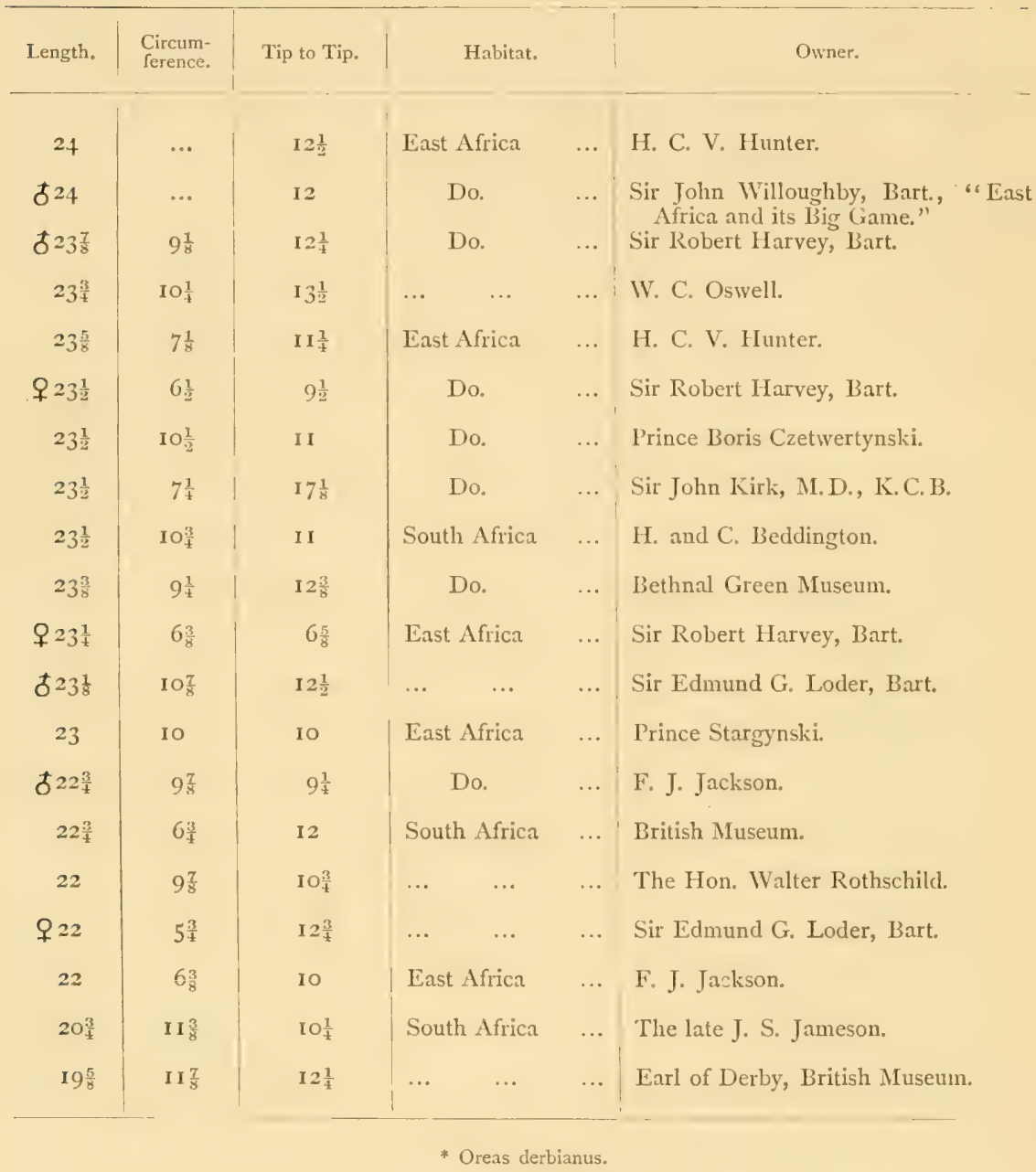




\section{CHAIMOIS (Rupicapra tragus).}

Habitat-Mountains of Europe, from the Pyrenees to Caucasus.

\begin{tabular}{|c|c|c|c|c|c|c|}
\hline Length. & $\begin{array}{l}\text { Circum- } \\
\text { ference. }\end{array}$ & Tip to Tip. & & Iabitat. & & Owner. \\
\hline $10 \frac{1}{4}$ & $3 \frac{1}{2}$ & $5^{\frac{1}{t}}$ & $\cdots$ & $\ldots$ & $\ldots$ & E. N. Buxton, \\
\hline $10 \frac{1}{4}$ & $4 \frac{1}{8}$ & $3 \frac{7}{3}$ & Caucas & & $\cdots$ & Sir Edmund G. Loder, Bart. \\
\hline $9 \frac{7}{8}$ & $3 \frac{1}{4}$ & $4 \frac{1}{x}$ & $\ldots$ & $\cdots$ & $\cdots$ & H.R.H. Duke of Edinburgh. \\
\hline$* 9 \frac{3}{4}$ & $3 \frac{1}{2}$ & $5 \frac{1}{8}$ & $\cdots$ & $\ldots$ & $\cdots$ & $\begin{array}{l}\text { H.I. and R.H. Crown Prince Rudolph } \\
\text { of Austria, British Museum. }\end{array}$ \\
\hline$* 9 \frac{3}{4}$ & $3 \frac{1}{4}$ & $3 \frac{7}{8}$ & Transy & Ivania & $\cdots$ & C. G. Danford, British Museum. \\
\hline $9 \frac{5}{8}$ & $3 \frac{1}{4}$ & $4 \frac{1}{2}$ & Caucas & & $\cdots$ & St. George Littledale, I89I. \\
\hline $9 \frac{5}{8}$ & $3 \frac{3}{4}$ & $4^{\frac{3}{4}}$ & Piz Di & avel & $\cdots$ & E. N. Buxton, 1876 . \\
\hline $9 \frac{5}{8}$ & $3 \frac{5}{8}$ & 4 & $\cdots$ & $\cdots$ & $\cdots$ & Capt. Townley Parker. \\
\hline $9 \frac{5}{8}$ & 3 & $3 \frac{5}{8}$ & $\cdots$ & $\cdots$ & $\cdots$ & The Hon. Walter Rothschild. \\
\hline$* 9 \frac{5}{8}$ & $3 \frac{1}{2}$ & $3 \frac{1}{2}$ & Tyrol & ... & $\ldots$ & $\begin{array}{l}\text { H. I. and R.H. Crown Prince Rudolph } \\
\text { of Austria, British Museum. }\end{array}$ \\
\hline $9 \frac{3}{8}$ & $3^{\frac{1}{4}}$ & $2 \frac{5}{8}$ & $\cdots$ & $\cdots$ & $\cdots$ & J. Carr-Saunders. \\
\hline $9 \frac{1}{4}$ & $2 \frac{1}{2}$ & 4 & Alps & $\cdots$ & $\cdots$ & Gen. Hardwicke, British Museum. \\
\hline $9 \frac{1}{8}$ & $3 \frac{3}{8}$ & $3 \frac{7}{8}$ & $\cdots$ & $\cdots$ & $\cdots$ & H.R.H. Duke of Edinburgh. \\
\hline $9 \frac{1}{8}$ & $2 \frac{3}{4}$ & $4 \frac{1}{4}$ & $\cdots$ & $\cdots$ & $\cdots$ & Do. \\
\hline 9 & $3 \frac{1}{2}$ & $3^{\frac{3}{4}}$ & $\cdots$ & $\cdots$ & $\cdots$ & W. H. Briggs. \\
\hline ㅇ 9 & $2 \frac{3}{4}$ & $4 \frac{1}{2}$ & $\cdots$ & $\cdots$ & $\cdots$ & Maj.-Gen. Arthur Ellis, C.S.I. \\
\hline $8 \frac{3}{4}$ & $3 \frac{2}{8}$ & $4 \frac{1}{8}$ & $\cdots$ & $\cdots$ & $\cdots$ & Do. \\
\hline $8 \frac{3}{4}$ & $3 \frac{1}{2}$ & 4 & $\cdots$ & $\cdots$ & $\cdots$ & Rowland Ward's Collection. \\
\hline $8 \frac{3}{4}$ & $3 \frac{3}{8}$ & 3 & Caucas & & $\cdots$ & St. George Littledale. \\
\hline $8 \frac{3}{4} \times 5 \frac{1}{4}$ & $3 \frac{3}{8} \times 2 \frac{3}{4}$ & $\begin{array}{c}4 \frac{1}{8} \times 3 \frac{1}{8} \\
(4 \text { horns })\end{array}$ & $\ldots$ & $\ldots$ & $\ldots$ & Capt. Townley Parker. \\
\hline $8 \frac{3}{4}$ & 3 & $3 \frac{7}{8}$ & $\cdots$ & $\cdots$ & $\cdots$ & Gerald Buxton, I $88_{3}$. \\
\hline $8 \frac{3}{4}$ & $3 \frac{1}{2}$ & $3 \frac{3}{8}$ & Cauca & & ... & St. George Littledale. \\
\hline
\end{tabular}

* Rupicapra vulgaris. 
CHAIMOIS (Rupicapra tragus)-continued.

\begin{tabular}{|c|c|c|c|c|c|}
\hline Length. & $\begin{array}{l}\text { Circum- } \\
\text { ference. }\end{array}$ & Tip to Tip. & Habitat. & & Owner. \\
\hline $8 \frac{3}{4}$ & $2 \frac{7}{8}$ & 21 & $\ldots$ & $\ldots$ & The Hon. Walter Rothschild. \\
\hline $8 \frac{5}{8}$ & $3 \frac{3}{t}$ & $3 \frac{3}{4}$ & $\ldots$ & $\ldots$ & E. W. Reegan. \\
\hline $8 \frac{5}{8}$ & $3 \frac{1}{2}$ & $2 \frac{3}{8}$ & Caucasus & $\ldots$ & St. George Littledale, $\mathbf{I} 89 \mathbf{I}$. \\
\hline $8 \frac{1}{2}$ & $2 \frac{1}{2}$ & $3 \frac{7}{8}$ & Do. & $\ldots$ & Do. $\quad$ I887. \\
\hline$* 8 \frac{1}{2}$ & $2 \frac{5}{8}$ & 4 & Alps $\quad \ldots$ & $\ldots$ & J. Gould, British Museum. \\
\hline 오 $8 \frac{3}{8}$ & $2 \frac{1}{t}$ & $4 \frac{3}{3}$ & Pyrenees & $\ldots$ & Sir Victor Brooke's Collection. \\
\hline$\delta_{x}^{3}$ & 3 & $3 \frac{3}{7}$ & $\ldots$ & $\ldots$ & British Museum. \\
\hline $8 \frac{3}{8}$ & $3 \frac{1}{8}$ & $3 \frac{1}{8}$ & Caucasus & $\ldots$ & St. George Littledale, $\mathbf{1} 887$. \\
\hline$\oint_{\frac{1}{4}}$ & $3 \frac{3}{8}$ & $2 \frac{33}{4}$ & Do. & $\ldots$ & Do. $\quad$ I $89 \mathrm{I}$. \\
\hline $8 \frac{1}{4}$ & $2 \frac{7}{8}$ & 4 & $\begin{array}{ll}\cdots & \cdots\end{array}$ & $\ldots$ & H.R.H. Duke of Edinburgh. \\
\hline $8 \frac{1}{4}$ & $3 \frac{3}{8}$ & $3 \frac{1}{8}$ & Alps $\quad \ldots$ & $\ldots$ & Gen. Hardwicke, British Museum. \\
\hline 오 $S_{\frac{1}{8}}$ & $2 \frac{5}{8}$ & $4 \frac{7}{8}$ & $\ldots$ & $\ldots$ & Maj.-Gen. Arthur Ellis, C.S.I. \\
\hline $8_{8}^{1}$ & $2 \frac{7}{8}$ & $3 \frac{1}{2}$ & $\begin{array}{ll}\cdots & \ldots\end{array}$ & $\ldots$ & E. N. Buxton. \\
\hline 옹 & 3 & 2 & Caucasus & $\ldots$ & St. George Littledale, I 887 . \\
\hline $8 \frac{1}{8}$ & $3 \frac{1}{1}$ & $3 \frac{3}{8}$ & Do. & $\ldots$ & ISS6. \\
\hline q $s$ & $2 \frac{1}{2}$ & 3 & $\ldots$ & $\ldots$ & Maj.-Gen. Arthur Ellis, C.S.I \\
\hline 8 & $3 \frac{3}{8}$ & $2 \frac{7}{8}$ & Caucasus & $\ldots$ & St. George Littledale, ISS7. \\
\hline q S & $2 \frac{3}{4}$ & $3 \frac{3}{8}$ & Do. & $\cdots$ & IS86. \\
\hline $7 \frac{7}{8}$ & $3 \frac{1}{2}$ & $1 \frac{5}{8}$ & Do. & $\cdots$ & I89r. \\
\hline $7 \frac{3}{4}$ & $3 \frac{3}{8}$ & $3 \frac{1}{2}$ & Do. & $\ldots$ & 1887. \\
\hline $7 \frac{3}{4}$ & $2 \frac{3}{t}$ & $3 \frac{5}{8}$ & $\cdots$ & $\ldots$ & E. N. Buxton, I 883 . \\
\hline $7 \frac{3}{4}$ & $2 \frac{7}{8}$ & 3 & Alps $\quad \ldots$ & $\cdots$ & Dr. J. E. Gray, British Museum. \\
\hline 오 $7 \frac{3}{ \pm}$ & $2 \frac{3}{4}$ & $3 \frac{7}{8}$ & Caucasus & $\ldots$ & St. George Littledale, I887. \\
\hline 오 $7 \frac{5}{8}$ & $2 \frac{7}{8}$ & $2 \frac{3}{8}$ & Do. & $\ldots$ & 1887. \\
\hline$*_{7 \frac{1}{2}}$ & $2 \frac{3}{4}$ & $2 \frac{7}{8}$ & Do. & $\ldots$ & British Museum. \\
\hline $7^{\frac{1}{2}}$ & $3 \frac{3}{8}$ & $3 \frac{1}{2}$ & Do. & $\cdots$ & St. George Littledale, I $\$ 87$. \\
\hline
\end{tabular}


CHAMOIS (Rupicapra tragus)-continued.

\begin{tabular}{|c|c|c|c|c|c|}
\hline Length。 & $\begin{array}{l}\text { Circum- } \\
\text { ference. }\end{array}$ & Tip to Tip. & Habitat. & & Owner. \\
\hline † $7 \frac{1}{2}$ & $2 \frac{3}{4}$ & 5 & Caucasus & ... & St. George Littledale, I\$gI. \\
\hline 궁 & $2 \frac{7}{8}$ & $3 \frac{1}{2}$ & Do. & ... & Do. $\quad \mathbf{I} 887$ \\
\hline $7 \frac{1}{ \pm}$ & $3 \frac{3}{8}$ & $3 \frac{3}{8}$ & Pyrenees & $\ldots$ & British Museum. \\
\hline $7 \frac{1}{4}$ & $2 \frac{5}{8}$ & I $\frac{5}{8}$ & Especieres & $\ldots$ & E. N. Buxton, IS\&2. \\
\hline 7 & $2 \frac{5}{8}$ & 2 & Alps & ... & Gen, Hardwicke, British Museum. \\
\hline ㅇ 7 & $2 \frac{3}{8}$ & $3 \frac{1}{2}$ & Pyrenees & .. & Sir Victor Brooke's Collection. \\
\hline $6 \frac{7}{8}$ & $3 \frac{1}{ \pm}$ & $2 \frac{1}{8}$ & Alps & $\ldots$ & Dr. J. E. Gray, British Museum. \\
\hline ㅇ $6 \frac{7}{8}$ & $2 \frac{5}{8}$ & $2 \frac{3}{4}$ & Caucasus & ... & St. George Littledale, British. Mu- \\
\hline $6 \frac{3}{4}$ & $3 \frac{1}{8}$ & $2 \frac{1}{8}$ & Alps & $\cdots$ & Dr. J. E. Gray, British Museuz. \\
\hline ㅇ $6 \frac{1}{2}$ & $2 \frac{1}{8}$ & $3 \frac{\pi}{8}$ & Pyrenees & ... & Sir Victor Brooke's Collection.. \\
\hline \& 6 & $2 \frac{33}{4}$ & $3 \frac{1}{8}$ & Caucasus & $\cdots$ & St. George Littledale, I 886 . \\
\hline 6 & $2 \frac{5}{8}$ & $2 \frac{5}{8}$ & $\ldots$ & $\cdots$ & Sir Victor Brooke's Collection. \\
\hline
\end{tabular}

GOORAL or HIMALAYAN CHAIMOIS (Nemorhœdus goral).

Habitat-Himalayas, from Bhotan and Sikim to Kashmir.

\begin{tabular}{|c|c|c|c|c|c|c|}
\hline Length. & $\begin{array}{l}\text { Circum- } \\
\text { ference. }\end{array}$ & $\begin{array}{l}\text { Tip to } \\
\text { Tip. }\end{array}$ & \multicolumn{3}{|c|}{ Habitat. } & Owner. \\
\hline $7 \frac{5}{8}$ & $3^{\frac{3}{4}}$ & $3 \frac{1}{2}$ & India & $\ldots$ & $\ldots$ & Hume Collection, British Museum \\
\hline 7 & $3 \frac{3}{*}$ & $2 \frac{3}{4}$ & Do. & $\ldots$ & $\ldots$ & Capt. J. A. Orr-Ewing. \\
\hline 7 & $3 \frac{1}{2}$ & $2 \frac{7}{8}$ & Do. & $\ldots$ & $\ldots$ & J. M. Nicolls. \\
\hline $6 \frac{3}{4}$ & $3 \frac{1}{2}$ & I & Do. & $\ldots$ & .. & Capt. G. Campbell. \\
\hline $6 \frac{5}{3}$ & $3 \frac{1}{2}$ & $2 \frac{1}{8}$ & Do. & $\cdots$ & .. & Hume Collection, British Museum. \\
\hline $6 \frac{3}{8}$ & $3 \frac{1}{8}$ & $2 \frac{7}{8}$ & Do. & $\ldots$ & $\cdots$ & J. Carr-Saunders. \\
\hline $6 \frac{1}{4}$ & $3 \frac{3}{8}$ & $3 \frac{1}{2}$ & Do. & $\ldots$ & $\ldots$ & Sir Victor Brooke's Collection. \\
\hline
\end{tabular}


GOORAL or HIMALAYAN CHAIMOIS (Nemorhœdus goral) continued.

\begin{tabular}{|c|c|c|c|c|c|}
\hline Length. & $\begin{array}{l}\text { Circum- } \\
\text { ference. }\end{array}$ & Tip to Tip. & \multicolumn{2}{|l|}{ Habitat. } & Owner. \\
\hline$* 6 \frac{1}{4}$ & 4 & $3 \frac{1}{2}$ & \multicolumn{2}{|c|}{ North Mongolia } & Messrs. Dorries, British Museum. \\
\hline 6 & $2 \frac{5}{8}$ & I & $\cdots$ & ... & Hume Collection, British Museum. \\
\hline 6 & $\cdots$ & $\cdots$ & $\cdots$ & $\cdots$ & B. H. Hodgson, P.Z.S. $18_{34}$. \\
\hline $5 \frac{7}{8}$ & $3 \frac{1}{2}$ & $2 \frac{3}{4}$ & $\begin{array}{c}\text { Kussowlie, I } \\
\text { India. }\end{array}$ & North & Sir Victor Brooke's Collection. \\
\hline $5^{\frac{3}{4}}$ & $2 \frac{1}{2}$ & 2 & $\cdots \quad \cdots$ & ... & Do. \\
\hline $5 \frac{5}{8}$ & $3 \frac{5}{8}$ & $2 \frac{1}{2}$ & $\cdots$ & $\cdots$ & H. F. Campbell. \\
\hline $5 \frac{1}{4}$ & $3 \frac{1}{8}$ & $2 \frac{3}{4}$ & Nepal $\ldots$ & $\cdots$ & British Museum. \\
\hline $5 \frac{1}{8}$ & $3 \frac{3}{8}$ & 3 & Do. $\quad \ldots$ & $\cdots$ & Do. \\
\hline$*_{4} \frac{3}{4}$ & $3 \frac{3}{8}$ & $I \frac{1}{2}$ & Pekin ... & $\cdots$ & F. W. Styer, British Museum. \\
\hline$*_{4} \frac{5}{8}$ & 3 & 2 & North China & $\ldots$ & British Museum. \\
\hline $4 \frac{1}{2}$ & $3 \frac{1}{8}$ & $2 \frac{1}{2}$ & $\begin{array}{l}\text { Kussowlie, I } \\
\text { India. }\end{array}$ & North & Sir Victor Brooke's Collection. \\
\hline
\end{tabular}

JAPANESE GOORAL (Nemorhœdus crispus).

Habitat-Japan.

\begin{tabular}{|c|c|c|c|c|c|}
\hline Length. & $\begin{array}{l}\text { Circum- } \\
\text { ference. }\end{array}$ & $\begin{array}{l}\text { Tip to } \\
\text { Tip. }\end{array}$ & Habitat. & & Owner. \\
\hline $5 \frac{1}{4}$ & $3 \frac{1}{8}$ & $3 \frac{1}{8}$ & Japan ... & ... & British Museum. \\
\hline
\end{tabular}


SUMATRAN SEROW (Nemorhœdus sumatrensis).

Native name-"Cambing-Utan."

Habitat-Sumatra.

\begin{tabular}{|c|c|c|c|c|c|}
\hline Length. & $\begin{array}{l}\text { Circum- } \\
\text { ference. }\end{array}$ & $\begin{array}{l}\text { Tip to } \\
\text { Tip. }\end{array}$ & \multicolumn{2}{|c|}{ Habitat. } & Owner. \\
\hline 9 & 5 & 2 & Sumatra & ... & Hume Collection, British Museum. \\
\hline $8 \frac{3}{4}$ & $5 \frac{1}{4}$ & $4 \frac{1}{2}$ & $\cdots$ & $\cdots$ & Do. \\
\hline $8 \frac{3}{8}$ & $4 \frac{7}{8}$ & $4 \frac{1}{4}$ & .., & $\because$ & Do, \\
\hline $8 \frac{1}{8}$ & $5 \frac{1}{2}$ & $4 \frac{1}{8}$ & $\ldots$ & $\ldots$ & Do. \\
\hline $8 \frac{1}{8}$ & $4 \frac{7}{8}$ & $3 \frac{1}{4}$ & $\cdots$ & $\ldots$ & Do. \\
\hline 8 & 5 & $3 \frac{7}{8}$ & $\cdots$ & $\cdots$ & Do. \\
\hline $7 \frac{7}{8}$ & $5 \frac{1}{8}$ & $2 \frac{3}{4}$ & $\ldots$ & $\ldots$ & Do. \\
\hline $7^{\frac{3}{4}}$ & $4 \frac{5}{8}$ & $3 \frac{1}{8}$ & $\because$ & $\cdots$ & Do. \\
\hline $7 \frac{1}{8}$ & $4 \frac{1}{2}$ & $4 \frac{1}{8}$ & $\cdots$ & $\cdots$ & Do. \\
\hline 7 & $4 \frac{3}{4}$ & I $\frac{7}{8}$ & $\cdots$ & $\cdots$ & Do. \\
\hline $6 \frac{1}{2}$ & $4 \frac{5}{8}$ & $3 \frac{1}{2}$ & $\cdots$ & $\cdots$ & Do. \\
\hline $6 \frac{1}{2}$ & $4 \frac{1}{2}$ & $2 \frac{5}{8}$ & $\ldots$ & $\cdots$ & Do. \\
\hline $5 \frac{7}{8}$ & $3 \frac{3}{ \pm}$ & $3 \frac{5}{8}$ & \multicolumn{2}{|c|}{$\begin{array}{l}\text { Moutmein, Tenas- } \\
\text { serion. }\end{array}$} & Lieut. R. C. Beavan, British Museum. \\
\hline $5^{\frac{3}{4}}$ & $4 \frac{1}{ \pm}$ & 4 & $\cdots$ & $\cdots$ & Hume Collection, British Museum. \\
\hline 5 & $4 \frac{1}{8}$ & $3 \frac{1}{4}$ & $\cdots$ & $\ldots$ & Do. \\
\hline $4 \frac{5}{8}$ & $3 \frac{1}{4}$ & $3 \frac{7}{8}$ & $\cdots$ & $\ldots$ & British Museum. \\
\hline
\end{tabular}




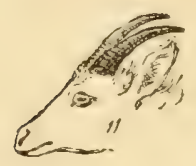

\section{HIMAIAYAN SEROW or FOREST GOAT (Nemorhœdus bubalinus).}

Habitat-Himalayas, from Kashmir to Sikim.

\begin{tabular}{|c|c|c|c|c|c|c|}
\hline \multirow[b]{2}{*}{ Length. } & \multirow[b]{2}{*}{$\begin{array}{l}\text { Circum- } \\
\text { ference. }\end{array}$} & \\
\hline & & $\begin{array}{l}\text { Tip to } \\
\text { Tip. }\end{array}$ & \multicolumn{3}{|c|}{ Habitat. } & Owner. \\
\hline $10 \frac{1}{2}$ & $5^{\frac{3}{8}}$ & $3 \frac{1}{2}$ & $\ldots$ & $\ldots$ & $\ldots$ & Hon. Charles Ellis. \\
\hline $9 \frac{7}{8}$ & $5 \frac{5}{8}$ & $4 \frac{1}{2}$ & $\ldots$ & $\ldots$ & $\ldots$ & Sir Victor Brooke's Collection. \\
\hline $9 \frac{3}{4}$ & $5 \frac{1}{8}$ & 6 & P'ir Pun & njal & $\cdot \ldots$ & R. Lydekker, British Museum. \\
\hline $9 \frac{1}{2}$ & $5 \frac{5}{8}$ & $4^{\frac{3}{4}}$ & Kashmir & & $\ldots$ & St. George Littledale, 1877 . \\
\hline $9 \frac{1}{2}$ & $4 \frac{7}{6}$ & $3 \frac{1}{4}$ & Nepal & $\cdots$ & $\ldots$ & B. H. Hodgson, British Museum. \\
\hline $9 \frac{1}{4}$ & $4 \frac{1}{2}$ & $3 \frac{3}{4}$ & $\cdots$ & $\cdots$ & $\cdots$ & Hume Collection, British Museum. \\
\hline $9 \frac{1}{8}$ & $5^{\frac{1}{4}}$ & $5^{\frac{1}{2}}$ & $\cdots$ & $\cdots$ & $\cdots$ & Do. \\
\hline $8 \frac{7}{8}$ & $5 \frac{1}{4}$ & $5^{\frac{3}{4}}$ & $\ldots$ & $\ldots$ & $\ldots$ & Do. \\
\hline 8 & $4^{\frac{3}{4}}$ & $4 \frac{3}{4}$ & Near D & Darjeeli1 & & R. Lydekker, British Museum. \\
\hline 8 & $4 \frac{1}{2}$ & $4 \frac{3}{8}$ & Nepal & $\cdots$ & $\cdots$ & B. H. Hodgson, British Museum. \\
\hline $7 \frac{7}{8}$ & $4 \frac{5}{8}$ & $4 \frac{1}{2}$ & Do. & $\cdots$ & $\cdots$ & Do. \\
\hline $7 \frac{3}{*}$ & 5 & $4 \frac{1}{4}$ & $\cdots$ & $\ldots$ & $\cdots$ & Hume Collection, British Museum. \\
\hline$* 6 \frac{1}{2}$ & $3 \frac{1}{4}$ & $2 \frac{3}{8}$ & Ichang & $\ldots$ & $\cdots$ & P. Montgomery, British Museum. \\
\hline
\end{tabular}

* Nemorhœdus cinereus. 


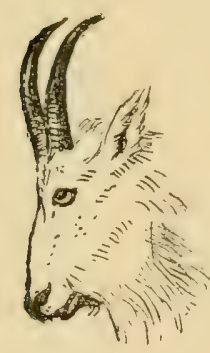

ROCKY-MOUNTAIN GOAT (Haploceros montanus).

Habitat-North America.

\begin{tabular}{|c|c|c|c|c|c|}
\hline Length. & $\begin{array}{l}\text { Circum- } \\
\text { ference. }\end{array}$ & Tip to Tip. & \multicolumn{2}{|l|}{ Habitat. } & Owner. \\
\hline $10 \frac{1}{8}$ & $6 \frac{1}{2}$ & (owner's & North America & & Arthur Pearse. \\
\hline $10 \frac{1}{8}$ & 5 & $6 \frac{1}{8}$ & Do. & $\cdots$ & E. N. Buxton. \\
\hline 10 & $5 \frac{3}{8}$ & 5 & Do, & ... & J. C. L. Knight-Bruce. \\
\hline $9 \frac{1}{2}$ & $5^{\frac{1}{2}}$ & $6 \frac{1}{x}$ & Do. & $\cdots$ & St. George Littledale. \\
\hline $9 \frac{1}{2}$ & $4 \frac{1}{4}$ & ... & Do. & $\cdots$ & J. D. Cobbold. \\
\hline $9 \frac{3}{8}$ & $5 \frac{1}{1}$ & $6 \frac{1}{2}$ & Do. & $\cdots$ & J. C. L. Knight-Bruce. \\
\hline $9 \frac{\bar{T}}{4}$ & $5^{\frac{1}{2}}$ & ... & Do. & ... & Earl of Lonsdale. \\
\hline $9 \frac{1}{8}$ & $\ldots$ & 6 & Montana & $\cdots$ & Thomas Bate, British Museum. \\
\hline $8 \frac{3}{4}$ & 5 & $5^{\frac{1}{2}}$ & North America & $\cdots$ & St. George Littledale. \\
\hline $8 \frac{1}{2}$ & $5 \frac{1}{8}$ & $5^{\frac{3}{4}}$ & Do. & $\cdots$ & J. C. I. Knight-Bruce. \\
\hline 8 & 5 & $3 \frac{3}{8}$ & Rocky Mountai & & J. K. Lord, British Museum. \\
\hline 8 & $4 \frac{7}{8}$ & $6 \frac{3}{8}$ & North America & $\ldots$ & F. J. Horniman. \\
\hline
\end{tabular}


TAKIN (Budorcas taxicolor),

Habitat-Northern India.

\begin{tabular}{|c|c|c|c|c|c|c|}
\hline Length. & $\begin{array}{l}\text { Circum- } \\
\text { ference. }\end{array}$ & Tip to Tip. & \multicolumn{3}{|c|}{ Habitat. } & Owner. \\
\hline $22 \frac{3}{8}$ & $10 \frac{5}{8}$ & $14 \frac{3}{4}$ & Assam & $\ldots$ & $\ldots$ & British Museum. \\
\hline $20 \frac{7}{8}$ & II $\frac{3}{8}$ & II $\frac{7}{8}$ & India & $\ldots$ & $\cdots$ & Hume Collection, British Museum. \\
\hline $20 \frac{3}{4}$ & II $\frac{7}{8}$ & $12 \frac{1}{2}$ & Do. & $\ldots$ & ... & A. O. Hume, Private Collection. \\
\hline $20 \frac{3}{4}$ & $9 \frac{5}{8}$ & $13 \frac{3}{5}$ & Do. & $\cdots$ & $\cdots$ & Hume Collection, British Museum. \\
\hline $20 \frac{1}{2}$ & $10 \frac{7}{8}$ & $12 \frac{3}{8}$ & Nepal & $\cdots$ & $\cdots$ & B. H. Hodgson, British Museum. \\
\hline $19 \frac{3}{8}$ & II & 15 & India & $\ldots$ & ... & Sir Edmund G. Loder, Bart. \\
\hline IS & $10 \frac{1}{4}$ & 8 & Do. & $\ldots$ & ... & British Museum. \\
\hline $16 \frac{1}{8}$ & $8_{\frac{3}{4}}$ & $10 \frac{3}{4}$ & Do. & $\cdots$ & $\ldots$ & Do. \\
\hline $15 \frac{1}{4}$ & $9 \frac{3}{8}$ & $5 \frac{3}{4}$ & Mishmi & i Mounta & hins & B. H. Hodgson, British Museum. \\
\hline $14 \frac{3}{3}$ & $\oint_{\frac{3}{4}}$ & $8 \frac{7}{8}$ & Upper & Assam & $\ldots$ & Lieut.-Col. Graham. \\
\hline
\end{tabular}




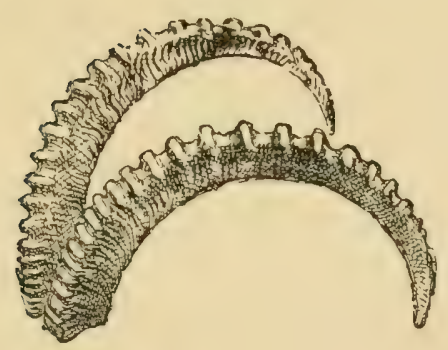

HIMALAYAN IBEX (Capra sibirica).

Habitat-Himalayas from Kashmir to Nepal, and northwards to Turkestan.

\begin{tabular}{|c|c|c|c|c|c|}
\hline I.ength. & $\begin{array}{l}\text { Circum- } \\
\text { ference. }\end{array}$ & $\begin{array}{l}\text { Tip to } \\
\text { Tip. }\end{array}$ & Habitat. & & Owner. \\
\hline $5^{\mathrm{I} \frac{1}{2}}$ & (owner's & $\ldots$ & Kashmir & $\cdots$ & Martyn Kennard. \\
\hline $5 \mathrm{I} \frac{1}{4}$ & $10 \frac{1}{3}$ & I 6 & India $\quad \ldots$ & ... & Blyth, P.Z.S. I 840 , p. 8 o. \\
\hline $5 \mathrm{I}$ & $9 \frac{1}{8}$ & $2 S \frac{1}{2}$ & Do. $\quad \ldots$ & ... & Sir Victor Brooke's Collection. \\
\hline $5^{\circ}$ & 9 & $\cdots$ & $\cdots$ & ... & Capt. John Brickley. \\
\hline $49 \frac{3}{4}$ & $10 \frac{3}{8}$ & $25 \frac{1}{4}$ & India $\quad \ldots$ & $\cdots$ & Sir Edmund G. Loder, Bart. \\
\hline $49 \frac{1}{2}$ & $10 \frac{1}{8}$ & 42 & Kashmir & $\cdots$ & Martyn Kennard, I 887. \\
\hline $48 \frac{1}{2}$ & $9 \frac{5}{8}$ & $30 \frac{1}{3}$ & India $\quad \ldots$ & $\cdots$ & A. O. Hume, Private Collection. \\
\hline $47 \frac{7}{8}$ & $9 \frac{1}{8}$ & $2 \mathrm{O}_{3}^{3}$ & Do. & $\ldots$ & Do. \\
\hline $47^{\frac{1}{2}}$ & $10 \frac{1}{4}$ & 20 & Do. & $\cdots$ & Rowland Ward's Collection. \\
\hline 47 & II $\frac{3}{4}$ & $\ldots$ & $\ldots$ & $\cdots$ & Carl Hagenback. \\
\hline 47 & $10 \frac{1}{2}$ & 293 & Kashmir & $\ldots$ & Martyn Kennard. \\
\hline 47 & 10 & $\cdots$ & India $\quad \ldots$ & $\cdots$ & Manners Smith. \\
\hline $46 \frac{3}{4}$ & $10 \frac{1}{2}$ & $24 \frac{1}{1}$ & Dangloongma & $\cdots$ & Martyn Kennard, I $\$ S \&$. \\
\hline $46 \frac{1}{2}$ & I I & $24 ?$ & India $\quad \ldots$ & ... & Hon. Charles Ellis. \\
\hline $46 \frac{1}{8}$ & $10 \frac{1}{4}$ & 20 & Kashmir & $\cdots$ & Martyn Kennard. \\
\hline 46 & $10 \frac{3}{4}$ & 25 & India $\quad \ldots$ & $\ldots$ & Col. G. Jackson, 1867. \\
\hline $4^{6}$ & $9 \frac{3}{4}$ & 25 & Do. & $\ldots$ & Sir Victor Brooke's Collection. \\
\hline
\end{tabular}




\section{HIMALAYAN IBEX (Capra sibirica)-continued.}

\begin{tabular}{|c|c|c|c|c|c|c|}
\hline Length. & $\begin{array}{l}\text { Circum- } \\
\text { ference. }\end{array}$ & Tip to Tip. & & Iabitat. & & Owner. \\
\hline $45 \frac{5}{8}$ & 9 & $9 \frac{3}{8}$ & Kashmir & & $\ldots$ & Martyn Kennard. \\
\hline $45 \frac{1}{2}$ & $10 \frac{1}{2}$ & $15 \frac{1}{4}$ & Do. . & $\cdots$ & $\cdots$ & Do. \\
\hline $45^{\frac{1}{2}}$ & $9 \frac{1}{2}$ & $\cdots$ & India . & $\cdots$ & $\cdots$ & Capt. Townley Parker. \\
\hline $45^{3}$ & $9 \frac{1}{2}$ & $22 \frac{1}{4}$ & Kashmir & & $\cdots$ & Martyn Kennard. \\
\hline 45 & II & $3^{6}$ & India .. & ... & ... & Col. G. Jackson, I 867. \\
\hline $44 \frac{5}{8}$ & $10 \frac{3}{4}$ & 30 & Do. . & $\cdots$ & ... & A. O. Hume, Private Collection. \\
\hline $44 \frac{1}{2}$ & II $\frac{3}{4}$ & 26 & Do. & ... & $\cdots$ & Capt. Orr-Ewing. \\
\hline $43^{\frac{3}{4}}$ & I $1 \frac{1}{2}$ & 16 & Do. . & .. & $\ldots$ & J. Carr-Saunders. \\
\hline $43 \frac{3}{ \pm}$ & $10 \frac{1}{4}$ & $25 \frac{1}{8}$ & Kashmir & & $\cdots$ & Martyn Kennard. \\
\hline $43 \frac{5}{8}$ & $10 \frac{1}{4}$ & $24 \frac{3}{4}$ & India . & ... & $\cdots$ & Sir Robert Harvey, Bart. \\
\hline $43 \frac{1}{2}$ & $9 \frac{3}{4}$ & 19 & Do. . & $\cdots$ & $\cdots$ & Hume Collection, British Museum. \\
\hline $43 \frac{1}{2}$ & $\begin{array}{c}\text { (owner's } \\
\text { measurement) }\end{array}$ & ... & & ... & ... & Capt. A. A. Kinloch (his book). \\
\hline $43^{\frac{1}{4}}$ & IO $\frac{1}{8}$ & $27 \frac{1}{8}$ & Kashmir & & $\cdots$ & Martyn Kennard. \\
\hline 43 & $12 \frac{1}{2}$ & $35^{\frac{1}{2}}$ & India . & $\cdots$ & $\cdots$ & T. W. H. Greenfield. \\
\hline 43 & $\cdots$ & $\cdots$ & Do. . & $\cdots$ & $\cdots$ & Lewis Flower. \\
\hline $42 \frac{7}{8}$ & II $\frac{1}{8}$ & $25 \frac{3}{4}$ & Do. . & $\cdots$ & $\cdots$ & A. O. Hume, Private Collection. \\
\hline $42 \frac{7}{8}$ & $10_{2}^{\frac{1}{2}}$ & $\cdots$ & Do. . & $\cdots$ & $\cdots$ & H. C. V. Hunter. \\
\hline $42 \frac{7}{8}$ & $10 \frac{3}{8}$ & $20 \frac{3}{4}$ & Do. & ... & $\cdots$ & Sir Robert Harvey, Bart. \\
\hline $42^{3}$ & $9 \frac{1}{8}$ & $25 \frac{3}{8}$ & Do. . & $\cdots$ & $\cdots$ & Do. \\
\hline $42 \frac{1}{2}$ & $9 \frac{1}{2}$ & 20 & Do. . & $\cdots$ & $\cdots$ & J. D. Cobbold. \\
\hline $42 \frac{1}{8}$ & $9 \frac{5}{8}$ & $20 \frac{7}{8}$ & Kashmir & & $\ldots$ & Martyn Kennard. \\
\hline 42 & $6 \frac{1}{2}$ & 27 & Indin . & & $\ldots$ & Capt. Townley Parker. \\
\hline $41 \frac{7}{8}$ & $10 \frac{1}{8}$ & $20 \frac{1}{2}$ & Kashmir & & $\cdots$ & Martyn Kennard. \\
\hline $41 \frac{5}{8}$ & $10 \frac{3}{4}$ & $22 \frac{2}{4}$ & Do. . & ... & $\ldots$ & Do. \\
\hline $41 \frac{5}{8}$ & 10 & $2 \mathrm{I} \frac{1}{8}$ & Do. . & $\cdots$ & $\cdots$ & Do. \\
\hline $41 \frac{1}{2}$ & $10 \frac{1}{2}$ & $23 \frac{1}{2}$ & India . & ... & $\cdots$ & C. Spedding. \\
\hline $41^{\frac{1}{2}}$ & $9 \frac{5}{8}$ & $17 \frac{3}{x}$ & Kashmir & & $\cdots$ & Martyn Kennard. \\
\hline $4 \mathrm{I}_{\frac{3}{8}}^{\frac{3}{2}}$ & $10 \frac{1}{4}$ & 203 & Do. . & ... & $\cdots$ & Do. \\
\hline
\end{tabular}


HIMALAYAN IBEX (Capra sibirica)-continued.

\begin{tabular}{|c|c|c|c|c|c|}
\hline Length. & $\begin{array}{l}\text { Circum- } \\
\text { ference. }\end{array}$ & Tip to Tip. & \multicolumn{2}{|c|}{ Habitat. } & Owner. \\
\hline $41 \frac{1}{4}$ & $9 \frac{1}{2}$ & $33 \frac{1}{8}$ & Kashmir & ... & Martyn Kennard. \\
\hline $4 I \frac{1}{8}$ & $10 \frac{1}{2}$ & $12 \frac{5}{8}$ & Do. ... & $\ldots$ & Do. \\
\hline $4 I$ & $\begin{array}{c}\text { (owner's } \\
\text { measurement) }\end{array}$ & ... & India $\ldots$ & $\ldots$ & Capt. A. A. Kinloch. \\
\hline $4^{I}$ & Io & $26 \frac{3}{4}$ & Kashmir & $\ldots$ & Martyn Kennard. \\
\hline $40 \frac{5}{8}$ & $10^{3}$ & $33^{\frac{3}{4}}$ & India $\ldots$ & $\cdots$ & Sir Edmund G. Loder, Bart. \\
\hline $40 \frac{1}{2}$ & II $\frac{1}{4}$ & $21 \frac{1}{2}$ & Do. $\quad \ldots$ & $\ldots$ & Hon. Charles Ellis. \\
\hline $40 \frac{1}{2}$ & ro & $22 \frac{1}{8}$ & Kashmir & $\ldots$ & Martyn Kennard. \\
\hline $40 \frac{1}{2}$ & $9 \frac{7}{8}$ & $22 \frac{3}{4}$ & India $\ldots$ & $\ldots$ & Sir Robert Harvey, Bart. \\
\hline $40 \frac{1}{8}$ & $8 \frac{1}{2}$ & $25 \frac{3}{8}$ & Kashmir & ... & Martyn Kennard. \\
\hline 40 & $10 \frac{1}{4}$ & I $7 \frac{3}{4}$ & Do. ... & $\ldots$ & Do. \\
\hline 40 & I0 & 27 & India $\ldots$ & $\ldots$ & W. F. Templer. \\
\hline 40 & $9 \frac{3}{4}$ & $18 \frac{3}{4}$ & Do. ... & $\cdots$ & Sir Robert Harvey, Bart. \\
\hline 40 & $8 \frac{3}{4}$ & 23 & Do. $\quad \ldots$ & $\cdots$ & Hume Collection, British Museum. \\
\hline $39 \frac{7}{8}$ & $10 \frac{5}{8}$ & $24 \frac{5}{8}$ & Jay Nai & & British Museum. \\
\hline $39 \frac{7}{8}$ & $10 \frac{1}{2}$ & I $8 \frac{5}{8}$ & Kashmir & $\ldots$ & Martyn Kennard. \\
\hline $39 \frac{5}{8}$ & $9 \frac{7}{8}$ & $13 \frac{1}{ \pm}$ & $\cdots$ & ... & Reginald Beech. \\
\hline $39 \frac{5}{8}$ & 9 & $2 I \frac{1}{8}$ & Mongolia & $\ldots$ & St. George Littledale. \\
\hline $39 \frac{1}{2}$ & $9 \frac{7}{8}$ & I $7 \frac{x}{2}$ & Himalayas & $\ldots$ & Sir Victor Brooke's Collection. \\
\hline $39 \frac{1}{2}$ & $9 \frac{1}{2}$ & $22 \frac{1}{2}$ & Ladak ... & ... & Capt. Strachey, British Museum. \\
\hline $39 \frac{1}{2}$ & $8 \frac{3}{8}$ & 26 & Kashmir & ... & Martyn Kennard. \\
\hline $39 \frac{1}{4}$ & $9 \frac{7}{8}$ & $15 \frac{1}{2}$ & Do. ... & $\cdots$ & Do. \\
\hline $39 \frac{x}{4}$ & $9 \frac{3}{8}$ & $24 \frac{1}{2}$ & Astor $\quad \ldots$ & ... & Sir Victor Brooke's Collection, I875. \\
\hline 39 & $9 \frac{1}{2}$ & $14 \frac{1}{4}$ & India $\ldots$ & $\cdots$ & Hume Collection, British Museum. \\
\hline 39 & 9 & $25 \frac{7}{8}$ & Kashmir & ... & Martyn Kennard. \\
\hline $38 \frac{7}{8}$ & $8 \frac{7}{8}$ & ... & India $\quad \ldots$ & $\ldots$ & F. Morrice. \\
\hline $3^{8 \frac{3}{4}}$ & $9 \frac{7}{8}$ & $30 \frac{1}{4}$ & Do. & $\ldots$ & Sir Robert Harvey, Bart. \\
\hline $38 \frac{5}{8}$ & $10 \frac{1}{8}$ & $30 \frac{1}{8}$ & Himalayas & $\ldots$ & Capt. Townley Parker. \\
\hline $38 \frac{1}{2}$ & ro & $25 \frac{1}{2}$ & India $\ldots$ & $\ldots$ & Capt. M. Murphy. \\
\hline
\end{tabular}


HIMALAYAN IBEX (Capra sibirica)-continued.

\begin{tabular}{|c|c|c|c|c|c|c|}
\hline Length. & $\begin{array}{l}\text { Circum- } \\
\text { ference. }\end{array}$ & $\begin{array}{l}\text { Tip to } \\
\text { ' 'ip. }\end{array}$ & \multicolumn{3}{|c|}{ Habitat. } & Owner. \\
\hline $38 \frac{1}{2}$ & $9 \frac{3}{8}$ & $15 \frac{7}{8}$ & Yarkund & & $\ldots$ & Dr. Bellew, British Museum. \\
\hline $38 \frac{1}{2}$ & 9 & $24 \frac{1}{2}$ & India & $\ldots$ & $\ldots$ & Rowland Ward's Collection. \\
\hline $38 \frac{3}{8}$ & $9 \frac{1}{2}$ & I $S_{\frac{3}{4}}$ & Kashmir & & $\ldots$ & R. Lydekker, British Museum. \\
\hline $38 \frac{1}{4}$ & $10 \frac{3}{8}$ & $22 \frac{1}{2}$ & India & $\ldots$ & $\cdots$ & Hon. Walter Rothschild. \\
\hline $37 \frac{7}{8}$ & $10 \frac{7}{8}$ & $22 \frac{3}{4}$ & Do. & $\ldots$ & $\cdots$ & British Museum. \\
\hline $37 \frac{7}{8}$ & $10_{4}^{\frac{1}{4}}$ & $15 \frac{7}{8}$ & Kashmir & & $\ldots$ & Martyn Kennard. \\
\hline $37 \frac{3}{4}$ & $10 \frac{3}{6}$ & $24 \frac{1}{4}$ & India & $\ldots$ & $\ldots$ & H.R.H. Duke of Edinburgh. \\
\hline $37 \frac{5}{8}$ & II $\frac{1}{8}$ & $27 \frac{3}{4}$ & Do. & $\cdots$ & $\ldots$ & Bethnal Green Museum. \\
\hline $37 \frac{5}{8}$ & IO & $12 \frac{1}{8}$ & Do. & $\cdots$ & $\cdots$ & Reginald Beech. \\
\hline $37 \frac{5}{8}$ & $9 \frac{5}{8}$ & $18 \frac{1}{2}$ & Kashmir & & $\ldots$ & Martyn Kennard. \\
\hline $37 \frac{1}{2}$ & IO & $177 \frac{7}{8}$ & India & $\cdots$ & $\cdots$ & Sir Robert Harvey, Bart. \\
\hline $37 \frac{3}{8}$ & $9 \frac{1}{4}$ & 23 & Do. . & $\cdots$ & $\cdots$ & Hume Collection, British Museun. \\
\hline $37 \frac{1}{t}$ & $10 \frac{1}{8}$ & $1 S_{\frac{3}{8}}$ & Kashmir & & $\cdots$ & Martyn Kennard. \\
\hline $37 \frac{1}{8}$ & $\delta_{\frac{3}{4}}$ & $\mathrm{I}^{\frac{1}{2}}$ & Do. . & $\cdots$ & $\cdots$ & St. George Littledale. \\
\hline $36 \frac{7}{8}$ & $9 \frac{1}{2}$ & $3 \mathrm{O}_{4}^{3}$ & India . & $\cdots$ & $\ldots$ & British Museum. \\
\hline $36 \frac{3}{4}$ & $1 I_{4}^{\frac{1}{4}}$ & 24 & Kashair & & $\cdots$ & Sir Victor Brooke's Collection. \\
\hline $36 \frac{3}{4}$ & $9 \frac{1}{4}$ & 26 & India . & $\cdots$ & $\cdots$ & Hume Collection, British Museum. \\
\hline $36 \frac{5}{8}$ & $9 \frac{1}{8}$ & $22 \frac{1}{2}$ & Kashmir & & $\cdots$ & St. George Littledale, $\mathbf{I} S 77$. \\
\hline $36 \frac{5}{8}$ & $9 \frac{1}{8}$ & $13 \frac{3}{8}$ & India & $\ldots$ & $\ldots$ & II. C. V. Hunter. \\
\hline $3^{6 \frac{1}{2}}$ & $9 \frac{1}{2}$ & $27 \frac{1}{2}$ & Do. & ... & $\ldots$ & J. Carr-Saunders. \\
\hline $3^{6 \frac{1}{2}}$ & $9 \frac{1}{8}$ & $20 \frac{7}{8}$ & Do. . & $\cdots$ & $\cdots$ & Hume Collection, British Museum. \\
\hline $36 \frac{3}{5}$ & $9 \frac{3}{8}$ & $17 \frac{3}{4}$ & Kashmir & & ... & Martyn Kennard. \\
\hline $36 \frac{3}{8}$ & $9 \frac{1}{8}$ & 23 & Do. . & $\cdots$ & $\cdots$ & St. George Littledale. \\
\hline $36 \frac{3}{8}$ & $9 \frac{3}{8}$ & $13 \frac{1}{ \pm}$ & India . & $\cdots$ & $\cdots$ & H. de Burgh. \\
\hline $36 \frac{3}{8}$ & $8 \frac{7}{8}$ & $2 I \frac{1}{2}$ & Do. & $\cdots$ & $\cdots$ & F. J. Jackson. \\
\hline $36 \frac{1}{8}$ & $\$ \frac{7}{8}$ & $13 \frac{1}{2}$ & Do. . & $\cdots$ & $\cdots$ & H. de Burgh. \\
\hline $36 \frac{1}{8}$ & $9 \frac{1}{8}$ & $244^{3}$ & Kashmir & & $\cdots$ & Martyn Kennard. \\
\hline $3^{6}$ & $10 \frac{3}{5}$ & I $S \frac{1}{2}$ & India . & $\cdots$ & $\cdots$ & Hume Collection, British Museum. \\
\hline
\end{tabular}


HIMALAYAN IBEX (Capra sibirica)-continued.

\begin{tabular}{|c|c|c|c|c|c|c|}
\hline Length. & $\begin{array}{l}\text { Circum- } \\
\text { fe:ence. }\end{array}$ & $\begin{array}{l}\text { Tip to } \\
\text { Tip. }\end{array}$ & \multicolumn{3}{|c|}{ Habitat. } & Owner. \\
\hline $35^{\frac{3}{4}}$ & $8_{8}^{5}$ & I $7 \frac{1}{8}$ & Kashmir & & ... & R. Lydekker, British Museum. \\
\hline $35^{\frac{1}{2}}$ & $10 \frac{1}{2}$ & 23 & India & $\ldots$ & ... & Capt. M. Murphy. \\
\hline $35 \frac{3}{8}$ & $9 \frac{7}{8}$ & 20 & Do. & $\ldots$ & ... & British Museum. \\
\hline $35^{\frac{1}{4}}$ & $9 \frac{1}{2}$ & $8 \frac{1}{2}$ & Kashmir & & $\ldots$ & Martyn Kennard. \\
\hline $35 \frac{1}{8}$ & 9 & II & $\begin{array}{l}\text { Saar Mo } \\
\text { Altai. }\end{array}$ & ountains & & St. George Littledale, British Museun. \\
\hline 35 & $\cdots$ & $\cdots$ & India & & $\cdots$ & Major Lloyd Dickson. \\
\hline 35 & $I I \frac{1}{8}$ & $17 \frac{7}{8}$ & Himalay & hyas & ... & British Museum. \\
\hline $34 \frac{1}{2}$ & $10 \frac{1}{2}$ & $\cdots$ & India & $\cdots$ & $\cdots$ & Rowland Ward's Collection. \\
\hline $34 \frac{1}{2}$ & $9 \frac{3}{8}$ & II $\frac{3}{4}$ & Kashmir & & $\cdots$ & St. George Littledale, $x 877$. \\
\hline $34 \frac{1}{2}$ & $8 \frac{5}{8}$ & $10 \frac{3}{t}$ & India & $\cdots$ & $\ldots$ & British Museum. \\
\hline $33 \frac{5}{8}$ & $8 \frac{5}{8}$ & $13 \frac{3}{4}$ & Kasharis & & $\cdots$ & St. George Littledale. \\
\hline $33 \frac{3}{8}$ & $9 \frac{3}{4}$ & $28 \frac{1}{2}$ & Do. & $\cdots$ & ... & St. Ceorge Littledale, $\mathbf{I} \oint_{77}$. \\
\hline $33 \frac{3}{8}$ & $9 \frac{5}{8}$ & $14 \frac{1}{ \pm}$ & India & $\cdots$ & $\ldots$ & Hume Collection, British Museum. \\
\hline 33 & 9 & $20 \frac{1}{4}$ & Kashmi & & $\ldots$ & St. George Littledale. \\
\hline 33 & $\cdots$ & .. & India & $\cdots$ & $\cdots$ & Major Lloyd Dickson. \\
\hline $32 \frac{7}{8}$ & $10 \frac{1}{8}$ & $13 \frac{1}{t}$ & Do. & $\cdots$ & $\cdots$ & Hume Collection, British Museum. \\
\hline $32 \frac{1}{2}$ & $9 \frac{1}{2}$ & $26 \frac{1}{2}$ & Do. & $\cdots$ & $\ldots$ & Capt. G. Campbell. \\
\hline $32 \frac{1}{2}$ & $9 \frac{3}{8}$ & $10 \frac{1}{8}$ & $\begin{array}{c}\text { Thian } \\
\text { Cent }\end{array}$ & $\begin{array}{l}\text { Shan, } \\
\text { tral Asi: }\end{array}$ & & British Museum. \\
\hline $30 \frac{7}{8}$ & $8 \frac{1}{4}$ & $30 \frac{1}{2}$ & India & $\cdots$ & $\cdots$ & Hume Collection, British Museum. \\
\hline $30 \frac{7}{8}$ & $7 \frac{5}{8}$ & $13 \frac{5}{8}$ & Siberia & $\cdots$ & $\cdots$ & British Museum. \\
\hline $30 \frac{5}{8}$ & $9 \frac{3}{8}$ & $17 \frac{5}{8}$ & India & $\cdots$ & $\ldots$ & F. J. Jackson. \\
\hline 29 & $8 \frac{7}{8}$ & $\begin{array}{l}\text { (mal- } \\
\text { formed) }\end{array}$ & Kashmir & & ... & Martyn Kennard. \\
\hline $28 \frac{7}{8}$ & $9 \frac{7}{8}$ & $14 \frac{7}{8}$ & India & ... & $\ldots$ & F. J. Jackson. \\
\hline $28 \frac{1}{2}$ & $8 \frac{1}{8}$ & Io & Siberia & $\cdots$ & $\cdots$ & British Museum. \\
\hline $25 \frac{5}{8}$ & $7 \frac{3}{8}$ & $15 \frac{1}{2}$ & India & $\cdots$ & $\cdots$ & Do. \\
\hline $23 \frac{1}{4}$ & $8 \frac{1}{2}$ & $10 \frac{1}{2}$ & Do. & $\cdots$ & .. & Giwynne Griffiths. \\
\hline I $8 \frac{3}{8}$ & 8 & $9 \frac{7}{8}$ & Do. & $\cdots$ & $\cdots$ & Hume Collection, British Museum. \\
\hline $12 \frac{1}{2}$ & $4 \frac{7}{8}$ & $8 \frac{1}{2}$ & Do. & $\ldots$ & $\ldots$ & British Museum. \\
\hline
\end{tabular}


CAUCASIAN IBEX (Capra caucasica).

Habitat-Caucasus.

\begin{tabular}{|c|c|c|c|c|c|c|}
\hline Length. & $\begin{array}{l}\text { Circum- } \\
\text { ference. }\end{array}$ & Tip to Tip. & Habitat. & & \multicolumn{2}{|l|}{ Owner. } \\
\hline $40 \frac{1}{8}$ & $12 \frac{5}{8}$ & $15 \frac{1}{4}$ & Caucasus & $\ldots$ & St. George Littledale. & \\
\hline $36 \frac{7}{8}$ & II $1 \frac{5}{8}$ & $27 \frac{5}{8}$ & Do. & $\ldots$ & Do. & \\
\hline $34 \frac{3}{4}$ & $11 \frac{5}{8}$ & $22 \frac{7}{8}$ & Do. & $\ldots$ & Do. & 1891. \\
\hline $33^{\frac{1}{4}}$ & $10 \frac{5}{8}$ & $26 \frac{3}{4}$ & Do. & $\cdots$ & Do. & Do. \\
\hline 33 & $12 \frac{1}{4}$ & $22 \frac{3}{4}$ & Do. & $\cdots$ & Do. & 1887 \\
\hline $32 \frac{7}{8}$ & I I & $26 \frac{7}{8}$ & Do. & $\ldots$ & Do. & Do. \\
\hline $31 \frac{7}{8}$ & $10 \frac{5}{8}$ & 20 & Do. & $\ldots$ & Do. & Do. \\
\hline $3^{I \frac{1}{4}}$ & $x I \frac{1}{2}$ & $21 \frac{1}{2}$ & Do. & $\cdots$ & Do. & Do. \\
\hline $30 \frac{5}{8}$ & III & $16 \frac{3}{8}$ & Do. & $\cdots$ & Sir Edmund G. Loder, & , Bart. \\
\hline $30 \frac{1}{4}$ & II $\frac{3}{4}$ & $20 \frac{5}{8}$ & Do. & $\cdots$ & St. George Littledale, I & I 891. \\
\hline $29 \frac{1}{2}$ & 12 & $22 \frac{1}{8}$ & Do. & $\cdots$ & Do. & 1887. \\
\hline $29 \frac{1}{4}$ & $11 \frac{1}{8}$ & $20 \frac{1}{8}$ & Do. & $\ldots$ & Do. & Do. \\
\hline$*_{22 \frac{3}{4}}$ & $10 \frac{7}{8}$ & $22 \frac{1}{4}$ & Do. & $\cdots$ & Do. & I 886. \\
\hline $19 \frac{7}{8}$ & $10 \frac{5}{8}$ & I $6 \frac{1}{4}$ & Do. & $\ldots$ & P. A. Holt, British Mu & useum. \\
\hline 15 & $8 \frac{3}{4}$ & $12 \frac{5}{8}$ & Do. & $\cdots$ & Do. & \\
\hline 9 & $4 \frac{1}{8}$ & $4 \frac{1}{1}$ & Do. & $\cdots$ & British Museum. & \\
\hline
\end{tabular}

* Cross between Capra caucasica and Capra pallasi.

\section{ALPINE IBEX (Capra ibex).}

Habitat-Switzerland Alps, Savoy and the Tyrol.

\begin{tabular}{|c|c|c|c|c|}
\hline Length. & $\begin{array}{l}\text { Circurn- } \\
\text { ference. }\end{array}$ & Tip to Tip. & Habitat. & Owner. \\
\hline $3^{1} \frac{3}{4}$ & $9 \frac{1}{8}$ & I $S_{\frac{3}{5}}$ & The Alps, Savoy... & Sir Edmund G. Loder, Bart. \\
\hline $26 \frac{3}{4}$ & $\delta \frac{3}{8}$ & $22 \frac{1}{8}$ & The Alps & British Museum. \\
\hline $21 \frac{1}{2}$ & $8 \frac{5}{8}$ & $14 \frac{7}{8}$ & Do. & Do. \\
\hline
\end{tabular}


PYRENEAN IBEX (Capra pyrenaica).

Habitat-Pyrenees, High ranges of Central Spain, Andalusia and Portugal.

\begin{tabular}{|c|c|c|c|c|c|}
\hline Length. & $\begin{array}{l}\text { Circum- } \\
\text { ference. }\end{array}$ & Tip to Tip. & Habitat. & & Owner. \\
\hline 28 & $9 \frac{1}{8}$ & $23 \frac{3}{8}$ & Spain ... & ... & British Museum. \\
\hline$*_{27 \frac{3}{4}}$ & 9 & 25 & Do. $\quad \ldots$ & $\ldots$ & Sir Edmund G. Loder, Bart. \\
\hline $27 \frac{1}{2}$ & $10_{\frac{3}{8}}^{3}$ & $19 \frac{1}{2}$ & Pyrenees & $\ldots$ & British Museum. \\
\hline$*_{25} \frac{5}{8}$ & $8 \frac{1}{2}$ & $16 \frac{5}{8}$ & Spain .... & $\ldots$ & Hon. Walter Rothschild. \\
\hline $24 \frac{1}{2}$ & 10 & 14 & Val d'Arras & $\ldots$ & E. N. Buxton, I 885 . \\
\hline $24 \frac{3}{8}$ & $9 \frac{1}{8}$ & $18 \frac{7}{8}$ & Do. & $\ldots$ & Do. Do. \\
\hline$*_{22}$ & $7^{\frac{3}{4}}$ & 14 & Spain ... & $\ldots$ & Pablo Larios. \\
\hline$*_{12 \frac{1}{2}}$ & $6 \frac{3}{8}$ & $18 \frac{1}{2}$ & Do. $\quad \ldots$ & ... & Do. \\
\hline$\nmid 10 \frac{1}{8}$ & 7 & 6 & Do. $\quad \ldots$ & $\ldots$ & Professor Owen, British Museum. \\
\hline 워난 & $5 \frac{3}{8}$ & $7 \frac{5}{8}$ & Val d'Arras & $\ldots$ & E. N. Buxton, $188_{3}$. \\
\hline 우 $9 \frac{1}{2}$ & $5 \frac{3}{3}$ & $6 \frac{1}{4}$ & Do. & $\ldots$ & Do. Do. \\
\hline
\end{tabular}

\section{ARABIAN IBEX (Capra sinaitica).}

[Rev. H. B. Tristram on the Mammals of Palestine, P.Z.S., I866, p. 85.]

The Syrian Ibex, or Bedeb of the Arabs. Only found in Palestine in the neighbourhood of the Dead Sea, especially about Engedi. Very abundant on the east side in the mountains of Moab, and still more so throughout the Sinaitic peninsula. It formerly extended to the Lebanon, where its teeth have been found in cave-breccia by ourselves and by M. Lartet. (See Bull, Sve. Geol. de France, 22, p. 543.) Quite as wild, nimble and wary as the Ibex of the Alps. It drops its kid in March or April, and has but one at a birth. Several young ones were brought to us and reared for some time by hand. The horns of the female are much smaller than those of the male. The flesh is excellent, far superior to that of the Gazelle. The horns are subquadrangular, sharply emarginated at the inner anterior angle, and with from twenty to twenty-four (in fully adult specimens) bold ridges on the front face only.

Habitat-Upper Egypt, Sinaitic Peninsula and Palestine.

\begin{tabular}{c|c|c|c|c}
\hline Length. & $\begin{array}{c}\text { Circum- } \\
\text { ference. }\end{array}$ & Tip to Tip. & Habitat. & Owner. \\
\hline $38 \frac{7}{8}$ & $7 \frac{1}{2}$ & I2 $\frac{1}{4}$ & $\begin{array}{c}\text { Upper Egypt, Sinaitic } \\
\text { Peninsula \& Palestine. }\end{array}$ & Sir Edmund G. Loder, Bart. \\
\hline
\end{tabular}


WILD GOAT (Capra ægagrus).

Habitat-South-Eastern Europe.

\begin{tabular}{|c|c|c|c|c|c|}
\hline Length. & $\begin{array}{l}\text { Circum- } \\
\text { ference. }\end{array}$ & Tip to Tip. & \multicolumn{2}{|l|}{ Habitat. } & Owne:- \\
\hline 53 & $\cdots$ & $\begin{array}{c}\text { (owner's mea- } \\
\text { surement) }\end{array}$ & $\begin{array}{l}\text { Sumbak Hil } \\
\text { Sind. }\end{array}$ & & Col. F. Marston. \\
\hline $52 \frac{3}{8}$ & $7 \frac{7}{8}$ & $8 \frac{3}{4}$ & $\cdots \quad \cdots$ & & A. O. Hume, Private Collection. \\
\hline $48 \frac{1}{1}$ & $8 \frac{3}{8}$ & I $3 \frac{7}{8}$ & Caucasus & $\ldots$ & British Museum. \\
\hline $45^{\frac{1}{1}}$ & $9 \frac{1}{2}$ & $14 \frac{3}{8}$ & South Asia Mir & inor & Capt. Townley Parker. \\
\hline $44 \frac{1}{2}$ & $8 \frac{7}{8}$ & $2 \mathrm{I} \frac{3}{8}$ & Caucasus & $\ldots$ & British Museum. \\
\hline $44 \frac{1}{2}$ & $8 \frac{5}{8}$ & II & $\ldots \quad \ldots$ & $\cdots$ & Hume Collection, British Museum. \\
\hline $43 \frac{1}{2}$ & $8 \frac{1}{8}$ & $22 \frac{3}{8}$ & $\cdots \quad \cdots$ & $\ldots \mid$ & J. Carr-Saunders. \\
\hline $43 \frac{3}{8}$ & $8 \frac{3}{8}$ & $14 \frac{5}{8}$ & $\ldots \quad \ldots$ & $\ldots \mid$ & A. O. Hume, Private Collection. \\
\hline 43 & 9 & I $5 \frac{3}{4}$ & $\begin{array}{l}\text { Taurus Mou } \\
\text { tains. }\end{array}$ & un- & Sir Edxund G. Loder, Bart. \\
\hline $4 r^{\frac{3}{8}}$ & $8 \frac{1}{8}$ & $7 \frac{1}{8}$ & $\ldots \quad \cdots$ & $\ldots$ & Hume Collection, British Museum. \\
\hline $40 \frac{1}{8}$ & $8 \frac{1}{2}$ & $7 \frac{7}{8}$ & India $\ldots$ & $\ldots$ & British Museum. \\
\hline $39 \frac{7}{8}$ & $9 \frac{5}{8}$ & $I_{\frac{1}{4}}$ & Erzeroum & $\ldots$ & Do. \\
\hline $39 \frac{7}{8}$ & $7 \frac{7}{8}$ & 27 & $\ldots$ & $\cdots$ & A. O. Hume, Private Collection. \\
\hline $38 \frac{1}{2}$ & $7 \frac{3}{4}$ & 19 & $\cdots$ & $\cdots$ & Iume Collection, British Museum. \\
\hline $38 \frac{1}{4}$ & $7 \frac{7}{8}$ & I0 & $\cdots \quad \cdots$ & $\ldots$ & Do. \\
\hline $35 \frac{5}{8}$ & $9 \frac{1}{8}$ & $13 \frac{3}{8}$ & Mount Ararat & & British Museum. \\
\hline $34 \frac{1}{2}$ & $9 \frac{1}{2}$ & IS & Beluchistan & ... & $\begin{array}{l}\text { Sir Victor Brooke's Collection. (Shot } \\
\text { by Maj. Hogg). }\end{array}$ \\
\hline $33 \frac{7}{8}$ & $6 \frac{7}{8}$ & $14 \frac{5}{8}$ & $\cdots$ & $\cdots$ & IIume Collection, British Museum. \\
\hline $33 \frac{3}{t}$ & $7 \frac{5}{8}$ & $10 \frac{1}{2}$ & $\cdots$ & $\cdots$ & Do. \\
\hline $33 \frac{5}{8}$ & $9^{\frac{3}{4}}$ & $14 \frac{1}{2}$ & $\cdots$ & $\cdots$ & British Museum. \\
\hline $30 \frac{3}{4}$ & $6 \frac{7}{8}$ & 14 & $\cdots \quad \cdots$ & $\cdots$ & Hume Collection, British Museum. \\
\hline $29 \frac{5}{8}$ & $9 \frac{1}{1}$ & $8 \frac{3}{8}$ & $\begin{array}{l}\text { Cilecian Taurt } \\
\text { Asia Minor. }\end{array}$ & rus, & C. G. Danford, British Museum. \\
\hline $26 \frac{1}{3}$ & $7 \frac{1}{2}$ & $12 \frac{1}{2}$ & Beluchistan & & $\begin{array}{l}\text { Sir Victor Brooke's Collection. (Shot } \\
\text { by Maj. Hogg). }\end{array}$ \\
\hline $25 \frac{3}{1}$ & $6 \frac{1}{2}$ & $99^{3}$ & $\cdots$ & $\cdots$ & Hume Collection, British Museum. \\
\hline $25 \frac{5}{8}$ & $\cdots$ & $5 \frac{1}{2}$ & $\begin{array}{l}\text { Solomon Mtus } \\
\text { Asia Minor. }\end{array}$ & & British Museum. \\
\hline $1 S_{\frac{1}{2}}$ & $7 \frac{3}{4}$ & 9 & $\cdots \quad \cdots$ & & E. N. Buxton. \\
\hline I $8 \frac{3}{8}$ & $7 \frac{1}{8}$ & $9 \frac{1}{2}$ & $\cdots$ & $\cdots$ & ISgo. \\
\hline
\end{tabular}


NORTH AFRICAN IBEX.

WILD GOAT (Capra ægagrus)-continued.

\begin{tabular}{|c|c|c|c|c|}
\hline Length. & $\begin{array}{l}\text { Circum- } \\
\text { ference. }\end{array}$ & Tip to Tip. & Habitat. & Owner. \\
\hline 16 & $7 \frac{1}{2}$ & 7 & Perșia $\quad \ldots$ & R. J. Kennedy. \\
\hline 16 & 7 & $6 \frac{3}{4}$ & $\begin{array}{l}\text { Cilecian Taurus, } \\
\text { Asia Minor. }\end{array}$ & C. G. Danford, British Museum. \\
\hline $12 \frac{5}{8}$ & $5 \frac{5}{8}$ & $7 \frac{1}{4}$ & Europe $\quad \ldots$ & British Museum. \\
\hline II $\frac{1}{8}$ & $4 \frac{3}{8}$ & $3 \frac{5}{8}$ & Cilecian Taurus, & C. G. Danford, British Museum. \\
\hline 오 $10 \frac{7}{8}$ & $4 \frac{3}{8}$ & $3 \frac{5}{8}$ & Caucasus $\quad \ldots$ & Sir Victor Brooke's Collection. \\
\hline $8_{\frac{5}{8}}$ & $4 \frac{5}{8}$ & $4^{\frac{3}{4}}$ & Maimun Dagh & E. N. Buxton, I 890. \\
\hline $8 \frac{1}{8}$ & $3 \frac{5}{8}$ & $4^{\frac{3}{4}}$ & Do. & Do. \\
\hline $7 \frac{7}{8}$ & $3 \frac{5}{8}$ & $3 \frac{7}{8}$ & Do. & Do. \\
\hline
\end{tabular}

NORTH AFRICAN IBEX (Capra nubiana).

\begin{tabular}{|c|c|c|c|c|c|}
\hline Length. & $\begin{array}{l}\text { Circum- } \\
\text { ference. }\end{array}$ & Tip to Tip. & Habitat. & & Owner. \\
\hline $46 \frac{1}{8}$ & 8 & $\cdots$ & North Africa & ... & British Museum. \\
\hline $42 \frac{1}{4}$ & $9^{\frac{1}{4}}$ & $15 \frac{1}{4}$ & $\ldots$ & ... & Capt. Bartelot. \\
\hline $4 \mathrm{I}_{\frac{3}{4}}$ & $7 \frac{5}{8}$ & $17 \frac{5}{8}$ & North Africa & $\ldots$ & Capt. W. H. Besant. \\
\hline $35^{\frac{3}{4}}$ & 6 & $10 \frac{7}{8}$ & Do. & $\ldots$ & British Museum. \\
\hline $35 \frac{1}{8}$ & $7 \frac{1}{8}$ & 12 & $\ldots$ & $\cdots$ & W. P. Gore Graham. \\
\hline $33 \frac{1}{8}$ & 7 & 20 & $\ldots$ & $\cdots$ & Do. \\
\hline $14 \frac{3}{8}$ & $6 \frac{1}{8}$ & $6 \frac{3}{4}$ & North Africa & $\ldots$ & British Museum. \\
\hline
\end{tabular}

DOMESTIC GOAT (Capra hircus).

\begin{tabular}{|c|c|c|c|c|c|}
\hline Length. & $\begin{array}{l}\text { Circum- } \\
\text { ference. }\end{array}$ & Tip to Tip. & Habitat. & & Owner. \\
\hline $52 \frac{1}{2}$ & IO $\frac{1}{2}$ & $40 \frac{5}{8}$ & Great Britain & $\ldots$ & Sir Edmund G. Loder, Bart. \\
\hline $26 \frac{1}{8}$ & $8 \frac{3}{x}$ & 7 & Asia Minor & $\ldots$ & C. G. Danford, British Museum. \\
\hline 14 & $5^{\frac{1}{2}}$ & 8 & $\ldots$ & $\ldots$ & British Museum. \\
\hline 오 10 & $3 \frac{7}{8}$ & $4 \frac{5}{8}$ & Asia Minor & $\ldots$ & C. G. Danford, British Museum. \\
\hline $7 \frac{7}{8}$ & $4 \frac{5}{4}$ & $8 \frac{3}{3}$ & $\ldots$ & ... & British Museum. \\
\hline
\end{tabular}




\section{NILGHIRI IBEX or WILD GOAT (Capra hylocrius).}

Habitat_India, Nilghiri Hills.

\begin{tabular}{|c|c|c|c|c|c|}
\hline Length. & $\begin{array}{l}\text { Circum- } \\
\text { ference. }\end{array}$ & Tip to Tip. & Habitat. & & Owner. \\
\hline $16 \frac{3}{4}$ & $8 \frac{7}{8}$ & $5 \frac{5}{8}$ & India ... & $\cdots$ & A. O. Hume, Private Collection. \\
\hline $16 \frac{1}{2}$ & ... & $\begin{array}{c}\text { (owner's } \\
\text { (ownement) }\end{array}$ & Do. ... & $\cdots$ & St. George Littledale. \\
\hline $15 \frac{1}{2}$ & $8 \frac{5}{8}$ & $7 \frac{7}{8}$ & Do. ... & $\cdots$ & Sir Edmund G. Loder, Bart. \\
\hline $15 \frac{3}{8}$ & $8 \frac{3}{4}$ & $6 \frac{3}{4}$ & Do. ... & $\ldots$ & Martyn Kennard. \\
\hline $15 \frac{3}{8}$ & $\delta_{\frac{1}{4}}$ & 6 & Nilghiri Hill & & St. George Littledale, $\mathbf{I} 875$. \\
\hline $15 \frac{1}{8}$ & $8 \frac{5}{8}$ & $4 \frac{3}{4}$ & India... & $\cdots$ & Martyn Kennard. \\
\hline I $5 \frac{1}{8}$ & $S_{\frac{5}{8}}$ & $4 \frac{3}{5}$ & Nilghiri Hill & & St. George Littledale, $\mathbf{I} 87 \mathbf{I}$. \\
\hline $14 \frac{3}{2}$ & $S_{4}^{3}$ & $6 \frac{1}{4}$ & Do. ... & .. & British Museun. \\
\hline $14 \frac{1}{2}$ & $S_{\frac{\pi}{6}}^{\frac{\pi}{6}}$ & $5 \frac{1}{8}$ & India ... & ... & Sir Victor Brooke's Collection. \\
\hline $14 \frac{3}{8}$ & 8 & $4 \frac{5}{8}$ & Do. ... & $\cdots$ & T. W. H. Greenfield. \\
\hline $14 \frac{1}{8}$ & $8 \frac{5}{8}$ & $5: \frac{1}{2}$ & Do. ... & $\cdots$ & Sir Victor Brooke's Collection. \\
\hline $14 \frac{1}{3}$ & $S_{2}^{1}$ & $6 \frac{1}{2}$ & Do. ... & $\cdots$ & Do. \\
\hline 14 & $\delta_{\frac{5}{5}}^{5}$ & $5 \frac{1}{2}$ & Nilghiri Hill & & British Museum. \\
\hline 14 & S & $5 \frac{1}{2}$ & India ... & $\cdots$ & Martyn Kennard. \\
\hline $13 \frac{3}{4}$ & S & $6 \frac{1}{8}$ & Do. ... & $\cdots$ & Do. \\
\hline $13 \frac{5}{8}$ & 9 & $4 \frac{5}{8}$ & Do. ... & ... & Do. \\
\hline $13 \frac{5}{8}$ & 85 & (one horn & Nilghiri Hill & & St. George Littledale. \\
\hline $13 \frac{1}{2}$ & $S \frac{1}{3}$ & $5^{\frac{3}{4}}$ & India ... & $\cdots$ & Sir Victor Brooke's Collection. \\
\hline $13 \frac{1}{2}$ & S & $5 \frac{1}{2}$ & Do. ... & $\cdots$ & W. C. Oswell. \\
\hline $13 \frac{3}{8}$ & S! & $5 \frac{1}{8}$ & Do. ... & $\cdots$ & A. O. Hume, Private Collection. \\
\hline I3 & $9 \frac{1}{8}$ & $4 \stackrel{3}{1}$ & Do. ... & $\cdots$ & Sir Victor Brooke's Collection. \\
\hline 13 & $S_{\frac{1}{8}}$ & $5 ?$ & $D 0 . \ldots$ & $\ldots$ & T. IV. II. Greenfield. \\
\hline $12 \frac{3}{4}$ & $7 \frac{7}{8}$ & 37 & 1)o. ... & $\cdots$ & Martyn Kennard. \\
\hline $12 \frac{3}{4}$ & $7 \frac{5}{8}$ & 53 & Do. ... & $\ldots$ & T. W. H. Greenfield. \\
\hline
\end{tabular}


NILGHIRI IBEX or WILD GOAT (Capra hylocrius)-continute.

\begin{tabular}{|c|c|c|c|c|c|}
\hline Length. & $\begin{array}{l}\text { Circum- } \\
\text { ference. }\end{array}$ & Tip to Tip. & \multicolumn{2}{|l|}{ Habitat. } & Owner. \\
\hline $12 \frac{1}{2}$ & 8 & $4 \frac{5}{8}$ & India... & $\cdots$ & Hume Collection, Brilish Museum. \\
\hline $12 \frac{1}{2}$ & $7 \frac{3}{4}$ & $4 \frac{3}{4}$ & Do. ... & ... & Do. \\
\hline $12 \frac{1}{4}$ & $7 \frac{3}{8}$ & $3 \frac{3}{4}$ & Do. ... & $\ldots$ & Sir Robert Harvey, Bart. \\
\hline 12 & $7 \frac{1}{2}$ & $4 \frac{3}{4}$ & Do. ... & $\cdots$ & Hume Collection, British Museum. \\
\hline II $\frac{3}{4}$ & $5 \frac{7}{8}$ & $5 \frac{5}{8}$ & Nilghiri Hills & & British Museum. \\
\hline II $\frac{5}{8}$ & $7 \frac{7}{8}$ & $5 \frac{3}{4}$ & India ... & $\cdots$ & Sir Victor Brooke's Collection. \\
\hline I I & $5 \frac{5}{8}$ & $4 \frac{1}{8}$ & Do. ... & $\cdots$ & Hume Collection, British Museum. \\
\hline $10 \frac{5}{8}$ & 6 & $3 \frac{7}{8}$ & Do. $\ldots$ & ... & Sir Robert Harvey, Bart. \\
\hline $10 \frac{1}{4}$ & $5 \frac{7}{8}$ & $3 \frac{1}{4}$ & Do. ... & $\cdots$ & J. Carr-Saunders. \\
\hline 우이 & $5 \frac{1}{2}$ & 4 & Nilghiri Hills & & St. George Littledale, I 875 . \\
\hline 9 & $5 \frac{7}{8}$ & $3 \frac{1}{4}$ & Do. & & British Museum. \\
\hline $8 \frac{5}{8}$ & 5 & $3 \frac{5}{8}$ & Do. & & Hume Collection, British Museum. \\
\hline
\end{tabular}




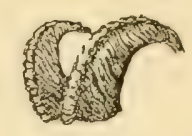

THAR (Capra jemlaica).

Weight about 200 lbs.

Habitat-Himalayas from Kashmir to Bhuton.

\begin{tabular}{|c|c|c|c|c|c|c|}
\hline Length. & $\begin{array}{l}\text { Circum- } \\
\text { ference. }\end{array}$ & Tip to Tip. & \multicolumn{3}{|c|}{ Habitat. } & Owner. \\
\hline $14 \frac{2}{8}$ & $8 \frac{7}{8}$ & $6 \frac{5}{8}$ & $\cdots$ & $\cdots$ & $\ldots$ & A. O. Hume, Private Collection. \\
\hline $13 \frac{7}{8}$ & 9 & $8 \frac{3}{8}$ & $\ldots$ & ... & $\cdots$ & Gwynne Griffiths. \\
\hline $13 \frac{3}{t}$ & 9 & II $\frac{7}{4}$ & $\cdots$ & $\cdots$ & $\cdots$ & Hume Collection, British Museum. \\
\hline $13 \frac{3}{4}$ & $8 \frac{3}{8}$ & $6 \frac{7}{8}$ & $\cdots$ & $\ldots$ & $\ldots$ & A. O. Hume, Private Collection. \\
\hline $13 \frac{5}{8}$ & 8 & $6 \frac{1}{4}$ & $\cdots$ & $\cdots$ & $\ldots$ & Hume Collection, British Museum. \\
\hline $13 \frac{1}{2}$ & 87 & $6 \frac{1}{8}$ & $\cdots$ & $\cdots$ & $\cdots$ & British Museum. \\
\hline $13 \frac{1}{2}$ & $8 \frac{1}{2}$ & $6 \frac{3}{4}$ & $\cdots$ & $\cdots$ & $\cdots$ & Rowland Ward's Collection. \\
\hline $13 \frac{3}{8}$ & $9 \frac{1}{8}$ & $5^{\frac{3}{4}}$ & $\cdots$ & $\cdots$ & $\cdots$ & Hon. Walter Rothschild. \\
\hline $13 \frac{1}{4}$ & $9 \frac{1}{2}$ & $7 \frac{3}{x}$ & $\cdots$ & $\cdots$ & $\cdots$ & A. O. Hume, Private Collection. \\
\hline $13 \frac{1}{x}$ & $8 \frac{7}{8}$ & $5^{\frac{3}{4}}$ & $\cdots$ & $\ldots$ & $\cdots$ & Sir Victor Brooke's Collection. \\
\hline $13 \frac{1}{4}$ & $8 \frac{1}{4}$ & $6 \frac{7}{8}$ & $\cdots$ & $\cdots$ & $\ldots$ & Sir Edmund G. Loder, Bart. \\
\hline $13 \frac{1}{8}$ & S $\frac{7}{8}$ & $6 \frac{5}{8}$ & $\ldots$ & ... & $\cdots$ & J. Carr-Saunders. \\
\hline 13 & $7 \frac{7}{8}$ & $5 \frac{1}{2}$ & $\cdots$ & $\cdots$ & $\cdots$ & British Museum. \\
\hline $12 \frac{7}{8}$ & $S_{\frac{3}{4}}^{3}$ & $6 \frac{1}{2}$ & $\cdots$ & $\cdots$ & $\cdots$ & J. M. Nicolls. \\
\hline $12 \frac{3}{4}$ & $8 \frac{1}{8}$ & $6 \frac{7}{8}$ & Himala & yas & $\ldots$ & Capt. Townley Parker. \\
\hline $12 \frac{5}{8}$ & $8 \frac{7}{3}$ & $7 \frac{1}{1}$ & $\cdots$ & $\ldots$ & $\ldots$ & Hume Collection, British Museum. \\
\hline $12 \frac{1}{2}$ & 8 & $6 \frac{3}{5}$ & Nepal & .. & $\ldots$ & B. H. Hodgson, British Museum. \\
\hline $12 \frac{3}{8}$ & $8 \frac{3}{8}$ & $7 \frac{1}{4}$ & ... & ... & $\ldots$ & Sir Victor Brooke's Collection. \\
\hline $12 \frac{1}{t}$ & $8 \frac{7}{8}$ & $4^{\frac{3}{4}}$ & $\cdots$ & ... & $\ldots$ & Sir Robert Harvey, Bart. \\
\hline $12 \frac{1}{t}$ & $8 \frac{1}{4}$ & $6 \frac{1}{2}$ & $\cdots$ & $\ldots$ & $\ldots$ & H.R.H. Duke of Edinburgh. \\
\hline $12 \frac{x}{4}$ & $7 \frac{5}{8}$ & $6 \frac{1}{8}$ & $\cdots$ & ... & $\ldots$ & Hume Collection, British Museum. \\
\hline
\end{tabular}


THAR.

THAR (Capra jemlaica)-continued.

\begin{tabular}{|c|c|c|c|c|c|c|}
\hline Length. & $\begin{array}{l}\text { Circum- } \\
\text { ference. }\end{array}$ & Tip to Tip. & & Iabitat. & & Owner. \\
\hline I $2 \frac{1}{8}$ & 9 & $6 \frac{1}{4}$ & ... & ... & $\ldots$ & Gwynne Griffiths. \\
\hline I 2 & $8 \frac{1}{8}$ & $5 \frac{7}{8}$ & $\ldots$ & ... & ... & Sir Robert Harvey, Bart. \\
\hline II $\frac{7}{8}$ & $8 \frac{7}{8}$ & $6 \frac{3}{8}$ & ... & $\ldots$ & ... & Sir Victor Brooke's Collection. \\
\hline $11 \frac{5}{8}$ & $8 \frac{1}{4}$ & $7 \frac{1}{2}$ & Nepal & $\ldots$ & ... & $\begin{array}{l}\text { H.H. the Mahrajah Duleep Singh, } \\
\text { British Museum. }\end{array}$ \\
\hline II $\frac{1}{2}$ & $\cdots$ & $\cdots$ & $\cdots$ & $\cdots$ & $\cdots$ & Capt. J. A. Orr-Ewing. \\
\hline II $\frac{1}{8}$ & $8 \frac{5}{8}$ & $5 \frac{3}{8}$ & $\cdots$ & $\cdots$ & $\cdots$ & Sir Robert Harvey, Bart. \\
\hline II & $8 \frac{1}{2}$ & $6 \frac{1}{8}$ & ... & $\ldots$ & $\cdots$ & H. F. Campbell. \\
\hline $10 \frac{3}{4}$ & 9 & $7 \frac{1}{2}$ & $\cdots$ & $\ldots$ & $\cdots$ & Major W. G. Forbes. \\
\hline 10 & $8 \frac{1}{4}$ & $6 \frac{7}{8}$ & $\cdots$ & $\cdots$ & $\cdots$ & J. Carr-Saunders. \\
\hline $9 \frac{7}{8}$ & $7 \frac{5}{8}$ & $5^{\frac{1}{2}}$ & $\cdots$ & $\cdots$ & $\cdots$ & British Museum. \\
\hline $8 \frac{7}{8}$ & 8 & $6 \frac{3}{8}$ & $\cdots$ & $\cdots$ & $\cdots$ & Sir Victor Brooke's Collection. \\
\hline 8 & $\cdots$ & $\cdots$ & $\cdots$ & $\cdots$ & $\cdots$ & B. H. Hodgson, P.Z.S., I834, p. 86. \\
\hline
\end{tabular}

CAUCASIAN BURHEL (Capra pallasi, Capra cylindricornis). Habitat-Eastern Caucasus.

\begin{tabular}{|c|c|c|c|c|c|}
\hline Length. & $\begin{array}{l}\text { Circum- } \\
\text { ference. }\end{array}$ & Tip to Tip. & Habitat. & & Owner. \\
\hline $\begin{array}{c}36 \\
\text { (about) }\end{array}$ & 15 & ... & $\cdots$ & $\ldots$ & Blyth, P.Z.S., I 840. \\
\hline $34 \frac{1}{2}$ & $10 \frac{7}{8}$ & $13 \frac{1}{2}$ & $\cdots$ & $\cdots$ & Major Talbot. \\
\hline $33 \frac{3}{8}$ & 12 & $19 \frac{3}{4}$ & Caucasus & $\ldots$ & British Museum. \\
\hline $28 \frac{1}{2}$ & II & $16 \frac{1}{2}$ & Do. & $\ldots$ & Sir Victor Brooke's Collection. \\
\hline $26 \frac{1}{2}$ & $10 \frac{5}{x}$ & I 8 & Do. & $\ldots$ & Sir Edmund G. Loder, Bart. \\
\hline $25 \frac{5}{8}$ & $10_{3}^{3}$ & $15 \frac{7}{8}$ & Do. & $\ldots$ & British Museum. \\
\hline $20 \frac{1}{2}$ & 10 & I $1 \frac{1}{2}$ & Do. & ... & H.R.H. Duc d'Orleans. \\
\hline
\end{tabular}




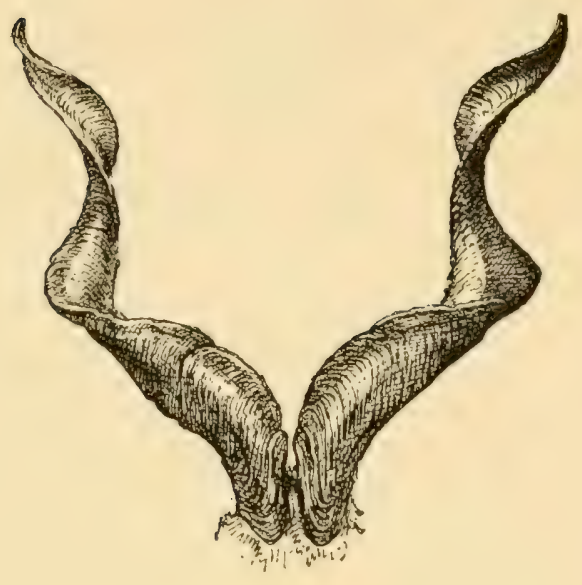

IMARKHOR (Capra megaceros).

Habitat-India, Astor, Gilgit, and Scardo.

\begin{tabular}{|c|c|c|c|c|c|c|}
\hline \multicolumn{2}{|c|}{ Length. } & \multirow{2}{*}{$\begin{array}{l}\text { Circum- } \\
\text { ference. }\end{array}$} & \multirow{2}{*}{ Tip to Tip. } & \multirow{2}{*}{\multicolumn{2}{|c|}{ Habitat. }} & \multirow{2}{*}{ Owner. } \\
\hline $\begin{array}{l}\text { Round } \\
\text { Curve. }\end{array}$ & $\begin{array}{l}\text { Straight } \\
\text { line. }\end{array}$ & & & & & \\
\hline $54 \frac{1}{4}$ & $\cdots$ & $10 \frac{1}{2}$ & $26 \frac{1}{2}$ & India & $\ldots$ & A. O. Hume, Private Col- \\
\hline 54 & ... & $10 \frac{5}{8}$ & $33^{\frac{3}{4}}$ & Do. & $\ldots$ & Martyn Kennard. \\
\hline $53^{\frac{1}{4}}$ & $42 \frac{1}{2}$ & II $1 \frac{1}{2}$ & $5^{52}$ & Do. & $\ldots$ & $\begin{array}{l}\text { Sir Victor Brooke's Collec- } \\
\text { tion, I } 875 \text {. }\end{array}$ \\
\hline $53 \frac{1}{2}$ & ... & ... & $37^{\frac{1}{2}}$ & Do. & $\ldots$ & Rowland Ward's Collection. \\
\hline 53 & $24 \frac{1}{4}$ & $9^{\frac{1}{4}}$ & $30 \frac{1}{4}$ & Do. & $\cdots$ & A. O. Hume, Private Collec \\
\hline $52 \frac{3}{4}$ & $399^{\frac{3}{4}}$ & $12 \frac{1}{8}$ & $33^{\frac{3}{4}}$ & Do. & $\ldots$ & Martyn Kennard. \\
\hline $51 \frac{3}{8}$ & $37 \frac{1}{8}$ & 10 & 33 & Do. & $\cdots$ & Sir Edmund G. Loder, Bart. \\
\hline $5^{1 \frac{1}{8}}$ & $\cdots$ & $11 \frac{3}{8}$ & $49 \frac{3}{8}$ & Do. & $\ldots$ & Martyn Kennard. \\
\hline $5^{1}$ & $\cdots$ & Io & 34 & Do. & $\cdots$ & Col. G. Jackson, I864. \\
\hline 50 & 38 & $11 \frac{3}{4}$ & 36 & Do. & $\ldots$ & Sir Edmund G. Loder, Bart. \\
\hline
\end{tabular}


MARKHOR (Capra megaceros)-continued.

\begin{tabular}{|c|c|c|c|c|c|c|}
\hline \multicolumn{2}{|c|}{ Length. } & \multirow{2}{*}{$\begin{array}{l}\text { Circum- } \\
\text { ference. }\end{array}$} & \multirow{2}{*}{ Tip to Tip. } & \multirow{2}{*}{\multicolumn{2}{|c|}{ Habitat. }} & \multirow{2}{*}{ Owner. } \\
\hline $\begin{array}{l}\text { Round } \\
\text { Curve. }\end{array}$ & $\begin{array}{l}\text { Straight } \\
\text { line. }\end{array}$ & & & & & \\
\hline 50 & ... & II $\frac{5}{8}$ & $35 \frac{1}{4}$ & India & $\ldots$ & Martyn Kennard. \\
\hline 50 & ... & $\begin{array}{l}\text { (owner's mea- } \\
\text { surement) }\end{array}$ & $\cdots$ & Do. & $\ldots$ & Capt. H. V. Olivier, R. E. \\
\hline $48 \frac{1}{2}$ & $\cdots$ & II $\frac{1}{2}$ & 45 & Do. & $\cdots$ & Capt. M. Murphy. \\
\hline $48 \frac{1}{2}$ & $\cdots$ & $7^{\frac{3}{4}}$ & $\begin{array}{c}\text { (odd horn, } \\
\text { Suleiman race) }\end{array}$ & Do. & $\cdots$ & British Museum. \\
\hline $48 \frac{1}{4}$ & ... & II $\frac{7}{8}$ & $29 \frac{3}{8}$ & Do. & $\cdots$ & A. O. Hume, Private Collec- \\
\hline $47 \frac{5}{8}$ & $37 \frac{5}{8}$ & I I $\frac{1}{4}$ & 29 & Do. & $\cdots$ & Martyn Kennard. \\
\hline $47 \frac{1}{2}$ & $36 \frac{3}{4}$ & $10 \frac{1}{2}$ & $22 \frac{3}{4}$ & Do. & $\cdots$ & Major Talbot. \\
\hline 47 & 36 & II $\frac{5}{8}$ & 39 & Do. & $\ldots$ & $\begin{array}{l}\text { Sir Victor Brooke's Collec- } \\
\text { tion, I } 875 \text {. }\end{array}$ \\
\hline $46 \frac{1}{2}$ & ... & I I $\frac{1}{2}$ & $\begin{array}{l}\text { (odd horn, } \\
\text { Astor race) }\end{array}$ & Astor Valle & & British Museum. \\
\hline $46 \frac{1}{4}$ & $32 \frac{1}{4}$ & 12 & $44^{\frac{3}{4}}$ & India & $\ldots$ & $\begin{array}{l}\text { A. O. Hume, Private Collec- } \\
\text { tion. }\end{array}$ \\
\hline 46 & $\cdots$ & $10 \frac{1}{2}$ & $\cdots$ & Do. & $\cdots$ & Manners Smith. \\
\hline 44 & $33 \frac{7}{8}$ & $10 \frac{1}{2}$ & $3 \mathbf{I}$ & Do. & $\cdots$ & J. Carr-Saunders. \\
\hline 44 & $\cdots$ & $\cdots$ & 35 & Do. & $\cdots$ & Lloyd Anstruther. \\
\hline 44 & $\cdots$ & $10 \frac{1}{4}$ & $3 I^{\frac{1}{4}}$ & Do. & $\cdots$ & Martyn Kennard. \\
\hline $43 \frac{7}{8}$ & $\cdots$ & II $\frac{1}{8}$ & $32 \frac{7}{8}$ & Do. & $\cdots$ & Do. \\
\hline $43^{\frac{3}{4}}$ & $\cdots$ & $10 \frac{1}{2}$ & $32 \frac{7}{8}$ & Do. & $\cdots$ & Hon. Charles Ellis. \\
\hline $43^{\frac{1}{2}}$ & $\cdots$ & II $\frac{1}{2}$ & 32 & Do. & $\cdots$ & Martyn Kennard. \\
\hline $43 \frac{1}{8}$ & $\cdots$ & II $\frac{1}{8}$ & $30 \frac{1}{8}$ & Do. & $\cdots$ & Do. \\
\hline 43 & $\cdots$ & 12 & 33 & Kashmir & $\cdots$ & Rowland Ward's Collection. \\
\hline 43 & $\cdots$ & $10 \frac{1}{2}$ & 36 & India & $\cdots$ & C. Spedding. \\
\hline 43 & $\cdots$ & $\cdots$ & 29 & Do. & $\cdots$ & Lloyd Anstruther. \\
\hline $42 \frac{3}{4}$ & $\cdots$ & 12 & $32 \frac{1}{2}$ & Do. & $\cdots$ & Sir Robert Harvey, Bart. \\
\hline $42 \frac{1}{2}$ & $32 \frac{1}{2}$ & II $\frac{1}{4}$ & $32 \frac{5}{8}$ & Do. & $\cdots$ & Hon. Walter Rothschild. \\
\hline $42 \frac{1}{2}$ & $\cdots$ & 11 & 35 & Do. & $\cdots$ & Martyn Kennard. \\
\hline $42 \frac{3}{8}$ & $\cdots$ & $10 \frac{3}{4}$ & $32 \frac{7}{8}$ & Do. & $\cdots$ & Sir Edmund G. Loder, Bart. \\
\hline $4 \mathrm{I}_{4}^{\frac{1}{4}}$ & $\cdots$ & ro & $30 \frac{7}{8}$ & Do. & $\ldots$ & St. George Littledale. \\
\hline
\end{tabular}


MARKHOR (Capra megaceros)-continued.

\begin{tabular}{|c|c|c|c|c|c|c|}
\hline \multicolumn{2}{|c|}{ Length. } & \multirow{2}{*}{$\begin{array}{l}\text { Circum- } \\
\text { ference. }\end{array}$} & \multirow[b]{2}{*}{ Tip to Tip. } & \multirow{2}{*}{\multicolumn{2}{|c|}{ Habitat. }} & \multirow{2}{*}{ Owner. } \\
\hline $\begin{array}{l}\text { Round } \\
\text { Curve. }\end{array}$ & $\begin{array}{l}\text { Straight } \\
\text { line. }\end{array}$ & & & & & \\
\hline $4 \mathrm{I}$ & ... & $12 \frac{2}{4}$ & $42 \frac{3}{8}$ & \multirow{3}{*}{\multicolumn{2}{|c|}{\begin{tabular}{|l} 
River Indus, \\
Kashmir. \\
Pir Pingal, \\
Kashmirgoooft. \\
India ...
\end{tabular}}} & $\begin{array}{l}\text { Capt. M. Murphy, British } \\
\text { Museum. }\end{array}$ \\
\hline $4 I$ & $\cdots$ & I0 & $27 \frac{3}{8}$ & & & $\begin{array}{l}\text { R. Lydekker, British Mu- } \\
\text { seum. }\end{array}$ \\
\hline $40 \frac{3}{8}$ & $\cdots$ & $12 \frac{3}{8}$ & $27 \frac{1}{2}$ & & & Sir Edmund G. Loder, Bart. \\
\hline $40 \frac{1}{8}$ & $\cdots$ & I $1 \frac{3}{8}$ & $26 \frac{3}{4}$ & Do. & $\cdots$ & Martyn Kennard. \\
\hline $40 \frac{1}{8}$ & $\cdots$ & $10 \frac{3}{8}$ & $30 \frac{1}{2}$ & Do. & $\ldots$ & Sir Edmund G. Loder, Bart. \\
\hline $39 \frac{3}{4}$ & $\cdots$ & II $\frac{5}{8}$ & $19 \frac{3}{7}$ & Do. & $\ldots$ & H. C. V. Hunter. \\
\hline $39 \frac{3}{8}$ & $\cdots$ & $10 \frac{1}{4}$ & $24 \frac{1}{4}$ & Afghanista & & Col. Grant, British Museum. \\
\hline $39 \frac{1}{4}$ & ... & $9 \frac{1}{2}$ & $21 \frac{3}{4}$ & India & $\ldots$ & British Museum. \\
\hline 39 & $\cdots$ & II & $31 \frac{1}{2}$ & Do. & ... & Martyn Kennard. \\
\hline 39 & $\cdots$ & $10 \frac{7}{8}$ & $30 \frac{1}{2}$ & Do. & ... & Hon. Charles Ellis. \\
\hline $38 \frac{1}{4}$ & ... & $10 \frac{1}{4}$ & (one horn & Do. & ... & Martyn Kennard. \\
\hline $37 \frac{1}{2}$ & ... & $10 \frac{3}{8}$ & $25^{\frac{1}{8}}$ & Kashmir & $\cdots$ & $\begin{array}{l}\text { Lieut. Abbott, British Mu- } \\
\text { seum. }\end{array}$ \\
\hline 37 & $\cdots$ & 12 & $18 \frac{3}{4}$ & India & $\cdots$ & H. C. V. Hunter. \\
\hline $35^{\frac{3}{4}}$ & 28 & $10 \frac{1}{4}$ & $27 \frac{7}{8}$ & Do. & $\cdots$ & J. Carr-Saunders. \\
\hline $35 \frac{3}{8}$ & $\begin{array}{c}\text { (Suleiman } \\
\text { race) }\end{array}$ & $9 \frac{7}{8}$ & $20 \frac{3}{8}$ & Paghura & & $\begin{array}{l}\text { Lieut. Abbott, British Mu- } \\
\text { seum. }\end{array}$ \\
\hline $35 \frac{1}{x}$ & $\cdots$ & $10 \frac{7}{8}$ & 25 & India & $\cdots$ & Sir Robert Harvey, Bart. \\
\hline $30 \frac{1}{4}$ & ... & $8 \frac{1}{4}$ & $13 \frac{3}{4}$ & $\begin{array}{l}\text { Peshawar, } \\
\text { North In }\end{array}$ & & $\begin{array}{l}\text { Lieut. Abbott, British Mu. } \\
\text { seum. }\end{array}$ \\
\hline $2 \mathrm{I} \frac{1}{2}$ & $\cdots$ & $8 \frac{1}{2}$ & $21 \frac{5}{8}$ & Kashmir & ... & $\begin{array}{l}\text { R. Lydekker, British Mu- } \\
\text { seum. }\end{array}$ \\
\hline ㅇ $16 \frac{1}{4}$ & I $3 \frac{3}{8}$ & $5 \frac{3}{8}$ & I3 & India & ... & Martyn Kennard. \\
\hline
\end{tabular}




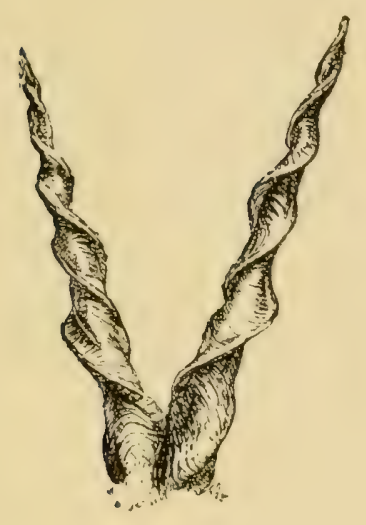

\section{TRANS-INDUS MARKHOR (Capra falconeri).}

Habitat-India, Pir Pinjal Range, Afghanistan, Suleiman Range.

\begin{tabular}{|c|c|c|c|c|c|}
\hline \multicolumn{2}{|c|}{ Length. } & \multirow{2}{*}{$\begin{array}{l}\text { Circum- } \\
\text { ference. }\end{array}$} & \multirow{2}{*}{ Tip to Tip. } & \multirow{2}{*}{ Habitat. } & \multirow{2}{*}{ Owner. } \\
\hline $\begin{array}{l}\text { Straight } \\
\text { line. }\end{array}$ & $\begin{array}{l}\text { Round } \\
\text { curve. }\end{array}$ & & & & \\
\hline $3^{8 \frac{1}{2}}$ & $\ldots$ & $10 \frac{1}{2}$ & $23 \frac{7}{8}$ & India & H.R.H. Duke of Edinburgh. \\
\hline 33 & $47 \frac{7}{8}$ & $9 \frac{1}{4}$ & $19 \frac{3}{4}$ & Afghanistan & Sir Edmund G. Loder, Bart. \\
\hline $32 \frac{1}{4}$ & ... & $9 \frac{3}{4}$ & 28 & India & A. O. Hume, Private Collec- \\
\hline $30 \frac{3}{4}$ & $45^{\frac{1}{t}}$ & $8 \frac{3}{4}$ & $2 I \frac{1}{2}$ & Do. & $\begin{array}{l}\text { Sir Victor Brooke's Collection, } \\
\text { I } 875 \text {. }\end{array}$ \\
\hline $27 \frac{1}{8}$ & 39 & $8 \frac{3}{8}$ & 21 & Do. & Sir Edmund G. Loder, Bart. \\
\hline 27 & ... & ro & $22 \frac{3}{4}$ & Do. & $\begin{array}{l}\text { A. O. Hume, Private Collec- } \\
\text { tion. }\end{array}$ \\
\hline $25 \frac{1}{2}$ & ... & $\begin{array}{l}\text { (one horn } \\
\text { malformed) }\end{array}$ & $\cdots$ & Do. & $\begin{array}{l}\text { G. Landseer, "The Field," } \\
\text { I } 873 \text {. }\end{array}$ \\
\hline $25 \frac{3}{8}$ & ... & $8 \frac{3}{8}$ & $21 \frac{1}{2}$ & $\begin{array}{lll}\cdots & \cdots & \cdots\end{array}$ & $\begin{array}{l}\text { A. O. Hume, Private Collec- } \\
\text { tion. }\end{array}$ \\
\hline 24 & ... & $\begin{array}{c}\text { (owner's mea- } \\
\text { surement) }\end{array}$ & ... & $\begin{array}{l}\text { Sheikh Boo- } \\
\text { deen. }\end{array}$ & Capt. A. A. Kinloch. \\
\hline $22 \frac{1}{2}$ & $\ldots$ & $10 \frac{1}{4}$ & $15 \frac{3}{t}$ & India $\quad \ldots$ & J. E. Penton. \\
\hline
\end{tabular}




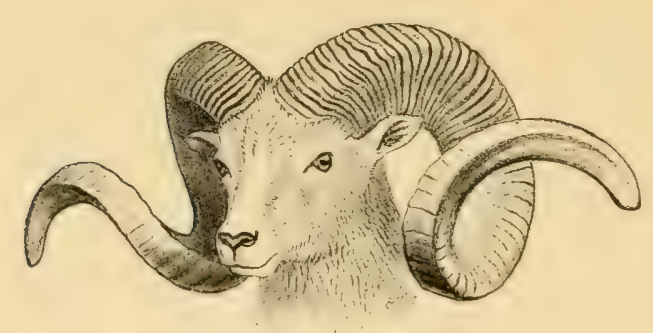

MARCO POLO'S SHEEP (Ovis poli).

[Sir Victor Brooke, Bart., and Mr. Basil Brooke, P.Z.S. 1875, p. 514.]

THE horns are very large, pressed in from the sides, the edges, with the exception of the fronto-nuchal edge, being rounded. The orbital surface is pressed in, and commences to get narrower only during the last third of its length. The horn is more than four times the length of the skull. The basal and terminal axes of the horns are not parallel, the latter being directed more horizontally. All round the neck there is a pure white mane, and there is a dark line along the spiral column from the shoulders to the loins. The light greyish brown of the sides shades off into white towards the belly. A pure white anal disk surrounds the tail; above this disk is bordered by a rather dark line, but below it extends largely over the hinder parts of the thighs, shading gradually into the brown colour of the legs. I did not discover any soft short hair under the long winter hair during the month of October.

Height, 46 inches; length of horns, 57 inches.

Habitat-Central Asia, the Pamir, and Thian Shan of Turkestan.

\begin{tabular}{|c|c|c|c|c|c|c|}
\hline Length. & $\begin{array}{l}\text { Circum- } \\
\text { ference. }\end{array}$ & Tip to Tip. & & Habitat. & & Ouner. \\
\hline 75 & I6 & $54 \frac{1}{2}$ & $\ldots$ & $\cdots$ & $\ldots$ & $\begin{array}{l}\text { H. E, Gen. Sir Fredk, Roberts, } \\
\text { "The Asian," Nov, 13th, ISoI. }\end{array}$ \\
\hline 73 & 15 & $\mid 48$ (owner's $\mid$ & Little & Pamir & $\ldots$ & Col. Tanner, I88I. \\
\hline $68 \frac{1}{2}$ & 15 & $\left|35^{\frac{3}{4}}\right|$ & $\ldots$ & ... & $\ldots$ & Lewis Flower. \\
\hline 68 & 17 & 43 & $\ldots$ & $\ldots$ & $\ldots$ & Col. R. Pole Carew. \\
\hline
\end{tabular}


IMARCO POLO'S SHEEP (Ovis poli)-continued.

\begin{tabular}{|c|c|c|c|c|c|c|}
\hline Length. & $\begin{array}{l}\text { Circum- } \\
\text { ference. }\end{array}$ & Tip to Tip. & \multicolumn{3}{|c|}{ Habitat. } & Owner. \\
\hline 68 & 16 & $5^{2}$ & $\cdots$ & $\cdots$ & $\cdots$ & Indian Museum, "The Asian," \\
\hline 67 & $16 \frac{1}{2}$ & 42 & $\ldots$ & $\cdots$ & $\ldots$ & Col. R. Pole Carew. \\
\hline $66 \frac{7}{8}$ & $15 \frac{5}{8}$ & 46 & $\cdots$ & $\cdots$ & ... & Sir Edmund G. Loder, Bart. \\
\hline $66 \frac{7}{8}$ & I $3 \frac{7}{8}$ & $\cdots$ & $\cdots$ & $\cdots$ & $\ldots$ & A. O. Hume, Private Collection. \\
\hline 66 & $15 \frac{1}{t}$ & 44 & $\cdots$ & $\cdots$ & $\ldots$ & Hume Collection, British Museum. \\
\hline $65 \frac{7}{8}$ & 16 & $4^{8}$ & $\cdots$ & $\cdots$ & $\cdots$ & Do. \\
\hline $65 \frac{1}{2}$ & I6 & 53 & $\begin{array}{l}\text { Great }) \\
\quad(I 6,\end{array}$ & $\begin{array}{l}\text { amir } \\
\text { ooft.) }\end{array}$ & & Col. T. E. Gordon, British Museum. \\
\hline $65 \frac{3}{8}$ & $16 \frac{1}{4}$ & $\cdots$ & $\cdots$ & $\cdots$ & $\cdots$ & A. O. Hume, Private Collection. \\
\hline 65 & $16 \frac{1}{2}$ & $49 \frac{1}{2}$ & $\cdots$ & $\cdots$ & $\cdots$ & Capt. Blane. \\
\hline 65 & $\begin{array}{c}\text { (owner's } \\
\text { measurement) }\end{array}$ & ... & $\ldots$ & $\cdots$ & ... & $\begin{array}{l}\text { St. George Littledale. (Presented } \\
\text { to the Empress of Russia.) }\end{array}$ \\
\hline 65 & $\cdots$ & $\cdots$ & $\cdots$ & $\cdots$ & $\cdots$ & Rowland Ward's Collection. \\
\hline $64 \frac{7}{8}$ & I $5 \frac{5}{8}$ & 46 & Great & amir & $\cdots$ & Hume Collection, British Museum. \\
\hline $64^{\frac{3}{4}}$ & $16 \frac{1}{2}$ & $4 I$ & $\cdots$ & $\cdots$ & $\cdots$ & W. Lawrence. \\
\hline $64 \frac{1}{4}$ & I $5 \frac{1}{4}$ & 39 & $\cdots$ & $\cdots$ & $\cdots$ & H. C. V. Hunter. \\
\hline $63 \frac{5}{8}$ & $16 \frac{1}{8}$ & $42 \frac{1}{2}$ & $\cdots$ & $\cdots$ & $\cdots$ & Hon. Walter Rothschild. \\
\hline $63 \frac{1}{2}$ & $15 \frac{7}{8}$ & $\cdots$ & $\cdots$ & $\cdots$ & $\cdots$ & A. O. Hume, Private Collection. \\
\hline $62 \frac{1}{2}$ & $16 \frac{1}{4}$ & $\cdots$ & $\cdots$ & $\cdots$ & $\cdots$ & H. C. V. Hunter. \\
\hline $62 \frac{1}{2}$ & $15 \frac{1}{4}$ & 41 & Great & amir & $\cdots$ & Hume Collection, British Museum. \\
\hline 62 & $15 \frac{1}{4}$ & 40 & $\cdots$ & $\cdots$ & $\cdots$ & Hon. Charles Ellis. \\
\hline $6 \mathrm{I} \frac{3}{5}$ & $15 \frac{7}{8}$ & $4 I \frac{3}{8}$ & $\cdots$ & $\cdots$ & $\cdots$ & Do. \\
\hline $61 \frac{1}{8}$ & $15 \frac{1}{2}$ & $47 \frac{1}{8}$ & $\cdots$ & $\cdots$ & $\cdots$ & Do. \\
\hline $61 \frac{1}{8}$ & $15 \frac{3}{8}$ & 46 & $\cdots$ & $\cdots$ & $\cdots$ & Do. \\
\hline $60 \frac{3}{4}$ & $15 \frac{3}{t}$ & $46 \frac{7}{8}$ & Pamir & $\cdots$ & $\cdots$ & St. George Littledale. \\
\hline $60 \frac{1}{4}$ & $14 \frac{1}{2}$ & $50 \frac{5}{8}$ & $\cdots$ & $\cdots$ & $\cdots$ & Hon. Charles Ellis. \\
\hline $59 \frac{7}{8}$ & 16 & 43 & Great 1 & amir & $\cdots$ & Hume Collection, British Museum. \\
\hline $59 \frac{1}{2}$ & $17 \frac{3}{8}$ & $45^{\frac{3}{8}}$ & $\cdots$ & $\cdots$ & $\cdots$ & Hon. Charles Ellis. \\
\hline 59 & 16 & $\cdots$ & $\cdots$ & $\cdots$ & $\cdots$ & A. O. Hume, Private Collection. \\
\hline
\end{tabular}


IMARCO POLO'S SHEEP (Ovis poli)-rontinucd.

\begin{tabular}{|c|c|c|c|c|c|c|}
\hline Length. & $\begin{array}{l}\text { Circum- } \\
\text { ference. }\end{array}$ & Tip to Tip. & \multicolumn{3}{|c|}{ Habitat. } & Owner. \\
\hline 59 & $15 \frac{3}{t}$ & $\stackrel{4 I}{\text { (about) }}$ & $\cdots$ & $\cdots$ & $\cdots$ & Martyn Kennard. \\
\hline $5^{S_{4}^{3}}$ & I5 & $5^{2}$ & Great $P$ & azir & $\ldots$ & IIume Collection, British Museum. \\
\hline $58 \frac{5}{8}$ & $14 \frac{1}{2}$ & $39 \frac{5}{8}$ & Do & & $\ldots$ & Do. \\
\hline $58 \frac{1}{2}$ & I $5 \frac{1}{4}$ & $5^{1}$ & $\cdots$ & ... & $\ldots$ & Hon. Charles Elliz. \\
\hline $58 \frac{1}{4}$ & $15 \frac{1}{4}$ & $46 \frac{3}{4}$ & $\cdots$ & $\cdots$ & $\cdots$ & Do. \\
\hline $57^{\frac{3}{4}}$ & I5 & $45 \frac{3}{4}$ & $\cdots$ & $\cdots$ & $\cdots$ & Maj.-Gen. Arthur Ellis, C.S.I. \\
\hline $57^{\frac{3}{4}}$ & $14^{\frac{3}{4}}$ & 50 & $\cdots$ & ... & $\cdots$ & Reginald Beech. \\
\hline $57 \frac{1}{2}$ & $16 \frac{1}{8}$ & $43 \frac{1}{4}$ & $\cdots$ & $\cdots$ & $\ldots$ & IV. Lawrence. \\
\hline $57^{\frac{1}{2}}$ & $14 \frac{3}{4}$ & 48 & $\cdots$ & $\cdots$ & $\cdots$ & Reginald Beech. \\
\hline $57 \frac{3}{8}$ & $15 \frac{5}{8}$ & $43^{\frac{3}{4}}$ & $\cdots$ & $\cdots$ & $\cdots$ & Hon. Charles Ellis. \\
\hline $56 \frac{7}{8}$ & 16 & $42 \frac{7}{8}$ & $\cdots$ & $\ldots$ & $\ldots$ & Do. \\
\hline $56 \frac{7}{8}$ & I $5 \frac{1}{8}$ & $35 \frac{1}{2}$ & Pamir & $\cdots$ & $\cdots$ & St. George Littledale. \\
\hline $5^{6}$ & $14 \frac{1}{t}$ & 45 & $\cdots$ & $\cdots$ & $\cdots$ & Blyth, P.Z.S. I84o, p. 63 . \\
\hline $5^{6}$ & $\cdots$ & $\cdots$ & $\ldots$ & $\cdots$ & $\cdots$ & C. Hunter. \\
\hline $55^{\frac{3}{4}}$ & $15 \frac{3}{4}$ & 43 & $\cdots$ & $\cdots$ & $\ldots$ & J. Carr-Saunders. \\
\hline $55^{\frac{1}{8}}$ & $14 \frac{1}{4}$ & $43 \frac{3}{4}$ & $\cdots$ & $\ldots$ & $\cdots$ & British Museum. \\
\hline 55 & 16 & 44 & $\cdots$ & $\ldots$ & ... & H.R.H. Duc d'Orleans. \\
\hline $54 \frac{7}{8}$ & $16 \frac{3}{8}$ & $42 \frac{5}{8}$ & Pamir & $\cdots$ & $\cdots$ & St. George Littledale. \\
\hline $54 \frac{7}{8}$ & $15 \frac{3}{8}$ & $46 \frac{5}{8}$ & Do. & ... & ... & Do. \\
\hline $54 \frac{7}{8}$ & $15 \frac{1}{4}$ & $48 \frac{3}{8}$ & Do. & $\cdots$ & $\cdots$ & Sir Victor Brooke's Collection. \\
\hline $54 \frac{3}{4}$ & $16 \frac{7}{8}$ & $38 \frac{1}{2}$ & $\cdots$ & $\cdots$ & $\cdots$ & Hon. Charles Ellis. \\
\hline $54 \frac{1}{2}$ & $14 \frac{3}{4}$ & $40 \frac{1}{2}$ & Pamir & $\cdots$ & $\cdots$ & St. George Littledale. \\
\hline $54 \frac{1}{8}$ & $14 \frac{1}{2}$ & 41 & Do. & $\cdots$ & $\cdots$ & I 886. \\
\hline $53 \frac{7}{8}$ & $15 \frac{5}{8}$ & $43^{\frac{3}{4}}$ & Lake $\mathrm{K}$ & Carakol & $\cdots$ & Do. \\
\hline $53 \frac{1}{2}$ & $14 \frac{1}{2}$ & $40 \frac{7}{8}$ & $\ldots$ & $\ldots$ & $\cdots$ & Reginald Beech. \\
\hline $52 \frac{3}{4}$ & $15 \frac{5}{8}$ & $48 \frac{1}{4}$ & Alichur & $\cdots$ & $\cdots$ & St. George Littledale. \\
\hline $52 \frac{1}{2}$ & $15 \frac{5}{8}$ & $45^{\frac{1}{2}}$ & $\cdots$ & $\cdots$ & $\cdots$ & Reginald Beech. \\
\hline
\end{tabular}


IMARCO POLO'S SHEEP (Ovis poli)-continued.

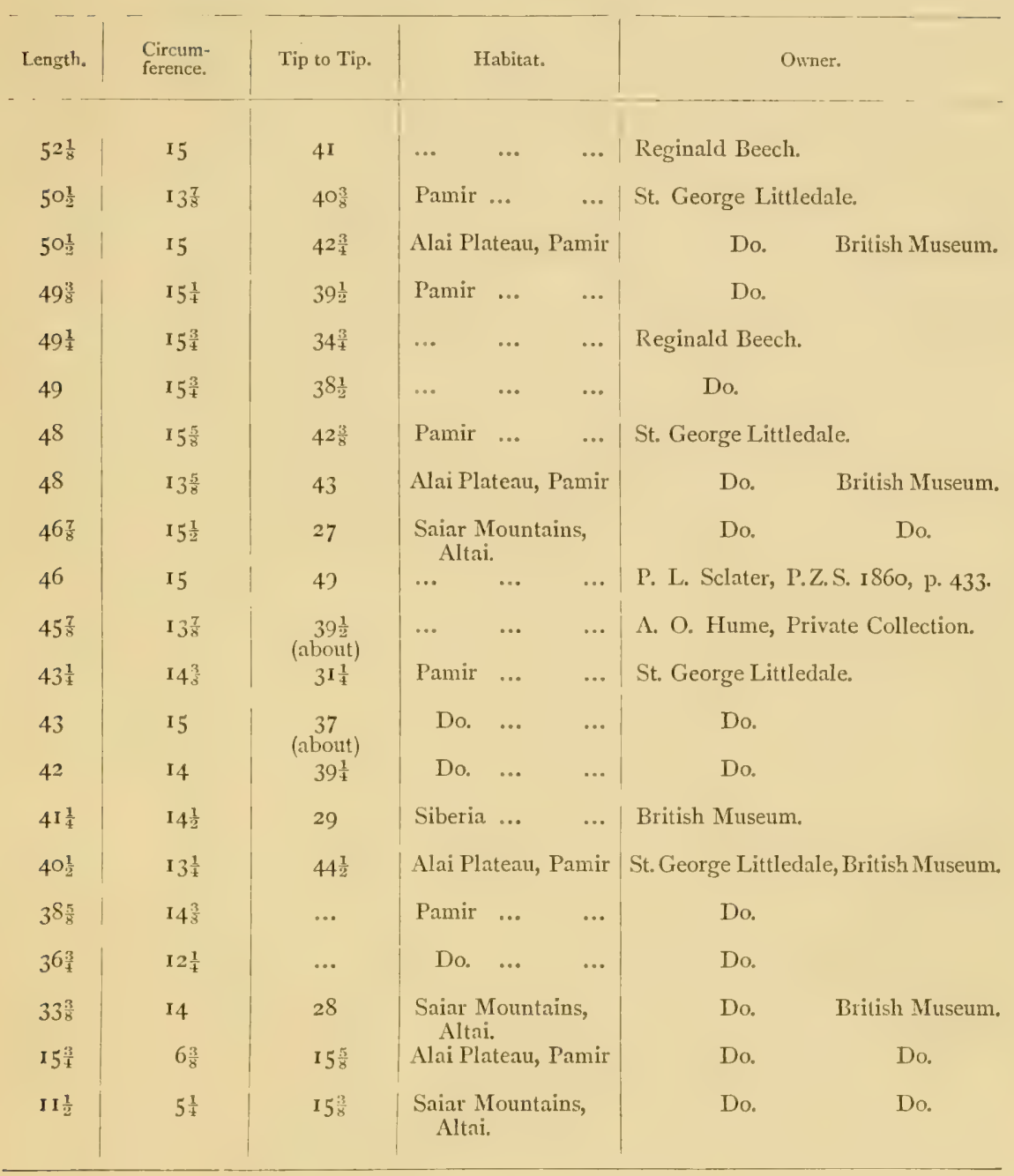




\section{WILD SHEEP OF THIAN SHAN (Ovis karelini).}

[Sir Victor Brooke, Bart., and Mr. B. BROOKE, P.Z.S. 1875, p. 512.]

THE horns are moderately thick, with rather rounded edges; frontal surface very prominent; orbital surface rather flat, narrowing only in the last third of its length. The horns are three times as long as the skull. The basal and terminal axes of the horn rise parallel with each other; the median axis is parallel with the axis of the skull. The neck is covered by a white mane, shaded with greyish brown. The light brown of the back and sides is separated from the yellowish white of the belly by a wide dark line. The light brown of the upper parts gets gradually lighter towards the tail, where it becomes greyish white, but does not form a sharply marked anal disk. On the back there is a sharply marked dark line running from the shoulders to the loins. I did not find any soft hair under the long winter hair in October.

Height at the shoulder, $3 \mathrm{ft} .6$ in. ; length of the horns, from 44 in. to 45 in.

Range.-Ovis karelini inhabits all the Semiretchinsk Altai, and also the Sapliskey Altai, but is not so common there as in the mountains between Tamgali (?) and Kaskelen; but it is partly driven from this latter locality by the Cossack sportsmen, and has gone to a higher elevation, namely the Kebin Steppe, above the range of trees. East of Tamgali (?) (Turgeli) (?), on the bare mountains and plains near the rivers Chilik and Kelen, Ovis karelini is very abundant, but not on the mountains covered with trees; it extends from this locality as far as Santash. Further it inhabits all the neighbourhood of Issik $\mathrm{Kul}$; is rather rare on the northern parts of the Thian Shan, which are thickly wooded. I also met with numerous flocks in the steppes of the Narin, where they find abundance of food and shelter at an elevation of about 12,000 or 13,000 feet above the level of the sea. 
This species is also met with on the mountains separating the Narin from its tributary the Atpash, as far as the plains between the rivers Kurtka and Chatir Kul ; but from the eastern sources of the Atpash as far as the Chatir Kul it is found only in company with Ovis polii.

\begin{tabular}{|c|c|c|c|c|c|}
\hline Length. & $\begin{array}{l}\text { Circum- } \\
\text { ference. }\end{array}$ & Tip to Tip. & Habitat. & & Oxner. \\
\hline $54 \frac{1}{4}$ & $16 \frac{1}{8}$ & $34 \frac{3}{4}$ & West Chinese & Mon- & St. George Littledale. \\
\hline $49 \frac{1}{4}$ & $14 \frac{1}{2}$ & $34 \frac{5}{8}$ & Do. & & Do. \\
\hline 49 & $15 \frac{3}{5}$ & $3 \mathrm{I} \frac{1}{2}$ & Do. & & I889. \\
\hline $48 \frac{1}{4}$ & $13 \frac{1}{4}$ & $33^{\frac{3}{4}}$ & Thian Shan & $\ldots$ & Yarkund Mission, British Museun. \\
\hline $46 \frac{1}{2}$ & $15 \frac{3}{8}$ & $29 \frac{1}{2}$ & Do. & $\ldots$ & Do. \\
\hline 42 & $14 \frac{1}{4}$ & 34 & Do. & $\ldots$ & Do. \\
\hline $41 \frac{5}{8}$ & $13 \frac{7}{8}$ & 19 & West Chinese & Mon- & St. George Littledale. \\
\hline $27 \frac{1}{8}$ & $\cdots$ & $2 I \frac{1}{2}$ & Thian Shan & ... & Yarkund Mission, British Museum. \\
\hline $23 \frac{3}{8}$ & $10 \frac{1}{2}$ & 24 & Do. & ... & Sir Victor Brooke's Collection. \\
\hline $16 \frac{3}{4}$ & $\ldots$ & 18 & Do. & $\ldots$ & Yarkund Mission, British Museum. \\
\hline
\end{tabular}




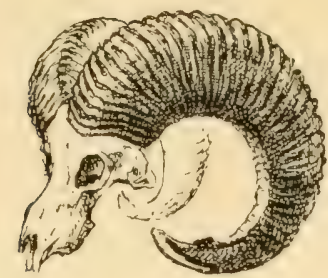

OVIS AMIMON.

Habitat-Northern Asia.

\begin{tabular}{|c|c|c|c|c|c|c|}
\hline Length. & $\begin{array}{l}\text { Circum- } \\
\text { ference. }\end{array}$ & Tip to Tip. & \multicolumn{3}{|c|}{ Habitat. } & Owner. \\
\hline $47^{\frac{3}{4}}$ & 19 & $3 \mathrm{I} \frac{1}{4}$ & Siberi: & & $\ldots$ & British Museum. \\
\hline $46 \frac{1}{2}$ & $19 \frac{3}{4}$ & $\begin{array}{c}20 \\
\text { (about) }\end{array}$ & $\cdots$ & & $\cdots$ & A. O. Hume, Private Collection. \\
\hline $45^{\frac{1}{2}}$ & $16 \frac{1}{4}$ & 17 & India & $\cdots$ & ... & H. R.H. Duke of Teck. \\
\hline $43^{\frac{1}{2}}$ & $16 \frac{5}{8}$ & (loose horns) & ... & $\ldots$ & $\ldots$ & H. C. V. Hunter. \\
\hline $42 \frac{1}{2}$ & $16 \frac{1}{2}$ & $\begin{array}{c}19 \\
\text { (aiout) }\end{array}$ & $\cdots$ & $\ldots$ & $\ldots$ & A. O. Hume, Private Collection. \\
\hline $42 \frac{1}{2}$ & 16 & I 8 & $\cdots$ & $\cdots$ & $\cdots$ & Hume Collection, British Museux. \\
\hline $42 \frac{1}{2}$ & 16 & I8 & $\cdots$ & $\ldots$ & $\cdots$ & Rowland Ward's Collection. \\
\hline $42 \frac{1}{2}(?)$ & $15 \frac{1}{t}$ & $\cdots$ & ... & $\cdots$ & $\ldots$ & J. Carr-Saunders. \\
\hline $42 \frac{3}{8}$ & $16 \frac{1}{4}$ & 20 & $\cdots$ & $\ldots$ & $\ldots$ & H.R.H. Duke of Edinburgh. \\
\hline $42 \frac{1}{8}$ & $16 \frac{3}{4}$ & $14 \frac{1}{4}$ & $\ldots$ & $\cdots$ & $\ldots$ & Hume Collection, British Musetrin. \\
\hline $42 \frac{1}{8}$ & $16 \frac{1}{2}$ & $\begin{array}{c}\text { IS } \\
\text { (about) }\end{array}$ & $\cdots$ & $\ldots$ & $\ldots$ & A. O. Hume, Private Collecion. \\
\hline 42 & $16 \frac{3}{4}$ & $17 \frac{1}{4}$ & $\cdots$ & $\cdots$ & $\cdots$ & Hume Collection, British Museuz. \\
\hline $41 \frac{7}{8}$ & I6 & $\ldots$ & $\cdots$ & $\ldots$ & $\cdots$ & Hon. Walter Rothschild. \\
\hline $4 I^{\frac{1}{4}}$ & $17 \frac{1}{2}$ & $\begin{array}{c}16 \\
\text { (about) }\end{array}$ & $\cdots$ & $\ldots$ & $\ldots$ & A. O. Hume, Private Collection. \\
\hline $40 \frac{1}{2}(?)$ & $14 \frac{1}{2}$ & $26 \frac{1}{4}$ & $\cdots$ & $\cdots$ & $\cdots$ & $\begin{array}{l}\text { St. George Littledale, British Mu- } \\
\text { seum. }\end{array}$ \\
\hline $40 \frac{3}{8}$ & $17 \frac{5}{8}$ & $20 \frac{3}{5}$ & $\cdots$ & $\cdots$ & $\cdots$ & Hon. Charles Ellis. \\
\hline $40 \frac{1}{4}$ & $17 \frac{1}{4}$ & 20 & $\cdots$ & $\cdots$ & $\ldots$ & IIume Collection, British Museum. \\
\hline 40 & $16 \frac{3}{4}$ & $19 \frac{1}{2}$ & $\cdots$ & $\ldots$ & $\ldots$ & Do. \\
\hline
\end{tabular}


OVIS AIMIMON-continued.

\begin{tabular}{|c|c|c|c|c|c|c|}
\hline Length. & $\begin{array}{l}\text { Circum- } \\
\text { ference. }\end{array}$ & Tip to Tip. & & labitat. & & Owner. \\
\hline 40 & 16 & I $\$ \frac{1}{2}$ & $\cdots$ & $\cdots$ & $\ldots$ & Hume Collection, British Museum. \\
\hline $39 \frac{1}{2}$ & $16 \frac{1}{2}$ & I $8 \frac{1}{2}$ & $\ldots$ & ... & $\cdots$ & Sir Victor Brooke's Collection. \\
\hline $39 \frac{1}{4}$ & 17 & $14 \frac{38}{4}$ & $\ldots$ & .. & $\ldots$ & Hon. Charles Ellis. \\
\hline 39 & I 7 & I9 & $\ldots$ & $\cdots$ & $\ldots$ & Sir Robert Harvey, Bart. \\
\hline $38 \frac{7}{8}$ & $14 \frac{7}{8}$ & $\begin{array}{l}\text { (one horn } \\
\text { broken) }\end{array}$ & Altai & $\cdots$ & $\ldots$ & Sir Victor Brooke's Collection, I $87 \mathrm{I}$. \\
\hline $3^{S \frac{3}{4}}$ & 16 & 20 & $\cdots$ & $\cdots$ & $\ldots$ & Maj-Gen. Arthur Ellis, C.S.I. \\
\hline $38 \frac{1}{2}$ & I6 & $\begin{array}{c}\text { (owner's mea- } \\
\text { surement) }\end{array}$ & $\cdots$ & $\cdots$ & $\cdots$ & Capt. A. A. Kinloch. \\
\hline $3^{8 \frac{1}{4}}$ & $17 \frac{1}{8}$ & I $8 \frac{1}{4}$ & Himal: & layas & $\cdots$ & Col. Congreve, British Museum. \\
\hline 38 & 18 & $19 \frac{1}{4}$ & $\cdots$ & $\cdots$ & $\cdots$ & Hon. Charles Ellis. \\
\hline $3^{8}$ & $17 \frac{1}{2}$ & $19 \frac{1}{2}$ & $\cdots$ & $\cdots$ & $\cdots$ & H. C. V. Hunter. \\
\hline $3^{8}$ & I6 & $23 \frac{1}{2}$ & $\ldots$ & $\cdots$ & $\ldots$ & $\begin{array}{l}\text { East India Company, British Mu- } \\
\text { seum. }\end{array}$ \\
\hline $37 \frac{3}{4}$ & $16 \frac{1}{2}$ & $\cdots$ & $\cdots$ & $\cdots$ & $\cdots$ & Sir Robert Harvey, Bart. \\
\hline $37 \frac{1}{2}$ & $18 \frac{1}{4}$ & $16 \frac{1}{2}$ & $\ldots$ & $\cdots$ & $\ldots$ & G. McMicking. \\
\hline $37 \frac{1}{2}$ & $16 \frac{1}{4}$ & 17 & $\ldots$ & $\cdots$ & $\cdots$ & Capt. M. Murphy. \\
\hline $36 \frac{7}{8}$ & $16 \frac{1}{4}$ & $16 \frac{3}{4}$ & $\ldots$ & $\cdots$ & $\ldots$ & Sir Edmund G. Loder, Bart. \\
\hline $36 \frac{1}{2}$ & I $5 \frac{1}{4}$ & $19 \frac{1}{2}$ & Ladak & & $\ldots$ & Col. Congreve, British Museun. \\
\hline 36 & $16 \frac{3}{4}$ & $\cdots$ & $\ldots$ & $\ldots$ & $\ldots$ & H. C. V. Hunter. \\
\hline 36 & $15^{\frac{3}{4}}$ & 20 & $\ldots$ & $\cdots$ & $\cdots$ & G. McMicking. \\
\hline $3^{6}$ & $15 \frac{1}{3}$ & 20 & $\ldots$ & $\cdots$ & $\ldots$ & Capt. G. Campbell. \\
\hline $35 \frac{1}{2}$ & $16 \frac{3}{4}$ & $\mathbf{1} 5$ & $\ldots$ & $\cdots$ & $\cdots$ & H. C. V. Hunter. \\
\hline $35 \frac{1}{4}$ & $17 \frac{3}{4}$ & $21 \frac{1}{4}$ & Ladak & $\cdots$ & $\ldots$ & British Museum. \\
\hline $34^{\frac{3}{4}}$ & $15 \frac{1}{2}$ & $20 \frac{1}{2}$ & $\ldots$ & $\cdots$ & $\cdots$ & H. C. V. Hunter. \\
\hline $33^{\frac{1}{2}}$ & $15 \frac{7}{8}$ & $\cdots$ & $\cdots$ & $\cdots$ & $\cdots$ & Do. \\
\hline 33 & I6 & $I 7 \frac{1}{2}$ & $\cdots$ & $\cdots$ & $\cdots$ & | Reginald Beech. \\
\hline $20 \frac{1}{4}$ & $7 \frac{3}{8}$ & I7 & Siberia & & $\cdots$ & British Museum. \\
\hline 오 I 8 & 7 & $19 \frac{3}{4}$ & Nepal & $\cdots$ & $\cdots$ & B. H. Hodgson, British Museum. \\
\hline \& $12 \frac{1}{2}$ & 5 & $15 \frac{5}{8}$ & $\ldots$ & $\ldots$ & $\ldots$ & Sir Victor Brooke's Collection. \\
\hline
\end{tabular}




\section{OVIS HODGSONI.}

[Sir Victor Brooke, Bart., and Mr. B. Brooke, P.Z.S. i875, p. 520].

Adult male, winter, Colebrooke collection. Obtained within 30 miles of Leh in the winter of 1873 .

Hair of body about 2 inches in length, coarse and close-set; on the sides and lower surface of the neck the hair is lengthened into a long rich ruff-like mane of a snow-white colour. Along the median line of the upper neck there is a narrow band of rather shorter hair, which, however, is about twice as long as that on the body, and, being continued as far back as the withers, forms a short dorsal mane; general colour of the body dark brown, mixed with grey; anterior parts of the face, belly, limbs below the carpi and tarsi internally, and a small anal disk surrounding the tail and rump, dirty white; cheeks and forearms darker than the rest of the body; ears and tail short, the latter with a narrow dark line along its upper surface; horns massive and rather short, their terminal curve being but slightly developed.

Female, much paler than the male, with little or no mane. No distinct anal disk.

Range.-Little Thibet (Kinloch, \&c.); Cachar region, Nepaul (Hodgson).

Habitat-Nepal.

\begin{tabular}{|c|c|c|c|c|c|c|}
\hline Length. & $\begin{array}{l}\text { Circum- } \\
\text { ference. }\end{array}$ & Tip to $T ! p$. & \multicolumn{3}{|c|}{ Habitat. } & Owner. \\
\hline $44 \frac{5}{8}$ & $17 \frac{1}{2}$ & $22 \frac{1}{2}$ & Nepal & $\cdots$ & $\ldots$ & B. H. Hodgson, British Museum. \\
\hline $41 \frac{1}{8}$ & $16 \frac{3}{4}$ & 19 & Ladak & ... & ... & Sir Victor Brooke's Collection. \\
\hline $38 \frac{3}{8}$ & $15 \frac{3}{8}$ & IS & Do. & $\cdots$ & $\ldots$ & Do. \\
\hline $35 \frac{1}{4}$ & $16 \frac{1}{2}$ & $15 \frac{1}{2}$ & Do. & ... & $\ldots$ & R. Lydekker, British Museum. \\
\hline q $14 \frac{1}{2}$ & $7 \frac{1}{2}$ & $17 \frac{1}{2}$ & Do. & $\ldots$ & $\ldots$ & Sir Victor Brooke's Collection. \\
\hline
\end{tabular}




\section{OVIS BROOKEI.}

[Sir Victor Brooke, Bart., and Mr. B. Brooke, P.Z.S. i875, p. 521 ].

THIs species is based upon a skull and horns in our own collection. The locality from which the specimen was obtained in not so certain as could be desired, but we have strong reasons for believing that the animal was shot in the vicinity of Leh, in Ladak. In its much smaller size it differs from Ovis hodgsoni, and in its deeply sulcated horns, the angles of which are very much rounded and the terminal curve but slightly developed, it differs as decidedly from Ovis karelini. It is not improbable that the species will be found to inhabit the Kuenluen Mountains.

$$
\text { Habitat-Ladak. }
$$

Dimensions of the specimen :

Length of skull, measured from highest point between the horn $\quad \ldots \quad \ldots . \quad \ldots \quad \ldots . \quad \ldots \quad \ldots$ II

Smallest breadth between orbits $\quad \ldots \quad \ldots \quad \ldots \quad 4 \quad 4 \frac{5}{8}$

Length of horns, round the curve $\quad \ldots \quad \ldots \quad \ldots \quad 33 \frac{1}{2}$

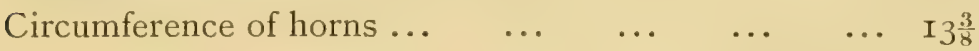

\begin{tabular}{|c|c|c|c|c|c|}
\hline Length. & $\begin{array}{l}\text { Circum- } \\
\text { ference. }\end{array}$ & Tip to Tip. & Habitat. & & Owner. \\
\hline $33 \frac{1}{2}$ & $13 \frac{1}{2}$ & I $8 \frac{1}{2}$ & Ladak ... & $\ldots$ & Sir Victor Brooke's Collection. \\
\hline
\end{tabular}

OVIS BLANFORDI.

\begin{tabular}{r|c|c|cc|c|c}
\hline Length. & $\begin{array}{c}\text { Circum- } \\
\text { ference. }\end{array}$ & Tip to Tip. & Habitat. & Owner. \\
\hline $36 \frac{1}{2}$ & $9 \frac{5}{8}$ & $\begin{array}{c}\text { (single } \\
\text { lorn) }\end{array}$ & $\ldots$ & $\ldots$ & $\ldots$ & Hume Collection, British Museum. \\
$33^{\frac{3}{6}}$ & $9 \frac{3}{8}$ & $13^{\frac{3}{4}}$ & $\ldots$ & $\ldots$ & $\ldots$ & Do. \\
$23 \frac{5}{8}$ & $9 \frac{1}{2}$ & $17^{\frac{3}{4}}$ & $\ldots$ & $\ldots$ & $\ldots$ & Do.
\end{tabular}




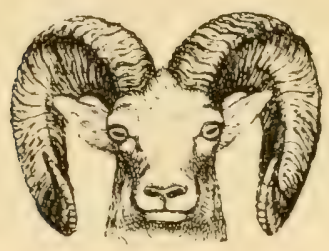

BIGHORN SHEEP (Ovis canadensis'.

Habitat-North America.

\begin{tabular}{|c|c|c|c|c|c|c|}
\hline Length. & $\begin{array}{l}\text { Circum- } \\
\text { ference. }\end{array}$ & Tip to Tip. & \multicolumn{3}{|c|}{ Habitat. } & Owner. \\
\hline 41 & I $7 \frac{1}{4}$ & 26 & $\ldots$ & $\ldots$ & $\ldots$ & Blyth, P.Z.S. 1840, p. 64. \\
\hline$\ldots$ & $17 \frac{1}{4}$ & (owner's men- & .. & $\ldots$ & ... & T. IV. H. Clarke. \\
\hline $40 \frac{3}{1}$ & $x 6 \frac{1}{2}$ & ... & $\ldots$ & $\ldots$ & $\ldots$ & $\begin{array}{l}\text { British Museum (American Exhilition } \\
\text { Catalogue). }\end{array}$ \\
\hline $40 \frac{1}{4}$ & $15 \frac{1}{4}$ & $20 \frac{1}{4}$ & ... & $\ldots$ & $\ldots$ & Sir Edmund G. Loder, Bart. \\
\hline $39 \frac{2}{2}$ & $15 \frac{3}{8}$ & $\cdots$ & & rado & $\ldots$ & St. George Littledale. \\
\hline $39 \frac{1}{2}$ & $16 \frac{1}{2}$ & $24 \frac{3}{4}$ & & h Americ & & British Museum. \\
\hline 39 & $15 \frac{3}{4}$ & $\ldots$ & ... & $\ldots$ & & $\begin{array}{l}\text { IV. A. Baillie Grohman (American } \\
\text { Exhibition Catalogue. }\end{array}$ \\
\hline 39 & $14 \frac{1}{8}$ & I $8 \frac{1}{2}$ & .. & .. & $\ldots$ & The late J. S. Jameson. \\
\hline $3^{S: 3}$ & $15 \frac{1}{2}$ & 22 & .. & $\ldots$ & .. & E. N. Buxten. \\
\hline $3^{8}+\frac{1}{1}$ & $15 \frac{1}{4}$ & $19 \frac{1}{4}$ & & $\operatorname{tana}$ & $\ldots$ & St. George Littledale. \\
\hline $3^{S i}$ & $16 \frac{3}{8}$ & $\ldots$ & $\ldots$ & $\ldots$ & $\ldots$ & $\begin{array}{l}\text { H. Seton Karr (American Exhibition } \\
\text { Catalogue). }\end{array}$ \\
\hline $3^{S}$ & I 7 & $\cdots$ & . & $\cdots$ & $\cdots$ & Capt. A. Pike. \\
\hline $3^{S}$ & I5 & $\ldots$ & & $\ldots$ & $\ldots$ & Capt. F. Cookson. \\
\hline $37:$ & I $5 \frac{7}{8}$ & $23 \frac{3}{8}$ & & ico & $\ldots$ & J. A. H. Drought. \\
\hline $37 \frac{3}{4}$ & I $4 \frac{1}{8}$ & $15 \frac{3}{4}$ & & ch Columl & bia & Capt. F. Cockson. \\
\hline $37 \frac{1}{3}$ & 16 & $19 \frac{1}{8}$ & $\ldots$ & ... & $\cdots$ & Sir Edmund G. Loder, Bart. \\
\hline 37 & I $6 \frac{1}{4}$ & $\cdots$ & $\ldots$ & $\cdots$ & $\ldots$ & $\begin{array}{l}\text { Major Maitland Kirwin (American } \\
\text { Exhibition Catalogue). }\end{array}$ \\
\hline 36 & $14 \frac{3}{4}$ & $14 \frac{3}{ \pm}$ & ... & ... & $\ldots$ & F. J. Horniman. \\
\hline
\end{tabular}


BIGHORN SHEEP (Ovis canadensis)-continued.

\begin{tabular}{|c|c|c|c|c|c|c|}
\hline Length. & $\begin{array}{l}\text { Circum- } \\
\text { ference. }\end{array}$ & Tip to Tip. & & tabitat. & & Owner. \\
\hline $36 \frac{1}{2}$ & $15 \frac{1}{2}$ & $\cdots$ & $\cdots$ & $\cdots$ & $\cdots$ & $\begin{array}{l}\text { Major Maitland Kirwin (American } \\
\text { Exhibition Catalogue). }\end{array}$ \\
\hline $36 \frac{1}{2}$ & $14 \frac{1}{2}$ & $\cdots$ & $\cdots$ & $\cdots$ & $\cdots$ & $\begin{array}{l}\text { Gerald Buxton (American Exhibition } \\
\text { Catalogue). }\end{array}$ \\
\hline $3^{6 \frac{1}{2}}$ & I $4 \frac{1}{2}$ & $\cdots$ & $\cdots$ & $\cdots$ & $\cdots$ & $\begin{array}{l}\text { Thos. Bate (American Exhibition } \\
\text { Catalogue). }\end{array}$ \\
\hline $36 \frac{1}{2}$ & 14 & $\cdots$ & $\cdots$ & $\cdots$ & $\cdots$ & J. D. Cobbold. \\
\hline $3^{6 \frac{1}{2}}$ & $14 \frac{3}{8}$ & $13 \frac{1}{2}$ & $\cdots$ & $\cdots$ & $\cdots$ & J. C. L. Knight Bruce. \\
\hline $36 \frac{3}{4}$ & $15 \frac{1}{\frac{1}{t}}$ & $22 \frac{1}{2}$ & $\cdots$ & $\cdots$ & $\cdots$ & Moreton Frewen. \\
\hline $36 \frac{1}{4}$ & $14 \frac{3}{8}$ & $18 \frac{1}{2}$ & $\ldots$ & $\cdots$ & $\cdots$ & E. N. Buxton. \\
\hline 36 & $14 \frac{1}{2}$ & $I 6 \frac{1}{2}$ & Mont & ana & $\cdots$ & R. H. Sawyer. \\
\hline $3^{6}$ & $15 \frac{1}{2}$ & $\cdots$ & $\cdots$ & $\cdots$ & $\cdots$ & Capt. A. Pike. \\
\hline $35^{\frac{3}{4}}$ & $13 \frac{7}{8}$ & $\begin{array}{c}19 \\
\text { (about) }\end{array}$ & $\ldots$ & $\ldots$ & $\cdots$ & E. N. Buxton. \\
\hline $35 \frac{3}{8}$ & $13 \frac{1}{4}$ & 18 & Mont & ana & $\cdots$ & St. George Littledale. \\
\hline $35^{\frac{1}{4}}$ & $12 \frac{1}{4}$ & I6 & Calif & ornia & $\cdots$ & Sir Victor Brooke's Collection. \\
\hline $35^{\frac{1}{4}}$ & $16 \frac{1}{2}$ & $16 \frac{1}{2}$ & Britis & h Colum & abia & Capt. F. Cookson, I 885. \\
\hline 35 & 14 & $\cdots$ & $\cdots$ & $\cdots$ & $\cdots$ & Sir Michael Culme Seymour, Bart. \\
\hline 35 & I $5 \frac{3}{1}$ & $I 7 \frac{3}{4}$ & $\cdots$ & $\cdots$ & $\cdots$ & Hon. Charles Ellis. \\
\hline $34^{\frac{3}{4}}$ & $15 \frac{1}{2}$ & $\cdots$ & Color & ado & $\cdots$ & St. George Littledale. \\
\hline $34^{\frac{3}{4}}$ & $14 \frac{1}{4}$ & IS & $\cdots$ & $\cdots$ & $\cdots$ & The late J. S. Jameson. \\
\hline $34 \frac{1}{2}$ & $14 \frac{1}{2}$ & $\cdots$ & $\cdots$ & $\cdots$ & $\cdots$ & G. P. Fitzgerald. \\
\hline 34 & 15 & $\cdots$ & $\cdots$ & $\cdots$ & $\cdots$ & Do. \\
\hline $33 \frac{7}{8}$ & $14 \frac{3}{x}$ & 17 & $\cdots$ & $\cdots$ & $\cdots$ & J. C. L. Knight Bruce. \\
\hline $33 \frac{1}{2}$ & $15 \frac{1}{4}$ & $20 \frac{1}{x}$ & $\cdots$ & $\cdots$ & $\cdots$ & Sir Victor Brooke's Collection. \\
\hline $33^{\frac{1}{2}}$ & $14 \frac{1}{2}$ & $\cdots$ & $\cdots$ & $\cdots$ & $\cdots$ & Sir Michael Culme Seymour. \\
\hline $33 \frac{1}{2}$ & 14 & $\cdots$ & $\cdots$ & $\cdots$ & $\cdots$ & Rowland Ward's Collection. \\
\hline $33 \frac{1}{2}$ & I6 & $18 \frac{1}{2}$ & $\cdots$ & $\cdots$ & $\cdots$ & J. Carr-Saunders. \\
\hline 33 & $14 \frac{1}{2}$ & $\cdots$ & $\cdots$ & $\cdots$ & $\cdots$ & G. P. Fitzgerald. \\
\hline $32 \frac{7}{8}$ & $15 \frac{3}{8}$ & $19 \frac{1}{2}$ & $\cdots$ & $\cdots$ & $\cdots$ & Sir Victor Brooke's Collection. \\
\hline $32 \frac{1}{2}$ & I $4 \frac{1}{2}$ & $16 \frac{3}{4}$ & $\cdots$ & $\cdots$ & $\cdots$ & The late J. S. Jameson. \\
\hline
\end{tabular}


BIGHORN SHEEP (Ovis canadensis)-continued.

\begin{tabular}{|c|c|c|c|c|c|c|}
\hline Length. & $\begin{array}{l}\text { Circum* } \\
\text { ference. }\end{array}$ & Tip to Tip. & \multicolumn{3}{|c|}{ Habitat. } & Owner. \\
\hline $32 \frac{1}{2}$ & $13 \frac{1}{2}$ & $\cdots$ & $\cdots$ & ... & $\ldots$ & J. D. Cobbold. \\
\hline $32 \frac{3}{8}$ & 14 & $\begin{array}{c}20 \frac{1}{2} \\
\text { (about) }\end{array}$ & $\cdots$ & $\cdots$ & $\cdots$ & E. N. Buxton. \\
\hline $32 \frac{3}{8}$ & $12 \frac{7}{8}$ & $16 \frac{1}{2}$ & $\cdots$ & $\cdots$ & $\cdots$ & J. C. L. Knight Bruce. \\
\hline $32 \frac{1}{8}$ & $13 \frac{1}{2}$ & $17 \frac{1}{2}$ & $\ldots$ & $\cdots$ & $\ldots$ & Do. \\
\hline 32 & $16 \frac{1}{2}$ & ... & $\ldots$ & $\cdots$ & $\ldots$ & Rowland Ward's Collection. \\
\hline 32 & $14 \frac{3}{4}$ & I $7 \frac{1}{4}$ & Yell & $\begin{array}{l}\text { wstone } \\
\text { ver. }\end{array}$ & & British Museum. \\
\hline 32 & I $3 \frac{1}{2}$ & $\cdots$ & $\cdots$ & $\cdots$ & $\cdots$ & J. D. Cobbold. \\
\hline $31 \frac{1}{4}$ & $15 \frac{3}{\frac{1}{4}}$ & $\cdots$ & $\ldots$ & ... & $\cdots$ & $\begin{array}{l}\text { G. D. Whatman (American Exhibition } \\
\text { Catalogue). }\end{array}$ \\
\hline $3 \mathrm{I}$ & $16 \frac{1}{2}$ & ... & $\cdots$ & $\cdots$ & $\cdots$ & $\begin{array}{l}\text { Frank Cooper (American Exhibition } \\
\text { Catalogue). }\end{array}$ \\
\hline $30_{\frac{3}{4}}^{3}$ & I5 & $\begin{array}{c}23 \\
\text { (about) }\end{array}$ & $\cdots$ & $\cdots$ & $\cdots$ & Hon. Walter Rothschild. \\
\hline $30 \frac{1}{2}$ & 15 & $\cdots$ & $\cdots$ & $\cdots$ & $\cdots$ & Wm. Marr. \\
\hline 30 & 13 & $\ldots$ & $\cdots$ & $\cdots$ & $\cdots$ & J. D. Cobbold. \\
\hline $28 \frac{5}{6}$ & $13 \frac{5}{8}$ & ... & Brit & h Columl & bia & Capt. F. Cookson. \\
\hline $28 \frac{3}{8}$ & $13 \frac{5}{8}$ & $19 \frac{3}{4}$ & $\ldots$ & ... & $\cdots$ & Hon. T. A. Brassey. \\
\hline 28 & $14 \frac{1}{2}$ & .. & $\ldots$ & $\cdots$ & $\cdots$ & IV. C. Beaumont. \\
\hline $27 \frac{3}{4}$ & $14 \frac{1}{2}$ & I $S \frac{1}{2}$ & Colc & ado & $\ldots \mid$ & $\begin{array}{l}\text { Sir Victor Brooke's Collection (from } \\
\text { J. H. Batty's Collection). }\end{array}$ \\
\hline $27 \frac{1}{2}$ & $14 \frac{1}{2}$ & $\begin{array}{l}15 \\
\text { (about) }\end{array}$ & $\ldots$ & ... & $\cdots$ & Col. Ralph Vivian. \\
\hline $27 \frac{3}{8}$ & $14 \frac{1}{2}$ & $\cdots$ & $\cdots$ & $\cdots$ & $\cdots$ & E. N. Buxton. \\
\hline $26 \frac{3}{4}$ & I $3 \frac{1}{2}$ & $\cdots$ & $\cdots$ & $\cdots$ & $\cdots$ & A. H. Harrison. \\
\hline $25 \frac{1}{2}$ & $13 \frac{1}{4}$ & ... & $\cdots$ & $\cdots$ & $\cdots$ & J. D. Cobbold. \\
\hline 25 & I $3 \frac{1}{7}$ & $\ldots$ & Brit & h Colum & bia & Capt. F. Cookson. \\
\hline 25 & $14 \frac{3}{4}$ & $\cdots$ & $\cdots$ & $\ldots$ & $\cdots$ & W. C. Beaumont. \\
\hline $21 \frac{7}{8}$ & $13 \frac{1}{8}$ & $20 \frac{1}{2}$ & Cali & rnia & $\ldots$ & British Museum. \\
\hline ㅇ 13 & $6 \frac{7}{8}$ & $13 \frac{3}{x}$ & Mex & & $\ldots 1$ & J. A. H. Drought. \\
\hline 오 4 & $3^{\frac{3}{4}}$ & 6 & $\begin{array}{r}\text { Yell } \\
\mathrm{R}\end{array}$ & $\begin{array}{l}\text { wstone } \\
\text { yer. }\end{array}$ & & British Museum. \\
\hline
\end{tabular}




\section{KAMSCHATKA WILD SHEEP OP WILD SHEEP OF ALASKA (Ovis nivicola).}

[Sir Victor Brooke, Bart., and Mr. B. BrookE, P.Z.S. I875, p. 521.$]$

Adult male (winter), Mus. Lugd., Kamschatka.

Hair very long and woolly, and not lengthened into a mane on the neck; general colour grizzly brown; an indefinitely bounded patch on the face below the eyes, and all four limbs anteriorly rich uniform dark brown; space round the muzzle, upper and under lip, rump, and posterior part of the haunches, centre of the belly, and the limbs posteriorly pure white. The white on the posterior parts of the limbs give place very suddenly to the brown of their anterior surfaces. The white of the rump does not surround the tail, which on its upper surface is darker than the back. Ears and tail very short.

Horns in form closely resembling those of Ovis montana. Frontal and nuchal surfaces convex; orbital surface flat; fronto-nuchal and edges very greatly rounded; fronto-orbital edge strongly defined, a deep groove lying between it and the orbital surface; the terminal curve of the horns well developed, and directed upwards and outwards.

Skull remarkably short and broad, and strongly anchylosed; a shallow anteorbital fossa. Length from between horns to end of præmaxillæ Io inches, its greatest width across the orbits $6 \frac{3}{4}$ inches; height at the shoulder, 37 inches; length of horns round curve, 33 inches, their circumference $12^{\frac{3}{4}}$ inches.

Adult male, Mus. Strassburg. Only differs from the former in being considerably paler in colour. Length of horns 27 inches, their circumference $13 \frac{1}{2}$ inches.

Adult female, Mus. Lugd. Is darker than the male, and has short compressed horns, about 9 inches in length. The distribution of colour is the same as in the male.

Range.-Kamschatka (Eschscholtz); Stanovoi Mountains, as far south as the sources of the Utschur (Middendorff).

Habitat-Kamschatka. 
KAIMSCHATKA WILD SHEEP Or WILD SHEEP OF ALASKA (Ovis nivicola)-continued.

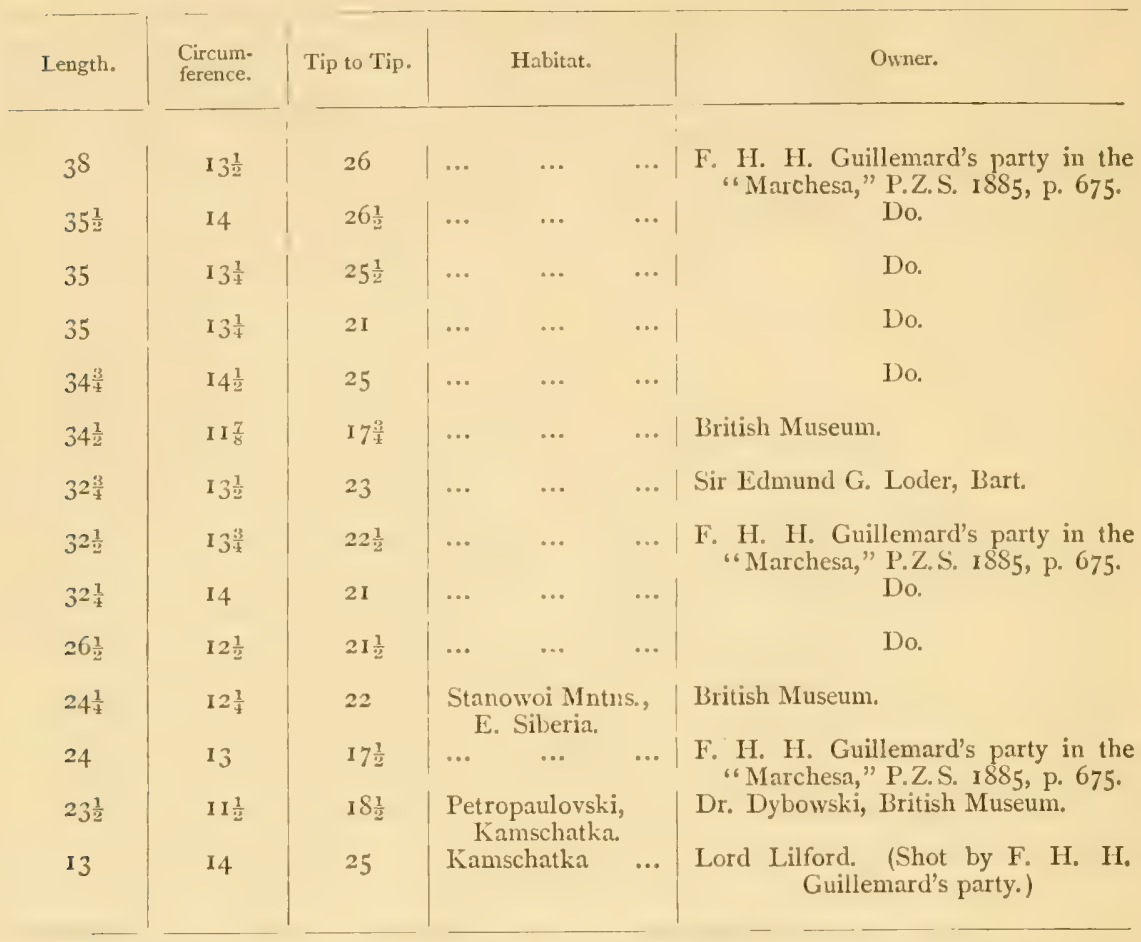

The horns can be readily distinguished from O. Montana by the slenderness at the points. 


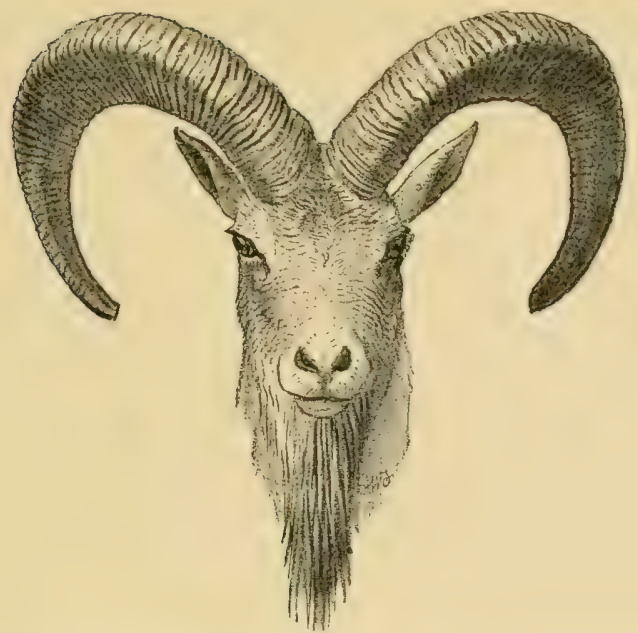

OORIAL (Ovis cycloceros)-SHAPOO (Ovis vignei). Habitat-North-West India.

\begin{tabular}{|c|c|c|c|c|c|c|}
\hline Length. & $\begin{array}{l}\text { Circum- } \\
\text { ference. }\end{array}$ & Tip to Tip. & & fabitat. & & Owner. \\
\hline$*_{3} 8 \frac{5}{8}$ & $12 \frac{1}{4}$ & $\operatorname{II} \frac{1}{4}$ & India & $\ldots$ & ... & J. Carr-Saunders. \\
\hline$*_{37 \frac{1}{3}}$ & $10 \frac{1}{4}$ & $\begin{array}{c}\text { II } \\
\text { (about) }\end{array}$ & $\cdots$ & $\ldots$ & $\cdots$ & A. O. Hume, Private Collection. \\
\hline$*_{3} 6 \frac{3}{8}$ & $9 \frac{1}{8}$ & 17 & $\cdots$ & $\cdots$ & $\cdots \mid$ & Do. \\
\hline$* 36 \frac{1}{4}$ & II $\frac{1}{4}$ & $\cdots$ & $\cdots$ & $\cdots$ & $\cdots$ & Do. \\
\hline$* 35 \frac{1}{2}$ & $10 \frac{1}{2}$ & 16 & Gulran, & Afghani: & $\operatorname{stan}$ & Dr. J. Aitchison, British Museum. \\
\hline$* 33 \frac{5}{8}$ & $9 \frac{1}{8}$ & $\ldots$ & $\ldots$ & $\cdots$ & $\cdots$ & A. O. Hume, Private Collection. \\
\hline$* 33 \frac{1}{8}$ & I I $\frac{1}{2}$ & $10 \frac{5}{8}$ & $\ldots$ & $\cdots$ & $\ldots$ & Sir Edmund G. Loder, Bart. \\
\hline$* 32 \frac{1}{2}$ & II & 8 & $\ldots$ & $\cdots$ & $\cdots$ & Blyth, P.Z.S. I 840 , p. 7 I. \\
\hline$\dagger 32 \frac{1}{2}$ & IO & $\operatorname{II} \frac{1}{2}$ & $\ldots$ & $\cdots$ & $\cdots$ & Sir Victor Brooke's Collection. \\
\hline$*_{32 \frac{1}{4}}$ & $10 \frac{1}{2}$ & $I I \frac{3}{4}$ & $\cdots$ & $\cdots$ & $\ldots$ & Do. \\
\hline$* 32 \frac{1}{4}$ & 10 & II $\frac{1}{2}$ & $\cdots$ & $\cdots$ & $\cdots$ & Hume Collection, British Museum. \\
\hline$\dagger 3 I^{\frac{3}{4}}$ & $9 \frac{1}{4}$ & $5 \frac{1}{4}$ & $\cdots$ & $\cdots$ & $\cdots$ & Do. \\
\hline$\dagger 3 I \frac{1}{4}$ & $8 \frac{1}{2}$ & I6 & $\cdots$ & $\cdots$ & $\cdots$ & Do. \\
\hline$*_{3} \mathbf{r}$ & 12 & $9 \frac{3}{4}$ & ... & ... & $\ldots$ & A. O. Hume, Private Collection. \\
\hline
\end{tabular}


OORIAL (Ovis cycloceros)-SHAPOO (Ovis vignei)-continud.

\begin{tabular}{|c|c|c|c|c|c|c|}
\hline Length. & $\begin{array}{l}\text { Circum- } \\
\text { ference. }\end{array}$ & Tip to Tip. & & Tabitat. & & Owner. \\
\hline$\dagger 3 I$ & 9 & $\ldots$ & $\cdots$ & $\ldots$ & $\ldots$ & Col. Western. \\
\hline$* 3 \circ \frac{7}{8}$ & $10 \frac{1}{2}$ & I3 & Ladak & $\ldots$ & $\ldots \mid$ & R. Lydekker, British Museum. \\
\hline$\dagger 30 \frac{7}{8}$ & $9 \frac{7}{8}$ & $20 \frac{1}{8}$ & Jhelum, & Salt $R$ & ange ! & Sir Victor Brooke's Collection. \\
\hline$* 30 \frac{1}{2}$ & 12 & $\ldots$ & $\ldots$ & $\ldots$ & $\ldots !$ & Rowland Ward's Collection. \\
\hline$*_{30} \frac{1}{2}$ & $\ldots$ & $\mid \begin{array}{c}\text { (owner's } \mid \\
\text { measurement) }\end{array}$ & ... & $\cdots$ & $\ldots$ & C. Hunter. \\
\hline$* 30 \%$ & $10 \frac{3}{1}$ & $10 \frac{1}{2}$ & $\cdots$ & $\cdots$ & $\ldots$ & Hume Collection, British Museum. \\
\hline$* 30$ & II $\frac{3}{4}$ & 16 & $\ldots$ & $\ldots$ & $\ldots$ & Hon. Walter Rothschild. \\
\hline$\dagger 30$ & IO.1. & 10 & $\ldots$ & $\cdots$ & $\ldots$ & IIume Collection, British Museum. \\
\hline$\uparrow 30$ & $9 \frac{3}{5}$ & I I $\frac{1}{1}$ & $\cdots$ & $\cdots$ & $\cdots$ & Sir Victor Brooke's Collection. \\
\hline$* 30$ & $S_{\frac{3}{4}}$ & 14 & $\ldots$ & $\cdots$ & $\ldots$ & P'rof. Oldham, British Museun. \\
\hline$\dagger 29 \frac{7}{5}$ & $\mathrm{IO}_{\mathrm{s}}^{3}$ & $\cdots$ & $\ldots$ & $\cdots$ & $\ldots$ & II. C. V. Hunter. \\
\hline$+293_{4}^{3}$ & $9 \frac{1}{t}$ & $8: 3$ & \multicolumn{2}{|c|}{ Punjaub... } & $\ldots$ & British Museum. \\
\hline$+29 \frac{3}{8}$ & $9 \frac{7}{8}$ & $14 \frac{1}{2}$ & ... & $\cdots$ & $\ldots$ & Hume Collection, British Museum. \\
\hline$* 29 \frac{1}{4}$ & $10 \frac{7}{8}$ & $13 \frac{1}{2}$ & $\cdots$ & $\cdots$ & $\ldots$ & Do. \\
\hline$* 29 \frac{1}{4}$ & $10 \frac{7}{8}$ & $11 \frac{3}{4}$ & ... & $\cdots$ & $\ldots$ & British Museum. \\
\hline$\nmid 29 \frac{1}{t}$ & 9 & $13 \frac{1}{2}$ & $\ldots$ & $\cdots$ & $\ldots$ & Hume Collection, British Museum. \\
\hline$* 29$ & II $\frac{5}{8}$ & $\mathbf{I} S_{\frac{1}{2}}^{1}$ & $\cdots$ & $\ldots$ & $\ldots$ & Do. \\
\hline $\begin{array}{l}* 29 \\
\text { (shortest) }\end{array}$ & I I $\frac{1}{8}$ & $\ldots$ & $\ldots$ & $\cdots$ & $\ldots$ & Sir Robert Harvey, Bart. \\
\hline $28 \frac{1}{3}$ & $8 \frac{5}{x}$ & $19 \frac{1}{3}$ & Ladak & $\cdots$ & $\ldots \mid$ & Prof. Oldham, British Museum. \\
\hline$*_{2} S_{\frac{1}{4}}$ & 9 & $15 \frac{1}{2}$ & \multicolumn{3}{|c|}{ Kusan, Afghanistan | } & Dr. J. Aitchison, British Museum. \\
\hline$*_{27 \frac{1}{2}}$ & $11 \frac{1}{8}$ & I $8 \frac{1}{4}$ & $\ldots$ & $\cdots$ & $\cdots$ & Martyn Kennard. \\
\hline $127 \frac{3}{5}$ & $9 \frac{3}{4}$ & $17 \frac{1}{2}$ & $\ldots$ & $\ldots$ & $\ldots$ & Hume Collection, British Museum. \\
\hline$*_{27}$ & II $1 \frac{3}{8}$ & I $\$ \frac{1}{8}$ & \multicolumn{3}{|c|}{ Cya Mullah, Ladak } & $\begin{array}{l}\text { Sir Victor Brooke's Collection, } \\
\text { I } 875 \text {. }\end{array}$ \\
\hline$*_{2} 6_{4}^{3}$ & 103 & $12 \frac{5}{6}$ & $\ldots$ & $\cdots$ & $\cdots$ & Martyn Kennard. \\
\hline$\dagger 26 \frac{1}{2}$ & $10 \frac{1}{2}$ & 13 & Ladak & $\cdots$ & $\ldots$ & St. George Littledale, $\mathbf{I} S_{77}$. \\
\hline$*_{26 \frac{3}{2}}$ & $10 \frac{3}{8}$ & II $\frac{5}{8}$ & $\ldots$ & $\ldots$ & $\ldots$ & Martyn Kennard. \\
\hline$*_{26} 6 \frac{1}{2}$ & $9 \frac{1}{2}$ & $23 \frac{1}{2}$ & ... & $\cdots$ & $\cdots$ & Sir Victor Brooke's Collection. \\
\hline
\end{tabular}


OORIAL (Ovis cycloceros)-SHAPOO (Ovis vignei)-continucd.

\begin{tabular}{|c|c|c|c|c|c|c|}
\hline Lengtll. & $\begin{array}{l}\text { Circum- } \\
\text { ference. }\end{array}$ & Tip to Tip. & \multicolumn{3}{|c|}{ Habitat. } & Owner. \\
\hline$\nmid 26 \frac{1}{4}$ & II $\frac{1}{2}$ & $12 \frac{3}{4}$ & \multicolumn{3}{|c|}{ Ladak ... } & St. George Littledale, $\mathbf{I} \$ 77$. \\
\hline$* 26$ & $10 \frac{1}{2}$ & $17 \frac{1}{2}$ & ... & $\cdots$ & ... & Martyn Kennard. \\
\hline$*_{26}$ & $9 \frac{1}{4}$ & $13 \frac{1}{8}$ & $\cdots$ & $\cdots$ & $\ldots$ & Do. \\
\hline$\dagger 26$ & (owner's' & ... & \multicolumn{3}{|c|}{$\begin{array}{l}\text { Kolet Khoond, Salt } \\
\text { Range. }\end{array}$} & Capt. A. A. Kinloch. \\
\hline$*_{25 \frac{3}{4}}$ & $10 \frac{7}{8}$ & 19 & $\cdots$ & $\cdots$ & $\cdots$ & Hume Collection, British Museum. \\
\hline$* 25 \frac{1}{2}$ & II $\frac{1}{t}$ & $15 \frac{5}{8}$ & $\cdots$ & $\cdots$ & $\cdots$ & Hon. Charles Ellis. \\
\hline$*_{25 \frac{1}{2}}$ & $9 \frac{3}{4}$ & 12 & $\cdots$ & $\cdots$ & $\cdots$ & British Museum. \\
\hline$\uparrow 25 \frac{3}{8}$ & $9^{\frac{3}{4}}$ & $13 \frac{3}{4}$ & $\cdots$ & $\cdots$ & $\cdots$ & Sir Edmund G. Loder, Bart. \\
\hline$\uparrow 25 \frac{1}{8}$ & $9 \frac{5}{8}$ & $17 \frac{1}{2}$ & \multicolumn{3}{|c|}{ Kohrod, Persia ... } & Sir Victor Brooke's Collection. \\
\hline$* 24 \frac{3}{4}$ & $10 \frac{1}{2}$ & 18 & $\cdots$ & $\cdots$ & $\cdots$ & British Museum. \\
\hline$* 24 \frac{5}{8}$ & $9 \frac{7}{8}$ & $20 \frac{1}{2}$ & $\cdots$ & $\cdots$ & $\cdots$ & Martyn Kennard. \\
\hline$+24 \frac{1}{2}$ & $8 \frac{7}{8}$ & $\cdots$ & $\cdots$ & $\cdots$ & $\cdots$ & H. C. V. Hunter. \\
\hline$*_{24 \frac{3}{8}}$ & $9 \frac{1}{8}$ & 13 & $\cdots$ & $\cdots$ & $\cdots$ & Martyn Kennard. \\
\hline$\nmid 23 \frac{7}{8}$ & 9 & $10 \frac{1}{3}$ & $\cdots$ & $\cdots$ & $\cdots$ & Hume Collection, British Museum. \\
\hline$*_{2} 3 \frac{3}{4}$ & I I & I 8 & Astor & $\ldots$ & $\cdots$ & $\begin{array}{l}\text { Sir Victor Broolie's Collection, } \\
\text { I } 875 \text {. }\end{array}$ \\
\hline$\nmid 23 \frac{1}{2}$ & IO & ... & $\cdots$ & $\cdots$ & $\cdots$ & Col. Marten. \\
\hline$\dagger 23 \frac{3}{5}$ & 87 & $9 \frac{5}{8}$ & $\cdots$ & $\cdots$ & $\cdots$ & H. C. V. Hunter. \\
\hline$+23 \frac{1}{8}$ & $8 \frac{7}{8}$ & $\cdots$ & $\cdots$ & $\cdots$ & $\cdots$ & Do. \\
\hline$*_{23}$ & $10 \frac{7}{8}$ & $20 \frac{1}{4}$ & $\cdots$ & $\cdots$ & $\cdots$ & Martyn Kecnard. \\
\hline$\uparrow 23$ & $10 \frac{9}{4}$ & $15 \frac{3}{4}$ & $\cdots$ & $\cdots$ & $\cdots$ & Sir Robert Harvey, Bart. \\
\hline$\dagger 23$ & $9 \frac{1}{2}$ & $\cdots$ & $\cdots$ & $\cdots$ & $\cdots$ & H. C. V. Hunter, I $S 89$. \\
\hline$\dagger 22 \frac{3}{x}$ & 97 & $16 \frac{7}{8}$ & $\cdots$ & $\cdots$ & $\cdots$ & Sir Robert Harvey, Bart. \\
\hline$\left\lceil 22 \frac{8}{4}\right.$ & $9 \frac{1}{4}$ & $\begin{array}{c}16 \\
\text { (tips broken) }\end{array}$ & $\cdots$ & $\ldots$ & $\cdots$ & Rowland Ward's Collection. \\
\hline$\dagger 22 \frac{1}{4}$ & $8 \frac{1}{2}$ & 20 & Punjauk & & $\cdots$ & British Museum. \\
\hline$\dagger 22$ & $10 \frac{1}{2}$ & 20 & $\cdots$ & $\cdots$ & $\cdots$ & Capt. M. Murphy. \\
\hline$* 21 \frac{1}{8}$ & $10 \frac{5}{8}$ & I $9 \frac{1}{4}$ & $\cdots$ & $\cdots$ & $\cdots$ & British Museum. \\
\hline$*_{20}$ & $10 \frac{5}{8}$ & $19 \frac{1}{2}$ & Astor & $\cdots$ & $\cdots$ & $\begin{array}{l}\text { Sir Victor Brooke's Collection, } \\
\text { I } 875 \text {. }\end{array}$ \\
\hline
\end{tabular}




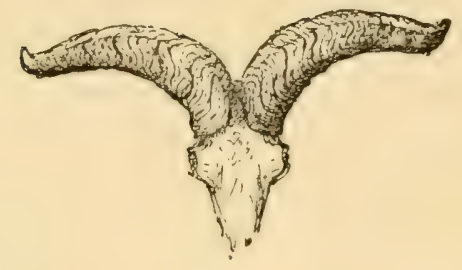

\section{BURHEL (Ovis nahura).}

Habitat-Himalayas.

\begin{tabular}{|c|c|c|c|c|c|c|}
\hline Length. & $\begin{array}{l}\text { Circum- } \\
\text { ference. }\end{array}$ & Tip to Tip. & & Cabitat. & & Owner. \\
\hline 32 & ... & .. & India & $\cdots$ & ... & B. H. Hodgson, P.Z.S. I840, p. 66 . \\
\hline $30 \frac{7}{8}$ & $12 \frac{1}{4}$ & $21 \frac{7}{8}$ & Do. & ... & $\ldots$ & A. O. Hume, Private Collection. \\
\hline 30 & $12 \frac{1}{4}$ & $22 \frac{1}{2}$ & Do. & $\cdots$ & $\ldots$ & Do. \\
\hline $29 \frac{1}{2}$ & II $\frac{5}{8}$ & $25 \frac{1}{2}$ & Do. & $\cdots$ & $\cdots$ & H.R.H. Duke of Edinburgh. \\
\hline 28 & II & $20 \frac{1}{4}$ & Do. & $\cdots$ & $\cdots$ & IIume Collection, British Museum. \\
\hline $27 \frac{3}{4}$ & $10 \frac{1}{4}$ & IO & Do. & $\cdots$ & $\cdots$ & Capt. G. Campbell. \\
\hline $27 \frac{1}{4}$ & I I & $21 \frac{1}{2}$ & Ladak & $\cdots$ & $\cdots$ & St. Geo. Littledale, I 877 . \\
\hline $26 \frac{1}{4}$ & II $\frac{7}{8}$ & 23 & India & $\cdots$ & $\cdots$ & Sir Victor Brooke's Collection. \\
\hline $26 \frac{1}{4}$ & 12 & $21 \frac{1}{4}$ & Do. & $\cdots$ & $\cdots$ & Hon. Walter Rothschild. \\
\hline $26 \frac{1}{4}$ & $\mathrm{IO}_{4}^{3}$ & 22 & Do. & ... & ... & Sir Edmund G. Loder, Bart. \\
\hline $26 \frac{1}{8}$ & $12 \frac{3}{6}$ & 25 & Do. & $\cdots$ & $\cdots$ & Hume Collection, British Museum. \\
\hline 26 & 12 & $20 \frac{1}{2}$ & Do. & ... & ... & Rowland Ward's Collection. \\
\hline $25 \frac{1}{2}$ & I I $\frac{1}{2}$ & ... & Do. & $\cdots$ & ... & $\begin{array}{l}\text { Zoo. Society's Museum (P.Z.S. IS40, } \\
\text { p. } 67 \text { and } 68 \text { ). }\end{array}$ \\
\hline $25 \frac{3}{8}$ & I I $\frac{1}{4}$ & $25 \frac{1}{t}$ & Ladak & $\cdots$ & $\cdots$ & St. George Littledale, 1877 . \\
\hline $24 \frac{1}{2}$ & $12 \frac{1}{4}$ & 26 & India & $\cdots$ & $\cdots$ & J. Carr-Saunders. \\
\hline 24 & II & ... & Ladak & $\ldots$ & ... & $\begin{array}{l}\text { Mr. Leadbeater, Zoo. Society's Mu- } \\
\text { seum (P.Z.S. I } 840 \text {, p. } 67 \text { and 68). }\end{array}$ \\
\hline 24 & 10 & $22 \frac{1}{4}$ & India & $\cdots$ & $\cdots$ & Kowland Waras Conection. \\
\hline 24 & I I & $22 \frac{3}{4}$ & Do. & $\cdots$ & $\cdots$ & B. H. Hodgson, British Museum. \\
\hline $23^{\frac{3}{4}}$ & $10 \frac{5}{8}$ & $26 \frac{1}{8}$ & Ladak & $\cdots$ & $\cdots$ & R. Lydekker, British Museum. \\
\hline $23 \frac{3}{8}$ & II $\frac{3}{4}$ & $24 \frac{1}{t}$ & India & $\cdots$ & $\cdots$ & Sir Robt. Harvey, Bart. \\
\hline
\end{tabular}


BURHEL (Ovis nahura)-continued.

\begin{tabular}{|c|c|c|c|c|c|c|}
\hline Length. & $\begin{array}{l}\text { Circum- } \\
\text { ference. }\end{array}$ & Tip to Tip. & & Fabitat. & & Owner. \\
\hline $23^{\frac{1}{4}}$ & II $\frac{1}{4}$ & $25 \frac{3}{4}$ & India & $\cdots$ & $\cdots$ & Hume Collection, British Museum. \\
\hline 23 & 12 & 26 & Do. & $\ldots$ & $\ldots$ & H.R.H. Duke of Edinburgh. \\
\hline $22 \frac{3}{8}$ & $10^{3}$ & $22 \frac{3}{4}$ & Do. & $\cdots$ & $\ldots$ & B. H. Hodgson, British Museum. \\
\hline $22 \frac{1}{4}$ & $10 \frac{1}{2}$ & 23 & Ladak & $\cdots$ & $\cdots$ & St. George Littledale, 1877 . \\
\hline $22 \frac{1}{8}$ & $10 \frac{5}{8}$ & $\cdots$ & India & $\cdots$ & $\ldots$ & Sir Robert Harvey, Bart. \\
\hline 22 & $\cdots$ & $\cdots$ & Do. & $\cdots$ & $\ldots$ & Do. \\
\hline 22 & $10 \frac{5}{8}$ & $25 \frac{1}{2}$ & Do. & $\cdots$ & $\cdots$ & Do. \\
\hline 22 & $10 \frac{1}{2}$ & $21 \frac{1}{4}$ & Nepal & $\cdots$ & $\cdots$ & B. H. Hodgson, British Museum. \\
\hline 22 & II & 24 & $\cdots$ & $\cdots$ & $\cdots$ & Capt. G. Campbell. \\
\hline $21 \frac{3}{4}$ & $10 \frac{1}{4}$ & 23 & India & $\cdots$ & $\ldots$ & Capt. G. McMicking. \\
\hline $2 \mathrm{I} \frac{3}{4}$ & $11 \frac{3}{8}$ & $25 \frac{3}{4}$ & Do. & $\cdots$ & $\cdots$ & Hume Collection, British Museum. \\
\hline $2 \mathrm{I} \frac{1}{2}$ & II & $\cdots$ & Do. & $\cdots$ & $\cdots$ & H. C. V. Hunter. \\
\hline $21 \frac{1}{8}$ & I I & $23^{\frac{3}{t}}$ & Do. & $\cdots$ & $\cdots$ & Hume Collection, British Museum. \\
\hline 20 & IO & 25 & Do. & $\cdots$ & $\ldots$ & $\begin{array}{l}\text { Zoo. Society's Museum (P.Z.S. } 1840 \text {, } \\
\text { p. } 67 \text { and } 68 \text { ). }\end{array}$ \\
\hline $19 \frac{7}{8}$ & $10 \frac{3}{8}$ & $23^{\frac{1}{4}}$ & Ladak & $\cdots$ & $\cdots$ & St. George Littledale, I 877 . \\
\hline $19 \frac{1}{4}$ & $10 \frac{1}{2}$ & 24 & India & $\cdots$ & $\cdots$ & B. H. Hodgson, British Museum. \\
\hline $19 \frac{1}{4}$ & I I $\frac{1}{4}$ & 27 & Thibet & $\cdots$ & $\cdots$ & Sir Victor Brooke's Collection. \\
\hline I9 & $10 \frac{1}{2}$ & 26 & India & $\cdots$ & $\cdots$ & Reginald Beech. \\
\hline $18 \frac{3}{4}$ & II $\frac{7}{8}$ & $24 \frac{1}{2}$ & Nepal & $\cdots$ & $\cdots$ & B. H. Hodgson, British Museum. \\
\hline
\end{tabular}

OVIS ARGALI.

Habitat-India.

\begin{tabular}{|c|c|c|c|c|c|c|}
\hline Length. & $\begin{array}{l}\text { Circum- } \\
\text { ference. }\end{array}$ & Tip to Tip. & & abitat. & & Owner. \\
\hline $40 \frac{1}{2}$ & 17 & I I & Nepal & $\cdots$ & $\ldots$ & B. H. Hcdgson, British Museum. \\
\hline $37 \frac{1}{4}$ & I6 & $17 \frac{1}{2}$ & Do. & $\ldots$ & $\cdots$ & Do. \\
\hline
\end{tabular}




\section{MOUFFLON (Ovis musimon).*}

[BLYTH, P.Z.S. 1840 , p. 72.]

IT is unnecessary to give a detailed description of this beautiful little species, though I may mention that the fine living male in the gardens measures 39 in. from nose to tail, the tail 5 in.; from nose to base of horn 7 in.; ears 4 in.; neck, from posterior base of horn to the abrupt angle of its insertion, 8 in., and thence to base of tail $2 \mathrm{I}$ in.; height at the shoulder $2 \frac{1}{4} \mathrm{ft}$.

The female has seldom any horns, which, when they exist, are ordinarily about 2 in. long.

\section{Habitat-Sardinia and Corsica.}

\begin{tabular}{|c|c|c|c|c|c|}
\hline Length & $\begin{array}{l}\text { Circum- } \\
\text { ference. }\end{array}$ & Tip to Tip. & \multicolumn{2}{|l|}{ Habitat. } & Owner. \\
\hline 287 & $8 \frac{1}{8}$ & ... & Sardinia & $\ldots$ & E. N. Buxton. \\
\hline $2 S$ & $8_{4}^{3}$ & $9 \frac{3}{3}$ & Do. $\ldots$ & $\ldots$ & Do. \\
\hline 26 & 7 & 12 & $\ldots \quad \ldots$ & $\cdots$ & Blyth, P.Z.S. IS 40 , pt. 8, p. 72. \\
\hline $25 \frac{1}{2}$ & $8 \frac{1}{4}$ & 10 & $\ldots$ & .. & Hon. Walier Rothschild. \\
\hline $24 \frac{1}{2}$ & $9 \frac{1}{8}$ & $9 \frac{7}{8}$ & $\begin{array}{l}\text { South - West } \\
\text { dinia. }\end{array}$ & Sar- & Sir Victor Brooke's Collection. \\
\hline 24 & 9 & 12 & $\cdots$ & $\cdots$ & R. J. Kennedy. \\
\hline $22 \frac{7}{8}$ & S & $5 \frac{1}{8}$ & $\ldots$ & $\cdots$ & Zoological Society, British Museum. \\
\hline $2 I \frac{1}{4}$ & $8 \frac{7}{8}$ & $\ldots$ & Sardinia & $\ldots$ & E. N. Buxton. \\
\hline $2 I$ & $8 \frac{1}{4}$ & 9 & South Sardinia & a $\ldots$ & Sir Victor Brooke's Collection. \\
\hline 21 & $8 \frac{1}{4}$ & 6 & $\ldots$ & $\cdots$ & Blyth, P.Z.S. I $\delta_{40}$, pt. S, p. 72. \\
\hline $20 \frac{3}{4}$ & $7 \frac{5}{8}$ & 12 & North Africa & $\cdots$ & British Museum. \\
\hline $8 \frac{5}{8}$ & $5^{\frac{7}{5}}$ & $9 \frac{5}{8}$ & Sardinia & $\cdots$ & E. N. Buxton. \\
\hline
\end{tabular}

* The Moufflon sheep of Corsica and Sarclinia, but not, there is reason to suspect, of the Levantine Countries. 
CYPRIAN WILD SHEEP OY MOUFFLON (Ovis ophion).

Habitat-Cyprus.

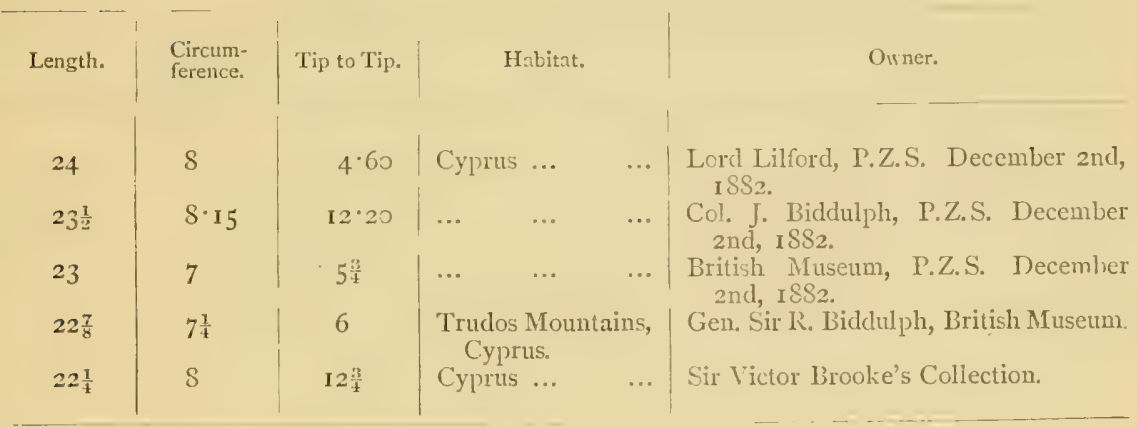

AOUDAD (Ovis tragelaphus).

[BLyTh, P.Z.S. I840, p. 75.]

THIs animal appears to vary considerably in size-some exceeding a Fallow Deer in stature, while others are much smaller. It has no beard on the chin, like the true goats, but is remarkable for the long hanging hair in front of the neck and on the upper part of the fore-limbs, the former attaining in fine males to about a foot in length, and the latter to 9 in.; there is also some lengthened hair at the setting on of the head, and a dense nuchal mane, the hairs of which are 3 in. long, continued over the withers till lost about the middle of the back; general colour yellow-brown; horns moderately stout, turning outwards, backwards, and so inwards, with the tips inclining towards each other.

This species is well known as the "Aoudad" of the Moors, and the "Kebsh" of the Egyptians; it is also, according to Rüppell, the "Tedal" of the inhabitants of Nubia, which is doubtless the same as "Teybal," applied by Burkhardt to the wild goat of that region, in addition to the word "Beden," which (in common with Rüppell and others) he also assigns to the latter. 
AOUDAD (Ovis tragelaphus)-continuct.

Habitat-Mountain-ranges of North Africa.

\begin{tabular}{|c|c|c|c|c|c|}
\hline Length. & $\begin{array}{l}\text { Circum- } \\
\text { ference at } \\
\text { base. }\end{array}$ & Tip to Tip. & Habitat. & & Owner. \\
\hline $26 \frac{3}{3}$ & $1 \mathrm{O}_{4}^{\frac{3}{4}}$ & $15 \frac{1}{4}$ & North Africa & $\ldots$ & British Museum. \\
\hline 26 & II & $17^{\frac{3}{4}}$ & Do. & $\ldots$ & Do. \\
\hline 26 & $10 \frac{7}{8}$ & ... & Do. & $\ldots$ & Do. \\
\hline 25 & $10 \frac{1}{2}$ & 15 & Do. & $\ldots$ & Blyth, P.Z.S. I 840, p. 76. \\
\hline 23 & II $\frac{1}{8}$ & 5 & Do. & $\ldots$ & British Museum. \\
\hline $22 \frac{1}{2}$ & $10 \frac{3}{4}$ & $12 \frac{3}{4}$ & Do. & $\cdots$ & Lord Edward Cecil. \\
\hline $19 \frac{7}{8}$ & I I $\frac{1}{4}$ & $14 \frac{1}{4}$ & Do. & $\cdots$ & British Musenm. \\
\hline $19 \frac{1}{4}$ & $9 \frac{1}{4}$ & $16 \frac{3}{4}$ & Djobel Matlili & $\ldots$ & E. N. Buxton. \\
\hline I $8 \frac{1}{2}$ & II $\frac{5}{8}$ & $18 \frac{1}{2}$ & North Africa & $\ldots$ & Sir Edmund G. Loder, Bart. \\
\hline I $S_{\frac{1}{4}}$ & $7 \frac{1}{2}$ & $14 \frac{1}{1}$ & Do. & $\ldots$ & Rowland Ward's Collection. \\
\hline I 8 & $7 \frac{3}{4}$ & 15 & Do. & $\cdots$ & British Museum. \\
\hline $16 \frac{3}{4}$ & $7 \frac{7}{8}$ & $16 \frac{3}{4}$ & Do. & $\ldots$ & Do. \\
\hline $16 \frac{1}{2}$ & $7 \frac{1}{4}$ & 15 & Djobel Matlili & $\ldots$ & E. N. Buxton. \\
\hline 16 & $7 \frac{1}{2}$ & $17 \frac{1}{ \pm}$ & North Africa & $\ldots$ & Blyth, P.Z.S. I 840 , p. $7^{6}$. \\
\hline
\end{tabular}

ASIA MINOR SHEEP (Ovis gmelini).

Habitat-Persia and Asia Minor.

\begin{tabular}{|c|c|c|c|c|c|c|}
\hline Length. & $\begin{array}{l}\text { Circum- } \\
\text { ference. }\end{array}$ & Tip to Tip. & & Habitat. & & Owner. \\
\hline $40 \frac{1}{x}$ & $10 \frac{1}{2}$ & $5 \frac{1}{2}$ & $\ldots$ & $\cdots$ & $\cdots$ & $\begin{array}{l}\text { W. Burchart Barker, British Museum, } \\
\text { P.Z.S. Feb. I } 880 \text {. }\end{array}$ \\
\hline $36 \frac{1}{x}$ & $10 \frac{3}{8}$ & $5 \frac{3}{4}$ & $\cdots$ & $\cdots$ & .. & British Museum. \\
\hline $30 \frac{1}{2}$ & $10 \frac{5}{8}$ & IS & $\ldots$ & $\cdots$ & $\ldots$ & Sir Victor Brooke's Collection. \\
\hline $29 \frac{5}{8}$ & $9 \frac{1}{8}$ & I $1 \frac{3}{4}$ & $\ldots$ & $\ldots$ & $\ldots$ & Do. \\
\hline
\end{tabular}


ASIA MINOR SHEEP (Ovis gmelini)-continued.

\begin{tabular}{|c|c|c|c|c|c|c|}
\hline Length. & $\begin{array}{l}\text { Circum- } \\
\text { ference. }\end{array}$ & Tip to Tip. & & Habitat & & Owner. \\
\hline $26 \cdot 10$ & $8 \frac{1}{2}$ & $12 \cdot 20$ & ... & $\cdots$ & $\ldots$ & C. G. Danford, P.Z.S. Feb. I88o. \\
\hline $24^{\circ} 40$ & Io & $12 \cdot 80$ & $\ldots$ & $\ldots$ & $\ldots$ & Do. \\
\hline $23 \frac{1}{8}$ & 9 & $15 \frac{1}{2}$ & ... & ... & $\ldots$ & Sir Victor Brooke's Collection. \\
\hline $2 I^{\circ} 40$ & I $0 \frac{1}{t}$ & $2 \mathrm{I}$ & ... & $\ldots$ & $\cdots$ & British Museum, P.Z.S. Feb, I88o. \\
\hline 20 & 10 & $2 \mathrm{I}$ & $\cdots$ & $\cdots$ & ... & Blyth, P.Z.S. I840, p. 7o. \\
\hline I $8 \frac{1}{2}$ & $8 \frac{1}{2}$ & I I & $\ldots$ & $\ldots$ & $\ldots$ & British Museum, P.Z.S. Feb. I88o. \\
\hline
\end{tabular}

DOIMESTIC SHEEP (Ovis aries).

\begin{tabular}{|c|c|c|c|c|c|}
\hline Length. & $\begin{array}{l}\text { Circum- } \\
\text { ference. }\end{array}$ & Tip to Tip. & \multicolumn{2}{|l|}{ Habitat. } & Owner. \\
\hline $39 \frac{1}{2}$ & $8 \frac{3}{4}$ & $2 \mathrm{I}$ & ... & $\cdots$ & H. E. Surtees. \\
\hline $35^{\frac{1}{2}}$ & 8 & $16 \frac{7}{8}$ & ... & ... & Sir Victor Brooke's Collection. \\
\hline 33 & II & $22 \frac{1}{4}$ & Yarkund & $\cdots$ & Hume Collection, British Museum. \\
\hline 32 & $8 \frac{5}{8}$ & $27 \frac{3}{4}$ & Do. & .. & Do. \\
\hline 32 & $8 \frac{3}{8}$ & $17 \frac{7}{8}$ & ... & ... & British Museum. \\
\hline $28 \frac{3}{4}$ & 8 & $2 I \frac{1}{2}$ & Scotland & $\ldots$ & Rowland Ward's Collection. \\
\hline $22 \frac{5}{8}$ & $9 \frac{1}{2}$ & $20 \frac{1}{4}$ & Yarkund & ... & Hume Collection, British Museum. \\
\hline $22 \frac{3}{8}$ & $9 \frac{1}{4}$ & $22 \frac{1}{2}$ & Do. & $\ldots$ & Do. \\
\hline I8 & $7 \frac{3}{4}$ & $16 \frac{1}{4}$ & (Fezzan sheep) & ... & British Museum. \\
\hline $17 \frac{7}{8} \times 14 \frac{1}{8}$ & $7 \frac{5}{8} \times 4 \frac{3}{1}$ & $4 \frac{1}{2} \times 6 \frac{3}{4}$ & (four horns) & .. & Hume Collection, British Museum. \\
\hline $17 \times 11 \frac{3}{4}$ & $6 \frac{3}{4} \times 5$ & $6 \times 8 \frac{1}{2}$ & Do. & $\cdots$ & British Museum. \\
\hline $13 \frac{1}{4} \times 10 \frac{7}{8}$ & $5^{\frac{3}{4}} \times 4^{\frac{1}{2}}$ & $\ldots$ & (five horns) & $\ldots$ & Hume Collection, British Museum. \\
\hline $\mathbf{I} 3 \times 9 \frac{7}{8}$ & $7 \frac{5}{8} \times 5$ & $14^{\frac{3}{4}}$ & (four horns) & $\ldots$ & A. O. Hume, Private Collection. \\
\hline $12 \frac{1}{2}$ & ... & $\ldots$ & West Africa & $\ldots$ & British Museum. \\
\hline $12 \times 9 \frac{3}{4}$ & $6 \frac{1}{2} \times 4$ & $17 \frac{1}{4}$ & (four horns) & ... & A. O. Hume, Private Collection. \\
\hline
\end{tabular}




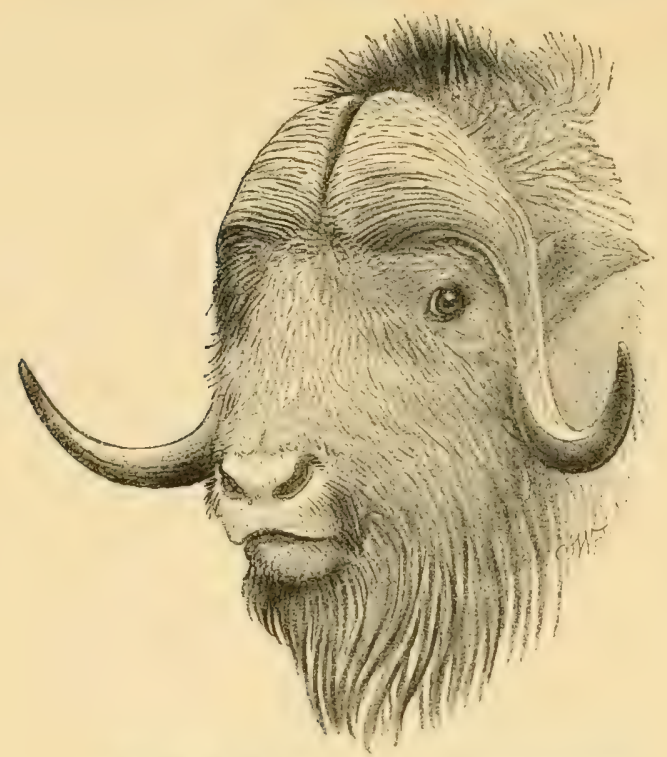

MUSK OX (Ovibos moschatus).

Habitat-Arctic Regions.

\begin{tabular}{|c|c|c|c|c|}
\hline Length. & $\begin{array}{l}\text { Breadth of } \\
\text { Palm. }\end{array}$ & Tip to Tip. & Habitat. & Owner. \\
\hline $27 \frac{1}{4}$ & $12 \frac{1}{2}$ & 27 & North America ... & Earl of Lonsdale. \\
\hline $26 \frac{7}{8}$ & II & 27 & $\begin{array}{l}\text { Barren Ground of } \\
\text { Northern Canada. }\end{array}$ & Warburton Pike. \\
\hline $26 \frac{3}{4}$ & $12 \frac{3}{5}$ & $\cdots$ & North America ... & J. Rae, British Museum. \\
\hline $26 \frac{1}{4}$ & $13 \frac{1}{8}$ & $27 \frac{\pi}{8}$ & Do. & British Museum. \\
\hline $24 \frac{3}{4}$ & II & $25 \frac{1}{2}$ & $\begin{array}{l}\text { Barren Ground of } \\
\text { Northern Canada. }\end{array}$ & Warburton Pike. \\
\hline $24 \frac{1}{2}$ & $12 \frac{3}{6}$ & $24 \frac{1}{2}$ & North America ... & British Museum. \\
\hline $24 \frac{1}{4}$ & $10 \frac{1}{2}$ & 26 & $\begin{array}{l}\text { Barren Ground of } \\
\text { Northern Canada. }\end{array}$ & Warburton Pike. \\
\hline 24 & $\begin{array}{l}20 \text { (circum- } \\
\text { ference) }\end{array}$ & 30 & North America ... & Earl of Lonsdale. \\
\hline 24 & $9 \frac{33}{4}$ & $23 \frac{1}{8}$ & Do. $\quad \ldots$ & Sir Edmund G. Loder, Bart. \\
\hline $22 \frac{3}{1}$ & $9 \frac{1}{4}$ & $19 \frac{1}{4}$ & Grinnell Land ... & $\begin{array}{l}\text { Capt. W. H. Fielden, Brilish Mu- } \\
\text { seum. }\end{array}$ \\
\hline $22 \frac{1}{2}$ & $\begin{array}{c}8 \frac{3}{4} \\
\text { (about) }\end{array}$ & $\cdots$ & Discovery Bay ... & British Museum. \\
\hline$q 18 \frac{\pi}{8}$ & $4 \frac{1}{4}$ & $\cdots$ & North America ... & A. G. Dallas, British Museun. \\
\hline q $18 \frac{1}{4}$ & $4 \frac{1}{8}$ & $\cdots$ & Do. & Do. \\
\hline$q 16$ & $3 \frac{1}{2}$ & ... & $\cdots$ & British Museum. \\
\hline
\end{tabular}




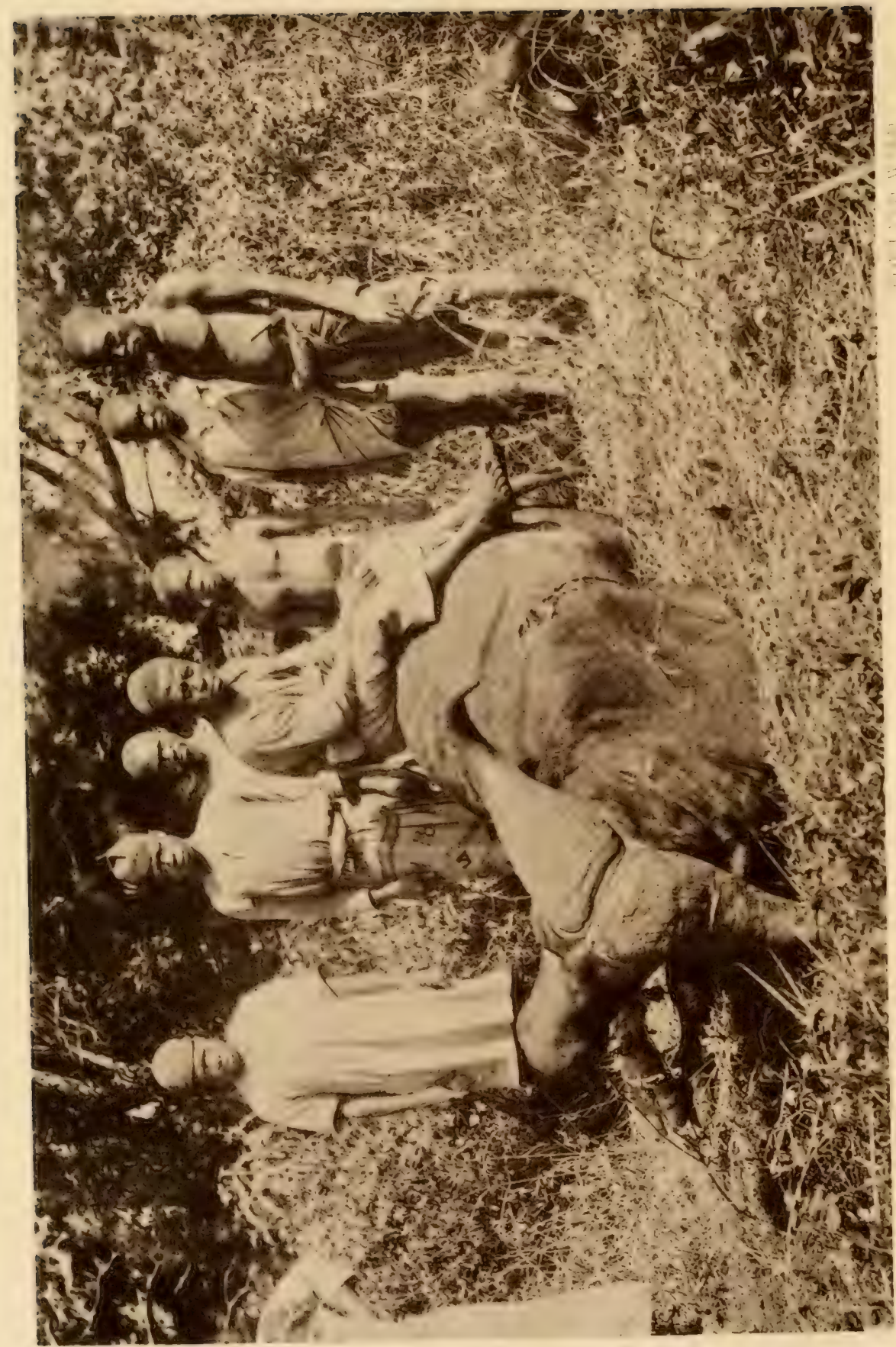




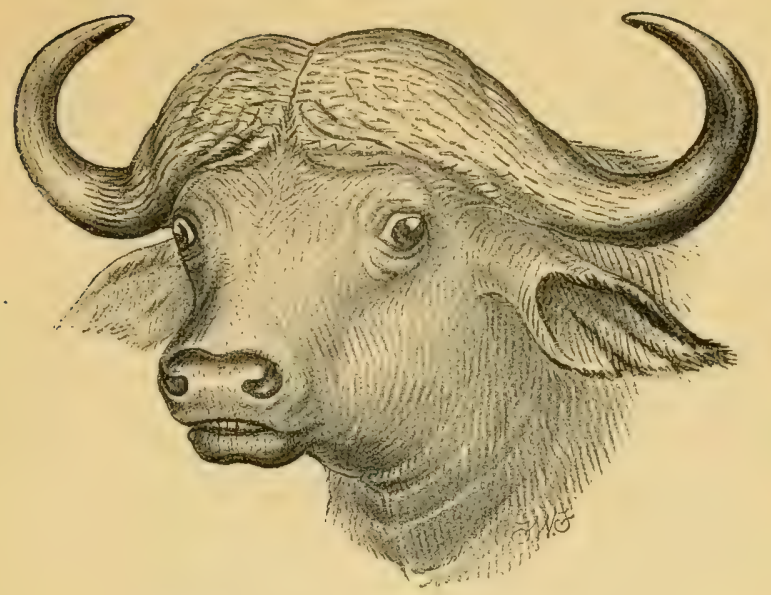

CAPE BUFFALO (Bos caffer).

Bechuana nane-" Nari."

Lower Zambesi name—“Nyati" or "Njati."

Habitat-Africa.

\begin{tabular}{|c|c|c|c|c|c|}
\hline \multicolumn{2}{|c|}{ Greatest Width. } & \multirow{2}{*}{$\begin{array}{l}\text { Tip to } \\
\text { Tip. }\end{array}$} & \multirow{2}{*}{$\begin{array}{l}\text { Width of } \\
\text { Palm. }\end{array}$} & \multirow{2}{*}{ Habitat. } & \multirow{2}{*}{ Owner. } \\
\hline Inside. & Outside. & & & & \\
\hline 42 & $\ldots$ & $\ldots$ & 17 & $\begin{array}{l}\text { Near River Ra- } \\
\text { mokwebani, } \\
\text { South Africa. }\end{array}$ & $\begin{array}{l}\text { F. C. Selous, "A Hunter's } \\
\text { Wanderings." }\end{array}$ \\
\hline$\ldots$ & $45 \frac{1}{2}$ & $\ldots$ & I $1 \frac{1}{2}$ & $\begin{array}{l}\text { Kilimanjaro, } \\
\text { East Africa. }\end{array}$ & H. C. V. Hunter. \\
\hline $41 \frac{1}{4}$ & $45^{\frac{3}{4}}$ & $37 \frac{1}{8}$ & $\cdots$ & South Africa ... & British Museum. \\
\hline 41 & $45 \frac{1}{2}$ & 30 & $\ldots$ & East Africa & W. Astor Chanler. \\
\hline $40 \frac{7}{8}$ & 47 & $\ldots$ & $12 \frac{1}{4}$ & Do. & F. J. Jackson. \\
\hline $40 \frac{1}{2}$ & $\cdots$ & 26 & $\cdots$ & Do. & Prince Boris Czetwertynski. \\
\hline 40 & $\ldots$ & $\ldots$ & 7 & $\begin{array}{l}\text { Linyanti, River } \\
\text { Cholic. }\end{array}$ & $\begin{array}{l}\text { F. C. Selous, "A Hunter's } \\
\text { Wanderings." }\end{array}$ \\
\hline $39 \frac{3}{4}$ & $45^{\frac{33}{4}}$ & $\cdots$ & $12 \frac{1}{8}$ & East $A$ frica $\quad \ldots$ & H. C. V. Hunter. \\
\hline $39 \frac{5}{8}$ & $44^{\frac{3}{4}}$ & $27 \frac{1}{2}$ & $\mathrm{II}_{4}^{1}$ & 1). $\ldots$ & Sir Robert Harvey, Bart. \\
\hline 39 & $43 \frac{7}{8}$ & $29 \frac{1}{8}$ & $13 \frac{1}{2}$ & South Africa ... & F. C. Selous. \\
\hline $38 \frac{1}{4}$ & $43^{\frac{1}{4}}$ & $24 \frac{1}{2}$ & $13 \frac{1}{4}$ & Do. & Sir John Willoughby, Bart. \\
\hline
\end{tabular}


CAPE BUFFALO (Bos caffer)-continued.

\begin{tabular}{|c|c|c|c|c|c|c|}
\hline \multicolumn{2}{|c|}{ Greatest Width. } & \multirow{2}{*}{$\begin{array}{l}\text { Tip to } \\
\text { Tip. }\end{array}$} & \multirow{2}{*}{$\begin{array}{l}\text { Width of } \\
\text { Palm. }\end{array}$} & \multirow{2}{*}{\multicolumn{2}{|c|}{ Habitat. }} & \multirow{2}{*}{ Owner. } \\
\hline Inside. & Outside. & & & & & \\
\hline$\cdots$ & 44 & ... & ... & East Africa & $\ldots$ & $\begin{array}{l}\text { Joseph Thomson, "Through } \\
\text { Masai Land." }\end{array}$ \\
\hline $38 \frac{1}{4}$ & $42 \frac{3}{8}$ & $28 \frac{1}{8}$ & $13 \frac{1}{4}$ & Do. & $\ldots$ & Sir Robert Harvey, Bart. \\
\hline 38 & $42 \frac{1}{2}$ & $\cdots$ & $12 \frac{1}{4}$ & Do. & $\ldots$ & H. C. V. Hunter. \\
\hline $37 \frac{1}{4}$ & $41 \frac{3}{4}$ & $29 \frac{5}{8}$ & I3 & South Africa & $\ldots$ & J. Carr-Saunders. \\
\hline $37^{\frac{3}{4}}$ & $43 \frac{5}{8}$ & $26 \frac{1}{2}$ & $12 \frac{3}{7}$ & East Africa & $\cdots$ & IIon. Walter Rothschild. \\
\hline $37 \frac{1}{8}$ & $41 \frac{1}{2}$ & 30 & 12 & Do. & $\cdots$ & Sir Robert Harvey, Bart. \\
\hline $37 \frac{1}{8}$ & $41 \frac{3}{4}$ & 34 & $12 \frac{3}{x}$ & Do. & $\cdots \mid$ & Do. \\
\hline 37 & 42 & 34 & $\cdots$ & Do. & $\ldots$ & Prince Stargynski. \\
\hline 37 & $41 \frac{1}{2}$ & 24 & $12 \frac{3}{4}$ & South Africa & $\ldots$ & W. C. Oswell. \\
\hline 37 & 40 & 35 & $\cdots$ & East Africa & $\cdots$ & Gen. Mathews. \\
\hline 37 & 40 & 32 & $11 \frac{7}{8}$ & Abyssinia & $\cdots$ & E. Lort-Phillips. \\
\hline $36 \frac{7}{8}$ & $4 I \frac{1}{2}$ & 28 & $\cdots$ & $\cdots \quad \ldots$ & $\cdots \mid$ & British Museum. \\
\hline $36 \frac{7}{8}$ & $40 \frac{1}{4}$ & $30 \frac{7}{8}$ & III $\frac{3}{4}$ & East Africa & $\ldots$ & Sir Robert Harvey, Bart. \\
\hline $36 \frac{3}{4}$ & $4^{1}$ & $27 \frac{5}{8}$ & $10 \frac{1}{4}$ & $\cdots$ & $\cdots$ & Bethnal Green Museum. \\
\hline $36 \frac{3}{4}$ & $42 \frac{3}{8}$ & $29 \frac{1}{2}$ & $12 \frac{1}{2}$ & $\cdots \quad \cdots$ & $\ldots$ & $\begin{array}{l}\text { Sir Victor Brooke's Collec- } \\
\text { tion. }\end{array}$ \\
\hline $36 \frac{1}{4}$ & $4 I$ & $\cdots$ & $11 \frac{3}{4}$ & East Africa & $\ldots$ & H. C. V. Hunter. \\
\hline $36 \frac{1}{8}$ & $41 \frac{1}{8}$ & $\cdots$ & $25 \frac{3}{4}$ & South Africa & $\cdots$ & British Museum. \\
\hline 36 & $\cdots$ & $2 \mathrm{I}$ & $\begin{array}{l}\text { (owner's mea- } \\
\text { surement) }\end{array}$ & $\cdots \quad \cdots$ & $\cdots$ & Sir John Willoughby, Bart. \\
\hline $3^{6}$ & $42 \frac{1}{8}$ & $\cdots$ & $22 \frac{1}{4}$ & South Africa & $\cdots$ & British Museum. \\
\hline 36 & $41 \frac{1}{2}$ & 22 & $\cdots$ & Do. & $\cdots$ & A. W. Davis. \\
\hline 36 & $41 \frac{1}{4}$ & $29 \frac{1}{2}$ & I I $\frac{3}{4}$ & Do. & $\cdots$ & A. Beit. \\
\hline 36 & $40 \frac{1}{2}$ & 26 & $\cdots$ & East Africa & $\cdots \mid$ & Prince Boris Czetwertynski. \\
\hline 36 & 40 & $28 \frac{1}{2}$ & $\mid \begin{array}{l}\text { (shot by H. } \\
\text { H. Johnson) }\end{array}$ & South Africa & $\ldots \mid$ & Sir Philip Currie, Bart. \\
\hline$\cdots$ & $4 \mathrm{I}$ & 30 & $\cdots$ & $\cdots \quad \cdots$ & $\cdots$ & Rowland Ward's Collection. \\
\hline $35^{\frac{7}{8}}$ & $4 \mathrm{I}$ & $28 \frac{3}{4}$ & $\cdots$ & Mashona Lanc & & $\begin{array}{l}\text { F. C. Selous, British Mu- } \\
\text { seum. }\end{array}$ \\
\hline $35 \frac{\frac{1}{4}}{4}$ & $4^{\circ}$ & ... & $14 \frac{5}{8}$ & East Africa & $\cdots$ & F. J. Jackson. \\
\hline
\end{tabular}




\section{CAPE BUFFALO (Bos caffer)-continued.}

\begin{tabular}{|c|c|c|c|c|c|c|}
\hline \multicolumn{2}{|c|}{ Greatest Width. } & \multirow{2}{*}{$\begin{array}{l}\text { Tip to } \\
\text { Tip. }\end{array}$} & \multirow{2}{*}{$\begin{array}{l}\text { Width of } \\
\text { Palm. }\end{array}$} & \multirow{2}{*}{\multicolumn{2}{|c|}{ Habitat. }} & \multirow{2}{*}{ Owner. } \\
\hline Inside. & Outside. & & & & & \\
\hline $35 \frac{5}{8}$ & $39 \frac{3}{t}$ & $30 \frac{1}{t}$ & $10 \frac{3}{4}$ & East Africa & $\ldots$ & Sir Robert Harvey, Bart. \\
\hline 35 & 40 & 25 & $15 \frac{1}{2}$ & South Africa & $\ldots$ & F. C. Selous. \\
\hline $34 \frac{7}{8}$ & $38 \frac{1}{2}$ & $29 \frac{1}{8}$ & $13 \frac{3}{t}$ & East Africa & $\ldots$ & H. C. V. Hunter. \\
\hline$\cdots$ & $\cdots$ & $26 \frac{1}{2}$ & $\cdots$ & $\ldots \quad \ldots$ & $\cdots$ & J. A. Jameson. \\
\hline 34 & 39 & 26 & $\cdots$ & $\cdots \quad \cdots$ & $\ldots$ & R. P. Carrol. \\
\hline 34 & $38 \frac{1}{4}$ & $27 \frac{3}{8}$ & II $\frac{7}{8}$ & South Africa & $\ldots$ & F. C. Selous. \\
\hline 34 & $\begin{array}{c}\text { along front } \\
\text { curve one horn }\end{array}$ & $32 \frac{1}{2}$ & $13 \frac{1}{2}$ & East Africa & $\ldots$ & T. W. H. Greenfield. \\
\hline $33 \frac{7}{8}$ & 39 & $23 \frac{1}{8}$ & I $3 \frac{1}{t}$ & Do. & $\cdots$ & Sir Robert Harvey, Bart. \\
\hline $33 \frac{7}{8}$ & $38 \frac{1}{4}$ & $26 \frac{5}{8}$ & $12 \frac{3}{4}$ & Do. & $\cdots$ & H. C. V. Hunter. \\
\hline $33 \frac{1}{2}$ & $37 \frac{1}{4}$ & $32 \frac{3}{4}$ & $12 \frac{1}{2}$ & Do. & $\cdots$ & Sir John Kirk, M. D., K. C. B. \\
\hline $33 \frac{1}{4}$ & $38 \frac{3}{4}$ & ... & I6 & Do. & $\cdots$ & F. J. Jackson. \\
\hline 33 & 39 & $2 \mathrm{I}$ & $\cdots$ & South Africa & $\cdots$ & Rowland Ward's Collection. \\
\hline $32 \frac{\pi}{8}$ & $3^{6 \frac{1}{2}}$ & $23^{\frac{3}{4}}$ & $12 \frac{1}{t}$ & East Africa & $\ldots$ & Sir Robert Harvey, Bart. \\
\hline $32 \frac{1}{2}$ & 37 & $24 \frac{1}{2}$ & $I I \frac{1}{2}$ & Do. & $\cdots$ & Do. \\
\hline $32 \frac{1}{2}$ & $38 \frac{1}{t}$ & $19 \frac{3}{4}$ & 13 & $\ldots \quad \ldots$ & $\cdots$ & Sir Edmund G. Loder, Bart. \\
\hline 32 & $38 \frac{3}{4}$ & I6 & $\begin{array}{c}\mathrm{IO} \frac{1}{2} \\
\text { (about) }\end{array}$ & South Africa & $\ldots$ & A. Beit. \\
\hline 32 & $36 \frac{5}{8}$ & $20 \frac{1}{8}$ & $13 \frac{3}{8}$ & East Africa & $\cdots$ & Sir Robert Harvey, 13art. \\
\hline $31 \frac{7}{8}$ & $37 \frac{1}{8}$ & $23 \frac{5}{8}$ & I3 & $\cdots \quad \cdots$ & $\cdots$ & H. R. H. Duke of Edinburgh. \\
\hline $3 I \frac{1}{2}$ & $35^{\frac{3}{4}}$ & $24 \frac{1}{4}$ & $13 \frac{1}{4}$ & South Africa & $\ldots$ & $\begin{array}{l}\text { Dr. Knight Bruce, Bishop } \\
\text { of Mashuna Land. }\end{array}$ \\
\hline $3 I^{\frac{1}{4}}$ & $35^{\frac{3}{4}}$ & $\cdots$ & $12 \frac{1}{4}$ & East Africa & $\cdots$ & H. C. V. Hunter. \\
\hline $3 \mathbf{I}$ & $35^{\frac{5}{8}}$ & $24 \frac{1}{2}$ & $12 \frac{1}{2}$ & Do. & $\ldots$ & Sir Robert Harvey, Bart. \\
\hline $30 \frac{3}{4}$ & 36 & I9 & I0 & Do. & $\ldots$ & Sir John Kirk, M. D., K. C. B. \\
\hline $30 \frac{1}{2}$ & $34 \frac{1}{2}$ & $22 \frac{7}{8}$ & $x 4 \frac{1}{2}$ & Do. & $\ldots$ & Sir Robert Harvey, Bart. \\
\hline $30 \frac{1}{8}$ & $33 \frac{3}{8}$ & $26 \frac{3}{4}$ & $\cdots$ & South Africa & $\cdots$ & British Museum. \\
\hline $29 \frac{1}{4}$ & 34 & ... & $14 \frac{1}{8}$ & East Africa & $\ldots$ & H. C. V. Hunter. \\
\hline $28 \frac{7}{8}$ & $33 \frac{1}{8}$ & $22 \frac{1}{2}$ & $10 \frac{5}{8}$ & South Africa & ... & $\begin{array}{l}\text { Dr. Knight Bruce, Bishop of } \\
\text { Mashunaland. }\end{array}$ \\
\hline
\end{tabular}




\section{CAPE BUFFALO (Bos caffer)-continued.}

\begin{tabular}{|c|c|c|c|c|c|}
\hline \multicolumn{2}{|c|}{ Greatest Width. } & \multirow{2}{*}{$\begin{array}{l}\text { Tip to } \\
\text { Tip. }\end{array}$} & \multirow{2}{*}{$\begin{array}{l}\text { Width of } \\
\text { Palm. }\end{array}$} & \multirow{2}{*}{ Habitat. } & \multirow{2}{*}{ Owner. } \\
\hline Inside. & Outside. & & & & \\
\hline $28 \frac{1}{2}$ & 34 & ... & $28 \frac{3}{4}$ & Mashuna Land & F. C. Selous, British Mu- \\
\hline $28 \frac{1}{8}$ & $32 \frac{7}{8}$ & $18 \frac{1}{8}$ & $13 \frac{1}{8}$ & East Africa $\quad \ldots$ & Sir Robert Harvey, Bart. \\
\hline 28 & 32 & 22 & $6 \frac{3}{8}$ & Buse Country ... & F. L. James. \\
\hline $27 \frac{1}{2}$ & $32 \frac{3}{4}$ & $15 \frac{1}{2}$ & $10 \frac{1}{4}$ & East Africa $\quad \ldots$ & Sir Robert Harvey, Bart. \\
\hline $26 \frac{1}{2}$ & $3 \mathbf{I} \frac{1}{2}$ & $21 \frac{1}{4}$ & $9 \frac{1}{4}$ & $\cdots \quad \cdots$ & $\begin{array}{l}\text { C. P. V. Aylmer and W. D. } \\
\text { James, ISSI. }\end{array}$ \\
\hline $24 \frac{5}{8}$ & $28 \frac{3}{8}$ & ... & $21 \frac{3}{4}$ & Algoa Bay & British Museum. \\
\hline
\end{tabular}

\section{CENTRAL AFRICAN BUFFALO (Bos Centralis).}

Habitat-North and Central Africa.

\begin{tabular}{|c|c|c|c|c|c|c|}
\hline \multicolumn{2}{|c|}{ Greatest width. } & \multirow{2}{*}{$\begin{array}{l}\text { Tip to } \\
\text { Tip. }\end{array}$} & \multirow{2}{*}{$\begin{array}{l}\text { Width of } \\
\text { Palm. }\end{array}$} & \multirow{2}{*}{\multicolumn{2}{|c|}{ Habitat. }} & \multirow{2}{*}{ Owner. } \\
\hline Inside. & Outside. & & & & & \\
\hline $36 \frac{1}{2}$ & 39 & $34 \frac{1}{2}$ & $\ldots$ & Soudan ... & $\ldots$ & Rowland Ward's Collection. \\
\hline $2 \delta_{4}^{1}$ & $31 \frac{3}{4}$ & $25 \frac{3}{8}$ & $8 \frac{1}{2}$ & Do. $\ldots$ & $\ldots$ & Col. Ralph Vivian. \\
\hline 28 & $33 \frac{1}{4}$ & I9 & $8 \frac{3}{4}$ & Do. $\quad \ldots$ & ... & Do. \\
\hline $26 \frac{7}{8}$ & $31 \frac{3}{8}$ & $24 \frac{3}{8}$ & $\ldots$ & Bogos Land, A & bys- & British Museum. \\
\hline $25 \frac{5}{8}$ & $28 \frac{1}{2}$ & 24 & $\ldots$ & Do. & & Do. \\
\hline
\end{tabular}

BUBALUS RECLINUS.

\begin{tabular}{|c|c|c|c|c|c|}
\hline \multicolumn{2}{|c|}{ Greatest width. } & \multirow{2}{*}{ Tip to Tip. } & \multirow{2}{*}{\multicolumn{2}{|c|}{ Habitat. }} & \multirow{2}{*}{ Owner. } \\
\hline Inside. & Outside. & & & & \\
\hline 18 & $24 \frac{1}{4}$ & II $\frac{3}{8}$ & $\ldots$ & $\ldots$ & British Museun. \\
\hline
\end{tabular}




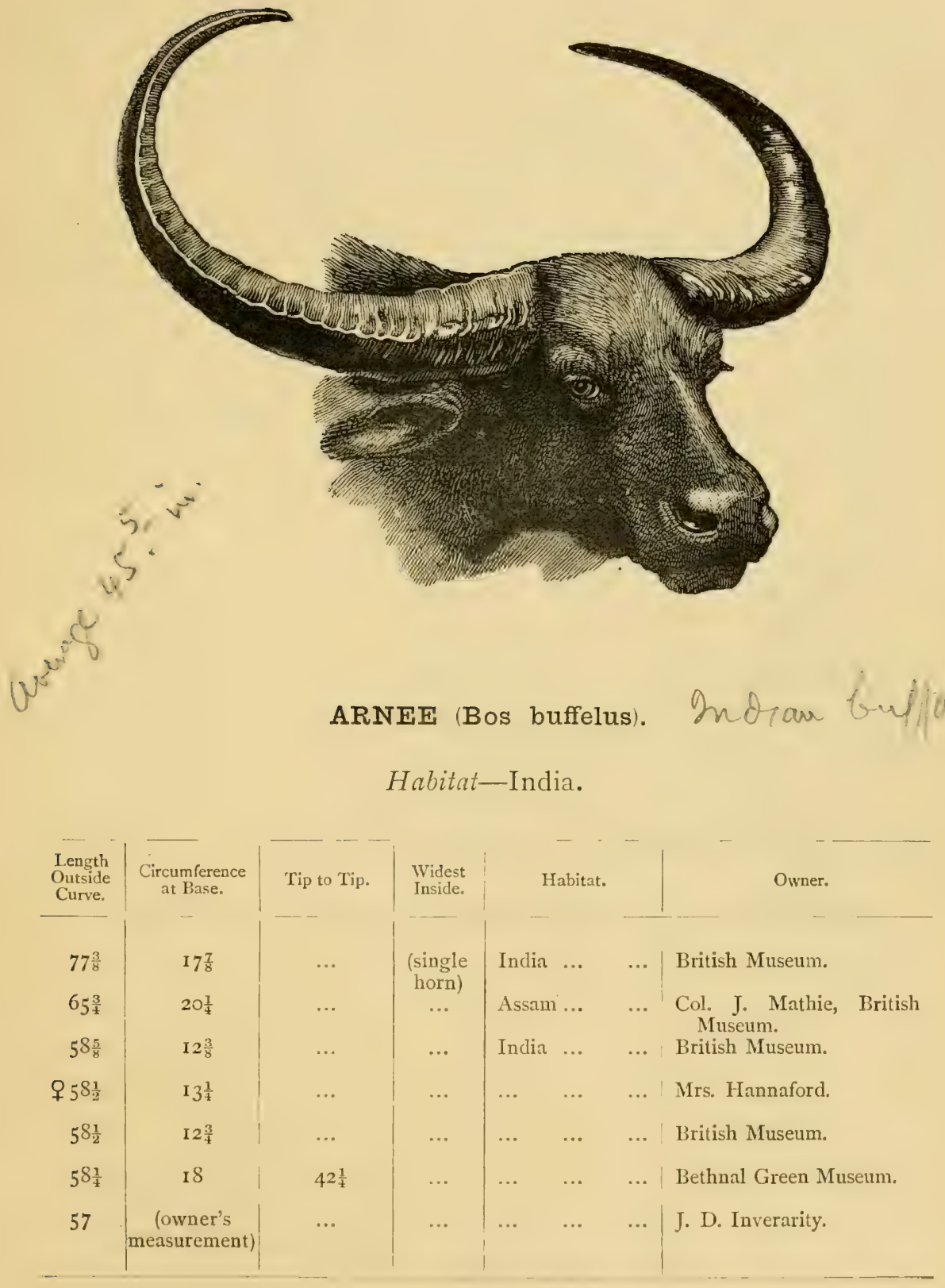


ARNEE (BOS buffelus)-continued.

\begin{tabular}{|c|c|c|c|c|c|c|c|}
\hline $\begin{array}{l}\text { Length } \\
\text { Outside } \\
\text { Curve. }\end{array}$ & $\begin{array}{l}\text { Circumference } \\
\text { at Base. }\end{array}$ & Tip to Tip. & $\begin{array}{l}\text { Widest } \\
\text { Inside. }\end{array}$ & & Habitat. & & Owner. \\
\hline 56 & $15 \frac{1}{2}$ & $55^{\frac{1}{2}}$ & $5^{8}$ & Kuch & Behar & $\ldots$ & Eyre Coote. \\
\hline $55^{\frac{1}{2}}$ & $18 \frac{1}{2}$ & 29 & 44 & India & $\ldots$ & $\cdots$ & J. Carr-Saunders. \\
\hline $54 \frac{1}{2}$ & I $8 \frac{1}{8}$ & $3^{\varepsilon \frac{1}{4}}$ & $48 \frac{7}{8}$ & Do. & $\ldots$ & $\ldots$ & $\begin{array}{l}\text { Hume Collection, British } \\
\text { Museum. }\end{array}$ \\
\hline $53 \frac{7}{8}$ & 12 & $\cdots$ & $\cdots$ & $\cdots$ & $\cdots$ & $\cdots$ & $\begin{array}{l}\text { A. O. Hume, Private Col- } \\
\text { lection. }\end{array}$ \\
\hline $53 \frac{1}{1}$ & $2 I$ & $26 \frac{7}{8}$ & $\cdots$ & $\cdots$ & $\cdots$ & $\cdots$ & | Baron de Nolde. \\
\hline $53^{\frac{1}{4}}$ & $12 \frac{1}{8}$ & $23 \frac{1}{4}$ & $40 \frac{5}{8}$ & $\ldots$ & .. & $\cdots$ & $\begin{array}{l}\text { Hume Collection, British } \\
\text { Museum. }\end{array}$ \\
\hline $53 \frac{1}{8}$ & $12 \frac{1}{2}$ & ... & $\cdots$ & Nepal & $1 \ldots$ & $\cdots$ & $\begin{array}{l}\text { B. H. Hodgson, British } \\
\text { Museum. }\end{array}$ \\
\hline 53 & $12 \frac{1}{8}$ & $\cdots$ & $\cdots$ & Inclia & $\cdots$ & $\cdots$ & British Museum. \\
\hline $52 \frac{3}{4}$ & $1 S_{4}^{1}$ & $30 \frac{1}{2}$ & $44 \frac{1}{x}$ & Do. & ... & ... & $\begin{array}{l}\text { Hume Collection, British } \\
\text { Museum. }\end{array}$ \\
\hline 52 & $16 \frac{38}{4}$ & $\cdots$ & $\cdots$ & Do. & $\cdots$ & $\cdots$ & British Museum. \\
\hline $5 I^{\frac{1}{2}}$ & $16 \frac{1}{2}$ & $\cdots$ & $\cdots$ & Do. & $\ldots$ & ... & Do. \\
\hline $5^{1 \frac{1}{4}}$ & $16 \frac{1}{2}$ & $\ldots$ & $\ldots$ & Do. & $\ldots$ & $\ldots$ & Do. \\
\hline $5^{1 \frac{5}{8}}$ & $18 \frac{3}{8}$ & $44 \frac{1}{8}$ & $50 \frac{3}{8}$ & Nepal & $1 \ldots$ & $\ldots$ & $\begin{array}{l}\text { B. II. Hodgson, Bitish } \\
\text { Museum. }\end{array}$ \\
\hline $50 \frac{3}{4}$ & $19 \frac{1}{2}$ & 36 & $\ldots$ & India & $\ldots$ & .. & Lieut. St. John Brodrick. \\
\hline $5^{\circ \frac{1}{2}}$ & $17 \frac{1}{2}$ & $21 \frac{1}{2}$ & 37 & Do. & $\ldots$ & ... & Hon. Walter Rothschild. \\
\hline $50^{\frac{1}{4}}$ & $18 \frac{7}{8}$ & $\ldots$ & $\ldots$ & Do. & $\ldots$ & $\ldots$ & British Museum. \\
\hline 50 & $13 \frac{1}{4}$ & 33 & $38 \frac{1}{2}$ & Do. & ... & $\ldots$ & Noel Fenwick. \\
\hline $49 \frac{1}{2}$ & $12 \frac{1}{8}$ & $35^{\frac{3}{3}}$ & $47 \stackrel{3}{\circ}$ & Do. & $\ldots$ & $\ldots$ & British Museum. \\
\hline $49 \frac{5}{8}$ & $17 \frac{5}{8}$ & 22 & 40 & Do. & $\ldots$ & ... & Do. \\
\hline $48 \frac{7}{8}$ & 16 & $\ldots$ & ... & Do. & $\ldots$ & $\cdots$ & Do. \\
\hline $47 \frac{5}{8}$ & $17 \frac{7}{8}$ & $19 \frac{3}{4}$ & $\cdots$ & Do. & $\cdots$ & $\cdots$ & Do. \\
\hline $47 \frac{1}{8}$ & IS & $\cdots$ & .. & Do. & $\cdots$ & $\ldots$ & Do. \\
\hline 47 & 16 & $38 \frac{1}{2}$ & $46 \frac{1}{4}$ & Do. & $\cdots$ & $\cdots$ & Bethnal Green Museum. \\
\hline $45^{\frac{1}{8}}$ & 12 & $25 \frac{1}{2}$ & ... & Do. & $\ldots$ & $\ldots$ & British Museum. \\
\hline $43^{\frac{1}{4}}$ & IS & $46 \frac{3}{7}$ & $49 \frac{1}{4}$ & Do. & $\cdots$ & $\ldots$ & Major Talbot. \\
\hline 43 & 16 & 50 & $50 \frac{3}{4}$ & Do. & $\cdots$ & $\ldots$ & H.R. H. Duke of Edinburgh. \\
\hline 42 & $13 \frac{3}{4}$ & 33 & $38 \frac{1}{2}$ & Do. & $\ldots$ & $\cdots$ & Rowland Ward's Collection. \\
\hline
\end{tabular}


ARNEE (BOs buffelus)-continucd.

\begin{tabular}{|c|c|c|c|c|c|c|}
\hline $\begin{array}{l}\text { Length } \\
\text { outside } \\
\text { Curve. }\end{array}$ & $\begin{array}{l}\text { Circumference } \\
\text { at base. }\end{array}$ & Tip to Tip. & $\begin{array}{l}\text { Widest } \\
\text { inside. }\end{array}$ & Habitat. & & Owner. \\
\hline $40 \frac{1}{8}$ & II $\frac{2}{4}$ & 35 & $4^{I}$ & India $\ldots .$. & ... & British Museum. \\
\hline $39 \frac{5}{8}$ & $14 \frac{3}{4}$ & $35^{\frac{1}{8}}$ & 42 & Nepal ... & $\ldots$ & $\begin{array}{l}\text { B. H. Hodgson, British } \\
\text { Museum. }\end{array}$ \\
\hline $39 \frac{1}{2}$ & I $4 \frac{1}{2}$ & $35 \frac{1}{8}$ & $41 \frac{7}{8}$ & Do. $\ldots$ & $\cdots$ & Do. \\
\hline $3^{8}$ & $16 \frac{1}{2}$ & $35^{\frac{1}{2}}$ & $37 \mathrm{c}$ & India $\ldots$ & 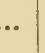 & Noel Fenwick. \\
\hline $3^{8}$ & I3 & $25 \frac{3}{4}$ & $34 \frac{7}{8}$ & Kuch Behar .. & $\cdots$ & Capt. H. Streatfield. \\
\hline $36 \frac{1}{4}$ & I I $\frac{1}{8}$ & $16 \frac{3}{4}$ & $24 \frac{1}{4}$ & Nepal ... & $\cdots$ & British Museum. \\
\hline 35 & 12 & $26 \frac{1}{2}$ & $4 I$ & India $\ldots . \quad$. & & E. Tennant. \\
\hline $32 \frac{3}{8}$ & II $\frac{1}{4}$ & $\cdots$ & ... & Nilghiri Hills. & & $\begin{array}{l}\text { Gen. Hardwicke, British } \\
\text { Museum. }\end{array}$ \\
\hline $30 \frac{3}{4}$ & 14 & 22 & 32 & Ceylon... & $\cdots$ & H. E. Lindsey. \\
\hline 29 & $9 \frac{3}{8}$ & $\cdots$ & $\cdots$ & $\cdots \quad \cdots$ & $\cdots$ & British Museum. \\
\hline $26 \frac{3}{8}$ & $14 \frac{3}{4}$ & $28 \frac{1}{8}$ & 25 & Formosa & $\cdots$ & R. Swinhoe, British Museum. \\
\hline $25 \frac{8}{4}$ & II $\frac{1}{8}$ & $\cdots$ & $\cdots$ & $\cdots \quad \cdots \quad-\cdots$ & $\cdots$ & British Museum. \\
\hline $25^{\frac{1}{4}}$ & I $7 \frac{1}{8}$ & 34 & $\cdots$ & $\therefore \quad \ldots$ & $\cdots$ & $\begin{array}{l}\text { Sir Stanford Raffles, British } \\
\text { Museum. }\end{array}$ \\
\hline 25 & $14 \frac{5}{8}$ & $22 \frac{1}{8}$ & 25 & Formosa & ... & R. Swinhoe, British Museum. \\
\hline $22 \frac{7}{8}$ & $10 \frac{3}{4}$ & $17 \frac{7}{8}$ & $20 \frac{5}{8}$ & Nepal ... & $\ldots$ & $\begin{array}{l}\text { B. H. Hodgson, British } \\
\text { Museum. }\end{array}$ \\
\hline 19 & 7 & $\begin{array}{c}\text { (owner's } \\
\text { measurement) }\end{array}$ & $\cdots$ & $\begin{array}{l}\text { Nicarawettya, } \\
\text { Ceylon. }\end{array}$ & & J. H. Mann. \\
\hline
\end{tabular}




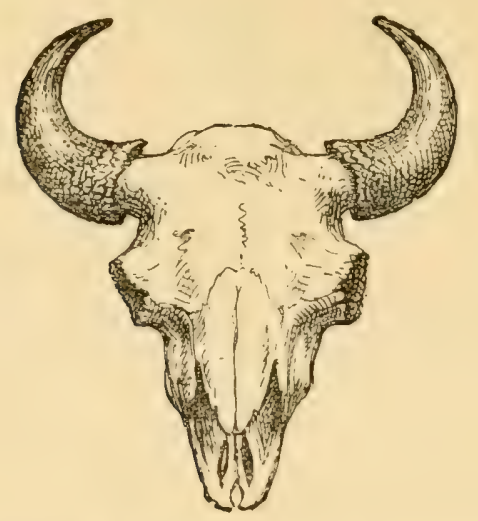

AMERICAN BISON (Bos Americanus).

Now nearly extinct.

Habitat-North America.

\begin{tabular}{|c|c|c|c|c|c|c|}
\hline Length. & $\begin{array}{l}\text { Circum- } \\
\text { ference. }\end{array}$ & Tip to Tip. & $\begin{array}{l}\text { Widest } \\
\text { insile. }\end{array}$ & \multicolumn{2}{|c|}{ Habitat. } & Owner. \\
\hline $20 \frac{7}{8}$ & 15 & $\ldots$ & $3 \circ \frac{1}{2}$ & Wyoming & $\ldots$ & Hon. F. Thellusson. \\
\hline $1 \$ \frac{7}{8}$ & $14 \frac{3}{4}$ & ... & $16 \frac{7}{8}$ & $\ldots$ & $\ldots$ & The late J. S. Jameson: \\
\hline $17 \frac{3}{k}$ & $12 \frac{3}{8}$ & $15 \frac{1}{8}$ & $\ldots$ & $\ldots$ & $\ldots$ & $\begin{array}{l}\text { H.R.H. Duke of Edin- } \\
\text { burgh. }\end{array}$ \\
\hline $17 \frac{1}{2}$ & 12 & $\ldots$ & $25^{\frac{1}{2}}$ & $\cdots$ & $\ldots$ & Rowland Ward's Collection. \\
\hline $17 \frac{1}{8}$ & II $\frac{3}{8}$ & $10 \frac{3}{8}$ & $17 \frac{2}{2}$ & $\ldots$ & $\ldots$ & British Museum. \\
\hline $16 \frac{5}{8}$ & $14 \frac{1}{1}$ & 24 & $\ldots$ & $\cdots$ & $\ldots$ & Moreton Frewen. \\
\hline $16 \frac{1}{2}$ & $12 \frac{1}{2}$ & $19 \frac{3}{x}$ & Hill Buffalo & $\ldots$ & $\cdots$ & Sir Edmund G. Loder, Bart. \\
\hline $16 \frac{1}{8}$ & $15 \frac{7}{8}$ & $25 \frac{3}{t}$ & $\cdots$ & $\cdots$ & $\cdots$ & Do. \\
\hline 16 & II $\frac{3}{4}$ & $15 \frac{3}{5}$ & $\cdots$ & $\cdots$ & $\cdots$ & F. J. Horniman. \\
\hline $15^{\frac{1}{2}}$ & $14 \frac{8}{8}$ & $\cdots$ & $19 \frac{3}{4}$ & Wyoming & $\cdots$ & St. George Littledale. \\
\hline 14 & $\cdots$ & $12 \frac{1}{4}$ & $\cdots$ & $\cdots$ & $\cdots$ & Col. Ralph Vivian. \\
\hline $13 \frac{3}{8}$ & 12 & $\cdots$ & $\cdots$ & $\cdots$ & $\cdots$ & Hon. Walter Rothschild. \\
\hline$I_{3} \frac{7}{8}$ & $12 \frac{3}{4}$ & $19 \frac{5}{8}$ & $\cdots$ & $\cdots$ & $\cdots$ & J. Carr-Saunders. \\
\hline $12 \frac{1}{4}$ & $12 \frac{1}{4}$ & $17 \frac{1}{4}$ & $17 \frac{3}{8}$ & $\begin{array}{l}\text { Mount Ye } \\
\text { stone. }\end{array}$ & ow- & British Museum. \\
\hline
\end{tabular}




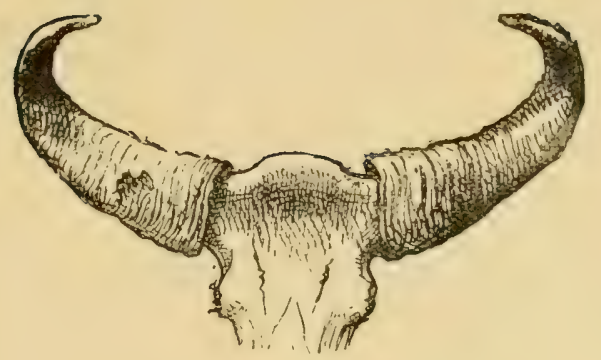

GAUR BISON (Bos Gaurus).

\section{Habitat-India.}

\begin{tabular}{|c|c|c|c|c|c|c|}
\hline Length. & $\begin{array}{l}\text { Circum- } \\
\text { ference. }\end{array}$ & Tip to Tip. & Widest inside. & \multicolumn{2}{|c|}{ Halitat. } & Owner. \\
\hline $33 \frac{\pi}{4}$ & $17 \frac{1}{4}$ & 24 & $\cdots$ & India & $\ldots$ & $\begin{array}{l}\text { J. D. Goldingham, Beth- } \\
\text { nal Green Museum. }\end{array}$ \\
\hline $33^{\frac{1}{2}}$ & I $8 \frac{1}{2}$ & 25 & $33 \frac{1}{4}$ & Do. & $\cdots$ & T. W. H. Greenfield. \\
\hline$\cdots$ & $\mid \begin{array}{c}\text { I9 (owner's } \\
\text { measurement) }\end{array}$ & $\ldots$ & $\cdots$ & Do. & $\ldots$ & J. D. Inverarity. \\
\hline $31 \frac{7}{8}$ & $17 \frac{1}{8}$ & $2 \mathrm{I} \frac{3}{8}$ & $32 \frac{1}{2}$ & Do. & ... & $\begin{array}{l}\text { A. Hume, Private } \\
\text { Collection. }\end{array}$ \\
\hline $3 I \frac{1}{2}$ & 18 & 29 & $\begin{array}{c}43 \\
\text { (outside) }\end{array}$ & Do. & $\ldots$ & $\begin{array}{l}\text { Bombay Nat. Hist. Suciety } \\
\text { ("Proceedings"). }\end{array}$ \\
\hline $31 \frac{1}{4}$ & $16 \frac{3}{8}$ & $12 \frac{5}{8}$ & $27 \frac{1}{2}$ & Do. & $\ldots$ & $\begin{array}{l}\text { J. D. Goldingham, Beth- } \\
\text { nal Green Museum. }\end{array}$ \\
\hline $30 \frac{1}{2}$ & $16 \frac{1}{2}$ & I $3 \frac{1}{2}$ & $\begin{array}{c}33 \frac{2}{4} \\
\text { (outside) }\end{array}$ & Do. & $\ldots$ & Lieut.-Col. Sandys. \\
\hline $30 \frac{1}{8}$ & $17 \frac{7}{8}$ & $33 \frac{3}{8}$ & $40 \frac{1}{4}$ & Do. & $\cdots$ & J. Carr-Saunders. \\
\hline $29 \frac{7}{8}$ & $18 \frac{1}{4}$ & 30 & 34 & Do. & $\ldots$ & $\begin{array}{l}\text { Sir Edmund G. Loder, } \\
\text { Bart. }\end{array}$ \\
\hline $29 \frac{3}{4}$ & $1 S \frac{\pi}{8}$ & $25 \frac{1}{4}$ & $30 \frac{3}{8}$ & $\begin{array}{l}\text { Hass } \\
\text { Hills, }\end{array}$ & dia. & $\begin{array}{l}\text { Sir V. Brooke's Coll. (Shot } \\
\text { by Col. D. Hamilton). }\end{array}$ \\
\hline $29 \frac{5}{8}$ & $18 \frac{1}{2}$ & I $6 \frac{1}{4}$ & $26 \frac{1}{2}$ & $\underset{\text { Sout }}{\text { Annan }}$ & s, & Do. \\
\hline $29 \frac{1}{2}$ & $\begin{array}{c}\text { I } 8 \text { (owner's } \\
\text { measurement) }\end{array}$ & $\cdots$. & $\begin{array}{c}33 \\
\text { (outside) }\end{array}$ & India & ... & J. D. Inverarity. \\
\hline $29 \frac{\pi}{4}$ & $12 \frac{7}{8}$ & $\ldots$ & 18 & Do. & $\ldots$ & $\begin{array}{l}\text { Gen. Hardwicke, British } \\
\text { Museum. }\end{array}$ \\
\hline $29 \frac{1}{8}$ & $16 \frac{5}{8}$ & $20 \frac{3}{8}$ & $29 \frac{1}{4}$ & Nepal & ... & $\begin{array}{l}\text { B. H. Hodgson, British } \\
\text { Museum. }\end{array}$ \\
\hline 29 & 22 & $\begin{array}{c}\text { (owner's } \\
\text { measurement) }\end{array}$ & $\ldots$ & India & ... & Otho Shaw. \\
\hline $28 \frac{3}{4}$ & $15 \frac{3}{4}$ & $30 \frac{5}{8}$ & 35 & Do. & $\cdots$ & British Museum. \\
\hline $28 \frac{5}{8}$ & $17 \frac{3}{8}$ & $24 \frac{1}{2}$ & $28 \frac{3}{8}$ & $\begin{array}{l}\text { Sahayd } \\
\text { Sout }\end{array}$ & $\begin{array}{l}\text { ts., } \\
\text { idia. }\end{array}$ & $\begin{array}{l}\text { Capt. W. Thompson, } \\
\text { British Museum. }\end{array}$ \\
\hline
\end{tabular}


GAUR BISON (BOS gaurus)-continued.

\begin{tabular}{|c|c|c|c|c|c|}
\hline Length. & $\begin{array}{l}\text { Circum- } \\
\text { ference. }\end{array}$ & Tip to Tip. & Wilest inside. & Habitat. & Owner. \\
\hline $2 S_{\dddot{3}}^{3}$ & IS & $25 \frac{3}{8}$ & $3 I_{\frac{1}{4}}$ & $\begin{array}{l}\text { Annamalies, } \\
\text { South India. }\end{array}$ & $\begin{array}{l}\text { Sir V. Brooke's Coll. (Shot } \\
\text { by Col. D. Hamilton.) }\end{array}$ \\
\hline $28 \frac{5}{8}$ & $15^{\frac{3}{4}}$ & $37^{\frac{3}{4}}$ & $40 \frac{1}{2}$ & India $\quad \ldots$ & A. O. Hume, I'rivate \\
\hline $2 S \frac{1}{2}$ & $15^{\frac{3}{4}}$ & $18 \frac{5}{8}$ & $28 \frac{5}{8}$ & Do. & $\begin{array}{l}\text { Maj.-Gen. Arthur Ellis, } \\
\text { C.S.I. }\end{array}$ \\
\hline $28 \frac{1}{2}$ & $15 \frac{1}{t}$ & 17 & 27 & Do. & $\begin{array}{l}\text { Hume Collection, British } \\
\text { Museum. }\end{array}$ \\
\hline $2 \$_{1}^{1}$ & IS & $15^{\frac{3}{4}}$ & $\begin{array}{c}\text { (owner's } \\
\text { measurement) }\end{array}$ & Do. $\quad \ldots$ & F. W. Wodehouse. \\
\hline $28 \frac{1}{4}$ & I $8 \frac{3}{x}$ & $24 \frac{3}{4}$ & $3 I^{\frac{1}{2}}$ & $\cdots \quad \ldots \quad \ldots$ & $\begin{array}{l}\text { Hume Collection, British } \\
\text { Musemm. }\end{array}$ \\
\hline $2 S$ & 17 & $24 \frac{3}{x}$ & $3 \mathrm{I} \frac{6}{8}$ & Palnis, India & $\begin{array}{l}\text { Sir V. Brooke's Coll. (Shot } \\
\text { by Col. D. Hamilton.) }\end{array}$ \\
\hline $2 S$ & $15^{\frac{3}{4}}$ & $19 \frac{1}{4}$ & 30 & $\begin{array}{l}\text { Annainalies, } \\
\text { South India. }\end{array}$ & Do. \\
\hline 28 & 16 & $10 \frac{1}{4}$ & 24 & India $\quad \ldots$ & Martyn Kennard. \\
\hline $27 \frac{7}{8}$ & $18 \frac{3}{8}$ & $20 \frac{3}{4}$ & $27 \frac{\pi}{k}$ & $\begin{array}{l}\text { Annamalies, } \\
\text { South India. }\end{array}$ & $\begin{array}{l}\text { Sir V. Brooke's Coll. (Shot } \\
\text { by Col. D. Hamilton.) }\end{array}$ \\
\hline $27 \frac{7}{8}$ & $17 \frac{1}{x}$ & $32 \frac{1}{4}$ & $32 \frac{3}{4}$ & India $\quad \ldots$ & Major Cumberland. \\
\hline $27 \frac{9}{7}$ & $16 \frac{5}{x}$ & $32 \frac{1}{2}$ & $3^{6}$ & Do. & $\begin{array}{l}\text { Iume Collection, British } \\
\text { Museum. }\end{array}$ \\
\hline $27 \frac{1}{3}$ & $18 \frac{5}{x}$ & $18 \frac{5}{4}$ & $28 \frac{5}{x}$ & Do. & Do. \\
\hline $27 \frac{3}{8}$ & $14 \frac{1}{2}$ & $21 \frac{3}{5}$ & $28 \frac{5}{8}$ & Do. & $\begin{array}{l}\text { Dr. Falconer, British } \\
\text { Museum. }\end{array}$ \\
\hline 27 & IS & $19 \frac{1}{2}$ & $27^{\frac{1}{2}}$ & $\begin{array}{l}\text { Annamalies, } \\
\text { South India. }\end{array}$ & $\begin{array}{l}\text { Sir V. Brooke's Coll. (Shot } \\
\text { by Col. D. IImilton.) }\end{array}$ \\
\hline 27 & I 6 & 16 & $\ldots$ & $\begin{array}{lll}\cdots & \cdots & \cdots\end{array}$ & $\begin{array}{l}\text { Rowland Ward's Collec- } \\
\text { tion. }\end{array}$ \\
\hline 267 & $15 \frac{7}{8}$ & $16 \frac{1}{4}$ & 27 & India $\quad \ldots$ & Hon. Walter Rothschild. \\
\hline $26 \frac{5}{x}$ & $13 \frac{1}{1}$ & $10 \frac{5}{x}$ & $20 \frac{\pi}{x}$ & Do. & British Museum. \\
\hline $26 \frac{1}{2}$ & $16 \frac{1}{4}$ & I $S \frac{1}{2}$ & $26 \frac{\pi}{x}$ & Madras & $\begin{array}{l}\text { Dr. Christie, British Mu- } \\
\text { seum. }\end{array}$ \\
\hline $26 \frac{1}{2}$ & $15 \frac{1}{2}$ & 16 & $\ldots$ & India & $\begin{array}{l}\text { Rowland Ward's Collec- } \\
\text { tion. }\end{array}$ \\
\hline $26 \frac{3}{8}$ & $14 \frac{7}{8}$ & $13 \frac{5}{8}$ & $24 \frac{1}{4}$ & $\begin{array}{l}\text { Sahaydri Mts, } \\
\text { South India. }\end{array}$ & $\begin{array}{l}\text { Capt. IV. Thompson, } \\
\text { British Museum. }\end{array}$ \\
\hline 26 & $17 \frac{1}{\frac{1}{4}}$ & $19 \frac{3}{8}$ & $\cdots$ & India $\quad \ldots$ & Lord Wolverton. \\
\hline 26 & $16 \frac{7}{8}$ & 27 & $30 \frac{1}{x}$ & $\begin{array}{l}\text { Bori Forest, } \\
\text { Hosangobod, } \\
\text { Cent. Provs. }\end{array}$ & $\begin{array}{l}\text { Sir John H. Morris, } \\
\text { K.C.S.I. }\end{array}$ \\
\hline $25^{\frac{3}{4}}$ & $15 \frac{3}{1}$ & $26 \frac{1}{2}$ & $29 \frac{5}{8}$ & $\begin{array}{lll}\cdots & \ldots & \ldots\end{array}$ & $\begin{array}{l}\text { Hume Collection, British } \\
\text { Museum. }\end{array}$ \\
\hline $25 \frac{3}{4}$ & $16 \frac{5}{8}$ & $20 \frac{7}{8}$ & $26 \frac{3}{4}$ & $\begin{array}{l}\text { Malay Penin- } \\
\text { sula. }\end{array}$ & H. C. V. Hunter. \\
\hline $25 \frac{1}{2}$ & $17 \frac{1}{2}$ & 19 & $\ldots$ & $\begin{array}{l}\text { Straits Settle- } \\
\text { ments. }\end{array}$ & T. S. Thompson. \\
\hline
\end{tabular}


GAUR BISON (Bos gaurus)-continued.

\begin{tabular}{|c|c|c|c|c|c|}
\hline Length. & $\begin{array}{l}\text { Circum- } \\
\text { ference. }\end{array}$ & 'lip to Tip. & Widest inside. & Habitat. & Owner. \\
\hline $25^{\frac{3}{8}}$ & $13 \frac{5}{8}$ & $19 \frac{1}{4}$ & $26 \frac{33}{7}$ & West Siam ... & British Museum. \\
\hline $25 \frac{3}{8}$ & $15 \frac{1}{1}$ & $13 \frac{5}{8}$ & $25 \frac{1}{4}$ & India & $\begin{array}{l}\text { Major A. A. Kinloch, } \\
\text { British Museum. }\end{array}$ \\
\hline $25 \frac{1}{1}$ & $13^{\frac{1}{4}}$ & $19^{\frac{1}{2}}$ & $26_{4}^{5}$ & Rajhpootna & $\begin{array}{l}\text { Col. J. Evans, British } \\
\text { Museum. }\end{array}$ \\
\hline $25 \frac{1}{4}$ & I $5 \frac{3}{3}$ & $19 \frac{1}{2}$ & $26 \frac{7}{8}$ & Mysore & $\begin{array}{l}\text { Bosworth Smith, British } \\
\text { Museum. }\end{array}$ \\
\hline 25 & 15 & I $4 \frac{1}{2}$ & $\cdots$ & $\begin{array}{l}\text { Straits Setıle- } \\
\text { ments. }\end{array}$ & T. S. Thompson. \\
\hline $24^{\frac{3}{4}}$ & 17 & $34 \frac{1}{2}$ & $34: 3$ & $\begin{array}{l}\text { Annamalies, } \\
\text { South India. }\end{array}$ & $\begin{array}{l}\text { Sir V. Brooke's Coll. (Shot } \\
\text { by Col. D. Hamilton.) }\end{array}$ \\
\hline $24 \frac{1}{2}$ & I6 & $28 \frac{1}{2}$ & $\cdots$ & $\begin{array}{lll}\cdots & \cdots & \cdots\end{array}$ & $\begin{array}{l}\text { Hume Collection, British } \\
\text { Museum. }\end{array}$ \\
\hline 24 & $\left|\begin{array}{c}\text { I } 8 \text { (owner's } \\
\text { measurement) }\end{array}\right|$ & 34 & $\begin{array}{c}{ }^{3} S_{\frac{1}{2}} \\
\text { (outside) }\end{array}$ & India & J. D. Inverarity. \\
\hline 24 & 16 & $25 \frac{1}{2}$ & $30 \frac{1}{8}$ & $\begin{array}{ll}\cdots & \cdots\end{array}$ & $\begin{array}{l}\text { Hume Collection, British } \\
\text { Museum. }\end{array}$ \\
\hline $23 \frac{3}{4}$ & $15 \frac{7}{8}$ & $20 \frac{1}{8}$ & 25 뭄 & Nepal & $\begin{array}{l}\text { B. H. Hodgson, British } \\
\text { Museum. }\end{array}$ \\
\hline $23 \frac{1}{2}$ & $14 \frac{5}{8}$ & 28 & $311^{3}$ & $\begin{array}{ll}\cdots & \cdots\end{array}$ & H. C. V. Hunter. \\
\hline $23 \frac{1}{ \pm}$ & $15 \frac{1}{8}$ & 20 & $25: \frac{3}{8}$ & South India & $\begin{array}{l}\text { Sir Victor Brooke's Col- } \\
\text { lection. }\end{array}$ \\
\hline $23^{\frac{1}{t}}$ & $12 \frac{38}{4}$ & I $3 \frac{1}{4}$ & .. & $\cdots \quad \cdots$ & A. O. Hume, Private \\
\hline $22 \frac{1}{2}$ & 16 & $2 I$ & $\cdots$ & $\begin{array}{lll}\cdots & \cdots & \cdots\end{array}$ & Surgeon-Major Briggs. \\
\hline $22 \frac{1}{8}$ & 15 & I $5 \frac{7}{8}$ & $22 \frac{1}{4}$ & $\begin{array}{l}\text { Malay Penin- } \\
\text { sula. }\end{array}$ & British Museum. \\
\hline $21 \frac{7}{8}$ & $15^{\frac{1}{2}}$ & I $S_{\frac{5}{8}}$ & $23 \frac{1}{8}$ & India & $\begin{array}{l}\text { Capt. W. E. Robinson, } \\
\text { British Museum. }\end{array}$ \\
\hline 오 21 $\frac{7}{8}$ & I I $\frac{1}{2}$ & $7 \frac{5}{8}$ & 18 & West Ghauts & Capt. W. Thompson, \\
\hline $2 \mathrm{I} \frac{5}{8}$ & $14 \frac{7}{8}$ & 20 & $25 \frac{1}{t}$ & Nepal & $\begin{array}{l}\text { B. H. Hodgson, British } \\
\text { Museum. }\end{array}$ \\
\hline $2 \mathrm{I} \frac{1}{2}$ & 17 & $22 \frac{1}{2}$ & $\ldots$ & $\cdots \quad \cdots$ & Rowland Ward's Collec- \\
\hline $2 \mathrm{I} \frac{1}{8}$ & 16 & $26 \frac{1}{4}$ & 29 & India & Major Talbot. \\
\hline $21 \frac{1}{8}$ & II $\frac{7}{8}$. & I 5 & $20_{5}^{3}$ & Mysore & $\begin{array}{l}\text { Bosworth Smith, British } \\
\text { Museum. }\end{array}$ \\
\hline $20 \frac{1}{2}$ & 15 & 12 & $\ldots$ & India & $\begin{array}{l}\text { Rowland Ward's Collec- } \\
\text { tion. }\end{array}$ \\
\hline $20 \frac{3}{8}$ & $11 \frac{2}{8}$ & $5 \frac{5}{8}$ & $I 6 \frac{1}{8}$ & Uo. & British Museum. \\
\hline I $8 \frac{1}{8}$ & $10 \frac{7}{8}$ & $10 \frac{3}{4}$ & $17 \frac{5}{5}$ & Do. & $\begin{array}{l}\text { R. C. Beavan, British } \\
\text { Museum. }\end{array}$ \\
\hline $17 \frac{6}{8}$ & $10 \frac{7}{8}$ & I $3 \frac{5}{8}$ & $17 \frac{1}{5}$ & $\begin{array}{l}\text { West Central } \\
\text { India. }\end{array}$ & $\begin{array}{l}\text { Major A. A. Kinlock, } \\
\text { British Museum. }\end{array}$ \\
\hline $17 \frac{1}{4}$ & III & $7 \frac{3}{4}$ & $15 !$ & $\cdots \quad \cdots$ & $\begin{array}{l}\text { B. H. Hodgson, British } \\
\text { Museum. } \\
\text { Col. G. P. Sanderson, } \\
\text { "Wild Beasts of India." }\end{array}$ \\
\hline
\end{tabular}


GAYAL (Bos frontalis).

Habitat-Assam Chittaging Districts.

\begin{tabular}{c|r|r|rr|r|r} 
Length. & $\begin{array}{l}\text { Circum. } \\
\text { ference. }\end{array}$ & Tip to Tip. & Habitat. & Owner. \\
\hline I5 & $11 \frac{1}{2}$ & $26 \frac{3}{8}$ & North India & $\ldots$ & British Museum. \\
$12 \frac{5}{6}$ & $13 \frac{1}{4}$ & $27 \frac{3}{4}$ & India $\ldots$ & $\ldots$ & Do. \\
\hline
\end{tabular}

\section{DWARF BUFFALO (Bos pumillus).}

[Sir VICTOR BROOKE, Bart., P.Z.S. I875, p. 455.]

Female about three years of age, all the permanent incisors except the two external teeth being in place (Zoological Gardens, Antwerp, received from Senegal) :-

Hair harsh, on the body and limbs of moderate length, longer on the withers and upper and under parts of the neck; general colour bright yellow; on the shoulders and anterior parts of the body there is a strong tinge of brown, caused by an intermixture of brown and yellow hairs; on the flanks and belly the yellow shades gradually into deep rufous; upper parts and sides of the head and neck pure yellow, lower surface of head and neck paler; entire upper and lower lip, fore limbs from above the carpi, and hind limbs from below the tarsi downwards black; ears about ro in. in length and 6 in. in breadth, narrowed gradually towards their tips, which, from their flexibility and length, curl back upon the ear-conches; internally the upper rim of the earconch is covered by pure yellow hair of about 5 in. length, hair of similar length and colour forming two marked lines along their inner surface; hair on the lower rim of the ear-conch and on the tip black; tail bare, excepting the terminal tuft, which is black. 
Height at the shoulder about 42 in.; horns slightly compressed at their bases, and directed upwards; the head is remarkably long in proportion to the body, which is compact and powerful, the limbs short.

Male, Adult.-Generally darker than the female (Du Chaillu); horns greatly compressed from above downwards and broad at their bases, which are transversed in unworn specimens by numerous sharply-marked irregular ridges; beyond these ridges the horns are suddenly contracted into round smooth points, which are directed upwards and sometimes boldly backwards.

The basal portion of the horn in most adult specimens rises slightly but decidedly from the horizontal upon leaving the skull; in other specimens, however, as for example in the type, the entire horn is directed upwards; length of horns round the curve about $2 I$ in., their diameter at the base 7 in.

Female, Adult.-In the female the horns are much less compressed at the base than in the male; as in the male the direction of the points of the horns is very variable; length of horns round the curve I7 in., their diameter at the base $4 \frac{1}{4}$ in.

RANGE.-Western Equatorial, and the rorthern parts of Central Africa.

Habitat-Africa.

\begin{tabular}{|c|c|c|c|c|c|}
\hline Length. & $\begin{array}{l}\text { Circum- } \\
\text { ference. }\end{array}$ & Tip to Tip. & Habitat. & & Owner. \\
\hline $21 \frac{1}{8}$ & $12 \frac{3}{4}$ & $2 \frac{1}{4}$ & West Africa & ... & British Museum. \\
\hline I $8 \frac{1}{4}$ & $1 \mathrm{O}_{4}^{3}$ & $5 \frac{1}{2}$ & Central Africa & $\cdots$ & $\begin{array}{l}\text { Col. Denham and Col. Clapperton. } \\
\text { British Museum. }\end{array}$ \\
\hline I7 & III $\frac{3}{8}$ & $6 \frac{3}{4}$ & Do. & $\ldots$ & Do. \\
\hline I $4 \frac{?}{4}$ & $12 \frac{1}{4}$ & $10 \frac{5}{8}$ & $\cdots$ & $\ldots 1$ & Sir Victor Brooke's Collection. \\
\hline $13 \frac{1}{2}$ & $13 \frac{5}{8}$ & $13 \frac{3}{4}$ & $\cdots$ & $\ldots 1$ & Do. \\
\hline $10 \frac{5}{8}$ & $9 \frac{3}{8}$ & $10 \frac{7}{8}$ & Gambia & $\ldots$ & Earl of Derby, British Museum. \\
\hline
\end{tabular}


BOS BRACHYCEROS.

Habitat-Africa.

\begin{tabular}{|c|c|c|c|c|c|}
\hline Length. & $\begin{array}{l}\text { Circum- } \\
\text { ference. }\end{array}$ & Tip to Tip. & Habitat. & & Owner. \\
\hline $15 \frac{3}{4}$ & II & $9 \frac{1}{4}$ & $\cdots$ & $\ldots$ & Sir Edmund G. Loder, Bart. \\
\hline $14 \frac{7}{8}$ & $9 \frac{5}{8}$ & $7 \frac{7}{8}$ & West Africa & $\ldots$ & British Museum. \\
\hline I $3 \frac{1}{8}$ & $8 \frac{7}{8}$ & 7 & Do. & ... & Do. \\
\hline II $\frac{33}{4}$ & $8 \frac{1}{2}$ & $9 \frac{7}{8}$ & $\ldots$ & ... & Sir Edmund G. Loder, Bart. \\
\hline
\end{tabular}

BOS DANTE.

Habitat-Africa.

\begin{tabular}{|c|c|c|c|c|}
\hline Length. & $\begin{array}{l}\text { Circum- } \\
\text { ference. }\end{array}$ & Tip to Tip. & Habitat. & Owner. \\
\hline $13 \frac{7}{8}$ & $10 \frac{1}{\frac{1}{4}}$ & $28 \frac{1}{2}$ & Gambia $\quad \ldots \quad \ldots$ & Earl of Derby, British Museum. \\
\hline
\end{tabular}

BOS MANUS.

Habitat-Africa.

\begin{tabular}{|c|c|c|c|c|}
\hline Length. & $\begin{array}{l}\text { Circum- } \\
\text { ference. }\end{array}$ & Tip to Tip. & Habitat. & Owner. \\
\hline $14 \frac{6}{8}$ & $8 \frac{1}{4}$ & $\frac{7}{3}$ & \multirow{3}{*}{$\begin{array}{l}\text { Sette Cama, West } \\
\text { Africa. } \\
\text { Do. } \\
\text { Do. }\end{array}$} & \multirow{2}{*}{$\begin{array}{c}\text { British Museum. } \\
\text { Do. }\end{array}$} \\
\hline $8 \frac{1}{2}$ & $5 \frac{8}{8}$ & $13 \frac{3}{8}$ & & \\
\hline $7 \frac{3}{8}$ & $6 \frac{1}{4}$ & 83 & & Do. \\
\hline
\end{tabular}

\section{ZEBU (Bos indicus).}

Habitat-India.

\begin{tabular}{|c|c|c|c|c|}
\hline Length. & $\begin{array}{l}\text { Circum- } \\
\text { ference. }\end{array}$ & Tip to Tip. & Habitat. & Owner. \\
\hline $17 \frac{1}{8}$ & $10 \frac{3}{4}$ & $25 \frac{1}{4}$ & India $\quad \ldots$ & British Museum. \\
\hline
\end{tabular}




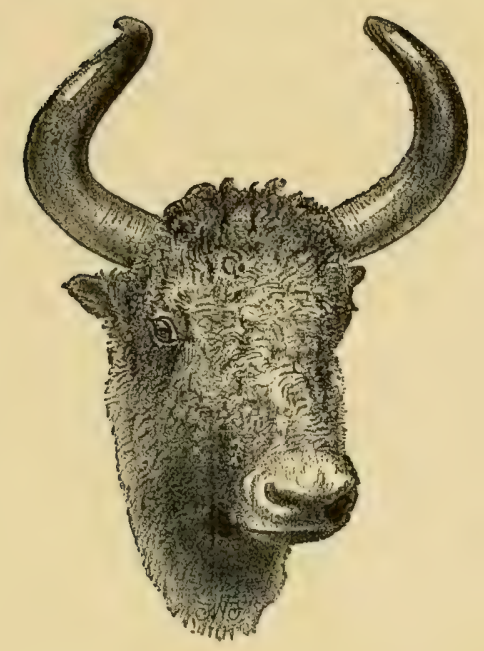

\section{YAK (Bos grunniens).}

\section{Habitat-Tibet.}

\begin{tabular}{|c|c|c|c|c|c|c|c|}
\hline Length. & $\begin{array}{l}\text { Circum- } \\
\text { ference. }\end{array}$ & $\begin{array}{l}\text { Tip to } \\
\text { Tip. }\end{array}$ & $\begin{array}{l}\text { Widest } \\
\text { inside. }\end{array}$ & \multicolumn{3}{|c|}{ Ilabitat. } & Uwner. \\
\hline $38 \frac{1}{4}$ & 17 & 19 & $3 \mathrm{I} \frac{1}{2}$ & $\cdots$ & $\cdots$ & $\cdots$ & Hume Collection, British Museum. \\
\hline $34 \frac{7}{8}$ & 15 & 16 & $27 \frac{3}{4}$ & ... & $\ldots$ & $\cdots$ & Do. \\
\hline 34 & 12 & $20 \frac{1}{2}$ & $\ldots$ & $\cdots$ & $\ldots$ & $\cdots$ & Hon. Walter Rothschild. \\
\hline $32 \frac{3}{8}$ & $13 \frac{3}{8}$ & $16 \frac{1}{2}$ & $26 \frac{3}{8}$ & ... & $\ldots$ & $\cdots$ & British Museum. \\
\hline 32 & I $3 \frac{7}{8}$ & $15 \frac{1}{4}$ & $22 \frac{1}{4}$ & $\cdots$ & $\ldots$ & $\cdots$ & H. C. V. Hunter. \\
\hline $30 \frac{3}{4}$ & I $3 \frac{1}{2}$ & $10 \frac{1}{2}$ & .. & ... & $\ldots$ & $\cdots$ & Sir Robert Harvey, Bart. \\
\hline $29 \frac{3}{4}$ & 14 & 12 & $25 \frac{1}{8}$ & ... & $\ldots$ & ... & Hume Collection, British Museum. \\
\hline $29 \frac{1}{2}$ & 12 & ... & $\cdots$ & $\cdots$ & $\ldots$ & $\cdots$ & Capt. G. Campbell. \\
\hline $28 \frac{1}{4}$ & 14 & $17 \frac{7}{8}$ & $26 \frac{3}{8}$ & India & $\ldots$ & ... & Capt. E. Smyth, British Museum. \\
\hline $27 \frac{7}{8}$ & II $\mathbf{I}_{4}^{3}$ & $18 \frac{3}{5}$ & $\begin{array}{c}27 \frac{33}{8} \\
\text { (wild) }\end{array}$ & Nepal & $\ldots$ & ... & B. H. Hodgson, British Museum. \\
\hline $27 \frac{1}{2}$ & $12 \frac{7}{8}$ & $14 \frac{33}{t}$ & $23 \frac{3}{4}$ & ... & $\ldots$ & $\cdots$ & Hume Collection, British Museum. \\
\hline $26 \frac{1}{2}$ & $10 \frac{7}{8}$ & 19 & $\begin{array}{c}22 \frac{33}{4} \\
\text { (wild) }\end{array}$ & ... & $\ldots$ & $\ldots$ & British Museum. \\
\hline 26 & $13 \frac{1}{2}$ & I 83 & $23 \frac{1}{2}$ & ... & $\ldots$ & ... & J. Carr-Saunders. \\
\hline
\end{tabular}


YAK (Bos grunniens)-continued.

\begin{tabular}{|c|c|c|c|c|c|c|c|}
\hline Length. & $\begin{array}{l}\text { Circum- } \\
\text { ference. }\end{array}$ & $\begin{array}{l}\text { Tip to } \\
\text { Tip. }\end{array}$ & $\begin{array}{l}\text { Width } \\
\text { inside. }\end{array}$ & \multicolumn{3}{|c|}{ Habitat. } & Owner. \\
\hline $22 \frac{3}{4}$ & $10 \frac{3}{8}$ & $12 \frac{1}{2}$ & $19 \frac{3}{4}$ & Nepal & $\cdots$ & ... & B. H. Hodgson, British Museum. \\
\hline $19 \frac{1}{8}$ & $9 \frac{1}{4}$ & $14 \frac{3}{4}$ & $\begin{array}{c}16 \frac{7}{8} \\
\text { (domestic) }\end{array}$ & Do. & $\ldots$ & ... & Do. \\
\hline $16 \frac{7}{8}$ & $6 \frac{1}{2}$ & $3 \frac{3}{8}$ & $\begin{array}{c}12 \frac{3}{8} \\
\text { (domestic) }\end{array}$ & Do. & $\ldots$ & $\ldots$ & Do. \\
\hline $16 \frac{3}{4}$ & 9 & $23 \frac{3}{t}$ & $\begin{array}{c}23^{\frac{3}{4}} \\
\text { (domestic) }\end{array}$ & Do. & ... & $\ldots$ & Do. \\
\hline $13 \frac{3}{8}$ & $5 \frac{5}{8}$ & $16 \frac{3}{8}$ & (domestic) & $\cdots$ & ... & $\cdots$ & British Museum. \\
\hline $11 \frac{3}{4}$ & $6 \frac{5}{8}$ & $9 \frac{1}{4}$ & $\begin{array}{c}\mathbf{I I} \frac{1}{2} \\
\text { (domestic) }\end{array}$ & Nepal & $\ldots$ & $\ldots$ & B. H. Hodgson, British Museum. \\
\hline
\end{tabular}

\section{JAVA OX (Bos banting.)}

Habitat-Java.

\begin{tabular}{|c|c|c|c|c|c|c|c|}
\hline Length. & $\begin{array}{l}\text { Circum- } \\
\text { ference. }\end{array}$ & $\begin{array}{l}\text { Tip to } \\
\text { Tip. }\end{array}$ & $\begin{array}{l}\text { Widest } \\
\text { inside. }\end{array}$ & & labitat. & & Owner. \\
\hline $24 \frac{3}{4}$ & $12 \frac{1}{4}$ & $15 \frac{33}{4}$ & $24^{\frac{1}{4}}$ & Java & $\cdots$ & $\cdots$ & British Museum. \\
\hline $21 \frac{3}{8}$ & $12 \frac{1}{4}$ & $13 \frac{1}{8}$ & $19 \frac{1}{4}$ & Borneo & $\ldots$ & $\cdots$ & H. B. Low, British Museum. \\
\hline $20 \frac{5}{8}$ & $12 \frac{1}{4}$ & $18 \frac{1}{8}$ & $22 \frac{1}{8}$ & Do. & $\ldots$ & $\ldots$ & Do. \\
\hline $19 \frac{1}{4}$ & II $\frac{1}{4}$ & $10 \frac{1}{8}$ & $15 \frac{3}{4}$ & Do. & ... & $\cdots$ & Do. \\
\hline I $S_{\frac{1}{4}}$ & $10 \frac{7}{8}$ & $14^{\frac{1}{2}}$ & $18 \frac{5}{8}$ & Do. & ... & ... & Do. \\
\hline IS & $12 \frac{1}{4}$ & $13 \frac{3}{8}$ & $16 \frac{5}{8}$ & Do. & ... & $\cdots$ & Do. \\
\hline $17 \frac{7}{8}$ & II & $2 \frac{3}{4}$ & $11 \frac{1}{4}$ & Do. & $\cdots$ & $\ldots$ & Do. \\
\hline $17 \frac{5}{8}$ & $10 \frac{3}{8}$ & $7 \frac{1}{8}$ & I $4 \frac{1}{t}$ & $\begin{array}{c}\text { British } \\
\text { neo. }\end{array}$ & North & Bor- & W. Pryer, British Museum. \\
\hline $17 \frac{1}{2}$ & $10 \frac{1}{8}$ & $17 \frac{1}{4}$ & $20 \frac{3}{4}$ & Siam & $\cdots$ & $\cdots$ & H. C. V. Hunter. (See Illustration.) \\
\hline $17 \frac{1}{2}$ & $9 \frac{7}{8}$ & $15^{3}$ & I $7 \frac{3}{4}$ & India & ... & $\cdots$ & British Museum. \\
\hline $16 \frac{1}{2}$ & $10 \frac{3}{4}$ & $9 \frac{1}{2}$ & $13 \frac{3}{8}$ & Sarawak & & ... & Do. \\
\hline $15 \frac{7}{8}$ & $10 \frac{1}{8}$ & $15 \frac{1}{4}$ & $x 6 \frac{1}{8}$ & Borneo & $\cdots$ & ... & H. B. Low, British Museum. \\
\hline $15 \frac{7}{8}$ & $9 \frac{3}{8}$ & $17 \frac{1}{x}$ & $\cdots$ & $\cdots$ & $\cdots$ & $\cdots$ & British Museum. \\
\hline $14 \frac{7}{8}$ & $10 \frac{3}{4}$ & $8 \frac{7}{8}$ & $13 \frac{1}{8}$ & Borneo & ... & ... & H. B. Low, British Museum. \\
\hline
\end{tabular}




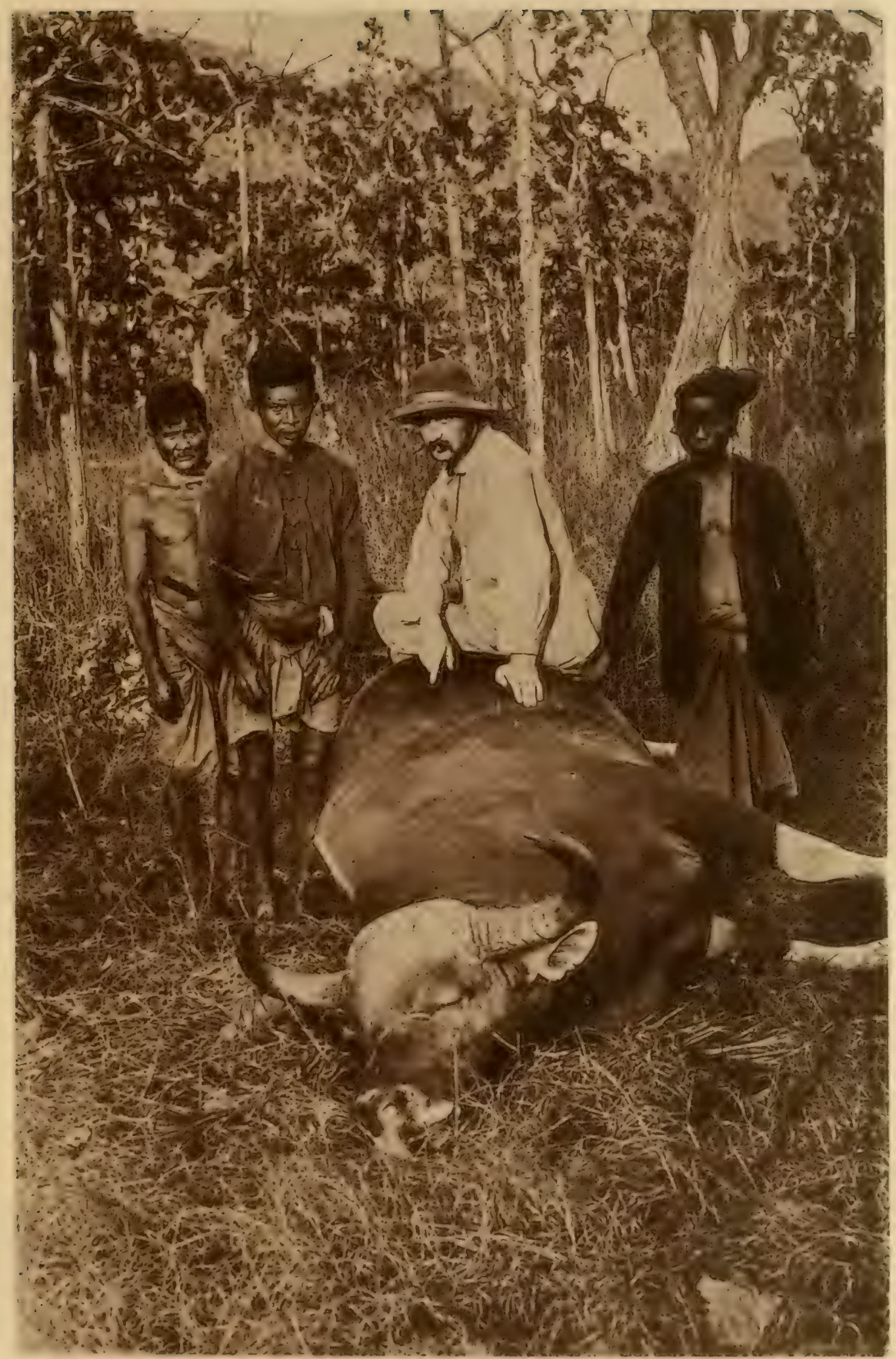





\section{SONDAIC OX (BANTENG) (Bos sondaicus).}

\section{Habitat-Java.}

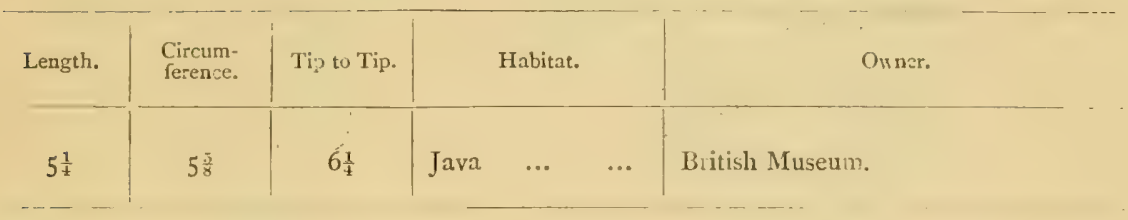

ANOA (Bos depressicornis).

Habitat-Celebes.

\begin{tabular}{|c|c|c|c|c|c|c|}
\hline Length. & $\begin{array}{l}\text { Circum- } \\
\text { ference. }\end{array}$ & Tip to Tip. & \multicolumn{3}{|c|}{ Habitat. } & Owner. \\
\hline $12 \frac{3}{8}$ & 6 & $6 \frac{1}{2}$ & Celebes & $\cdots$ & ... & British Museum. \\
\hline $12 \frac{1}{4}$ & $6 \frac{1}{4}$ & $8 \frac{3}{4}$ & Do. & $\ldots$ & $\ldots$ & Do. \\
\hline $11 \frac{7}{8}$ & $5 \frac{7}{8}$ & 7 & Do. & $\cdots$ & $\cdots$ & Do. \\
\hline$I I \frac{1}{2}$ & $5 \frac{3}{8}$ & $7 \frac{1}{4}$ & Do. & $\cdots$ & ... & Gen. Hardwicke, British Museum. \\
\hline $10 \frac{1}{3}$ & $6 \frac{1}{8}$ & $4 \frac{7}{3}$ & Do. & $\cdots$ & $\ldots$ & British Museum. \\
\hline 10 & $5 \frac{7}{8}$ & $5 \frac{\pi}{8}$ & Do. & $\cdots$ & $\ldots$ & Sir Victor Brooke's Collection. \\
\hline $8 \frac{7}{8}$ & $4 \frac{3}{8}$ & $4 \frac{3}{4}$ & Do. & $\cdots$ & $\ldots$ & Sir Edmund G. Loder, Bart. \\
\hline $7 \frac{5}{8}$ & $4 \frac{3}{8}$ & 4 & Do. & $\cdots$ & $\cdots$ & British Museum. \\
\hline $5 \frac{\pi}{8}$ & $4 \frac{1}{6}$ & 5 & Do. & ... & $\ldots$ & Do. \\
\hline
\end{tabular}




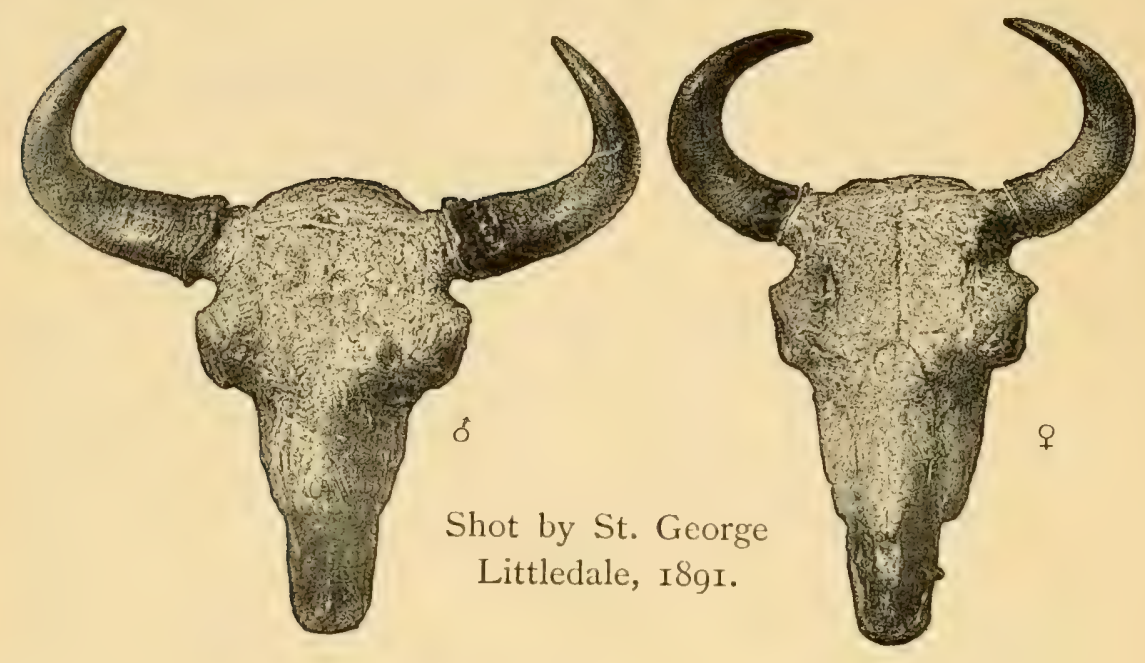

\section{AUROCHS (Bos urus).}

Habitat-Lithuania.

\begin{tabular}{|c|c|c|c|c|c|c|}
\hline I ength. & $\begin{array}{l}\text { Circum- } \\
\text { ference. }\end{array}$ & $\begin{array}{l}\text { Tip to } \\
\text { Tip. }\end{array}$ & $\begin{array}{l}\text { Widest } \\
\text { inside. }\end{array}$ & Habitat. & & Owner. \\
\hline I $8 \frac{1}{4}$ & $12 \frac{1}{8}$ & $13 \frac{3}{4}$ & $19 \frac{1}{2}$ & Lithuania & ... & $\begin{array}{l}\text { British Museum. (Presented by the } \\
\text { Emperor of Russia.) }\end{array}$ \\
\hline
\end{tabular}

\section{DOMESTIC OX (Bos taurus).}

\begin{tabular}{|c|c|c|c|c|c|}
\hline Length. & $\begin{array}{l}\text { Circum- } \\
\text { ference. }\end{array}$ & $\begin{array}{l}\text { Tip to } \\
\text { Tip. }\end{array}$ & $\begin{array}{l}\text { Widest } \\
\text { inside. }\end{array}$ & Habitat. & Owner. \\
\hline $\mathrm{SI}_{4} \times 78 \frac{1}{2}$ & $18 \frac{1}{4}$ & $103 \frac{1}{2}$ & .. & Lake 'Ngami & IV. C. Oswell. \\
\hline 47 & $15 \frac{5}{8}$ & $21 \frac{1}{8}$ & ... & Abyssinia (Galla Ox) & H. Salt, British Museum. \\
\hline 44 & $12 \frac{7}{2}$ & $\begin{array}{c}70 \frac{1}{2} \\
\text { (abuut) }\end{array}$ & $\ldots$ & $\ldots$ & British Museum. \\
\hline
\end{tabular}


DOIMESTIC OX (Bos taurus)-continud.

\begin{tabular}{|c|c|c|c|c|c|c|}
\hline Length. & $\begin{array}{l}\text { Circum- } \\
\text { fercunce. }\end{array}$ & $\begin{array}{l}\text { Tip to } \\
\text { Tip. }\end{array}$ & $\begin{array}{l}\text { Wifth } \\
\text { Inside. }\end{array}$ & Habitat. & & Owner. \\
\hline $42 \frac{1}{8}$ & $23 \frac{5}{8}$ & $22 \frac{1}{8}$ & $\cdots$ & Central Africa & ... & Col. Denham, British Museum. \\
\hline $4 I \frac{3}{4}$ & 14 & $52 \frac{3}{4}$ & ... & Madagascar & ... & Bethnal Green Museum. \\
\hline $40 \frac{1}{4}$ & I $2 \frac{1}{4}$ & $60 \frac{7}{8}$ & ... & Vienna (Polish Bu & ulI) & British Museum. \\
\hline $39 \frac{5}{8}$ & $15 \frac{7}{8}$ & 33 & $\cdots$ & $\cdots$ & ... & Do. \\
\hline $3^{S \frac{1}{2}}$ & $10 \frac{1}{2}$ & $54 \frac{3}{4}$ & $\cdots$ & Italy $\quad \ldots$ & .. & Bethnal Green Museum. \\
\hline $35^{\frac{3}{4}}$ & $12 \frac{3}{4}$ & $59 \frac{1}{2}$ & ... & Cape of Good Hop & pe & Do. \\
\hline $30 \frac{1}{8}$ & $12 \frac{3}{8}$ & 35 & $\cdots$ & Spain $\ldots$ & $\cdots$ & Do. \\
\hline $29 \frac{3}{8}$ & II $\frac{1}{8}$ & $28 \frac{7}{8}$ & ... & Gambia... & ... & Earl of Derby, British Museum. \\
\hline $18 \frac{1}{2}$ & $10 \frac{3}{8}$ & 20 & $21 \frac{1}{8}$ & Chillingham Park & & Earl of Tankerville, British Museum. \\
\hline$q$ I $8+$ & 7 & $10 \frac{1}{8}$ & $15 \frac{3}{8}$ & Do. & & Do. \\
\hline I $7 \frac{1}{2}$ & $10 \frac{3}{5}$ & $30 \frac{1}{2}$ & ... & $\begin{array}{l}\text { Buenos Ayres } \\
\text { (Niata Cattle). }\end{array}$ & & G. Claraz, British Museum. \\
\hline I $7 \frac{1}{4}$ & $10_{\frac{1}{4}}^{\frac{1}{4}}$ & $25 \frac{1}{8}$ & $\cdots$ & Gambia ... & -.. & Early of Derby, British Museum. \\
\hline $15 \frac{1}{ \pm}$ & $9 \frac{5}{8}$ & I $7 \frac{3}{8}$ & I $8 \frac{1}{8}$ & Northumberland & & Duke of Hamilton, British Museum. \\
\hline $12 \frac{1}{8}$ & 8 & $16 \frac{1}{8}$ & $\cdots$ & Angola, West Afri & ica & Sir Victor Brooke's Collection. \\
\hline $12 \frac{1}{8}$ & $6 \frac{1}{8}$ & $16 \frac{3}{4}$ & $16 \frac{7}{8}$ & England & $\cdots$ & British Museum. \\
\hline I $\frac{1}{2}$ & $7 \frac{1}{2}$ & $\begin{array}{l}\text { (single } \\
\text { horn) }\end{array}$ & $\ldots$ & Nepal ... & $\cdots$ & B. H. Hodgson, British Museum. \\
\hline $8 \frac{1}{8}$ & 8 & I $8 \frac{1}{4}$ & $\cdots$ & Do. & $\cdots$ & Do. \\
\hline
\end{tabular}




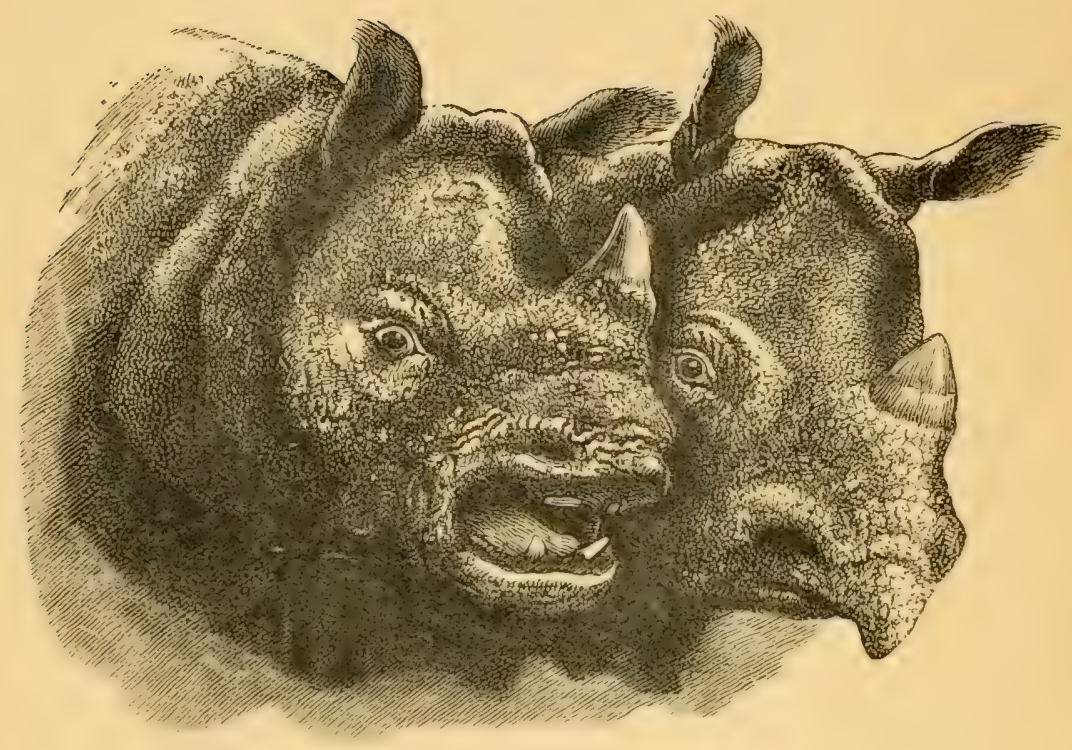

INDIAN RHINOCEROS (Rhinoceros unicornis).

Habitat-India.

\begin{tabular}{|c|c|c|c|c|c|c|}
\hline I.ength. & $\begin{array}{l}\text { Circum- } \\
\text { ference. }\end{array}$ & Tip to Tip. & & Habitat. & & Owner. \\
\hline 24 & $\ldots$ & $\cdots$ & $\ldots$ & $\cdots$ & ... & T. C. Jerdon, "Mammals of India." \\
\hline $19 \frac{1}{8}$ & $22 \frac{3}{8}$ & $\cdots$ & India & $\cdots$ & $\cdots$ & British Museum. \\
\hline $5^{\frac{1}{2}}$ & $14 \frac{1}{3}$ & $\cdots$ & Kuch & Behar & $\ldots$ & Capt. Henry Streatfield. \\
\hline
\end{tabular}




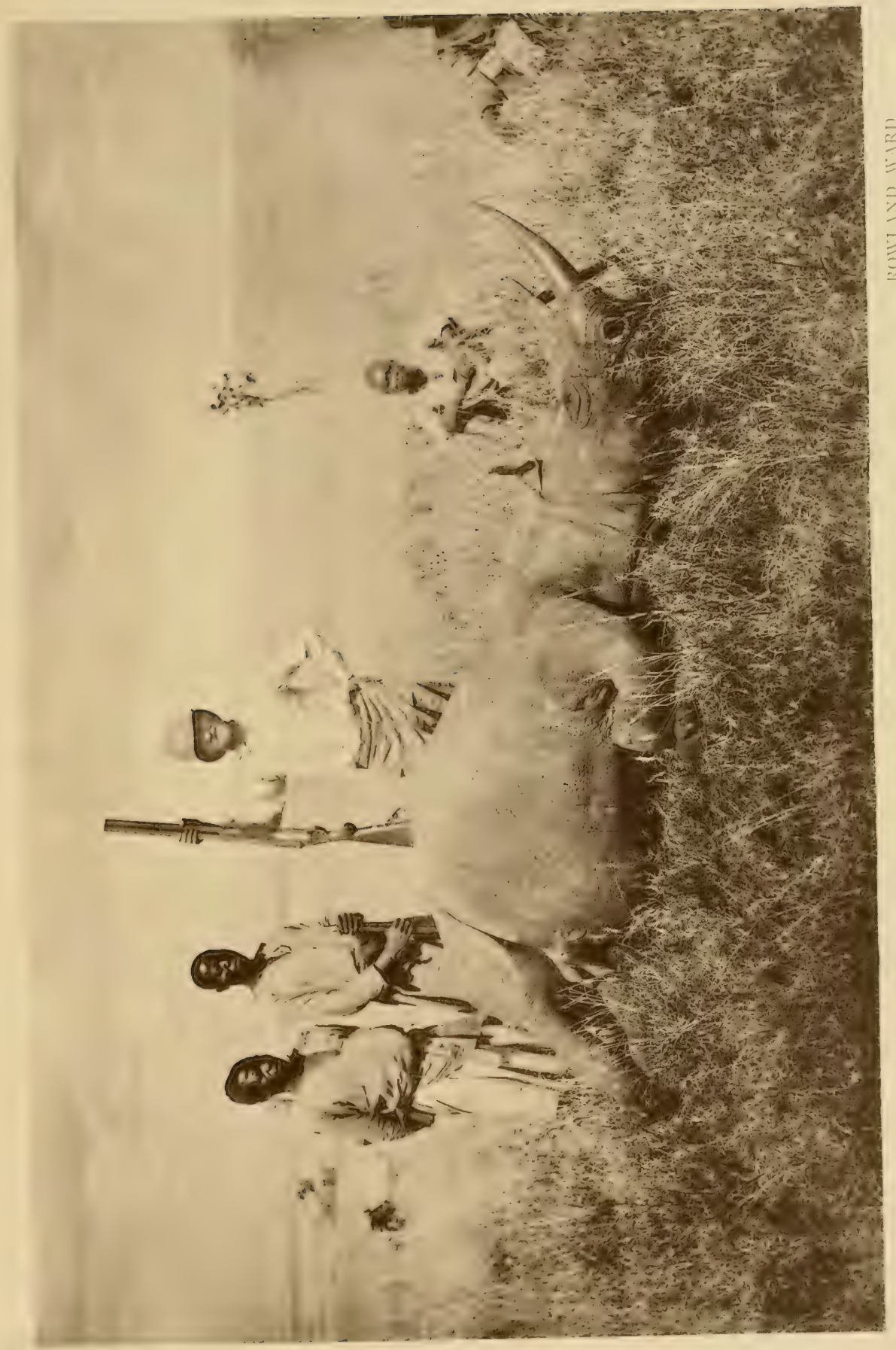

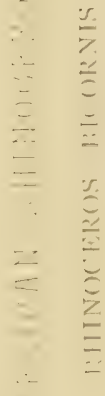





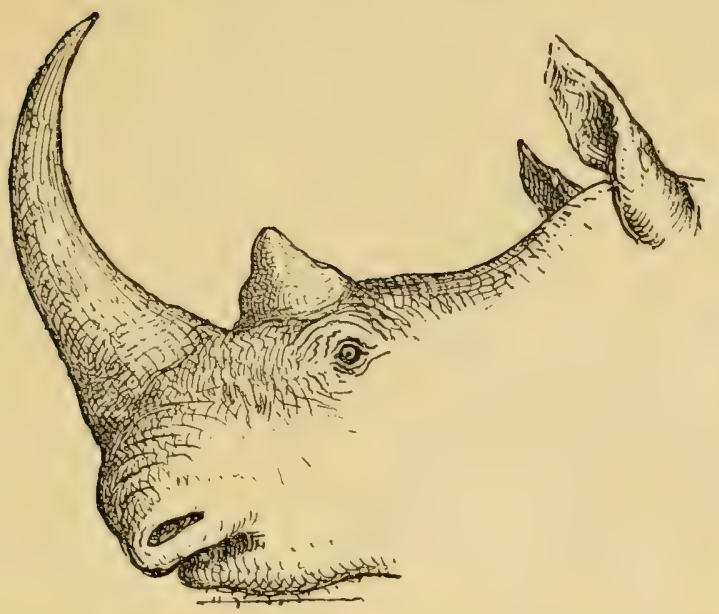

\section{AFRICAN RHINOCEROS (Rhinoceros bicornis).}

\section{Bechunana Name-"Borele."}

\section{Habitat-Africa.}

\begin{tabular}{|c|c|c|c|c|c|c|c|}
\hline \multicolumn{2}{|c|}{ Length. } & \multicolumn{2}{|c|}{ Circumference. } & \multirow{2}{*}{\multicolumn{3}{|c|}{ Habitat. }} & \multirow{2}{*}{ Owner. } \\
\hline $\begin{array}{l}\text { Front } \\
\text { Horn. }\end{array}$ & $\begin{array}{c}\text { Rear } \\
\text { Horn. }\end{array}$ & $\begin{array}{l}\text { Front } \\
\text { Horn. }\end{array}$ & $\begin{array}{l}\text { Rear } \\
\text { Horn. }\end{array}$ & & & & \\
\hline$*_{5} 6 \frac{1}{2}$ & $\ldots$ & $23 \frac{1}{2}$ & $\ldots$ & $\cdots$ & $\cdots$ & $\ldots$ & British Museum. \\
\hline 54 & $\cdots$ & $\ldots$ & ... & Sou & Africa & $\ldots$ & F. C. Selous (has seen). \\
\hline$*_{44}$ & $\cdots$ & 20 & $\ldots$ & ... & $\ldots$ & $\ldots$ & British Museum. \\
\hline 44 & $\cdots$ & $\ldots$ & $\cdots$ & Eas & frica & $\ldots$ & F. Holmwood. \\
\hline 43 & $\cdots$ & $\cdots$ & $\cdots$ & $\cdots$ & $\ldots$ & $\cdots$ & F. C. Selous, "A Hunter's Wan- \\
\hline$\dagger 42 \frac{5}{4}$ & $\cdots$ & $25 \frac{5}{8}$ & $\ldots$ & I in & $\begin{array}{l}\text { po, Sout } \\
\text { ca. }\end{array}$ & & \\
\hline $4 I$ & $\cdots$ & $\cdots$ & $\cdots$ & Ea & frica & $\cdots$ & F. Holmwood. \\
\hline *40 & $\cdots$ & I $5 \frac{3}{4}$ & $\cdots$ & $\cdots$ & $\cdots$ & $\cdots$ & British Museum. \\
\hline 40 & ... & $\cdots$ & .. & Eas & frica & $\ldots$ & F. Holmwood. \\
\hline $38 \frac{3}{4}$ & ... & $2 I$ & $\ldots$ & $\ldots$ & $\cdots$ & $\ldots$ & Hon. Walter Rothschild. \\
\hline Q $* 38 \frac{5}{8}$ & ... & $24 \frac{1}{2}$ & ... & $\ldots$ & $\ldots$ & $\ldots$ & W. C. Oswell. \\
\hline
\end{tabular}

* Rhinoceros simus (White or Burchell's Rhinoceros). † Rhinoceros oswellii. 
AFRICAN RHINOCEROS (Rhinoceros bicornis)-continud.

\begin{tabular}{|c|c|c|c|c|c|c|}
\hline \multicolumn{2}{|c|}{ Length. } & \multicolumn{2}{|c|}{ Circumference. } & \multirow{2}{*}{\multicolumn{2}{|c|}{ Habitat. }} & \multirow{2}{*}{ Owner. } \\
\hline $\begin{array}{l}\text { Front } \\
\text { Horn. }\end{array}$ & $\begin{array}{l}\text { Rear } \\
\text { Horn. }\end{array}$ & $\begin{array}{l}\text { Front } \\
\text { Hom. }\end{array}$ & $\begin{array}{l}\text { Rear } \\
\text { Hom. }\end{array}$ & & & \\
\hline $38 \frac{1}{3}$ & $\cdots$ & 19 & $\cdots$ & Masai Land & $\ldots$ & Sir John Kirk, M. D., K.C. B. \\
\hline$* 37 \frac{3}{8}$ & $17 \frac{7}{8}$ & $27 \frac{1}{8}$ & $\cdots$ & South Africa & $\cdots$ & F. C. Selous. \\
\hline$* 37 \frac{1}{4}$ & $\cdots$ & 24 & $\cdots$ & $\ldots$ & $\ldots$ & British Museum. \\
\hline 35 & $\cdots$ & $15 \frac{1}{8}$ & $\cdots$ & Masai Land & $\ldots$ & Sir John Kirk, M. D., K. C.B. \\
\hline$* 33 \frac{1}{2}$ & $\cdots$ & 21 & $\cdots$ & $\cdots$ & $\ldots$ & $\begin{array}{l}\text { British Museum (Sir Hans Sloane's } \\
\text { Museum). }\end{array}$ \\
\hline$* 33 \frac{9}{8}$ & $\cdots$ & 22 & $\cdots$ & $\cdots$ & $\ldots$ & British Museum. \\
\hline$* 33$ & $13 \frac{1}{8}$ & 231 & $\cdots$ & $\cdots$ & $\cdots$ & F. C. Selous. \\
\hline$* 32 \frac{3}{4}$ & $\cdots$ & $27 \frac{1}{3}$ & $\cdots$ & $\cdots$ & $\ldots$ & The late J. S. Jameson. \\
\hline$\delta * 32 \frac{3}{4}$ & $\cdots$ & 27 & $\cdots$ & $\cdots$ & $\cdots$ & W. C. Oswell. \\
\hline$+31 \frac{1}{2}$ & $\cdots$ & $\cdots$ & $\cdots$ & $\ldots$ & $\cdots$ & Dr. J. E. Gray, P.Z.S. 1854 , p. 250. \\
\hline 31 & $\cdots$ & I 8 & $\cdots$ & East Africa & $\ldots$ & R. P. Carrol. \\
\hline $3 \mathrm{I}$ & I $9 \frac{1}{2}$ & I6 & $16 \frac{1}{x}$ & Do. ... & $\ldots \mid$ & H. C. V. Hunter. \\
\hline$\$ 3 \mathbf{I}$ & $\cdots$ & $\cdots$ & $\cdots$ & $\begin{array}{l}\text { Central South } \\
\text { Africa. }\end{array}$ & 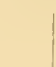 & P.Z.S. I 853, p. 46. \\
\hline$* 30^{\frac{3}{4}}$ & $\cdots$ & $19 \frac{33}{8}$ & $\cdots$ & $\begin{array}{ll}\ldots & \ldots\end{array}$ & $\ldots$ & British Museum. \\
\hline $30 \frac{1}{2}$ & $\cdots$ & $2 I_{\frac{1}{4}}^{\frac{1}{4}}$ & $\cdots$ & Zambezi Valley & $\cdots$ & Sir John Kirk, M.D., K.C.B. \\
\hline 29 & $\cdots$ & I9 & $\cdots$ & East Africa & $\cdots$ & H.H. Prince Boris Czetwertynski. \\
\hline$*_{2} S \frac{7}{8}$ & 9 & $22 \frac{5}{8}$ & $19 \frac{1}{4}$ & Lake 'Ngami & $\cdots$ & $\begin{array}{l}\text { WV. C. Oswell and Col. T. Stecle, } \\
\text { British Museum. }\end{array}$ \\
\hline $28 \frac{3}{4}$ & $15^{\frac{1}{4}}$ & $17 \frac{3}{8}$ & 178 & East Africa & $\ldots \mid$ & H. C. V. Hunter. \\
\hline $28 \frac{1}{4}$ & $8 \frac{3}{8}$ & I $\delta_{\frac{1}{8}}$ & $\cdots$ & $\cdots$ & $\cdots$ & F. C. Selous. \\
\hline 28 & $\cdots$ & $26 \frac{1}{4}$ & $\cdots$ & $\cdots$ & $\ldots$ & W. Fitzherbert. \\
\hline $27 \frac{7}{8}$ & $\cdots$ & $12 \frac{1}{8}$ & $\cdots$ & $\cdots$ & $\ldots \mid$ & J. Carr-Saunders. \\
\hline $27^{\frac{3}{4}}$ & $\cdots$ & I4 & $\cdots$ & $\ldots$ & $\ldots$ & $\mathrm{Dn}$ \\
\hline 27 & $\ldots$ & $20 \frac{1}{2}$ & $\ldots$ & East Africa & $\cdots$ & E. Gedge. \\
\hline 27 & $I 6 \frac{1}{2}$ & $17 \frac{7}{8}$ & I $7 \frac{1}{2}$ & Do. ... & $\ldots$ & Sir Robert Harvey, Bart. \\
\hline 27 & 12 & $\cdots$ & $\ldots$ & Do. ... & $\cdots$ & $\begin{array}{l}\text { Sir John Willoughby, Bart., " East } \\
\text { Africa and its Big Game." }\end{array}$ \\
\hline 26 & $\cdots$ & 23 & $\cdots$ & Do. ... & $\ldots$ & Do. \\
\hline
\end{tabular}

* Rhinoceros simus (White or Burchell's Rhinoceros). + Rhinoceros crossii. $\quad$ Rhinoceros oswellii. 
AFRICAN RHINOCEROS (Rhinoceros bicornis)-continutd.

\begin{tabular}{|c|c|c|c|c|c|c|}
\hline \multicolumn{2}{|c|}{ Length. } & \multicolumn{2}{|c|}{ Circumference. } & \multirow{2}{*}{\multicolumn{2}{|c|}{ Habitat. }} & \multirow{2}{*}{ Owner. } \\
\hline $\begin{array}{l}\text { Front } \\
\text { Horn. }\end{array}$ & $\begin{array}{l}\text { Rear } \\
\text { Horn. }\end{array}$ & $\begin{array}{l}\text { Front } \\
\text { Horn. }\end{array}$ & $\begin{array}{l}\text { Rear } \\
\text { Hom. }\end{array}$ & & & \\
\hline$*_{25 \frac{5}{8}}$ & ... & I $S_{\frac{3}{8}}$ & $\ldots$ & East Africa & $\ldots$ & British Museum. \\
\hline $25 \frac{1}{2}$ & II $1 \frac{3}{8}$ & $19 \frac{1}{2}$ & $\ldots$ & Do. ... & $\ldots$ & Sir Robert Harvey, Bart. \\
\hline $25 \frac{3}{8}$ & $9 \frac{1}{8}$ & $17 \frac{3}{8}$ & $\ldots$ & Do. ... & $\ldots$ & F. J. Jackson. \\
\hline $23 \frac{7}{8}$ & $13 \frac{1}{2}$ & $16 \frac{5}{8}$ & $17 \frac{3}{4}$ & Do. ... & $\ldots$ & Sir Robert Harvey, Bart. \\
\hline 23 & $\cdots$ & $22 \frac{3}{4}$ & $\cdots$ & South Africa & $\cdots$ & W. C. Oswell. \\
\hline 23 & $\cdots$ & $\ldots$ & $\cdots$ & $\begin{array}{ll}\cdots & \cdots\end{array}$ & $\ldots$ & $\begin{array}{l}\text { Sir Samuel Baker, F.R.S., "Wild } \\
\text { Beasts and their Ways." }\end{array}$ \\
\hline $22 \frac{7}{8}$ & I I $\frac{1}{2}$ & $16 \frac{3}{8}$ & ... & East Africa & $\ldots$ & F. J. Jackson. \\
\hline $22 \frac{3}{4}$ & $\ldots$ & $19 \frac{1}{4}$ & $\cdots$ & Do. ... & $\ldots$ & W. Astor Chanler. \\
\hline $22 \frac{3}{4}$ & $8 \frac{5}{8}$ & I7 & .. & Zambezi Valley & $\ldots$ & Sir John Kirk, M.D., K.C. B. \\
\hline $22 \frac{1}{4}$ & I I $\frac{1}{2}$ & I7 & ... & East Africa & $\ldots$ & Sir Robert Harvey, Bart. \\
\hline 22 & $17 \frac{7}{8}$ & I $6 \frac{3}{4}$ & $15 \frac{1}{4}$ & Do. ... & ... & Sir John Kirk, M.D., K.C.B. \\
\hline $21 \frac{3}{4}$ & $9 \frac{3}{8}$ & $19 \frac{1}{8}$ & $\ldots$ & Do. ... & $\ldots$ & Sir Robert Harvey, Bart. \\
\hline $2 I \frac{1}{2}$ & I $8 \frac{3}{4}$ & $22 \frac{1}{8}$ & $20 \frac{1}{2}$ & South Africa & $\ldots$ & Sir A. Smith, British Museum. \\
\hline $2 \mathbf{I} \frac{3}{8}$ & 17 & 14 & 16 & Abyssinia & $\ldots$ & British Museum. \\
\hline $20 \frac{1}{8}$ & $8 \frac{3}{8}$ & $16 \frac{1}{4}$ & $\ldots$ & East Africa & $\ldots$ & Sir Robert Harvey, Bart. \\
\hline $19 \frac{7}{8}$ & $7^{\frac{3}{4}}$ & I $8_{4}^{3}$ & $\cdots$ & Do. ... & $\ldots$ & Do. \\
\hline $17 \frac{1}{2}$ & $7 \frac{3}{4}$ & $19 \frac{1}{4}$ & $19 \frac{7}{8}$ & Abyssinia & $\ldots$ & British Museum. \\
\hline $16 \frac{3}{4}$ & $\cdots$ & $15 \frac{7}{8}$ & $\cdots$ & Soudan ... & $\cdots$ & Col. Ralph Vivian. \\
\hline $16 \frac{3}{8}$ & $12 \frac{5}{8}$ & I $3 \frac{3}{4}$ & $14 \frac{3}{4}$ & Abyssinia & $\cdots$ & W. Jessel, British Museum. \\
\hline 16 & $\cdots$ & I $7 \frac{1}{2}$ & $\cdots$ & Soudan ... & $\cdots$ & Col. Ralph Vivian. \\
\hline $15 \frac{7}{8}$ & Io & $19 \frac{5}{6}$ & $19 \frac{3}{4}$ & Do. $\quad \ldots$ & $\cdots$ & Do. \\
\hline $15 \frac{3}{4}$ & $\cdots$ & $20 \frac{1}{4}$ & $\cdots$ & South Africa & $\cdots$ & W. C. Oswell. \\
\hline I $4 \frac{5}{8}$ & $14 \frac{3}{4}$ & I7 & $\cdots$ & East Africa & $\cdots$ & Sir Robert İarvey, Bart. \\
\hline $13 \frac{5}{8}$ & 9 & $15^{\frac{3}{8}}$ & $16 \frac{1}{2}$ & Abyssinia & $\cdots$ & British Museum. \\
\hline $1 I \frac{1}{2}$ & $4^{\frac{1}{2}}$ & $13 \frac{7}{8}$ & I $2 \frac{1}{2}$ & South Africa & $\cdots$ & Earl of Derby, British Museum. \\
\hline 10 & $6 \times 3$ & (3 horns) & $\cdots$ & $\cdots$ & $\cdots$ & $\begin{array}{l}\text { Sir Joln Wrilloughby, J3art., "East } \\
\text { Africa and its Big Game." }\end{array}$ \\
\hline
\end{tabular}

* Rhinoceros simus (White or Burchell's Rhinoceros). 
SUIMATRAN RHINOCEROS (Rhinoceros sumatrensis).

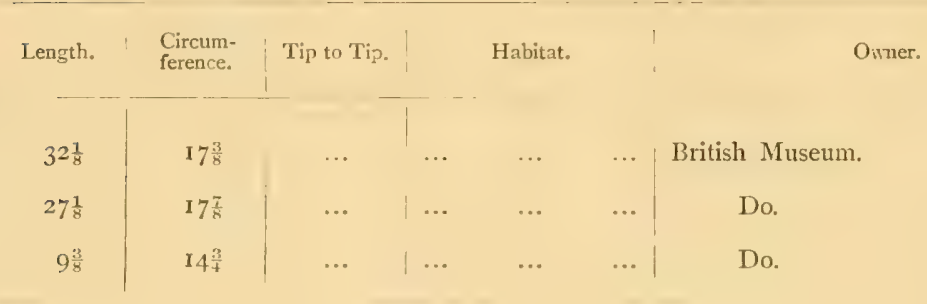

JAVAN RHINOCEROS (Rhinoceros sondaicus).

\begin{tabular}{l|c|c|c|c|c}
\hline Length. & $\begin{array}{l}\text { Circum- } \\
\text { ference. }\end{array}$ & Tip to Tip. & Habitat. & Owner. \\
\hline & & & & & \\
\hline
\end{tabular}




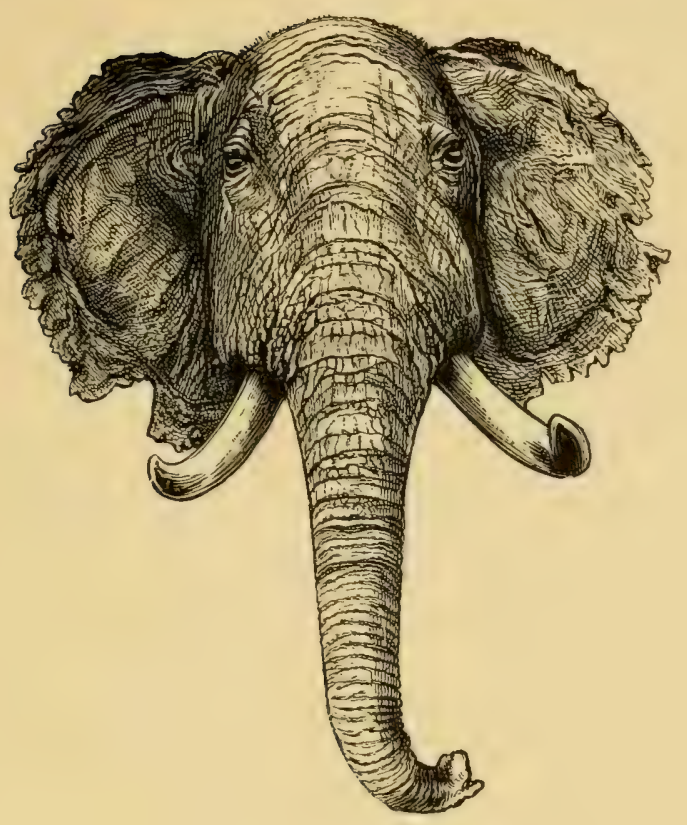

\section{ELEPHANT.}

LARGEST elephant ever killed by Col. G. P. Sanderson, vertical height at shoulder $9 \mathrm{ft}$. $7 \mathrm{in}$.; length from tip of trunk to tip of tail $26 \mathrm{ft}$. $2 \frac{1}{2}$ in.; tusks protruding from gum $2 \mathrm{ft} .4 \frac{1}{2} \mathrm{in}$.; tusks when taken out $5 \mathrm{ft}$.; tusk circumference at gum I ft. $4 \frac{1}{2}$ in.; tusk weight of each $37 \frac{1}{2}$ lbs.

Indian Elephants at Zoological Society's Gardens (P.Z.S. I879 and I88I).

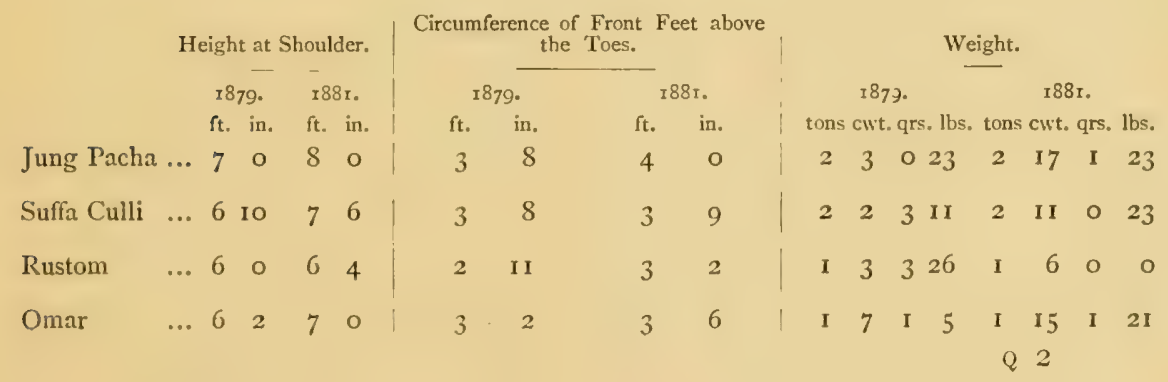


Height of African Elephant, foot to shoulder, Io ft.; F. C. Selous, "A Hunter's Wanderings."

Dimensions of Male Elephant killed on Lake Nyassa, gth September, I86I (Dr. Kirk, P.Z.S. I864, p. 655).

\begin{tabular}{|c|c|c|c|c|c|}
\hline Height at withers & & $\begin{array}{r}\text { in. } \\
3\end{array}$ & Horizontal diameter of ear & $\ldots$ & \\
\hline Circumference of fore foot ... & 5 & I & Perpendicular & $\ldots$ & \\
\hline From tip of trunk to crown of head & 9 & 0 & Semicircumference of belly & $\ldots$ & \\
\hline Crown of head to insertion of tail ... & 12 & 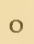 & of chest & $\ldots$ & \\
\hline Length of tail & 4 & 2 & Each tusk weighed & $301 \mathrm{lt}$ & \\
\hline
\end{tabular}

Dimensions of Elephant brought from South Africa by H.R.H. the Duke of Edinburgh.

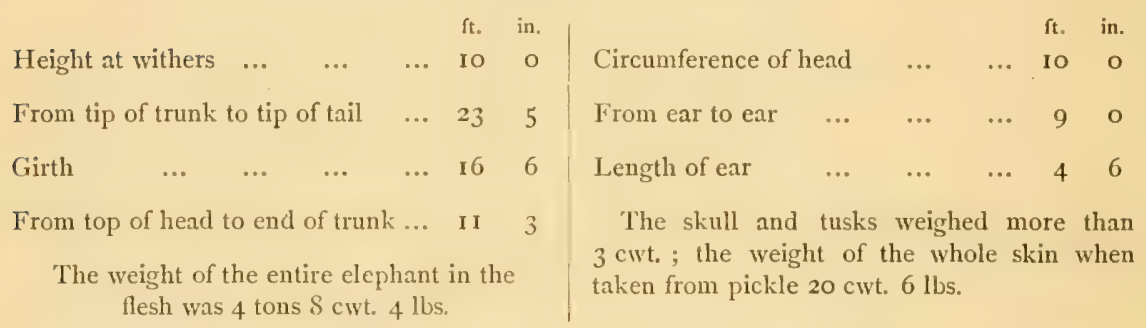

Weight of a Baby Elephant, when born, about $2 \mathrm{I}_{3}$ lbs. Period of gestation, 629 days. 



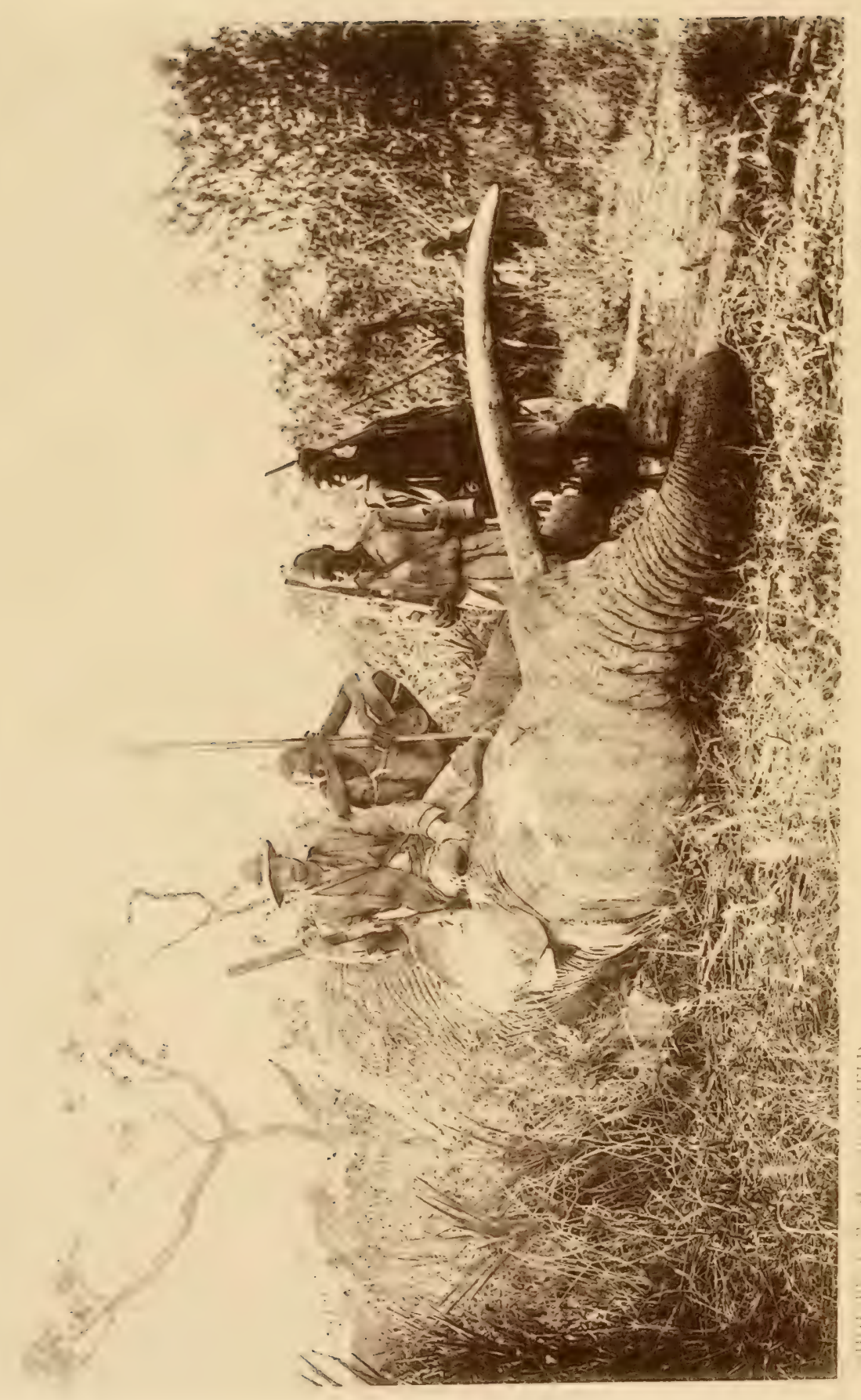




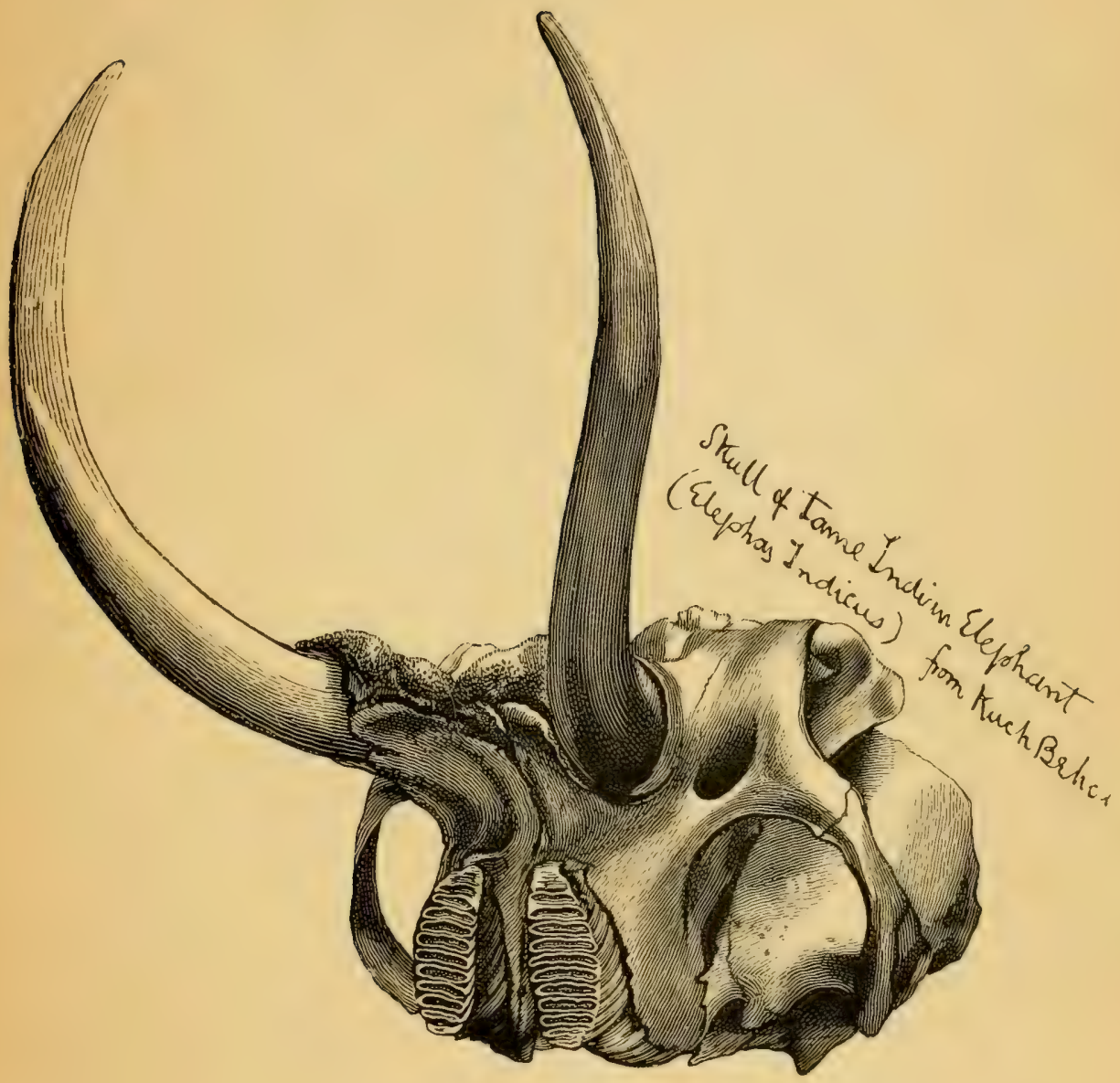

THis formed part of the Trophy of His Highness the Maharajalt of Kuch Behar exhibited at the Indian and Colonial Exhibition, I886. 
ELEPHANT TUSKS.

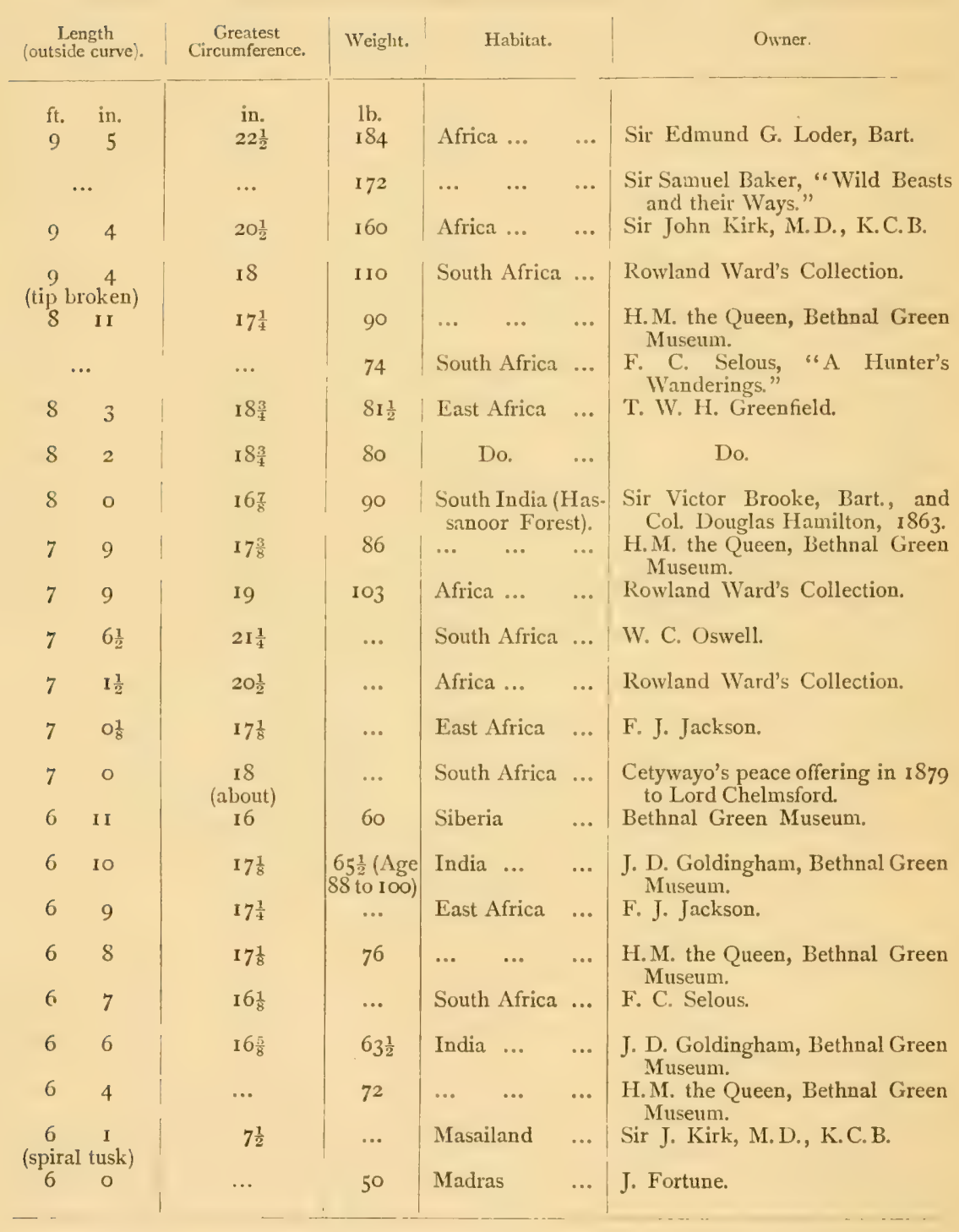


ELEPHANT TUSKS-continued.

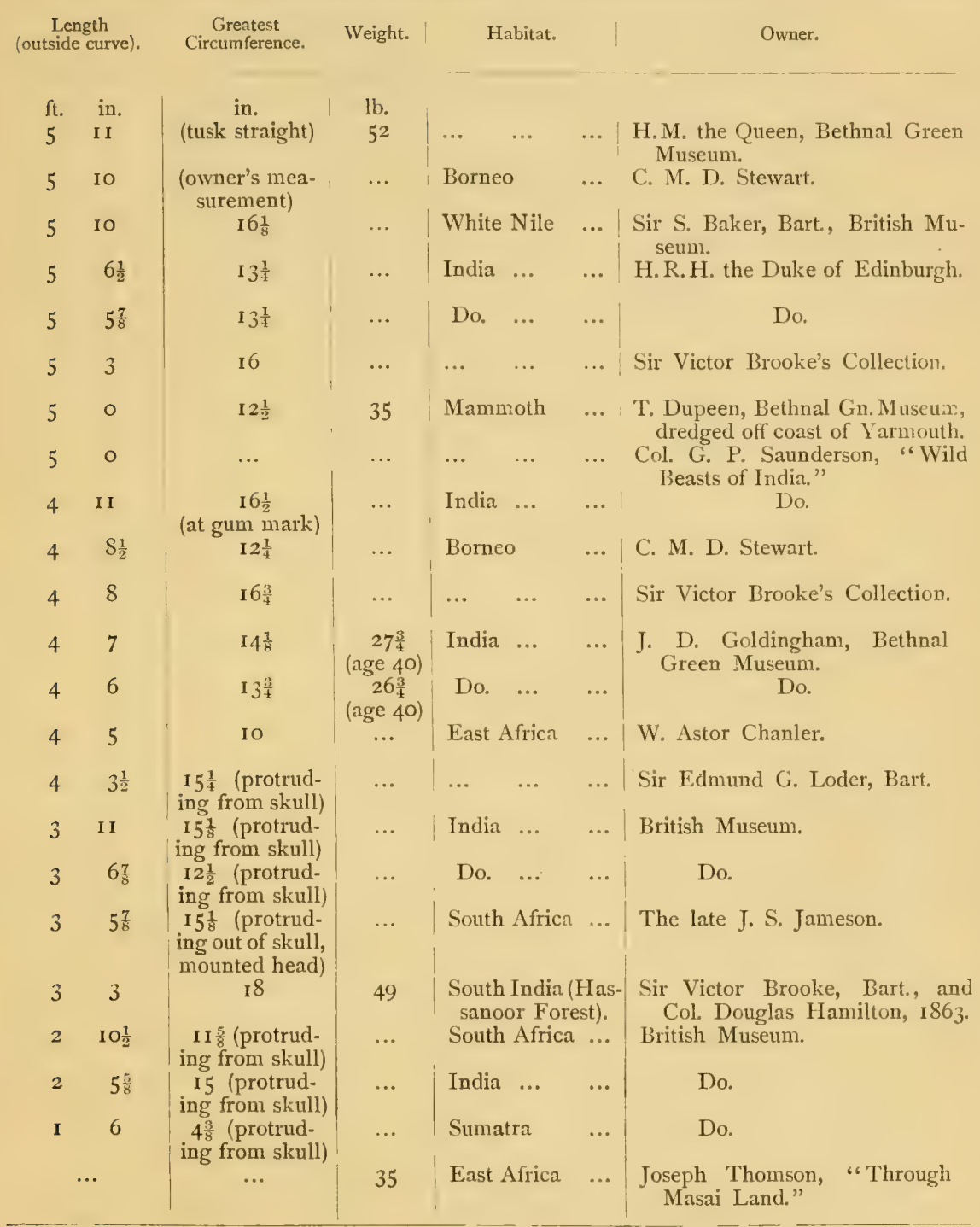




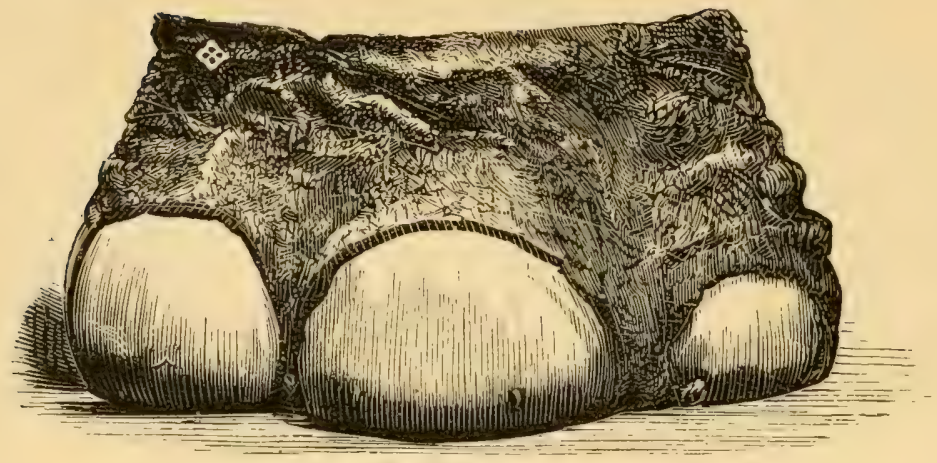

ELEPHANT FEET.

\begin{tabular}{|c|c|c|c|c|c|}
\hline $\begin{array}{l}\text { Circumference } \\
\text { at base. }\end{array}$ & $\begin{array}{l}\text { Width at bottom } \\
\text { back to fron:. }\end{array}$ & & Iabitat. & & Owner. \\
\hline $62 \frac{1}{2}$ & 20 & India & $\cdots$ & $\cdots$ & J. liortune. \\
\hline 54 & $I S \frac{1}{2}$ & Somali I & Land & $\cdots$ & Capt. J. S. Ashby. \\
\hline $53 \frac{7}{8}$ & $\mathbf{I} S_{\frac{1}{2}}$ & South Af & frica & $\cdots$ & The late J. S. Jameson. \\
\hline $53 \frac{1}{2}$ & IS & Ceylon & $\cdots$ & $\cdots$ & 11. Storey. \\
\hline $53 \frac{1}{4}$ & I $S_{\frac{1}{4}}$ & South Af & frica & $\cdots$ & The late J. S. Jameson. \\
\hline $52 \frac{1}{2}$ & 19 & Somali I & Land & $\cdots$ & Capt. J. S. $\Lambda$ shby. \\
\hline $52 \frac{1}{2}$ & 17 & Ceylon & $\cdots$ & $\cdots$ & I1. L. Lindsey. \\
\hline $52 \frac{1}{2}$ & 17 & Do. & $\cdots$ & $\cdots$ & Rowland Ward's Collection. \\
\hline $5 I \frac{1}{2}$ & $\cdots$ & Do. & $\cdots$ & $\cdots$ & Capt. C. Hill. \\
\hline 50 & I 7 & Do. & $\cdots$ & $\cdots$ & H. E. Lindsey. \\
\hline $49 \frac{1}{4}$ & $16 \frac{1}{2}$ & Last Afri & ica & $\cdots$ & W. Astor Chanler. \\
\hline 49 & $\cdots$ & Ceylon & $\cdots$ & $\cdots$ & Capt. C. Hill. \\
\hline $48 \frac{7}{8}$ & $\mathrm{I} 6 \frac{1}{x}$ & East Afri & ica & $\cdots$ & H. C. V. Hunter. \\
\hline $48 \frac{5}{8}$ & 16 & Do. & $\ldots$ & $\because$ & Do. \\
\hline $45^{\frac{1}{2}}$ & $15 \frac{1}{2}$ & Mysore & & $\cdots$ & W. G. Deeds. \\
\hline 45 & 17 & India & $\cdots$ & $\cdots$ & R. Stirling. \\
\hline $44 \frac{3}{4}$ & $14 \frac{3}{4}$ & Soudan & $\cdots$ & $\ldots$ & Col. Ralph Vivian. \\
\hline 44 & 14 & Ceylon & $\cdots$ & $\cdots$ & J. 13. Gardyne. \\
\hline $43 \frac{1}{2}$ & $13 \frac{1}{2}$ & Do. & $\ldots$ & $\ldots$ & Do. \\
\hline
\end{tabular}




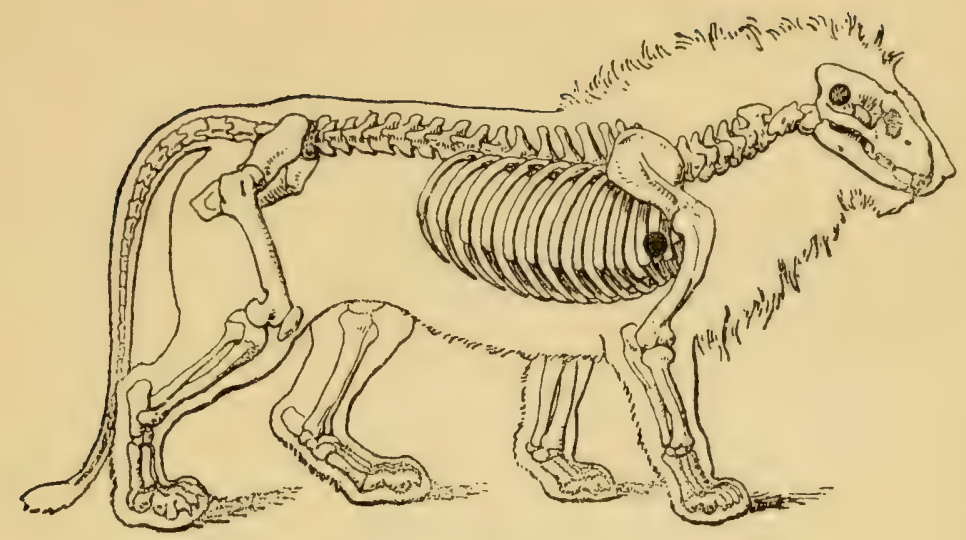

LION (Felis leo).

Habitat-India (nearly extinct) and Africa.

F. C. Selous, in “A Hunter's Wanderings," says weight $500 \mathrm{lb}$.

"The Field," July I3th, I89o, weight $563 \mathrm{lb}$.

Weight, one killed by J. S. Jameson in Mashuna Land, South Africa, November I887, $385 \mathrm{lb}$.

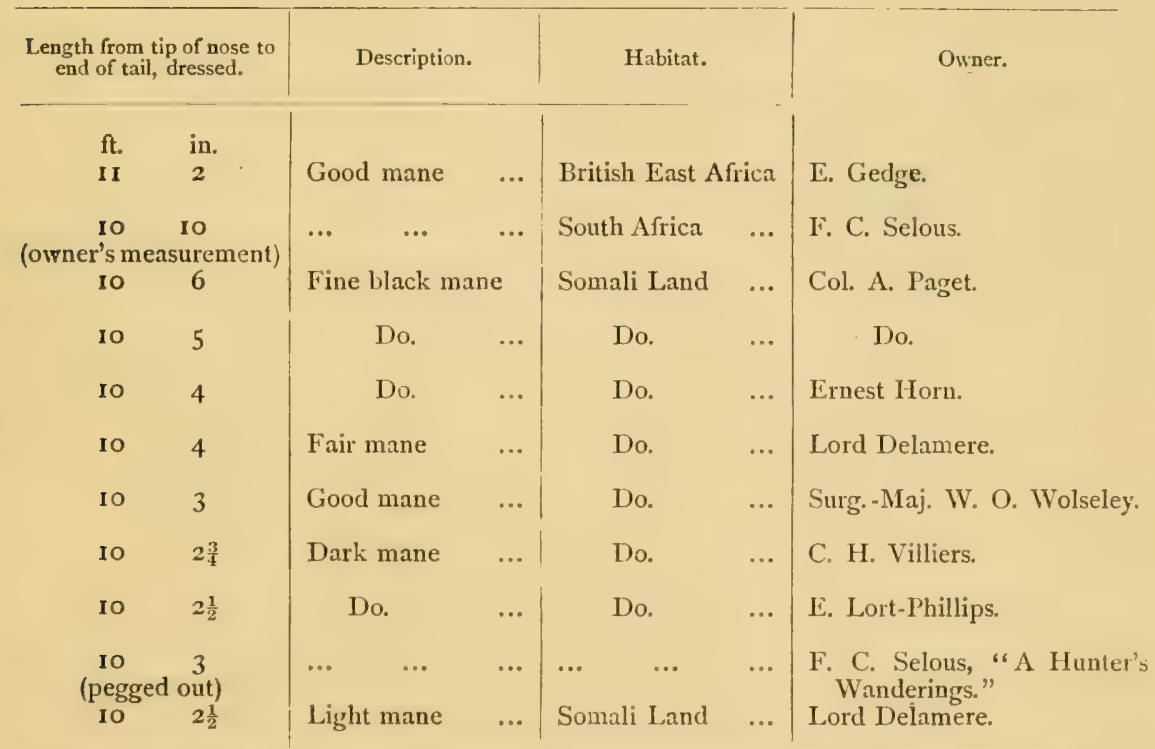


LION (Felis 1eo)-continued.

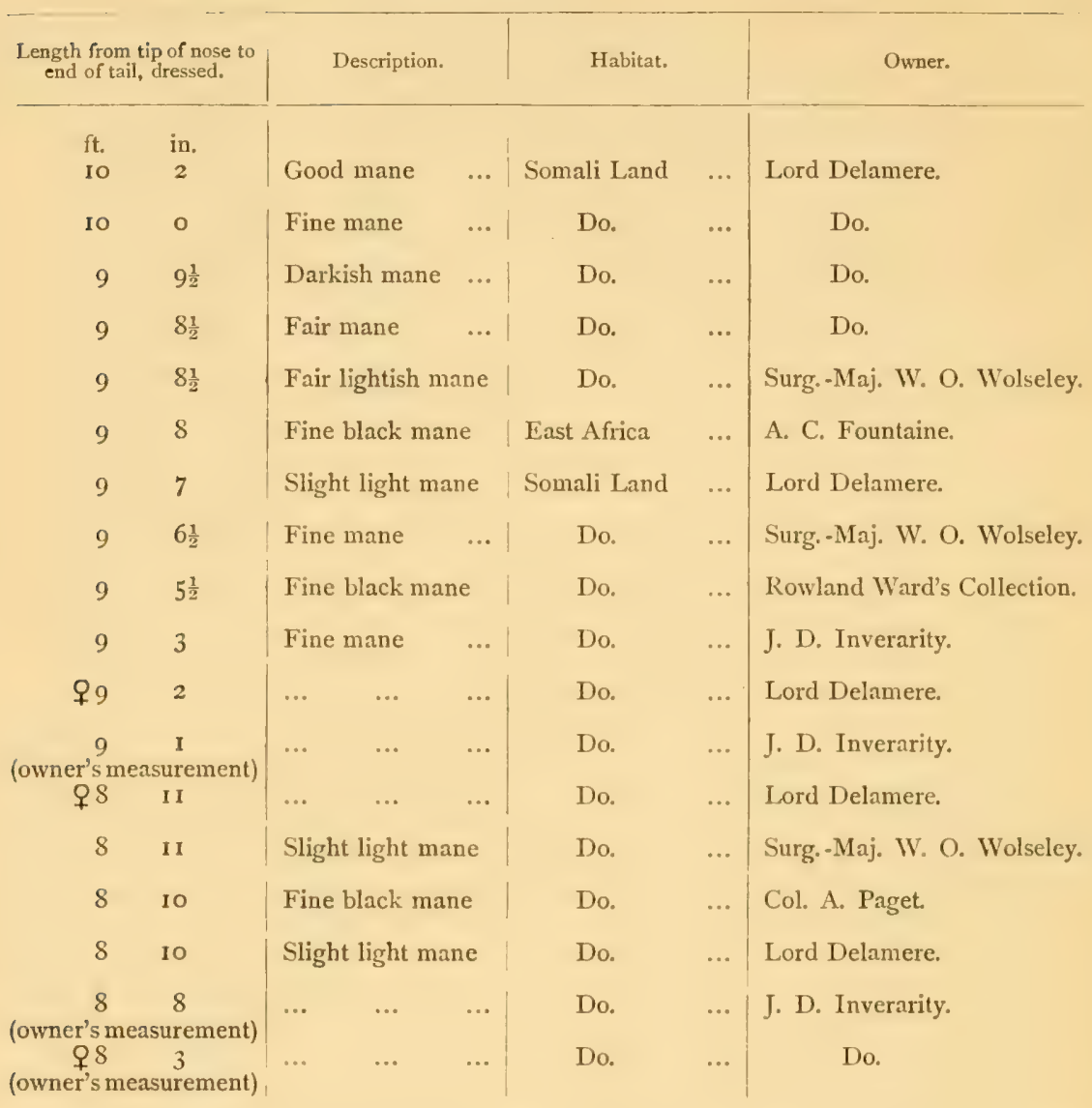


LION SKULLS (Felis leo).

\begin{tabular}{c|c|c|c|c|c}
$\begin{array}{c}\text { Length between } \\
\text { two lines. }\end{array}$ & $\begin{array}{c}\text { Width between } \\
\text { two lines. }\end{array}$ & $\begin{array}{c}\text { Weight, } \\
\text { cleaned. }\end{array}$ & Habitat. & Owner. \\
$\begin{array}{c}\text { I6 (end } \\
\text { broken) } \\
\text { I5 }\end{array}$ & $10 \frac{1}{4}$ & $5 \mathrm{lb}$. & East Africa & $\ldots$ & E. Gedge. \\
$13^{\frac{3}{4}}$ & 10 & $\ldots$ & Somali Land & $\ldots$ & Col. A. Paget. \\
\hline
\end{tabular}




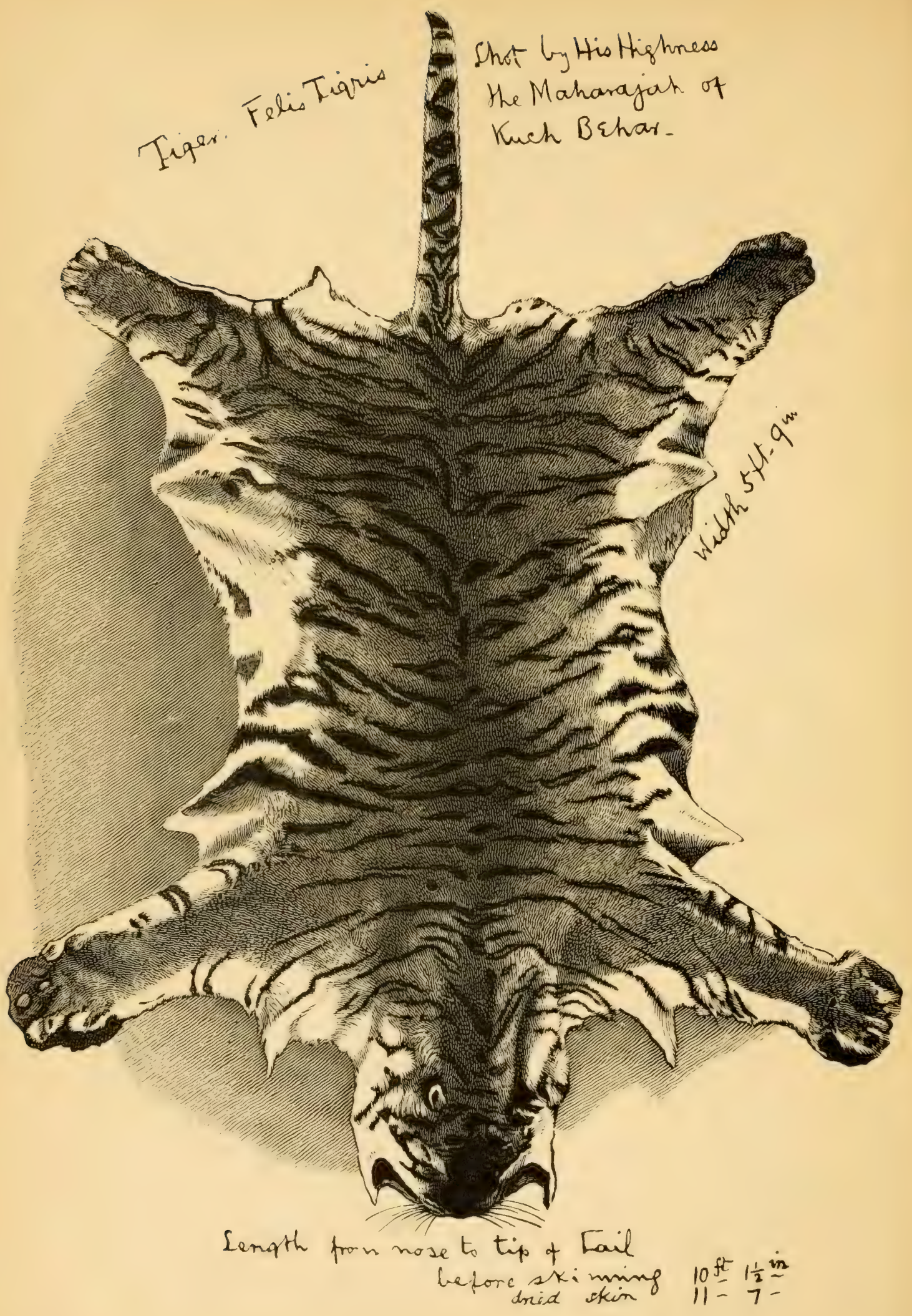




\section{TIGER (Felis tigris).}

\section{Habitat-Eastern Asia.}

\begin{tabular}{|c|c|c|c|c|c|c|}
\hline $\begin{array}{l}\text { Length before } \\
\text { skinning. }\end{array}$ & $\begin{array}{l}\text { Length after } \\
\text { dressing. }\end{array}$ & Width. & Weight. & Habitat. & & Owner. \\
\hline $\begin{array}{l}\mathrm{ft} . \text { in. } \\
\ldots\end{array}$ & $\begin{array}{l}\text { ft. in. } \\
\text { I } 3\end{array}$ & ft. in. & $\begin{array}{l}\text { lb. } \\
\cdots\end{array}$ & Chinese & & A. Bignold. \\
\hline IO $2 \frac{1}{2}$ & $\cdots$ & fowner's & 540 & $\cdots \quad \cdots$ & $\cdots$ & Maharajah of Kuch Behar, I S9o. \\
\hline IO $2 \frac{1}{2}$ & $\ldots$ & Do. & 493 & $\ldots \quad \ldots$ & $\cdots$ & I 89 I. \\
\hline I0 $\quad I_{2}^{\frac{1}{2}}$ & II 7 & 5 10 & $\ldots$ & $\ldots$ & $\ldots$ & Do. \\
\hline$\ldots$ & $11 \quad 7$ & $\ldots$ & $\ldots$ & $\ldots$ & $\ldots$ & II.R.H. Duc d'Orleans. \\
\hline & $\ldots$ & $\begin{array}{c}\text { (owner's } \\
\text { measurement) }\end{array}$ & 445 & $\ldots$ & $\ldots$ & Maharajah of Kuch Behar. \\
\hline 100 & $\ldots$ & Do. & $\cdots$ & $\ldots$ & $\cdots$ & Maharajah of Tikumgurh. \\
\hline 98 & $\cdots$ & Do. & 467 & $\cdots$ & $\cdots$ & Maharajah of Kuch Behar. \\
\hline 97 & II 4 & Do. & 437 & $\cdots$ & $\cdots$ & Sir Samuel Baker, F.R.S. \\
\hline 96 & $\cdots$ & Do. & 455 & $\cdots$ & $\cdots$ & Maharajah of Kuch Behar. \\
\hline 96 & $\cdots$. & Do. & $45^{\circ}$ & $\cdots$ & $\cdots$ & Dó. \\
\hline 96 & $\cdots$ & Do. & 400 & $\cdots$ & $\cdots$ & Sir Samuel Baker, F. R.S. \\
\hline 95 & $\cdots$ & Do. & $\cdots$ & $\cdots$ & $\cdots$ & J. D. Inverarity. \\
\hline 93 & $\cdots$ & Do. & $\ldots$ & Kewah & $\cdots$ & H. C. V. Hunter. \\
\hline$\cdots$ & II I & Do. & $\cdots$ & $\ldots \quad \ldots$ & $\ldots$ & Rowland Ward's Collection. \\
\hline 9 I & $\cdots$ & Do. & $\cdots$ & Iudia ... & $\cdots$ & J. D. Inverarity. \\
\hline 90 & $\cdots$ & Do. & $\cdots$ & $\ldots \quad \ldots$ & $\cdots$ & Do. \\
\hline$\cdots$ & 10 $10 \frac{1}{2}$ & $\cdots$ & $\cdots$ & $\cdots$ & $\cdots$ & Campbell Wynne. \\
\hline 984 & $\ldots$ & $\begin{array}{c}\text { (owner's } \\
\text { measurement) }\end{array}$ & $\ldots$ & $\cdots \quad \cdots$ & $\ldots$ & J. D. Inverarity. \\
\hline 98 I & $\cdots$ & Do. & $\ldots$ & $\ldots$ & $\cdots$ & Do. \\
\hline
\end{tabular}




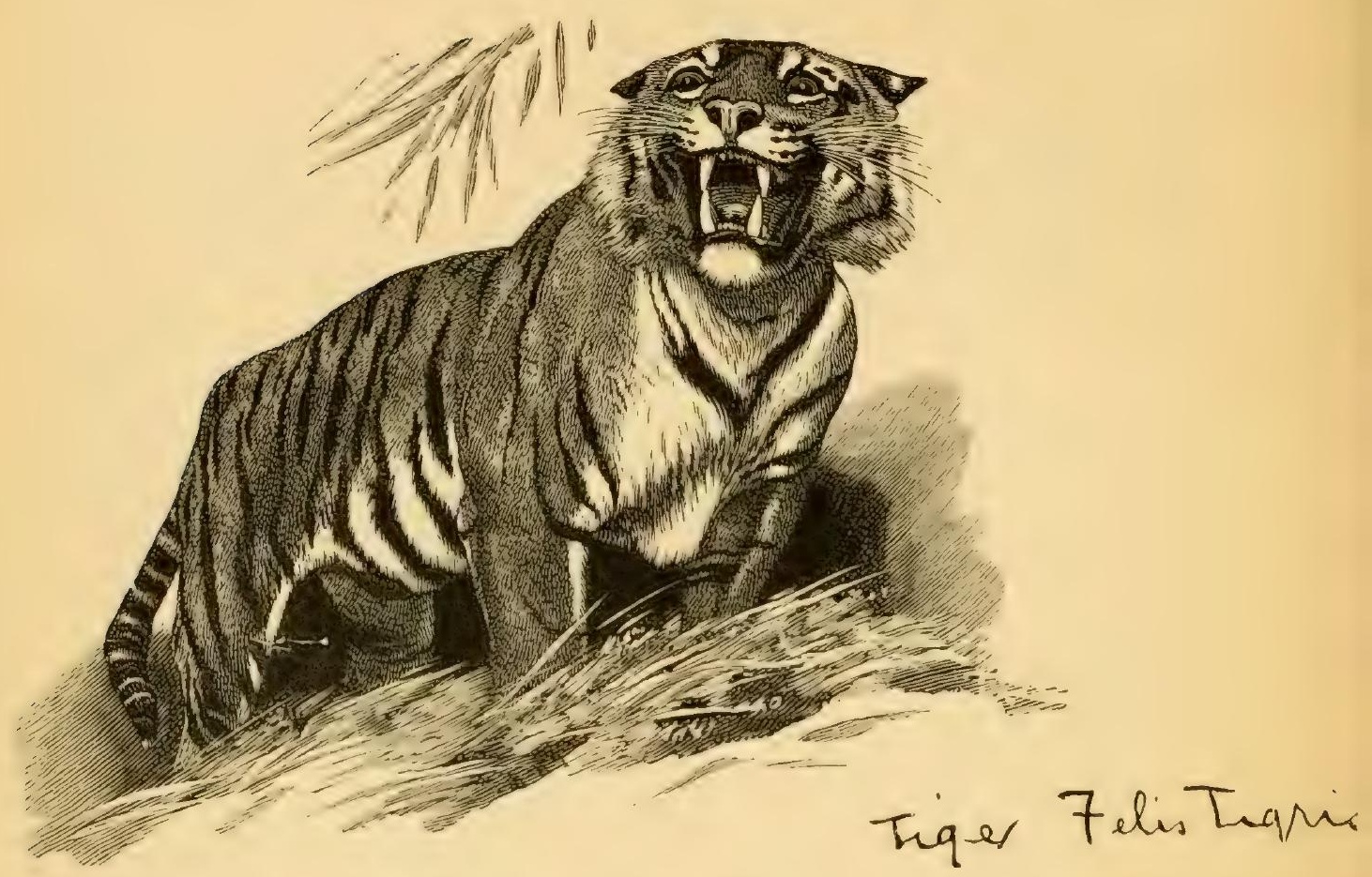

Shot by His Highness the Maharajah of Kuch Behar.

\section{TIGER SKULLS.}

\begin{tabular}{|c|c|c|c|c|c|c|c|}
\hline $\begin{array}{l}\text { Length } \\
\text { between two } \\
\text { lines, from } \\
\text { back to front. }\end{array}$ & $\begin{array}{c}\text { Breadth } \\
\text { between two } \\
\text { lines. }\end{array}$ & & $\begin{array}{l}\text { ght } \\
\text { aed. }\end{array}$ & & abita & & Owner. \\
\hline $14 \frac{1}{2}$ & IO & & $\begin{array}{l}\mathrm{cz} . \\
\mathbf{I} 3\end{array}$ & India & $\ldots$ & $\ldots$ & Rowland Ward's Collection. \\
\hline I $3 \frac{7}{8}$ & $9 \frac{3}{8}$ & 3 & 15 & Do. & ... & ... & Lieut.-Col. Boileau. \\
\hline $13 \frac{5}{8}$ & $10 \frac{1}{4}$ & 4 & Io & Do. & $\ldots$ & $\cdots$ & Rowland Ward's Collection. \\
\hline $13 \frac{1}{2}$ & $9 \frac{7}{8}$ & 4 & 3 & Do. & $\ldots$ & $\cdots$ & Capt. A. P. Longfield. \\
\hline
\end{tabular}




\section{BEAR SKULLS (Ursus).}

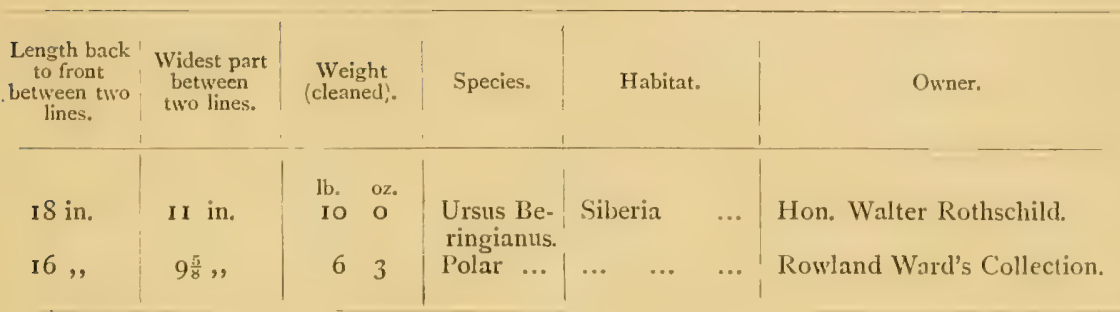

BEAR SKINS (Ursus).

\begin{tabular}{|c|c|c|c|c|}
\hline $\begin{array}{l}\text { Length from } \\
\text { nose to tip of } \\
\text { tail. }\end{array}$ & Weight. & Species. & Habitat. & Owner. \\
\hline $\begin{array}{ll}\text { ft. } & \text { in. } \\
8 & 7\end{array}$ & $\begin{array}{l}\text { lb. } \\
1600\end{array}$ & Polar ... & $\begin{array}{lll}\cdots & \cdots & \cdots\end{array}$ & Capt. Lyon, "Museum of Animated \\
\hline 80 & 1600 & Do. & Arctic Seas & P. Lamont. \\
\hline$\cdots$ & 1400 & Grizzly & $\cdots \quad \cdots$ & Sir Thomas Hesketh, Bart. \\
\hline 70 & $\cdots$ & Black ... & North America & T. W. H. Clarke. \\
\hline 610 & 423 & $\cdots$ & $\begin{array}{l}\text { India (owner's } \\
\text { measurements) }\end{array}$ & Maharajah of Kuch Behar. \\
\hline 6 nearly & 280 & ... & Do. & $\begin{array}{l}\text { Col. G. P. Sanderson, "Wild Beasts } \\
\text { of India." }\end{array}$ \\
\hline
\end{tabular}




ERRATA.

Prge 257 for "malches" read "machlis."

, 257 ,, "gaauus" ,, "gaurus."

, 258 ," "doriee" ,, "doriœ,"

, 260 ," "canma" ," “canna."

,2 26 I ," "rupocapra" ,, "rupicapra."

, 26 I ", "kurdu" , "kudu." 


\section{N D E X.}

$A$

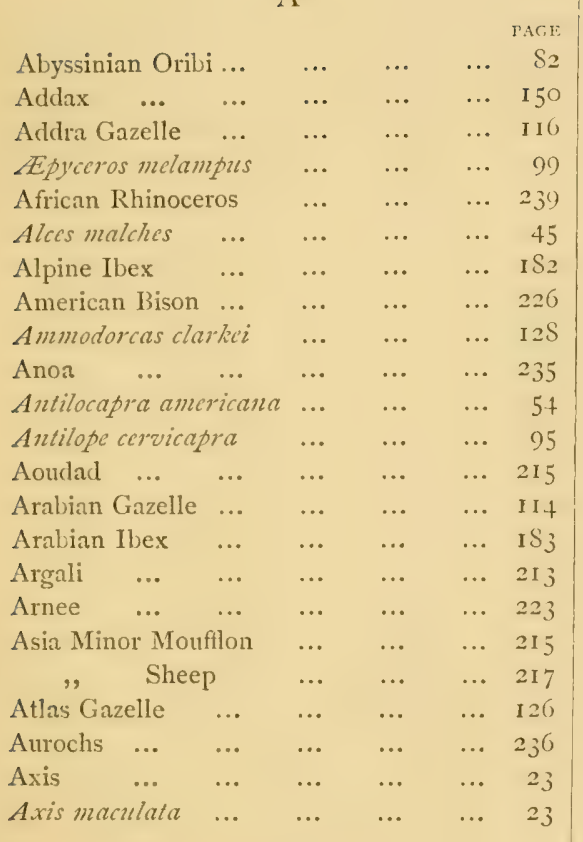

B

$\begin{array}{lllll}\text { Baker's Antelope ... } & \ldots & \ldots & \ldots & \text { I } 42\end{array}$

$\begin{array}{lllll}\text { Banded Bushbuck } & \ldots & \ldots & \ldots & 77\end{array}$

$\begin{array}{llllll}\text { Barasingh Deer } & \ldots & \ldots & \ldots & \ldots & 3^{8}\end{array}$

$\begin{array}{llllll}\text { Barbary Deer } & \ldots & \ldots & \ldots & \ldots & 27\end{array}$

$\begin{array}{llllll}\text { Barking Deer } & \ldots & \ldots & \ldots & \ldots & 5\end{array}$

$\begin{array}{lllllll}\text { Bears } \quad \ldots & \ldots & \ldots & \ldots & \ldots & 255\end{array}$

Beatrix Antelope ... $\quad \ldots \quad \ldots \quad \ldots \quad$ I 4 S

$\begin{array}{lllllll}\text { Beisa } & \ldots & \ldots & \ldots & \ldots & \ldots & \text { I } 46\end{array}$

$\begin{array}{llllll}\operatorname{Big} \text { Hcrn } \ldots & \ldots & \ldots & \ldots & \ldots & 204\end{array}$

$\begin{array}{lllllll}\text { Bison } & \ldots & \ldots & \ldots & \ldots & \ldots & 226\end{array}$

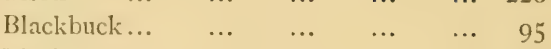

Black-tailed Deer... $\quad \ldots \quad \ldots \quad \ldots \quad$ 5I

$\begin{array}{llllll},, & \text { Gazelle } & \ldots & \ldots & \ldots & \mathbf{1} 26\end{array}$

$\begin{array}{lllllll}\text { Blessbok } & \ldots & \ldots & \ldots & \ldots & \ldots & 6 \mathrm{~S}\end{array}$

$\begin{array}{lllllll}\text { Bontebok } & \ldots & \ldots & \ldots & \ldots & \ldots & 69\end{array}$

$\begin{array}{llllll}\text { Bos americanuls } & \ldots & \ldots & \ldots & \ldots & 226\end{array}$

$\begin{array}{llllll}\text {, banting } & \ldots & \ldots & \ldots & \ldots & 234\end{array}$

$\begin{array}{llllll}\text {, brachyceros } & \ldots & \ldots & \ldots & \ldots & 232\end{array}$

$\begin{array}{lllllll}\text { ", buffelus } & \ldots & \ldots & \ldots & \ldots & 223\end{array}$

$\begin{array}{lllllll} & \text { " caffer } \ldots & \ldots & \ldots & \ldots & \ldots & 2 \text { I9 }\end{array}$

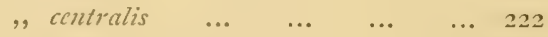

$\begin{array}{llllll}, \text { dante } \ldots & \ldots & \ldots & \ldots & \ldots & 232\end{array}$

$\begin{array}{llllll}\text {, depressicornis } & \ldots & \ldots & \ldots & 235\end{array}$

$\begin{array}{llllll}\text {, frontalis } & \ldots & \ldots & \ldots & \ldots & 230\end{array}$

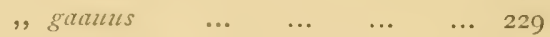

$\begin{array}{llllll}\text {, grunnicns } & \ldots & \ldots & \ldots & \ldots & 233\end{array}$

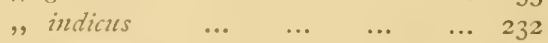

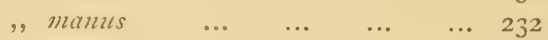

$\begin{array}{llllll}\text {,p pumilus } & \ldots & \ldots & \ldots & \ldots & 230\end{array}$

$\begin{array}{llllll}\text {,, sondaicus } & \ldots & \ldots & \ldots & \ldots & 235\end{array}$

$\begin{array}{llllll}\text {,, taurus } & \ldots & \ldots & \ldots & \ldots & 237\end{array}$

$\begin{array}{lllllll}\text {, } \text { urus } & \ldots & \ldots & \ldots & \ldots & \ldots & 236\end{array}$

Boselaphus tragocamelus... $\quad \ldots \quad \ldots . \quad$ I 5 I

$\begin{array}{llllll}\text { Brindled Gnu } & \ldots & \ldots & \ldots & \ldots & 72\end{array}$

$\begin{array}{lllll}\text { Bubaline Antelope } & \ldots & \ldots & \ldots & 55\end{array}$

$\begin{array}{lllll}\text { Bubalis albifrons } & \ldots & \ldots & \ldots & 6 \mathrm{~S}\end{array}$

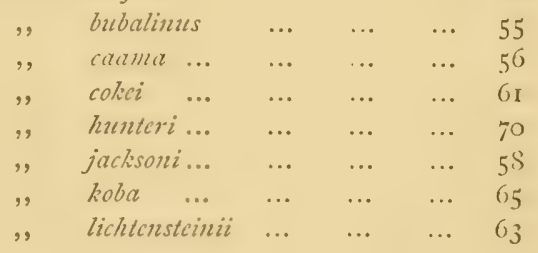




\begin{tabular}{|c|c|c|c|c|}
\hline Bubalis & lunatus ... & $\ldots$ & $\ldots$ & $\ldots$ \\
\hline ," & pygargus & $\ldots$ & $\ldots$ & $\ldots$ \\
\hline , & senegalensis & $\ldots$ & $\ldots$ & $\ldots$ \\
\hline , & swaynei ... & $\ldots$ & $\cdots$ & $\cdots$ \\
\hline & tora & $\cdots$ & $\ldots$ & $\cdots$ \\
\hline Bubalus & s reclinus ... & $\ldots$ & $\ldots$ & $\ldots$ \\
\hline Budorca & as taxicolor & $\ldots$ & $\ldots$ & $\ldots$ \\
\hline Burhel & $\ldots$ & ... & ... & $\ldots$ \\
\hline Burmese & e Stag & $\ldots$ & $\ldots$ & $\ldots$ \\
\hline Bushbuc & $\mathrm{ck} \ldots$ & $\ldots$ & $\ldots$ & $\ldots$ \\
\hline
\end{tabular}

C

$\begin{array}{lllll}\text { Canma Hartebeeste } & \ldots & \ldots & \ldots & 56\end{array}$

$\begin{array}{llllll}\text { Cape Buffalo } & \ldots & \ldots & \ldots & \ldots & 219\end{array}$

$\begin{array}{llllll}\text { Capra agagrues } & \ldots & \ldots & \ldots & \ldots & \mathrm{I} 84\end{array}$

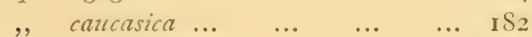

", cylindricornis $\quad \ldots \quad \ldots \quad \ldots \quad \ldots \quad$ I $S_{9}$

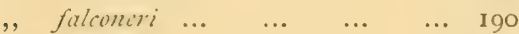

$\begin{array}{llllll}\text { ", hircus } & \ldots & \ldots & \ldots & \ldots & \mathbf{1 8 5}\end{array}$

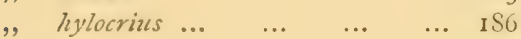

$\begin{array}{lllllll} & \| \text { ibex } & \ldots & \ldots & \ldots & \ldots & \mathrm{I} 82\end{array}$

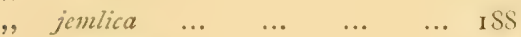

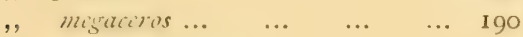

$\begin{array}{lllllll} & , 1 \text { mubiana } & \ldots & \ldots & \ldots & \ldots & \mathbf{1} 85\end{array}$

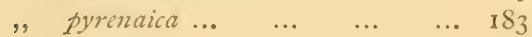

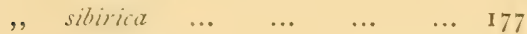

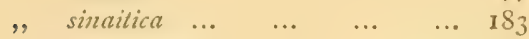

$\begin{array}{lllll}\text { Capreolus pygargus } & \ldots & \ldots & \ldots & 47\end{array}$

$\begin{array}{lllll}\text { Cariacus antisiensis } & \ldots & \ldots & \ldots & 52\end{array}$

$\begin{array}{llllll}, & \text { campestris } & \ldots & \ldots & \ldots & 52\end{array}$

$\begin{array}{llllll} & \text { macrotis } & \ldots & \ldots & \ldots & 53\end{array}$

$\begin{array}{llllll},, & \text { virginianus } & \ldots & \ldots & \ldots & 49\end{array}$

$\begin{array}{lllllll}\text { Cariboo } & \ldots & \ldots & \ldots & \ldots & \ldots & 42\end{array}$

$\begin{array}{lllll}\text { Caucasian Burhel... } & \ldots & \ldots & \ldots & \mathbf{I} \delta 9\end{array}$

$\begin{array}{lllllll}, & \text { Ibex } & \ldots & \ldots & \ldots & \ldots & \text { I } 82\end{array}$

Central African Buffalo $\ldots \quad \ldots \quad \ldots \quad \ldots \quad 222$

Central and Southern Indian Sambur ... 10

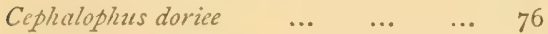

$\begin{array}{llllll}, & \text { grimini } & \ldots & \ldots & \ldots & 76\end{array}$

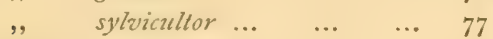

$\begin{array}{lllll}\text { Cervicapra arundineum ... } & \ldots & \ldots & 93\end{array}$

$\begin{array}{llllll}, & \text { bohor } \ldots & \ldots & \ldots & \ldots & 93\end{array}$

$\begin{array}{lllll}\text { Cervulus crinifrons } & \ldots & \ldots & \ldots & 5\end{array}$

$\begin{array}{llllll} & \text { muntjac } & \ldots & \ldots & \ldots & 5\end{array}$

$\begin{array}{llllll},, & \text { sclateri } \ldots & \ldots & \ldots & \ldots & 5\end{array}$

$\begin{array}{llllll}\text { Cervus affinis } & \ldots & \ldots & \ldots & \ldots & 39\end{array}$
Cervus canadensis . PAGE

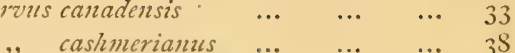

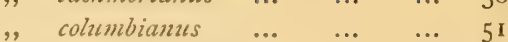

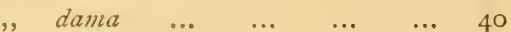

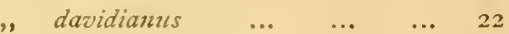

$\begin{array}{llllll} & , \text { duvaucelli } & \ldots & \ldots & \ldots & 17\end{array}$

$\begin{array}{lllllll}, & \text { elaphus } & \ldots & \ldots & \ldots & \ldots & 28\end{array}$

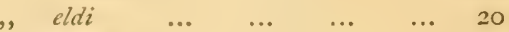

$\begin{array}{lllllll}\text {, cquinus } & \ldots & \ldots & \ldots & \ldots & \text { 10 }\end{array}$

$\begin{array}{llllll} & , \text { giganteus ... } & \ldots & \ldots & \ldots & 4 \mathrm{I}\end{array}$

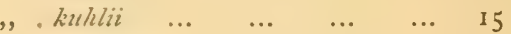

$\begin{array}{llllll}\text {," mantchuricus } & \ldots & \ldots & \ldots & 25\end{array}$

$\begin{array}{llllll} & \text { marianus... } & \ldots & \ldots & \ldots & \text { I3 }\end{array}$

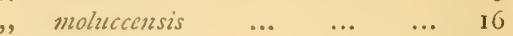

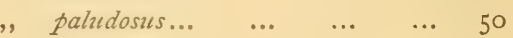

$\begin{array}{llllll} & , \text { philippinus } & \ldots & \ldots & \ldots & 12\end{array}$

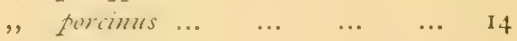

$\begin{array}{lllllll} & \text { rusa } & \ldots & \ldots & \ldots & \ldots & 22\end{array}$

$\begin{array}{llllll}, & \text { schomburgki } & \ldots & \ldots & \ldots & 19\end{array}$

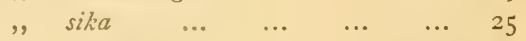

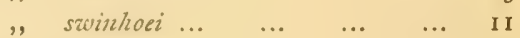

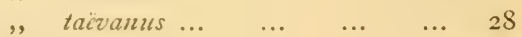

$\begin{array}{lllllll}\text { Chamois } & \ldots & \ldots & \ldots & \ldots & \ldots & 169\end{array}$

$\begin{array}{lllllll}\text { Chiru } & \ldots & \ldots & \ldots & \ldots & \ldots & 102\end{array}$

$\begin{array}{llllll}\text { Clarke's Gazelle } & \ldots & \ldots & \ldots & \ldots & 12 S\end{array}$

$\begin{array}{llllll}\text { Cobus defassus } & \ldots & \ldots & \ldots & \ldots & S 9\end{array}$

$\begin{array}{llllll}, & \text { ellipsipynnnus } & \ldots & \ldots & \ldots & \text { S6 }\end{array}$

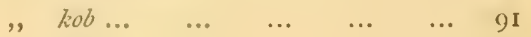

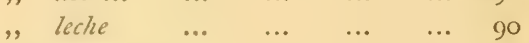

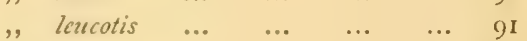

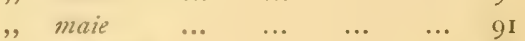

$\begin{array}{lllllll}, " & \sin \% \sin \% & \ldots & \ldots & \ldots & \ldots & S_{9}\end{array}$

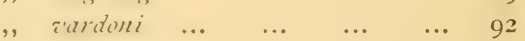

$\begin{array}{lllll}\text { Cokes Hartebeeste } & \ldots & \ldots & \ldots & 6 \mathbf{I}\end{array}$

$\begin{array}{llllll}\text { Connochactus gnu } & \ldots & \ldots & \ldots & 74\end{array}$

$\begin{array}{llllll}, & \text { taurina } & \ldots & \ldots & \ldots & 72\end{array}$

$\begin{array}{lllllll}\text { Corinne } & \ldots & \ldots & \ldots & \ldots & \ldots & 127\end{array}$

\section{D}

$\begin{array}{lllllll}\text { Deer } & \ldots & \ldots & \ldots & \ldots & \ldots & 28\end{array}$

$\begin{array}{llllll}\text { Domestic Goat } & \ldots & \ldots & \ldots & \ldots & \mathbf{1} 85\end{array}$

$\begin{array}{lllllll}, & \mathrm{Ox} & \ldots & \ldots & \ldots & \ldots & 237\end{array}$

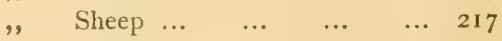

$\begin{array}{llllll}\text { Dorcas Gazelle } & \ldots & \ldots & \ldots & \ldots & \text { II } 4\end{array}$

$\begin{array}{lllllll}\text { Duiker } & \ldots & \ldots & \ldots & \ldots & \ldots & 76\end{array}$

$\begin{array}{llllll}\text { Dwarf Buffalo } & \ldots & \ldots & \ldots & \ldots & 230\end{array}$ 


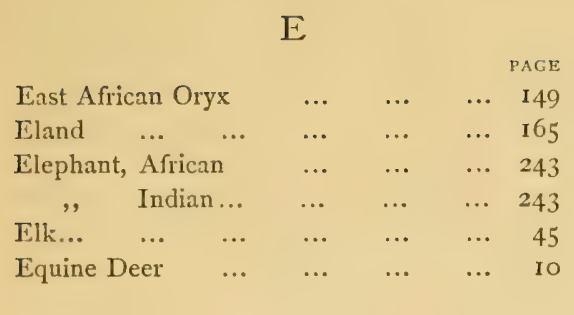

F

$\begin{array}{lllllr}\text { Fallow Deer } & \ldots & \ldots & \ldots & \ldots & 40 \\ \text { Felis } \quad \ldots & \ldots & \ldots & \ldots & \ldots & 253 \\ \text { Forest Goat } & \ldots & \ldots & \ldots & \ldots & \text { I } 7 \mathrm{I} \\ \text { Formosa Deer } & \ldots & \ldots & \ldots & \ldots & 26 \\ \text { Four Horned Antelope } & \ldots & \ldots & \ldots & 78\end{array}$

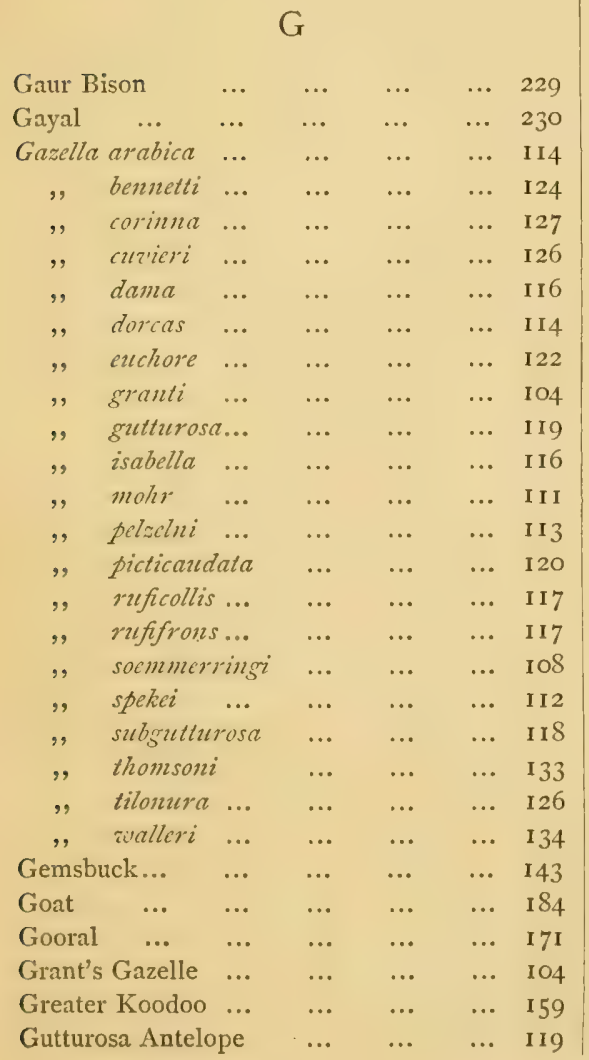

H

UAafloceros montanus

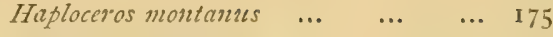

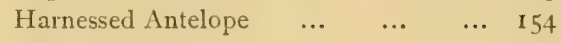

$\begin{array}{llllll}\text { Hartebeeste } & \ldots & \ldots & \ldots & \ldots & 56\end{array}$

Himalayan Chamois $\quad \ldots \quad \ldots \quad \ldots$. I 7 I

$\begin{array}{rlllll}, & \text { Ibex } \ldots & \ldots & \ldots & \ldots & 171 \\ & & \ldots & \ldots & 177\end{array}$

$\begin{array}{llllll},, & \text { Serow } & \ldots & \ldots & \ldots & \text { I } 74\end{array}$

$\begin{array}{llllll}\text { Hippopotamus } & \ldots & \ldots & \ldots & \ldots & 3\end{array}$

$\begin{array}{lllll}\text { Hippotragus bakeri } & \ldots & \ldots & \ldots & \mathrm{I} 42\end{array}$

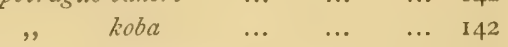

$\begin{array}{llllll} & \text { leucophous } \ldots & \ldots & \ldots & 140 \\ & \text { niger } & \ldots & \ldots & \ldots & 137\end{array}$

$\begin{array}{llllll}\text { Hog Deer ... } & \ldots & \ldots & \ldots & \ldots & \text { I4 }\end{array}$

Hunter's Antelope $\quad \ldots \quad \ldots \quad \ldots \quad 70$

\section{I}

$\begin{array}{lllllll}\text { Ibex } & \ldots & \ldots & \ldots & \ldots & \ldots & \text { I } 77\end{array}$

$\begin{array}{lllllll}\text { Impala } & \ldots & \ldots & \ldots & \ldots & \ldots & 99\end{array}$

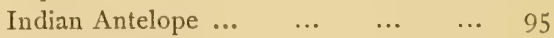

$\begin{array}{lllllll},, & \text { Gazelle } & \ldots & \ldots & \ldots & \ldots & 124\end{array}$

,, Rhinoceros $\quad \ldots \quad \ldots \quad \ldots \quad \ldots \quad 238$

$\begin{array}{lllllll}\text { Inyala } & \ldots & \ldots & \ldots & \ldots & \ldots & \mathrm{I} .57\end{array}$

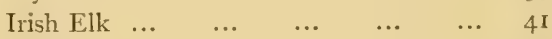

$\begin{array}{llllll}\text { Isabella Gazelle } & \ldots & \ldots & \ldots & \ldots & \text { II6 }\end{array}$

$\begin{array}{lllllll}\text { Ivory } & \ldots & \ldots & \ldots & \ldots & \ldots & 243\end{array}$

$\begin{array}{lllll}\text { Jackson's Hartebeeste } & \ldots & \ldots & \ldots & 5^{8}\end{array}$

$\begin{array}{llllll}, & \text { Wildebeeste } & \ldots & \ldots & \ldots & 75\end{array}$

$\begin{array}{llllll}\text { Japanese Deer } & \ldots & \ldots & \ldots & \ldots & 25\end{array}$

$\begin{array}{lllllll}\text { Java } & , & \ldots & \ldots & \ldots & \ldots & 22\end{array}$

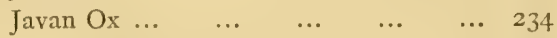

$\begin{array}{llllll}\text {, } & \text { Rhinoceros } & \ldots & \ldots & \ldots & 24\end{array}$

\section{K}

$\begin{array}{llll}\text { Kamschatka Wild Sheep } \quad \ldots & \ldots & 207\end{array}$

$\begin{array}{llllll}\text { Kashmir Stag } & \ldots & \ldots & \ldots & \ldots & 3\end{array}$

$\begin{array}{llllll}\text { Kirk's Antelope } & \ldots & \ldots & \ldots & \ldots & 79\end{array}$

$\begin{array}{llllll}\text { Klipspringer } & \ldots & \ldots & \ldots & \ldots & 8_{4}\end{array}$

$\begin{array}{lllllll}\text { Koodoo } & \ldots & \ldots & \ldots & \ldots & \ldots & \text { I59 }\end{array}$

$\begin{array}{llllll}\text { Kuhl's Stag } & \ldots & \ldots & \ldots & \ldots & \text { I5 }\end{array}$ 


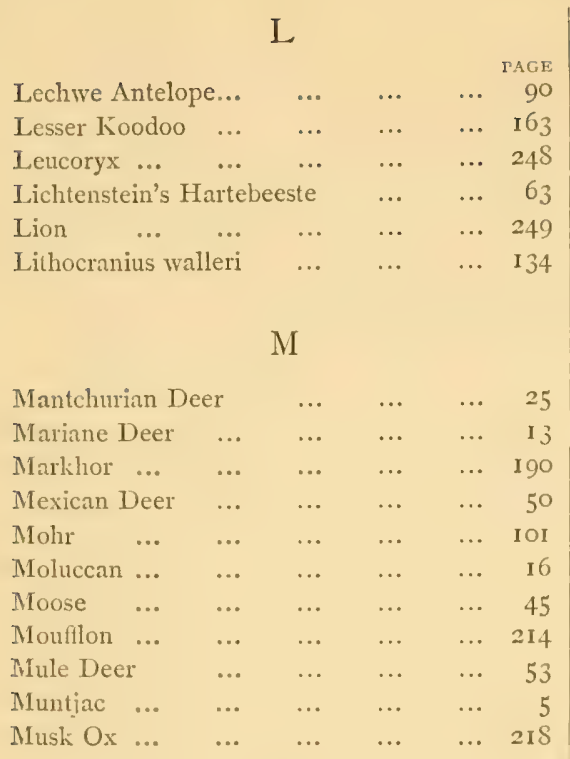

\section{$\mathrm{N}$}

$\begin{array}{lllllll}\text { Nakong } & \ldots & \ldots & \ldots & \ldots & \ldots & 155\end{array}$

Nanotragus montanus $\quad \ldots \quad \ldots \quad \ldots \quad \ldots \quad$ S2

, moschatus ... $\quad \ldots \quad$... $\quad$ So

$\begin{array}{lllll}" & \text { nigricaudatus } & \ldots & \ldots & \text { SO }_{1} \\ , & \text { orcotagus } & \ldots & \ldots & S_{I}\end{array}$

$\begin{array}{llllll}, & \text { oreotragus } & \ldots & \ldots & \ldots & 8_{4}\end{array}$

$\begin{array}{llllll}, & \text { scoparios } & \ldots & \ldots & \ldots & S_{I}\end{array}$

$\begin{array}{llllll}, & \text { tragulus } & \ldots & \ldots & \ldots & S_{3}\end{array}$

$\begin{array}{llllll}\text { Natal Bushbuck } & \ldots & \ldots & \ldots & \ldots & 77\end{array}$

$\begin{array}{lllll}\text { Nemoradus bubalinus } & \ldots & \ldots & \ldots & \text { I } 74\end{array}$

$\begin{array}{lllll}\text { Nemorhadus goral } & \ldots & \ldots & \ldots & \text { I } 71\end{array}$

$\begin{array}{lllll} & \text { sumatronsis } & \ldots & \ldots & 173\end{array}$

$\begin{array}{llllll}\text { Neotragus kirki } & \ldots & \ldots & \ldots & \ldots & 79\end{array}$

$\begin{array}{llllll}, & \text { sallianus } & \ldots & \ldots & \ldots & \text { So }\end{array}$

$\begin{array}{lllllll}\text { Nilghai } & \ldots & \ldots & \ldots & \ldots & \ldots & \text { I } 5 \text { I }\end{array}$

Nilghiri Wild Goat $\quad \ldots \quad \ldots . \quad \ldots \quad$ IS6

, Ibex $\quad \ldots \quad \ldots \quad \ldots$. ISo

$\begin{array}{lllll}\text { North African Ibex } & \ldots & \ldots & \ldots & 1 S_{5}\end{array}$

\section{$\mathrm{O}$}

$\begin{array}{lllllll}\text { Oorial } & \ldots & \ldots & \ldots & \ldots & \ldots & 209\end{array}$

$\begin{array}{llllll}\text { Oreas canma } & \ldots & \ldots & \ldots & \ldots & \mathrm{I} 65\end{array}$

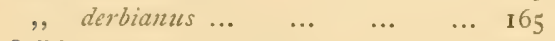

$\begin{array}{lllllll}\text { Oribi } & \ldots & \ldots & \ldots & \ldots & \ldots & \delta_{\text {I }}\end{array}$

Oribos moschatus... $\quad \ldots \quad \ldots \quad \ldots \quad 2 \mathrm{I} 8$

I'AT,E

$\begin{array}{llllll}\text { Oryx beatrix } & \ldots & \ldots & \ldots & \ldots & \text { I } 4 \mathrm{~S}\end{array}$

$\begin{array}{lllllll},, & b c i s a & \ldots & \ldots & \ldots & \ldots & 146\end{array}$

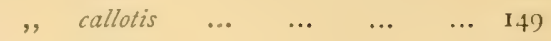

$\begin{array}{llllll}, \text { gasella } & \ldots & \ldots & \ldots & \ldots & \mathbf{1} 43\end{array}$

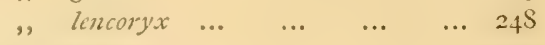

$\begin{array}{llllll}\text { Ovis ammon } & \ldots & \ldots & \ldots & \ldots & 200\end{array}$

$\begin{array}{lllllll},, \operatorname{argali} & \ldots & \ldots & \ldots & \ldots & 213\end{array}$

$\begin{array}{lllllll} & , \text { aries ... } & \ldots & \ldots & \ldots & \ldots & 217\end{array}$

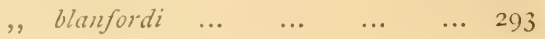

$\begin{array}{llllll}\text {,, brookci } & \ldots & \ldots & \ldots & \ldots & 293\end{array}$

$\begin{array}{llllll}, \text { canadensis } & \ldots & \ldots & \ldots & \ldots & 204\end{array}$

$\begin{array}{llllll}\text {," crilucires } & \ldots & \ldots & \ldots & \ldots & 209\end{array}$

$\begin{array}{lllllll},, & \text { gmelini } & \ldots & \ldots & \ldots & \ldots & 217\end{array}$

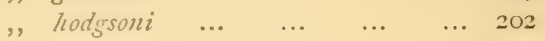

$\begin{array}{llllll}, \text { Karclini }_{1} & \ldots & \ldots & \ldots & \ldots & 19 \mathrm{~S}\end{array}$

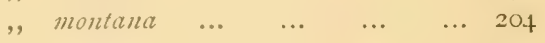

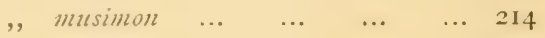

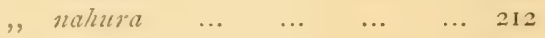

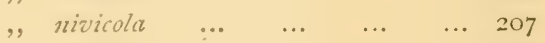

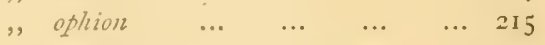

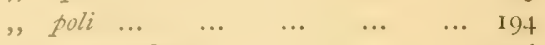

$\begin{array}{llllll}\text {, } & \text { tragelaphus ... } & \ldots & \ldots & \ldots & 216\end{array}$

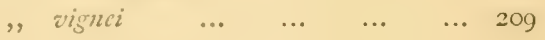

\section{P}

$\begin{array}{llllll}\text { Pampas Deer } & \ldots & \ldots & \ldots & \ldots & 52\end{array}$

$\begin{array}{lllllll}\text { Panolia } & \ldots & \ldots & \ldots & \ldots & \ldots & 20\end{array}$

$\begin{array}{lllll}\text { Pantholops hodgsoni } & \ldots & \ldots & \ldots & 102\end{array}$

$\begin{array}{llllll}\text { Pilca capreola } & \ldots & \ldots & \ldots & \ldots & \mathrm{S}_{5}\end{array}$

$\begin{array}{lllll}\text { Pere David's Deer } & \ldots & \ldots & \ldots & 22\end{array}$

$\begin{array}{llllll}\text { Persian Deer } & \ldots & \ldots & \ldots & \ldots & { }_{3} S\end{array}$

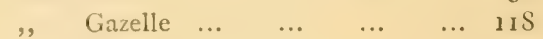

l'eruvian Roe Deer $\quad \ldots \quad \ldots \quad \ldots \quad 52$

$\begin{array}{llllll}\text { Philippine Deer } & \ldots & \ldots & \ldots & \ldots & \text { I2 }\end{array}$

$\begin{array}{llllll}\text { Polar Bear } & \ldots & \ldots & \ldots & \ldots & 255\end{array}$

$\begin{array}{lllllll}\text { Pookoo } & \ldots & \ldots & \ldots & \ldots & \ldots & 92\end{array}$

$\begin{array}{llll}\text { Prong Horned Antelope ... } & \ldots & \ldots & 54\end{array}$

$\begin{array}{llllll}\text { Pyrenean Ibex } & \ldots & \ldots & \ldots & \ldots & \mathrm{IS}_{3}\end{array}$

\section{$\mathrm{R}$}

$\begin{array}{lllll}\text { Rangifer tarandus } & \ldots & \ldots & \ldots & 42\end{array}$

$\begin{array}{llllll}\text { Ravine Deer } & \ldots & \ldots & \ldots & \ldots & 124\end{array}$

$\begin{array}{llllll}\text { Red Deer } \ldots & \ldots & \ldots & \ldots & \ldots & 27\end{array}$

$\begin{array}{llllll}\text { Reedbuck ... } & \ldots & \ldots & \ldots & \ldots & 93\end{array}$

$\begin{array}{lllllll}\text { Reh-Bok } & \ldots & \ldots & \ldots & \ldots & \ldots & S_{5}\end{array}$

$\begin{array}{lllllll}\text { Reindeer } & \ldots & \ldots & \ldots & \ldots & \ldots & 42\end{array}$ 


\begin{tabular}{|c|c|c|c|c|c|}
\hline \multirow{2}{*}{\multicolumn{2}{|c|}{ Rhinoceros bicornis }} & & & \multicolumn{2}{|c|}{ mige } \\
\hline & & $\ldots$ & $\ldots$ & $\ldots$ & 239 \\
\hline ", & indicus & $\ldots$ & $\ldots$ & $\ldots$ & 238 \\
\hline ," & sondaicus & $\ldots$ & $\ldots$ & $\ldots$ & 242 \\
\hline ," & sumatrensis & $\ldots$ & $\ldots$ & $\ldots$ & $2+2$ \\
\hline Roan Ante & elope $\quad .$. & $\ldots$ & $\ldots$ & $\ldots$ & 140 \\
\hline Rocky Mor & untain Goat & $\ldots$ & $\ldots$ & $\ldots$ & 175 \\
\hline & , Sheep & $\ldots$ & $\ldots$ & $\cdots$ & 204 \\
\hline Roe I)eer & $\ldots$ & $\ldots$ & $\ldots$ & $\ldots$ & 47 \\
\hline Rupocapra & tragus & $\ldots$ & $\ldots$ & $\ldots$ & 169 \\
\hline Rusa hippe & elaphus & $\ldots$ & $\ldots$ & $\ldots$ & IO \\
\hline ," maria & anus $\quad \ldots$ & $\ldots$ & $\ldots$ & $\ldots$ & I 3 \\
\hline
\end{tabular}

\section{$\mathrm{S}$}

\begin{tabular}{|c|c|c|c|c|c|}
\hline Sable Antelope & $\ldots$ & $\cdots$ & ... & $\ldots$ & 137 \\
\hline Saiga & $\ldots$ & $\cdots$ & $\cdots$ & $\ldots$ & 101 \\
\hline Salt's Antelope & $\ldots$ & $\ldots$ & ... & $\cdots$ & So \\
\hline Sambur Deer & $\ldots$ & $\ldots$ & $\ldots$ & $\ldots$ & 6 \\
\hline Sassaby $\quad \ldots$ & $\ldots$ & $\ldots$ & ... & $\ldots$ & 67 \\
\hline Schomburgk's De & & $\ldots$ & $\ldots$ & $\ldots$ & 19 \\
\hline Senegal Hartebee & este & $\ldots$ & ... & $\ldots$ & 64 \\
\hline Shapoo & $\cdots$ & $\cdots$ & $\ldots$ & $\ldots$ & 209 \\
\hline Sheep & $\cdots$ & $\cdots$ & $\ldots$ & $\cdots$ & 194 \\
\hline Shou & $\ldots$ & $\ldots$ & ... & $\cdots$ & 39 \\
\hline Siberian Roe De & & $\ldots$ & ... & $\ldots$ & 47 \\
\hline Sikkim Stag & $\cdots$ & $\cdots$ & $\cdots$ & $\cdots$ & 39 \\
\hline Sing-Sing Antelo & & $\cdots$ & ... & ... & Sy \\
\hline Situtunga ... & $\ldots$ & $\ldots$ & ... & $\ldots$ & 155 \\
\hline Soemmerring's Ga & azelle & $\ldots$ & ... & $\cdots$ & IOS \\
\hline Somali Lowland & Gazell & e... & $\ldots$ & $\ldots$ & II 2 \\
\hline , Plateau & ," & $\cdots$ & .. & $\cdots$ & II 3 \\
\hline Sondaic Ox & $\cdots$ & $\ldots$ & $\cdots$ & $\cdots$ & 235 \\
\hline Speke's Bushbuch & & $\ldots$ & $\ldots$ & $\ldots$ & 155 \\
\hline Springbuck & ... & $\ldots$ & $\ldots$ & ... & 122 \\
\hline Steinbuck ... & $\ldots$ & ... & ... & $\ldots$ & $s_{3}$ \\
\hline Strepsiceros inbe & rbis & $\ldots$ & $\ldots$ & $\cdots$ & $16_{3}$ \\
\hline kurd & & $\ldots$ & $\ldots$ & .. & I 59 \\
\hline Sumatra Rhinoce & eros & $\ldots$ & $\ldots$ & $\ldots$ & $2+2$ \\
\hline Sumatran Serow & $\ldots$ & $\ldots$ & $\ldots$ & $\ldots$ & I 73 \\
\hline Sus scrofa & $\cdots$ & $\cdots$ & $\ldots$ & $\cdots$ & 4 \\
\hline Swamp Deer & $\ldots$ & $\cdots$ & $\ldots$ & $\ldots$ & 17 \\
\hline Swayne's Hartebe & veeste & $\ldots$ & $\ldots$ & $\ldots$ & 60 \\
\hline Swinhoe's Deer & $\ldots$ & $\ldots$ & $\ldots$ & $\ldots$ & II \\
\hline
\end{tabular}

\section{$\mathrm{T}$}

$\begin{array}{lllllll}\text { Takin } & \ldots & \ldots & \ldots & \ldots & \ldots & 176\end{array}$ Tetraceros quadricornis... $\quad \ldots \quad \ldots \quad$... 78 $\begin{array}{lllllll}\text { Thar } & \ldots & \ldots & \ldots & \ldots & \ldots & \text { I }\end{array}$
PAGE

$\begin{array}{lllllll} & & & & & & \\ \end{array}$

$\begin{array}{llllll}\text { Thibet Antelope } & \ldots & \ldots & \ldots & \ldots & \text { I02 }\end{array}$

, $\quad$ Ravine Deer $\quad \ldots \quad \ldots \quad \ldots \quad$ I20

$\begin{array}{lllll}\text { Thomson's Gazelle } & \ldots & \ldots & \ldots & \text { I33 }\end{array}$

$\begin{array}{lllllll}\text { Tiger } & \ldots & \ldots & \ldots & \ldots & \ldots & 253\end{array}$

$\begin{array}{llllll}\text { Tora Antelope } & \ldots & \ldots & \ldots & \ldots & 59\end{array}$

$\begin{array}{lllll}\text { Tragelaphus angasi } & \ldots & \ldots & \ldots & 157\end{array}$

$\begin{array}{llllll}, & \text { curjutos } & \ldots & \ldots & \ldots & 158\end{array}$

$\begin{array}{llllll}, & \text { gratus } & \ldots & \ldots & \ldots & 156\end{array}$

$\begin{array}{llllll}, & \text { scriptus } & \ldots & \ldots & \ldots & \mathbf{1} 54\end{array}$

$\begin{array}{llllll}, & \text { spekei } & \ldots & \ldots & \ldots & \mathbf{1} 55\end{array}$

$\begin{array}{llllll},, & \text { sylvaticus } & \ldots & \ldots & \ldots & 153\end{array}$

$\begin{array}{lllll}\text { Tsessbe Antelope... } & \ldots & \ldots & \ldots & 67\end{array}$

$\begin{array}{lllllll}\text { Ursus } & \ldots & \ldots & \ldots & \ldots & \ldots & 255\end{array}$

V

$\begin{array}{llllll}\text { Virginian Deer } & \ldots & \ldots & \ldots & \ldots & 49\end{array}$

\section{W}

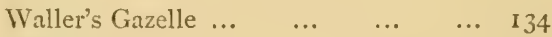

$\begin{array}{lllllll}\text { Wapiti } & \ldots & \ldots & \ldots & \ldots & \ldots & 33\end{array}$

$\begin{array}{llllll}\text { WVaterbuck } & \ldots & \ldots & \ldots & \ldots & \text { S6 }\end{array}$

$\begin{array}{lllll}\text { White-backed Bushbuck ... } & \ldots & \ldots & 77\end{array}$

$\begin{array}{llll}\text { White-eared Waterbuck ... } & \ldots & \ldots & 9 \text { I }\end{array}$

$\begin{array}{lllll}\text { White-tailed Deer } & \ldots & \ldots & \ldots & 49\end{array}$

$\begin{array}{llllll} & \text { Gnu } & \ldots & \ldots & \ldots & 72\end{array}$

$\begin{array}{llllll}\text { Wild Boar... } & \ldots & \ldots & \ldots & \ldots & 4\end{array}$

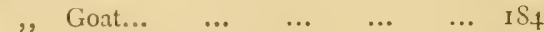

,, Sheep of Shian Shan $\quad \ldots \quad \ldots \quad$ IgS

Y

$\begin{array}{lllllll}\text { Yak } & \ldots & \ldots & \ldots & \ldots & \ldots & 233\end{array}$

$\begin{array}{lllll}\text { Yarkund Gazelle ... } & \ldots & \ldots & \ldots & \text { II } 8\end{array}$

\section{Z}

$\begin{array}{lllll}\text { Zanzibar Antelope } & \ldots & \ldots & \ldots & 8 \circ\end{array}$

$\begin{array}{lllllll}\text { Zebu } & \ldots & \ldots & \ldots & \ldots & \ldots & 232\end{array}$ 



\section{LIST OF ILLUSTRATIONS.}

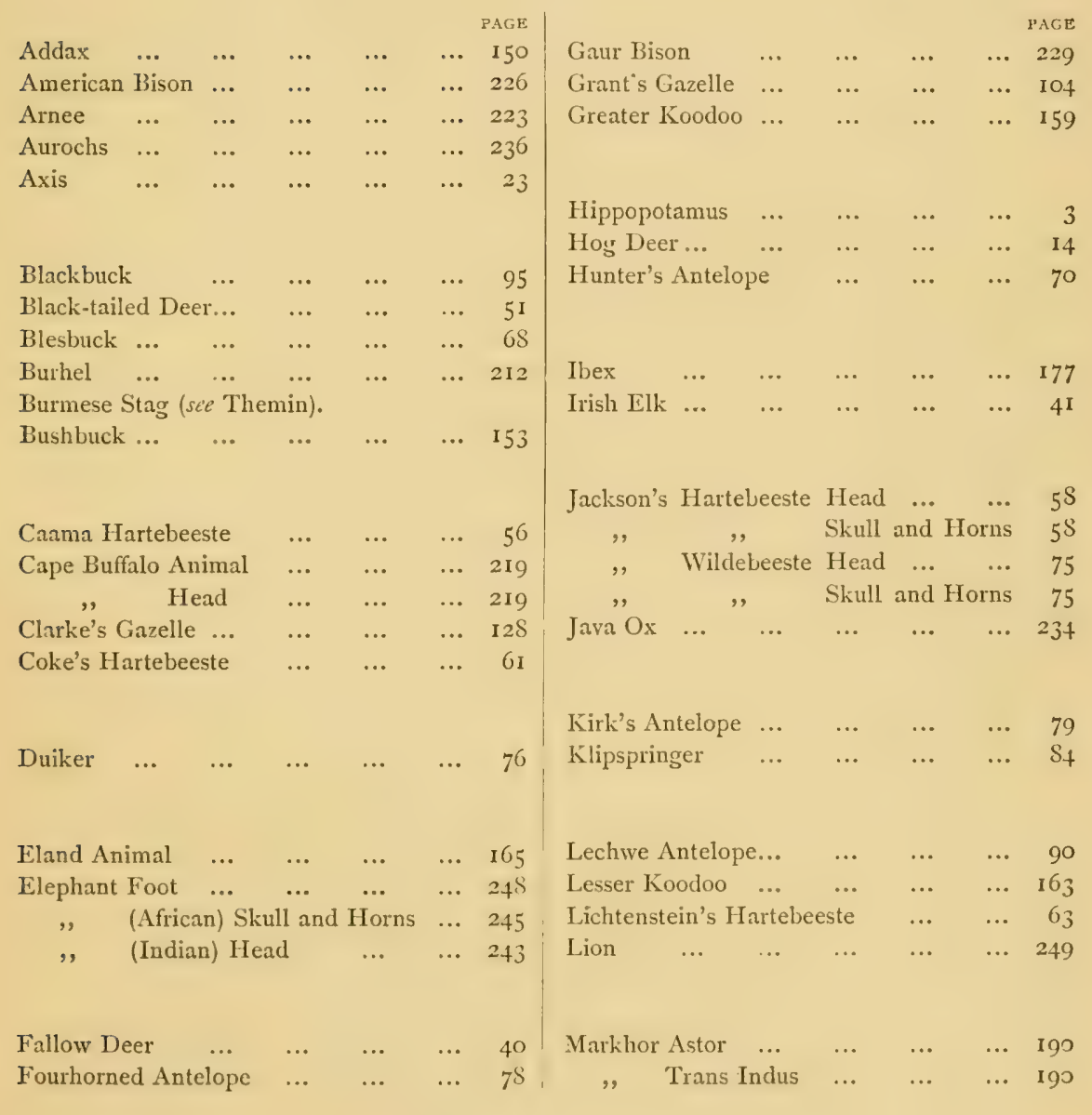




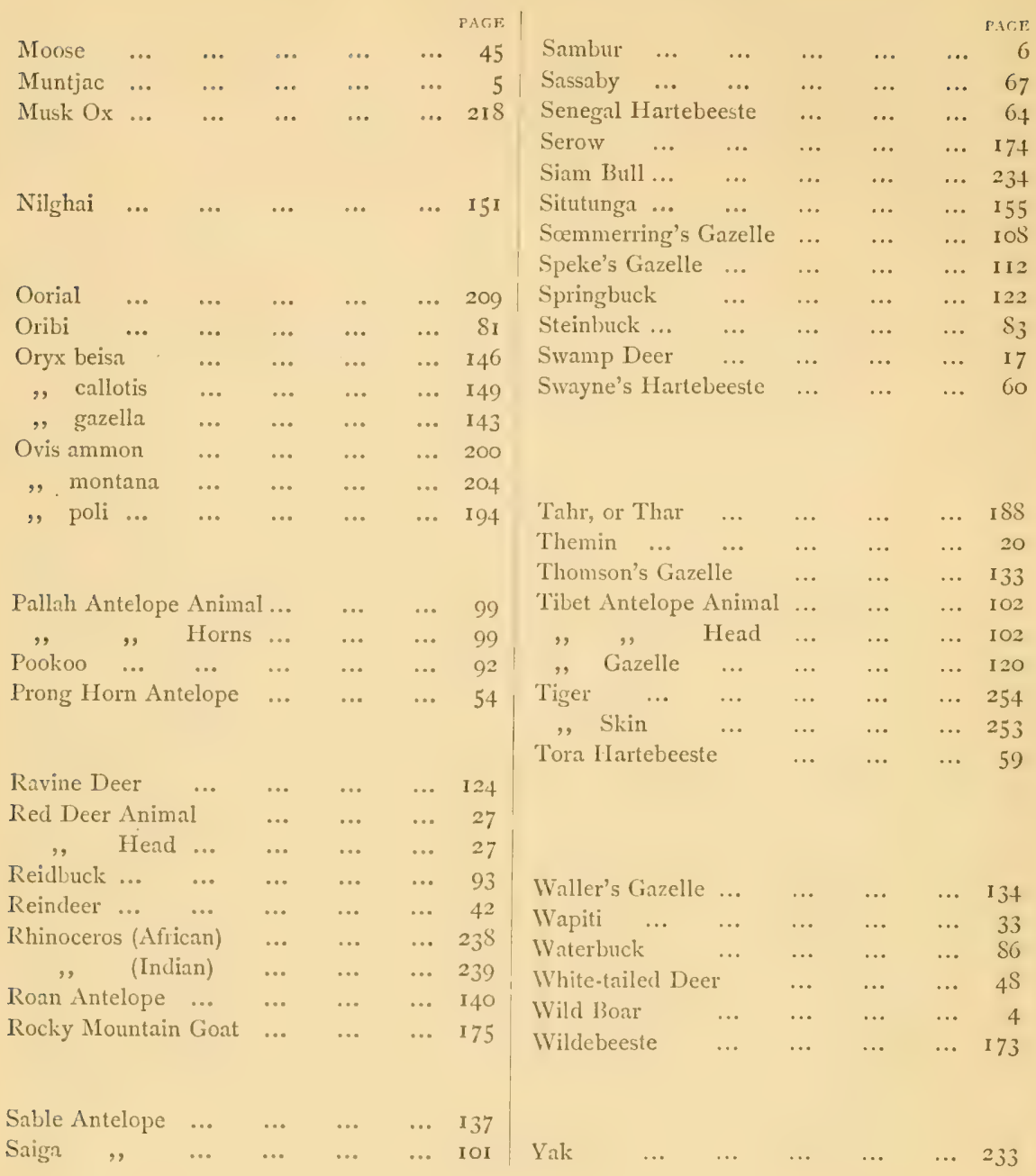




\section{ROWLAND WARD'S BOOK OF HORN MEASUREMENTS, WEIGHT, LENGTH, \&c.,} GREAT GAME OF THE WORLD :

BEING $\Lambda$ RECORD FOR THE USE OE

\section{SPORTSMEN AND NATURALISTS.}

BY ROWLAND WARD, F.Z.S., Author of "The Sportsman's Handbook," ENc.

LONDON: OF THE AUTHOR, 166, PICCADIIIY. One Vol., 4to., Cloth, Special Price, 21/- By Post, 21/6.

\section{ROWLAND WARD \& Co., Limited, NATURALISTS TO THE COURT,}

By Special Appointment to His Royal Highness the Prince of Wales, His Royal Highness the Duke of Edinburgh, and the Courts of Europe,

"THE JUNGLE," I66, PICCADILLY, LONDON, W.

TELEPHONE- 3644 .

Practical and Artistic Taxidermists, Designers of Trophies of Natural History, Preservers and Adapters of all Specimens of Animal Life. Natural Features of Animals adapted in Original Designs for Decorative Purposes and Every-day Uses. Furriers and Plumassiers, and Collectors in Natural History.

NOTICE.-RowLAND WARD, F.Z.S., is the only member left in the profession of the Ward Family, long unrivalled for their accumulated experience and their skill in Practical Taxidermy, especially in its artistic department.

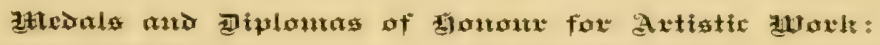

London International Exhibition, x862.

Paris International Exhibition, 1862.

Vienna International Exhibition, 1873.

London International Fisheries, 1883.

Calcutta International Exhibition, $188_{3}-84$.

London International Health Exhibition, 1884.

London Colonial and Indian Exhibition, 1886.

The Anglo-Danish Exhibition, South Kensington, 1888.
The Royal Military Exhibition (Army Medical Department), x8go.

$\Lambda$ the Calcutta International Exhibition, $188_{3}-84$, a Gold Medal and First Class Certificate were awarded to Arthur Crawford, Esq., Bombay Civil Service, for a Collection of the Birds of Western India that were Preserved and Mounted in original design by ROWLAND WARD, F.Z.S. 


\section{THE COLONIAL AND INDIAN EXHIBITION.}

\section{THE JUNGLE.}

"But everything else here is likely to be forgotten in presence of the wonderful jungle scene which Mr. Rowland Ward has constructed. . . This will certainly be the first of the many attractions to which visitors will turn. - They will find themselves in presence of a scene which is likely to keep their gaze for some time. Mr. Ward has made the most of his limited space, into which he has collected the scenery and life which, in reality, is found scattered over an area of many thousand square miles. On the right we have a trophy from Kooch Behar, formed by His Highness the Maharajah, the most prominent feature of which is a tiger hunt. We see a great group in the deep grass jungle. . Adjoining this are trophies designed to represent generally the Fauna and Flora of India, by representative animals and birds, picturesquely grouped in illustration of their life-habits." - Times.

"The visitors . Were lost in admiration of Mr. Rowland Ward's masterly designs, modellings, and general arrangement. The novelty is already known as "the Jungle." . . The deep grass jungle is occupied necessarily by many creatures which would not in their native wilds be found in such close companionship.

The scene is rendered with true tragic power." -Daily Nezus.

"These numerous beasts. Seem to illustrate the Fauna of India in a most vivid manner, and are very artistically prepared and arranged. . The entire trophy has been prepared by Mr. Rowland Ward. This group will unquestionably be one of the leading attractions of an exhibition which is already full of marvellous things." - Morning Post.

"Fitted up with the most perfect completeness-a jungle-the work of Mr. Rowland Ward. . . . The whole scene depicted is so life-like that one is startled by its vivid realism. . . This jungle alone is almost enough to make an exhibition. . Besides, Mr. Rowland Ward has designed and arranged such other scenes in comnection with several Colonial Courts."-Daily Chronicle.

"Mr. Rowland Ward, of Piccadilly, provides what will probably prove the most attractive feature of the exhibition, in the form of a series of picturesque trophies representing India, Ceylon, South Africa, Canada, and Queensland." "Spertsman.

"Perhaps the first place must be accorded to the jungle scene of Mr. Rowland Ward, which stands at the head of the Indian Courts, and which will certainly prove one of the favourite sights of the vast show." Daily lelegraph.

\section{THE SPORTSMAN'S HANDBOOK,}

"Sport, however, it must be bone in mind, is a thing of every climate and of all seasons, and the manual referred to- 'The Sportsman's Handbook,' by Kowland Ward, F.Z.S. - has a little to say of most regions, from the North of Scandinavia to the South of India. All knowledge is apt to come in useful; and even those of us who may never know the delight of facing a charge of the Cape buffalo-under some circumstances among the most dangerous experiences of the sportsman, we are told-may yet find a less rapturuus pleasure in learning how a real sportsman should entertain such a visitor. The next best thing to being able to shoot a lion in a workmanlike fashion is to know how the thing ought to be done, and that is among the items of instruction in this little book."-Daily Neres, Leading Article.

"With this in his portmanteau, no one fond of shooting and collecting need any longer lament his inability to preserve his trophies, since the directions given for skinning and preserving animals of all kinds are extremely clear and simple, and rendered all the more intelligible by the wood engravings by which they are accompanied. Quadrupeds, birds, fishes, reptiles, and insects, are all dealt with in zurns, and directions given not merely for skinning them, but also for mounting them, if desired, a year or two (it may be) after they have been procured." Firld.

"No one has a better right to speak with authority on questions of taxidermy than MIr. Ward, whose skill in the art is well known to everyone interested in the subject. In an introductory chapter he deals with much practical wisdom with the general equipment of a sportsman bent on big game, and gives some valuable particulars as to the places in which in various animals a well-aimed shot will be fatal. . . The middle of the book is occupied with directions as close and full as possible as to the skinning and preparation of various creatures; and, as we have said, no one is better qualified than Mr. Ward to give such directions. After this comes a synoptical guide to the Hunting Grounds of the World. A special feature in the arrangement of the book is found in the reservation of several blank pages at the end for memoranda."-Saturday Revieze.

"Mr. Ward's eminently clear and practical directions and hints come to us particularly well-timed. a thoroughly working little volume, and no sporting collector ought to be without it." - Laud and IVater.

It is

A valuable feature of this book is the directions (with anatomical diagrams) given for vital shots at great and dangerous game. This is original information, and so is the important division of the work that treats of the hunting fields of the world, and what fera. natura can be met with by the sportsman in each locality. . . The book is a valuable and well-written contribution to the literature of the subject of which it treats, and it will be welcomed by the gentleman sportsman, for whom it has been prepared, quite as much as by the aspirant in the arts of taxidermy, to whom some of its pages will convey a sort of revelation." - Corntry Gentleman.

"The text is made as clear as possible, but wherever the explanation can be assisted by an illustration one is introduced,"-Daily Chronicle.

"The name of Ward, father and sons, has acquired a world-wicle reputation. collector."-Calcutta Englishman.

"The traveller and the trader will find it a useful companion."-Cape Argus.

I Vol., Crown 8vo. Sirth Edition, with numerous additional illustrations. Bound in Crocodile Leather. Price $3^{s .6 d .}$ By Post, 3s. 9d.

Lั

OF THE AUTHOR, "THE JUNGLE," I66, PICCADILLY, SIMPKIN, MARSHALL \& Co., 4, STATIONERS' HALL COURT, AND OF ALL BOOKSELLERS. 







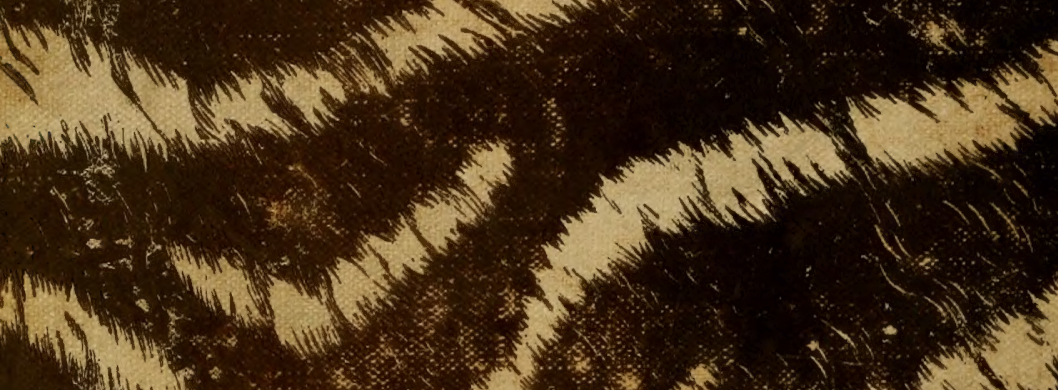
34 ?

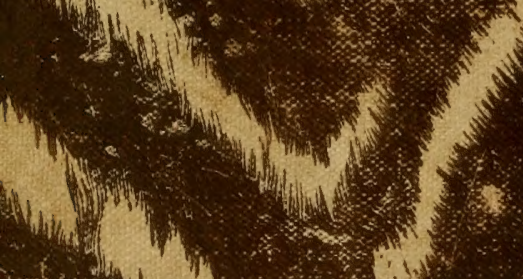

for. s

wh $x_{1} \times$ $x^{2} x^{2} y^{2}+y^{2}$

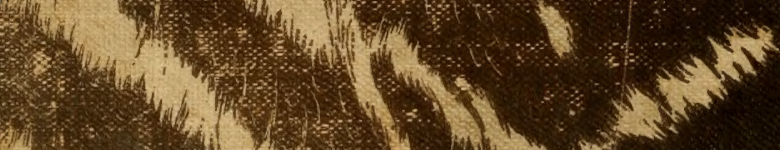
(x)

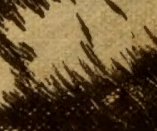

